AUS DEM INSTITUT FÜR ANATOMIE UND EMBRYOLOGIE

(PROF. DR. MED. C. VIEBAHN)

DER MEDIZINISCHEN FAKULTÄT DER UNIVERSITÄT GÖTTINGEN

\title{
Skorbutnachweis an frühmittelalterlichen und frühneuzeitlichen archäologischen Skeletfunden unter Anwendung eines kombinierten makroskopischen und lichtmikroskopischen Untersuchungsverfahrens
}

\author{
INAUGURAL-DISSERTATION \\ zur Erlangung des Doktorgrades \\ der Medizinischen Fakultät der \\ Georg-August-Universität zu Göttingen \\ vorgelegt von \\ Johanna Fischer-Gödde, geb. Gödde
}

aus Duisburg

Göttingen 2015 
Dekan:

I. Berichterstatter:

II. Berichterstatter/in:

III. Berichterstatter/in:

Tag der mündlichen Prüfung: $\quad$ 11. 10. 2017
Prof. Dr. rer. nat. H. K. Kroemer

Prof. Dr. phil. nat. Dr. med. M. Schultz

Prof. Dr. C. Stadelmann-Nessler

Prof. Dr. Margarete Schön 


\section{Inhaltsverzeichnis}

1. Einleitung 5

$\begin{array}{ll}1.1 \text { Geschichte des Skorbuts } & 6\end{array}$

1.2 Das Krankheitsbild des Skorbuts 11

$\begin{array}{ll}1.3 \text { Laborbefunde bei Skorbut } & 18\end{array}$

1.4 Biochemie und Physiologie des Vitamin C 21

$\begin{array}{ll}\text { a) Eigenschaften } & 21\end{array}$

b) Funktion $\quad 22$

c) Metabolismus/Aufnahme/Sekretion $\quad 27$

1.5 Pathophysiologie des Skorbuts 31

1.6 Therapie des Skorbuts 33

1.7 Empfehlungen zur täglichen Vitamin-C-Aufnahme 33

1.8 Vorkommen von Vitamin C 36

1.9 Nachweis von Skorbut an historischen Skeleten 39

1.10 Differentialdiagnosen 41

1.11 Untersuchungsmethoden 42

2. Material und Methoden $\quad 44$

2.1 Material 44

$2.1 \quad$ a) Das Gräberfeld der frühmittelalterlichen Siedlung von Barbing-Kreuzhof (Oberpfalz) 44

b) Das Gräberfeld Bácsalmás-Óalmás aus dem

16.-18. Jahrhundert (Ungarn) 46

c) Das Awaren-Gräberfeld von Sükösd-Ságod aus dem 7. Jahrhundert (Ungarn) 46

d) Der Schädel eines Kindes aus Kiszombor aus dem 11.-12. Jahrhundert

$\begin{array}{ll}\text { (Ungarn) } & 47\end{array}$

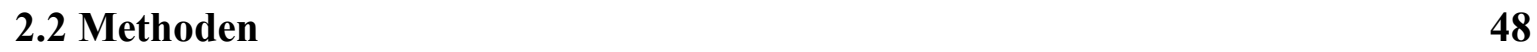

$\begin{array}{lll}2.2 & \text { a) Vorarbeit } & 48\end{array}$

b) Dokumentation des Bestands 48

c) Fotografie $\quad 48$

d) Altersbestimmung 49

e) Geschlechtsbestimmung 49

f) Makroskopische Untersuchung 49 
g) Endoskopie $\quad 50$

h) Radiologische Untersuchung 50

i) Lichtmikroskopie $\quad 51$

- Anfertigung der Präparate für die Lichtmikroskopie 53

- Untersuchung am Lichtmikroskop $\quad 54$

- Befundung der lichtmikroskopischen Präparate 54

j) Diagnosestellung 63

3. Ergebnisse $\quad 65$

$\begin{array}{ll}\text { Einzelbefunde } & 65\end{array}$

3.1 Untersuchungsbefunde der einzelnen Individuen des Gräberfelds Barbing-Kreuzhof 65

3.2 Untersuchungsbefunde der einzelnen Individuen des Gräberfelds Bácsalmás-Óalmás 109

3.3 Untersuchungsbefunde des Individuums Kiszombor $\quad 121$

3.4 Untersuchungsbefunde der einzelnen Individuen des Gräberfelds Sükösd-Ságod 123

$\begin{array}{ll}\text { Bildtafeln } & 127\end{array}$

$\begin{array}{ll}\text { Häufigkeit der unterschiedlichen Diagnosen } & 148\end{array}$

3.5 Häufigkeit der unterschiedlichen Diagnosen bei den Skeleten des Gräberfelds Barbing-Kreuzhof 148

3.6 Häufigkeit der unterschiedlichen Diagnosen bei den Skeleten aus Ungarn 151

3.7 Häufigkeit der unterschiedlichen Diagnosen bei allen untersuchten Skeleten 152

Häufigkeit und Verteilung der einzelnen Kardinalmerkmale für Skorbut $\quad 154$

3.8 Poröse Auflagerungen am äußeren Schädeldach (Kardinalmerkmal 1) 154

3.9 Poröse Auflagerungen am Orbitadach/Cribra orbitalia (Kardinalmerkmal 2) 158

3.10 Poröse Auflagerungen am äußeren Gesichtsschädel (Kardinalmerkmal 3) 163

3.11 Poröse Auflagerungen am Alveolarknochen (Kardinalmerkmal 4) 168

3.12 Poröse Auflagerungen an Langknochen (Kardinalmerkmal 5) 173

3.13 Poröse Auflagerungen am Schulterblatt (Kardinalmerkmal 6) 179

3.14 Poröse Auflagerungen am Os ilii (Kardinalmerkmal 7) 184

Häufigkeit und Verteilung der einzelnen Nebenmerkmale für Skorbut 189

3.15 Poröse Oberfläche bzw. Auflagerungen am harten Gaumen/Stomatitis

$\begin{array}{ll}\text { (Nebenmerkmal 1) } & 189\end{array}$

3.16 Poröse Auskleidungen der Alveolen (Nebenmerkmal 2) 195

3.17 Symmetrische poröse Auflagerungen in den Impressiones digitatae oder flächig am inneren Schädeldach (Nebenmerkmal 3) 200 
3.18 Poröse Oberflächen an Langknochen (Nebenmerkmal 4)

3.19 Poröse Außenfläche der Ala major ossis sphenoidalis/ der Squama temporalis (Nebenmerkmal 5)

Zusammenfassung: Häufigkeit der unterschiedlichen für Skorbut kennzeichnenden Merkmale bei den untersuchten Skeleten 215

3.20 Zusammenfassung: Kardinalmerkmale für Skorbut 215

3.21 Zusammenfassung: Nebenmerkmale für Skorbut

4. Diskussion

4.1 Merkmale für Skorbut am historischen Skelet 228

4.2 Skorbutische Läsionen am Schädel 230

4.3 Skorbutische Läsionen am Postcranium 239

4.4 Bedeutung der radiologischen Untersuchung 248

$\begin{array}{ll}4.5 \text { Differentialdiagnosen } & 249\end{array}$

a) Wachstum 249

b) Anämie 250

c) Meningeale Reizung 252

d) Rachitis 252

e) Lepra 255

f) Unspezifische Entzündungen: Periostitis, Ostitis und Osteomyelitis 257

$\begin{array}{ll}\text { g) Syphilis congenita } & 258\end{array}$

h) Infantile Corticale Hyperostose (Caffey's disease) 259

i) Hypertrophe Osteoarthropathie/Osteoperiostitis ossificans toxica/ $\begin{array}{ll}\text { Pierre-Marie-Bamberger-Syndrom } & 261\end{array}$

$\begin{array}{ll}4.6 \text { Skorbut heute } & 263\end{array}$

$\begin{array}{ll}\text { 5. Zusammenfassung } & 268\end{array}$

6. Literaturverzeichnis 272

\section{Anhang}

I. Befundbogen Langknochen

II. Befundbogen Schädel 


\section{Einleitung}

Bei der vorliegenden Arbeit handelt es sich um eine paläopathologische Untersuchung. Die Paläopathlogie ist die Lehre der Krankheiten von Menschen vergangener Zeiten. Das Untersuchungsmaterial sind menschliche Überreste, die aus archäologischen Grabungen, von alten Friedhöfen, die beispielsweise im Rahmen von Bauarbeiten entdeckt wurden, oder auch aus anatomischen Sammlungen in Universitäten und Museen stammen. Zumeist handelt es sich um Knochen, seltener stehen auch mumifizierte Weichteile zur Befundung zur Verfügung. In den letzten Jahren wurden die Untersuchungsmethoden der Paläopathologie weiterentwickelt, insbesondere der lichtmikroskopischen Untersuchung kommt dabei zunehmende Bedeutung zu (CARLI-THIELE 1996, KREUTZ 1997, SCHULTZ 1986, 1987, 1988a, 1988b, 1990, 1993, 2001, 2003, 2011, TEMPLIN 1993).

Im Rahmen der vorliegenden Arbeit werden die Ergebnisse der Untersuchung historischer Skelete unterschiedlicher Epochen und Herkunft auf Skorbut (chronischen schweren Vitamin-C-Mangel) präsentiert.

Dabei werden Diagnosekriterien für die paläopathologische Diagnostik von Skorbut vorgestellt.

Des Weiteren wird die Relevanz dieses Themas für die heutige Medizin diskutiert. Dabei sollen rezente Fälle vorgestellt und die Umstände besprochen werden, in denen es auch im 21. Jahrhundert zu Skorbuterkrankungen kommt.

In diesem Kapitel erfolgt zunächst ein Überblick über die Krankheit Skorbut und ihre Geschichte. Die Eigenschaften, der Metabolismus und die biologische Funktion des Vitamin C werden zusammengefasst und die Pathophysiologie des Skorbuts erläutert. Außerdem wird ein Überblick über den Vitamin-C-Bedarf des Menschen sowie die Deckungsmöglichkeiten durch verschiedene Nahrungsmittel gegeben.

Zuletzt werden Diagnosekriterien verschiedener Autoren vorgestellt, deren Anwendbarkeit auf historische Skelete es zu untersuchen gilt, sowie ein kurzer Überblick über zu erwartende Differentialdiagnosen gegeben. 


\subsection{Geschichte des Skorbuts}

Skorbut, die durch Vitamin-C-Mangel hervorgerufene Erkrankung, ist seit Jahrhunderten bekannt.

Schon in ägyptischen Papyri um 1500 vor Christus, bei Hippokrates um 400 vor Christus und bei Jacques de Vitry um 1210 findet Skorbut Erwähnung (AUFDERHEIDE und RODRÍGUEZ-MARTÍN 1998, HODGES 1980, WEISE PRINZO 1999).

Im Mittelalter scheint Skorbut vor allem in Nordeuropa ein großes gesundheitliches Problem dargestellt zu haben (AUFDERHEIDE und RODRÍGUEZ-MARTÍN 1998, WEISE PRINZO 1999).

Es gibt Berichte, dass Skorbut in den Niederlanden bei der entlang der Nordseeküste lebenden Bevölkerung in den Jahrhunderten vor den großen Seefahrten des 17. und 18. Jahrhunderts ein wiederkehrendes Problem war, das regelmäßig am Ende des Winters oder zu Frühlingsanfang oder auch nach Belagerungen oder Naturkatastrophen endemisch auftrat (MAAT 1982, MAAT 2004). Eine ganz neue Bedeutung bekam die Krankheit jedoch durch die großen Seefahrten der Europäer in die ganze Welt ab dem Ende des 16. Jahrhunderts. Die Seeleute, die teilweise viele Monate unterwegs und damit weitgehend abgeschnitten von frischer Nahrung waren, erkrankten in großer Zahl an Skorbut, so dass man bald von einer Seemannskrankheit sprach (AUFDERHEIDE und RODRÍGUEZ-MARTÍN 1998, HIRSCHMANN und RAUGI 1999, MAAT 1982, MAAT 2004). Einige berühmte Beispiele sind der portugiesische Seefahrer Vasco da Gama, der bei seiner Umsegelung des Kaps der Guten Hoffnung um das Jahr 1500100 von 166 Mann Besatzung an den Skorbut verlor, oder Lord Anson, der um 1740 mit sechs Schiffen und knapp 1000 Mann Besatzung nach Südamerika segelte und nach neun Monaten durch den Verlust von zwei Dritteln der Mannschaft gezwungen war, die Hälfte seiner Schiffe aufzugeben (HIRSCHMANN und RAUGI 1999, WEISE PRINZO 1999, ZELLWEGER und ADOLPH 1954).

Dabei wurde schon im auslaufenden 16. Jahrhundert ein Zusammenhang zwischen dem Fehlen frischer Nahrung und dem Auftreten von Skorbut erkannt. So schrieb der berühmte Arzt VAN FOREEST bereits 1558 ,scurvy tended to prevail during hot weather, when fresh water was lacking, in men whose diet consisted of salted meat, rancid bacon, smelly fish, musty hard tack, onions and old beer“ (FORESTUS 1590, zitiert nach: MAAT 2004, S. 78) und empfahl einen Skorbut-Trunk aus Skorbutkraut (Echtes Löffelkraut, Cochlearia officinalis) und Bachbunge (Veronica beccabunga). Und schon 1554 wurden durch Dodonaeus erstmals Apfelsinen als Therapie des Skorbuts vorgeschlagen (MAAT 2004). 
Im Oxford Dictionary fand sich bereits 1565 das Wort scurvy (AUFDERHEIDE und RODRÍGUEZ-MARTÍN 1998). Die Bezeichnung „Skorbut“, englisch scurvy, französisch scorbut, latein scorbutus, deutsch früher „Scharbock“, kommt vermutlich vom niederländischen schorbuk, das sich zusammensetzt aus schoren, „brechen“, und buk, „Bauch“ (GRIMM und GRIMM 1905, KLUGE 1989, HIRSCHMANN und RAUGI 1999). Übertragen bedeutet das „Krankheit, bei welcher der Bauch aufbricht“. Dies könnte als Anspielung auf das häufiger beschriebene Phänomen des Aufbrechens alter Wunden interpretiert werden. Allerdings wird auch ein Zusammenhang mit dem alten englischen Adjektiv „scurvy“ gesehen. Dies bedeutet „unehrenwert“ oder „gemein“, aber auch „schuppig“ (HIRSCHMANN und RAUGI 1999).

Skorbut blieb insbesondere unter Seefahrern noch Jahrhunderte lang ein ungelöstes Problem, vor allem, weil die damaligen Methoden zur Konservierung der Lebensmittel, wie etwa das Destillieren, Einkochen und Pökeln, sowie die Lagerungsdauer das - zu diesem Zeitpunkt natürlich noch unbekannte - Vitamin $\mathrm{C}$ unvermeidlich zerstören (AUFDERHEIDE und RODRÍGUEZ- MARTÍN 1998, HIRSCHMANN und RAUGI 1999, MAAT 2004, TEMPLIN 1993).

Die europäischen Eroberer, die in Amerika landeten, litten häufig unter Skorbut. Jacques Cartier traf auf nordamerikanische Ureinwohner, die einen aus Blättern und Rinde des Lebensbaums (Thuja occidentalis) gebrauten Saft als hilfreiche Medizin - interessanterweise sowohl gegen Skorbut als auch gegen die venerische Syphilis der Europäer - einsetzten (HODGES 1980, ORTNER und PUTSCHAR 1981 $)$. Dies lässt darauf schließen, dass auch auf dem amerikanischen Kontinent Skorbut schon seit Jahrhunderten eine Rolle spielte.

In den 1740ern führte Lind, ein britischer Marinearzt, das erste belegte Experiment zur Therapie von Skorbut an seiner Crew durch. Im Rahmen dessen teilte er an Skorbut erkrankte Männer in Paare ein. Eines bekam weiterhin die Standardverpflegung, eines zusätzlich Apfelsinen- und Zitronensaft, das letzte Standardverpflegung und Apfelwein. Das zweite Paar erfuhr eine prompte Besserung, das mit Apfelwein versorgte Paar eine Milderung der Symptome, während die lediglich Schiffskost konsumierenden beiden Männer weiterhin schwer erkrankt waren. Allerdings erkannte Lind nicht das Vorliegen eines Mangels als Krankheitsursache, sondern vermutete den Grund im kalten und feuchten Klima (HIRSCHMANN und RAUGI 1999, WEISE PRINZO 1999).

Erst vierzig Jahre später wurde auf Betreiben von Sir Gilbert Blane, einem schottischen Arzt, Zitronensaft bei der britischen Marine als Skorbutpräventionsmittel eingeführt

\section{${ }^{1}$ S. 272}


(AUFDERHEIDE und RODRÍGUEZ-MARTÍN 1998, HIRSCHMANN und RAUGI 1999, MAAT 2004).

Der berühmte Kapitän Cook setzte neben einer maximalen Fahrzeit von 17 Wochen auf große Mengen an Obst und Gemüse, darunter Sauerkraut, um die gefürchtete Krankheit bei seiner Mannschaft zu verhindern (HIRSCHMANN und RAUGI 1999).

Auch die Einführung der Kartoffel in den europäischen Speiseplan half, wenn auch eher durch die Haltbarkeit als die Höhe des Vitamin-C-Gehalts bedingt, das Problem Skorbut zu bekämpfen. Dies wurde deutlich bei der Großen Kartoffelfäulnis in Irland 1845-48, bei der Skorbut um sich griff (AUFDERHEIDE und RODRÍGUEZ-MARTÍN 1998, HIRSCHMANN und RAUGI 1999, WEISE PRINZO 1999).

Während des Goldrauschs in Kalifornien 1848-1850, als sich etwa 100000 Goldsucher in die Rocky Mountains aufmachten, starben ca. 10000 Menschen an Skorbut (HIRSCHMANN und RAUGI 1999, WEISE PRINZO 1999).

Die französische Armee verlor 1854 im Krimkrieg 23000 Männer durch Skorbut (AUFDERHEIDE und RODRÍGUEZ-MARTÍN 1998, HIRSCHMANN und RAUGI 1999).

Im Jahre 1883 veröffentlichte der britische Arzt Barlow eine Studie, in der er zu dem Schluss kam, dass es sich bei vielen Fällen der so genannten akuten Rachitis (so beschrieben unter anderen durch den deutschen Arzt Möller) in erster Linie um infantilen Skorbut handelte (BARLOW 1883). Daher findet sich auch in der Literatur häufig die Bezeichnung „MöllerBarlow'sche Krankheit“ für den infantilen Skorbut. Barlow gelang es, einige der von ihm behandelten Kinder zu heilen, er verschrieb Orangen-/Zitronensaft, frisches Gemüse und Säfte aus frischem Fleisch sowie lokale Kompressen, darüber hinaus empfahl er den Aufenthalt im Freien (BARLOW 1883²). In den Fällen, in denen jede Hilfe zu spät kam, führte er Autopsien durch, deren Ergebnisse im Unterkapitel 1.9 Nachweis von Skorbut an historischen Skeleten und in der Diskussion (Kapitel 4) beschrieben werden.

Zwar sah BARLOW schon 1883 den infantilen Skorbut als unabhängig von oder neben der Rachitis auftretende eigenständige Erkrankung, dennoch wurde in den folgenden Jahrzehnten immer wieder eine rachitische Grunderkrankung für infantilen Skorbut vermutet. Der deutsche Pathologe und Bakteriologe Fraenkel widmete sich Anfang des 20. Jahrhunderts ausführlich dem Studium der Möller-Barlow'schen Krankheit (FRAENKEL 1908). Er ergänzte die vorangegangenen Untersuchungen durch ausführliche pathologisch-anatomische, vor allem aber durch histologische und röntgenologische Untersuchungen, deren Ergebnisse im Unterkapitel 1.9 Nachweis von Skorbut an historischen Skeleten und in der Diskussion

\footnotetext{
${ }^{2}$ S. $204 f$
} 
(Kapitel 4) beschrieben werden, seine röntgenologischen Befunde stellen die Grundlage der im Kapitel 2.2 h) Radiologische Untersuchung vorgestellten radiologischen Untersuchungen der vorliegenden Arbeit dar. FRAENKEL (1908) erklärte im Sinne BARLOWs (1883), dass die Möller-Barlow'sche Krankheit als eine Erkrankung sui generis zu betrachten sei, die auch ohne jegliche - insbesondere rachitische - Grunderkrankung auftreten könne und dem Skorbut des Erwachsenen entspreche (FRAENKEL $1908^{3}$ ).

Man ging zunehmend davon aus, dass dem Skorbut ein Mangelzustand zugrunde lag. Immer wieder versuchten Forscher, den in der Ernährung fehlenden Faktor zu finden, der die Erkrankung auslöste. Besonders weit ging der englische Arzt William Stark, der sich im 18. Jahrhundert selbst verschiedenen extremen Diäten unterzog. Er starb im Verlauf seines Experiments - vermutlich an Skorbut (WEISE PRINZO 1999).

Um die Jahrhundertwende des 19./20. Jahrhunderts führten die Ärzte den (infantilen) Skorbut auf eine einseitige Ernährung, insbesondere mit Muttermilchersatzprodukten auf Basis von Kuhmilchpulver, zurück, ohne den entscheidenden fehlenden Inhaltsstoff - das Vitamin C benennen zu können. Daher traten Skorbutfälle zu dieser Zeit keinesfalls nur in armen Familien mit mangelhaftem Zugang zu Nahrungsmitteln auf, sondern auch oder gerade in vergleichsweise gut situiertem Umfeld (BARLOW 1883, FRAENKEL 1908 HIRSCHMANN und RAUGI 1999, LEWIS 2002, WEISE PRINZO 1999). Außerdem war es im 18. und 19. Jahrhundert bei wohlhabenden Frauen üblich, eine große Zahl Kinder in sehr kurzen Abständen (begünstigt durch den Verzicht auf das Stillen) zu gebären, was zu Mangelerscheinungen bei den Müttern führen kann.

Eine weitere Ursache für Skorbut wurde in Grunderkrankungen gesehen, welche die Nahrungsaufnahme bzw. -resorption beeinträchtigen (FRAENKEL 19085 ).

$\mathrm{Zu}$ Beginn des 20. Jahrhunderts suchte der Biochemiker Zilva intensiviert nach dem antiskorbutischen Faktor in Nahrungsmitteln; er erkannte, dass dieser wasserlöslich, hitzeempfindlich und stark reagibel bei Kontakt mit Sauerstoff und Metallen wie Eisen oder Kupfer sein musste (HODGES 1980). Er gab der gesuchten Substanz den Namen „Vitamin C“. Das Wort „Vitamin“ geht auf den polnischen Wissenschaftler Funk zurück, der mit der Kurzform für „Vitalamine“ die fälschlicherweise für Amine gehaltenen Substanzen bezeichnete, deren Fehlen Rachitis, Pellagra und Beriberi verursachte (HIRSCHMANN und RAUGI 1999).

\footnotetext{
${ }^{3}$ S. 3f, S. 31

${ }^{4}$ BARLOW 1883: S. 189, FRAENKEL 1908: S. 8, S. 35f

${ }^{5}$ S. 36
} 
Erst 1928 gelang die chemische Isolation der Ascorbinsäure parallel durch den amerikanischen Biochemiker King und den ungarischen Physiologen Szent-Györgyi (HODGES 1980, MAAT 2004). Szent-Györgyi, der sich später auch als Politiker und Widerstandskämpfer gegen die Nationalsozialisten einen Namen machte, wurde dafür 1937 mit dem Nobelpreis für Medizin und Physiologie ausgezeichnet (BÄSSLER et al. 2002). Der Name „Ascorbinsäure“ wurde hergeleitet vom englischen ,a-scorb[ut]ic acid“, bedeutet also „antiskorbutische Säure“ (HIRSCHMANN und RAUGI 1999).

Im Jahr 1932 wurde die therapeutische Potenz der Ascorbinsäure bestätigt (MAAT 2004). Dem polnischen Chemiker Reichstein und seiner Arbeitsgruppe gelang 1933 die Synthese von Ascorbinsäure (HOGDES 1980). Unabhängig von ihnen entwickelten auch die britischen Chemiker Haworth und Hirst ein Syntheseverfahren, Haworth erhielt dafür 1937 den Nobelpreis für Chemie (Bässler et al. 2002).

Dennoch wird auch im 20. Jahrhundert immer wieder über das Auftreten von Skorbut berichtet.

So starb das Team von Robert Falcon Scott, das 1912 zum Südpol segelte, teilweise an Skorbut, obwohl die Prävention dieser Erkrankung schon seit über hundert Jahren bekannt war (HODGES 1980).

Im Ersten Weltkrieg trat Skorbut immer wieder endemisch auf und wurde Gegenstand einer großen pathologischen Studie durch ASCHOFF und KOCH (1919).

Während der Belagerung von Leningrad 1941/42 starben Menschen an Unterernährung und Skorbut.

Bei im Zweiten Weltkrieg von Japanern gefangen gehaltenen Engländern in Hongkong trat ebenfalls Skorbut auf.

Von 1945 bis 1965 trat gehäuft infantiler Skorbut in Kanada auf, wo arme Familien ihren Säuglingen die billigere Kondensmilch an Stelle von Frischmilch oder Säuglingsnahrung gaben (LEWIS 2002).

1950 wurden in den USA bei 5\% aller Autopsien im ersten und zweiten Lebensjahr Feinzeichen für Skorbut gefunden; klinisch wurde nur ein Zehntel dieser Fälle erkannt (KÜBLER 1965).

Heutzutage spielt der „epidemische“ Skorbut in den Industrieländern keine Rolle mehr. Allerdings treten immer wieder Fälle von sporadischem Skorbut auf. Häufig betroffen sind ältere, meist ärmere, alleinstehende Männer, die sich ihre Mahlzeiten überwiegend aus Konserven zubereiten; dieses Phänomen wird als „bachelor scurvy“ bezeichnet (ZELLWEGER und ADOLPH 1954). 
Weitere Risikofaktoren sind Alkohol- und Drogenabhängigkeit.

Auch durch sehr einseitige Diäten, z. B. bei Magengeschwüren, ausgeprägten Nahrungsmittelallergien oder aus weltanschaulichen Gründen, kann heutzutage ein Vitamin-C-Mangel entstehen (ZELLWEGER und ADOLPH 1954, BÄSSLER et al. 2002).

Natürlich muss auch bei chronischen Durchfallerkrankungen und Resorptionsstörungen auf den Vitamin-C-Haushalt geachtet werden.

Ein wesentliches Problem bei rezenten Skorbutfällen ist, dass die behandelnden Ärzte die Erkrankung nicht erkennen, da sie wegen des seltenen Auftretens überhaupt nicht dafür sensibilisiert sind.

Anders als in den Industrieländern spielt Skorbut in weniger entwickelten Ländern und vor allem in Krisengebieten nach wie vor eine Rolle. So findet sich auf der Homepage der World Health Organization eine Anleitung zur Prävention von Skorbut in Katastrophenfällen, die u.a. konkrete Anweisungen zur Gestaltung und Versorgung von Flüchtlingslagern gibt (WEISE PRINZO 1999).

\subsection{Das Krankheitsbild des Skorbuts}

Skorbut entwickelt sich latent über Monate, lässt sich zwar durch Laboruntersuchungen und manchmal auch radiologisch bereits nachweisen, führt aber nur zu unspezifischen Symptomen wie Abgeschlagenheit, Muskelschmerzen oder Verdauungsstörungen (BÄSSLER et al. 2002, HODGES et al. 1969). Es wird eine ausgeprägte Antriebslosigkeit und Müdigkeit in der frühen Krankheitsphase beschrieben (HIRSCHMANN und RAUGI 1999). Die typischen Symptome, insbesondere die Blutungsneigung, entwickeln sich dann recht plötzlich innerhalb weniger Tage (JAFFE $1972^{6}$ ).

Eine Geschlechtsbevorzugung gibt es nicht (FRAENKEL 19087). Erwachsene und Kinder können gleichermaßen an Skorbut erkranken, wobei erste Symptome nach mehreren Monaten Vitamin-C-Mangels auftreten. Vermutlich kann dies bei kleinen Kindern aufgrund der hohen Kollagensyntheserate in der Wachstumsphase auch schneller der Fall sein, nämlich schon nach zwei bis vier Monaten (JAFFE $1972^{8}$ ). Auch bei den Seefahrern des 18. Jahrhunderts werden erste Symptome schon nach zwei Monaten Fahrt beschrieben (MAAT 2004), wobei man vermuten kann, dass die wiederholten Seefahrten die Reserven stark geschwächt haben (MAAT 1982). Außerdem ist die Zeitspanne bis zur Entwicklung vermutlich abhängig von

\footnotetext{
${ }^{6}$ S. 449

${ }^{7}$ S. 8

${ }^{8}$ S. 449
} 
der Menge des zugeführten Vitamin C; je geringer sie ist, desto schneller kommt es zum manifesten Skorbut (HODGES et al. 1969, LANG 1979).

Besonders anfällig für Skorbut scheinen Säuglinge und Kleinkinder zwischen ca. sechs Monaten und dem vollendeten zweiten Lebensjahr zu sein; es gibt nur wenige Berichte über jüngere Kinder, die skorbutische Symptome zeigen (BARLOW 1883, BRICKLEY und IVES 2006, FRAENKEL 1908, JAFFE 1972, KÜBLER 1965, ORTNER 2003, ORTNER und PUTSCHAR 19819). JAFFE (1972) vermutet in diesen Fällen einen mütterlichen Vitamin-CMangel in der Schwangerschaft, der zu unzureichender Vitamin-Speicherung beim Fetus führt (JAFFE 1972 ${ }^{10}$ ). Die Gründe für das gehäufte Auftreten in dieser Altersgruppe liegen zum einen in der Umstellung der Ernährung von Muttermilch auf feste Kost (LEWIS 2002, SCHULTZ und SCHMIDT-SCHULTZ 2013), zum anderen im wachstumsbedingten hohen Kollagenumsatz. Ein weiteres Risiko für die Entwicklung eines infantilen Skorbuts kann eine exzessive Vitamin-C-Zufuhr in der Schwangerschaft sein, die durch eine Steigerung des Metabolismus zu einem relativen Vitamin-C-Mangel beim Neugeborenen führt, auch wenn normale Mengen an Ascorbinsäure zugeführt werden. Ähnliches kann auch bei Erwachsenen auftreten, wenn eine sehr Vitamin-C-reiche Kost plötzlich durch eine normale Dosierung ersetzt wird (WEISE PRINZO 1999).

Gestillte Kinder scheinen einen Schutz vor Skorbut zu genießen. Es wurde festgestellt, dass der Vitamin-C-Gehalt der Muttermilch oft deutlich höher ist als der Vitamin-C-Plasmaspiegel der Mutter. Es scheint also zumindest eine Konzentration von Vitamin C in der Muttermilch zugunsten des Kindes zu geben, es gibt sogar Vermutungen, dass die mütterliche Brust möglicherweise doch im begrenzten Maße dazu in der Lage ist, Vitamin $\mathrm{C}$ zu synthetisieren (WEISE PRINZO 1999, siehe auch Kapitel 1.4 Biochemie und Physiologie des Vitamin C,

\subsection{Empfehlungen zur täglichen Vitamin-C-Aufnahme und 1.8 Vorkommen von Vitamin C).}

Besonders häufig werden Symptome im Winter und Frühjahr beschrieben, zu Zeiten, wo frisches Obst und Gemüse verhältnismäßig schlecht zugänglich sind (FRAENKEL $1908^{11}$, MAAT 1982, MAAT 2004), sie können sich aber - abhängig von der Ernährung - im gesamten Jahr manifestieren.

An Skorbut erkrankte Personen werden als sehr blass beschrieben (FRAENKEL 1908, JAFFE $1972^{12}$, VILTER et al. 1946), sie können kachektisch, jedoch auch augenscheinlich

\footnotetext{
${ }^{9}$ BARLOW 1883: S. 189, FRAENKEL 1908: S. 7, JAFFE 1972: S.449

ORTNER/PUTSCHAR 1981: S. 270

${ }^{10}$ S. 449

${ }^{11}$ S. 36

${ }^{12}$ FRAENKEL 1908: S. 9, S. 13, JAFFE 1972: S. 450, S. 454
} 
wohlgenährt sein. Die Muskulatur ist meist geschwächt und atrophiert (BARLOW 1883 ${ }^{13}$ ). Die Erkrankten neigen zum Schwitzen, vor allem am Kopf (BARLOW $1883^{14}$ ).

Die Patienten sind extrem berührungsempfindlich und insbesondere Kinder scheinen unter ständigen starken Schmerzen zu leiden (BARLOW 1883, FRAENKEL $1908^{15}$ ).

Klinisch fallen Schwellungen der Extremitäten auf, vor allem der unteren. Sie sind gespannt und schmerzhaft, weisen aber in der Regel keine Entzündungszeichen wie Überwärmung und Rötung auf (BARLOW 1883, FRAENKEL 1908 ${ }^{16}$, HIRSCHMANN und RAUGI 1999, HEYMANN 2007, VILTER et al. 1946). Diese Schwellungen sind durch subperiostale Blutungen bedingt, darüber hinaus werden Ödeme beschrieben, markanterweise stärker am Oberschenkel als am Unterschenkel und im Knöchelbereich (BARLOW 1883, JAFFE 1972, KÜBLER $1965^{17}$ ). Gelegentlich wird über Fieberschübe berichtet, die während der Entstehung neuer subperiostaler Blutungen auftreten (BARLOW 1883, FRAENKEL $1908^{18}$, KÜBLER 1965, HEYMANN 2007).

Es wird auch eine Verbreiterung der Metaphysenplatten durch organisierte Hämatome an den Handgelenken, Knien und Fußgelenken von kleinen Kindern beschrieben (AUFDERHEIDE und RODRÍGUEZ-MARTÍN 1998).

Darüber hinaus kann es schon bei minimalen Traumata zu sogenannten Infraktionen kommen. Dies sind auf die Corticalis beschränkte Rupturen des Knochens (AUFDERHEIDE und RODRÍGUEZ-MARTÍN 1998, FRAENKEL 1908, JAFFE $1972^{19}$, MAAT 1982, MAAT 2004).

Die Schmerzen der Extremitäten bringen die Patienten dazu, sich so wenig wie möglich zu bewegen, sie verfallen in eine so genannte Pseudo-Paralyse, dabei liegen Kinder meist auf dem Rücken, die Beine leicht anteflektiert und außenrotiert, die Arme oft leicht abgespreizt. Sie neigen dazu, zu weinen, sobald sich jemand nähert, aus Angst vor Berührung (BARLOW 1883, FRAENKEL 1908, JAFFE 1972 20). Bei einem Betasten der Femurepikondylen kommt es aus nahezu bewegungsloser Schonhaltung heraus zu einer heftigen Schmerzreaktion, die als „Hampelmannphänomen“ bezeichnet wird (KÜBLER 1965).

\footnotetext{
${ }^{13}$ S. 169

${ }^{14}$ S. 170

${ }^{15}$ BARLOW 1883: S. 166, FRAENKEL 1908: S. 9, S. 14

${ }^{16}$ BARLOW 1883: S. 166, FRAENKEL 1908: S. 10

${ }^{17}$ BARLOW 1883: S. 166, JAFFE 1972: S. 452, S. 454

${ }^{18}$ BARLOW 1883: S. 171, FRAENKEL 1908: S. 14

${ }^{19}$ FRAENKEL 1908: S. 18, JAFFE 1972: S. 455

${ }^{20}$ BARLOW 1883: S. 166, FRAENKEL 1908: S. 14, JAFFE 1972: S. 450, S. 452
} 
Durch Einblutungen zwischen Schaft und Epiphyse kann es bei Kindern zu Epiphysendislokationen kommen, die sich radiologisch (KÜBLER 1965, HEYMANN 2007) und klinisch gelegentlich durch einen Crepitus nachweisen lassen (AUFDERHEIDE und RODRÍGUEZ-MARTÍN 1998, BARLOW 1883²1).

Bei Kindern sind die Gelenke in der Regel nicht betroffen; bei Erwachsenen kommt es häufiger zur Ausbildung eines Hämarthros (AUFDERHEIDE und RODRÍGUEZ-MARTÍN 1998, FRAENKEL 1908, HIRSCHMANN und RAUGI 1999, JAFFE $1972^{22}$, MAAT 1982, MAAT 2004, VILTER et al. 1946).

Häufig fällt bei Kindern am Thorax ein „Skorbutischer Rosenkranz“ auf. Dieser kommt durch subperiostale Blutungen an den sternalen Enden der Rippenknochen zustande, die eine Trennung der costochondralen Verbindung, ein Verschieben des Rippenknorpels gegen den Rippenknochen und ein Zurücksinken des Sternums bedingen, wodurch die knöchernen Rippenenden und die aufgetriebenen Rippenknorpel als schmerzende kleine Buckel unter der Haut sicht- und tastbar sind (AUFDERHEIDE und RODRÍGUEZ-MARTÍN 1998, BARLOW 1883, FRAENKEL 1908, JAFFE $1972^{23}$ ). Auch beim Erwachsenen sind Veränderungen an den Rippen beschrieben (ASCHOFF und KOCH 1919).

Am Schädeldach lassen sich subperiostale Blutungen nachweisen, die sich in schmerzhaften Schwellungen von Os occipitale, parietale, temporale, frontale und zygomaticum äußern (BARLOW 1883, FRAENKEL $1908^{24}$ ).

Ebenso werden Schwellungen über den Scapulae beschrieben, insbesondere über der Fossa infraspinata; subperiostale Hämatome auf der Ventral- und Dorsalseite der Scapulae sind bekannt (BARLOW 1883, FRAENKEL $1908^{25}$ ).

Auch die Schleimhäute sind betroffen, vor allem im Mund. Es wird von aufgetriebenem spongiösen Zahnfleisch berichtet, das zu Blutungen neigt (ASCHOFF und KOCH 1919, AUFDERHEIDE und RODRÍGUEZ-MARTÍN 1998, FRAENKEL 1908, HEYMANN 2007, JAFFE $1972^{26}$, MAAT 1982, MAAT 2004, SCHULTZ et al. 2003, VILTER et al. 1946). Schwellungen können auch die Lippen befallen. Assoziiert sind diese Veränderungen gelegentlich mit eitrigem Mundgeruch und Zahnausfall (AUFDERHEIDE und RODRÍGUEZ-MARTÍN 1998, BARLOW $1883^{27}$, MAAT 1982). Die Kiefer können durch

\footnotetext{
${ }^{21}$ S. 167

${ }^{22}$ FRAENKEL 1908: S. 9, S. 23, JAFFE 1972: S. 455

${ }^{23}$ BARLOW 1883: S. 183, FRAENKEL 1908: S. 16f, JAFFE 1972: S. 450, S.452, S. 454f

${ }^{24}$ BARLOW 1883: S. 169, FRAENKEL 1908: S. 10

${ }^{25}$ BARLOW 1883: S. 168, S. 184, S. 188, FRAENKEL 1908: S. 10

${ }^{26}$ FRAENKEL 1908: S. 10f, S. 28, JAFFE: S. 454

${ }^{27}$ S. 173 , S. $198 \mathrm{f}$
} 
teilweise massive Blutergüsse geschwollen erscheinen (BARLOW 1883, FRAENKEL 1908, JAFFE 1972 ${ }^{28}$ ). Ebenso kann es zu Blutungen im Bereich des Afters und der Darmschleimhaut kommen, die sich durch Blut im Stuhl manifestieren; auch Hämatemesis durch Blutungen im oberen Verdauungstrakt wird beschrieben. Häufiger kommt es durch Schleimhautblutungen im Bereich des Urogenitaltrakts zur (Mikro-) Hämaturie (FRAENKEL 1908, HIRSCHMANN und RAUGI 1999, JAFFE 1972, KÜBLER 1965²9).

Die Patienten klagen über Zahnfleisch- und Nasenbluten, es wird über Ecchymosis der Augenlider, konjunktivale Hämorrhagien und Proptosis eines Auges durch retrobulbäre Hämatome berichtet; am Körper fallen Hämatome, Petechien und Einblutungen in die Haut auf (ASCHOFF und KOCH 1919, AUFDERHEIDE und RODRÍGUEZ-MARTÍN 1998, BARLOW 1883, FRAENKEL 1908, HIRSCHMANN und RAUGI 1999, HEYMANN 2007, JAFFE $1972^{30}$, KÜBLER 1965, MAAT 2004, VILTER et al. 1946).

Bei Erwachsenen sind Petechien im Bereich der Haarfollikel, ,perifollikuläre Purpura“, die vermutlich durch bei Bewegungen der Haare ausgelöste Rupturen der extrem brüchigen perifollikulären Blutgefäße bedingt sind, ein typisches Symptom für Vitamin-C-Mangel (HIRSCHMANN und RAUGI 1999, HODGES 1980, HODGES et al. 1969, VILTER et al. 1946). Auch Splitter-Blutungen im Nagelbett werden häufig beobachtet (HIRSCHMANN und RAUGI 1999, VILTER et al. 1946).

Lebensbedrohlich können Blutungen in den Verdauungstrakt und in die unteren Extremitäten sein (MAAT 2004). In Autopsien werden Einblutungen in die Muskulatur sowie im Verdauungstrakt, im Urogenitaltrakt, im Perikard, der Pleura und dem Peritoneum gefunden. Bei Kindern erscheint das Nervensystem unbeteiligt; bei Erwachsenen werden häufiger Einblutungen in periphere Nervenscheiden gefunden, die Symptome einer peripheren Polyneuropathie hervorrufen (AUFDERHEIDE und RODRÍGUEZ-MARTÍN 1998, HODGES 1980, JAFFE 1972 ${ }^{31}$ ). Hämorrhagien um die großen Beinvenen herum können diese komprimieren und zu Thrombosen und Lungenembolien führen (AUFDERHEIDE und RODRÍGUEZ-MARTÍN 1998, VILTER et al. 1946).

Es wird gehäuft über eine Rechtsherzverbreiterung berichtet, die möglicherweise zum Teil auf den durch die Brustkorbveränderungen erhöhten Atemwiderstand zurückzuführen ist (KÜBLER 1965).

\footnotetext{
${ }^{28}$ BARLOW 1883: S. 169, FRAENKEL 1908: S. 11, JAFFE 1972: S. 450, S. 452

${ }^{29}$ FRAENKEL 1908: S. 12, JAFFE 1972: S. 450

${ }^{30}$ BARLOW 1883: S. 169f, S. 195f, FRAENKEL 1908: S. 11-13, S. 29, JAFFE 1972: S. 452

${ }^{31}$ S. 454
} 
Häufig geht Skorbut mit einer Anämie einher, die allerdings nicht ausschließlich auf den Vitamin-C-Mangel zurückzuführen, sondern vermutlich zum Teil durch begleitende Mangelzustände bedingt ist (HIRSCHMANN und RAUGI 1999, HEYMANN 2007, VILTER et al. 1946, ZUELZER et al. 1949).

Weniger gravierend, aber sehr häufig, ist eine vor allem bei Erwachsenen auftretende Hyperkeratosis der Haarfollikel, die zu Korkenzieherhaaren führt (HIRSCHMANN und RAUGI 1999, HODGES 1980, HODGES et al. 1969, JAFFE 1972², VILTER et al. 1946). Diese Veränderungen gehen auf eine Reduktion der Tätigkeit der Talgdrüsen sowie eine gestörte Proteinsynthese zurück (HODGES 1980).

Auch die Aktivität der Tränen- und Speicheldrüsen ist herabgesetzt, was zu einem dem Sjörgen-Syndrom entsprechenden Bild führt (HIRSCHMANN und RAUGI 1999, HODGES 1980).

HODGES (1980) beschreibt eine „neurotische Trias“ aus Depression, Hysterie und Hypochondrie, die durch Vitamin-C-Substitution prompte Besserung erfährt (HODGES 1980, siehe auch HIRSCHMANN und RAUGI 1999).

Die Wundheilung ist verzögert, es gibt sogar Berichte über lang verheilte Wunden, die sich wieder öffnen (AUFDERHEIDE und RODRÍGUEZ-MARTÍN 1998, BÄSSLER et al. 2002, HIRSCHMANN und RAUGI 1999, HODGES 1980, KÜBLER 1965, LANG 1979, ZELLWEGER und ADOLPH 1954).

Eine vorbestehende Akne kann durch Vitamin-C-Mangel verstärkt werden (HIRSCHMANN und RAUGI 1999).

BARLOW (1883) summiert die Hauptmerkmale des (infantilen) Skorbuts folgendermaßen: ,[...] the five constituents, viz. pallor, wasting, immobility, swelling of limbs, and fretfulness, [...], with or without swelling of gums [...]“(BARLOW 1883, S. 175).

FRAENKEL (1908) kommt zu dem Ergebnis, „daß den durch die klassische Symptomentrias, Zahnfleischblutungen, Schmerzhaftigkeit bei Bewegungen, Auftreibung und Deformierung der Röhrenknochen, besonders der unteren Extremitäten gekennzeichneten, leicht diagnostizierbaren Fällen eine nicht geringe Anzahl anderer gegenübersteht, welche eines oder das andere dieser, in ihrer Gesamtheit als pathognomonisch anzusehenden, Merkmale vermissen lassen, und bei denen es neben der, als wichtig anzusehenden, hochgradigen Blässe, Störungen allgemeiner Art, wie Abnahme der Eßlust, Abneigung gegen aktive und passive Bewegungen [gibt]“" (FRAENKEL 1908, S. 14).

${ }^{32}$ S. 452 
Die Krankheitsdauer liegt zwischen einem und sechs Monaten, aber auch eine Dauer von mehr als einem Jahr wird beschrieben. Eine komplette Heilung ist möglich, bei schwerem Befall der Knochen mit Brüchen und Dislokationen sind Residuen, wie etwa Varus/Valgusstellungen der unteren Extremität, nicht auszuschließen (BARLOW 1883, FRAENKEL $1908^{33}$ ).

Ebenso ist ein letaler Verlauf möglich (ASCHOFF und KOCH 1919, BARLOW 1883, FRAENKEL 1908, HIRSCHMANN und RAUGI 1999).

Laut FRAENKEL (1908) führt aber meist nicht der Skorbut selbst zum Tode, sondern komplizierende Erkrankungen wie Darmkatarrhe und Infektionen der Luftwege (s. auch AUFDERHEIDE und RODRÍGUEZ-MARTÍN 1998, JAFFE 1972 ${ }^{34}$ ). Er weist darauf hin, dass diese Krankheiten sowohl auf der allgemeinen Schwächung des Organismus basierende Folgen des Skorbuts sein können, als auch zur Entstehung bzw. Verschlimmerung des Leidens beitragen können, indem sie Appetit und Nahrungsresorption herabsetzen (FRAENKEL $1908^{35}$ ).

MAAT (2004) vermutet einen klinischen Verlauf von generalisierter Schwäche über einen Volumenmangelschock durch den Blutverlust bis zum Herzstillstand (MAAT 2004). Es gibt Berichte über das Entstehen von Lungenödemen, Hirnblutungen und Blutungen in die Herzmuskulatur oder das Perikard, die natürlich lebensbedrohlich sind (AUFDERHEIDE und RODRÍGUEZ-MARTÍN 1998, HIRSCHMANN und RAUGI 1999, WEISE PRINZO 1999). Immer wieder wird über plötzlichen Herztod bei Skorbutkranken berichtet (HIRSCHMANN und RAUGI 1999). Auch Synkopen, neu auftretende Blockbilder im EKG, Herzrhythmusstörungen und Angina pectoris werden beobachtet (HIRSCHMANN und RAUGI 1999).

VILTER (1946) und seine Kollegen haben bei Patienten, die in den 1940er Jahren mit manifestem Skorbut in ihre Klinik eingewiesen wurden, zunächst eine Vitamin-C-freie Kost fortgesetzt, um den weiteren Krankheitsverlauf zu beobachten. Dabei kam es gehäuft zu Blutdruckabfällen bis hin zu Schockzuständen, die in den meisten Fällen durch hoch dosierte Gaben reiner Ascorbinsäure rasch gebessert werden konnten (VILTER et al. 1946). Im fortgeschrittenen Krankheitsverlauf wird auch eine Cheyne-Stokes-Atmung beobachtet (HIRSCHMANN und RAUGI 1999, VILTER et al. 1946).

Der Vasotonus ist reduziert, da für die Synthese der Katecholamine Vitamin C benötigt wird (HODGES 1980, siehe auch Kapitel 1.4 Biochemie und Physiologie des Vitamin C,

\footnotetext{
${ }^{33}$ BARLOW 1883: S. 176, FRAENKEL 1908: S. 19, S. $33 \mathrm{f}$

${ }^{34}$ S. 450

${ }^{35}$ S. 30
} 
Unterkapitel b) Funktion). Außerdem scheint die Reaktionsfähigkeit der durch die gestörte Kollagensynthese defekten Blutgefäße auf adrenerge Stimulation deutlich herabgesetzt zu sein. Dies könnte neben den mechanischen Problemen der Blutungen, wie etwa der Perikardtamponade und dem Volumenmangel, eine Ursache für die Häufung kardialer Probleme sein (HIRSCHMANN und RAUGI 1999).

\subsection{Laborbefunde bei Skorbut}

Selbstverständlich lassen sich bei historischen Skeleten keine Blutuntersuchungen durchführen, aber der Vollständigkeit halber und für ein besseres Verständnis der Erkrankung werden die typischen Veränderungen hier kurz vorgestellt.

Es gibt zwei Laborparameter, die über den Vitamin-C-Status einer Person Auskunft geben. Zum einen kann man den Ascorbinsäuregehalt im Plasma bestimmen. Dieser sinkt jedoch schon nach wenigen Tagen Vitamin-C-freier Ernährung unter die Nachweisgrenze von 0,1 mg/dl (JAFFE 1972 ${ }^{36}$, LANG 1979), wobei man schon bei $0,2 \mathrm{mg} / \mathrm{dl}$ von massivem Mangel sprechen kann (HODGES 1980), auch wenn noch genügend Ascorbinsäure im Gewebe gespeichert ist. Ein optimaler Wert und Ziel einer Skorbut-Therapie wären 0,7 mg/dl (JAFFE 1972) oder sogar noch höher bei 1,0-1,4 mg/dl (BÄSSLER et al. 2002, LANG 1979). Heymann (2007) setzt eine kritische Grenze von $<11 \mu$ mol/L Ascorbinsäure im Blutserum an, unterhalb welcher er einen Skorbut für wahrscheinlich hält.

Erst unmittelbar vor der klinischen Manifestation des Skorbuts zeigt sich auch ein signifikanter Abfall der klinisch wichtigeren Ascorbinsäurekonzentration in den Leukozyten. Dieser Wert liegt bei an Skorbut erkrankten Personen meist unter $4 \mathrm{mg} / 100 \mathrm{~g}$ und sollte etwa 25-35 mg/100 g betragen, wobei ab einer Konzentration von um die $15 \mathrm{mg} / 100 \mathrm{~g}$ von einer ungenügenden Vitamin-C-Zufuhr ausgegangen werden kann (JAFFE 1972 ${ }^{37}$, LANG 1979, WEISE PRINZO 1999).

Hodges teilt die Entwicklung eines manifesten Vitamin-C-Mangels in vier Stadien ein. Stadium 1 entspricht dabei einer ausgewogenen täglichen Vitamin-C-Zufuhr bei vollständig gefüllten Reserven (Body-Pool ca. 1500 mg), also dem Optimalzustand. Stadium 2 tritt ein, wenn über längere Zeit zuwenig Vitamin C zugeführt wird. Dann werden die Reserven abgebaut, die Plasmawerte bleiben auf Normalniveau und klinisch zeigen sich keinerlei Symptome. Wird weiterhin kein Vitamin C zugeführt, kommt es zu Stadium 3, nun sinken auch die Blutwerte für Vitamin C, klinische Symptome werden noch nicht beobachtet. Sinkt

\footnotetext{
${ }^{36}$ S. 453, S. 464

${ }^{37}$ S. 453, S. 464
} 
der Body-Pool auf $300 \mathrm{mg}$ oder weniger, fällt die Verstoffwechselungsrate auf $9 \mathrm{mg}$ oder weniger. In diesem Stadium 4 tritt manifester Skorbut auf (HODGES 1980).

Einschätzung/Bewertung des Vitamin C-Status nach Hodges

\begin{tabular}{lllll}
\hline & Plasma $(\mathbf{m g} / \mathbf{d l})$ & Vollblut $(\mathbf{m g} / \mathbf{d l})$ & Leukozyten $\left(\boldsymbol{\mu g} / \mathbf{1 0} \mathbf{8}^{\text {Zellen})}\right.$ & Metabolischer Body-Pool (mg) \\
\hline Hoch & $0.60-1.40$ & $>1.0$ & $>16$ & 1500 \\
Normal & $0.40-0.59$ & $0.60-0.99$ & $11-15$ & $600-1499$ \\
Niedrig & $0.20-0.39$ & $0.30-0.59$ & $2-10$ & $300-599$ \\
Mangel & $<0.20$ & $<0.30$ & $<2$ & $<300$ \\
\hline
\end{tabular}

Tab. 1: Einschätzung/Bewertung des Vitamin C-Status nach Hodges. Modifiziert nach HODGES 1980, S. 85

Darüber hinaus zeigt sich häufig eine - auch klinisch beschriebene - Anämie (s. auch Kapitel 1.2 Das Krankheitsbild des Skorbuts). Diese kann sowohl mikrozytär als auch megaloblastisch und sogar hämolytischen Ursprungs sein (JAFFE $1972^{38}$, VILTER et al. 1946).

FRAENKEL (1908) hält die Anämie für eines der klinischen Hauptsymptome des Skorbuts, seine Blut- und Knochenmarksuntersuchungen an betroffenen Kindern ergeben in den meisten Fällen eine normo- oder megaloblastäre Anämie bei ansonsten unverändertem Blutbild (FRAENKEL 1908 ${ }^{39}$ ). Dies spricht eher gegen eine auf Blutverlust oder alimentäre Unterversorgung basierende Eisenmangelanämie, bei der sich mikrozytäre Erythrozyten finden würden. Eher lässt dieser Befund in Richtung eines Vitamin-B12- oder Folsäuremangels denken, was bei der durch FRAENKEL (1908) geschilderten einseitigen Ernährung seiner Patienten durchaus möglich scheint. LEWIS (2002) führt das Auftreten einer Anämie im Rahmen des Skorbuts auf abnorme erythroblastische Regionen zurück, in denen rotes Knochenmark durch Fettmark ersetzt wird (LEWIS 2002).

ZUELZER et al. (1949) beobachten makrozytäre, normozytäre, normochrome und mikrozytäre hypochrome Anämien bei einer umfassenden hämatologischen Untersuchung von 36 Fällen infantilen Skorbuts. Sie führen die Anämien auf drei Mechanismen zurück: Hämorrhagien, Infektionen und koexistente Mangelzustände (z.B. Eisen, Folsäure, Leberenzyme). Sie stellen fest, dass sich diese Anämien durch Substitution der jeweils fehlenden Substanz auch unter Fortbestand des Vitamin-C-Mangels heilen lassen, wohingegen die alleinige Substitution von Ascorbinsäure ohne Therapie der anderen Mängel

\footnotetext{
${ }^{38}$ S. 452

${ }^{39}$ S. 9, S. 13
} 
keine Besserung der Anämie bringt. Die Anämie ist also nicht primär durch den Skorbut bedingt (ZUELZER et al. 1949).

VILTER et al. (1946) kommen diesbezüglich zu einem anderen Ergebnis, sie beobachten bei ihren Patienten eine Normalisierung der Erythropoese durch alleinige Substitution von Vitamin C (VILTER et al. 1946).

Möglicherweise verstärkt Skorbut eine anderweitig verursachte Anämie (ZELLWEGER und ADOLPH 1954). Es scheint eine Steigerung der Hämolyse mit Verkürzung der Lebensdauer der Erythrozyten zu geben. Die Ursache hierfür liegt, wie Transfusionsversuche zeigen, wohl nicht in den Erythrozyten, sondern möglicherweise in den veränderten Blutgefäßen (COX et al. 1967, HIRSCHMANN und RAUGI 1999, VILTER et al. 1946). Dennoch scheint die Hämolyse nicht die Ursache für die häufig beobachtete skorbutische Anämie zu sein (COX et al. 1967).

Da Vitamin C Einfluss auf die Eisenresorption hat und es außerdem durch die gehäuften Blutungen im Rahmen von Skorbut zu Eisenverlusten kommen kann, erscheint es sinnvoll, im Rahmen einer Therapie auch Eisen zu substituieren. In skorbutischen Patienten wird häufig ein Eisenmangel beobachtet, allerdings wird in den meisten Fällen allein durch den Ausgleich des Vitamin C-Mangels eine Normalisierung des Eisenhaushaltes erreicht.

Auch am Folatstoffwechsel ist Ascorbinsäure beteiligt (s. auch Kapitel 1.4 b) Funktionen des Vitamin (), allerdings ist sie hier ersetzbar. Vitamin $\mathrm{C}$ allein kann das Entstehen einer megaloblastären Anämie durch Folatmangel nicht verhindern; fehlendes Vitamin C kann allerdings einen bestehenden Folatmangel verschärfen, weil dann die Stabilisierung der Methyltetrahydrofolsäure durch Ascorbinsäure entfällt (COX et al. 1967, NATHAN und OSKI 1993).

Verschiedene Untersuchungen des Knochenmarks zeigen keinen einheitlichen Befund bei Skorbutkranken. Es sind sowohl normoblastische als auch megaloblastische Zellbilder beschrieben, meist liegt ein hyperzelluläres Bild vor, aber auch hypozelluläre Befunde sind beschrieben. Dies erschwert die Erkenntnis eines direkten Zusammenhangs (COX et al. 1967). Es scheint allerdings zu einer Steigerung der Erythropoese zu kommen. Der Umsatz von radioaktiv markiertem Eisen ist in skorbutischen Situationen deutlich gesteigert (COX et al. 1967).

Bei ihrem freiwilligen Vitamin-C-Entzugs-Versuch an Gefängnisinsassen stellen HODGES und Mitarbeiter 1969 trotz ausreichender Zufuhr einen Abfall der Vitamin-A-Konzentration fest, die nach Vitamin-C-Gabe schnell wieder steigt. Eine Erklärung dafür findet sich derzeit nicht (HODGES et al. 1969). 
Eine Veränderung des übrigen Blutbilds liegt, außer während der häufigen Sekundärinfektionen, nicht vor. Auch die Calcium- und Phosphatwerte liegen im Normalbereich.

Die Blutgerinnung ist unverändert. Für die Blutungen beim Skorbut muss also die mechanische Instabilität der Blutgefäße verantwortlich gemacht werden (HODGES et al. 1969).

Bei manifestem Skorbut ist die alkalische Phosphatase erniedrigt, sie steigt nach Ascorbinsäuregabe rasch wieder an (JAFFE $1972^{40}$, LANG 1979).

\subsection{Biochemie und Physiologie des Vitamin C}

\section{a) Eigenschaften}

Vitamin C, die L-Ascorbinsäure, wird aus D-Glucose gebildet. Sie ist ein abgewandeltes Monosaccharid und hat die Formel $\mathrm{C}_{6} \mathrm{H}_{8} \mathrm{O}_{6}$ (siehe auch $A b b$. 2). Es gibt vier mögliche Stereoisomere, von denen zwei biologisch aktiv sind, nämlich die L-Ascorbinsäure selbst und die D-Araboascorbinsäure, während D-Ascorbinsäure und L-Araboascorbinsäure inaktiv sind. Daraus lässt sich schließen, dass für die biologische Aktivität die Konfiguration am C-Atom 4 ausschlaggebend ist, wo der Lactonring, bei konventioneller Schreibweise, auf der rechten Seite gelegen sein muss. Die Konfiguration am C-Atom 5 und die Länge der C-Atomkette dagegen scheinen für die biologische Funktion keine entscheidende Rolle zu spielen (KÜBLER 1965, LANG 1979).

In reiner Form ist Vitamin C ein weißes kristallines Pulver, dessen Siedepunkt bei $192^{\circ} \mathrm{C}$ liegt. Es ist leicht autoxidabel, aber in kristalliner Form und in sauren wässrigen Lösungen $(\mathrm{pH}<6)$ auch in Gegenwart von Luftsauerstoff stabil. Dagegen folgt in alkalischen Lösungen und in Gegenwart von Schwermetallionen, v. a. Kupfer, die rasche Oxidation (BÄSSLER et al. 2002, KÜBLER 1965). Schutzstoffe wie Zitronensäure, Mono- und Polysaccharide, Flavonide und Peptide machen Vitamin C haltbar (BÄSSLER et al. 2002).

Ascorbinsäure ist wasserlöslich, weniger gut löslich in Ethanol und annähernd unlöslich in unpolaren Fettlösungen (HODGES 1980). Sie bildet Salze, u.a. Natrium-, Calcium- und Magnesiumsalz (BÄSSLER et al. 2002).

L-Ascorbinsäure reagiert durch Dissoziation der beiden enolischen Hydroxylgruppen als zweibasige Säure $\left(\mathrm{pK}_{1}=4,1, \mathrm{pK}_{2}=11,8\right)$ und ist ein sehr wirksames Reduktionsmittel (BÄSSLER et al. 2002). Sie bildet ein Redoxsystem mit Dehydroascorbinsäure (siehe auch Abb. 1). Man könnte beide Redoxpartner zusammen als biologisch aktive Form des Vitamin 
$\mathrm{C}$ bezeichnen, da sie biologisch nicht voneinander $\mathrm{zu}$ trennen und gleichermaßen antiskorbutisch wirksam sind (HODGES 1980).

\section{b) Funktion}

Anders als andere wasserlösliche Vitamine stellt Vitamin C in der Regel kein Koenzym dar, sondern schützt, erleichtert und reguliert die Funktionen von Enzymen. Es schützt andere Antioxidantien (HODGES 1980).

Die wichtigste Funktion des Vitamin $\mathrm{C}$ ist die Hydroxilierung von Prolin und Lysin, was essentiell für die Synthese von Kollagen ist (siehe $A b b$. 3). Die Konsequenzen der fehlerhaften Kollagensynthese sind im Kapitel 1.5 Pathophysiologie des Skorbuts ausführlich behandelt.

Vermutlich ist Vitamin $\mathrm{C}$ an der Aktivierung der Folsäure zu Tetrahydrofolat beteiligt (HODGES 1980, KREUTZIG 1997 ${ }^{41}$ ).

Beim Abbau der Aminosäuren Phenylalanin und Tyrosin wird Vitamin C für die Hydroxylierung und Decarboxylierung des p-Hydroxyphenylpyruvat zu Homogentisinsäure benötigt (HODGES 1980, KREUTZIG 1997, KÜBLER 1965, LANG 1979, LÖFFLER $\left.2001^{42}\right)$.

Außerdem spielt Vitamin C eine Rolle bei der Hydroxilierung von Tryptophan zu 5Hydroxytryptophan, das wiederum durch Decarboxylierung $\mathrm{zu}$ Serotonin (5Hydroxytryptamin) wird (HODGES 1980, KREUTZIG 1997, LANG 1979³).

Auch an der Synthese von Noradrenalin und damit auch Adrenalin ist Vitamin C beteiligt. Es ist wie $\mathrm{Cu}^{2+}$ notwendig zur Hydroxilierung des aus Tyrosin abgeleiteten Dopamins zu Norardrenalin durch die Dopamin-Hydroxilase (BÄSSLER et al. 2002, HODGES 1980, LANG 1979).

Damit ist Vitamin C wichtig für die Synthese vasoaktiver Substanzen, was zumindest teilweise den Verlust der vaskulären Reagibilität, die bei Skorbut beobachtet wird, erklären könnte (HIRSCHMANN und RAUGI 1999, HODGES 1980, KÜBLER 1965).

In Hydroxilierungsreaktionen, die auf der reduzierten Form von Kupfer-Ionen beruhen, werden diese von Ascorbinsäure stabilisiert. Ähnliches gilt für Reaktionen, in denen Wasserstoffionen transferiert werden (HODGES 1980).

So kann Ascorbinsäure bei wichtigen Monooxygenase-Reaktionen, die Kupfer und molekularen Sauerstoff benötigen, das Reduktionsmittel darstellen. Diese sind beteiligt an der Entstehung von z.B. Bombesin, Calcitonin, Cholecystokinin, Corticotropin-releasing factor,

\footnotetext{
${ }^{41}$ S. 306

${ }^{42}$ KREUTZIG 1997: S. 53, LÖFFLER 2001: S. 272

${ }^{43}$ S. 306
} 
Gastrin, Wachstumshormon-releasing factor, Thyreotropin-releasing Hormon, Melanotropin, Oxytocin und Vasopressin (BÄSSLER et al. 2002). Diese Substanzen sind für den Organismus von großer Wichtigkeit, allerdings kann Vitamin $\mathrm{C}$ hier durch andere Reduktionsmittel ersetzt werden; deshalb kann man über die Konsequenzen eines Vitamin-CMangels für die Synthese nur Vermutungen anstellen.

Im Eisenstoffwechsel wird Vitamin C ebenso wie ATP für den Einbau von Eisen in Ferritin, das Speicher- und Transportprotein, benötigt. Damit wird die Phagozytose in Lysosomen verhindert, in denen Eisen in Hämosiderin umgewandelt wird, was seine Verfügbarkeit entscheidend senkt. Auch an der Aufnahme des Eisens ist Ascorbinsäure beteiligt: Die die Resorption steigernde Reduktion von $\mathrm{Fe}^{2+} \mathrm{zu} \mathrm{Fe}{ }^{3+}$ wird durch Vitamin $\mathrm{C}$ gefördert (BÄSSLER et al. 2002, HIRSCHMANN und RAUGI 1999, HODGES 1980, KREUTZIG 1997, LANG 1979).

Verschiedene Cytochrome können Ascorbinsäure $\mathrm{zu}$ Semidehydroascorbat (Monodehydroascorbinsäure) reduzieren. Dieses ist ein kurzlebiges Radikal und ermöglicht Einzelelektronenreaktionen. Es dient als Quelle freier Radikale für andere Reaktionen. Somit spielt Vitamin C eine Rolle bei der mikrosomalen Hydroxilierung, die vor allem für Entgiftungsvorgänge wichtig ist. Abbildung 1 zeigt die Funktion von Ascorbinsäure im Elektronentransportsystem des Cytochrom P-450. Im Tierversuch sinken die Konzentrationen der Cytochrome P-450 und $b_{5}$ unter Ascorbinsäuremangel (LANG 1979). Also beeinflusst Vitamin C vermutlich die Metabolisierung (giftiger) Substanzen, z.B. vieler Medikamente.

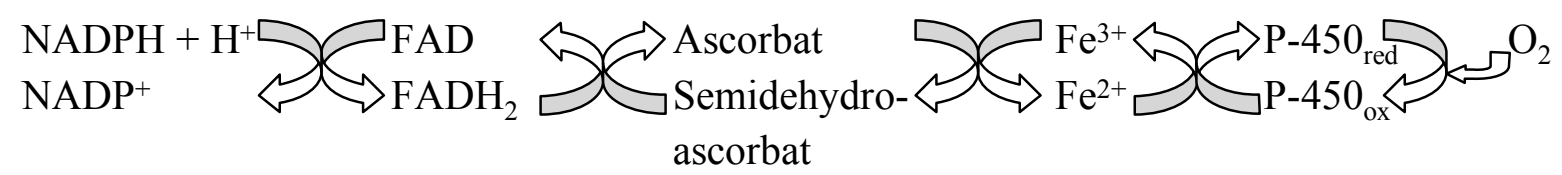

Abb. 1: Beteiligung von Vitamin C an mikrosomalen Hydroxilierungsreaktionen (nach: BÄSSLER et al. 2002, S. 249).

Ein Einfluss auf den Cholesterinspiegel durch Vitamin C könnte auf eine Beteiligung an der $7 \alpha$-Hydroxylierung von Cholesterin beim Abbau zu Gallensäuren zurückzuführen sein. Bei Skorbut wird eine Steigerung des Cholesterinspiegels beobachtet (BÄSSLER et al. 2002).

Auf nicht genau geklärte Art und Weise hemmt Ascorbinsäure die Nitrosaminbildung aus Nitrit und Aminen. Damit schützt sie den Körper vor hepatotoxischen und cancerogenen Verbindungen (BÄSSLER et al. 2002). 
Neben den bekannten Funktionen gibt es viele Spekulationen über weitere Wirkungen von Vitamin C. So wird Vitamin C häufig als „Stress-Vitamin“ bezeichnet, da in der Nebenniere relativ hohe Konzentrationen von Vitamin $\mathrm{C}$ gefunden werden, die im Tierversuch unter Stimulation der Nebennierenrinde recht schnell sinken (HODGES 1980). Die Synthese von

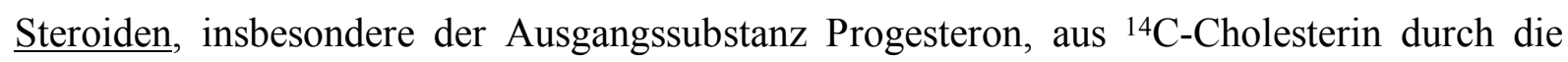
Mitochondrien der Nebennierenrinde wird durch Ascorbinsäuregabe, die allerdings nur in katalytischen Mengen gebraucht wird, gesteigert (LANG 1979). Es ist unbestritten, dass Stresssituationen körperlicher und psychischer Natur mit einem erhöhten Vitamin-C-Bedarf einhergehen (HODGES et al. 1969), vermutlich aufgrund der oben erwähnten ascorbinsäureabhängigen Katecholaminsynthese (BÄSSLER et al. 2002). Ein Benefit durch Megadosen Vitamin C für Menschen, die physischem Stress ausgesetzt wurden, konnte aber nicht nachgewiesen werden (HODGES 1980).

Ähnliches gilt für die Vermutungen, dass Vitamin C Erkältungen verhindere, $\underline{\text { Atherosklerose }}$ aufhalte, Tumorwachstum stoppe oder psychische Erkrankungen positiv beeinflusse (HODGES 1980). Allerdings ist der Ascorbinsäure-Umsatz des Menschen während Erkältungskrankheiten gesteigert. Sie wird unter diesen Umständen aus den Leukozyten freigesetzt und reichert sich in den entzündeten Membranen des Respirationstrakts an (LANG 1979).

Es gibt verschiedene Vermutungen hinsichtlich der Beeinflussung des Immunsystems durch Vitamin C. Eine der wenigen belegten Wirkungen ist der Schutz der Zellmembran von Phagozyten vor Selbstzerstörung durch im Rahmen des „respiratory burst“ zur Zerstörung phagozytierter Zellen gebildete aggressive Sauerstoffspezies. Außerdem kommt es bei Ascorbinsäuremangel nachweisbar zu einem Absinken der chemotaktischen Antwort (BÄSSLER et al. 2002, KÜBLER 1965).

Auch die Beteiligung des Vitamin C an der Synthese von Thyreotropin-releasing Hormon hat einen Einfluss auf das Immunsystem. Denn das daraufhin sezernierte TSH (Thyroideastimulierendes Hormon) stimuliert die spezifischen Abwehrzellen der Darmschleimhaut (BÄSSLER et al. 2002).

Gegenstand weiterer Forschung ist die Frage, ob Vitamin C die Arbeitsökonomie des Menschen verbessert. Es gibt Berichte über eine Steigerung von Konzentration, Auffassung und Koordination nach Ascorbinzufuhr (LANG 1979).

In Tierversuchen wird auch über eine Beeinflussung der Fertilität berichtet (KÜBLER 1965). 
Abb. 2 (I. und II.): Kollagenbiosynthese

Erstellt nach LÖFFLER 2001, LÖFFLER et al. 2007, KREUTZIG 1997, PROBST 2004)

\section{I. im Fibroblasten}

1. Zunächst erfolgt die Verknüpfung von $\alpha_{1}-\alpha_{2}$-Polypeptidketten. Es entsteht Protokollagen in Form einer linksdrehenden Helix.

Dabei ist ca. jede dritte Aminosäure Glycin, dessen H-Rest nach innen gerichtet ist. Alle anderen Reste sind nach außen gerichtet, weil die Helix zu eng ist.

An Position 2 (x genannt) findet sich häufig Prolin, dessen Ringstruktur die Helix in eine steile Form zwingt.

An Position 3 (y genannt) kommt gehäuft Lysin vor, was für die späteren Vernetzungsschritte von Bedeutung ist.

Am Anfang und am Ende der Helix befinden sich Telopeptide, die für die spätere Quervernetzung wichtig sind, und Propeptide, die eine vorzeitige Zusammenlagerung der Helices verhindern.

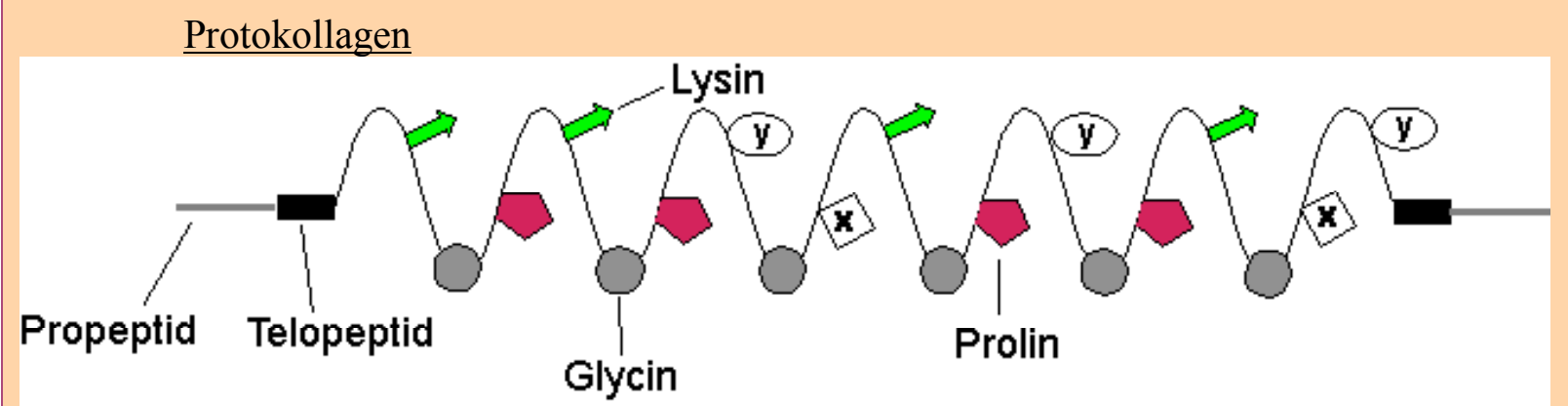

2. Im nächsten Schritt erfolgt die Hydroxilierung von Lysin- und Prolinresten. Diese Reaktion wird katalysiert durch das Enzym Protokollagenhydroxylase und ist Vitamin-C-abhängig. Dabei dient das Vitamin $\mathrm{C}$ der Stabilisierung des $\mathrm{Fe}^{2+}$ in reduzierter Form.

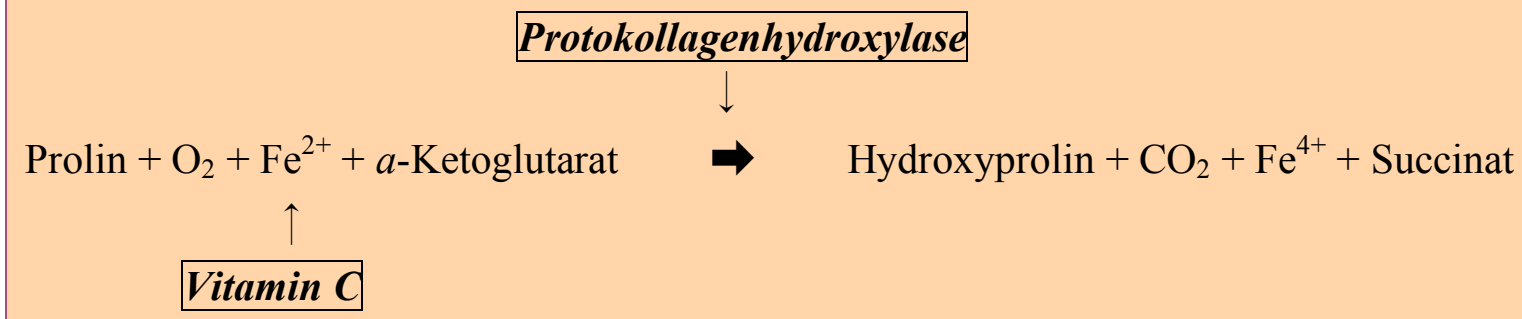

Das Hydroxyprolin steigert den Schmelzpunkt des Protokollagens von ca. $25^{\circ} \mathrm{C}$ auf über $37^{\circ} \mathrm{C}$, ist also sehr bedeutsam für die Stabilität des Kollagens. Das Hydroxylysin dient der Anlagerung von Zuckern und der späteren Quervernetzung der Helices.

\section{Hydroxyliertes Protokollagen}

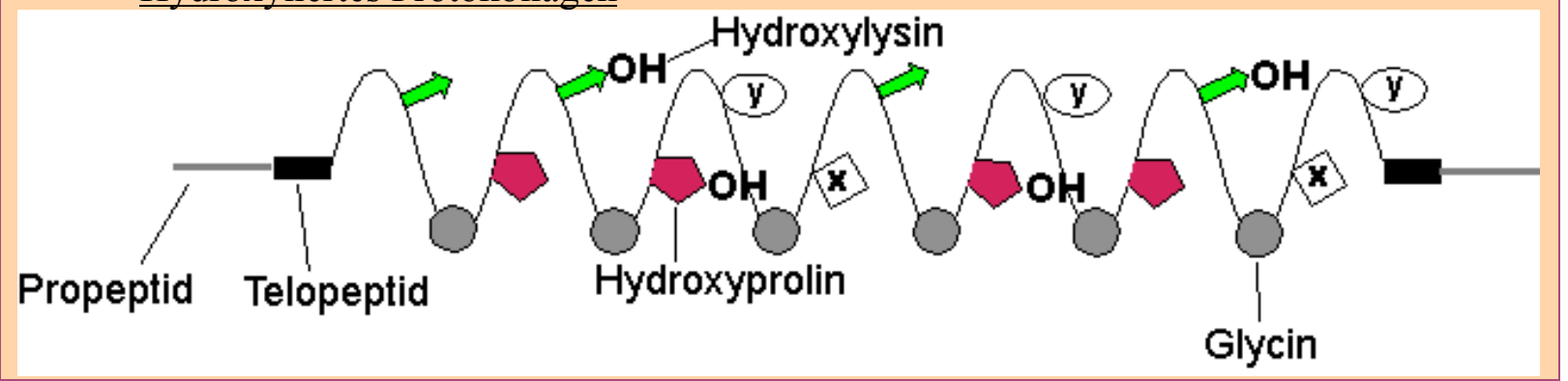


3. Der dritte Syntheseschritt besteht in der Glykosylierung von Hydroxylysinresten.

Hydroxyliertes und glykosyliertes Protokollagen

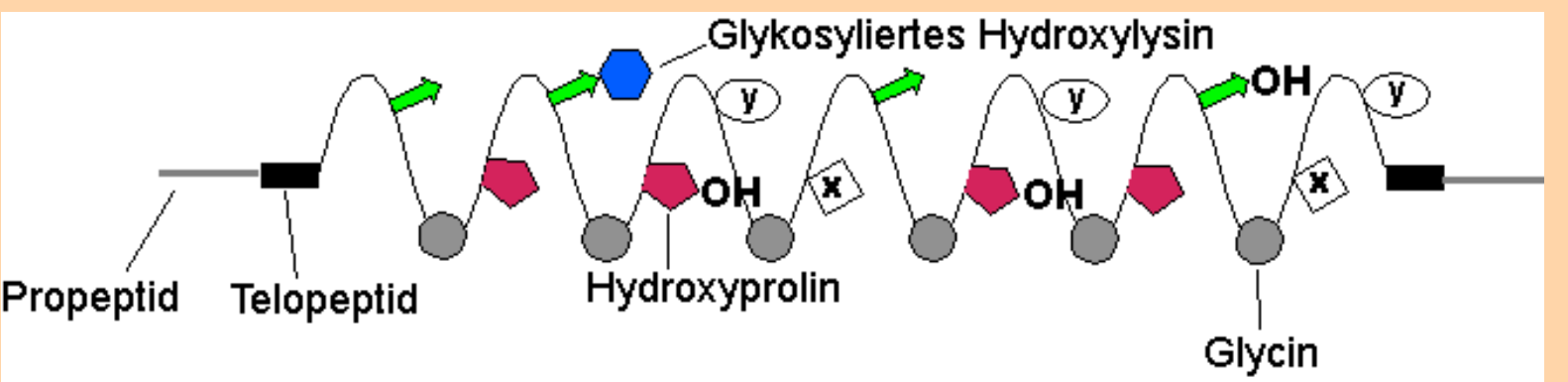

4. Als nächstes bilden drei Protokollagenhelices zusammen eine rechtsdrehende Trippel(Super-) Helix. Diese wird als Prokollagen bezeichnet.

Dabei zeigen nur die H-Reste nach innen. Die Stabilisierung der Trippelhelix erfolgt durch Wasserstoff- und Disulfidbrücken.

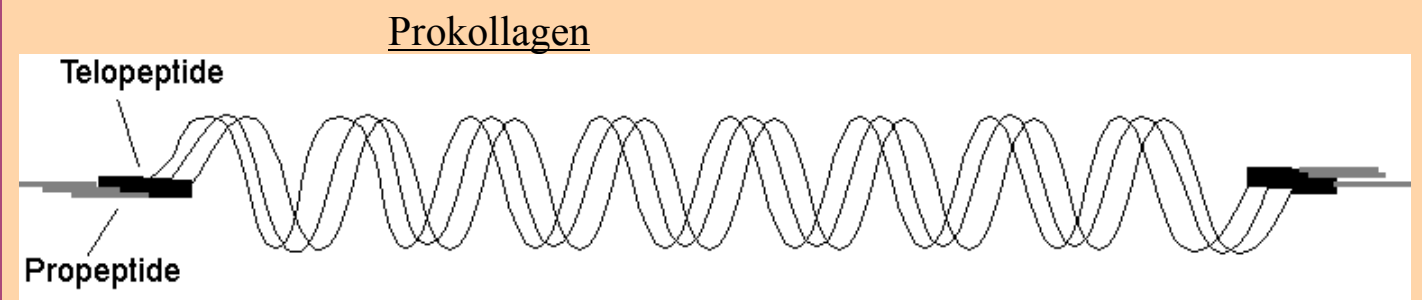

\section{II. im Extrazellulärraum}

5. Das Prokollagen wird in den Extrazellulärraum sezerniert. Dort erfolgt die Abspaltung der Propeptide durch Peptidasen und abhängig vom Kollagentyp eine weitere Glykosylierung.

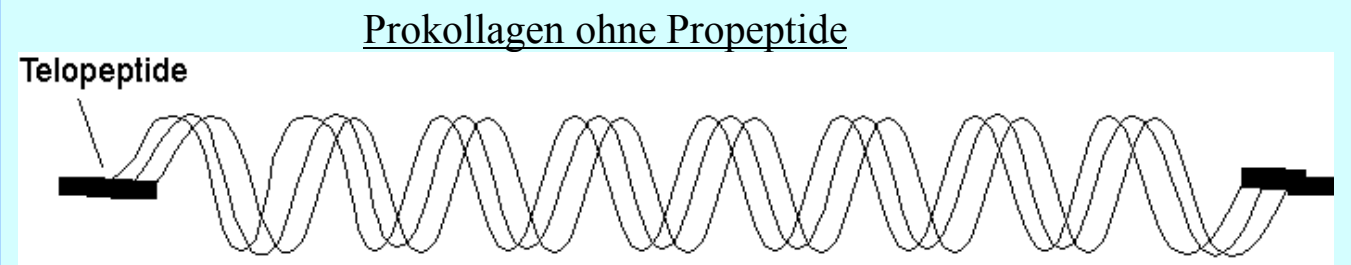

Den letzten Syntheseschritt bildet die Selbstassoziation von fünf Trippelhelices zu einer Kollagenmikrofibrille. Dabei ist die Anordnung parallel versetzt. Das führt in regelmäßigen Abständen zu sehr dichten und weniger dichten Bereichen, was sich im Elektronenmikroskop als Streifung darstellt.

Stabilität wird vor allem durch die Oxydierung der Lysilreste an den Telopeptiden erreicht. Dafür sorgt die Lysiloxydase unter Bildung reaktiver Aldehydgruppen. Diese reagieren beispielsweise mit den $\varepsilon$-Aminogruppen benachbarter Trippelhelices unter Bildung von Schiff'schen Basen.

Kollagenmikrofibrille

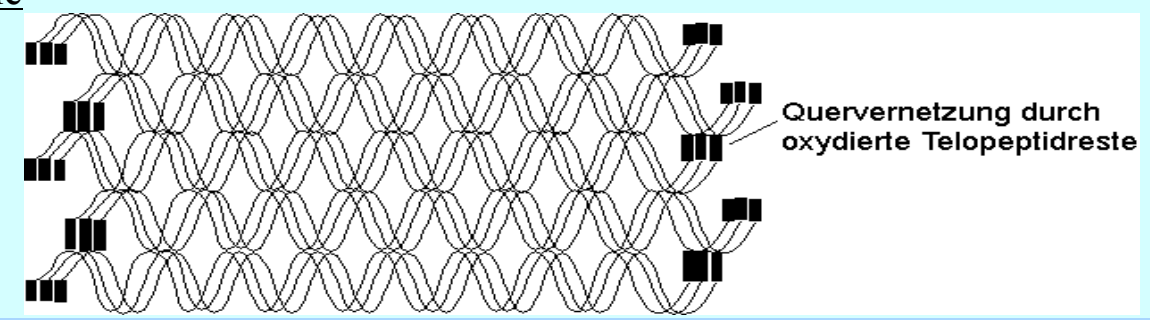




\section{c) Metabolismus/Aufnahme/Sekretion}

Es gibt verschiedene Stoffwechselwege zur Herstellung von Vitamin C, wobei man davon ausgeht, dass Pflanzen unterschiedliche nutzen können, wohingegen Tiere stets den in Abb. 2 dargestellten Weg über L-Gulonolacton gebrauchen. Menschen, Meerschweinchen und einige wenige andere Tiere sind nicht in der Lage, L-Gulonolacton in L-Ascorbinsäure umzuwandeln, weil ihnen die L-Gulonolacton-oxidase fehlt (HODGES 1980, KÜBLER 1965, LANG 1979).

Allerdings werden immer wieder Vermutungen geäußert, dass schwangere bzw. stillende Frauen möglicherweise doch in der Lage sind, Vitamin $\mathrm{C}$ zu synthetisieren, weil selbst in Mangelsituationen eigentlich keine skorbutischen Kinder geboren werden und auch gestillte Kinder von mangelernährten Frauen selten Skorbut entwickeln. Dafür spricht auch der unverhältnismäßig hohe Vitamin-C-Gehalt der Muttermilch (ca. $65 \mathrm{mg} / \mathrm{l}$ ), der auch bei Müttern, die selbst verminderte Vitamin C-Plasmaspiegel haben, erreicht wird (HODGES 1980, WEISE PRINZO 1999, siehe auch Kapitel 1.2 Das Krankheitsbild des Skorbuts, 1.7 Empfehlungen zur täglichen Vitamin-C-Aufnahme, 1.8 Vorkommen von Vitamin C).

Diese Vermutungen lassen sich bisher nicht wissenschaftlich erklären (HODGES 1980). Auf jeden Fall wird Vitamin $\mathrm{C}$ durch einen aktiven Transportmechanismus diaplacentar auf den Fetus übertragen (BÄSSLER et al. 2002).

Vitamin C wird - in physiologischen Dosen - fast vollständig absorbiert. Die Aufnahme erfolgt im Duodenum und proximalen Jejunum sowie zu geringen Teilen über die Mundschleimhaut.

Ähnlich wie bei der Aufnahme von Glucose existiert ein $\mathrm{Na}^{+}$- und Glucoseabhängiger Transportmechanismus, der niedrige Dosen Ascorbinsäure aufnimmt. Hohe Dosen sowie das Oxidationsprodukt Dehydroascorbinsäure werden durch Diffusion aufgenommen (BÄSSLER et al. 2002).

Wenn über einen längeren Zeitraum sehr hohe Dosen von Vitamin C zugeführt werden, so sinkt die Absorptionsrate. Bei einer normalen Aufnahme von bis zu $180 \mathrm{mg} / \mathrm{d}$ werden 80-90\% resorbiert, bei einer Zufuhr von $1 \mathrm{~g} / \mathrm{d}$ ca. 65-75\%, bei $12 \mathrm{~g}$ nur noch 16\% (BÄSSLER et al. 2002). Das im Darmlumen verbleibende Vitamin C wird durch die Dickdarmflora zu CO und organischen Säuren abgebaut; dies hat einen osmotischen Effekt und kann wässrige Durchfälle erzeugen (BÄSSLER et al. 2002, HODGES 1980, WEISE PRINZO 1999). Die normale Ascorbinsäureausscheidung über den Stuhl liegt bei <5 mg/d (LANG 1979).

Auch in der Niere wird die Ascorbinsäure ähnlich wie Glucose behandelt. Bei niedrigen Konzentrationen wird Vitamin $\mathrm{C}$ über $\mathrm{Na}^{+}$- abhängige Carrier aktiv von den Zellen des 
proximalen Tubulus reabsorbiert, bei erhöhter Plasmakonzentration steigt der Verlust über den Urin. Bei einem normalen Plasmawert von etwa $0.6 \mathrm{mg} / \mathrm{dl}$ wird so gut wie kein Vitamin C über den Urin ausgeschieden; der Grenzwert, ab dem die Exkretion dosisabhängig steigt, liegt bei etwa 1,4 mg/dl (BÄSSLER et al. 2002, HODGES 1980, HODGES et al. 1969).

Es gibt Berichte, dass Menschen, die vor einem Vitamin-C-Mangelzustand gewohnt waren, hohe Dosen Ascorbinsäure zu sich zu nehmen, schneller skorbutische Symptome entwickeln als Menschen, die von jeher an niedrige Zufuhr gewöhnt sind (HODGES 1980); allerdings sind diese Theorien umstritten, zumal im Tierversuch gegensätzliche Ergebnisse erzielt wurden (LANG 1979). Offensichtlich gibt es eine Down-Regulation der Vitamin-CAufnahme, die zu hohe Dosen des Vitamins im Körper vermeiden soll. Möglicherweise gibt es sogar einen aktiven Zerstörungsmechanismus (HODGES 1980). Das mag einer der Gründe sein, weshalb Überdosierung von Vitamin C meist folgenlos bleibt. Allerdings gibt es durchaus Berichte über Übelkeit und Erbrechen, Interferenzen mit dem Glucosestoffwechsel bei Diabetikern und sogar Aborte durch Vitamin-C-Intoxikation. Außerdem kann eine Vitamin-C-Überdosierung die Aufnahme und Funktion anderer bedeutsamer Substanzen, wie z.B. von Vitamin-K-Antagonisten, hemmen (HODGES 1980).

Nach der Aufnahme verteilt sich Ascorbinsäure in den wässrigen Körperkompartimenten (HODGES 1980); in den Organen liegen nur 1-3\% der Gesamtascorbinsäure in Form von Dehydroascorbinsäure vor, im Blut mehr als 20\% (LANG 1979). Vitamin-C-reiche Organe des Menschen sind Hypophyse, Gehirn, Nebennieren, Augenlinse, Leber, Milz, Magen, Pankreas sowie Leukozyten und Lymphozyten, in denen sich das Vitamin C meist im Cytosol befindet (BÄSSLER 2002).

Der biologisch aktive Pool an Vitamin C wird auf etwa $1500 \mathrm{mg}$ bis zu max. $3000 \mathrm{mg}$ bei einem gesunden Mann mittleren Alters geschätzt. Der tägliche Verbrauch wird bei etwa 3\% dieses Pools vermutet, also bei ca. 40-80 mg pro Tag. Diese Rechnung stellt die Grundlage für verschiedene Empfehlungen zur angemessenen Vitamin-C-Zufuhr dar. Nach höheren Dosen steigt der Turn-over an und die Halbwertszeit nimmt ab, eine nennenswerte Speicherung erfolgt nicht (AUFDERHEIDE und RODRÍGUEZ-MARTÍN 1998, BÄSSLER et al. 2002, HODGES 1980).

Der Abbau der L-Ascorbinsäure erfolgt entweder durch Oxidation zu Dehydroascorbinsäure oder zu Oxalsäure, L-Threonsäure, L-Xylolose und Ascorbinsäure-2-sulfat. Die biologische Halbwertszeit des Vitamin C schwankt aufgrund der homöostatischen Regulation zwischen acht und 40 Tagen (BÄSSLER et al. 2002). 
Die Hauptmetaboliten des Vitamin C sind 2,3-Diketogulonsäure und Oxalsäure. Sie werden wie auch Ascorbinsäure und Dehydroascorbinsäure renal ausgeschieden, wobei Oxalsäure mit einem Anteil von ca. 40\% das Hauptausscheidungsprodukt im Harn darstellt (BÄSSLER et al. 2002, HODGES 1980, LANG 1979).

Befürchtungen bezüglich eines gehäuften Auftretens von Harnsteinen durch den Metaboliten Oxalsäure haben sich nicht bestätigt; die Konzentration im Harn steigt auch bei Überdosen Vitamin C nicht signifikant an (LANG 1979); außer bei Nierenfunktionsstörungen stellt die Oxalsäure kein Problem dar (BÄSSLER et al. 2002).

Ein weiterer im Urin zu findender Metabolit der Ascorbinsäure, das Ascorbinsäure-2-sulfat, war Gegenstand verschiedener Spekulationen. Es gab Vermutungen, dass es sich hierbei ebenfalls um eine biologisch aktive Form des Vitamin $\mathrm{C}$ handele. Als mögliche Funktion wurde die Sulfatisierung von Glykosaminoglykanen angenommen. Außerdem sollte das Ascorbinsäuresulfat die Passage von Vitamin C über die Blut-Hirn-Schranke erleichtern und über die Sulfatisierung von Cholesterol atherosklerotische Plaques löslicher machen. Allerdings konnten diese Vermutungen in Studien nicht bestätig werden; in Primaten konnte auch kein antiskorbutischer Effekt nachgewiesen werden. Man geht heute davon aus, dass es sich bei Ascorbinsäuresulfat um ein metabolisches Endprodukt handelt, das über den Urin ausgeschieden wird (HODGES 1980). 
Abb. 3: Synthese der Ascorbinsäure

modif. nach: LANG 1979, S. $588 f$

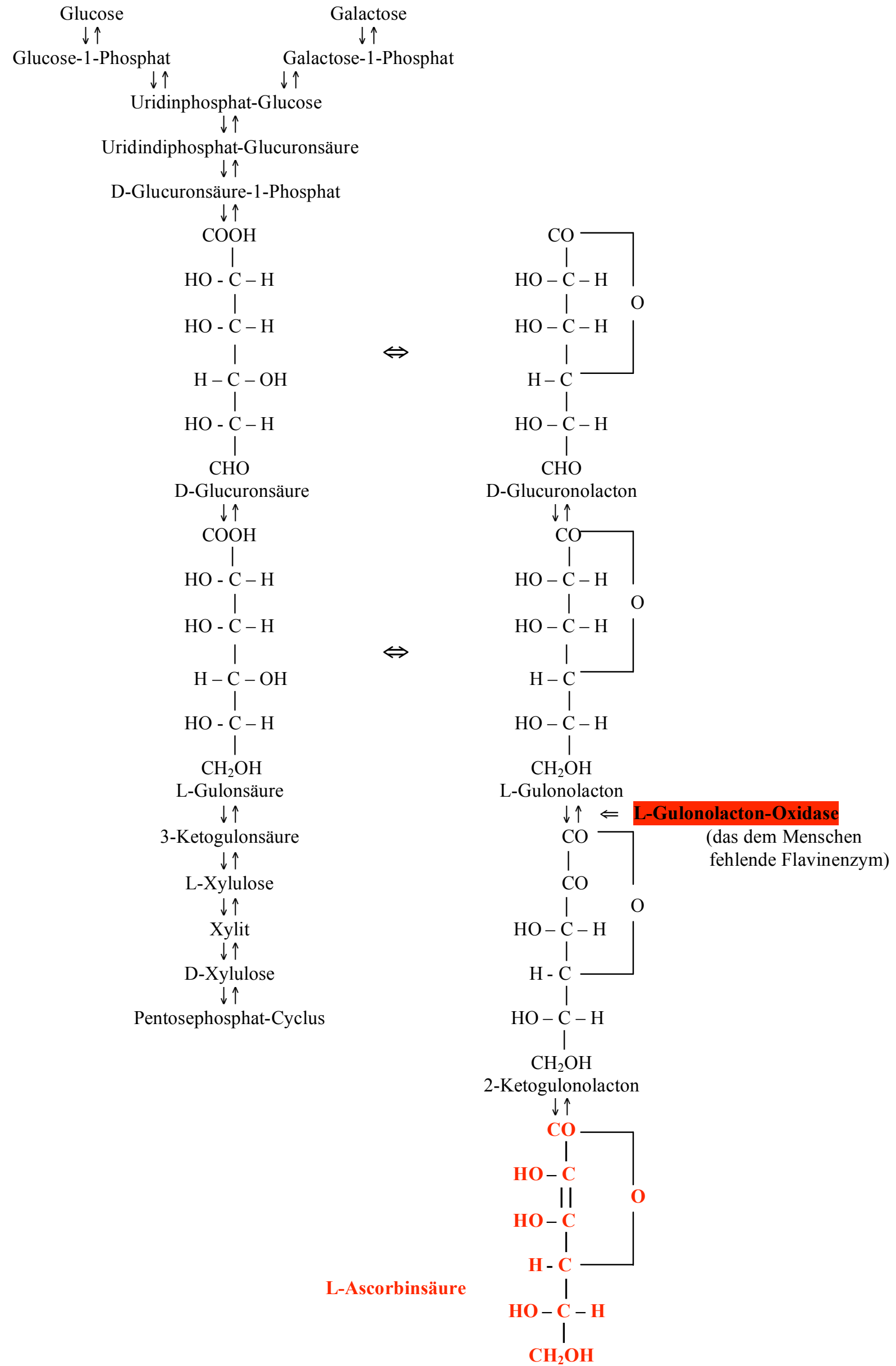




\subsection{Pathophysiologie des Skorbuts}

Ascorbinsäure, meist Vitamin C genannt, gehört zu den Substanzen, die Menschen zwingend durch ihre Ernährung zu sich nehmen müssen, da der menschliche Organismus - anders als die meisten Säugetiere - nicht in der Lage ist, Glucose über Gulonolaktonoxidat in Vitamin C umzuwandeln. Dies jedoch ist für den Körper essentiell. Es gibt viele Vermutungen über unterschiedliche Funktionen des Vitamins, beispielsweise wird seine Rolle in der Infektabwehr immer wieder diskutiert (LANG 1979). Gesichert ist die Bedeutung der Ascorbinsäure für die Kollagensynthese (s. auch Kapitel 1.4 Biochemie und Physiologie des Vitamin C).

Da Kollagen für viele Strukturen im Körper das Grundgerüst darstellt, hat die schadhafte Synthese teilweise weit reichende Folgen. Das auffälligste Symptom des Skorbuts ist die Blutungsneigung, die auf einer erhöhten Fragilität der Blutgefäße beruht. Diese wird bedingt durch den Einbau defekten Kollagens (Kollagen-Typen III, VIII, XV, XVIII) in die Gefäßwände (LÖFFLER et. al 2007). Oft wird auch von einer gestörten Synthese der extrazellulären Matrix (,Zement“) des Endothels gesprochen. HODGES (1980) vermutet jedoch, dass es sich dabei um eine Fehlinterpretation einer Studie von S. B. Wolbach und P. R. Howe aus dem Jahre 1926 handelt, in der eigentlich das defekte Kollagen beschrieben sei, die jedoch zu dem Missverständnis führe, dass es eine nicht genauer definierte extrazelluläre Kittsubstanz - eben den „Zement“ - gebe, für deren Synthese Vitamin C benötigt werde. Laut Hodges lässt sich dafür in elektronenmikroskopischen Untersuchungen kein Anhalt finden (HODGES 1980). Die mechanische Instabilität führt schon bei Mikrotraumata zu Verletzungen der Gefäße und konsekutiven Hämorrhagien (AUFDERHEIDE und RODRÍGUEZ-MARTÍN 1998, JAFFE $1972^{44}$, MAAT und UYTTERSCHAUT 1984, ORTNER und ERICKSEN 1997, ORTNER et al. 1999, ORTNER et al. 2001). Besonders häufig finden sich subperiostale Blutungen, die sich sowohl durch Verletzung der zahlreichen periostalen Gefäße als auch durch Eröffnung der Module des roten Knochenmarks in der Spongiosa im Rahmen von Infraktionen (MAAT und UYTTERSCHAUT 1984) erklären lassen.

Auf zellulärer Ebene führt der Vitamin-C-Mangel zu einer Herabsetzung der Osteoblastenaktivität. Das bewirkt eine verlangsamte Ossifikation der enchondralen Wachstumszonen, was insbesondere bei Kindern Wachstumsverzögerungen und Knocheninstabilität zur Folge hat (AUFDERHEIDE und RODRÍGUEZ-MARTÍN 1998).

${ }^{44}$ S. 453 
Diese Veränderungen können auch histologisch im Übergang zwischen Knochensubstanz und enchondraler Wachstumszone beobachtet werden, wo sich ein typisches Bild mit modifizierten Osteoblasten und desorganisiertem Knorpel- und Knochengewebe bietet $\left(\right.$ JAFFE $\left.1972^{45}\right)$.

Nach Ausheilung der Krankheit werden auch Wachstumsschübe beobachtet (BARLOW $1883^{46}$ ). Es kann durch Störungen der Knochenbildung und subperiostale Blutungen im Bereich der Epiphysenfugen zu Epiphysendislokationen kommen (BARLOW 1883 ${ }^{47}$ ), die sich radiologisch nachweisen lassen (JAFFE $1972^{48}$ ).

Die Resorption durch die Osteoklasten dagegen ist nicht beeinträchtigt, sondern kann sogar gesteigert sein, was zu einer Reduktion der Knochensubstanz von Substantia corticalis und spongiosa führt, wodurch das Trabekelwerk und die Wände ausgedünnt erscheinen und der Knochen brüchig wirkt (BARLOW 1883 ${ }^{49}$ ). Im Mikroskop lassen sich vermehrt Howship'sche Lakunen nachweisen (MAAT und UYTTERSCHAUT 1984).

Die mechanische Instabilität führt bei kleinen Traumata zu Infraktionen, also Frakturen der Spongiosabälkchen und der Corticalis, welche die Kontinuität des Knochens nicht unterbrechen. Im Bereich dieser Frakturen fehlen Zeichen von Reparaturprozessen wie Callusbildung (MAAT und UYTTERSCHAUT 1984), was sich auch durch die herabgesetzte Osteoblastenaktivität erklären lässt.

Im Tierversuch mit Meerschweinchen, die ebenso wie Menschen nicht in der Lage sind, Vitamin C zu synthetisieren, zeigt sich, dass unter Ascorbinsäuremangel die Odontoblasten die Dentinsynthese stoppen, sodass die Zähne zeitweise zu wachsen aufhören (JAFFE $1972^{50}$ ). Dies ähnelt dem Phänomen der bei Menschen beobachteten (transversalen) Schmelzhypoplasien, das im Kapitel 4.2 Skorbutische Läsionen am Schädel $\rightarrow$ Transversale Schmelzhypoplasien beschrieben wird.

\footnotetext{
${ }^{45}$ S. 453 , Seite 456

${ }^{46}$ S. 167

${ }^{47}$ S. 167

${ }^{48}$ S. 460

${ }^{49}$ S. 186

${ }^{50}$ S. 453
} 


\subsection{Therapie des Skorbuts}

Die Behandlung des Skorbuts besteht in Vitamin-C-Substitution. Dies kann sowohl in Form künstlich hergestellter Ascorbinsäure oder in Form von Saft, z. B. von Zitrusfrüchten oder Tomaten, erfolgen. Vorteil der reinen Ascorbinsäure ist die Möglichkeit der oralen, intravenösen oder intramuskulären Applikation, was bei geschwächten Individuen oder Hinweisen auf eine Beeinträchtigung der intestinalen Resorption nützlich sein kann. Außerdem ist hiermit eine bessere Kontrolle der tatsächlich zugeführten Vitamin-C-Menge möglich. Eine Zufuhr von 500-600 mg/d (JAFFE 1972 ${ }^{51}$ ) sollte in wenigen Wochen zu einer Normalisierung der Laborwerte führen, wobei für eine klinische Heilung selbst so geringe Dosen wie $10 \mathrm{mg} / \mathrm{d}$ ausreichen (LANG 1979).

Eine klinische Besserung tritt oft schon 48 Stunden nach Therapiebeginn ein, Symptomfreiheit ist meist nach drei bis vier Wochen erreicht. Die vollständige Ausheilung der Skeletveränderungen kann Jahre dauern (HIRSCHMANN und RAUGI 1999, JAFFE 1972).

Während der Therapie sollte besonderes Augenmerk auf der Vermeidung von Superinfektionen liegen, da diese oft komplikationsreich sind. Wenn eine Anämie vorliegt, kann eine orale Eisensubstitution erfolgen. Solange Symptome bestehen, müssen die Patienten äußerst vorsichtig bewegt werden, um Schmerzen und weitere Blutungen zu vermeiden.

Im Anschluss an eine Therapie sollte auf eine Ernährung geachtet werden, bei der die empfohlene Tagesdosis von $25 \mathrm{mg} / \mathrm{d}$ zugeführt wird (JAFFE $1972^{52}$ ).

\subsection{Empfehlungen zur täglichen Vitamin-C-Aufnahme}

Die minimale Vitamin-C-Dosis, die am Tag zugeführt werden muss, damit kein Skorbut entsteht, liegt bei ca. 10 mg/d (HODGES 1980, BÄSSLER et al. 2002).

Über die optimale tägliche Vitamin-C-Dosis herrscht Uneinigkeit (BÄSSLER et al. 2002, HODGES 1980). Es gibt Verfechter einer täglichen Zufuhr im Grammbereich (darunter der populäre Nobelpreisträger Linus Pauling), die ihre Forderungen u. a. auf die hypothetische Syntheserate von Vitamin C durch den Menschen, wenn er über das Enzym Gulonolactonoxidase (s. Abb. 2 in Kapitel 1.4 Biochemie und Physiologie des Vitamin C) verfügen würde, stützen. Ausgehend von Tierversuchen vermutet man eine Syntheserate von täglich $2-4 \mathrm{~g}$, unter Stressbedingungen sogar 15 g (BÄSSLER et al. 2002). Es gibt jedoch auch Warnungen

\footnotetext{
${ }^{51}$ S. 464, S. 466

${ }^{52}$ S. 464, S. 466
} 
vor möglichen schädlichen Wirkungen von Vitamin C bei Überdosierung (HODGES 1980). Gefährdet sind Menschen mit eingeschränkter Nierenfunktion, bei denen die renale Ausscheidung der Ascorbinsäure und möglicherweise über kompetitive Blockade der Transportsysteme auch die Ausscheidung anderer harnpflichtiger Substanzen eingeschränkt sein kann (vgl. WEISE PRINZO 1999).

Ein Benefit bezüglich der Infektionsprophylaxe durch Vitamin-C-Megadosen konnte bisher nicht nachgewiesen werden (WEISE PRINZO 1999).

Die DACH-Referenzwerte ${ }^{53}$ basieren auf der Überlegung, wie ein Gesamtkörper-Pool von etwa 1500 g erreicht werden kann, der auch bei Phasen ascorbinsäurefreier Ernährung ein Auftreten von Mangelsymptomen für ca. zwei Monate verhindert. Dies wäre bei ca. $50 \mathrm{mg} / \mathrm{d}$ erreicht. Aus präventiven Überlegungen wird eine durchschnittliche Zufuhr von $100 \mathrm{mg} / \mathrm{d}$ empfohlen (s. Tab. 2). In den USA liegen die Empfehlungen bei 75 mg für Frauen und $90 \mathrm{mg}$ für Männer. Da Raucher signifikant erniedrigte Vitamin-C-Werte haben, wird ihnen eine höhere Zufuhr empfohlen. Schwangeren und stillenden Müttern wird ebenfalls eine erhöhte Aufnahme von Vitamin C nahegelegt, da man davon ausgeht, dass der Bedarf im dritten Trimenon der Schwangerschaft gesteigert ist bzw. dass die Frau augrund des vergleichsweise hohen Vitamin-C-Gehalts der Muttermilch (ca. $65 \mathrm{mg} / \mathrm{l}$ ) sonst selbst unterversorgt sein könnte. Außerdem wirkt sich Ascorbinsäuregabe günstig auf den in der Schwangerschaft oft zu hohen Histaminspiegel aus (BÄSSLER et al. 2002, WEISE PRINZO 1999).

Es konnte ein Zusammenhang zwischen dem Vitamin-C-Plasmaspiegel stillender Mütter und dem Vitamin-C-Gehalt der Muttermilch nachgewiesen werden. Bei einem mütterlichen Mangel sinkt auch die Vitamin-C-Sekretion in die Muttermilch (SALMENPERÄ 1984, SALMENPERÄ et al. 1988). Allerdings scheint es hier eine Verteilung zugunsten des Kindes zu geben, weil die bei gestillten Kindern gemessenen Vitamin-C-Plasmaspiegel auch bei sinkender Sättigung der Mutter noch im Normalbereich liegen (SALMENPERÄ 1984, SALMENPERÄ et al. 1988).

Neugeborene haben eine ca. doppelt so hohe Vitamin-C-Konzentration wie ihre Mütter, da eine aktive Sekretion in die Plazenta stattfindet. Trotz dieses hohen Ausgangswertes steigt der Plasmaspiegel voll gestillter Kinder weiter an. Dabei ist die Konzentration des Vitamin C in der Muttermilch unabhängig vom produzierten Milchvolumen (SALMENPERÄ 1984).

53 „DACH-Referenzwerte: Neuauflage der Referenzwerte für Nährstoffzufuhr, die die deutschen Empfehlungen ablösen sollen. Sie wurden erstmals 2000 von den Gesellschaften für Ernährung in Deutschland (D), Österreich (A) und der Schweiz (CH) gemeinsam herausgegeben.“ (aus: BÄSSLER et al. 2002, S. 686) 
Nach langer Stillphase sinkt der Vitamin-C-Gehalt der Muttermilch. Besonders hoch ist der Vitamin-C-Gehalt im Colostrum (Vormilch), nach etwa zwei Monaten sinkt er deutlich und fortan kontinuierlich (SALMENPERÄ 1984). Die Vitamin-C-Abgabe in die Muttermilch scheint aktiv reguliert zu werden, da bis zu 48 fach höhere Werte in der Milch als im mütterlichen Plasma gemessen werden. Es gibt sogar Vermutungen, dass stillende Mütter auf bisher nicht bekannte Art und Weise Vitamin C synthetisieren können (SALMENPERÄ 1984).

Eine zusätzliche Vitamin-C-Einnahme gut ernährter stillender Frauen führt nicht zu einer Steigerung der Sekretion in die Muttermilch über das normale Maß hinaus (SALMENPERÄ 1984, SALMENPERÄ et al. 1988). Besonders gefährdet für einen Vitamin-C-Mangel sind stillende Mütter ca. zwei Monate postpartal, weil in dieser Phase die Ausschüttung in die Muttermilch besonders hoch ist, und im Frühjahr, vermutlich weil die Reserven durch die geringere Verfügbarkeit im Winter aufgebraucht sind (SALMENPERÄ 1984).

SALMENPERÄ (1984) berichtet nur über ein gestilltes Kind, bei dem ein zu niedriger Vitamin-C-Plasmaspiegel nachgewiesen wurde, in diesem Fall hatte die Mutter aufgrund eines atopischen Ekzems eine annähernd Vitamin-C-freie Kost zu sich genommen.

Dieser Fall lässt vermuten, dass Säuglinge manifest skorbutischer Frauen auch einen relevanten Mangel entwickeln können, obwohl die Umverteilungsvorgänge zugunsten des Kindes dies vermutlich lange verhindern (siehe auch Kapitel 1.2 Das Krankheitsbild des Skorbuts, 1.4 Biochemie und Physiologie des Vitamin C und 1.8 Vorkommen von Vitamin C). Auch Infektionserkrankungen steigern den Vitamin-C-Bedarf, sodass eine zusätzliche Zufuhr empfehlenswert erscheint.

Um eine vollständige Gewebssättigung (Body-Pool ca. $3000 \mathrm{mg}$ ) zu erreichen, wären Vitamin-C-Dosen von ca. $200 \mathrm{mg} / \mathrm{d}$ erforderlich. Allerdings erfolgt bei einer so hohen Zufuhr keine vollständige Resorption mehr. Auch bei Tieren, die selbst Ascorbinsäure synthetisieren können, wird keine Gewebssättigung nachgewiesen. Sie scheint also für eine normale Funktion des Organismus nicht notwendig zu sein (BÄSSLER et al. 2002).

Für die Skorbutprävention in Krisenregionen wird empfohlen, mindestens $30 \mathrm{mg}$ Vitamin C pro Tag zu gewährleisten (WEISE PRINZO 1999). 


\begin{tabular}{|l|l|}
\hline Alter & Vitamin C mg/d \\
\hline Säuglinge & \\
\hline 0-4 Monate & 50 \\
\hline 4-12 Monate & 55 \\
\hline Kinder & \\
\hline 1-4 Jahre & 60 \\
\hline 4-7 Jahre & 70 \\
\hline 7-10 Jahre & 80 \\
\hline 10-13 Jahre & 90 \\
\hline 13-15 Jahre & 100 \\
\hline Jugendliche & 100 \\
\hline Erwachsene & 100 \\
\hline Raucher & 150 \\
\hline Schwangere & 110 \\
\hline Stillende & 150 \\
\hline
\end{tabular}

Tab. 2: DACH-Referenzwerte aus dem Jahr 2000 für die empfohlene tägliche Vitamin-C-Zufuhr (nach: BÄSSLER et al. 2002, S. 253)

\subsection{Vorkommen von Vitamin C}

Die meisten höheren Pflanzen und Tiere können Vitamin C aus Glucose synthetisieren (für den Syntheseweg siehe Kapitel 1.4 Biochemie und Physiologie des Vitamin C); daher findet sich das Vitamin sowohl in tierischen als auch in pflanzlichen Nahrungsprodukten. Allerdings speichern Tiere nur geringe Mengen von Ascorbinsäure, weshalb tierische Produkte, mit Ausnahme von Leber und Nieren, keine guten Quellen für den Menschen darstellen (HODGES 1980, BÄSSLER et al. 2002).

Tabelle 3 gibt den Vitamin-C-Gehalt einiger geläufiger Lebensmittel wieder. Dabei muss man allerdings beachten, dass Ascorbinsäure aufgrund ihrer Sauerstoff- und Lichtempfindlichkeit sowie ihrer Unbeständigkeit in Gegenwart von Schwermetallen und in alkalischen Medien nur bedingt lagerungsfähig ist. Durch längere Lagerungszeiten kann der Vitamin-C-Gehalt um bis zu 75\% sinken (BÄSSLER et al. 2002).

Bei Grüngemüsen werden nach der Ernte Blattoxydasen freigesetzt, die für einen Großteil der Vitamin-C-Verluste beim Lagern verantwortlich sind (KÜBLER 1965). Weitere Enzyme in pflanzlichen Produkten, die Ascorbinsäure zerstören, sind Ascorbasen, Polyphenolasen und Peroxidasen, wobei die beiden letzteren nicht direkt die Ascorbinsäure angreifen, sondern über die Bildung von Chinonen (ZELLWEGER und ADOLPH 1954).

Ein weiteres Problem stellt die Zubereitung der Speisen dar: Beim Schälen wird ausgerechnet der Vitamin-C-reichste Teil der meisten Obst- und Gemüsesorten entfernt, lange Koch- und 
Warmhaltezeiten führen zu weiterer Zerstörung. Der Vitamin-C-Verlust durch Lagerung und Zubereitung kann bis zu 100\% betragen (BÄSSLER et al. 2002).

Heutzutage gibt es Techniken, Nahrungsmittel schonend zu lagern (z.B. durch Einfrieren) und zuzubereiten, außerdem kann durch Schutzsubstanzen ein Vitamin-C-Verlust vermieden werden. Man kann sich aber gut vorstellen, dass bei den Möglichkeiten, die den Menschen in vergangenen Zeiten zur Verfügung standen, Nahrungsmittel insbesondere für den Winter zu konservieren, kaum eine ausreichende Vitamin-C-Sättigung erreicht werden konnte.

\begin{tabular}{ll}
\hline & $\begin{array}{l}\text { Vitamin-C-Gehalt } \\
\text { ( } \mathbf{m g / 1 0 0 g )}\end{array}$ \\
\hline Gemüse & \\
Paprika (gelb) & 294 \\
Paprika (rot) & 150 \\
Paprika (grün) & 139 \\
Broccoli & 47 \\
Grünkohl & 47 \\
Feldsalat & 30 \\
Blumenkohl & 30 \\
Kohlrabi & 25 \\
Tomate & 22 \\
Spinat & 21 \\
Weißkohl & 19 \\
Kartoffel & 14 \\
Obst & \\
Johannisbeere & 180 \\
Kiwi & 80 \\
Zitrone & 61 \\
Apfelsine & 50 \\
Stachelbeere & 35 \\
Pflaume & 5 \\
Birne & 4 \\
Milch & \\
Vollmilch & $2^{*}$ \\
Fleisch & \\
Rinderleber & 25 \\
Schweineleber & 23 \\
Fisch & \\
Lachs & 3 \\
Forelle & 2 \\
\hline
\end{tabular}

Tab. 3: Vitamin-C-Gehalt verschiedener Lebensmittel (nach: BÄSSLER et al. 2002, S. 246)

\footnotetext{
* Es gibt unterschiedliche Angaben zum Vitamin-C-Gehalt der verschiedenen Lebensmittel. BÄSSLER et al. (2002) geben einen höheren Vitamin-C-Gehalt für Kuhmilch an als ELMADFA et al. (1989), SALMENPERÄ et al. (1988) finden höhere Werte in der Muttermilch.
} 


\section{Vitamin-C-Gehalt der Muttermilch}

Der Vitamin-C-Gehalt der menschlichen Muttermilch ist höher als bei den meisten anderen Säugern, da Kinder anders als Jungtiere das fürs Wachstum in großen Mengen benötigte Vitamin C nicht synthetisieren können.

Dabei variiert der Vitamin-C-Gehalt abhängig von der Stilldauer. Besonders hoch ist er im Colostrum (Vormilch), nach etwa zwei Monaten sinkt er deutlich und fortan kontinuierlich (SALMENPERÄ 1984). Tabelle 4 a zeigt die sinkenden Werte.

Früher war es ein großes Problem, Säuglinge, die nicht gestillt werden konnten (z.B. weil die Mutter peripartal verstorben war), adäquat zu versorgen. Tabelle $4 b$ zeigt, dass der VitaminC-Gehalt von Kuhmilch nicht ausreicht, um den Bedarf eines Säuglings zu decken. Zwar stellen Schafs-, Ziegen- und Stutenmilch diesbezüglich eine bessere Alternative dar, allerdings unterscheiden sich die unterschiedlichen Milchsorten auch in der sonstigen Zusammensetzung, insbesondere in den Fett-, Protein- und Kohlenhydratanteilen, deutlich von Muttermilch (ELMADFA 1989), sodass fraglich ist, ob die Verträglichkeit für Säuglinge überhaupt ausreichend ist. Vermutlich war für verwaiste Säuglinge eine Amme früher die einzige wirkliche Überlebenschance (siehe auch Kapitel 1.2 Das Krankheitsbild des Skorbuts, 1.4 Biochemie und Physiologie des Vitamin C und 1.7 Empfehlungen zur täglichen VitaminC-Aufnahme). Der Vitamin-C-Gehalt in der Milch einer Amme hängt dabei natürlich vom Abstand zur Geburt des eigenen letzten Kindes ab.

\begin{tabular}{|l|l|}
\hline Dauer der Stillzeit & Vitamin-C-Gehalt der Muttermilch $\mathbf{( m g / 1 0 0 m I )}$ \\
\hline 0 Monate & 6,22 \\
\hline 2 Monate & 5,93 \\
\hline 4 Monate & 4,97 \\
\hline 6 Monate & 4,68 \\
\hline 7,5 Monate & 4,68 \\
\hline
\end{tabular}

Tab. 4a: Vitamin-C-Gehalt der Muttermilch in Abhängigkeit von der Laktationsdauer. Durchschnittswerte aus einer Studie mit 47 Frauen (nach: SALMENPERÄ et al. 1988)

\begin{tabular}{|l|l|l|l|l|l|}
\hline & Muttermilch & $\begin{array}{l}\text { Kuhmilch } \\
(\mathbf{3 , 5} \% \text { Fett })\end{array}$ & Schafmilch & Stutenmilch & Ziegenmilch \\
\hline $\begin{array}{l}\text { Vitamin-C-Gehalt } \\
\text { pro } \mathbf{1 0 0} \mathbf{~ m g}\end{array}$ & $4 \mathrm{mg}^{*}$ & $1 \mathrm{mg}^{*}$ & $4 \mathrm{mg}$ & $15 \mathrm{mg}$ & $10 \mathrm{mg}$ \\
\hline
\end{tabular}

Tab. 4b: Vitamin-C-Gehalt verschiedener für den Menschen verzehrsfertiger Milchsorten im Vergleich (nach: ELMADFA et al. 1989)

* Es gibt unterschiedliche Angaben zum Vitamin-C-Gehalt der verschiedenen Lebensmittel. BÄSSLER et al. (2002) geben einen höheren Vitamin-C-Gehalt für Kuhmilch an als ELMADFA et al. (1989), SALMENPERÄ et al. (1988) finden höhere Werte in der Muttermilch. 


\subsection{Nachweis von Skorbut an historischen Skeleten}

Man vermutet, dass sich Skorbut bei Kindern schneller als bei Erwachsenen am Skelet manifestiert, da die hohe Wachstumsaktivität den Mangel an Vitamin C für die Kollagensynthese und Osteoblastentätigkeit schneller deutlich werden lässt. Deshalb konzentrieren sich viele Autoren auf Kinder und Jugendliche bei der Untersuchung historischer Skelete auf Skorbut (BRICKLEY und IVES 2006, LEWIS 2002, MELIKIAN und WALDRON 2003, ORTNER und ERICKSEN 1997, ORTNER et al. 1999, ORTNER et al. 2001, SCHULTZ et al. 2007, SCHULTZ und SCHMIDT-SCHULTZ 2013, WEIHMANN 2010). Es gibt jedoch auch Nachweise von Skorbut an Skeleten von Erwachsenen (MAAT 2004). In der vorliegenden Arbeit sollen ausdrücklich sowohl adulte Individuen als auch Kinder untersucht werden.

Im Wesentlichen gibt es zwei Formen von Veränderungen am Knochen, die auf Skorbut zurückgeführt werden.

Zum einen kann an bestimmten Regionen eine erhöhte Porosität der Knochenoberfläche nachgewiesen werden. Diese ist definiert durch lokal begrenzte feine Gefäßforamina, die ohne Vergrößerungsglas sichtbar sind, im Durchmesser typischerweise weniger als einen Millimeter betragen und die lamelläre Knochenoberfläche penetrieren. Dabei kann es sich bei der porösen Struktur um die normale Knochenoberfläche oder um eine Auflagerung handeln (ORTNER und ERICKSEN 1997, ORTNER et al. 1999, ORTNER et al. 2001, SCHULTZ 1990). Diese Porosität wird auf die bei Skorbut auftretenden Hämorrhagien mit folgender chronischer Inflammation und die dadurch bedingte erhöhte Vaskularisierung zurückgeführt (BRICKLEY und IVES 2006, ORTNER and ERICKSEN 1997, ORTNER et al. 1999, ORTNER et al. 2001). Differentialdiagnostisch ist diese Porosität abzugrenzen von der porösen Hyperostose, die auf einer Hyperplasie des hämatopoetischen Knochenmarks beruht (ORTNER und ERICKSEN 1997) sowie von postmortaler Zerstörung im Sinne der Diagenese.

Die zweite sichtbare Veränderung ist die reaktive Knochenneubildung, makroskopisch erkennbar als feinporöse Auflagerung. Sie wird zurückgeführt auf subperiostale Hämatome, die durch Läsionen der skorbutös geschädigten Blutgefäße entstehen. Das Abheben des Periosts von der Knochenoberfläche stellt einen Wachstumsreiz für Osteoblasten dar, es kommt zur reaktiven Knochenneubildung (BARLOW $1883^{54}$, ORTNER et al. 1999, ORTNER et al. 2001, SCHULTZ et al. 2007, SCHULTZ 2011, WEIHMANN 2010).

\footnotetext{
${ }^{54}$ S. 186
} 
BRICKLEY und IVES (2006) beschreiben eine gesteigerte Porosität an der Lamina externa des Os parietale und an der Lamina interna des Os occipitale, an letzterem auch Auflagerungen (BRICKLEY und IVES 2006). Auch BARLOW (1883) weist bei Autopsien poröse Knochenoberflächen unter subperiostalen Hämatomen am Os parietale nach (BARLOW $1883^{55}$ ).

Neben den Veränderungen am Schädel finden verschiedene Autoren erhöhte Porosität und Hyperostosen im Bereich der Fossa infra- und supraspinata der Scapula, wo die Blutgefäße ähnlich wie in der Temporalregion unterhalb der Muskulatur dicht am Knochen verlaufen (BARLOW 1883, BRICKLEY und IVES 2006, ORTNER et al. $2001^{56}$ ).

Auch an den Metaphysen der Langknochen werden poröse Läsionen nachgewiesen (ORTNER et al. 2001). BARLOW (1883) beschreibt bei Autopsien ausgeprägte subperiostale Hämatome im Bereich der Langknochen, teilweise mit darüber liegenden Knochenneubildungen und Epiphysendislokationen (BARLOW 1883 ${ }^{57}$, siehe auch FRAENKEL 1908).

MAAT (2004) kann an den durch Kälte hervorragend konservierten Überresten niederländischer Seefahrer auf Spitzbergen sogar schwarze Verfärbungen an Langknochen und Zahnwurzeln erkennen, in denen er denaturiertes Hämoglobin nachweist. Außerdem findet er zahlreiche Infraktionen (MAAT 2004).

MELIKIAN und WALDRON (2003) legen die von ORTNER (ORTNER und ERICKSEN 1997, ORTNER et al. 1999, ORTNER et al. 2001) und seinen verschiedenen Koautoren aufgestellten diagnostischen Kriterien zugrunde, um Schädel aus verschiedenen Grabungen in Großbritannien und Peru auf Skorbut zu untersuchen; wo es möglich ist, fügen sie bei Befall des Schädels die Untersuchung des Postcraniums hinzu. Anschließend vergleichen sie die Befunde mit Skeleten aus dem Museum of the Royal College of Surgeons, bei denen intravital die Diagnose Skorbut gestellt worden war, darunter Originalfälle von Barlow. MELIKIAN und WALDRON (2003) finden bei der Mehrzahl der untersuchten Schädel erhöhte Porositäten, können jedoch an den Postcranien keine pathologischen Veränderungen nachweisen und stellen beim Vergleich mit den klinisch bekannten Skorbutfällen fest, dass keinerlei Übereinstimmung in Verteilung und Ausprägung der Veränderungen vorliegt. Sie kommen daher zu dem Schluss, dass die Diagnosekriterien unzulänglich sind, und bezweifeln, dass eine eindeutige Diagnosestellung Skorbut in paläopathologischen Untersuchungen

\footnotetext{
${ }^{55}$ S. 169, S. 182, S. 188

${ }^{56}$ S. 168, S. 184, S. 188

${ }^{57}$ BARLOW 1883 S. 167, S. 168, S. 180, S. 183, S. 184, S. 185, S. 186, S. 187f, FRAENKEL 1908 S. 2f, S. 17f, S. 20-25
} 
überhaupt möglich ist (MELIKIAN und WALDRON 2003). BRICKLEY und IVES (2006) halten dagegen, dass MELIKIAN und WALDRON (2003) klinisch stark ausgeprägte Fälle zur Grundlage ihres Vergleichs nehmen. Sie vermuten, dass es sich bei den von ORTNER (ORTNER und ERICKSEN 1997, ORTNER et al. 1999, ORTNER et al. 2001) und seinen verschiedenen Koautoren beschriebenen und von MELIKIAN und WALDRON (2003) vielfach nachgewiesenen Veränderungen um eine schwächere Ausbildung des Skorbuts handelt, weshalb sie nicht die deutlichen Merkmale der klinisch bekannten Fälle aufweisen (BRICKLEY und IVES 2006).

Ein bisher in paläopathologischen Untersuchungen nicht allzu beachteter Hinweis auf Skorbut liegt in der Veränderung des Knochenaufbaus, bei dem die Corticalis ausgedünnt und das Trabekelwerk reduziert erscheinen (FRAENKEL 1908 ${ }^{58}$ ). Auch hierauf soll bei der vorliegenden Studie ein Augenmerk gelegt werden.

Zusätzlich $\mathrm{zu}$ den typischerweise mit Skorbut assoziierten Merkmalen soll auch eine Untersuchung auf unspezifische Stressindikatoren wie transversale Schmelzhypoplasien (siehe auch Kapitel 1.5 Pathophysiologie des Skorbuts und Kapitel 4.2 Skorbutische Läsionen am Schädel $\rightarrow$ Transversale Schmelzhypoplasien) der Zähne und Harris-Linien (siehe auch Kapitel 4.3 Skorbutische Läsionen am Postcranium $\rightarrow$ Harris-Linien) an den Langknochen erfolgen.

\subsection{Differentialdiagnosen}

Es gibt einige Differentialdiagnosen für die beschriebenen Veränderungen der Knochenoberfläche, die stets in Erwägung gezogen werden sollten.

Oft ist es sehr schwierig, eine pathologisch gesteigerte Porösität vom physiologischen Remodeling während des normalen Wachstums bei Kindern abzugrenzen (BRICKLEY and IVES 2006, ORTNER et al. 1999, ORTNER et al. 2001). Auch unter dem Mikroskop ist die Unterscheidung zwischen hämorrhagischen Auflagerungen und physiologischem appositionellen Wachstum oft schwierig (SCHULTZ 1986).

Osteomyelitis kann ebenfalls reaktive Knochenneubildung durch Periostabhebung hervorrufen, dies wird vor allem an den Diaphysen der unteren Extremität beobachtet (AUFDERHEIDE und RODRÍGUEZ-MARTÍN 1998 59 ).

Anämie führt zu porösen Oberflächenveränderungen und Hyperostosen am Schädeldach, vor allem durch Cribra orbitalia und Cribra cranii externa kann es zu Verwechslungen mit

\footnotetext{
${ }^{58}$ S. 19 f, S. 26

${ }^{59}$ Seite 173
} 
Skorbut kommen (SCHULTZ 1986, 2001, 2003, 2011, SCHULTZ et al. 2007). Allerdings beruht die Knochenbildung bei der Anämie auf einer Hypertrophie des Knochenmarks (ORTNER und ERICKSEN 1997, ORTNER et al. 2001), sodass zumindest mikroskopisch eine Abgrenzung möglich sein sollte (SCHULTZ 1986, 2001, 2003, 2011, WAPLER et al. 2004, WEIHMANN 2010). Denn beim Skorbut erfolgt die Auflagerung auf die - in der Regel - gesunde Knochenoberfläche, die unter dem Mikroskop auch noch als solche zu erkennen ist (SCHULTZ 2001, 2003, 2011, WEIHMANN 2010).

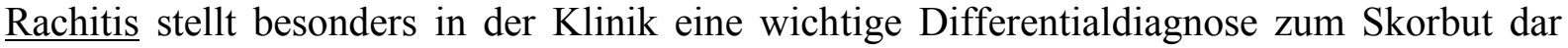
und wurde - wie im Kapitel 1.1 Geschichte des Skorbuts dargestellt - lange Zeit als zugrunde liegende Erkrankung gewertet. Sie kann ebenfalls zu Auflagerungen am Orbitadach und am äußeren Schädeldach führen (SCHULTZ 2001).

\section{Meningeale Reizung}

Viele Formen der meningealen Reizung können zu porösen Oberflächenveränderungen und Auflagerungen an der Lamina interna des Schädeldachs führen. Bakterielle und hämorrhagische Meningitiden, epidurale Hämatome (durch Traumata, z. B. auch Geburtstraumata), Irritationen der venösen Sinus und Neoplasien können makroskopisch den skorbutischen Läsionen ähnliche Bilder erzeugen, die ohne mikroskopische Untersuchung schwer zu unterscheiden sind (SCHULTZ 1990, 2001, 2003, 2011, SCHULTZ et al. 2007).

Syphillis (congenita)

Syphillis wird vor allem in der älteren Literatur häufig als Differentialdiagnose zum Skorbut erwähnt (BARLOW 1883, FRAENKEL $1908^{60}$ ).

Möglichkeiten zur Abgrenzung der Differentialdiagnosen werden in der Diskussion (Kapitel 4.5 Differentialdiagnosen) erörtert.

\subsection{Untersuchungsmethoden in der Literatur}

FRAENKEL (1908) und JAFFE (1972) fertigen mikroskopische Präparate von Autopsiefällen an, in denen sie subperiostale Blutungen, Knochenneubildungen und die „Trümmerfeldzone“ - einen mit Detritus verfüllten Bereich der epiphysennahen Diaphysen an Langknochen und Rippen - sowie Hämorrhagien ins Periost der Orbita mit Ausbildung knöcherner Auflagerungen nachweisen (FRAENKEL 1908, JAFFE 1972 ${ }^{61}$ ).

Einige Untersucher verwenden ein Rasterelektronenmikroskop, zum Beispiel, um die Tiefe der Löcher der porösen Strukturen zu überprüfen (BRICKLEY und IVES 2006, ORTNER et al. 2001).

\footnotetext{
${ }^{60}$ BARLOW 1883: S. 190, FRAENKEL 1908: S. 33

${ }^{61}$ FRAENKEL 1908: S. 16, JAFFE 1972: S. 450
} 
M. Schultz betont die Wichtigkeit der Lichtmikroskopie an Knochendünnschliffen nicht entkalkter rezenter und (prä-)historischer Knochenproben zur Abgrenzung der Differentialdiagnosen (SCHULTZ 1986, 2001, 2011).

G. J. R. Maat und H. T. Uytterschaut fertigen lichtmikroskopische Präparate, an denen ihnen durch immunoenzymatische Färbungen der Nachweis von denaturiertem Hämoglobin an den Resten subperiostaler Hämatome gelingt (MAAT 2004, MAAT und UYTTERSCHAUT 1984).

Im Kapitel 2.2 Methoden erfolgt eine Vorstellung der in dieser Arbeit verwendeten Methoden. 


\section{Material und Methoden}

\subsection{Material}

Es wurden insgesamt 143 Individuen untersucht. Davon stammen 134 aus Barbing, die übrigen wurden der Arbeitsgruppe Paläopathologie Göttingen im Rahmen eines DAADMÖB-Projekts für diese Arbeit von der Universität Szeged zur Untersuchung zur Verfügung gestellt.

Im Folgenden sollen die unterschiedlichen Populationen kurz vorgestellt werden.

\section{a) Das Gräberfeld der frühmittelalterlichen Siedlung von Barbing-Kreuzhof (Oberpfalz)}

Das Gräberfeld wurde 1975 bei den Bauarbeiten für die Großkläranlage der Stadt Regensburg entdeckt. Im Anschluss wurden die Gräber 1-4 (Grabungsfläche „W“ Abb. 4) durch D. Klonk (Bayerisches Landesamt für Denkmalpflege) geborgen. Im Jahr 1976 wurden die übrigen Skelete durch das Bayerische Landesamt für Denkmalpflege ausgegraben. Sie lagen auf einem Friedhof, der zu der im Ostteil gelegenen Kirche gehört (Grabungsfläche „B“ Abb. 4). Eine archäologische Untersuchung des Friedhofs erfolgte durch GEISLER (1984). Er datiert die Gräber in das vierte bis fünfte, eventuell das sechste Jahrhundert nach Christus.

Durch das Museum der Stadt Regensburg erfolgte eine grobe Reinigung der Skelete, wobei es vereinzelt zu Verwechslungen der ursprünglichen Grabnummern kam (DETKEN 1991).

Eine erste anthropologische Untersuchung durch Schultz legt einen gallo-romanischen bzw. keltischen Hintergrund der Bevölkerung nahe (DETKEN 1991).

Die Skelete wurden zu osteologischen Untersuchungen ins Zentrum für Anatomie der GeorgAugust-Universität in Göttingen und waren bereits Bestandteil verschiedener Arbeiten (z.B. DETKEN 1991, SCHLOMM 2000).

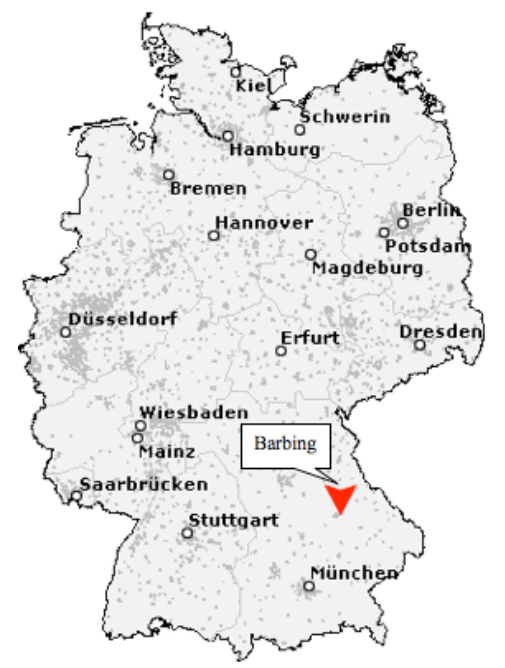

Abb. 4: Lage des heutigen Barbing

(Quelle: http://www.postleitzahl.org/bayern/images/karte_barbing.png, Stand 13.12.2011, modifiziert) 

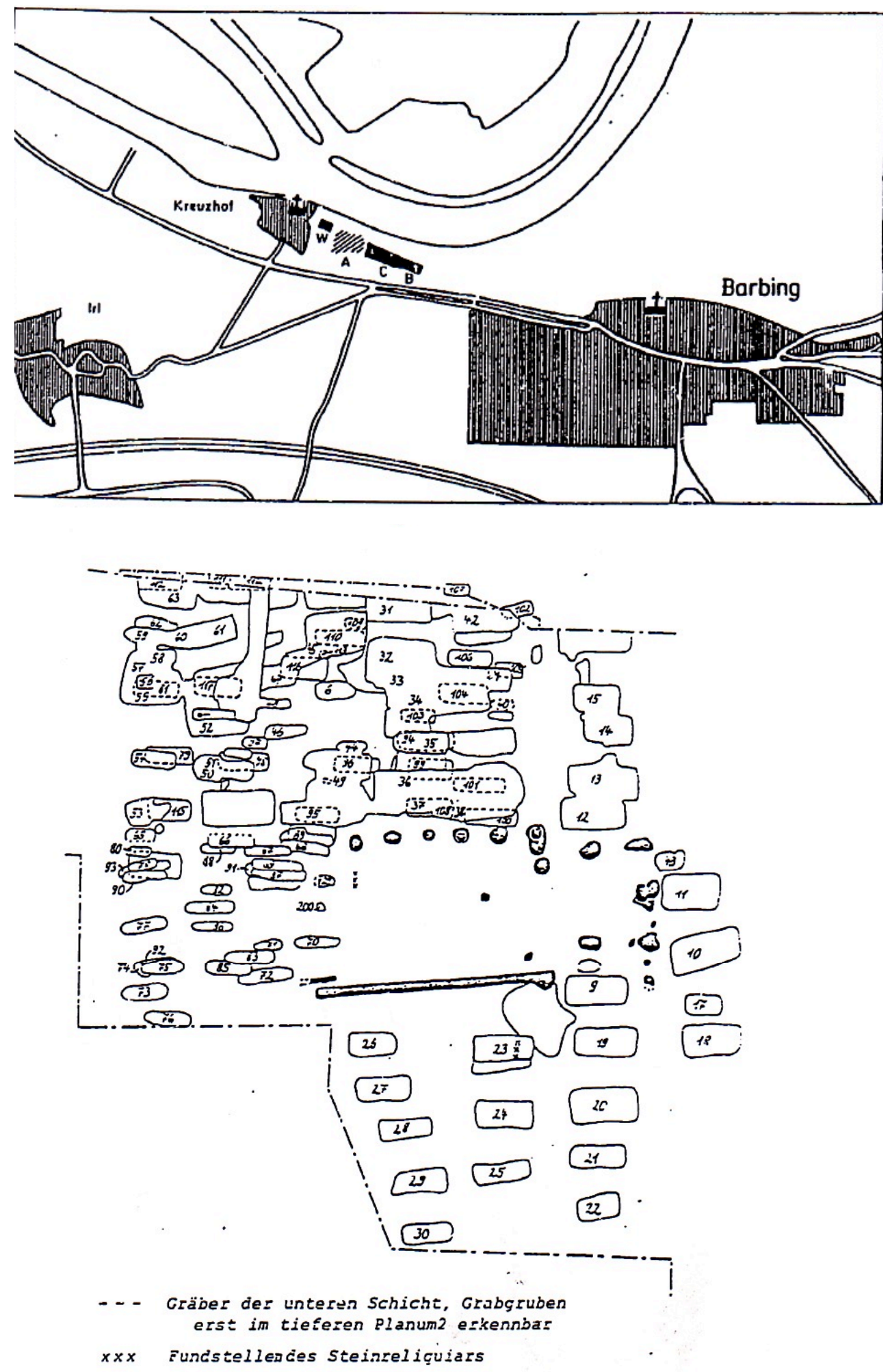

Abb. 5 a (oben): Lage der Grabungsflächen von Barbing-Kreuzhof. Die untersuchten Skelete entstammen den Grabungsflächen W und B. Das weiße Kreuz bei B markiert eine abgegangene Kirche.

Abb. 5 b (unten): Gräberfeldplan der Grabung von Barbing-Kreuzhof.

Übernommen von DETKEN 1991 (S. 6, S. 8) 


\section{b) Das Gräberfeld Bácsalmás-Óalmás aus dem 16.-18. Jahrhundert (Ungarn)}

Im Rahmen des DAAD-MÖB-Projekts Nr. 32 wurden der Autorin sechs Skelete dieser ca. 480 Individuen umfassenden Population zur Bearbeitung zur Verfügung gestellt. Sie waren ausgewählt worden, weil in früheren Untersuchungen der Verdacht auf Skorbut bestand.

Der Ort Bácsalmás liegt im südlichen Bereich der Donau-Theiß-Wasserscheide. In einem Vorort namens Óalmás fand ein Archäologe des Türr István Museums von Baja 1964 eine mittelalterliche Kirche und Teile eines sie umgebenden Friedhofs.

Zwei Kilometer von dieser Grabungsstätte entfernt fand Erika Wicker, Archäologin des Katona József Museums Kecskemét, 1993 weitere Gräber in einer Sandmine. Sie barg und untersuchte alle 480 Individuen. Der Friedhof war vom späten 16. Jahrhundert bis zum frühen 18. Jahrhundert in Gebrauch, also zur Zeit der türkischen Besatzung; vermutlich wurde er von mindestens drei Generationen genutzt. Anders als zu der Zeit üblich, wurde trotz sehr guten Erhaltungszustands des Friedhofs keine Kirche gefunden. Dafür zeigten die Gräber Spuren islamischer Bestattungsriten. Daher wird vermutet, dass der Friedhof von Muslimen oder einer durch die Türken beherrschten ethnischen Gruppierung genutzt wurde.

Die Gräber sind nur in einer Lage angeordnet und ost-west-orientiert. Es handelt sich ausschließlich um Einzelgräber. Die meisten Gräber haben eingelassene Cineraria oder seltener Seitennischen. Die Verstorbenen wurden meist nur in Leichentücher gewickelt, Särge sind selten. Es wurden nur wenige Grabbeigaben gefunden, darunter Bronzenadeln, Muschelschalen, Bronzeketten, eiserne Verschlüsse für Kleidung, Kleinigkeiten aus Silber und Bronze und Knöpfe aus Tierknochen.

Die Skelete werden am Department of Anthropology der Universität in Szeged, Ungarn, untersucht (MARCSIK und BERECZKI 2008).

c) Das Awaren-Gräberfeld von Sükösd-Ságod aus dem 7. Jahrhundert (Ungarn)

Im Rahmen des DAAD-MÖB-Projekts Nr. 32 wurden der Autorin zwei Individuen mit Verdacht auf Skorbut aus dieser Population zur Bearbeitung vorgelegt.

Das Gräberfeld befindet sich ca. 14 km vom heutigen Ort Sükösd entfernt im Bereich eines ca. 20 m hohen Löss-Hügels. Zwischen 1967 und 1969 wurden etwa 170 Gräber des südwestlichen Teils ausgegraben. Vermutlich handelt es sich hierbei nur um ca. 20 Prozent des gesamten Friedhofs. Die ausgehobenen Gräber waren in drei großen Gruppen angeordnet, wobei die Frauengräber einen Kreis um die Kindergräber bildeten, während die Männergräber ein Stück abseits lokalisiert waren. Die Verstorbenen wurden meist in Leichentüchern bestattet. Auch wenn typische awarische Dekorationen fehlen, wird das Gräberfeld in die Awarenzeit datiert, es wird vermutet, dass es zwischen 640 und 660 nach Christus angelegt 
und dann für etwa drei Generationen genutzt wurde. Anthropologische und archäologische Untersuchungen wurden durch KÖHEGYI und MARCSIK (1971) durchgeführt.

Weitere Ausgrabungen wurden zwischen 1979 und 1981 durch Székely und Wicker vorgenommen, sodass insgesamt 396 früh- und spätawarische Gräber freigelegt wurden.

Als Grabbeigaben fanden sich Waffen (Schwert, Lanzenspitze, Bogen, Streitaxt), aus Blech gepresste und gegossene Gürtelbeschläge, Löffel und eine Kette aus Bronze, Schmuck (Ohrgehänge mit Kugelanhängern, Augenperlen) und eine große geschliffene Glaskristallperle (SZENTPÉTERI 2002).

\section{d) Der Schädel eines Kindes aus Kiszombor aus dem 11.-12. Jahrhundert (Ungarn)}

Dieser Schädel aus dem Bestand der Universität Szeged stammt aus dem südungarischen Ort Kiszombor. Er wurde im Rahmen einer Grabung durch Ferenc Móra im Sommer 1928 geborgen, bei dem 227 Schädel von Individuen unterschiedlicher Epochen ausgegraben wurden. Dieser Kinderschädel wird auf die Arpadenzeit (11. bis 12. Jahrhundert nach Christus) datiert (MOLNÁR 2014).

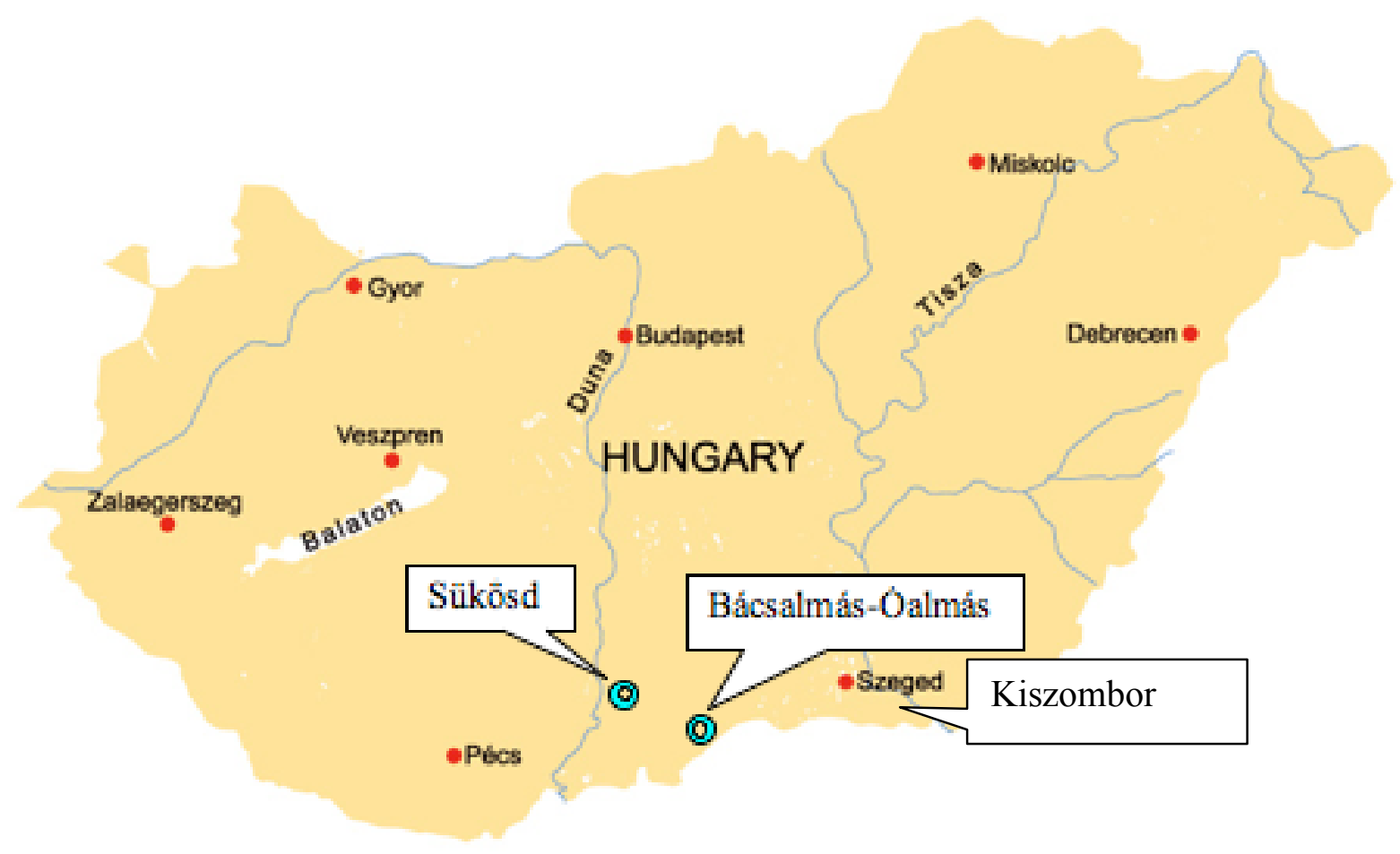

Abb. 6: Lage der ungarischen Gräberfelder (Quelle: http://www.weltatlas.info/front/pics/Landkarten/landkarteungarn-gross.jpg, Stand 13.12.2011, modifiziert). 


\subsection{Methoden}

\section{a) Vorarbeit}

Zur makroskopischen Beurteilung wurden die einzelnen Knochenteile bei Bedarf vorsichtig gereinigt und in den anatomischen Zusammenhang gelegt. Auf das Zusammenkleben von Fragmenten wurde verzichtet, um eine Verfälschung von lichtmikroskopischen und radiologischen Untersuchungen zu vermeiden. Der Schädel Kiszombor aus der Sammlung der Universität Szeged war schon im Vorfeld geklebt worden.

\section{b) Dokumentation des Bestands}

Zur einheitlichen Erfassung des Bestands wurden basierend auf Zeichnungen aus dem „Taschenatlas der Anatomie“ (PLATZER 1991) verschiedene Befundbögen entwickelt.

Dabei wurden die erhaltenen Langknochen und Schädel sowohl zeichnerisch als auch tabellarisch erfasst.

Grau eingezeichnete Strukturen sind erhalten, grau schraffierte Bereiche sind zwar erhalten, durch Zerstörung der Oberfläche jedoch nur eingeschränkt beurteilbar. Weiß belassene Bereiche sind nicht erhalten.

Spuren pathologischer Prozesse wurden möglichst genau eingezeichnet und in den entsprechenden Tabellen beschrieben. Dabei wurden Auflagerungen auf die ursprüngliche Knochenoberfläche rot eingezeichnet, Bereiche mit gesteigerter Porosität blau. Rot bzw. blau schraffierte Bereiche bedeuten, dass man zwar pathologische Strukturen ausmachen kann, die Oberfläche jedoch nur unvollständig erhalten ist. Auffällige Gefäßimpressionen wurden ebenfalls blau eingezeichnet.

Die Bögen erfassen nur Lang- und Schädelknochen, da hier die Wahrscheinlichkeit für skorbutische Läsionen am höchsten ist. Selbstverständlich wurde jedoch das gesamte Skelet untersucht. Fielen dabei an anderen Strukturen Veränderungen auf, so wurden diese zusätzlich vermerkt und die Befundbögen um die in der Arbeitsgruppe Paläopathologie üblichen Übersichtsbögen ergänzt.

Beispielhafte Befundbögen finden sich im Anhang S. 281-282.

\section{c) Fotografie}

Alle erhobenen Befunde wurden fotografisch dokumentiert. 


\section{d) Altersbestimmung}

Die Altersbestimmung der Barbinger Skelete wurde großenteils von DETKEN (1991) und SCHLOMM (2000) durchgeführt. Bei einigen Individuen ergab sich die Notwendigkeit einer erneuten Altersbestimmung, welche nach dem Zahndurchbruchsschema von UBELAKER (1978) und anhand der Diaphysenlänge der Langknochen nach STLOUKAL und HANÁKOVÁ (1978) unter Berücksichtigung der Empfehlungen von FEREMBACH et al. (1979) und SZILVÁSSY (1988) erfolgte. Abweichungen von den vorherigen Untersuchungen ergaben sich bei Sk 1371 und Sk 1372.

Die Altersbestimmung der ungarischen Skelete erfolgte im Rahmen dieser Arbeit ebenfalls anhand der oben angeführten Methoden.

\section{e) Geschlechtsbestimmung}

Die Geschlechtsbestimmung der Barbinger Skelete wurde von DETKEN (1991) und SCHLOMM (2000) übernommen. Bei Individuen von unter 12 Jahren wurde keine Geschlechtsbestimmung durchgeführt. Daher entfiel diese bei den ungarischen Skeleten. Im Befundteil wird weiblich mit $\mathrm{w}$, männlich mit $\mathrm{m}$ abgekürzt, $\mathrm{m}=\mathrm{w}$ bedeutet, dass keine eindeutige Geschlechtszuweisung möglich ist.

\section{f) Makroskopische Untersuchung}

Alle Skelete wurden makroskopisch mit Hilfe einer Lupe (von Krüss, 18mm Triplet 10x) untersucht. Bei Unklarheiten oder sehr kleinen Strukturen wurde ein Lupenmikroskop mit maximal 50facher Vergrößerung (ZEISS, M5) verwendet.

Dabei wurde an Langknochen und Schädeln gezielt nach Oberflächenveränderungen wie feinporösen Knochenauflagerungen oder gesteigerter Porosität gesucht, die einen Hinweis auf subperiostale Hämatome im Rahmen einer Skorbuterkrankung darstellen. Wurden derartige Veränderungen gefunden, wurde das entsprechende Skelet auch radiologisch und lichtmikroskopisch untersucht.

Alle Schädel wurden auf das Vorhandensein von Cribra orbitalia, also auf poröse Knochenoberflächen beziehungsweise -auflagerungen im Bereich des Orbitadaches, untersucht. Dabei wurde zur Befundung die folgende Einteilung nach SCHULTZ (1988) verwendet:

Grad I: Leichte beginnende Veränderungen in Form einzelner, feinporöser Lochdefekte.

Grad II: Auffällig poröses Orbitadach; deutlich ausgeprägte Lochdefekte treten miteinander in Verbindung; geringgradige, d. h. sehr flache Vorwölbung der porösen Knochen- 
neubildungen.

Grad III: Kräftiges trabekuläres Wachstum aus der Diploë des Orbitadaches in die Augenhöhle.

\section{g) Endoskopie}

Von außen nicht befundbare Hohlräume, z. B. vollständig erhaltene Schädel oder pneumatisierte Räume des Schädels, wurden mit einem Stabendoskop (Volpi) untersucht.

\section{h) Radiologische Untersuchung}

Von allen Individuen mit Verdacht auf Skorbut wurden ausgewählte Knochen mit dem Vollschutz-Röntgengerät Faxitron (Hewlett Packard) durchleuchtet. Bei besonderen Befunden wurden auch Röntgenbilder angefertigt. Die Bedeutung der radiologischen Untersuchung für die Paläopathologie legt SCHULTZ $(1987,1993)$ dar.

Bei der radiologischen Untersuchung wurden folgende in der Literatur beschriebenen Phänomene gesucht:

Laut FRAENKEL (1908) lassen sich bei skorbutischen Langknochen verschiedene Formen der Läsionen nachweisen, die jedoch nicht immer vorliegen. Dazu zählen parallel zum Schaft verlaufende Schatten, die subperiostalen Hämatomen entsprechen (FRAENKEL 1908 ${ }^{62}$ ). Selbstverständlich können diese am archäologischen Knochenfund nur erwartet werden, wenn eine Knochenneubildung erfolgte (und diese nicht postmortal zerstört wurde), da die Weichteile nicht erhalten sind. Solche Neubildungen sind sowohl bei adulten Individuen als auch bei Kindern radiologisch beschrieben (JAFFE $1972^{63}$, KÜBLER 1965, ZELLWEGER und ADOLPH 1954).

Außerdem wurden die Langknochen auf die von FRAENKEL (1908) beschriebene und als sehr charakteristisch erachtete „Trümmerfeldzone“ im endständigen Diaphysenbereich untersucht, wobei sich die Frage stellte, inwieweit dieses Phänomen, das FRAENKEL (1908) auf ein „wirres Durcheinander von regellos angeordneten Kalkbälkchen, schmächtigen Knochentrabekeln , Kalk- und Knochentrümmern, sowie von mit Blut- und Pigmentmassen durchsetztes Gerüstmark“ (FRAENKEL 1908 ${ }^{64}$ ) zurückführt, am mazerierten Knochen nachweisbar ist. Die Wahrscheinlichkeit hierfür wird dadurch eingeschränkt, dass dieses Phänomen bei beginnender Heilung schnell schwindet (FRAENKEL $1908^{65}$ ). Dennoch gibt

\footnotetext{
${ }^{62}$ S. $15 f$

${ }^{63}$ S. 460

${ }^{64}$ S. 16

${ }^{65}$ S. 18
} 
es diesbezügliche paläopathologische Schilderungen (AUFDERHEIDE und RODRÍGUEZMARTÍN 1998).

JAFFE (1972) beschreibt eine im Röntgenbild erkennbare Ausdünnung der Corticalis und Rarifizierung der Trabeculae im Bereich der Metaphysen, die er als „Milchglas-Atrophie“ bezeichnet (s. auch CAFFEY 1973, JAFFE 1972, KÜBLER $1965{ }^{66}$ ). Diese Veränderungen können auch am mazerierten Knochen erkennbar sein (AUFDERHEIDE und RODRÍGUEZMARTÍN 1998).

Beim ausgeheilten Skorbut kann man im Röntgenbild noch über Jahre ein grob strukturiertes Band im Bereich der ehemaligen Trümmerfeldzone, die „Skorbut-Linie““, erkennen, weil das „Remodeling“ lange dauert und neu gebildeter Knochen den Bereich zunehmend von der Wachstumsplatte separiert (AUFDERHEIDE und RODRÍGUEZ-MARTÍN 1998, CAFFEY 1973, ORTNER und PUTSCHAR 1981 ${ }^{67}$, TEMPLIN 1993). Auch dies könnte bei historischen Skeleten nachweisbar sein.

Ein weiteres radiologisches Zeichen für Skorbut ist der sogenannte „Wimberger Ring“, ein radiodenser Doppelring, verursacht durch Osteoporose der sekundären Ossifikationszentren, umgeben von vermehrt mineralisiertem Knorpel in der wachsenden Peripherie (KÜBLER 1965, LEWIS 2002, ORTNER 2003, ORTNER und PUTSCHAR $1981^{68}$, ZELLWEGER und ADOLPH 1954).

Im Metaphysenbereich kann es zu einer Spornbildung an den seitlichen Ecken der Metaphysenabschlüsse kommen; dieses so genannte „corner sign“ wird ebenfalls auf Skorbut zurückgeführt (CAFFEY 1973, CARLI-THIELE 1996, KÜBLER 1965, TEMPLIN 1993, ZELLWEGER und ADOLPH $1954^{69}$, WEIHMANN 2010).

J. Caffey beschreibt eine Eindellung der Metaphysenplatten als Folge von Epiphysendyslokationen, die über Monate radiologisch zu erkennen bleibt (CAFFEY 1973).

Des Weiteren erfolgte eine Untersuchung der Langknochen auf Infraktionen und Harris-

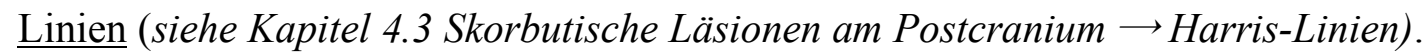

\section{i) Lichtmikroskopie}

Am archäologischen Skeletfund kann man in der Regel keine Weichteile befunden und keine zytologischen Untersuchungen durchführen, doch zumindest die mineralisierten und auch einige organische Strukturen wie Kollagen lassen sich noch beurteilen. Aufgrund dieser

\footnotetext{
${ }^{66}$ S. 458

${ }^{67}$ S. 272

${ }^{68}$ S. 272

${ }^{69}$ S. 188
} 
Unterschiede zwischen rezenten und paläopathologischen Präparaten ist es nötig, eigene Diagnosekriterien für die Paläopathologie zu entwickeln (SCHULTZ 1986, 1987, 1988a, 1988b, 1993, 2001, 2011).

Die Lichtmikroskopie erlaubt eine detaillierte Betrachtung der unterschiedlichen Gewebsschichten; in der Tiefe gelegene Strukturen können sichtbar gemacht werden. Sehr hilfreich ist sie auch bei der Beurteilung von Knochenneubildungen und Auflagerungen (WAPLER et al. 2004, SCHULTZ 2011).

Ein weiterer Vorteil der mikroskopischen Untersuchung ist die Möglichkeit, Veränderungen in einem Stadium zu beobachten, in dem makroskopisch noch kaum Auffälligkeiten zu erkennen sind (SCHULTZ 1986, 1987, 1988a, 1993, 2001).

Durch den Einsatz von polarisiertem Licht und eines Hilfsobjekts Rot 1. Ordnung (Quarz, $\lambda-$ Plättchen) als Kompensator kann vor allem die Kollagenstruktur des mazerierten Knochens gut beurteilt werden. Besonders deutlich wird hier die Ausrichtung und Anordnung der Kollagenfasern, was Aussagen über den Organisationsgrad des Knochens ermöglicht. Dabei ist von Vorteil, dass das Kollagen in großen Mengen an den Knochenapatit gebunden und auch in archäologischen Knochen meist gut erhalten ist (SCHULTZ 1986, 1987, 1988a, 1993, 2011, WAPLER et al. 2004).

In der mikroskopischen Untersuchung können proliferative und resorptive Prozesse unterschieden werden. Die Anzahl und Verteilung von Howship'schen Lakunen kann dabei Aufschluss über die Natur eines osteoklastischen Geschehens geben (SCHULTZ 2001, 2011). Darüber hinaus lassen sich auch diagenetisch eingebrachte Strukturen wie Kristalle oder florale und faunale Residuen, besonders im polarisierenden Licht, gut erkennen und abgrenzen (SCHULTZ 1986, 2001, TEMPLIN 1993, WAPLER et al. 2004).

Sind nur einzelne Knochenfragmente erhalten, erlaubt die Analyse der Knochenstruktur unter dem Mikroskop eine Unterscheidung zwischen menschlichen und tierischen Knochen (SCHULTZ 1986).

Die Struktur des Knochens kann auch Aufschlüsse über das Alter des Individuums erlauben; so findet man beispielsweise bei Säuglingen typischerweise Schalenknochen (SCHULTZ 1986, 2001). Der Organisationsgrad einer Neubildung kann Auskunft über die Dauer des Bestehens der Veränderung geben; sind beispielsweise Auflagerungen faserknöchern organisiert, sind sie jüngeren Alters, als wenn sie bereits zu Lamellenknochen umgebaut sind. Auf eine rasterelektronenmikroskopische Untersuchung wird in dieser Arbeit verzichtet, da sie häufig für die Differentialdiagnose weniger hilfreich ist als die Lichtmikroskopie. 


\section{- Anfertigung der Präparate für die Lichtmikroskopie}

Für die mikroskopische Untersuchung archäologischer Skeletfunde ist die Anfertigung von geeigneten Präparaten erforderlich. Im Falle der Population aus Barbing konnte dabei großenteils auf bereits bestehende Präparate aus den Arbeiten von SCHLOMM (2000) und DETKEN (1991) zurückgegriffen werden. Für die ungarischen Skelete mussten sämtliche Präparate neu angefertigt werden.

Dafür wurde das von M. Schultz und M. Brandt entwickelte Verfahren genutzt (CARLITHIELE 1996, GRESKY 2008, SCHULTZ 1986, 1988a, 1993, 2001, 2011). Dies besteht aus folgenden Schritten:

1. Dokumentation

Da für die Anfertigung eines Präparats ein Stück Knochen entnommen wird, muss vorher eine ausführliche Dokumentation des intakten Knochens erfolgen. Dies geschieht durch Zeichnungen im Rahmen der Befundbögen, Messungen (v.a. bei Langknochen), Fotografie, Röntgenaufnahmen und, wenn nötig, endoskopische Aufnahmen.

2. Probeentnahme

Aus dem zu untersuchenden Knochen wird ein Stück herausgesägt. Dabei empfiehlt es sich, jeweils einen Teil pathologischen und einen Teil gesunden Gewebes mit anzuschneiden, um eine bessere Vergleichbarkeit zu erzielen. Am besten eignen sich vollständige Querschnitte. Ziel ist es, zwei Dünnschliffe unterschiedlicher Schliffstärke (üblicherweise 50 bzw. $70 \mu \mathrm{m}$ ) aus unterschiedlichen Ebenen der Knochenprobe zu gewinnen, um Veränderungen in allen drei Dimensionen beurteilen zu können.

Wenn es - beispielsweise zur Stabilitätssicherung oder aus sammlungskonservatorischen Gründen - notwendig ist, das zurückbleibende Loch im Knochen zu verfüllen, kann dies unter anderem durch Gips oder einen originalgetreuen Abguss erfolgen.

\section{Einbettung}

Wenn nötig, erfolgt zunächst eine Dehydrierung durch Einbringen der Proben in die aufsteigende Alkoholreihe. Anschließend werden sie in Dichlormethan eingelegt, das als Intermedium dient. Als Einbettungssubstanz hat sich das Epoxidharz Biodur®, entwickelt durch VON HAGENS (1979), bewährt. Die Plastination beruht auf der Dampfdruckdifferenz zwischen Dichlormethan und Epoxidharz. Die in Dichlormethan eingelegten Proben geben im Exsikkator Dichlormethan ab und nehmen dafür Epoxidharz auf.

Anschließend muss der Kunststoff aushärten. 


\section{Anfertigung der Dünnschliffe}

Die entstandenen Kunststoffblöckchen werden auf eine geeignete Größe getrimmt und auf Objektträger gebracht. Danach werden sie auf 50 bzw. $70 \quad \mu \mathrm{m}$ Schliffdicke heruntergeschliffen und mit einem Deckglas abgedeckt. Dünnere Schliffe, wie in der histopathologischen Untersuchung rezenter Knochen üblich, bei denen auch das Gewebe mitbeurteilt wird, sind nicht empfehlenswert, da dann in der Regel die Kollagenstrukturen im polarisierten Licht nicht mehr gut zu erkennen sind (SCHULTZ und ROBERTS 2002).

Der gesamte Präparationsprozess dauert ca. sechs Wochen.

\section{- Untersuchung am Lichtmikroskop}

Die Untersuchung der Dünnschliffe erfolgte zunächst am Universal-Sichtgerät nach Kraus/Lanooy mit 10,5- bis 48facher Vergrößerung. Die mikroskopische Untersuchung erfolgte am Polarisationsmikroskop LEICA-DM-RXP, an welchem auch die Fotos angefertigt wurden. Die Präparate wurden im einfachen und polarisierenden Durchlicht mit einem Hilfsobjekt Rot 1. Ordnung als Kompensator betrachtet.

\section{- Befundung der lichtmikroskopischen Präparate}

Zur Befundung der Knochendünnschliffpräparate wurde in Anlehnung an die Literatur eine einfache Klassifizierung entwickelt, in welche die Oberflächenveränderungen, entsprechend ihrer vermutlichen Ätiologie, eingeordnet wurden. Es wurden fünf Typen von Auflagerungen/Oberflächenveränderungen gewählt, welche die häufigsten Differentialdiagnosen für die makroskopischen Veränderungen darstellen. Im Folgenden werden die unterschiedlichen Typen und die zu erwartenden histologischen Phänomene erläutert. Die Reihenfolge der Durchnummerierung ist zufällig und erlaubt keine Rückschlüsse über Häufigkeit oder Wichtigkeit der Erkrankung beziehungsweise des pathologischen Prozesses. Lediglich aus praktischen Gründen wurde die bei Skorbut auftretende Auflagerung als Typ I bezeichnet.

Es ist zu beachten, dass durchaus verschiedene Formen der Veränderungen, beispielsweise hämorrhagische und entzündliche Prozesse, zusammen auftreten können (SCHULTZ 2001).

\section{Typ I - Hämorrhagische Auflagerung (unter anderem bei Skorbut)}

Bei Skorbut lassen sich Auflagerungen an der Knochenoberfläche nachweisen, die auf subperiostale Hämatome zurückzuführen sind. Bei diesen Auflagerungen ist die darunter liegende Lamina externa beziehungsweise die äußere Generallamelle intakt; auch die Substantia compacta ist in der Regel unverändert. 
Es lassen sich unterschiedliche Ausprägungen der Auflagerungen beobachten, je nachdem, ob es sich um kleinflächige Veränderungen, den Randbereich oder um einen Anschnitt mitten in einer großen Auflagerung handelt.

Im Randbereich oder in kleinen Läsionen erkennt man eine sehr dünne Schicht neu gebildeten Knochens, die auf die ehemalige Lamina externa beziehungsweise auf die äußere Generallamelle aufgelagert ist (SCHULTZ 2001, SCHULTZ 2011).

Großflächige starke Auflagerungen bestehen im Zentrum aus radial von der ehemaligen Knochenoberfläche abgehenden kurzen plumpen Trabekeln mit brückenförmigen Deckplatten, welche die neue Knochenoberfläche darstellen (MAAT und UYTTERSCHAUT 1984, SCHULTZ 2001, 2011, SCHULTZ und SCHMIDT-SCHULTZ 2013).

Auch der Nachweis einer Mehrphasigkeit der Erkrankung ist möglich. So lassen sich manchmal mehrere Lagen von Auflagerungen vom Typ I erkennen. Dies ist auf die Bildung neuer Hämatome auf der älteren Knochenneubildung, vermutlich im Rahmen eines neuen Krankheitsschubs, zurückzuführen (SCHULTZ 2001, MAAT 2004).

Bei der endgültigen Diagnosestellung sollte daran gedacht werden, dass auch andere Blutungsursachen als Skorbut ein solches organisiertes Hämatom hervorrufen können, z.B. Traumata (SCHULTZ 2001).

An der Lamina interna lassen sich ebenfalls hämorrhagische Prozesse nachweisen, insbesondere in den Impressiones digitatae, aber auch großflächig und strukturübergreifend. Auch am Schädel liegen die Neubildungen auf dem ursprünglichen Knochen auf, ohne dass dieser sich verändert. Allerdings kann es durch den erhöhten Schädelinnendruck zu einer Atrophie der Lamina interna kommen. Bei Kindern erfüllt die Dura mater die Aufgaben des Periosts und reagiert sehr ähnlich, sodass die mikroskopische Differenzierung unterschiedlicher Ätiologien genauso wie am Postcranium möglich ist (SCHULTZ 2001, 2011).

SCHULTZ (2001) unterscheidet drei Stadien der Organisation dieser Hämatome:

Beginnendes Remodeling: Es finden sich fleckförmige umschriebene Stellen mit verzweigten Blutgefäßimpressionen.

Fortgeschrittenes Remodeling: Poröse Platten von neugebildetem Knochen bedecken die oben beschriebenen Blutgefäßimpressionen. Bei ausgeprägten Hämatomen können die Neubildungen recht dick sein.

Weit fortgeschrittenes Remodeling: Man erkennt kleine Gruppen von schuppenförmigen feinen Knochenplättchen, die durch ausgeprägte netzartig ausgebreitete Impressionen kleiner Blutgefäße getrennt werden (SCHULTZ 2001). 
Der neugebildete Knochen ist zunächst faserknöchern und wird im Verlauf des Heilungsprozesses in Lamellenknochen umgewandelt.

Auch am äußeren Schädeldach lassen sich organisierte Hämatome finden. Makroskopisch erscheinen sie als poröse Hyperostose, die auch bei vielen Erkrankungen (z. B. entzündlichen Prozessen, bei Hämatomen nach Traumata, tumorösen Erkrankungen, vielen Anämieformen, Rachitis) zu sehen ist. Deshalb ist eine lichtmikroskopische Differenzierung wichtig. Bei Skorbut sind diese Hämatome oft deutlich ausgeprägt und können auch sehr groß sein (SCHULTZ 2001).

In vielen Präparaten von Skeleten mit Verdacht auf Skorbut lässt sich eine osteoklastische Aktivität in Form von Howship'schen Lakunen nachweisen (MAAT und UYTTERSCHAUT 1984).

Auch Infrakturen der Langknochen lassen sich erkennen, wenn sie bei der Präparation mit angeschnitten werden. Hinweise auf Reparaturvorgänge fehlen (MAAT und UYTTERSCHAUT 1984).

FRAENKEL (1908) und JAFFE (1972) beschreiben bei frischen Schnitten im Bereich der „Trümmerfeldzonen“ (siehe auch Kapitel 2.2h) Radiologische Untersuchung) Veränderungen der Knochenmarkstruktur, die für die Untersuchung am mazerierten Knochen nicht geeignet sind. Darüber hinaus wird in diesem Bereich eine auch nach klinischer Besserung noch lange bestehende Ausdünnung der Corticalis und eine Armut an regelrechten Trabekeln bei Persistenz irregulärer Kalkbälkchen beschrieben (FRAENKEL 1908, JAFFE 1972 ${ }^{70}$ ); diese Beobachtungen lassen sich möglicherweise auch am historischen Knochen anstellen. Am Orbitadach beschreibt FRAENKEL (1908) Knochenneubildungen, die einem Typ I zu entsprechen scheinen (FRAENKEL $1908^{71}$ ).

Zur Veranschaulichung der skorbutischen Veränderungen am Schädeldach siehe Abb. 7.b.

Typ II - Anämie

Bei einer ausgeprägten Anämie findet sich eine Hyperplasie des erythropoeitischen Knochenmarks mit aufgeweiteten Markräumen. Der Druck des Marks führt dazu, dass die Lamina externa des Schädels ausdünnt und eröffnet wird. Dadurch wird der Blick auf die Diploë freigegeben, was zum makroskopisch porösen Aspekt führt. Die neu entstehenden Hohlräume sind in Größe und Anordnung sehr regelmäßig. Insbesondere in der externen Hälfte der Diploë sind die Trabeculae vergrößert und annähernd rechtwinklig zur ehemaligen

\footnotetext{
${ }^{70}$ FRAENKEL 1908: S. 19f, S. 26, S. 34, JAFFE 1972: S. $455 \mathrm{f}$

${ }^{71}$ S. 24
} 
Knochenoberfläche angeordnet, was als „Bürstenschädel“ oder „Hair-on-end-Phänomen“ bezeichnet wird. Im fortgeschrittenen Stadium erscheint der Knochen deutlich verdickt (WAPLER et al. 2004, SCHULTZ 2001, 2011 SCHULTZ und SCHMIDT-SCHULTZ 2013). SCHULTZ (2001) unterscheidet drei Stadien bei der Entwicklung einer anämischen porösen Hyperostose: Stadium 1 ist charakterisiert durch die punktuelle Resorption der Lamina externa und das Entstehen von sekundären Foraminae, die mit den Modulen des Markraums verschmelzen. Der Knochen ist dabei nicht verdickt.

Stadium 2 weist makroskopisch eine zunehmende Porosität auf. Mikroskopisch ist die Lamina externa über große Strecken nicht mehr erhalten. Zwischen den sekundären Eröffnungen entstehen kurze plumpe Trabeculae, die aus der Diploë herauswachsen. Der Knochen ist leicht verdickt.

In Stadium 3 fallen makroskopisch eine deutliche Porosität und Verdickung des Knochens auf. Die Lamina externa ist mikroskopisch nicht mehr zu erkennen. Das „Hair-on-endPhänomen“ beziehungsweise der „Bürstenschädel“ ist voll ausgebildet (SCHULTZ 2001).

Diese Veränderungen lassen sich am Schädeldach („Bürstenschädel“), insbesondere an den Tubera frontalia und parietalia und parallel zur Lambdanaht, und an der Orbita (Cribra orbitalia) nachweisen (SCHULTZ 2011, SCHULTZ und SCHMIDT-SCHULTZ 2013, WEIHMANN 2010).

Dabei gibt es altersabhängig Unterschiede. Wie bei vielen Erkrankungen lassen sich die Knochenveränderungen aufgrund der hohen Wachstumsrate am besten bei sehr kleinen Kindern nachweisen. Auch Remodelingprozesse können das mikroskopische Bild natürlich verändern (SCHULTZ 2001, SCHULTZ 2011).

Eine Differenzierung der unterschiedlichen Anämieformen (z. B. Eisenmangelanämie versus Thaläsämie) allein anhand von Knochenschliffen ist nicht möglich (WAPLER et al. 2004).

Ein Beispiel für einen anämischen Bürstenschädel findet sich bei Abb. 7.a.

\section{Typ III - Rachitis}

Auch Rachitis kann eine poröse Hyperostose des Schädeldachs hervorrufen, die makroskopisch kaum von einer leichten, geringgradig ausgebildeten skorbutischen Läsion zu unterscheiden ist. Mikroskopisch zeigt sich, dass - anders als bei hämorrhagischen Veränderungen - alle Knochenschichten betroffen sind. Im Bereich der desintegrierten Lamina externa finden sich schuppenartige Knochenplättchen, die Spongiosa erscheint vollkommen irregulär und auch die Lamina interna ist zersplittert (SCHULTZ 2001).

Die rachitischen Veränderungen des Schädeldachs zeigt Abb. 7.c. 


\section{Typ IV - Unspezifische Entzündung}

Eine Periostitis, ausgelöst z.B. durch eine Osteitis oder eine (hämatogene) Osteomyelitis, kann makroskopisch ein sehr ähnliches Bild erzeugen wie ein hämorrhagisches Geschehen. Mikroskopisch ist die Unterscheidung in der Regel gut möglich (SCHULTZ 1987, SCHULTZ 1993, SCHULTZ 2001, SCHULTZ 2011).

Anders als bei Hämorrhagien sind die Knochenneubildungen bei entzündlichen Prozessen nicht lediglich auf den ursprünglichen Knochen aufgelagert, sondern dieser ist ebenfalls affektiert. Zumindest die Knochenoberfläche ist verändert, oft aber auch tiefere Schichten. Bei kleinen Läsionen oder im Randbereich größerer Läsionen findet sich eine dünne Schicht feiner Knochenneubildungen. Bei größeren Läsionen finden sich lange dünne Trabeculae in einer meist leicht unregelmäßigen parallelen Anordnung radial zur Knochenoberfläche. Eine Brückenbildung ist nicht sehr ausgeprägt. Wenn allerdings, durch Rezidive, eine Mehrlagigkeit entsteht, sind Brücken an den Grenzen zwischen den einzelnen Phasen häufig zu sehen (SCHULTZ 2001).

Entzündungen führen zu einer Steigerung der Vaskularisierung. Dies kann anhand von Gefäßimpressionen an der Knochenoberfläche auch an archäologischem Material nachgewiesen werden (WAPLER et al. 2004, SCHULTZ 2011).

Im Dünnschliff des mazerierten Knochens ist gelegentlich eine Spaltbildung an der Basis von Knochenneubildungen sichtbar. Diese resultiert daraus, dass das entzündlich aktivierte Periost neue Knochensubstanz bildet, die in den basalen Schichten noch nicht mineralisiert und daher am mazerierten Knochen nicht erhalten ist (SCHULTZ 1986). Bei starker Periostreaktion kann es zum Phänomen der so genannten „Totenlade“ kommen; der ursprüngliche tote Knochen liegt dann beweglich in einer Hülle aus entzündlich bedingten Knochenneubildungen (SCHULTZ 1986, SCHULTZ 2011).

Wenn der Entzündungsprozess des Periosts auch auf den Knochen übergreift (Osteitis), was bei einer chronischen Periostitis meist der Fall ist, kann dies zu ausgeprägten Resorptionsprozessen führen (SCHULTZ 2001, 2011, WAPLER et al. 2004). Dies lässt sich anhand von Howship'schen Lakunen (Resorptionshöhlen der Osteoklasten) in gut erhaltenen Knochen mikroskopisch nachweisen (SCHULTZ 2001, 2011, WAPLER et al. 2004). Parallel findet auch ein schnelles Remodeling statt. So kann es passieren, dass große Stücke der ursprünglichen Knochensubstanz noch erhalten, aber völlig von neu gebildetem Knochen umgeben sind (SCHULTZ 2001, 2011). 
Wird die Markhöhle durch den Entzündungsprozess erreicht, spricht man von einer Osteomyelitis.

Natürlich gibt es auch den umgekehrten - weit häufigeren - Ausbreitungsweg: Bei der hämatogenen Osteomyelitis beginnt der Entzündungsprozess im Mark und breitet sich von dort nach außen aus (SCHULTZ 1986, 1987, 1993, 2001, 2011).

Um festzustellen, ob es sich um ein lokal entstandenes oder hämatogen gestreutes Entzündungsgeschehen handelt, kann eine Serie von dicht aufeinander folgenden Dünnschliffen rund um das affektierte Gebiet hilfreich sein (SCHULTZ 1986).

Auch am Schädeldach sind entzündliche Veränderungen, z.B. im Rahmen einer Meningitis, Meningo-Encephalitis oder Pachymeningitis, zu beobachten, insbesondere in den Impressiones digitatae. Wie am Postcranium ist auch hier die Knochenoberfläche in jedem Fall mitbetroffen, oft auch tiefere Knochenschichten (SCHULTZ 2001, 2011). Auch der Gesichtsschädel, insbesondere der Kieferbereich und das Jochbein, ist häufig von entzündlichen Prozessen betroffen, was unter anderem durch die Nähe zu den pneumatisierten Höhlen bedingt ist (SCHULTZ 1987, 1993).

SCHULTZ (2001) unterscheidet frische, im Stadium der floriden Entzündung befindliche und bereits organisierte Läsionen: Im frischen Stadium erkennt man flache stippchen- bis plättchenförmige Knochenneubildungen, die in der Regel tangential zur Knochenoberfläche angeordnet sind. Die einzelnen Plättchen können auch $\mathrm{zu}$ einer geschlossenen Lage verschmelzen. Auch eine Mehrlagigkeit durch Rezidive ist möglich. Im Stadium der Organisation verlieren die Konturen der Neubildung an Klarheit und der plättchenförmige Charakter wird aufgehoben (SCHULTZ 2001).

Vielfach findet man Mischformen mit hämorrhagischen und entzündlichen Anteilen. Bei diesen finden sich in den Sulci der venösen Sinus kleine Blutgefäßimpressionen und kleine, flache, gestielte Platten von Knochenneubildungen (SCHULTZ 2001).

Entzündliche Veränderungen, bedingt etwa durch eine Periostitis oder Entzündungen der Kopfschwarte, können auch am äußeren Schädeldach auftreten, makroskopisch dem unspezifischen Bild einer porösen Hyperostose entsprechend. In der Regel sind Porosität und Verdickung des Knochens hier verhältnismäßig schwach ausgeprägt (SCHULTZ 2001).

Abb. 7.d liefert ein Beispiel für eine unspezifische Osteomyelitis des Schädeldachs.

\section{Typ V - Spezifische Entzündung - Lepra}

Bei einer lepromatösen Periostitis finden sich polsterartige Neubildungen, die sich teilweise schalenförmig um den Knochen legen (SCHULTZ 2001, 2011, SCHULTZ und ROBERTS 
2002). Sie sind zumeist gut organisiert, was auf langjähriges Bestehen der Erkrankung hindeutet, und scheinen langsam zu wachsen. Rezidive lassen sich in Form zusätzlicher Auflagerungen nachweisen. Außerdem kann es eine Rarifizierung der Knochensubstanz im Sinne einer Inaktivitätsatrophie und Zeichen von Sekundärinfektionen geben (SCHULTZ 2011, SCHULTZ und ROBERTS 2002).

Ein ähnliches Bild findet sich bei treponematöser Periostitis, allerdings sind dort die Polster höher und schärfer geformt. Außerdem fehlt bei Lepra in der Regel der für Treponematosen typische „Grenzstreifen“ (SCHULTZ 2001, 2011, SCHULTZ und ROBERTS 2002). BLONDIAUX et al. (2002) kommen allerdings $\mathrm{zu}$ dem Schluss, dass die corticalen Veränderungen im Bereich von Tibia und Fibula durch Syphilis und Lepra identisch sind (BLONDIAUX et al. 2002).

\section{Typ VI - Spezifische Entzündung - Syphilis}

Bei der Syphilis fallen im Mikroskop relativ starke Auflagerungen in kräftiger Polsterform und ein Verlust oder eine Ausdünnung der darunter liegenden Substantia corticalis auf (SCHULTZ 2011, SCHULTZ und SCHMIDT-SCHULTZ 2013). Diese Polster bestehen aus pfeilerartig angeordneten parallelen Lamellen von auf dem Niveau des Periosts entstehenden Knochenneubildungen, die durch die - im Rahmen der Entzündung vermehrt gebildeten Blutgefäße des Periosts voneinander getrennt werden. Durch das verhältnismäßig langsame Wachstum erscheinen die Knochenneubildungen sehr regelmäßig (SCHULTZ 2001, 2011). Außerdem entsteht offenbar nur bei Treponematosen der sogenannte „Grenzstreifen“, eine bandartige Struktur, die sich aus den ehemaligen äußeren Generallamellen und den während des ersten Entzündungsschubs entstandenen neuen Knochenlamellen zusammensetzt (SCHULTZ 2001, 2011). Die Trabeculae der Spongiosa sind in der Regel ausgedünnt und häufig breccienartig aufgebaut (SCHULTZ 2011, SCHULTZ und SCHMIDT-SCHULTZ 2013).

Typ VII - Läsionen im Rahmen zirkulatorischer Prozesse (Pierre-Marie-Bamberger$\underline{\text { Syndrom) }}$

Eine im Rahmen der hypertrophen Arthropathie (Pierre-Marie-Bamberger-Erkrankung) auftretende Periostose hat makroskopisch und mikroskopisch große Ähnlichkeit mit den Veränderungen im Rahmen hämorrhagischer Prozesse. Man findet dicke Lagen von Knochenneubildungen auf unveränderter ursprünglicher Knochenoberfläche, die symmetrisch die Diaphysen der Röhrenknochen umschließen (SCHULTZ 1986). Formt diese neue 
Substanz Trabeculae, so sind diese kurz und plump und nicht von denen eines hämorrhagischen Geschehens zu unterscheiden. Einziges Unterscheidungskriterium kann die bei hypertropher Arthropathie höhere Dichte sein. Sehr häufig wird eine Mehrlagigkeit durch Rezidive beobachtet (SCHULTZ 1986, SCHULTZ 2001).

In schweren Krankheitsfällen lässt sich im Verlauf auch ein Befall der Compacta erkennen. Mit dem Überlagern neuer Knochenschichten auf ältere - sogenannter „Baumrindencharakter“ - werden die tiefer liegenden älteren Neubildungen $\mathrm{zu}$ soliderem, nahezu kompakten Knochengewebe umgebaut. Im weiteren Verlauf kommt es $\mathrm{zu}$ einer so genannten „Spongiosierung“ zunächst der umgebauten Schichten und im weiteren Verlauf sogar der ursprünglichen Compacta. Dabei entstehen aus ehemaligen Havers'schen Kanälen durch glattwandige Knochenresorption Lochdefekte. Es wird keine echte Spongiosa gebildet, sondern die ehemals kompakten Strukturen werden aufgelockert (SCHULTZ 1986, SCHULTZ 2011).

Offenbar ist das Pierre-Marie-Bamberger-Syndrom bei Wegfall der auslösenden Noxe voll reversibel (SCHULTZ 1986, 2011).

\section{Diagenese/Pseudopathologie}

Manche scheinbar pathologischen Prozesse entpuppen sich unter dem Mikroskop als postmortale Veränderungen. Ein Hinweis darauf ist die Desintegration von Kollagenfasern ohne erkennbare Reaktion des Knochengewebes (SCHULTZ 1986, WAPLER et al. 2004).

Pflanzenwurzeln formen Abdrücke, die makroskopisch wie Gefäßimpressionen aussehen. Algen und Pilze verursachen Löcher, Höhlen und tunnelartige Destruktionen, die als „Bohrkanäle“ bezeichnet werden. Diese Veränderungen lassen sich besonders im fluoreszierenden Licht und im Rasterelektronenmikroskop gut nachweisen (SCHULTZ 1986, 2001, 2011).

Somit kann die lichtmikroskopische Untersuchung auch genutzt werden, um Aussagen über den Erhaltungszustand eines Knochens zu machen (SCHULTZ 1986). 
Abb. 7 a-b: Zeichnungen erstellt anhand von Originalpräparaten, übernommen von SCHULTZ 2001 (S. 132, 134). Anhand dieser Abbildungen lassen sich die für die unterschiedlichen Erkrankungen spezifischen Veränderungen am Schädeldach gut differenzieren.

Abb. 7a Anämie

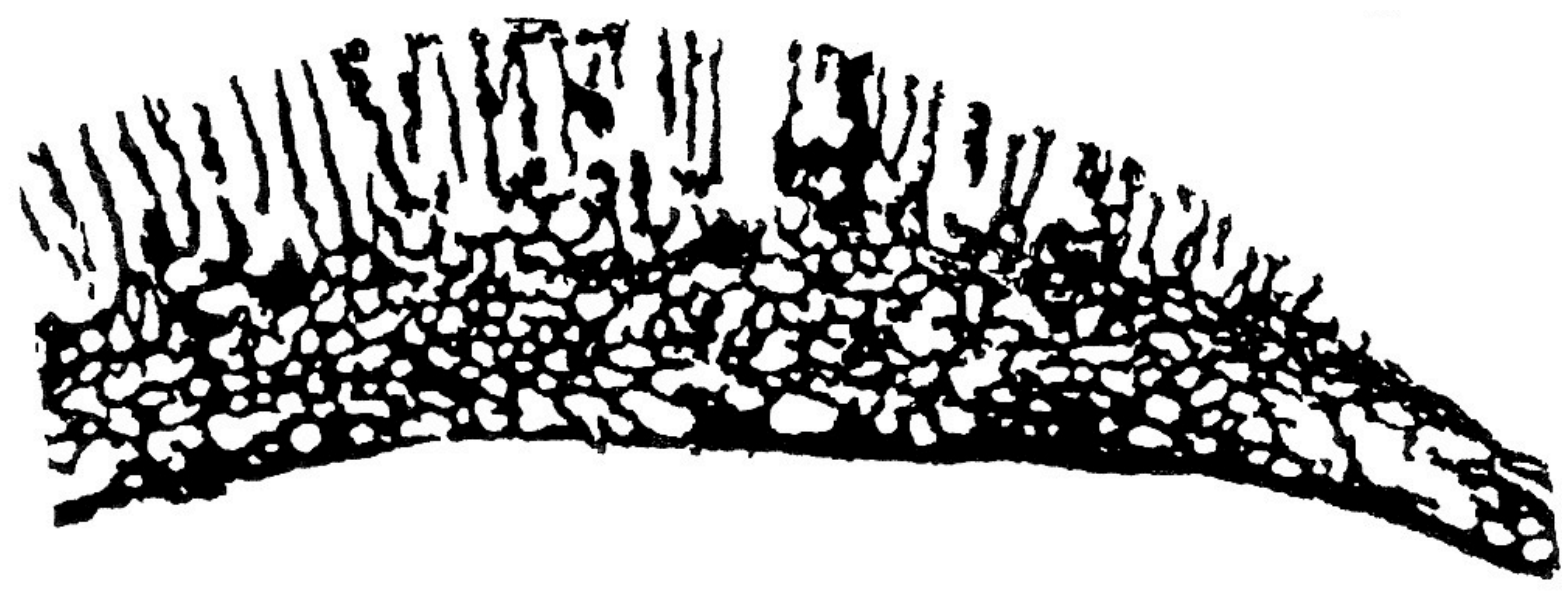

7.a Anämie: Schädeldach eines 9-12jährigen Kindes einer Grabstätte aus der Missionszeit in Santa Maria de Yamasee, Florida. Aufnahmevergrößerung etwa 1,75fach. Dargestellt sind fortgeschrittene Veränderungen (Stadium 3) des Schädelknochens durch chronische Anämie. Das Schädeldach ist verdickt und porös an der Außenseite. Die Lamina externa ist zerstört, rechtwinklig zu ihrem Verlauf finden sich parallel angeordnete, lange, schlanke Trabeculae. Dies entspricht dem typischen Bild eines „Bürstenschädels“/ „Hair-on-endPhänomen“.

Abb. 7b Skorbut

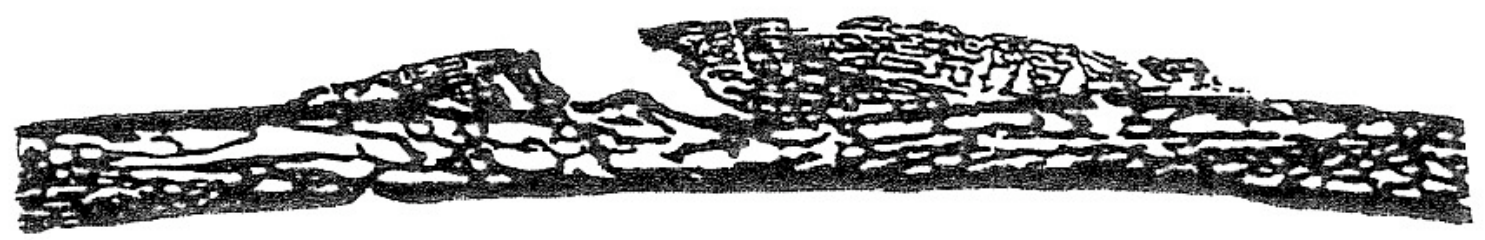

7.b Skorbut: Os frontale eines Kindes der präkolumbianischen Sundown Site, Arizona, USA. Aufnahmevergrößerung etwa 3,5fach. Dargestellt ist ein großes, knöchern organisiertes Hämatom im Rahmen von Skorbut. Die Lamina externa ist erhalten, darauf aufgelagert finden sich die typischen Knochenneubildungen in horizontalen Lagen. Die Diploë ist nicht affektiert. 
Abb. 7 c-d: Zeichnungen erstellt anhand von Originalpräparaten, übernommen von SCHULTZ 2001 (S. 132, 134). Anhand dieser Abbildungen lassen sich die für die unterschiedlichen Erkrankungen spezifischen Veränderungen am Schädeldach gut differenzieren.

Abb. 7c Rachitis

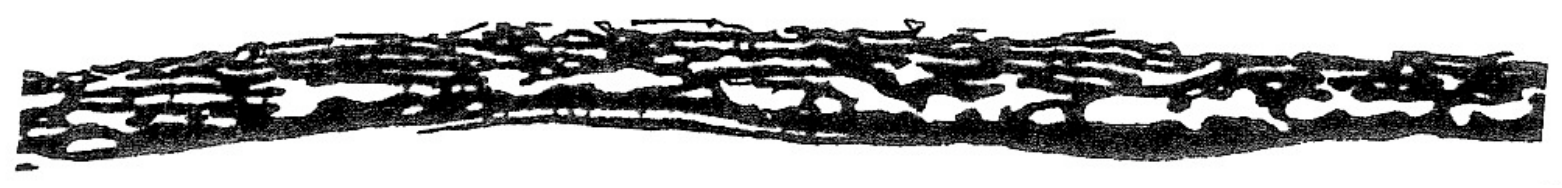

7.c Rachitits: Os parietale eines Säuglings der frühbronzezeitlichen Grabstätte von Ikiztepe, Türkei. Aufnahmevergrößerung etwa 5 fach. Hier zeigen sich die tief greifenden Veränderungen des Schädeldachs durch Rachitis. Die Lamina externa besteht nur noch aus squamösen Plättchen, die Substantia spongiosa ist völlig irregulär angeordnet und die Lamina interna zerstört.

Abb. 7d Unspezifische Entzündung

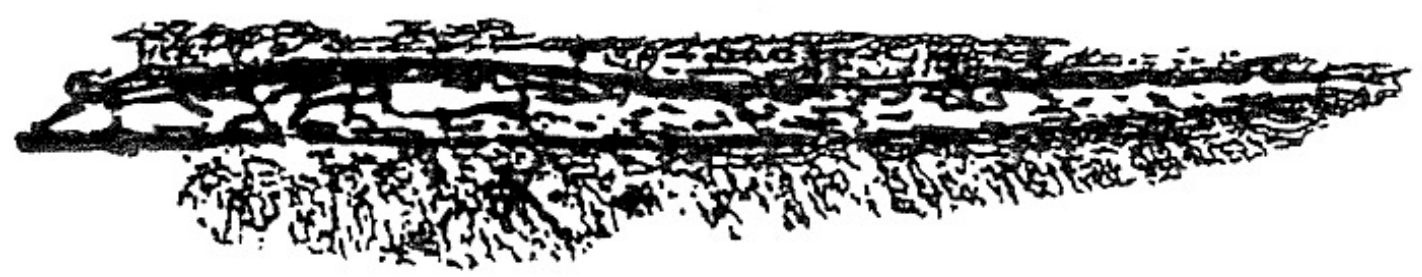

7.d Unspezifische Entzündung: Schädeldach eines Kleinkindes der präkolumbianischen Sundown Site, Arizona, USA. Aufnahmevergrößerung etwa 3,5fach. Der Knochen ist durch eine unspezifische Osteomyelitis fast vollständig zerstört. An Lamina interna und externa finden sich stark verästelte unregelmäßige Knochenneubildungen. Die Substanz der Diploë ist durch den inflammatorischen Prozess nahezu aufgezehrt.

\section{j) Diagnosestellung}

Im Anschluss an alle Untersuchungen wurden die Befunde in folgendes von SCHULTZ 1994 entwickeltes, bisher unveröffentlichtes Schema eingefügt, um die Wahrscheinlichkeit für eine Skorbuterkrankung zu ermitteln. 


\begin{tabular}{|l|l|l|}
\hline A & Kardinalmerkmale \\
\hline 1.$)$ & Poröse AL am äußeren Schädeldach & \\
\hline 2.$)$ & $\begin{array}{l}\text { Poröse AL am Orbitadach (Cribra } \\
\text { orbitalia) }\end{array}$ & \\
\hline 3.) & $\begin{array}{l}\text { Poröse AL am äußeren Gesichts- } \\
\text { schädel* }\end{array}$ & \\
\hline 4.$)$ & Poröse AL am Alveolarknochen* & \\
\hline 5.$)$ & Poröse AL an Langknochenschäften & \\
\hline 6.) & Poröse AL am Schulterblatt & \\
\hline 7.$)$ & Poröse AL am Darmbein & \\
\hline B & Nebenmerkmale & \\
\hline 1.) & $\begin{array}{l}\text { Poröse OB/AL am harten Gaumen } \\
\text { (Stomatitis)** }\end{array}$ & \\
\hline 2.) & Poröse Auskleidung der Alveolen & \\
\hline 3.) & $\begin{array}{l}\text { Symmetrische poröse AL am } \\
\text { inneren Schädeldach, flächig/in den } \\
\text { Impressiones digitatae }\end{array}$ & \\
\hline 4.) & Poröse OB der Langknochenschäfte & \\
\hline 5.) & $\begin{array}{l}\text { Poröse Außenfläche des Großen } \\
\text { Keilbeinflügels }\end{array}$ & \\
\hline C & Weitere Auffälligkeiten & \\
\hline
\end{tabular}

Tab. 5: Merkmalstabelle zur Diagnose von Skorbut an historischen Skeleten (nach SCHULTZ 1994). $\mathrm{AL}=$ Auflagerung, $\mathrm{OB}=$ Oberfläche

*Selbstverständlich ist auch der Alveolarknochen Teil des Gesichtsschädels. Da jedoch häufig nur der Alveolarbereich betroffen ist, wird dieser gesondert betrachtet. Um die Übersichtlichkeit der Tabelle zu erhalten, wird darauf verzichtet, beim Kardinalmerkmal 3 jeweils den Alveolarknochen auszunehmen.

** In der Paläopathologie bezeichnet man entzündliche Prozesse an der Schleimhaut im Bereich des harten Gaumens als Stomatitis. Diese ist unspezifisch; sie kann verschiedene Ursachen haben: Mangelnde Mundhygiene und Erkrankungen der Zähne und des Zahnhalteapparats, Pilz- und Virusinfektionen. Begünstigt wird eine Stomatitis durch systemische Erkrankungen wie Skorbut, Diabetes mellitus oder Leukämie (WEIHMANN 2010).

Zur Bewertung wurde folgende Einteilung verwendet:

Als erkrankt wurde ein Individuum gewertet, wenn mindestens

- zwei Kardinalmerkmale oder

- ein Kardinalmerkmal und zwei Nebenmerkmale oder

- drei Nebenmerkmale

positiv waren.

Als wahrscheinlich erkrankt wurde ein Individuum gewertet, wenn

- ein Kardinalmerkmal (+/- max. ein Nebenmerkmal) oder

- zwei Nebenmerkmale

positiv waren.

Im Rahmen dieser Arbeit soll diese Tabelle erprobt und ihre Anwendbarkeit überprüft werden. 


\section{Ergebnisse}

\section{Einzelbefunde}

\subsection{Untersuchungsbefunde der einzelnen Individuen des Gräberfelds Barbing-Kreuzhof}

Im folgenden Befundteil werden die makroskopischen und mikroskopischen Befunde eines jeden Individuums zusammengefasst und anhand der im Kapitel 2.2 j) Diagnosestellung beschriebenen Merkmalstabelle nach SCHULTZ (1994) eine abschließende Diagnose gestellt.

\section{$\begin{array}{llll}\text { Sk } 1272 & \text { Grab } 15 & \text { 12J }+/-30 ~ M & \text { m }=\mathbf{w}\end{array}$}

\section{Makroskopischer Befund}

Bei Sk 1272 handelt es sich um das recht gut erhaltene Skelet eines ca. 12 Jahre (+/- 30 Monate) alten Kindes, bei dem keine eindeutige Geschlechtszuordnung möglich ist. Sehr auffällig ist der stark deformierte rechte Humerus (Bildtafel 1, Bild 1 und 2), bei dem im distalen Bereich eine Verschmelzung der einzelnen Strukturen stattgefunden hat, so dass keine Differenzierung der Epicondylen und der Fossae radialis und coronoidea möglich ist. Stattdessen ist der distale Humerus plump verdickt und nach lateral und medial zugespitzt. Die ganze Knochenneubildung ist feinporös gestaltet und mit ausgeprägten Gefäßimpressionen versehen, wirkt jedoch recht gut organisiert; sowohl ventral als auch dorsal haben sich neue Gelenkflächen zur Artikulation mit den Unterarmknochen gebildet, so dass davon auszugehen ist, dass die Veränderungen intravital schon längere Zeit vor dem Tode bestanden haben. Der entsprechende Radius ist ebenfalls deutlich deformiert: Der Schaft ist deutlich zierlicher als das linke Gegenstück und derart gebogen, dass man auch vermuten könnte, dass es sich um den linken Radius handelt. Es ist kein Radiusköpfchen mehr zu erkennen, die proximale Spitze ist jedoch abgebrochen. Sollte sich hier intravital noch ein Radiusköpfchen befunden haben, so wäre der rechte Radius allerdings deutlich länger als sein linkes Gegenstück. Das Collum ist undifferenziert, und zumindest bei den obersten 1,5 cm des erhaltenen Knochens handelt es sich um eine Neubildung, die der am Humerus sehr ähnelt. Auch hier finden sich flache feinporöse Auflagerungen im Bereich des gesamten Schafts. Die rechte Ulna ist nicht erhalten, die Knochen des linken Arms sind normal gestaltet. Am ehesten ist hier von einem lokalen infektiösen Geschehen auszugehen, vielleicht einer Osteomyelitis nach einem Bruch des Arms oder auch von Knochentuberkulose.

Darüber hinaus finden sich an allen erhaltenen Langknochen sowie an einer Rippe feinporöse Auflagerungen. Sie scheinen auf ein hämorrhagisches Geschehen, beispielsweise im Rahmen von Skorbut, zurückzugehen. Die Auflagerungen wirken gut integriert; vermutlich hat das Kind die Erkrankung eine Weile überlebt. 
Eine weitere Auffälligkeit stellt die auf fast allen Langknochen vorhandene Längsstreifung dar. Am Schädel finden sich keine Auflagerungen, allerdings fallen vermehrte Gefäßimpressionen an der Lamina interna auf. Am linken Orbitadach liegt ein Cribrum Grad 1 vor, das rechte ist - soweit erhalten - unauffällig.

\section{Radiologischer Befund}

Im insgesamt schlecht erhaltenen rechten Femur fallen zwei Harris-Linien im Bereich der Diaphyse auf.

\section{Mikroskopischer Befund}

Es wurden Präparate des linken Humerus (Bildtafel 1, Bild 3), der linken Tibia sowie des linken Os parietale untersucht. Bei den Langknochen können eine typische Auflagerung vom Typ I sowie eine ausgeprägte Vaskularisierung nachgewiesen werden. Der makroskopische Verdacht auf ein hämorrhagisches Geschehen im Sinne von Skorbut kann also bestätigt werden. An der Lamina interna des Parietale fallen lediglich ausgeprägte Gefäßimpressionen wie bei einer meningealen Reizung auf.

\section{Gesamtbeurteilung}

\begin{tabular}{|c|c|c|}
\hline $\mathbf{A}$ & \multicolumn{2}{|l|}{ Kardinalmerkmale } \\
\hline 1.) & Poröse AL am äußeren Schädeldach & - \\
\hline 2.) & $\begin{array}{l}\text { Poröse AL am Orbitadach (Cribra } \\
\text { orbitalia) }\end{array}$ & Cribrum Grad 1 rechts \\
\hline 3.) & $\begin{array}{l}\text { Poröse AL am äußeren Gesichts- } \\
\text { schädel }\end{array}$ & - \\
\hline 4.) & Poröse AL am Alveolarknochen & - \\
\hline 5.) & Poröse AL an Langknochenschäften & $\begin{array}{l}\text { Humerus beidseits, Ulna links, } \\
\text { Femura, Tibiae }\end{array}$ \\
\hline 6.) & Poröse AL am Schulterblatt & - \\
\hline 7.) & Poröse AL am Darmbein & - \\
\hline B & Nebenmerkmale & \\
\hline 1.) & $\begin{array}{l}\text { Poröse OB/AL am harten Gaumen } \\
\text { (Stomatitis) }\end{array}$ & - \\
\hline 2.) & Poröse Auskleidung der Alveolen & - \\
\hline 3.) & $\begin{array}{l}\text { Symmetrische poröse AL am } \\
\text { inneren Schädeldach, flächig/in den } \\
\text { Impressiones digitatae }\end{array}$ & - \\
\hline 4.) & Poröse OB der Langknochenschäfte & Femura \\
\hline 5.) & $\begin{array}{l}\text { Poröse Außenfläche des Großen } \\
\text { Keilbeinflügels }\end{array}$ & - \\
\hline $\mathrm{C}$ & Weitere Auffälligkeiten & $\begin{array}{l}\text { Ausgeprägte Formveränderung von } \\
\text { Humerus und Radius, AL auf einer } \\
\text { Rippe, Harris-Linien im rechten } \\
\text { Femur, Längsstreifung }\end{array}$ \\
\hline
\end{tabular}

Tab. 6: Merkmalstabelle zur Diagnose von Skorbut (nach SCHULTZ 1994), angewandt auf Sk 1272 aus dem Gräberfeld Barbing-Kreuzhof

$\mathrm{AL}=$ Auflagerung, $\mathrm{OB}=$ Oberfläche, $-=$ Merkmal liegt nicht vor 
Die mikroskopische und makroskopische Befundung von Sk 1272 ergeben ein Kardinal- und zwei Nebenmerkmale, sodass eine Erkrankung an Skorbut wahrscheinlich ist. Daneben muss - wie bereits diskutiert - von einem Entzündungsprozess am linken Arm ausgegangen werden, der in keinem direkten kausalen Zusammenhang mit der Skorbuterkrankung zu sehen ist.
Sk 1279
Grab 22
3J $+/-\mathbf{1 2 M}$
$\mathbf{m}=\mathbf{w}$

\section{Makroskopischer Befund}

Sk 1279 ist das Skelet eines ca. 3 Jahre (+/-12Monate) alten Kindes, bei dem keine eindeutige Geschlechtsbestimmung möglich ist.

Bei der makroskopischen Untersuchung von Sk 1279 fallen frische feinporöse Auflagerungen mit profiliertem bis unterschnittenem Rand an den Medialseiten der Tibiae (Bildtafel 1, Bild 4) sowie an den lateralen Schaftseiten der proximalen Radii auf. Diese Auflagerungen sind hämorrhagischer Genese, könnten also durch Skorbut bedingt sein.

Auch am Boden der rechten Orbita lassen sich - allerdings durch Erosion großenteils zerstörte - hämorrhagisch bedingte Auflagerungen erkennen, ebenso beidseits an der Innenseite der Incisura mandibularis. Das Os palatinum zeigt poröse Auflagerungen entsprechend dem Bild einer Stomatitis; die Alveolarfortsätze von Maxilla und Mandibula zeigen um die Zähne herum feinporöse frische Auflagerungen sowie Reste von porösen Auskleidungen in den Alveolen.

\section{$\underline{\text { Radiologischer Befund }}$}

Im Röntgenbild der linken Tibia (Bildtafel 1, Bild 5) lässt sich im mittleren Teil des Schafts medial eine schmale Auflagerung erkennen. Außerdem sind in beiden Tibiae proximal sowie in der linken auch distal Harris-Linien nachweisbar.

\section{Mikroskopischer Befund}

Es liegt nur ein Präparat des makroskopisch unauffälligen rechten Femurs vor (Bildtafel 1, Bild 6 und 7). Dieses zeigt auch abgesehen von einer erhöhten Spongiosität keinen pathologischen Befund.

\section{Gesamtbeurteilung}

\begin{tabular}{|l|l|l|}
\hline A & Kardinalmerkmale \\
\hline 1.) & Poröse AL am äußeren Schädeldach & - \\
\hline 2.$)$ & $\begin{array}{l}\text { Poröse AL am Orbitadach (Cribra } \\
\text { orbitalia) }\end{array}$ & - \\
\hline 3.$)$ & $\begin{array}{l}\text { Poröse AL am äußeren Gesichts- } \\
\text { schädel }\end{array}$ & Orbitaboden \\
\hline
\end{tabular}




\begin{tabular}{|c|c|c|}
\hline 4.) & Poröse AL am Alveolarknochen & Maxilla und Mandibula \\
\hline 5.) & Poröse AL an Langknochenschäften & Tibia bds., Radius bds. \\
\hline 6.) & Poröse AL am Schulterblatt & - \\
\hline 7.) & Poröse AL am Darmbein & - \\
\hline B & Nebenmerkmale & \\
\hline 1.) & $\begin{array}{l}\text { Poröse OB/AL am harten Gaumen } \\
\text { (Stomatitis) }\end{array}$ & Poröse AL \\
\hline 2.) & Poröse Auskleidung der Alveolen & Maxilla und Mandibula \\
\hline 3.) & $\begin{array}{l}\text { Symmetrische poröse AL am } \\
\text { inneren Schädeldach, flächig/in den } \\
\text { Impressiones digitatae }\end{array}$ & - \\
\hline 4.) & Poröse OB der Langknochenschäfte & - \\
\hline 5.) & $\begin{array}{l}\text { Poröse Außenfläche des Großen } \\
\text { Keilbeinflügels }\end{array}$ & - \\
\hline $\mathrm{C}$ & Weitere Auffälligkeiten & AL an der Incisura mandibularis \\
\hline
\end{tabular}

Tab. 7: Merkmalstabelle zur Diagnose von Skorbut (nach SCHULTZ 1994), angewandt auf Sk 1279 aus dem Gräberfeld Barbing-Kreuzhof

$\mathrm{AL}=$ Auflagerung, $\mathrm{OB}=$ Oberfläche, $-=$ Merkmal liegt nicht vor

Zwar fehlt eine mikroskopische Bestätigung der Befunde, aber da die Auflagerungen radiologisch nachweisbar sind, können sie als gesichert gelten. Makroskopisch lassen sich somit drei Kardinalmerkmale und zwei Nebenmerkmale für Skorbut nachweisen, sodass mit großer Sicherheit von einem chronischen Vitamin-C-Mangel ausgegangen werden kann.

\section{Sk $1280 \quad$ Grab 23a $\quad 30-59$ J männlich}

\section{Makroskopischer Befund}

Bei Sk 1280 handelt es sich um das fast vollständige, aber stellenweise stark erodierte Skelet eines Mannes zwischen 30 und 60 Jahren. An den Langknochen lassen sich makroskopisch keine Krankheitszeichen nachweisen. Am Schädeldach fällt außen im Bereich des Os frontale und des Os parietale sowie des Os zygomaticum eine poröse, glatt verstrichene Oberfläche auf.

Im Schädelinneren sind beidseits eine erhöhte Porosität der Gefäßimpressionen im Os parietale und Os frontale sowie vermehrte Gefäßimpressionen im Sinus sigmoideus zu sehen. Im rechten Sinus maxillaris befindet sich ein glatt begrenzter, wulstiger Tumor von der Größe eines Weizenkorns. Das Os palatinum ist porös, allerdings sind keine frischen Auflagerungen zu sehen; vermutlich handelt es sich um eine abgeheilte Stomatitis.

\section{Mikroskopischer Befund}

Es existieren mikroskopische Präparate vom linken Femur und vom linken Os zygomaticum. Letzteres ist vollkommen unauffällig. Am Femur jedoch sind deutliche Auflagerungen vom 
Typ I zu erkennen, was angesichts des makroskopisch unauffälligen Aspekts überrascht. Dies könnte man so erklären, dass sich im ausgesägten Bereich eine kleine, lokal begrenzte Auflagerung befunden haben muss. Aufgrund der erodierten Oberflächen der Langknochen lässt sich natürlich nicht mit Sicherheit ausschließen, dass auch an anderer Stelle Auflagerungen bestanden haben.

\section{Radiologischer Befund}

Beim Durchleuchten ergab sich kein auffälliger Befund.

\section{Gesamtbeurteilung}

\begin{tabular}{|c|c|c|}
\hline $\mathbf{A}$ & \multicolumn{2}{|l|}{ Kardinalmerkmale } \\
\hline 1.) & Poröse AL am äußeren Schädeldach & - \\
\hline 2.) & $\begin{array}{l}\text { Poröse AL am Orbitadach (Cribra } \\
\text { orbitalia) }\end{array}$ & - \\
\hline 3.) & $\begin{array}{l}\text { Poröse AL am äußeren Gesichts- } \\
\text { schädel }\end{array}$ & - \\
\hline 4.) & Poröse AL am Alveolarknochen & - \\
\hline 5.) & Poröse AL an Langknochenschäften & Femur links \\
\hline 6.) & Poröse AL am Schulterblatt & - \\
\hline 7.) & Poröse AL am Darmbein & - \\
\hline B & Nebenmerkmale & \\
\hline 1.) & $\begin{array}{l}\text { Poröse OB/AL am harten Gaumen } \\
\text { (Stomatitis) }\end{array}$ & Poröse OB \\
\hline 2.) & Poröse Auskleidung der Alveolen & - \\
\hline 3.) & $\begin{array}{l}\text { Symmetrische poröse AL am } \\
\text { inneren Schädeldach, flächig/in den } \\
\text { Impressiones digitatae }\end{array}$ & - \\
\hline 4.) & Poröse OB der Langknochenschäfte & - \\
\hline 5.) & $\begin{array}{l}\text { Poröse Außenfläche des Großen } \\
\text { Keilbeinflügels }\end{array}$ & - \\
\hline $\mathrm{C}$ & Weitere Auffälligkeiten & Poröse OB Schädeldach \\
\hline
\end{tabular}

Tab. 8: Merkmalstabelle zur Diagnose von Skorbut (nach SCHULTZ 1994), angewandt auf Sk 1280 aus dem Gräberfeld Barbing-Kreuzhof

$\mathrm{AL}=$ Auflagerung, $\mathrm{OB}=$ Oberfläche, $-=$ Merkmal liegt nicht vor

Es lassen sich ein Kardinal- und ein Nebenmerkmal für Skorbut nachweisen. Allerdings muss man das Kardinalmerkmal der porösen Auflagerung an Langknochenschäften dadurch einschränken, dass nur am linken Femur eine kleine Auflagerung zu finden ist, obwohl alle Langknochen erhalten sind. Diese könnte sowohl der Überrest eines lokalen subperiostalen Hämatoms, verursacht beispielsweise durch eine stumpfe Verletzung, als auch Ausdruck eines systemischen Geschehens wie Skorbut sein. Auch das Nebenmerkmal der Stomatitis ist nicht wegweisend, da dies ein sehr unspezifisches Symptom unterschiedlicher Erkrankungen sein kann.

Insgesamt ist Skorbut bei diesem Individuum eher unwahrscheinlich. 
Makroskopischer Befund

Sk 1281 ist das Skelet eines 25-35 Jahre alten Mannes. Makroskopisch fallen massive Auflagerungen an den Ulnae (Bildtafel 1, Bild 8) und Radii über dem gesamten Schaft, an den Femura lateral der Linea aspera sowie an beiden Tibiae am medialen distalen Ende auf. Die Auflagerungen sind feinporös, mehrlagig und stark erhaben, der Rand erscheint profiliert bis unterschnitten, stellenweise ausgezackt. Insgesamt entsteht der Eindruck eines massiven hämorrhagischen Geschehens wie bei einer floriden Skorbut-Erkrankung. Dazu passen Auflagerungen am harten Gaumen, entsprechend einer Stomatitis, allerdings ist der Alveolarbereich von Mandibula und Maxilla blande. Der übrige Schädel zeigt keine Auflagerungen, lediglich im Bereich des Os frontale fallen starke Gefäßimpressionen sowie eine poröse Oberfläche an der Lamina interna auf.

\section{Mikroskopischer Befund}

Es gibt nur Schliffe der linken Ulna. In diesen zeigen sich deutlich mehrlagige Auflagerungen vom Typ I, die teilweise bereits faserknöchern organisiert sind (Bildtafel 2, Bild 1 und 2).

\section{Radiologischer Befund}

Das Durchleuchten ergab keine neuen Erkenntnisse.

\section{Gesamtbeurteilung}

\begin{tabular}{|c|c|c|}
\hline $\mathbf{A}$ & \multicolumn{2}{|l|}{ Kardinalmerkmale } \\
\hline 1.) & Poröse AL am äußeren Schädeldach & - \\
\hline 2.) & $\begin{array}{l}\text { Poröse AL am Orbitadach (Cribra } \\
\text { orbitalia) }\end{array}$ & - \\
\hline 3.) & $\begin{array}{l}\text { Poröse AL am äußeren Gesichts- } \\
\text { schädel }\end{array}$ & - \\
\hline 4.) & Poröse AL am Alveolarknochen & - \\
\hline 5.) & Poröse AL an Langknochenschäften & Ulnae, Radii, Femura, Tibiae \\
\hline 6.) & Poröse AL am Schulterblatt & - \\
\hline 7.) & Poröse AL am Darmbein & - \\
\hline B & \multicolumn{2}{|l|}{ Nebenmerkmale } \\
\hline 1.) & $\begin{array}{l}\text { Poröse OB/AL am harten Gaumen } \\
\text { (Stomatitis) }\end{array}$ & Stomatitis \\
\hline 2.) & Poröse Auskleidung der Alveolen & - \\
\hline 3.) & $\begin{array}{l}\text { Symmetrische poröse } \mathrm{AL} \text { am } \\
\text { inneren Schädeldach, flächig/in den } \\
\text { Impressiones digitatae }\end{array}$ & - \\
\hline 4.) & Poröse OB der Langknochenschäfte & - \\
\hline 5.) & $\begin{array}{l}\text { Poröse Außenfläche des Großen } \\
\text { Keilbeinflügels }\end{array}$ & - \\
\hline $\mathrm{C}$ & Weitere Auffälligkeiten & $\begin{array}{l}\text { Vermehrte Gefäßzeichnung und } \\
\text { poröse OB der Lamina interna }\end{array}$ \\
\hline
\end{tabular}

Tab. 9: Merkmalstabelle zur Diagnose von Skorbut (nach SCHULTZ 1994), angewandt auf Sk 1281 aus dem Gräberfeld Barbing-Kreuzhof 
In der makroskopischen und mikroskopischen Befundung lassen sich ein Kardinal- und ein Nebenmerkmal für Skorbut nachweisen. Damit ist eine Skorbuterkrankung nicht sicher, aber durchaus anzunehmen. Hinzu kommt, dass sowohl der makroskopische Aspekt als auch der lichtmikroskopische Befund sehr eindeutig sind, sodass Skorbut wahrscheinlicher ist, als die Merkmalstabelle annehmen lässt.

\section{Sk 1290 \\ Grab 32 \\ $4 \mathbf{J}+/-12 \mathrm{M}$ \\ $\mathbf{m}=\mathbf{w}$}

\section{Makroskopischer Befund}

Sk 1290 ist das Skelett eines ca. 4jährigen (+/- 12 Monate) Kindes. Man erkennt flache feinporöse Auflagerungen an den proximalen und distalen Schaftenden der Humeri, an den proximalen Schaftenden der Ulnae, am distalen rechten Femur sowie im mittleren Schaftbereich der Tibiae. Die Neubildungen imponieren faserknöchern, sind jedoch gut integriert.

Außerdem fällt in der Fossa subscapularis der rechten Scapula eine poröse Oberfläche auf, die aussieht, als hätte hier eine Auflagerung vorgelegen, die postmortal erodiert ist.

Des Weiteren sind alle erhaltenen linken Rippen auf der Innenseite mit ausgeprägten feinporösen Auflagerungen bedeckt (Bildtafel 2, Bild 3). Dieses Bild ließe sich mit einer einseitigen Pleuritis in Einklang bringen.

Die Impressiones digitatae des Schädeldachs und der Orbitadächer sind mit feinporösen Auflagerungen ausgekleidet, die von feinen Gefäßimpressionen durchzogen sind. Außerdem sind sie stark ausgeprägt, was an eine längere Zeit bestehende Hirndrucksymptomatik denken lässt. Hierzu passt auch die steile Inklination der Orbitadächer.

An der Innenseite des linken Parietale liegt eine flächige Auflagerung vor (Bildtafel 2, Bild 4), ebenso an der Ala minor links sowie an den Alae majores ossis sphenoidalis beidseits. Auch am Ramus mandibulae sind Auflagerungen an der Außenseite zu finden, die Alveolarfortsätze von Maxilla und Mandibula sind ebenfalls mit feinporösen Auflagerungen bedeckt.

Am linken Orbitadach liegt ein Cribrum Grad 1 vor, am rechten ist die Oberfläche stark porös, möglicherweise lag auch hier ein Cribrum vor, welches postmortal erodiert ist.

\section{Mikroskopischer Befund}

Es wurden Schliffe der sechsten Rippe links sowie der linken Tibia angefertigt. Die Rippe zeigt massive Auflagerungen vom Typ I, die in drei Krankheitsphasen entstanden zu sein scheinen. Außerdem fallen ausgeprägte Resorptionshöhlen unterhalb der Corticalis externa auf (Bildtafel 2, Bild 5 und 6). 
An der Tibia lassen sich nur noch kleine Reste einer Auflagerung vom Typ I erkennen, da die restliche Oberfläche postmortal zerstört ist. Doch scheint auch hier eine großflächige Auflagerung hämorrhagischer Genese vorgelegen zu haben.

\section{Radiologischer Befund}

Im Röntgenbild der 6. Rippe links sind die Auflagerungen deutlich als Doppelkontur an der inneren Curvatur zu erkennen (Bildtafel 2, Bild 7).

\section{Gesamtbeurteilung}

\begin{tabular}{|c|c|c|}
\hline $\mathbf{A}$ & \multicolumn{2}{|l|}{ Kardinalmerkmale } \\
\hline 1.) & Poröse AL am äußeren Schädeldach & - \\
\hline 2.) & $\begin{array}{l}\text { Poröse AL am Orbitadach (Cribra } \\
\text { orbitalia) }\end{array}$ & Cribrum Grad 1 links, evtl. auch rechts \\
\hline 3.) & $\begin{array}{l}\text { Poröse AL am äußeren Gesichts- } \\
\text { schädel }\end{array}$ & $\begin{array}{l}\text { Ala major ossis sphenoidalis, } \\
\text { Mandibula, Maxilla }\end{array}$ \\
\hline 4.) & Poröse AL am Alveolarknochen & Mandibula, Maxilla \\
\hline 5.) & Poröse AL an Langknochenschäften & Humeri, Ulnae, Femur rechts, Tibiae \\
\hline 6.) & Poröse AL am Schulterblatt & Fossa subscapularis rechts \\
\hline 7.) & Poröse AL am Darmbein & - \\
\hline B & Nebenmerkmale & \\
\hline 1.) & $\begin{array}{l}\text { Poröse OB/AL am harten Gaumen } \\
\text { (Stomatitis) }\end{array}$ & $\mathrm{AL}$ \\
\hline 2.) & Poröse Auskleidung der Alveolen & - \\
\hline 3.) & $\begin{array}{l}\text { Symmetrische poröse AL am } \\
\text { inneren Schädeldach, flächig/in den } \\
\text { Impressiones digitatae }\end{array}$ & $\begin{array}{l}\text { AL in Impressiones digitatae, flächig } \\
\text { im linken Os parietale }\end{array}$ \\
\hline 4.) & Poröse OB der Langknochenschäfte & - \\
\hline 5.) & $\begin{array}{l}\text { Poröse Außenfläche des Großen } \\
\text { Keilbeinflügels }\end{array}$ & $\mathrm{AL}$ \\
\hline $\mathrm{C}$ & Weitere Auffälligkeiten & $\begin{array}{l}\text { AL auf Rippeninnenseite links, an } \\
\text { Ramus mandibulae }\end{array}$ \\
\hline
\end{tabular}

Tab. 10: Merkmalstabelle zur Diagnose von Skorbut (nach SCHULTZ 1994), angewandt auf Sk 1290 aus dem Gräberfeld Barbing-Kreuzhof

$\mathrm{AL}=$ Auflagerung, $\mathrm{OB}=$ Oberfläche, $-=$ Merkmal liegt nicht vor

Es lassen sich fünf Kardinal- und drei Nebenmerkmale für Skorbut nachweisen, sodass mit hoher Wahrscheinlichkeit ein chronischer Vitamin-C-Mangel vorlag.

Die hohe Anzahl von befallenen Knochen lässt auf jeden Fall an eine systemische Erkrankung denken. Die Auflagerungen an den Rippen ließen sich auch durch eine Pleuritis erklären, die Hirndruckzeichen und Auflagerungen an der Lamina interna könnten auch durch eine Meningitis verursacht worden sein. Die Auflagerungen an den Langknochen, der Scapula und am äußeren Gesichtsschädel sowie die Cribra orbitalia deuten stark auf Skorbut hin. Man kann vermuten, dass das Kind einige Wochen, vielleicht sogar Monate unter Skorbut litt, was u.a. die mikroskopisch belegte Mehrphasigkeit der Auflagerungen an den Rippen nahelegt, 
und darüber hinaus, möglicherweise als Sekundärinfektion aufgrund der durch Vitamin-CMangel geschwächten Abwehr, an einer Pleuritis und Meningitis erkrankte. Dies führte vermutlich zum Tode.
Sk 1292
Grab 34
$15 \mathrm{~J}+/-36 \mathrm{M}$
$\mathbf{m}=\mathbf{w}$

\section{Makroskopischer Befund}

Sk 1292 ist das Skelet eines ca. 15 Jahre (+/- 36 Monate) alten Jugendlichen, bei dem keine genaue Geschlechtszuweisung möglich ist. Am rechten Femur fällt eine sehr poröse Oberfläche auf, in welche integriert sich sehr feine poröse Auflagerungen finden. Auch am Schaft der rechten Fibula finden sich feinporöse Auflagerungen in gut integriertem Zustand. An der linken Fibula befanden sich vermutlich ähnliche Auflagerungen, allerdings ist die Oberfläche hier zu erodiert für eine eindeutige Befundung. Es fehlen beide Tibiae und die linke Ulna, die übrigen Langknochen sind unauffällig. Am unvollständig erhaltenen und stark fragmentierten Schädel findet sich ein Cribrum Grad 1 am linken Orbitadach. Am Os parietale und Os frontale beider Seiten ist die Oberfläche im Bereich der Suturen außen sehr porös. Auch im Sulcus sinus sagittalis und an der Außenfläche der Ala major des rechten Os sphenoidale ist die Oberfläche feinporös. Alle Kiefer fehlen.

\section{Mikroskopischer Befund}

Da die makroskopischen Befunde diskret und der Erhaltungszustand der Knochen unzulänglich sind, wurde davon abgesehen, Schliffe anzufertigen.

\section{Radiologischer Befund}

Beim Durchleuchten von Femur und Fibulae konnten keine Auffälligkeiten festgestellt werden. Am proximalen und distalen Schaft beider Tibiae sind Harris-Linien nachweisbar.

\section{Gesamtbeurteilung}

\begin{tabular}{|c|c|c|}
\hline $\mathbf{A}$ & \multicolumn{2}{|l|}{ Kardinalmerkmale } \\
\hline 1.) & Poröse AL am äußeren Schädeldach & - \\
\hline 2.) & $\begin{array}{l}\text { Poröse AL am Orbitadach (Cribra } \\
\text { orbitalia) }\end{array}$ & Cribrum Grad 1 Orbita rechts \\
\hline 3.) & $\begin{array}{lccc}\text { Poröse } & \text { AL } & \text { am } & \text { äußeren } \\
\text { Gesichtsschädel } & & \end{array}$ & - \\
\hline 4.) & Poröse AL am Alveolarknochen & - \\
\hline 5.) & Poröse AL an Langknochenschäften & $\begin{array}{l}\text { Femur rechts, Fibula rechts, evtl. } \\
\text { Fibula links }\end{array}$ \\
\hline 6.) & Poröse AL am Schulterblatt & - \\
\hline 7.) & Poröse AL am Darmbein & - \\
\hline B & Nebenmerkmale & \\
\hline 1.) & $\begin{array}{l}\text { Poröse OB/AL am harten Gaumen } \\
\text { (Stomatitis) }\end{array}$ & - \\
\hline 2.) & Poröse Auskleidung der Alveolen & - \\
\hline
\end{tabular}




\begin{tabular}{|l|l|l|}
\hline 3.) & $\begin{array}{l}\text { Symmetrische poröse AL am } \\
\text { inneren Schädeldach, flächig/in den } \\
\text { Impressiones digitatae }\end{array}$ & \\
\hline 4.) & Poröse OB der Langknochenschäfte & Femur rechts \\
\hline 5.) & $\begin{array}{l}\text { Poröse Außenfläche des Großen } \\
\text { Keilbeinflügels }\end{array}$ & Ala major rechts (links nicht erhalten) \\
\hline C & Weitere Auffälligkeiten & $\begin{array}{l}\text { Poröse OB außen an Os parietale und } \\
\text { Os frontale, Längsstreifung der } \\
\text { Femura }\end{array}$ \\
\hline
\end{tabular}

Tab. 11: Merkmalstabelle zur Diagnose von Skorbut (nach SCHULTZ 1994) angewandt auf Sk 1292 aus dem Gräberfeld Barbing-Kreuzhof

$\mathrm{AL}=$ Auflagerung, $\mathrm{OB}=$ Oberfläche, $-=$ Merkmal liegt nicht vor

Es lassen sich zwei Kardinal- und zwei Nebenmerkmale für Skorbut nachweisen, sodass von einer Erkrankung ausgegangen werden darf. Allerdings muss man hinzufügen, dass aufgrund des schlechten Erhaltungszustandes und der fehlenden lichtmikroskopischen Bestätigung die Diagnose nicht sicher ist.

\section{$\begin{array}{llll}\text { Sk } 1293 & \text { Grab } 35 & \text { Alter 25-30 J } & \text { weiblich }\end{array}$}

\section{Makroskopischer Befund}

Bei Sk 1293 handelt es sich um das Skelet einer ca. 25 - 30 Jahre alten Frau. An den proximalen Schäften beider Humeri, am Übergang zur Metaphyse, finden sich netzartig angeordnete feinporöse Auflagerungen, die von Erosionen durchsetzt sind. Auch am Collum und in der Fossa poplitea beider Femura fallen gleichartige Auflagerungen auf. Über die Schäfte beider Tibiae und der rechten Fibula verteilt erkennt man disseminierte Flecken dieser Auflagerungen, die teilweise erodiert sind. Alle Auflagerungen scheinen noch recht frisch zu sein, man erkennt kleine Brücken über die Periostgefäße, die vermutlich aus Faserknochen bestehen.

Der Schädel ist von außen unauffällig, lediglich am Os zygomaticum fällt eine poröse Oberfläche auf, die jedoch noch als normal gewertet werden kann. Im Schädelinneren finden sich vermehrt Gefäßimpressionen, vor allem in den Sinus, die alle nach occipital zu weisen scheinen. In der Mandibula, im Bereich des zweiten Prämolaren rechts, liegt eine tiefe Höhle, umgeben von frischen Auflagerungen, vor, die wohl auf einen frischen Zahnabszess zurückzuführen ist (Bildtafel 2, Bild 8 und Bildtafel 3, Bild 1). Generell sind die Zähne in einem sehr schlechten Zustand, viele haben Löcher, die Schneidezähne weisen transversale Schmelzhypoplasien auf. 


\section{Mikroskopischer Befund}

Es wurden Schliffe aus dem Zahnabszess in der Mandibula und aus der linken Tibia angefertigt. An der Mandibula zeigen sich deutliche Auflagerungen vom Typ I, die teilweise zweilagig erscheinen. Außerdem scheint auch die Spongiosa entzündlich verändert, sodass von einem tiefreichenden entzündlichen, möglicherweise teilweise hämorrhagischen Prozess mit Affektion des Knochens ausgegangen werden kann (Bildtafel 3, Bild 3).

An der Tibia lässt sich eine kleine Auflagerung vom Typ I nachweisen, deren Ränder postmortal abgebrochen sind und die wahrscheinlich von größerer Ausdehnung war (Bildtafel 3, Bild 4).

\section{$\underline{\text { Radiologischer Befund }}$}

Im proximalen Schaftbereich der Tibia erkennt man schmale Auflagerungen.

An der Mandibula erkennt man den Zahnabszess radiologisch an der entzündlich veränderten Spongiosastruktur und flachen porösen Auflagerungen (Bildtafel 3, Bild 2).

\section{Gesamtbeurteilung}

\begin{tabular}{|c|c|c|}
\hline $\mathbf{A}$ & \multicolumn{2}{|l|}{ Kardinalmerkmale } \\
\hline 1.) & Poröse AL am äußeren Schädeldach & - \\
\hline 2.) & $\begin{array}{l}\text { Poröse AL am Orbitadach (Cribra } \\
\text { orbitalia) }\end{array}$ & - \\
\hline 3.) & $\begin{array}{l}\text { Poröse AL am äußeren Gesichts- } \\
\text { schädel }\end{array}$ & - \\
\hline 4.) & Poröse AL am Alveolarknochen & -, nur Abszess an Mandibula \\
\hline 5.) & Poröse AL an Langknochenschäften & Humeri, Femura, Tibiae, Fibula rechts \\
\hline 6.) & Poröse AL am Schulterblatt & - \\
\hline 7.) & Poröse AL am Darmbein & - \\
\hline B & Nebenmerkmale & \\
\hline 1.) & $\begin{array}{l}\text { Poröse OB/AL am harten Gaumen } \\
\text { (Stomatitis) }\end{array}$ & - \\
\hline 2.) & Poröse Auskleidung der Alveolen & - \\
\hline 3.) & $\begin{array}{l}\text { Symmetrische poröse AL am } \\
\text { inneren Schädeldach, flächig/in den } \\
\text { Impressiones digitatae }\end{array}$ & - \\
\hline 4.) & Poröse OB der Langknochenschäfte & - \\
\hline 5.) & $\begin{array}{l}\text { Poröse Außenfläche des Großen } \\
\text { Keilbeinflügels }\end{array}$ & - \\
\hline $\mathrm{C}$ & Weitere Auffälligkeiten & $\begin{array}{l}\text { Vermehrte Gefäßimpressionen an } \\
\text { Schädelinnenseite }\end{array}$ \\
\hline
\end{tabular}

Tab. 12: Merkmalstabelle zur Diagnose von Skorbut (nach SCHULTZ 1994) angewandt auf Sk 1293 aus dem Gräberfeld Barbing-Kreuzhof

$\mathrm{AL}=$ Auflagerung, $\mathrm{OB}=$ Oberfläche, $-=$ Merkmal liegt nicht vor

Es lässt sich nur ein Kardinalmerkmal für Skorbut nachweisen, nämlich die feinporösen Auflagerungen an den Langknochenschäften. Dies wird verstärkt durch den Umstand, dass 
die Auflagerungen sehr symmetrisch und an vielen verschiedenen Knochen auftreten, sodass ein systemisches Geschehen wie Skorbut wahrscheinlich ist.

Die Auflagerungen an der Mandibula sind nicht als Zeichen für Skorbut zu werten, sondern lediglich als Ausdruck einer lokalen Entzündung im Rahmen eines Zahnabszesses. Die transversalen Schmelzhypoplasien der Schneidezähne lassen zumindest auf Mangelernährung schließen.

Insgesamt lässt sich Skorbut nicht sicher bestätigen, ist jedoch auch nicht unwahrscheinlich.
Sk 1297
Grab 39
$4 \mathbf{J}+/-12$
$\mathbf{m}=\mathbf{w}$

Das Grab ist nicht auf dem Gräberfeldplan verzeichnet, da es durch Baumaschinen stark verschoben war (DETKEN 1991).

\section{Makroskopischer Befund}

Bei Sk 1297 handelt es sich um Teile des Skelets eines ca. 4 Jahre alten (+/- 12 Monate) Kindes. Eine Geschlechtsbestimmung ist nicht möglich.

An Langknochen sind nur der linke Radius, die linke Tibia und ein Teil der linken Fibula erhalten. Auch bei diesen Knochen ist die Oberfläche erodiert. Dennoch erkennt man an Radius und Tibia schwache feinporöse Auflagerungen. Sie sind teilweise abgetragen, wirken jedoch gut integriert.

Vom Schädeldach sind nur einige Fragmente des rechten Os parietale und frontale erhalten. Auch hier scheinen außen flache feinporöse Auflagerungen bestanden zu haben, die jedoch schon sehr gut integriert sind und eher als feinporöse Oberfläche erscheinen. Unter dem Binokular lässt sich ein gut verstrichener, aber erhabener Rand erkennen. Vergleichbare Spuren von Auflagerungen finden sich in den Impressiones digitatae des Os parietale, Os frontale und Os temporale sowie an der linken Ala major ossis sphenoidalis.

Im Oberkieferbereich ist nur ein Teil der rechten Maxilla erhalten, auch hier sind außen sehr flache, gut integrierte Auflagerungen zu erkennen. Im Sinus maxillaris sind deutliche mehrlagige, gut organisierte Auflagerungen $\mathrm{zu}$ sehen, entsprechend dem Bild einer abgeheilten Sinusitis. Der harte Gaumen ist mit feinporösen gut integrierten Auflagerungen wie bei einer verheilten Stomatitis bedeckt. Auch an der linken Incisura mandibularis und im Alveolarbereich der Mandibula beider Seiten sind Spuren feinporöser Auflagerungen erhalten (Bildtafel 3, Bild 5).

\section{Mikroskopischer Befund}

Die Anfertigung von Schliffen erschien angesichts des schlechten Erhaltungszustandes der Knochen und der weit fortgeschrittenen Integration der Auflagerungen nicht sinnvoll. 


\section{Radiologischer Befund}

Beim Durchleuchten fielen keine Krankheitszeichen auf.

\section{Gesamtbeurteilung}

\begin{tabular}{|l|l|l|}
\hline A & Kardinalmerkmale \\
\hline 1.) & Poröse AL am äußeren Schädeldach & $\begin{array}{l}\text { Parietale, Frontale, Occipitale, } \\
\text { Sphenoidale }\end{array}$ \\
\hline 2.) & $\begin{array}{l}\text { Poröse AL am Orbitadach (Cribra } \\
\text { orbitalia) }\end{array}$ & - \\
\hline 3.) & $\begin{array}{l}\text { Poröse AL am äußeren } \\
\text { Gesichtsschädel }\end{array}$ & Maxilla \\
\hline 4.) & Poröse AL am Alveolarknochen & Mandibula \\
\hline 5.) & Poröse AL an Langknochenschäften & Radius, Tibia \\
\hline 6.) & Poröse AL am Schulterblatt & - \\
\hline 7.) & Poröse AL am Darmbein & - \\
\hline B & Nebenmerkmale & $\begin{array}{l}\text { Poröse OB, vermutlich gut integrierte } \\
\text { 1.) }\end{array}$ \\
\hline Poröse OB/AL am harten Gaumen \\
\hline 2.) & Poröse Auskleidung der Alveolen & - \\
\hline 3.) & $\begin{array}{l}\text { Symmetrische poröse AL am } \\
\text { inneren Schädeldach, flächig/in den } \\
\text { Impressiones digitatae }\end{array}$ & Frontale, Parietale, Temporale \\
\hline 4.) & Poröse OB der Langknochenschäfte & - \\
\hline 5.) & $\begin{array}{l}\text { Poröse Außenfläche des Großen } \\
\text { Keilbeinflügels }\end{array}$ & + \\
\hline C & Weitere Auffälligkeiten & - \\
\hline
\end{tabular}

Tab. 13: Merkmalstabelle zur Diagnose von Skorbut (nach SCHULTZ 1994), angewandt auf Sk 1297 aus dem Gräberfeld Barbing-Kreuzhof

$\mathrm{AL}=$ Auflagerung, $\mathrm{OB}=$ Oberfläche, - = Merkmal liegt nicht vor

Es lassen sich vier Kardinal- und drei Nebenmerkmale für Skorbut nachweisen. Somit erscheint die Diagnose Skorbut recht sicher. Allerdings fehlt die lichtmikroskopische Bestätigung. Außerdem sollte man beachten, dass nur sehr wenige Knochen des Skelets erhalten sind, auch wenn diese ein einheitliches Bild zeigen. Die Auflagerungen sind sehr diskret und gut integriert, sodass man davon ausgehen kann, dass der Skorbut überlebt wurde und zum Todeszeitpunkt des Kindes beinahe vollständig ausgeheilt war.
Sk 1298
Grab 40
$3-4(5) J$
$\mathbf{m}=\mathbf{w}$

Das Grab befand sich auf dem nördlichen Teil des Gräberfelds (DETKEN 1991).

\section{Makroskopischer Befund}

Sk 1298 ist das Skelet eines drei- bis vier- (evtl. fünf-) jährigen Kindes. Bei der makroskopischen Untersuchung fallen flache feinporöse, strähnige Auflagerungen an den 
Diaphysen der Femura und der Tibiae auf, die allerdings durch Erosion teilweise zerstört sind. Auch am Os ilii sind feinporöse Auflagerungen zu erkennen. An den erhaltenen Resten der linken Scapula finden sich vergleichbare Auflagerungen in den Fossae supra- und infraspinata sowie am Unterrand der Spina scapulae.

Der Schädel ist stark fragmentiert. An der Innenseite des Os frontale im Sinus frontalis sowie im Bereich der Crista frontalis liegen beidseits kleine Flecken feinporöser Auflagerungen mit profiliertem Rand vor. Ähnliche Auflagerungen finden sich am Boden und der lateralen Wand der linken Orbita, deren Dach ebenso wie die rechte Orbita leider nicht erhalten ist. Auch im Gesichtsbereich der Maxillae fallen disseminierte kleine Auflagerungen auf. Der harte Gaumen ist feinporös belegt, entsprechend einer Stomatitis. An den Aveolen von Maxilla und Mandibula finden sich beidseits feine poröse Auflagerungen, ebenso an den Rami und Incisurae mandibulae.

Die rechte Ala major ossis sphenoidalis ist in der Fossa cranii media bedeckt mit mehrlagigen feinporösen Auflagerungen. Am linken Os temporale finden sich außen im Bereich der Squama, unmittelbar hinter dem Ansatz des Jochbogens, flache Auflagerungen, außerdem ist der gesamte Sulcus sinus sigmoidei links mit feinporösen, teilweise mehrlagigen Auflagerungen verfüllt. Das Os occipitale ist erodiert, dennoch sind an der Crista occipitalis externa sowie an der Protuberantia occipitalis schwache feinporöse Auflagerungen zu erkennen.

\section{Mikroskopischer Befund}

Es existieren nur Schliffe der rechten Tibia. Hier sind keine Pathologien zu erkennen, lediglich normales appositionelles Wachstum.

\section{Radiologischer Befund}

Im Röntgenbild der Tibia erkennt man am medialen Schaft eine dem ehemaligen Periost aufgesetzte Auflagerung (Bildtafel 3, Bild 6). Außerdem sind in beiden Tibiae proximal und distal je eine Harris-Linie zu sehen.

\section{Gesamtbeurteilung}

\begin{tabular}{|l|l|l|}
\hline A & Kardinalmerkmale \\
\hline 1.) & Poröse AL am äußeren Schädeldach & - \\
\hline 2.) & $\begin{array}{l}\text { Poröse AL am Orbitadach (Cribra } \\
\text { orbitalia) }\end{array}$ & - \\
\hline 3.$)$ & $\begin{array}{l}\text { Poröse AL am äußeren Gesichts- } \\
\text { schädel }\end{array}$ & Maxilla, Os temporale \\
\hline 4.) & Poröse AL am Alveolarknochen & Mandibula, Maxilla \\
\hline 5.) & Poröse AL an Langknochenschäften & Femura, Tibiae \\
\hline 6.) & Poröse AL am Schulterblatt & Fossa supra- und infraspinata, Spina \\
\hline 7.) & Poröse AL am Darmbein & + \\
\hline
\end{tabular}




\begin{tabular}{|l|l|l|}
\hline B & Nebenmerkmale \\
\hline 1.$)$ & $\begin{array}{l}\text { Poröse OB/AL am harten Gaumen } \\
\text { (Stomatitis) }\end{array}$ & OB \\
\hline 2.) & Poröse Auskleidung der Alveolen & - \\
\hline 3.$)$ & $\begin{array}{l}\text { Symmetrische poröse AL am } \\
\text { inneren Schädeldach, flächig/in den } \\
\text { Impressiones digitatae }\end{array}$ & Frontale, Parietale, Temporale \\
\hline 4.) & Poröse OB der Langknochenschäfte & - \\
\hline 5.$)$ & $\begin{array}{l}\text { Poröse Außenfläche des Großen } \\
\text { Keilbeinflügels }\end{array}$ & - \\
\hline C & Weitere Auffälligkeiten & - \\
\hline
\end{tabular}

Tab. 14: Merkmalstabelle zur Diagnose von Skorbut (nach SCHULTZ 1994), angewandt auf Sk 1298 aus dem Gräberfeld Barbing-Kreuzhof

$\mathrm{AL}=$ Auflagerung, $\mathrm{OB}=$ Oberfläche, $-=$ Merkmal liegt nicht vor

Es können fünf Kardinal- und zwei Nebenmerkmale für Skorbut nachgewiesen werden. Einschränkend muss man sagen, dass sich die makroskopisch sehr diskreten Auflagerungen lichtmikroskopisch - zumindest an der rechten Tibia - nicht nachweisen ließen. Aufgrund der Fülle von Befunden sollte man dennoch von einem manifesten chronischen Vitamin $\mathrm{C}-$ Mangel ausgehen.

Sk 1299

\section{Grab 41}

$0,5-1,5 \mathrm{~J}$

$\mathbf{m}=\mathbf{w}$

Dieses Grab war im nördlichen Teil des Gräberfelds in unmittelbarer Nähe der Gräber 104, 105 und 106 lokalisiert. In die Archivkiste wurden versehentlich Teile von Fußphalangen eines adulten Individuums einsortiert, die sich keinem Individuum zuweisen lassen (DETKEN 1991).

\section{Makroskopischer Befund}

Bei Sk 1299 handelt es sich um Teile des Skelets eines Säuglings im Alter von 6 bis 18 Monaten ohne Geschlechtszuweisung.

Vom Postcranium sind lediglich die Femura und Tibiae erhalten. Auf Letzteren zeigen sich an den Diaphysenschäften feinporöse Auflagerungen mit profiliertem Rand.

Auch am Schädel lassen sich Auflagerungen nachweisen: An der Innenseite des Os parietale fallen beidseits kleinflächige feinporöse Auflagerungen mit profiliertem Rand bei insgesamt erhöhter Porosität auf. Am Os frontale liegen Auflagerungen innen im Bereich der Crista frontalis vor, außen beidseitig an der Sutura frontozygomatica. Auch an der Innen- und teilweise Außenseite der Sutura coronalis und Sutura sagittalis (Bildtafel 4, Bild 2) beider Seiten sowie an Innen- und Außenseite der rechten Sutura squamosa finden sich ähnliche feinporöse Auflagerungen. 
An Dach und Hinterwand der rechten Orbita liegt ein Cribrum Grad 2 vor (Bildtafel 4, Bild 1), die linke Orbita ist kaum erhalten. Am Os sphenoidale ist die Außenfläche der linken Ala major mit feinporösen Auflagerungen bedeckt, der Rand ist hier verstrichen, sodass eine klare Abgrenzung zur lediglich porösen Oberfläche schwerfällt. Ein ähnliches Bild bietet das Jugum sphenoidale. Am Os temporale fallen eine erhöhte Porosität der Lamina interna sowie diffus verteilte kleine feinporöse Auflagerungen auf, die teilweise mehrlagig erscheinen und einen profilierten bis unterschnittenen Rand aufweisen.

Auch an der Außenseite des Os occipitale beider Seiten zeigen sich diese Auflagerungen, ebenso im linken Sulcus sinus transversi (Bildtafel 3, Bild 8).

An der Mandibula fallen beidseits feinporöse Auflagerungen im Alveolarbereich und an der Incisura mandibulae auf, außerdem finden sich poröse Auskleidungen der Alveolen. Die Maxillae sind nicht erhalten (Bildtafel 3, Bild 7).

\section{Mikroskopischer Befund}

Aufgrund des schlechten Erhaltungszustandes der Tibiae und der Kleinflächigkeit der Auflagerungen im Schädelbereich wurden keine Schliffe angefertigt.

\section{Radiologischer Befund}

Beim Durchleuchten zeigten sich keine Krankheitszeichen.

\section{Gesamtbeurteilung}

\begin{tabular}{|c|c|c|}
\hline $\mathbf{A}$ & \multicolumn{2}{|l|}{ Kardinalmerkmale } \\
\hline 1.) & Poröse AL am äußeren Schädeldach & Temporale, Occipitale, Frontale \\
\hline 2.) & $\begin{array}{l}\text { Poröse AL am Orbitadach (Cribra } \\
\text { orbitalia) }\end{array}$ & Cribrum Grad 2 rechts \\
\hline 3.) & $\begin{array}{l}\text { Poröse AL am äußeren Gesichts- } \\
\text { schädel }\end{array}$ & - \\
\hline 4.) & Poröse AL am Alveolarknochen & Mandibula \\
\hline 5.) & Poröse AL an Langknochenschäften & Tibiae \\
\hline 6.) & Poröse AL am Schulterblatt & - \\
\hline 7.) & Poröse AL am Darmbein & - \\
\hline B & Nebenmerkmale & \\
\hline 1.) & $\begin{array}{l}\text { Poröse OB/AL am harten Gaumen } \\
\text { (Stomatitis) }\end{array}$ & - \\
\hline 2.) & Poröse Auskleidung der Alveolen & Mandibula \\
\hline 3.) & $\begin{array}{l}\text { Symmetrische poröse AL am } \\
\text { inneren Schädeldach, flächig/in den } \\
\text { Impressiones digitatae }\end{array}$ & Temporale, Parietale, Frontale \\
\hline 4.) & Poröse OB der Langknochenschäfte & - \\
\hline 5.) & $\begin{array}{l}\text { Poröse Außenfläche des Großen } \\
\text { Keilbeinflügels }\end{array}$ & DD: AL mit verstrichenem Rand \\
\hline $\mathrm{C}$ & Weitere Auffälligkeiten & $\begin{array}{l}\text { Erhöhte Porösität der Lamina interna, } \\
\text { AL an Incisura mandibulae }\end{array}$ \\
\hline
\end{tabular}

Tab. 15: Merkmalstabelle zur Diagnose von Skorbut (nach SCHULTZ 1994), angewandt auf Sk 1299 aus dem Gräberfeld Barbing-Kreuzhof 
Es lassen sich vier Kardinal- und drei Nebenmerkmale für Skorbut nachweisen. Die Auflagerungen sind symmetrisch verteilt. Auch wenn keine mikroskopische Diagnostik erfolgt ist und der größte Teil des Postcraniums nicht mehr existiert, kann die Diagnose Skorbut als sehr wahrscheinlich angenommen werden.
Sk 1300
Grab 42
$4-5 \mathbf{J}$
$\mathbf{m}=\mathbf{w}$

Dieses Grab lag an der nördlichen Begrenzung des Gräberfelds über Grab 102 und war auf ganzer Körperlänge gestört (DETKEN 1991).

\section{Makroskopischer Befund}

Sk 1300 ist das Skelet eines 4 - 5 Jahre alten Kindes, dessen Erhaltungszustand schlecht ist. Vom Postcranium sind nur die Femura, die rechte Tibia und Fibula sowie ein Fragment der linken Fibula erhalten. An beiden Femura findet sich unterhalb des Trochanter major eine ca. $4 \mathrm{~cm}^{2}$ große feinporöse Auflagerung mit profiliertem Rand, am linken ist die Oberfläche dorsal stark porös. Die gesamte mediale Schaftseite der Tibia ist mit vergleichbaren Auflagerungen bedeckt (Bildtafel 4, Bild 3). Auch an der dorsolateralen Seite der rechten Fibula liegen strähnige feinporöse Auflagerungen mit profiliertem Rand vor. Die Oberfläche aller Knochen ist recht stark erodiert. Außerdem fällt eine starke ventral konvexe Biegung der Diaphysen auf.

Vom Schädel sind nur einige Fragmente erhalten. An diesen finden sich keine Auffälligkeiten.

\section{Mikroskopischer Befund}

Von der Anfertigung eines Knochendünnschliffs wurde abgesehen, da die Auflagerungen an den Langknochen schon zu sehr erodiert bzw. integriert sind.

\section{Radiologischer Befund}

Das Durchleuchten ergab keine Auffälligkeiten.

Gesamtbeurteilung

\begin{tabular}{|l|l|l|}
\hline A & Kardinalmerkmale \\
\hline 1.) & Poröse AL am äußeren Schädeldach & - \\
\hline 2.) & $\begin{array}{l}\text { Poröse AL am Orbitadach (Cribra } \\
\text { orbitalia) }\end{array}$ & - \\
\hline 3.$)$ & $\begin{array}{l}\text { Poröse AL am äußeren Gesichts- } \\
\text { schädel }\end{array}$ & - \\
\hline 4.) & Poröse AL am Alveolarknochen & - \\
\hline 5.) & Poröse AL an Langknochenschäften & Femura, Tibia, Fibula \\
\hline 6.) & Poröse AL am Schulterblatt & - \\
\hline 7.) & Poröse AL am Darmbein & - \\
\hline B & Nebenmerkmale & \multicolumn{2}{|l|}{} \\
\hline 1.) & Poröse OB/AL am harten Gaumen & - \\
\hline
\end{tabular}




\begin{tabular}{|l|l|l|}
\hline & (Stomatitis) & \\
\hline 2.) & Poröse Auskleidung der Alveolen & - \\
\hline 3.$)$ & $\begin{array}{l}\text { Symmetrische poröse AL am } \\
\text { inneren Schädeldach, flächig/in den } \\
\text { Impressiones digitatae }\end{array}$ & - \\
\hline 4.) & Poröse OB der Langknochenschäfte & Femur \\
\hline 5.) & $\begin{array}{l}\text { Poröse Außenfläche des Großen } \\
\text { Keilbeinflügels }\end{array}$ & - \\
\hline C & Weitere Auffälligkeiten & - \\
\hline
\end{tabular}

Tab. 16: Merkmalstabelle zur Diagnose von Skorbut (nach SCHULTZ 1994), angewandt auf Sk 1300 aus dem Gräberfeld Barbing-Kreuzhof

$\mathrm{AL}=$ Auflagerung, $\mathrm{OB}=$ Oberfläche, - = Merkmal liegt nicht vor

Es lassen sich je ein Kardinal- und Nebenmerkmal für Skorbut nachweisen. Für diese fehlt allerdings eine lichtmikroskopische Sicherung. In Anbetracht des schlechten Erhaltungszustandes des Skelets ist eine sichere Diagnose schwierig, dennoch ist Skorbut wahrscheinlich.
Sk 1301
Grab 43
$<36 \mathrm{M}$
$\mathbf{m}=\mathbf{w}$

Das Grab lag an der östlichen Kirchenmauer (DETKEN 1991).

\section{Makroskopischer Befund}

Bei Sk 1301 handelt es sich um Teile des Skelets eines unter drei Jahre alten Kleinkindes.

Am rechten Humerus, an beiden Femura sowie an beiden Tibiae fallen deutliche feinporöse Auflagerungen mit profiliertem Rand auf. Weitere Knochen des Postcraniums sind nicht erhalten.

An den Schädelfragmenten lassen sich sowohl an der Lamina interna als auch an der Lamina externa feinporöse Auflagerungen mit scharf profiliertem Rand nachweisen. Besonders ausgeprägt sind sie im Bereich der Suturen. So finden sich an der Innenseite der linken Suturae metopica, coronoidea, sagittale und lambdoidea sowie an der Außenseite der letzten beiden (Bildtafel 4, Bild 7) deutliche, teilweise mehrlagig erscheinende Auflagerungen.

Am linken Os parietale liegt medial und occipital des Tuber eine großflächige kranzförmige Auflagerung; sie ist feinporös und mehrlagig, der Rand ist unterschnitten, teilweise zungenartig. An der Lamina interna des linken Os parietale finden sich diffus in den Impressiones digitatae verteilte kleinflächige Auflagerungen, auch diese erscheinen feinporös und mehrlagig mit unterschnittenem Rand, der Aspekt ist teilweise strähnig.

Ein ganz ähnliches Bild bietet die Lamina interna des Os frontale (Bildtafel 4, Bild 4, 5 und 6). Auffällig sind außerdem Auflagerungen auf der Glabella. 
Am Dach der linken Orbita gibt es ein Cribrum Grad (1-) 2, das aus feinen Auflagerungen gebildet wird (Bildtafel 4, Bild 8). Die rechte Orbita ist nicht erhalten.

Im Os occipitale ist die Crista occipitalis interna ebenso wie die erhaltenen Teile der Sinus mit stark ausgeprägten mehrlagigen porösen Auflagerungen bedeckt, der Rand ist unterschnitten (Bildtafel 5, Bild 1). Die Squama occipitalis ist innen wie außen mit ebensolchen Auflagerungen versehen.

Weitere Teile des Schädels sind nicht vorhanden. Es fällt auf, dass jeder erhaltene Knochen dieses Individuums die charakteristischen Auflagerungen aufweist, wobei diese an den Langknochen diskreter und besser integriert erscheinen.

\section{Mikroskopischer Befund}

Es wurden Schliffe vom rechten Os femoris, dem Os frontale und dem Os parietale angefertigt. Am Femur zeigt sich ein physiologisches Bild ohne besondere Auffälligkeiten. Sowohl am Os frontale als auch am Os parietale (Bildtafel 5, Bild 2 und 3) lassen sich deutliche Auflagerungen vom Typ I an der Lamina interna nachweisen, die Lamina externa dagegen ist unauffällig.

\section{Radiologischer Befund}

Im Röntgenbild des Femurs erkennt man im anteroposterioren Strahlengang an beiden Seiten der Diaphyse feine Doppelkonturen.

\section{Gesamtbeurteilung}

\begin{tabular}{|c|c|c|}
\hline $\mathbf{A}$ & \multicolumn{2}{|l|}{ Kardinalmerkmale } \\
\hline 1.) & Poröse AL am äußeren Schädeldach & Frontale, Parietale, Occipitale \\
\hline 2.) & $\begin{array}{l}\text { Poröse AL am Orbitadach (Cribra } \\
\text { orbitalia) }\end{array}$ & Cribrum Grad (1-) 2 links \\
\hline 3.) & $\begin{array}{l}\text { Poröse AL am äußeren Gesichts- } \\
\text { schädel }\end{array}$ & Glabella \\
\hline 4.) & Poröse AL am Alveolarknochen & - \\
\hline 5.) & Poröse AL an Langknochenschäften & Humerus, Femura, Tibiae \\
\hline 6.) & Poröse AL am Schulterblatt & - \\
\hline 7.) & Poröse AL am Darmbein & - \\
\hline B & \multicolumn{2}{|l|}{ Nebenmerkmale } \\
\hline 1.) & $\begin{array}{l}\text { Poröse OB/AL am harten Gaumen } \\
\text { (Stomatitis) }\end{array}$ & - \\
\hline 2.) & Poröse Auskleidung der Alveolen & - \\
\hline 3.) & $\begin{array}{l}\text { Symmetrische poröse AL am } \\
\text { inneren Schädeldach, flächig/in den } \\
\text { Impressiones digitatae }\end{array}$ & Frontale, Parietale, Occipitale \\
\hline 4.) & Poröse OB der Langknochenschäfte & - \\
\hline 5.) & $\begin{array}{l}\text { Poröse Außenfläche der Ala majoris } \\
\text { ossis sphenoidalis }\end{array}$ & - \\
\hline $\mathrm{C}$ & Weitere Auffälligkeiten & - \\
\hline
\end{tabular}

Tab. 17: Merkmalstabelle zur Diagnose von Skorbut (nach SCHULTZ 1994), angewandt auf Sk 1301 aus dem Gräberfeld Barbing-Kreuzhof $\quad \mathrm{AL}=$ Auflagerung, $\mathrm{OB}=$ Oberfläche, $-=$ Merkmal liegt nicht vor 
Es lassen sich vier Kardinal- und ein Nebenmerkmal für Skorbut nachweisen. Diese können lichtmikroskopisch teilweise bestätigt werden. Der mikroskopische Nachweis der Auflagerungen am Femur könnte an der diskreten Ausbildung und guten Integration der Veränderung gescheitert sein. Was die Auflagerungen an der Lamina externa des Schädeldachs betrifft, so waren sie möglicherweise in beiden Präparaten nicht angeschnitten. Überall fällt eine große Regelmäßigkeit und Symmetrie der Auflagerungen auf; da sämtliche erhaltene Knochen befallen sind, kann man davon ausgehen, dass die Ausbreitung generalisiert war.

Insgesamt ist eine Skorbut-Erkrankung bei diesem Kind sehr wahrscheinlich.
Sk 1334
Grab 76
$23-28 \mathrm{~J}$
$\mathbf{m}=\mathbf{w}$

Das Grab befand sich auf dem westlichen Teil des Gräberfelds.

\section{Makroskopischer Befund}

Sk 1334 ist das Skelet eines ca. 23 bis 28 Jahre alten Individuums, dessen Geschlecht sich nicht bestimmen lässt.

Bei der Untersuchung des Postcraniums fallen massive großflächige Auflagerungen auf der Diaphyse der rechten Tibia auf. Sie sind feinporös und mehrlagig, deutlich erhaben und bilden schmale Wülste. Der Rand ist verstrichen (Bildtafel 5, Bild 4).

Außerdem erkennt man „Schnürfurchen“. Dabei handelt es sich um kurze, quer zum Schaft verlaufende Gefäßimpressionen der Periostgefäße. Dies lässt auf eine Reizung des Periosts schließen (WAPLER et al. 2004).

Auch auf der rechten Fibula lassen sich Auflagerungen nachweisen. Sie sind ebenfalls feinporös, jedoch flacher und glatter gestaltet, der Rand ist auch hier verstrichen. Außerdem besteht hier der Verdacht auf eine inkomplette Fraktur (Infraktion).

Die übrigen Langknochen sind - soweit erhalten - unauffällig.

Am Schädel fallen verstärkte Impressiones digitatae auf, in denen sich netzartige Gefäßimpressionen, umgeben von flachen feinporösen Auflagerungen, befinden. Ein ähnliches Bild bietet der rechte Sulcus sinus sigmoidei. Es entsteht der Eindruck eines gut abgeheilten hämorrhagischen Geschehens mit Hirndrucksymptomatik.

In der Mandibula fehlt der zweite Molar rechts, in dessen Alveole finden sich feinporöse Auskleidungen. Der übrige Alveolarbereich ist jedoch unauffällig. Die Prämolaren von Oberund Unterkiefer und die Molaren oben weisen beidseits transversale Schmelzhypoplasien auf. 


\section{Mikroskopischer Befund}

Es existieren ein Schliff durch den harten Gaumen und ein Präparat der rechten Tibia. Am Os palatinum lassen sich zapfenartig in den Mundraum einwachsende Auflagerungen nachweisen, die sich auf eine Stomatitis zurückführen lassen.

An der Tibia finden sich deutliche Auflagerungen vom Typ IV mit Auflösung der darunter liegenden Generallamelle. Es lassen sich auch Spaltbildungen unterhalb der Generallamelle nachweisen. Es bietet sich das Bild einer Osteomyelitis (Bildtafel 5, Bild 5).

\section{Radiologischer Befund}

Beim Durchleuchten fallen zwei Harris-Linien im distalen Schaft der rechten Tibia auf.

\section{Gesamtbeurteilung}

\begin{tabular}{|c|c|c|}
\hline A & \multicolumn{2}{|l|}{ Kardinalmerkmale } \\
\hline 1.) & Poröse AL am äußeren Schädeldach & - \\
\hline 2.) & $\begin{array}{l}\text { Poröse AL am Orbitadach (Cribra } \\
\text { orbitalia) }\end{array}$ & $\begin{array}{l}\text { Cribrum Grad } 2-3 \text { links, poröse } \\
\text { OB/Cribrum Grad } 1 \text { rechts }\end{array}$ \\
\hline 3.) & $\begin{array}{l}\text { Poröse AL am äußeren Gesichts- } \\
\text { schädel }\end{array}$ & - \\
\hline 4.) & Poröse AL am Alveolarknochen & - \\
\hline 5.) & Poröse AL an Langknochenschäften & Tibia rechts, Fibula rechts \\
\hline 6.) & Poröse AL am Schulterblatt & - \\
\hline 7.) & Poröse AL am Darmbein & - \\
\hline B & \multicolumn{2}{|l|}{ Nebenmerkmale } \\
\hline 1.) & $\begin{array}{l}\text { Poröse OB/AL am harten Gaumen } \\
\text { (Stomatitis) }\end{array}$ & $\mathrm{AL}$ \\
\hline 2.) & Poröse Auskleidung der Alveolen & 2. Molar Mandibula rechts \\
\hline 3.) & $\begin{array}{l}\text { Symmetrische poröse AL am } \\
\text { inneren Schädeldach, flächig/in den } \\
\text { Impressiones digitatae }\end{array}$ & $\begin{array}{l}\mathrm{AL} \text { und Gefäßzeichnungen in } \\
\text { Impressiones digitatae von Frontale, } \\
\text { Parietale, Temporale }\end{array}$ \\
\hline 4.) & Poröse OB der Langknochenschäfte & - \\
\hline 5.) & $\begin{array}{l}\text { Poröse Außenfläche des Großen } \\
\text { Keilbeinflügels }\end{array}$ & - \\
\hline $\mathrm{C}$ & Weitere Auffälligkeiten & Schnürfurchen Tibia rechts \\
\hline
\end{tabular}

Tab. 18: Merkmalstabelle zur Diagnose von Skorbut (nach SCHULTZ 1994) angewandt auf Sk 1334 aus dem Gräberfeld Barbing-Kreuzhof; $\mathrm{AL}=$ Auflagerung, $\mathrm{OB}=$ Oberfläche, - = Merkmal liegt nicht vor

Es lassen sich zwei Kardinal- und drei Nebenmerkmale für Skorbut nachweisen. Dennoch ist eine Skorbut-Erkrankung eher unwahrscheinlich. Zwar scheint das Kriterium der porösen Auflagerungen auf Langknochenschäften erfüllt, allerdings sind nur die Tibia und Fibula der rechten Seite befallen, alle anderen Knochen sind unauffällig. Außerdem ergab die lichtmikroskopische Diagnostik an der Tibia das typische Bild einer Osteomyelitis. Es ist wahrscheinlicher, dass es sich bei den Auflagerungen der Tibia um ein lokales Entzündungsgeschehen und bei denen der benachbarten Fibula um eine Begleitreaktion auf diesen Prozess handelt, als um eine systemische Erkrankung. 
Auch die isolierten Alveolarauskleidungen bei einem fehlenden Molaren scheinen nicht durch Skorbut, sondern durch einen lokalen Entzündungsprozess bedingt zu sein.

Lediglich das hämorrhagische Geschehen an der Lamina interna des Schädels sowie die Cribra orbitalia lassen eine Skorbut-Erkrankung wahrscheinlich erscheinen, wobei hier auch an Differentialdiagnosen gedacht werden sollte.

Zusammenfassend kann man sagen, dass eine Skorbut-Erkrankung möglich, aber nicht eindeutig belegbar ist.

\section{Sk $1340 \quad$ Grab 81a 25-45 Jahre männlich}

Bei der Archivierung der Individuen Sk 1339, Sk 1340 und Sk 1341 müssen Fehler passiert sein, da alle drei Individuen unter der Grabnummer 81 katalogisiert wurden. Vermutlich ist für Sk 1340 die korrekte Grabnummer 81a (DETKEN 1991).

\section{Makroskopischer Befund}

Sk 1340 ist das recht gut erhaltene Skelet eines 25 bis 45 Jahre alten Mannes. Bei der makroskopischen Untersuchung fallen kleinflächige feinporöse und bereits gut integrierte Auflagerungen auf. Diese finden sich im Übergang zwischen Metaphyse und Diaphyse, und zwar proximal am rechten Humerus, beiden Radii, beiden Femura und beiden Tibiae, distal am rechten Humerus und beiden Femura; bei den anderen Knochen ist die distale Metaphyse nicht vorhanden. Außerdem finden sich an den Femura Längsstreifen, die ein Relikt gut integrierter früherer Auflagerungen sein könnten, assoziiert mit Schnürfurchen, die von ehemaligen Periostgefäßen erzeugt wurden.

Am Schädel befinden sich flache feinporöse Auflagerungen in den Sulci der venösen Sinus sagittalis, sigmoideus und transversus (übergreifend auf die Crista occipitalis interna); in den Sulci sinus sigmoidei fallen vermehrte Gefäßimpressionen auf (Bildtafel 5, Bild 6).

Der knöcherne Gaumen ist feinporös gestaltet, vermutlich handelt es sich um ehemalige, gut verheilte Auflagerungen. An der Mandibula finden sich feinporöse Auflagerungen im Bereich der hinteren Molaren rechts. Die Alveolarränder sind teilweise eingeschmolzen und miteinander verbunden.

An der Außenseite der Ossa parietalia fällt eine feinporöse Oberfläche in der Scheitelgegend auf; auch die Ossa zygomatica sind feinporös gestaltet.

\section{Mikroskopischer Befund}

Es existieren zwei Schliffe aus dem Os occipitale. Darin lassen sich alte Auflagerungen im angeschnittenen Sulcus sinus transversus nachweisen. Vermutlich handelte es sich hierbei um 
Auflagerungen vom Typ I, also hämorrhagischer Genese. Allerdings ist die Integration bereits so weit fortgeschritten, dass sich dies nicht mehr mit Sicherheit sagen lässt.

\section{Radiologischer Befund}

Das Durchleuchten ergab keine Auffälligkeiten.

\section{Gesamtbeurteilung}

\begin{tabular}{|c|c|c|}
\hline $\mathbf{A}$ & \multicolumn{2}{|l|}{ Kardinalmerkmale } \\
\hline 1.) & Poröse AL am äußeren Schädeldach & - \\
\hline 2.) & $\begin{array}{l}\text { Poröse AL am Orbitadach (Cribra } \\
\text { orbitalia) }\end{array}$ & - \\
\hline 3.) & $\begin{array}{l}\text { Poröse AL am äußeren Gesichts- } \\
\text { schädel }\end{array}$ & - \\
\hline 4.) & Poröse AL am Alveolarknochen & Mandibula \\
\hline 5.) & Poröse AL an Langknochenschäften & $\begin{array}{l}\text { Humerus rechts, Radii, Ulnae, } \\
\text { Femura, Tibiae }\end{array}$ \\
\hline 6.) & Poröse AL am Schulterblatt & - \\
\hline 7.) & Poröse AL am Darmbein & - \\
\hline B & Nebenmerkmale & \\
\hline 1.) & $\begin{array}{l}\text { Poröse OB/AL am harten Gaumen } \\
\text { (Stomatitis) }\end{array}$ & $\mathrm{OB}$, vermutlich gut verheilte $\mathrm{AL}$ \\
\hline 2.) & Poröse Auskleidung der Alveolen & - \\
\hline 3.) & $\begin{array}{l}\text { Symmetrische poröse AL am } \\
\text { inneren Schädeldach, flächig/in den } \\
\text { Impressiones digitatae }\end{array}$ & AL in den Sulci der venösen Sinus \\
\hline 4.) & Poröse OB der Langknochenschäfte & OB Femura \\
\hline 5.) & $\begin{array}{l}\text { Poröse Außenfläche des Großen } \\
\text { Keilbeinflügels }\end{array}$ & - \\
\hline $\mathrm{C}$ & Weitere Auffälligkeiten & $\begin{array}{l}\text { Längsstreifung und Schnürfurchen der } \\
\text { Femura, poröse Oberfläche außen an } \\
\text { Parietale und zygomaticum }\end{array}$ \\
\hline
\end{tabular}

Tab. 19: Merkmalstabelle zur Diagnose von Skorbut (nach SCHULTZ 1994), angewandt auf Sk 1340 aus dem Gräberfeld Barbing-Kreuzhof

$\mathrm{AL}=$ Auflagerung, $\mathrm{OB}=$ Oberfläche, $-=$ Merkmal liegt nicht vor

Es lassen sich zwei Kardinal- und drei Nebenmerkmale für Skorbut nachweisen. Damit erscheint eine Skorbut-Erkrankung wahrscheinlich.

Die merkwürdige symmetrische Verteilung der Auflagerungen an den Langknochen scheint mit den Muskelansätzen assoziiert zu sein. Vermutlich musste der Mann trotz seiner Krankheit schwer körperlich arbeiten, möglicherweise hatte er eine Familie zu ernähren. Die starke Beanspruchung der Muskeln führte dann zu Schäden der durch den Vitamin C Mangel strukturell anfälligen kleinen Gefäße und zur Bildung subperiostaler Hämatome im Bereich der Muskelansätze. 
Alle beschriebenen Auflagerungen machen einen sehr gut integrierten und ausgeheilten Eindruck, sodass man davon ausgehen kann, dass dieser Mann die Skorbut-Erkrankung eine ganze Weile überlebte.
Sk 1342
Grab 82
$5 \mathbf{J}+/-16 \mathbf{M}$
$\mathbf{m}=\mathbf{w}$

Dieses Grab befand sich zwischen Westrand des Gräberfelds und Kirchenmauer (DETKEN 1991).

\section{Makroskopischer Befund}

Bei Sk 1342 handelt es sich um das Skelet eines ca. 5 Jahre alten Kindes (+/- 16 Monate).

Am Postcranium finden sich feinporöse Oberflächen beidseits an der Tuberositas radii, am dorsalen Schaft des rechten Femurs, an der medialen Schaftseite der rechten Tibia und an der distalen Schafthälfte beider Fibulae. Die Oberflächenveränderungen sind feinporös, teilweise strähnig, leicht erhaben und gut integriert. Es sieht aus, als hätten an diesen Stellen einmal feinporöse Auflagerungen vorgelegen. Vor allem an der Tibia entsteht der Eindruck, dass hier eine aberodierte und vorher bereits gut integrierte Auflagerung vorlag.

Außerdem fallen an den Femura verbreiterte Metaphysenplatten mit darüber liegenden Eindellungen des Schafts auf.

Am inneren Schädeldach finden sich in den Impressiones digitatae und den Sulci der venösen Sinus ähnliche Oberflächenveränderungen wie an den Langknochen, auch hier haben vermutlich Auflagerungen vorgelegen. Lediglich an der Mandibula lassen sich noch erhaltene Auflagerungen im Bereich des linken Ramus und des Alveolarfortsatzes nachweisen. Der knöcherne Gaumen zeigt eine feinporöse Oberfläche im Sinne einer ausgeheilten Stomatitis.

An der rechten Orbita liegt ein Cribrum Grad 1 vor, die linke ist nicht erhalten.

\section{Mikroskopischer Befund}

Es wurde ein Schliff von der rechten Tibia angefertigt, dessen Beurteilung durch den schlechten Erhaltungszustand des Knochens eingeschränkt ist. Die fraglichen Oberflächenveränderungen sind eher durch appositionelles Wachstum als durch Auflagerungen verursacht.

Dies überrascht insofern, als das Kind schon etwa 5 Jahre alt gewesen sein muss. Appositionelles Knochenwachstum ist vor allem bei jüngeren Kindern physiologisch.

\section{Radiologischer Befund}

Beim Durchleuchten fallen Harris-Linien am proximalen und distalen Ende beider Tibiae auf. 
Gesamtbeurteilung

\begin{tabular}{|c|c|c|}
\hline $\mathbf{A}$ & \multicolumn{2}{|l|}{ Kardinalmerkmale } \\
\hline 1.) & Poröse AL am äußeren Schädeldach & - \\
\hline 2.) & $\begin{array}{l}\text { Poröse AL am Orbitadach (Cribra } \\
\text { orbitalia) }\end{array}$ & Cribrum Grad 1 rechts \\
\hline 3.) & $\begin{array}{l}\text { Poröse AL am äußeren Gesichts- } \\
\text { schädel }\end{array}$ & - \\
\hline 4.) & Poröse AL am Alveolarknochen & Mandibula \\
\hline 5.) & Poröse AL an Langknochenschäften & - \\
\hline 6.) & Poröse AL am Schulterblatt & - \\
\hline 7.) & Poröse AL am Darmbein & - \\
\hline B & Nebenmerkmale & \\
\hline 1.) & $\begin{array}{l}\text { Poröse OB/AL am harten Gaumen } \\
\text { (Stomatitis) }\end{array}$ & Poröse OB \\
\hline 2.) & Poröse Auskleidung der Alveolen & - \\
\hline 3.) & $\begin{array}{l}\text { Symmetrische poröse } \mathrm{AL} \text { am } \\
\text { inneren Schädeldach, flächig/in den } \\
\text { Impressiones digitatae }\end{array}$ & - \\
\hline 4.) & Poröse OB der Langknochenschäfte & Radii, Femur und Tibia rechts, Fibulae \\
\hline 5.) & $\begin{array}{l}\text { Poröse Außenfläche des Großen } \\
\text { Keilbeinflügels }\end{array}$ & - \\
\hline $\mathrm{C}$ & Weitere Auffälligkeiten & $\begin{array}{l}\text { Poröse OB in den Impressiones } \\
\text { digitatae und in den Sulci sinus } \\
\text { venosus, verbreiterte Metaphysen- } \\
\text { platten am Femur }\end{array}$ \\
\hline
\end{tabular}

Tab. 20: Merkmalstabelle zur Diagnose von Skorbut (nach SCHULTZ 1994), angewandt auf Sk 1342 aus dem Gräberfeld Barbing-Kreuzhof

$\mathrm{AL}=$ Auflagerung, $\mathrm{OB}=$ Oberfläche,$-=$ Merkmal liegt nicht vor

Es lassen sich zwei Kardinal- und zwei Nebenmerkmale für Skorbut nachweisen. Der Verdacht, dass die poröse Oberfläche ein Relikt von hämorrhagischen Auflagerungen ist, lässt sich weder mikroskpisch noch radiologisch beweisen.

Allerdings ist das symmetrische Verteilungsmuster der Oberflächenveränderungen sehr typisch für Skorbut. Würden tatsächlich Auflagerungen vorliegen, so wäre die Diagnose sehr wahrscheinlich. Da dies nicht der Fall ist, kann man einen Vitamin-C-Mangel nicht eindeutig nachweisen.

\section{$\begin{array}{llll}\text { Sk } 1356 & \text { Grab } 95 & 60-80 ~ J & \text { weiblich }\end{array}$}

Das Grab befand sich an der nordwestlichen Begrenzung der Kirchenmauer (DETKEN 1991).

\section{Makroskopischer Befund}

Sk 1356 ist das Skelet einer etwa 60 bis 80 Jahre alten Frau.

Am Postcranium finden sich sehr ausgeprägte Muskel- und Bandansätze an allen Knochen. An den Tibiae fällt eine Längsstreifung auf, an deren Stelle sich möglicherweise einmal 
Auflagerungen befunden haben. Die rechte Tibia weist am distalen Schaftende lateral eine gut integrierte Auflagerung auf.

Die Schädelkalotte erscheint deutlich verdickt. Am Os frontale ca. $3 \mathrm{~cm}$ vor der Sutura coronoidea wurde ein Schliff entnommen, an dieser Stelle beträgt die Dicke des Schädeldachs $0,8 \mathrm{~cm}$. Ansonsten fallen entzündliche Auflagerungen im linken Sinus maxillaris auf, entsprechend einer Sinusitis. Auch am Os palatinum und im Alveolarbereich erkennt man entzündliche Veränderungen.

\section{Mikroskopischer Befund}

Es wurde ein Schliff aus dem verdickten Os frontale untersucht. Hier fällt vor allem eine deutliche Hypertrophie der Diploë zu Ungunsten der Lamina interna bei einer insgesamt unauffälligen Lamina externa auf. Des Weiteren sind die Markräume sehr ungleich gestaltet: Während sie im an die Lamina externa grenzenden Bereich stark vergrößert wirken, erscheinen sie an der Lamina interna sehr engporig (Bildtafel 5, Bild 7 und 8).

\section{Radiologischer Befund}

In der Röntgenaufnahme der rechten Tibia erkennt man am distalen Ende lateral, im Bereich der Syndesmose mit der Fibula eine kleine Auflagerung. Diese ist gut integriert, es scheint sich um ein altes Geschehen, vermutlich ein fast ausgeheiltes subperiostales Hämatom, zu handeln (Bildtafel 6, Bild 1). Die Lokalisation lässt an ein Trauma denken.

\section{Gesamtbeurteilung}

\begin{tabular}{|c|c|c|}
\hline $\mathbf{A}$ & \multicolumn{2}{|l|}{ Kardinalmerkmale } \\
\hline 1.) & Poröse AL am äußeren Schädeldach & - \\
\hline 2.) & $\begin{array}{l}\text { Poröse AL am Orbitadach (Cribra } \\
\text { orbitalia) }\end{array}$ & - \\
\hline 3.) & $\begin{array}{l}\text { Poröse AL am äußeren Gesichts- } \\
\text { schädel }\end{array}$ & - \\
\hline 4.) & Poröse AL am Alveolarknochen & Maxilla rechts \\
\hline 5.) & Poröse AL an Langknochenschäften & Tibia rechts \\
\hline 6.) & Poröse AL am Schulterblatt & - \\
\hline 7.) & Poröse AL am Darmbein & - \\
\hline B & Nebenmerkmale & \\
\hline 1.) & $\begin{array}{l}\text { Poröse OB/AL am harten Gaumen } \\
\text { (Stomatitis) }\end{array}$ & $\mathrm{AL}$ \\
\hline 2.) & Poröse Auskleidung der Alveolen & - \\
\hline 3.) & $\begin{array}{l}\text { Symmetrische poröse } \mathrm{AL} \text { am } \\
\text { inneren Schädeldach, flächig/in den } \\
\text { Impressiones digitatae }\end{array}$ & - \\
\hline 4.) & Poröse OB der Langknochenschäfte & Alle Langknochen \\
\hline 5.) & $\begin{array}{l}\text { Poröse Außenfläche des Großen } \\
\text { Keilbeinflügels }\end{array}$ & - \\
\hline
\end{tabular}




\begin{tabular}{|l|l|l|}
\hline C & Weitere Auffälligkeiten & $\begin{array}{l}\text { Stark ausgeprägte Muskelmarken an } \\
\text { allen Langknochen (auch Clavicula } \\
\text { und Scapula), Tibiae Längsstreifung }\end{array}$ \\
\hline
\end{tabular}

Tab. 21: Merkmalstabelle zur Diagnose von Skorbut (nach SCHULTZ 1994), angewandt auf Sk 1356 aus dem Gräberfeld Barbing-Kreuzhof

$\mathrm{AL}=$ Auflagerung, $\mathrm{OB}=$ Oberfläche, $-=$ Merkmal liegt nicht vor

Es lassen sich zwei Kardinal- und zwei Nebenmerkmale für Skorbut nachweisen. Dennoch erscheint die Diagnose Skorbut eher unwahrscheinlich. Die Auflagerungen an den Alveolarknochen beschränken sich auf die rechte Maxilla und scheinen eher auf Parodontopathien als auf einen hämorrhagischen Prozess zurückzugehen. Das Gleiche gilt für die Stomatitis und die Sinusitis.

Auflagerungen an den Langknochen lassen sich lediglich an der rechten Tibia nachweisen. Ein solch isolierter Befund dieser Lokalisation lässt ein Hämatom aufgrund eines Traumas wahrscheinlicher erscheinen als eine systemische Erkrankung.

Das mikroskopische Bild des Os frontale weist auf eine maligne Erkrankung wie Leukämie hin. BURKHARDT (1970) beschreibt sehr ähnliche Veränderungen am Schädeldach von an Leukämie verstorbenen Patienten. Wie bei dem hier vorliegenden Präparat weisen auch BURKHARDTs (1970) Fälle eine unauffällige Lamina externa des Schädeldachs auf, während die Markräume vor allem im äußeren Bereich irregulär erweitert sind und zu einer Osteophytbildung an der Lamina interna führen. Diese erscheint teilweise treppenförmig (BURKHARDT 1970), was in Ansätzen auch bei dem hier untersuchten Präparat zu erkennen ist.

Aufgrund dieser Beobachtungen wird für das Skelet SK 1356 die Diagnose einer nicht näher differenzierbaren Leukämie gestellt.

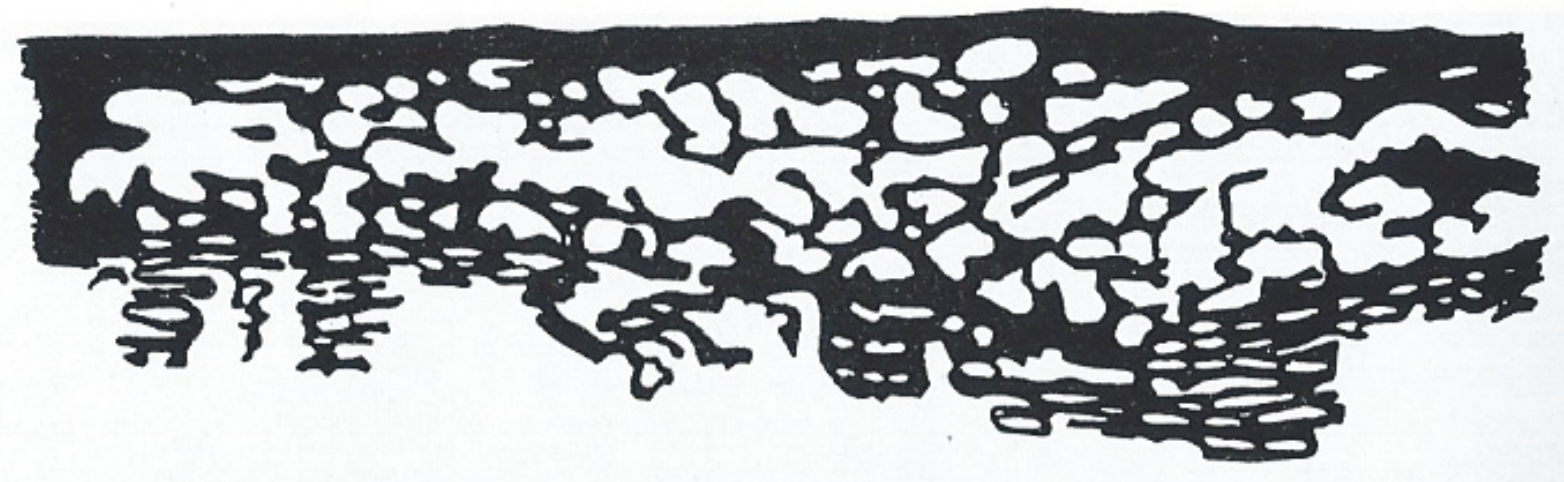

Abb. 8: Die Abbildung stammt von BURKHARDT 1970 (S. 292). Sie zeigt einen Schnitt durch das Schädeldach eines dreijährigen Kindes, das an Leukämie verstorben ist. Die Lamina externa ist unauffällig, an der Lamina interna sind treppenförmige Aufbauten zu sehen, die Markräume sind im Bereich der Lamina externa irregulär erweitert. 
Das Grab befand sich an der nordöstlichen Begrenzung des Gräberfeldes (DETKEN 1991).

\section{Makroskopischer Befund}

Sk 1363 ist das Skelet eines ca. 7jährigen Kindes (+/- 24 Monate). Am Postcranium fallen deutliche feinporöse Auflagerungen mit profiliertem Rand auf, die teilweise mehrlagig sind. Sie befinden sich an der dorsolateralen Schaftseite der proximalen Femura (Bildtafel 6, Bild 2), an den anterolateralen Tibiaschäften (Bildtafel 6, Bild 7) und ventral an der rechten Fibula. Auch am Darmbein sind beidseits Auflagerungen nachweisbar, die jedoch schon ziemlich erodiert sind.

Am inneren Schädeldach finden sich vergleichbare Auflagerungen symmetrisch in den Impressiones digitatae von Os frontale, Os parietale und Os temporale (Bildtafel 6, Bild 3, 4, 5 und 6).

Der knöcherne Gaumen weist eine feinporöse Oberfläche auf.

\section{Mikroskopischer Befund}

Es liegen Schliffe aus dem linken Os coxae sowie aus dem linken Femur vor. Am Os coxae lassen sich diskrete, etwas abgemürbte Auflagerungen vom Typ I nachweisen.

Am Femur fallen deutliche subperiostale Neubildungen im Sinne einer Auflagerung vom Typ I auf, die stellenweise zweilagig sind (Bildtafel 7, Bild 1 und 2).

\section{Radiologischer Befund}

Beim Durchleuchten fielen Harris-Linien am proximalen und distalen Schaft beider Tibiae auf.

\section{Gesamtbeurteilung}

\begin{tabular}{|c|c|c|}
\hline $\mathbf{A}$ & \multicolumn{2}{|l|}{ Kardinalmerkmale } \\
\hline 1.) & Poröse AL am äußeren Schädeldach & - \\
\hline 2.) & $\begin{array}{l}\text { Poröse AL am Orbitadach (Cribra } \\
\text { orbitalia) }\end{array}$ & - \\
\hline 3.) & $\begin{array}{l}\text { Poröse AL am äußeren Gesichts- } \\
\text { schädel }\end{array}$ & - \\
\hline 4.) & Poröse AL am Alveolarknochen & - \\
\hline 5.) & Poröse AL an Langknochenschäften & Femura, Tibiae, Fibula rechts \\
\hline 6.) & Poröse AL am Schulterblatt & - \\
\hline 7.) & Poröse AL am Darmbein & AL beidseits \\
\hline B & Nebenmerkmale & \\
\hline 1.) & $\begin{array}{l}\text { Poröse OB/AL am harten Gaumen } \\
\text { (Stomatitis) }\end{array}$ & OB \\
\hline 2.) & Poröse Auskleidung der Alveolen & - \\
\hline 3.) & $\begin{array}{l}\text { Symmetrische poröse AL am } \\
\text { inneren Schädeldach, flächig/in den } \\
\text { Impressiones digitatae }\end{array}$ & Frontale, Parietale, Temporale \\
\hline 4.) & Poröse OB der Langknochenschäfte & - \\
\hline
\end{tabular}




\begin{tabular}{|l|l|l|}
\hline 5.$)$ & $\begin{array}{l}\text { Poröse Außenfläche des Großen } \\
\text { Keilbeinflügels }\end{array}$ & - \\
\hline C & Weitere Auffälligkeiten & - \\
\hline
\end{tabular}

Tab. 22: Merkmalstabelle zur Diagnose von Skorbut (nach SCHULTZ 1994) angewandt auf Sk 1363 aus dem Gräberfeld Barbing-Kreuzhof

$\mathrm{AL}=$ Auflagerung, $\mathrm{OB}=$ Oberfläche, $-=$ Merkmal liegt nicht vor

Es lassen sich zwei Kardinal- und zwei Nebenmerkmale für Skorbut nachweisen. Diese können auch lichtmikroskopisch nachgewiesen werden. Die symmetrische Verteilung unterstützt den Verdacht, so dass Skorbut sehr wahrscheinlich erscheint.

\section{Sk $1365 \quad$ Grab $104 \quad>65 \mathrm{~J} \quad$ männlich}

Das Grab lag zwischen der nördlichen Begrenzung des Gräberfelds und der Kirche (DETKEN 1991).

\section{Makroskopischer Befund}

Bei Sk 1365 handelt es sich um das Skelet eines mindestens 65 Jahre alten Mannes. Bei der Begutachtung des Postcraniums fallen zunächst starke arthrotische Veränderungen, besonders an der linken oberen Extremität, auf. Vermutlich bestand hier eine Schultergelenksluxation, die nicht reponiert wurde und über Jahre bestehen blieb. Die Verletzung erfolgte wahrscheinlich schon in der Wachstumsphase, denn die Knochen des linken Unterarms sind deutlich kürzer als die der Gegenseite.

Außerdem fällt eine feinporöse Oberfläche der Tibiae (Bildtafel 7, Bild 5) und Femura auf. Diese weisen ferner eine Längsstreifung an den ventralen Diaphysen auf. An beiden befinden sich im distalen Bereich kleinflächige feinporöse Auflagerungen, die sich aber bei näherer Untersuchung unter dem Binokular als postmortale Veränderungen darstellen.

Deutliche feinporöse Auflagerungen mit profiliertem Rand von mehreren Zentimetern Ausdehnung lassen sich jedoch an der Lateralseite des rechten Calcaneus erkennen; sie könnten hämorrhagischen Ursprungs sein (Bildtafel 7, Bild 3 und 4).

Am Schädel fallen deutlich ausgeprägte Gefäßimpressionen an der Lamina externa im Frontalbereich und an der Lamina interna von Os frontale und Os parietale auf, auch Foveolae granulares finden sich in großer Zahl. Im Bereich der Crista frontalis sind feinporöse Oberflächenveränderungen erkennbar, die erodierte Auflagerungen sein könnten.

An den Orbitadächern befinden sich pinselstrichartige netzförmige Gefäßimpressionen. Der Sinus maxillaris und das Palatum durum beider Seiten zeigen eine feinporöse Oberflächengestaltung, wie nach Abheilung einer Entzündung. 


\section{Mikroskopischer Befund}

Es liegen keine mikroskopischen Präparate vor.

\section{Radiologischer Befund}

Im Röntgenbild fallen keine Pathologien auf.

\section{Gesamtbeurteilung}

\begin{tabular}{|c|c|c|}
\hline $\mathbf{A}$ & \multicolumn{2}{|l|}{ Kardinalmerkmale } \\
\hline 1.) & Poröse AL am äußeren Schädeldach & - \\
\hline 2.) & $\begin{array}{l}\text { Poröse AL am Orbitadach (Cribra } \\
\text { orbitalia) }\end{array}$ & $\begin{array}{l}\text { - (lediglich } \\
\text { Gefäßimpressionen) }\end{array}$ \\
\hline 3.) & $\begin{array}{l}\text { Poröse AL am äußeren Gesichts- } \\
\text { schädel }\end{array}$ & - \\
\hline 4.) & Poröse AL am Alveolarknochen & - \\
\hline 5.) & Poröse AL an Langknochenschäften & - \\
\hline 6.) & Poröse AL am Schulterblatt & - \\
\hline 7.) & Poröse AL am Darmbein & - \\
\hline $\mathbf{B}$ & Nebenmerkmale & \\
\hline 1.) & $\begin{array}{l}\text { Poröse OB/AL am harten Gaumen } \\
\text { (Stomatitis) }\end{array}$ & OB \\
\hline 2.) & Poröse Auskleidung der Alveolen & - \\
\hline 3.) & $\begin{array}{l}\text { Symmetrische poröse AL am } \\
\text { inneren Schädeldach, flächig/in den } \\
\text { Impressiones digitatae }\end{array}$ & - \\
\hline 4.) & Poröse OB der Langknochenschäfte & $\begin{array}{l}\text { Tibiae und Femura, zusätzlich } \\
\text { Längsstreifung }\end{array}$ \\
\hline 5.) & $\begin{array}{l}\text { Poröse Außenfläche des Großen } \\
\text { Keilbeinflügels }\end{array}$ & - \\
\hline $\mathrm{C}$ & Weitere Auffälligkeiten & $\begin{array}{l}\text { Längsstreifung (s.o.), Polyarthrose, } \\
\text { ausgeprägte Gefäßimpressionen des } \\
\text { Schädeldachs, Auflagerungen am } \\
\text { rechten Calcaneus }\end{array}$ \\
\hline
\end{tabular}

Tab. 23: Merkmalstabelle zur Diagnose von Skorbut (nach SCHULTZ 1994), angewandt auf Sk 1365 aus dem Gräberfeld Barbing-Kreuzhof

$\mathrm{AL}=$ Auflagerung, $\mathrm{OB}=$ Oberfläche, $-=$ Merkmal liegt nicht vor

Es lassen sich lediglich zwei Nebenmerkmale für Skorbut nachweisen. Damit ist eine Erkrankung eher unwahrscheinlich. Die Veränderungen der Gelenkflächen lassen sich durch eine im Alter häufig auftretende Polyarthrose erklären; die Deformation des linken Humeruskopfes und die Verkürzung des linken Unterarms gehen wohl auf ein Trauma in der Jugend und konsekutive Inaktivitätsatrophie zurück. Die Stomatitis und die Sinusitis maxillaris sind vermutlich als singuläre Entzündungen zu werten, die möglicherweise in einem kausalen Zusammenhang zu sehen sind. 
Die Auflagerungen am Calcaneus sind wohl durch ein recht kurz vor dem Tod des Individuums entstandenes organisiertes subperiostales Hämatom bedingt, das vermutlich eine traumatische Ursache hatte.
Sk 1368
Grab 104
$4 \mathrm{~J}+/-12 \mathrm{M}$
$\mathbf{m}=\mathbf{w}$

Das Grab war an der nördlichen Begrenzung des Gräberfelds lokalisiert. Das Postcranium ist, vermutlich aufgrund von Grabstörung, nur in Fragmenten erhalten (DETKEN 1991).

\section{Makroskopische Befundung}

Bei Sk 1368 handelt es sich um den stark fragmentierten Schädel eines ca. 4 Jahre alten (+/12 Monate) Kindes. Vom Postcranium sind nur der linke Humerus und einige Rippenfragmente erhalten; diese sind jedoch so erodiert, dass eine Befundung kaum möglich ist. Allerdings ist die Oberfläche, soweit erhalten, feinporös. Möglicherweise handelt es sich hier um erodierte Auflagerungen.

Am Palatum durum finden sich mehrlagige feinporöse Auflagerungen im Sinne einer Stomatitis (Bildtafel 7, Bild 6). Auch im Alveolarbereich von Maxilla und Mandibula beider Seiten finden sich feinporöse Auflagerungen in Form kurz vor dem Tode angelegter Platten. Sowohl außen um die Apertura piriformis beider Maxillae als auch außen an den Rami mandibulae sowie innen an den Incisurae mandibulae finden sich feinporöse Auflagerungen, diese sind jedoch schon stärker integriert als die im Alveolarbereich.

\section{Mikroskopischer Befund}

Von der Anfertigung von Schliffen wurde aufgrund des schlechten Erhaltungszustands der Knochen abgesehen.

\section{Radiologischer Befund}

Das Durchleuchten brachte keine weiteren Erkenntnisse.

\section{Gesamtbeurteilung}

\begin{tabular}{|l|l|l|}
\hline A & Kardinalmerkmale \\
\hline 1.) & Poröse AL am äußeren Schädeldach & - \\
\hline 2.) & $\begin{array}{l}\text { Poröse AL am Orbitadach (Cribra } \\
\text { orbitalia) }\end{array}$ & - \\
\hline 3.) & $\begin{array}{l}\text { Poröse AL am äußeren Gesichts- } \\
\text { schädel }\end{array}$ & Maxilla, Mandibula \\
\hline 4.) & Poröse AL am Alveolarknochen & Maxilla, Mandibula \\
\hline 5.$)$ & Poröse AL an Langknochenschäften & - \\
\hline 6.$)$ & Poröse AL am Schulterblatt & - \\
\hline 7.) & Poröse AL am Darmbein & - \\
\hline B & Nebenmerkmale & $\begin{array}{l}\text { Poröse OB/AL am harten Gaumen } \\
\text { (Stomatitis) }\end{array}$ \\
\hline 1.$)$ & AL \\
\hline
\end{tabular}




\begin{tabular}{|l|l|l|}
\hline 2.) & Poröse Auskleidung der Alveolen & - \\
\hline 3.$)$ & $\begin{array}{l}\text { Symmetrische poröse AL am } \\
\text { inneren Schädeldach, flächig/in den } \\
\text { Impressiones digitatae }\end{array}$ & - \\
\hline 4.) & Poröse OB der Langknochenschäfte & Humerus links \\
\hline 5.) & $\begin{array}{l}\text { Poröse Außenfläche des Großen } \\
\text { Keilbeinflügels }\end{array}$ & - \\
\hline C & Weitere Auffälligkeiten & - \\
\hline
\end{tabular}

Tab. 24: Merkmalstabelle zur Diagnose von Skorbut (nach SCHULTZ 1994), angewandt auf Sk 1368 aus dem Gräberfeld Barbing-Kreuzhof

$\mathrm{AL}=$ Auflagerung, $\mathrm{OB}=$ Oberfläche, - = Merkmal liegt nicht vor

Es lassen sich zwei Kardinalmerkmale und ein Nebenmerkmal für Skorbut nachweisen. Leider ist eine lichtmikroskopische Diagnostik nicht möglich. Selbstverständlich ist eine Diagnose ausschließlich anhand des Schädels nicht sehr valide. Dennoch erscheint eine Skorbut-Erkrankung möglich. Differentialdiagnostisch könnte an eine entzündliche Erkrankung des Mundraums gedacht werden, allerdings erscheint eine Streuung in den Bereich des Ramus mandibulae und der Incisura piriformis ungewöhnlich.
Sk 1371
Grab 110c
$\mathbf{1} \mathbf{J}+/-\mathbf{4} \mathbf{M}$
$\mathbf{m}=\mathbf{w}$

Das Grab war Teil einer Dreifachbestattung, bei der unten das Erwachsenengrab 110a, auf dessen rechten Bein das Kindergrab 110b (archiviert unter Sk 1372 Grab 111) und auf der linken Brust das Kindergrab 110c lagen. Die Gräber befanden sich am Nordrand des Gräberfelds zwischen den Gräbern 104, 109 und 113 (DETKEN 1991). Im Grab wurde ein Glasstück, vermutlich ein Zwickelstück aus einer Butzenscheibe, gefunden (GEISLER 1983).

\section{Makroskopischer Befund}

Bei Sk 1371 handelt es sich um Teile des Skelets eines Säuglings von etwa einem Jahr (+/- 4 Monate), bei dem keine Geschlechtsbestimmung möglich ist.

Am Postcranium fallen feinporöse Oberflächenveränderungen am linken Humerus und an den Tibiae auf, bei denen es sich vermutlich um postmortal erodierte Auflagerungen handelt. Am rechten Femur sind diese Auflagerungen an der medialen Schaftkante noch erhalten (Bildtafel 7, Bild 7), wenn auch nicht vollständig. Außerdem sind offenbar - vor allem an den Femura die Metaphysenplatten verbreitert (Bildtafel 7, Bild 7 und 8).

Vom Schädel sind nur einige kaum beurteilbare Fragmente erhalten. Am vorhandenen Teil der Mandibula sind flache feinporöse Auflagerungen mit profiliertem Rand zu erkennen.

\section{Radiologischer Befund}

Im Röntgenbild der Tibia erkennt man ventral eine flache Auflagerung. Auch dorsal ist distal eine Doppellinienstruktur wie bei einer Auflagerung zu sehen. Vermutlich handelt es sich hier 
jedoch eher um eine Überprojektion. Außerdem sind an beiden Tibiae proximal und distal Harris-Linien zu erkennen.

\section{Gesamtbeurteilung}

\begin{tabular}{|c|c|c|}
\hline A & \multicolumn{2}{|l|}{ Kardinalmerkmale } \\
\hline 1.) & Poröse AL am äußeren Schädeldach & - \\
\hline 2.) & $\begin{array}{l}\text { Poröse AL am Orbitadach (Cribra } \\
\text { orbitalia) }\end{array}$ & - \\
\hline 3.) & $\begin{array}{l}\text { Poröse AL am äußeren Gesichts- } \\
\text { schädel }\end{array}$ & - \\
\hline 4.) & Poröse AL am Alveolarknochen & Mandibula \\
\hline 5.) & Poröse AL an Langknochenschäften & Tibiae, Femura (Humerus) \\
\hline 6.) & Poröse AL am Schulterblatt & - \\
\hline 7.) & Poröse AL am Darmbein & - \\
\hline B & Nebenmerkmale & \\
\hline 1.) & $\begin{array}{l}\text { Poröse OB/AL am harten Gaumen } \\
\text { (Stomatitis) }\end{array}$ & - \\
\hline 2.) & Poröse Auskleidung der Alveolen & - \\
\hline 3.) & $\begin{array}{l}\text { Symmetrische poröse } \mathrm{AL} \text { am } \\
\text { inneren Schädeldach, flächig/in den } \\
\text { Impressiones digitatae }\end{array}$ & - \\
\hline 4.) & Poröse OB der Langknochenschäfte & Humerus, Tibiae, Femura \\
\hline 5.) & $\begin{array}{l}\text { Poröse Außenfläche des Großen } \\
\text { Keilbeinflügels }\end{array}$ & - \\
\hline $\mathrm{C}$ & Weitere Auffälligkeiten & Metaphysenplattenverbreiterung \\
\hline
\end{tabular}

Tab. 25: Merkmalstabelle zur Diagnose von Skorbut (nach SCHULTZ 1994), angewandt auf Sk 1371 aus dem Gräberfeld Barbing-Kreuzhof

$\mathrm{AL}=$ Auflagerung, $\mathrm{OB}=$ Oberfläche, - = Merkmal liegt nicht vor

Es lassen sich zwei Kardinalmerkmale und ein Nebenmerkmal für Skorbut nachweisen.

Eingeschränkt wird die Validität der Diagnose durch den schlechten Erhaltungszustand des Skelets, vor allem des Schädels, die symmetrische Anordnung der Auflagerungen an den Langknochen aber bestätigt ein systemisches hämorrhagisches Geschehen. Die Auflagerungen im Alveolarbereich sollten nicht überbewertet werden, da Veränderungen dieser Art - bis zu einem gewissen Grad - während des Zahnens auch physiologisch sein können.

Insgesamt erscheint eine Skorbut-Erkrankung wahrscheinlich.
Sk 1372
Grab 111
$1,5-2,5 \mathrm{~J}$
$\mathbf{m}=\mathbf{w}$

Das Grab war Teil der bei Sk 1371 geschilderten Dreifachbestattung.

\section{Makroskopischer Befund}

Bei Sk 1372 handelt es sich um die Überreste eines Kindes von etwa 1,5 bis 2,5 Jahren. Vom Postcranium sind nur die unteren Extremitäten erhalten. An den Femura fallen eine 
Längsstreifung am dorsalen Schaft und mehrere Flächen einer feinporösen Oberfläche auf, an denen sich möglicherweise einmal feinporöse Auflagerungen befunden haben.

Der Schädel ist stark fragmentiert. Es finden sich feinporöse Auflagerungen außen am Schädeldach an Os parietale und Os occipitale. Sie liegen in der Nähe der Suturen, sind teilweise mehrlagig und haben einen unterschnittenen Rand. Ein ähnliches Bild zeigt sich an der Innenseite des nur links erhaltenen Os parietale entlang der Sutura coronoidea. Auch im Sulcus sinus occipitalis und in den Sulci sinus transversi finden sich mehrlagige feinporöse Auflagerungen mit unterschnittenem Rand (Bildtafel 8, Bild 1). Es entsteht der Eindruck eines akuten hämorrhagischen Geschehens.

Der knöcherne Gaumen ist ebenfalls mit frischen feinporösen Auflagerungen bedeckt, und auch im Alveolarbereich der Mandibulae finden sich ähnliche Neubildungen.

\section{Mikroskopischer Befund}

Aufgrund der schlecht erhaltenen Knochensubstanz konnten keine Schliffe erstellt werden.

\section{Radiologischer Befund}

Beim Durchleuchten ergaben sich Harris-Linien am proximalen und distalen Schaft beider Tibiae.

\section{Gesamtbeurteilung}

\begin{tabular}{|l|l|l|}
\hline A & Kardinalmerkmale \\
\hline 1.) & Poröse AL am äußeren Schädeldach & Parietale, Occipitale \\
\hline 2.) & $\begin{array}{l}\text { Poröse AL am Orbitadach (Cribra } \\
\text { orbitalia) }\end{array}$ & - \\
\hline 3.$)$ & $\begin{array}{l}\text { Poröse AL am äußeren Gesichts- } \\
\text { schädel }\end{array}$ & - \\
\hline 4.) & Poröse AL am Alveolarknochen & Mandibula, Maxilla \\
\hline 5.) & Poröse AL an Langknochenschäften & - \\
\hline 6.) & Poröse AL am Schulterblatt & - \\
\hline 7.) & Poröse AL am Darmbein & - \\
\hline B & Nebenmerkmale & $\begin{array}{l}\text { AL } \\
\text { (Stomatitis) }\end{array}$ \\
\hline 1.) & $\begin{array}{l}\text { Poröse OB/AL am harten Gaumen } \\
\text { 2.) }\end{array}$ Poröse Auskleidung der Alveolen & - \\
\hline 3.) & $\begin{array}{l}\text { Symmetrische poröse AL am } \\
\text { inneren Schädeldach, flächig/in den } \\
\text { Impressiones digitatae }\end{array}$ & AL \\
\hline 4.) & Poröse OB der Langknochenschäfte & Femura \\
\hline 5.) & $\begin{array}{l}\text { Poröse Außenfläche des Großen } \\
\text { Keilbeinflügels }\end{array}$ & - \\
\hline C & Weitere Auffälligkeiten & Harris-Linien \\
\hline
\end{tabular}

Tab. 26: Merkmalstabelle zur Diagnose von Skorbut (nach SCHULTZ 1994), angewandt auf Sk 1372 aus dem Gräberfeld Barbing-Kreuzhof

$\mathrm{AL}=$ Auflagerung, $\mathrm{OB}=$ Oberfläche, $-=$ Merkmal liegt nicht vor 
Es lassen sich zwei Kardinal- und drei Nebenmerkmale für Skorbut nachweisen. Damit erscheint die Diagnose recht sicher, auch wenn keine mikroskopische Diagnostik vorgenommen werden kann. Zwar lassen sich am unvollständig erhaltenen Postcranium keine Auflagerungen nachweisen, aber die Oberflächenveränderungen weisen darauf hin. Sehr bedeutsam erscheint das gleichzeitige Vorliegen hämorrhagischer Prozesse an der Innen- und Außenseite der Schädelkalotte. Dies lässt auf eine systemische Ursache schließen.

Insgesamt ist eine Skorbut-Erkrankung wahrscheinlich.

Sk 1377

Grab 115b

$17-23 \mathrm{~J}$

weiblich

Das Grab lag an der westlichen Begrenzung des Gräberfeldes unter einem anderen Erwachsenengrab (DETKEN 1991).

\section{Makroskopischer Befund}

Bei Sk 1377 handelt es sich um die unteren Extremitäten eines vermutlich weiblichen Individuums im Alter zwischen 17 und 23 Jahren. Weitere Langknochen und der Schädel sind nicht erhalten. An den Schäften von Femura und Tibiae finden sich feinporöse Oberflächenveränderungen (Bildtafel 8, Bild 2). Sie sind vor allem ventral lokalisiert, reichen aber bis auf die dorsale Schaftseite. Sie haben zusätzlich zur Porosität ein wellenförmig gesträhntes Relief. Stellenweise wirkt die Oberfläche erhaben, möglicherweise handelt es sich hier um Auflagerungen.

\section{Mikroskopischer Befund}

Es wurden Schliffe vom linken Femur und von der linken Tibia angefertigt. Aus beiden ergibt sich kein Anhalt für einen hämorrhagischen Prozess. Die Corticalis ist stark ausgeprägt, es finden sich tiefe Gefäßimpressionen und annähernd polsterartige Neubildungen auf der Corticalis (Bildtafel 8, Bild 3). Insgesamt bietet sich ein Bild entsprechend AL vom Typ V, wie man sie bei Lepra findet.

\section{Radiologischer Befund}

Das Durchleuchten zeigte an beiden Tibiae proximal und distal Harris-Linien.

\section{Gesamtbeurteilung}

\begin{tabular}{|l|l|l|}
\hline A & Kardinalmerkmale \\
\hline 1.) & Poröse AL am äußeren Schädeldach & - \\
\hline 2.) & $\begin{array}{l}\text { Poröse AL am Orbitadach (Cribra } \\
\text { orbitalia) }\end{array}$ & - \\
\hline 3.) & $\begin{array}{l}\text { Poröse AL am äußeren Gesichts- } \\
\text { schädel }\end{array}$ & - \\
\hline 4.) & Poröse AL am Alveolarknochen & - \\
\hline 5.) & Poröse AL an Langknochenschäften & - \\
\hline 6.) & Poröse AL am Schulterblatt & - \\
\hline
\end{tabular}




\begin{tabular}{|l|l|l|}
\hline 7.) & Poröse AL am Darmbein & - \\
\hline B & Nebenmerkmale \\
\hline 1.$)$ & $\begin{array}{l}\text { Poröse OB/AL am harten Gaumen } \\
\text { (Stomatitis) }\end{array}$ & - \\
\hline 2.) & Poröse Auskleidung der Alveolen & - \\
\hline 3.) & $\begin{array}{l}\text { Symmetrische poröse AL am } \\
\text { inneren Schädeldach, flächig/in den } \\
\text { Impressiones digitatae }\end{array}$ & - \\
\hline 4.) & Poröse OB der Langknochenschäfte & OB, wellenartig gesträhnt \\
\hline 5.) & $\begin{array}{l}\text { Poröse Außenfläche des Großen } \\
\text { Keilbeinflügels }\end{array}$ & - \\
\hline C & Weitere Auffälligkeiten & - \\
\hline
\end{tabular}

Tab. 27: Merkmalstabelle zur Diagnose von Skorbut (nach SCHULTZ 1994), angewandt auf Sk 1377 aus dem Gräberfeld Barbing-Kreuzhof

$\mathrm{AL}=$ Auflagerung, $\mathrm{OB}=$ Oberfläche, - = Merkmal liegt nicht vor

Es lässt sich nur ein Nebenmerkmal für Skorbut nachweisen. Damit ist Skorbut annähernd ausgeschlossen. Die mikroskopische Untersuchung ergibt die Verdachtsdiagnose Lepra, die auch aufgrund des makroskopischen Aspekts sehr wahrscheinlich ist.
Sk 1380
Grab 117a
$23-25 \mathrm{~J}$
weiblich

Dieses Skelet entstammt einer Doppelbestattung im nordwestlichen Teil des Gräberfelds und lag unmittelbar auf dem Oberkörper eines männlichen adulten Individuums (DETKEN 1991).

\section{Makroskopischer Befund}

Sk 1380 ist das Skelet einer jungen Frau von etwa 23 bis 25 Jahren. Bei der Untersuchung des Postcraniums fallen feinporöse Auflagerungen an den Rippen auf. Sie sind teilweise mehrlagig, haben einen profilierten Rand und sind an der pleuralen Seite von Collum und Corpus an fast allen erhaltenen Rippen beider Seiten lokalisiert.

Ähnliche Auflagerungen finden sich beidseits am Os ilii in der Fossa iliaca, oberhalb des Acetabulums und an der Spina iliaca anterior inferior.

Weitere Auflagerungen liegen am medialen Tuber des rechten Calcaneus.

Auch an den distalen Tibiae sind lateral feinporöse Auflagerungen mit profiliertem Rand zu erkennen, ebenso an der rechten Fibula.

Des Weiteren fällt eine deutliche Längsstreifung an Femura und Tibiae auf.

Vom Schädel sind nur Teile des Schädeldachs und des Gesichtsschädels erhalten. Hier finden sich ausgeprägte Gefäßimpressionen an den Orbitadächern und an der Innenseite des Os frontale. Im Frontalbereich fallen auch ausgeprägte Foveolae granulares sowie eine deutliche Seitendifferenz der Schädeldicke (links $0,9 \mathrm{~cm}$, rechts $0,6 \mathrm{~cm}$ ) auf.

Der rechte Sinus maxillaris ist mit netzartigen Auflagerungen ausgekleidet. 


\section{Mikroskopischer Befund}

Es wurden Schliffe der linken Fibula (Bildtafel 8, Bild 6), der linken Tibia und einer Rippe (Bildtafel 8, Bild 5) angefertigt. An der Fibula und der Rippe lassen sich teilweise massive Auflagerungen vom Typ I nachweisen, die einen hämorrhagischen Prozess nahelegen.

Auch an der Tibia (Bildtafel 8, Bild 7) sind Auflagerungen erkennbar, allerdings sind diese sehr kurz und polsterartig, also entsprechend einem Typ V. Die Generallamelle ist erhalten und die gesamte Neubildung bereits lamellenknöchern organisiert, also vermutlich ausgeheilt.

\section{Radiologischer Befund}

Im Röntgenbild einer Rippe lassen sich die Auflagerungen gut erkennen (Bildtafel 8, Bild 4). Außerdem sind in beiden Tibiae proximal und distal Harris-Linien zu erkennen.

\section{Gesamtbeurteilung}

\begin{tabular}{|c|c|c|}
\hline A & \multicolumn{2}{|l|}{ Kardinalmerkmale } \\
\hline 1.) & Poröse AL am äußeren Schädeldach & - \\
\hline 2.) & $\begin{array}{l}\text { Poröse AL am Orbitadach (Cribra } \\
\text { orbitalia) }\end{array}$ & - \\
\hline 3.) & $\begin{array}{l}\text { Poröse AL am äußeren Gesichts- } \\
\text { schädel }\end{array}$ & - \\
\hline 4.) & Poröse AL am Alveolarknochen & - \\
\hline 5.) & Poröse AL an Langknochenschäften & Tibiae, Fibula rechts \\
\hline 6.) & Poröse AL am Schulterblatt & - \\
\hline 7.) & Poröse AL am Darmbein & Beidseits \\
\hline B & Nebenmerkmale & \\
\hline 1.) & $\begin{array}{l}\text { Poröse OB/AL am harten Gaumen } \\
\text { (Stomatitis) }\end{array}$ & - \\
\hline 2.) & Poröse Auskleidung der Alveolen & - \\
\hline 3.) & $\begin{array}{l}\text { Symmetrische poröse AL am } \\
\text { inneren Schädeldach, flächig/in den } \\
\text { Impressiones digitatae }\end{array}$ & - \\
\hline 4.) & Poröse OB der Langknochenschäfte & - \\
\hline 5.) & $\begin{array}{l}\text { Poröse Außenfläche des Großen } \\
\text { Keilbeinflügels }\end{array}$ & - \\
\hline $\mathrm{C}$ & Weitere Auffälligkeiten & $\begin{array}{l}\text { Längsstreifung Tibiae und Femura, } \\
\text { poröse AL Innenseite der Rippen, } \\
\text { ausgeprägte Gefäßimpressionen an } \\
\text { Orbitadächern, Auflagerungen am } \\
\text { Calcaneus }\end{array}$ \\
\hline
\end{tabular}

Tab. 28: Merkmalstabelle zur Diagnose von Skorbut (nach SCHULTZ 1994), angewandt auf Sk 1380 aus dem Gräberfeld Barbing-Kreuzhof

$\mathrm{AL}=$ Auflagerung, $\mathrm{OB}=$ Oberfläche, $-=$ Merkmal liegt nicht vor

Es lassen sich zwei Kardinalmerkmale und ein Nebenmerkmal für Skorbut nachweisen. Die hämorrhagischen Auflagerungen können auch lichtmikroskopisch bestätigt werden. Allerdings sind an der Tibia Auflagerungen zu erkennen, wie sie offenbar für Lepra typisch 
sind. Leider sind die knöchernen Strukturen um die Apertura piriformis, die Aufschluss über lepratypische Veränderungen im Gesichtsbereich geben könnten, nicht erhalten.

Für die vorliegende Befundkonstellation gibt es zwei Erklärungsmöglichkeiten: Man könnte davon ausgehen, dass diese junge Frau sowohl an Skorbut als auch an Lepra erkrankt war. Möglicherweise war sie aufgrund der Lepra-Erkrankung isoliert und hatte keinen Zugang zu frischen Lebensmitteln oder war nicht in der Lage, sie sich selbst zu beschaffen, was zum Vitamin-C-Mangel geführt haben könnte.

Man könnte sich die hämorrhagischen Auflagerungen jedoch auch anders erklären: Die Auflagerungen an den Rippen könnte man auf eine infektiöse Pleuritis zurückführen. Bei der Auflagerung an der Fibula könnte es sich um ein durch ein Trauma entstandenes organisiertes subperiostales Hämatom handeln. Das Gleiche könnte für die Auflagerungen am Calcaneus gelten, zumal Stolpertraumata bei Leprakranken aufgrund der eingeschränkten Sensibilität häufiger vorkommen. Möglicherweise handelt es sich bei den Auflagerungen am Calcaneus auch um lepröse Auflagerungen, auch wenn sie makroskopisch einen hämorrhagischen Aspekt zeigen. All diese Symptome könnten natürlich auch durch Skorbut hervorgerufen bzw. verstärkt werden. Durch die erhöhte Infektanfälligkeit steigt das Risiko von Pneumonien und Pleuritiden. Und auch Mikrotraumata können zu starken Blutungen führen.

Schwer fällt es, eine andere Erklärung als Skorbut für die hämorrhagischen Auflagerungen am Beckenknochen zu finden; da sie streng symmetrisch sind, erscheint ein Trauma als Erklärung unwahrscheinlich.

Eine weitere mögliche Differentialdiagnose ist das Pierre-Marie-Bamberger-Syndrom.

Insgesamt kann man sagen, dass vermutlich eine Lepraerkrankung vorlag. Eine zusätzliche Skorbuterkrankung ist wahrscheinlich.

\section{$\begin{array}{llll}\text { Sk } 1383 & \text { Grab } 120 & \text { (35) } 40-59 & \text { J }\end{array}$}

Dieses Grab ist im Gräberfeldplan nicht verzeichnet, da es nachträglich geborgen wurde. Vermutlich befand es sich mit einigen anderen Gräbern nördlich der Kirche in der unteren Schicht. Eine andere Möglichkeit wäre eine laut Plan nicht näher untersuchte Fläche südwestlich der Kirche, westlich der Gräberreihe 26-30 und südlich der Gräber 72 und 73 (GEISLER 1983, DETKEN 1991).

Das Grab selbst ist Teil einer Doppel- oder Nachbestattung mit einem adulten weiblichen Individuum. In der Archivkiste finden sich darüber hinaus Teile eines fetalen Individuums (DETKEN 1991). 


\section{Makroskopischer Befund}

Bei Sk 1383 handelt es sich um das Skelet eines 40 - 59 (einer anderen Schätzung zufolge 35) Jahre alten Mannes.

Bei der Untersuchung des Postcraniums fallen massive mehrlagige feinporöse Auflagerungen mit profiliertem Rand an den lateralen Schäften beider Tibiae auf (Bildtafel 9, Bild 2, 3, 4, 5 und 6).

Auch an den distalen Femura gibt es ventral vergleichbare Auflagerungen. Die dorsalen Femurschäfte zeigen längsstreifige feinporöse Oberflächenveränderungen.

An der distalen rechten Fibula finden sich ventral ähnliche Auflagerungen wie an den Tibiae. Auch in der Fossa subscapularis der linken Scapula sind feinporöse Auflagerungen mit profiliertem Rand lokalisiert (Bildtafel 8, Bild 8 und Bildtafel 9, Bild 1).

Vergleichbare Auflagerungen finden sich an beiden Ossa ilii unterhalb der Linea glutealis inferior.

\section{Mikroskopischer Befund}

Es liegen keine mikroskopischen Befunde vor.

\section{Radiologischer Befund}

Das Durchleuchten ergab keine weiteren Erkenntnisse.

\section{Gesamtbeurteilung}

\begin{tabular}{|l|l|l|}
\hline A & Kardinalmerkmale \\
\hline 1.) & Poröse AL am äußeren Schädeldach & - \\
\hline 2.$)$ & $\begin{array}{l}\text { Poröse AL am Orbitadach (Cribra } \\
\text { orbitalia) }\end{array}$ & - \\
\hline 3.$)$ & $\begin{array}{l}\text { Poröse AL am äußeren Gesichts- } \\
\text { schädel }\end{array}$ & - \\
\hline 4.) & Poröse AL am Alveolarknochen & - \\
\hline 5.$)$ & Poröse AL an Langknochenschäften & Femura, Tibiae, Fibula \\
\hline 6.) & Poröse AL am Schulterblatt & links \\
\hline 7.) & Poröse AL am Darmbein & beidseits \\
\hline B & Nebenmerkmale & \multicolumn{2}{|l|}{} \\
\hline 1.) & $\begin{array}{l}\text { Poröse OB/AL am harten Gaumen } \\
\text { (Stomatitis) }\end{array}$ & - \\
\hline 2.) & Poröse Auskleidung der Alveolen & - \\
\hline 3.) & $\begin{array}{l}\text { Symmetrische poröse AL am } \\
\text { inneren Schädeldach, flächig/in den } \\
\text { Impressiones digitatae }\end{array}$ & - \\
\hline 4.) & Poröse OB der Langknochenschäfte & Femura \\
\hline 5.) & $\begin{array}{l}\text { Poröse Außenfläche des Großen } \\
\text { Keilbeinflügels }\end{array}$ & - \\
\hline C & Weitere Auffälligkeiten & Längsstreifung Femura \\
\hline
\end{tabular}

Tab. 29: Merkmalstabelle zur Diagnose von Skorbut (nach SCHULTZ 1994) angewandt auf Sk 1383 aus dem Gräberfeld Barbing-Kreuzhof 
Es können drei Kardinalmerkmale und ein Nebenmerkmal für Skorbut nachgewiesen werden. Die symmetrische Anordnung und die typische Lokalisation an Langknochen, Scapula und Os ilii bestätigen den Verdacht, auch wenn eine mikroskopische Sicherung fehlt.

Die Auflagerungen erscheinen frisch und noch nicht gut integriert, sodass man davon ausgehen kann, dass das Inviduum während einer floriden Skorbuterkrankung starb.

\section{Sk 1385 \\ Grab 121a \\ $14-16 \mathrm{~J}$ \\ $\mathbf{m}=\mathbf{w}$}

Das Grab ist nicht auf dem Gräberfeld verzeichnet. Oberhalb des Beckens wurde das Skelet durch Baumaschinen zerstört (DETKEN 1991).

Makroskopischer Befund

Bei Sk 1385 handelt es sich um Teile des Postcraniums eines ca. 14 bis 16 Jahre alten Jugendlichen, bei dem eine Geschlechtsbestimmung unmöglich ist.

Bei der makroskopischen Untersuchung fallen an verschiedenen Stellen frische feinporöse Auflagerungen auf: An der linken Scapula finden sich mehrlagige Auflagerungen mit unterschnittenem Rand in der Fossa supraspinata am Margo superior und ventral entlang des Margo lateralis. An der rechten Scapula erkennt man ähnliche Auflagerungen in der Fossa subscapularis an der Grenze zum Collum scapulae. Weitere Knochen der oberen Extremität sind nicht erhalten.

Am Os ilii lassen sich beidseits Auflagerungen mit profiliertem Rand nachweisen. Links sind sie lateral zwischen Spina iliaca anterior superior und inferior gelegen, rechts an der Fossa iliaca vor der Facies auricularis und an der Facies lateralis im Bereich der Linea glutealis posterior. Außerdem fällt am rechten Becken ein zusätzliches großes Foramen nutritivum auf, in dessen Umfeld sich besonders ausgeprägte mehrlagige Auflagerungen erstrecken. Vermutlich ist hier das Gefäß, welches durch das Foramen trat, rupturiert und es kam so zu einem großen subperiostalen Hämatom (Bildtafel 9, Bild 7).

An beiden Femura finden sich mehrlagige feinporöse Auflagerungen mit profiliertem Rand großflächig über den Schaft und das Collum femoris verteilt (Bildtafel 9, Bild 8 und Bildtafel 10, Bild 1). Die Dorsalseite weist außerdem eine feinsträhnige Längsstreifung auf.

Auch im proximalen Drittel der linken Tibia finden sich ähnliche Auflagerungen an der lateralen Schaftkante.

Die Oberfläche der Fibulae ist stark erodiert, dennoch lassen sich auch hier Reste von feinporösen Auflagerungen über die gesamte Diaphysenlänge nachweisen (Bildtafel 10, Bild 2). 


\section{Mikroskopischer Befund}

Es liegen keine lichtmikroskopischen Befunde vor.

\section{Radiologischer Befund}

Beim Durchleuchten fielen an der linken Tibia Harris-Linien am proximalen und distalen Schaft auf.

\section{Gesamtbeurteilung}

\begin{tabular}{|c|c|c|}
\hline $\mathbf{A}$ & Kardinalmerkmale & \\
\hline 1.) & Poröse AL am äußeren Schädeldach & - \\
\hline 2.) & $\begin{array}{l}\text { Poröse AL am Orbitadach (Cribra } \\
\text { orbitalia) }\end{array}$ & - \\
\hline 3.) & $\begin{array}{l}\text { Poröse AL am äußeren Gesichts- } \\
\text { schädel }\end{array}$ & - \\
\hline 4.) & Poröse AL am Alveolarknochen & - \\
\hline 5.) & Poröse AL an Langknochenschäften & Femura, Tibia, Fibulae \\
\hline 6.) & Poröse AL am Schulterblatt & beidseits \\
\hline 7.) & Poröse AL am Darmbein & beidseits \\
\hline B & Nebenmerkmale & \\
\hline 1.) & $\begin{array}{l}\text { Poröse OB/AL am harten Gaumen } \\
\text { (Stomatitis) }\end{array}$ & - \\
\hline 2.) & Poröse Auskleidung der Alveolen & - \\
\hline 3.) & $\begin{array}{l}\text { Symmetrische poröse AL am } \\
\text { inneren Schädeldach, flächig/in den } \\
\text { Impressiones digitatae }\end{array}$ & - \\
\hline 4.) & Poröse OB der Langknochenschäfte & Femura \\
\hline 5.) & $\begin{array}{l}\text { Poröse Außenfläche des Großen } \\
\text { Keilbeinflügels }\end{array}$ & - \\
\hline $\mathrm{C}$ & Weitere Auffälligkeiten & Längsstreifung Femura \\
\hline
\end{tabular}

Tab. 30: Merkmalstabelle zur Diagnose von Skorbut (nach SCHULTZ 1994), angewandt auf Sk 1385 aus dem Gräberfeld Barbing-Kreuzhof

$\mathrm{AL}=$ Auflagerung, $\mathrm{OB}=$ Oberfläche, $-=$ Merkmal liegt nicht vor

Es lassen sich ein Neben- und drei Kardinalmerkmale für Skorbut nachweisen. Dies macht eine Skorbuterkrankung sehr wahrscheinlich. Zwar ist der Schädel nicht erhalten, aber die symmetrische Verteilung der hämorrhagischen Auflagerungen an allen vorliegenden Knochen des Postcraniums untermauert die Diagnose.

Die Auflagerungen erscheinen frisch, vermutlich starb das Individuum während der aktiven Skorbuterkrankung.
Sk 1386
Grab 122
$18 M+/-6 M$
$\mathbf{m}=\mathbf{w}$

Das Grab ist nicht im Gräberplan verzeichnet, es gehört zu der Gruppe von Gräbern, die nachträglich geborgen wurden. Siehe dazu auch bei Sk 1383 (DETKEN 1991). 


\section{Makroskopischer Befund}

Bei Sk 1386 handelt es sich um die Überreste eines ca. 18 Monate (+/- 6 Monate) alten Kleinkindes.

An Langknochen sind nur der rechte Humerus, der rechte Femur und die Tibiae erhalten. An diesen fallen feinporöse Oberflächenveränderungen auf, die den Eindruck erwecken, als seien hier Auflagerungen diagenetisch abgetragen worden. Diese Stellen liegen ventral am proximalen Humerus und am ventrolateralen Tibiaschaft rechts.

Am Schädel lassen sich teilweise mehrlagige feinporöse Auflagerungen mit profiliertem Rand an verschiedenen Stellen nachweisen.

Am Os occipitale finden sich Auflagerungen im Bereich der Crista occipitalis interna und der Sulci sinus transversi. Auch in den Impressiones digitatae liegen kleinflächige Auflagerungen, die durchzogen sind von pinselstrichartig verzweigten Gefäßimpressionen (Bildtafel 10, Bild 4 und 5).

Am Os sphenoidale ist die Oberfläche vielerorts, u.a. an den Alae beidseits (Bildtafel 10, Bild 8), feinporös gestaltet, es scheinen auch hier erodierte Auflagerungen vorgelegen zu haben, die stellenweise auch noch erhalten sind.

In den Sulci sinus sigmoidei des Os temporale finden sich mehrlagige feinporöse Auflagerungen, auch an der rechten Squama liegt eine flächig erscheinende Auflagerung. Außerdem fallen starke Gefäßimpressionen am gesamten Os temporale auf. Die äußeren Gehörgänge wirken beidseits deformiert.

Am Os parietale finden sich feinporöse Auflagerungen in den Impressiones digitatae, teilweise auch flächig konfluierend, sowie massive Gefäßimpressionen (Bildtafel 10, Bild 6 und 7). An der Außenseite über der rechten Sutura squamosa liegen morphologisch von der Norm abweichende kurze, teils parallel verlaufende, Gefäßimpressionen, dazwischen liegen zungenförmige kleine Platten.

Das Palatum durum ist feinporös gestaltet, der Processus alveolaris der Maxilla und die Pars alveolaris der Mandibula sind beidseits mit feinporösen Auflagerungen bedeckt, teilweise finden sich auch Alveolarauskleidungen (Bildtafel 10, Bild 3).

Auch am Ramus mandibulae links außen liegt eine kleinflächige Auflagerung.

Im Bereich des Meatus acusticus externus finden sich beidseits kleine Knochenneubildungen und Gefäßforamina. Möglicherweise bestand hier eine beidseitige Otitis media.

Mikroskopischer Befund

Angesichts des schlechten Erhaltungszustandes der Knochensubstanz und der Auflagerungen wurde von der Anfertigung mikroskopischer Präparate abgesehen. 


\section{Radiologischer Befund}

Das Durchleuchten zeigte Harris-Linien in den proximalen Tibiae.

\section{Gesamtbeurteilung}

\begin{tabular}{|c|c|c|}
\hline $\mathbf{A}$ & \multicolumn{2}{|l|}{ Kardinalmerkmale } \\
\hline 1.) & Poröse AL am äußeren Schädeldach & - \\
\hline 2.) & $\begin{array}{l}\text { Poröse AL am Orbitadach (Cribra } \\
\text { orbitalia) }\end{array}$ & - \\
\hline 3.) & $\begin{array}{l}\text { Poröse AL am äußeren Gesichts- } \\
\text { schädel }\end{array}$ & Mandibula \\
\hline 4.) & Poröse AL am Alveolarknochen & Mandibula, Maxilla \\
\hline 5.) & Poröse AL an Langknochenschäften & - \\
\hline 6.) & Poröse AL am Schulterblatt & - \\
\hline 7.) & Poröse AL am Darmbein & - \\
\hline B & Nebenmerkmale & \\
\hline 1.) & $\begin{array}{l}\text { Poröse OB/AL am harten Gaumen } \\
\text { (Stomatitis) }\end{array}$ & OB \\
\hline 2.) & Poröse Auskleidung der Alveolen & Mandibula, Maxilla \\
\hline 3.) & $\begin{array}{l}\text { Symmetrische poröse AL am } \\
\text { inneren Schädeldach, flächig/in den } \\
\text { Impressiones digitatae }\end{array}$ & Parietale, Temporale, Occipitale \\
\hline 4.) & Poröse OB der Langknochenschäfte & Humerus, Tibia \\
\hline 5.) & $\begin{array}{l}\text { Poröse Außenfläche des Großen } \\
\text { Keilbeinflügels }\end{array}$ & Beidseits \\
\hline $\mathrm{C}$ & Weitere Auffälligkeiten & $\begin{array}{l}\text { Auffällige Gefäßimpressionen, } \\
\text { deformierte Gehörgänge }\end{array}$ \\
\hline
\end{tabular}

Tab. 31: Merkmalstabelle zur Diagnose von Skorbut (nach SCHULTZ 1994), angewandt auf Sk 1386 aus dem Gräberfeld Barbing-Kreuzhof

$\mathrm{AL}=$ Auflagerung, $\mathrm{OB}=$ Oberfläche, - = Merkmal liegt nicht vor

Es lassen sich zwei Kardinal- und fünf Nebenmerkmale nachweisen. Damit erscheint die Diagnose Skorbut auch ohne mikroskopische Sicherung wahrscheinlich. Die Auflagerungen sind kaum integriert, vermutlich verstarb das Kind im Laufe der Erkrankung.

Sk 1398

Grab 2

$\mathbf{1 J}+/-6 \mathrm{M}$

$\mathbf{m}=\mathbf{w}$

Dieses Grab wurde zusammen mit einigen anderen abseits des eigentlichen Gräberfelds auf der westlichen Grabungsfläche geborgen (GEISLER 1983, DETKEN 1991).

\section{Makroskopischer Befund}

Bei Sk 1398 handelt es sich um die Überreste eines Säuglings oder Kleinkindes von etwa 12 Monaten. Vom Postcranium sind einige Rippen, der linke Femur und die linke Scapula erhalten. An diesen sind flache feinporöse Auflagerungen zu erkennen, die allerdings gut integriert und so erodiert sind, dass man sie nicht sicher gegenüber einer lediglich feinporösen 
Oberfläche ohne Neubildung abgrenzen kann. Lediglich in der Fossa subscapularis scheint es sich mit Sicherheit um gut integrierte Auflagerungen zu handeln.

Auch am Schädel finden sich feinporöse Oberflächenveränderungen, die teilweise sehr erodiert sind, stellenweise auch den Eindruck erwecken, gut integrierte Auflagerungen zu sein. Lokalisiert sind sie beidseits am Os parietale entlang der Sutura squamosa und der Sutura sagittale, sowohl an der Schädelaußenseite als auch an der Lamina interna.

Die kleinen erhaltenen Teile des Os temporale sehen ähnlich aus.

Die Außenfläche des Os occipitale ist sehr porös, vermutlich aber ohne Neubildungen. An der Innenseite befinden sich stark erodierte gut integrierte Auflagerungen im Sulcus sinus occipitalis und in den Resten des linken Sulcus sinus transversus.

Am rechten Orbitadach liegt ein Cribrum Grad 1 vor, das linke fehlt.

Es sind keine Kieferteile erhalten.

\section{Mikroskopischer Befund}

Angesichts des schlechten Erhaltungszustandes der Knochensubstanz und der Auflagerungen wurde von der Anfertigung mikroskopischer Präparate abgesehen.

\section{Radiologischer Befund}

Beim Durchleuchten fanden sich keine Auffälligkeiten.

\section{Gesamtbeurteilung}

\begin{tabular}{|c|c|c|}
\hline $\mathbf{A}$ & \multicolumn{2}{|l|}{ Kardinalmerkmale } \\
\hline 1.) & Poröse AL am äußeren Schädeldach & Parietale, Temporale \\
\hline 2.) & $\begin{array}{l}\text { Poröse AL am Orbitadach (Cribra } \\
\text { orbitalia) }\end{array}$ & Cribrum Grad 1 rechts \\
\hline 3.) & $\begin{array}{l}\text { Poröse AL am äußeren Gesichts- } \\
\text { schädel }\end{array}$ & - \\
\hline 4.) & Poröse AL am Alveolarknochen & - \\
\hline 5.) & Poröse AL an Langknochenschäften & Femur links \\
\hline 6.) & Poröse AL am Schulterblatt & Scapula links \\
\hline 7.) & Poröse AL am Darmbein & - \\
\hline B & Nebenmerkmale & \\
\hline 1.) & $\begin{array}{l}\text { Poröse OB/AL am harten Gaumen } \\
\text { (Stomatitis) }\end{array}$ & - \\
\hline 2.) & Poröse Auskleidung der Alveolen & - \\
\hline 3.) & $\begin{array}{l}\text { Symmetrische poröse AL am } \\
\text { inneren Schädeldach, flächig/in den } \\
\text { Impressiones digitatae }\end{array}$ & Parietale, Temporale, Occipitale \\
\hline 4.) & Poröse OB der Langknochenschäfte & Femur \\
\hline 5.) & $\begin{array}{l}\text { Poröse Außenfläche des Großen } \\
\text { Keilbeinflügels }\end{array}$ & - \\
\hline $\mathrm{C}$ & Weitere Auffälligkeiten & Fragliche Auflagerungen an Rippen \\
\hline
\end{tabular}

Tab. 32: Merkmalstabelle zur Diagnose von Skorbut (nach SCHULTZ 1994), angewandt auf Sk 1398 aus dem Gräberfeld Barbing-Kreuzhof $\mathrm{AL}=$ Auflagerung, $\mathrm{OB}=$ Oberfläche, $-=$ Merkmal liegt nicht vor 
Es lassen sich vier Kardinal- und zwei Nebenmerkmale für Skorbut nachweisen. Damit ist eine Skorbuterkrankung sehr wahrscheinlich. Allerdings muss man sagen, dass alle beschriebenen Auflagerungen aufgrund des schlechten Erhaltungszustandes der Knochen nur eingeschränkt beurteilbar sind.

\subsection{Untersuchungsbefunde der einzelnen Individuen des Gräberfelds Bácsalmás-Óalmás}

\section{Bácsalmás-Óalmás \\ Grab 285 \\ $6-7 \mathbf{J}$ \\ $\mathbf{m}=\mathbf{w}$}

Makroskopischer Befund

Bei dem Individuum aus Grab 285 der Grabung Bácsalmás-Óalmás handelt es sich um ein etwa 6 bis 7 Jahre altes Kind.

Am Postcranium lassen sich keine Auffälligkeiten feststellen.

An der Lamina interna von Os frontale, Os temporale, Os parietale und Os occipitale jedoch finden sich feinporöse Auflagerungen mit profiliertem Rand in den Impressiones digitatae, teilweise auch darüber hinaus konfluierend. Besonders ausgeprägt sind die Auflagerungen im Bereich des Sulcus sinus occipitalis.

Im Parietalbereich fallen drei Inkabeine auf, eines davon ist von feinporösen Auflagerungen an der Schädelaußenseite umgeben.

An beiden Orbitadächern liegen Cribra Grad 2 (-3) vor.

Die Außenseiten der Alae majores ossis sphenoidale haben feinporöse Oberflächen, die Squamae temporales sind - soweit beurteilbar - unauffällig.

Die Oberfläche des knöchernen Gaumens ist feinporös gestaltet. Im Alveolarbereich der rechten Maxilla gibt es feinporöse Auflagerungen.

In den Incisurae mandibulae fallen beidseits großflächige, feinporöse, von pinselstrichartigen Gefäßnetzen durchzogene Auflagerungen mit verstrichenem Rand auf (Bildtafel 11, Bild 1).

\section{Mikroskopischer Befund}

Es wurden Schliffe vom Os frontale und der linken Orbita angefertigt. Am Os frontale lassen sich feine Auflagerungen vom Typ I an der Lamina interna nachweisen. Sie sind gut integriert, der Umbau zu Lamellenknochen hat bereits eingesetzt (Bildtafel 11, Bild 2).

Am Orbitadach lassen sich nicht mit Sicherheit Auflagerungen nachweisen, auch wenn es stellenweise so aussieht, als hätten erodierte Auflagerungen vorgelegen. Vielmehr scheint der makroskopische Eindruck eines Cribrums durch starke Gefäßimpressionen hervorgerufen zu 
sein. Darüber hinaus sind an einigen Stellen Eröffnungen zu erkennen, wie bei einer beginnenden Anämie (Bildtafel 11, Bild 3).

\section{Gesamtbeurteilung}

\begin{tabular}{|c|c|c|}
\hline $\mathbf{A}$ & \multicolumn{2}{|l|}{ Kardinalmerkmale } \\
\hline 1.) & Poröse AL am äußeren Schädeldach & Parietale \\
\hline 2.) & $\begin{array}{l}\text { Poröse AL am Orbitadach (Cribra } \\
\text { orbitalia) }\end{array}$ & $\begin{array}{l}\text { - } \\
\text { Zwar entsteht makroskopisch der } \\
\text { Eindruck von Cribra Grad 2, dieser } \\
\text { lässt sich mikroskopisch nicht } \\
\text { bestätigen. }\end{array}$ \\
\hline 3.) & $\begin{array}{l}\text { Poröse AL am äußeren Gesichts- } \\
\text { schädel }\end{array}$ & - \\
\hline 4.) & Poröse AL am Alveolarknochen & Maxilla \\
\hline 5.) & Poröse AL an Langknochenschäften & - \\
\hline 6.) & Poröse AL am Schulterblatt & - \\
\hline 7.) & Poröse AL am Darmbein & - \\
\hline $\mathbf{B}$ & Nebenmerkmale & \\
\hline 1.) & $\begin{array}{l}\text { Poröse } \mathrm{OB} / \mathrm{AL} \text { am harten Gaumen } \\
\text { (Stomatitis) }\end{array}$ & $\mathrm{OB}$ \\
\hline 2.) & Poröse Auskleidung der Alveolen & - \\
\hline 3.) & $\begin{array}{l}\text { Symmetrische poröse AL am } \\
\text { inneren Schädeldach, flächig/in den } \\
\text { Impressiones digitatae }\end{array}$ & $\begin{array}{l}\text { Parietale, Frontale, Temporale, } \\
\text { Occipitale }\end{array}$ \\
\hline 4.) & Poröse OB der Langknochenschäfte & - \\
\hline 5.) & $\begin{array}{l}\text { Poröse Außenfläche des Großen } \\
\text { Keilbeinflügels }\end{array}$ & Beidseits \\
\hline $\mathrm{C}$ & Weitere Auffälligkeiten & - \\
\hline
\end{tabular}

Tab. 33: Merkmalstabelle zur Diagnose von Skorbut (nach SCHULTZ 1994), angewandt auf Skelet 285 aus dem Gräberfeld Bácsalmás-Óalmás

$\mathrm{AL}=$ Auflagerung, $\mathrm{OB}=$ Oberfläche, $-=$ Merkmal liegt nicht vor

Es lassen sich zwei Kardinal- und drei Nebenmerkmale für Skorbut nachweisen. Diese können teilweise mikroskopisch untermauert werden. Allerdings fällt auf, dass das Postcranium nicht befallen scheint. Außerdem entsteht bei der mikroskopischen Untersuchung eines Cribrum orbitale der Verdacht auf eine beginnende Anämie.

Insgesamt erscheint eine Skorbuterkrankung dennoch wahrscheinlich.

\section{Bácsalmás-Óalmás}

Grab 381

1,5 - $2 \mathrm{~J}$

$\mathbf{m}=\mathbf{w}$

\section{Makroskopischer Befund}

Bei dem Individuum aus Grab 381 der Grabung Bácsalmás-Óalmás handelt es sich um ein Kleinkind von 1,5 bis 2 Jahren. 
Bei der makroskopischen Untersuchung fallen frische feinporöse Auflagerungen mit profiliertem Rand auf. Sie finden sich an beiden Scapulae in den Fossae infraspinata und subscapularis und an den Metaphysen der Humeri (Bildtafel 11, Bild 7). Auch die proximalen Metaphysen und die Diaphysen der Radii und Ulnae (Bildtafel 12, Bild 4) sind befallen. Heftige Auflagerungen sind auch an den Metaphysen und dorsalen Diaphysen der Femura (Bildtafel 11, Bild 4,5 und 6), an der proximalen linken Fibula sowie an den medialen Tibiaschäften (Bildtafel 12, Bild 2 und 3) zu sehen.

Ebenso fallen Auflagerungen am Os ilium beidseits, an beiden Claviculae im Bereich der Muskelansätze sowie an den Ventral- und Dorsalseiten der oberen acht Rippen (Bildtafel 11, Bild 8 und Bildtafel 12, Bild 1) beider Seiten auf.

Am annähernd vollständig erhaltenen Schädel lassen sich sowohl an der Innen- als auch an der Außenseite massive feinporöse Auflagerungen nachweisen. Außen finden sie sich vor allem in Suturnähe, innen füllen sie die Impressiones digitatae und venösen Sulci, konfluieren aber auch großflächig (Bildtafel 12, Bild 5, 6 und 7, Bildtafel 13, Bild 1 und 2). Sowohl an der Maxilla als auch an der Mandibula liegen beidseits feinporöse Auflagerungen und Auskleidungen der Alveolen vor (Bildtafel 13, Bild 4, 5 und 6). Außerdem finden sich an beiden Seiten Auflagerungen an der Mandibula an Ramus und Corpus (Bildtafel 13, Bild 3), innen wie außen, und an der Maxilla am harten Gaumen und im Gesichtsbereich.

An den Orbitadächern finden sich Cribra Grad 2 -3.

Es ist nur die linke Ala major ossis sphenoidale erhalten, diese ist mit feinporösen Auflagerungen bedeckt (Bildtafel 12, Bild 8).

Mikroskopischer Befund

Es wurden Schliffe vom Os frontale, dem Os sphenoidale, der linken Orbita, der Mandibula, zweier Rippen und der rechten Tibia angefertigt.

Am Os frontale lassen sich Auflagerungen vom Typ I an der Lamina interna nachweisen, auch Howship'sche Lakunen als Zeichen eines aktiven Resorptionsprozesses sind zu erkennen (Bildtafel 14, Bild 2 und 3).

Am Os sphenoidale sind die Auflagerungen an der Lamina externa angeschnitten. Zwar ist sie teilweise erodiert, dennoch sind Auflagerungen vom Typ I zu erkennen (Bildtafel 13, Bild 7).

Die Mandibula zeigt das Bild einer Periostose infolge rezidivierender Hämorrhagien. Deutlich ist eine Mehrphasigkeit des Geschehens. Ganz außen sind frische faserknöcherne Auflagerungen vom Typ I zu sehen, darunter liegt eine ältere, schon gut organisierte Auflagerung, unterhalb derer sich Resorptionshöhlen befinden. Erst darunter befindet sich die ehemalige Lamina externa. Unterhalb dieser sind Resorptionshöhlen $\mathrm{zu}$ erkennen, die 
durchaus auch durch den physiologischen Umbau des kindlichen Kiefers zu erklären sind (Bildtafel 14, Bild 1).

Auch das Cribrum am Dach der linken Orbita lässt sich lichtmikroskopisch durch Auflagerungen vom Typ I bestätigen (Bildtafel 13, Bild 8).

Die Präparate der Rippen bieten einen unauffälligen Befund, allerdings sieht es stellenweise so aus, als seien ehemals vorhandene Auflagerungen weggebrochen.

Auch das Präparat der Tibia zeigt zu große Erosionen, als dass sich Auflagerungen mit Sicherheit nachweisen ließen.

\section{Gesamtbeurteilung}

\begin{tabular}{|c|c|c|}
\hline A & \multicolumn{2}{|l|}{ Kardinalmerkmale } \\
\hline 1.) & Poröse AL am äußeren Schädeldach & $\begin{array}{l}\text { Frontale, Parietale, Occipitale, } \\
\text { Sphenoidale, Temporale }\end{array}$ \\
\hline 2.) & $\begin{array}{l}\text { Poröse AL am Orbitadach (Cribra } \\
\text { orbitalia) }\end{array}$ & Cribra Grad 2-3 beidseits \\
\hline 3.) & $\begin{array}{l}\text { Poröse AL am äußeren Gesichts- } \\
\text { schädel }\end{array}$ & $\begin{array}{l}\text { Maxilla, Sphenoidale, Zygomaticum, } \\
\text { Mandibula }\end{array}$ \\
\hline 4.) & Poröse AL am Alveolarknochen & Mandibula, Maxilla \\
\hline 5.) & Poröse AL an Langknochenschäften & $\begin{array}{l}\text { Humeri, Ulnae, Radii, Femura, Tibiae, } \\
\text { Fibula }\end{array}$ \\
\hline 6.) & Poröse AL am Schulterblatt & beidseits \\
\hline 7.) & Poröse AL am Darmbein & beidseits \\
\hline B & Nebenmerkmale & \\
\hline 1.) & $\begin{array}{l}\text { Poröse OB/AL am harten Gaumen } \\
\text { (Stomatitis) }\end{array}$ & $\mathrm{AL}$ \\
\hline 2.) & Poröse Auskleidung der Alveolen & Mandibula, Maxilla \\
\hline 3.) & $\begin{array}{l}\text { Symmetrische poröse AL am } \\
\text { inneren Schädeldach, flächig/in den } \\
\text { Impressiones digitatae }\end{array}$ & $\begin{array}{l}\text { Frontale, Temporale, Occipitale, } \\
\text { Parietale, Sphenoidale }\end{array}$ \\
\hline 4.) & Poröse OB der Langknochenschäfte & alle \\
\hline 5.) & $\begin{array}{l}\text { Poröse Außenfläche des Großen } \\
\text { Keilbeinflügels }\end{array}$ & AL links \\
\hline $\mathrm{C}$ & Weitere Auffälligkeiten & $\begin{array}{l}\text { AL an Rippen innen wie außen, } \mathrm{AL} \text { an } \\
\text { Claviculae }\end{array}$ \\
\hline
\end{tabular}

Tab. 34: Merkmalstabelle zur Diagnose von Skorbut (nach SCHULTZ 1994), angewandt auf Skelet 381 aus dem Gräberfeld Bácsalmás-Óalmás

$\mathrm{AL}=$ Auflagerung, $\mathrm{OB}=$ Oberfläche, - = Merkmal liegt nicht vor

Es lassen sich alle Kardinal- und alle Nebenmerkmale für Skorbut nachweisen. Diese lassen sich großenteils mikroskopisch bestätigen.

Es lag also mit großer Sicherheit eine Skorbuterkrankung vor. Vermutlich durchlebte das Kind mehrere Krankheitsphasen, da einige Läsionen ausgeheilte tiefere Schichten aufweisen, und verstarb während des letzten, vermutlich sehr heftigen Krankheitsschubes. 


\section{Bácsalmás-Óalmás}

Grab 436

$\mathbf{0}-\mathbf{0 , 5} \mathbf{J}$

$\mathbf{m}=\mathbf{w}$

Makroskopischer Befund

Bei dem Individuum aus Grab 436 der Grabung Bácsalmás-Óalmás handelt es sich um einen Säugling von maximal 6 Monaten.

Bei der makroskopischen Untersuchung fallen hämorrhagische Auflagerungen an fast allen erhaltenen Knochen auf.

An beiden Scapulae finden sich feinporöse Auflagerungen mit profiliertem Rand in den Fossae supra- und infraspinata (Bildtafel 14, Bild 8), an der rechten Scapula auch am Margo medialis.

Vergleichbare Auflagerungen gibt es auch an den dorsalen Humerusschäften (Bildtafel 14, Bild 5 und 6), dorsal am rechten Radiusschaft sowie an der Diaphyse der linken Ulna, hier vor allem in der proximalen Hälfte.

An der unteren Extremität sind feinporöse Auflagerungen an der Dorsalseite des Os ilium beidseits lokalisiert (Bildtafel 14, Bild 4), außerdem an den Diaphysen des linken Femurs sowie beider Tibiae (Bildtafel 15, Bild 1). Weiterhin fällt auf, dass die Schäfte von Femur und Tibiae nach ventral konvex verbogen sind (Bildtafel 15, Bild 1).

Darüber hinaus sind sowohl die Ventral- als auch die Dorsalseite der Rippen beider Seiten mit Auflagerungen versehen.

Auch an der Ventralseite der linken Clavicula sind feine Auflagerungen zu erkennen.

Am Schädel fallen vor allem hämorrhagische Auflagerungen am äußeren Schädeldach auf. Sie erscheinen feinporös und teilweise mehrlagig, mit profiliertem bis unterschnittenem Rand, und befinden sich vor allem in Suturnähe an Os frontale (Bildtafel 15, Bild 2), Os parietale, Os temporale und Os occipitale.

Auch an der Lamina interna von Os frontale und Os parietale finden sich ähnliche Auflagerungen.

Besonders ausgeprägt sind die Auflagerungen am Os sphenoidale, hier sind beide Alae majores, beide Alae minores sowie das Corpus befallen (Bildtafel 15, Bild 4 und 5).

Auch an der Mandibula finden sich hämorrhagische Auflagerungen, diese sind an den Rami innen und außen, am Corpus nur außen zu finden. Der Alveolarfortsatz ist ebenso wie das knöcherne Palatinum mit frischen feinporösen Auflagerungen bedeckt.

An den Orbitadächern finden sich Cribra Grad 2-3. 


\section{Mikroskopischer Befund}

Am Postcranium wurden Schliffe der linken Clavicula (Bildtafel 16, Bild 5), einer Rippe, des linken Femurs (Bildtafel 17, Bild 2), des linken Os ilium, der linken Scapula (Bildtafel 16, Bild 7) und beider Tibiae entnommen.

Außerdem wurden Präparate von der linken Ala major ossis sphenoidalis, dem rechten Os frontale, der rechten Mandibula und des rechten Os occipitale (Bildtafel 16, Bild 6) sowie des rechten Os parietale und des linken Os temporale angefertigt.

In allen Präparaten des Postcraniums erkennt man das für das frühe Kindesalter physiologische appositionelle Knochenwachstum. Die Trabekel der Substantia compacta erscheinen reduziert und dünn, was sich wohl durch das sehr geringe Alter des Kindes erklären lässt. Darüber hinaus finden sich in fast allen Schliffen zumindest Hinweise auf Auflagerungen vom Typ I. Besonders ausgeprägt sind diese an den Tibiae, an der rechten Tibia sind sogar an zwei Seiten deutliche hämorrhagische Auflagerungen nachweisbar (Bildtafel 16, Bild 8 und Bildtafel 17, Bild 1). Nur am rechten Femur sowie einem der beiden Präparate der Rippe lassen sich keine Auflagerungen erkennen.

Am Os ilium fällt neben den Auflagerungen eine morphologisch von der Norm abweichende streifenförmige Anordnung der Spongiosabälkchen auf, die sich am ehesten durch mechanische Belastung, z.B. beim Laufen oder Krabbeln, erklären ließe, was allerdings in Anbetracht des sehr geringen Alters des Kindes unwahrscheinlich ist (Bildtafel 15,

Bild 6 und 7).

Auch am Schädel können hämorrhagische Auflagerungen nachgewiesen werden. An der Ala major ossis sphenoidalis (Bildtafel 16, Bild 4) und am Os parietale befinden sich Auflagerungen vom Typ I an der Lamina interna, am Os temporale sind sie an der Lamina externa lokalisiert und am Os frontale (Bildtafel 15, Bild 8 und Bildtafel 16, Bild 1, 2 und 3) und occipitale sowohl innen als auch außen.

Auch an der Mandibula sind Auflagerungen vom Typ I nachweisbar, diese erscheinen gut integriert.

\section{Gesamtbeurteilung}

\begin{tabular}{|l|l|l|}
\hline A & Kardinalmerkmale \\
\hline 1.) & Poröse AL am äußeren Schädeldach & $\begin{array}{l}\text { Frontale, Parietale, Temporale, } \\
\text { Occipitale }\end{array}$ \\
\hline 2.) & $\begin{array}{l}\text { Poröse AL am Orbitadach (Cribra } \\
\text { orbitalia) }\end{array}$ & Cribra Grad 2-3 beidseits \\
\hline 3.$)$ & $\begin{array}{l}\text { Poröse AL am äußeren Gesichts- } \\
\text { schädel }\end{array}$ & Frontale, Sphenoidale \\
\hline 4.) & Poröse AL am Alveolarknochen & Mandibula, Maxilla \\
\hline 5.) & Poröse AL an Langknochenschäften & Humeri, Radius rechts, Ulna links, \\
\hline
\end{tabular}




\begin{tabular}{|c|c|c|}
\hline & & Femur rechts, Tibiae \\
\hline 6.) & Poröse AL am Schulterblatt & beidseits \\
\hline 7.) & Poröse AL am Darmbein & beidseits \\
\hline B & Nebenmerkmale & \\
\hline 1.) & $\begin{array}{l}\text { Poröse OB/AL am harten Gaumen } \\
\text { (Stomatitis) }\end{array}$ & $\mathrm{AL}$ \\
\hline 2.) & Poröse Auskleidung der Alveolen & - \\
\hline 3.) & $\begin{array}{l}\text { Symmetrische poröse AL am } \\
\text { inneren Schädeldach, flächig/in den } \\
\text { Impressiones digitatae }\end{array}$ & $\begin{array}{l}\text { Frontale, Parietale, Temporale, } \\
\text { Occipitale }\end{array}$ \\
\hline 4.) & Poröse OB der Langknochenschäfte & - \\
\hline 5.) & $\begin{array}{l}\text { Poröse Außenfläche des Großen } \\
\text { Keilbeinflügels }\end{array}$ & Beidseits AL \\
\hline $\mathrm{C}$ & Weitere Auffälligkeiten & $\begin{array}{l}\text { Poröse AL an Rippen beider Seiten } \\
\text { innen und außen und Clavicula links }\end{array}$ \\
\hline
\end{tabular}

Tab. 35: Merkmalstabelle zur Diagnose von Skorbut (nach SCHULTZ 1994), angewandt auf Skelet 436 aus dem Gräberfeld Bácsalmás-Óalmás

$\mathrm{AL}=$ Auflagerung, $\mathrm{OB}=$ Oberfläche, $-=$ Merkmal liegt nicht vor

Es lassen sich alle Kardinal- und drei Nebenmerkmale für Skorbut nachweisen, die auch lichtmikroskopisch darstellbar sind. Damit ist die Diagnose Skorbut bestätigt.

Die Tatsache, dass alle erhaltenen Knochen Auflagerungen aufweisen, spricht auf jeden Fall für ein systemisches Geschehen. Auch die Lokalisation an den Diaphysen der Langknochen, in den Fossae der Scapulae, am Os ilium sowie an der Innen- und Außenseite von Rippen und Schädeldach lässt eigentlich keine andere Erklärung zu.

Überraschend ist das geringe Alter des Säuglings. In der Zeit kurz nach der Geburt sollte das Kind noch über den „Nestschutz“ der Mutter, also auch noch über Vitamin-C-Reserven aus der intrauterinen Ernährung verfügen. Darüber hinaus ist die menschliche Muttermilch Vitamin-C-haltig. Daraus könnte man schließen, dass die Mutter des Kindes entweder selbst so stark an Skorbut litt, dass sie dem Säugling keine Reserven mitgeben konnte, oder dass die Mutter verstorben ist oder auf andere Weise davon abgehalten wurde, das Kind zu stillen. Möglicherweise wurde der Säugling von anderen Menschen mit Kuhmilch gefüttert. Diese enthält kaum Vitamin C, da diese Tiere - wie die meisten Säuger - dazu in der Lage sind, Ascorbinsäure zu synthetisieren. Deshalb ist Skorbut bei Säuglingen, die statt Muttermilch Tiermilch erhalten, relativ häufig (siehe auch Kapitel 1.2 Das Krankheitsbild des Skorbuts, 1.4 Biochemie und Physiologie des Vitamin C, 1.7 Empfehlungen zur täglichen Vitamin-CAufnahme und 1.8 Vorkommen von Vitamin C). 


\section{Bácsalmás-Óalmás}

Grab 437

$1-1,5 \mathrm{~J}$

$\mathbf{m}=\mathbf{w}$

Makroskopischer Befund

Bei dem Individuum aus Grab 437 der Grabung Bácsalmás-Óalmás handelt es sich um ein Kleinkind von 12 bis 18 Monaten.

Bei der makroskopischen Untersuchung zeigen sich feinporöse, teilweise recht erodierte Auflagerungen an allen erhaltenen Knochen. Sie scheinen gut integriert zu sein.

Am linken Humerus befinden sie sich in den distalen zwei Schaftdritteln vor allem dorsal (Bildtafel 17, Bild 3), an den Radii sowohl an der Diaphyse als auch an den Metaphysen, an den Ulnae im Bereich der proximalen Metaphysen und Diaphysen.

An den Femura fallen Auflagerungen im dorsalen Schaftbereich auf, an den Tibiae am mittleren Diaphysendrittel ventral und rechts auch dorsal (Bildtafel 17, Bild 6).

Vom Schädel sind nur Teile der Mandibula (Bildtafel 17, Bild 4) und ein Occipitalfragment (Bildtafel 17, Bild 5) erhalten. An beiden sind massive feinporöse Auflagerungen zu erkennen, wobei auch hier die Diagnostik durch Erosion erschwert wird. Darüber hinaus findet man feinporöse Alveolarauskleidungen.

\section{Mikroskopischer Befund}

Es wurden Schliffe des linken Femurs, des rechten Radius und der Mandibula angefertigt.

Im Präparat des Femurs sind deutliche Auflagerungen vom Typ I zu erkennen. Sie zeigen das Bild eines mehrphasigen Geschehens, wobei die untersten, also ältesten Auflagerungsschichten sehr gut organisiert sind. Außerdem fällt eine stark ausgeprägte Linea aspera auf, der Verlauf der Sehnenfasern ist teilweise noch gut auszumachen.

Vermutlich wurde der Knochen mechanisch belastet, zum Beispiel durch Laufenlernen (Bildtafel 17, Bild 7).

Auch an der Mandibula lassen sich Auflagerungen vom Typ I nachweisen. Sie sind zarter und sehr gut faserknöchern integriert. Die ehemalige Lamina externa ist gut erhalten (Bildtafel 17, Bild 8 und Bildtafel 18, Bild 1).

Am rechten Radius lässt sich nicht mit Sicherheit sagen, ob es sich bei den Veränderungen an der Corticalis um erodierte Auflagerungen oder lediglich um Gefäßforamina mit postmortaler Absplitterung handelt.

\section{Gesamtbeurteilung}

\begin{tabular}{|l|l|l|}
\hline A & Kardinalmerkmale \\
\hline 1.$)$ & Poröse AL am äußeren Schädeldach & Occipitale \\
\hline 2.$)$ & $\begin{array}{l}\text { Poröse AL am Orbitadach (Cribra } \\
\text { orbitalia) }\end{array}$ & - \\
\hline 3.$)$ & $\begin{array}{l}\text { Poröse AL am äußeren Gesichts- } \\
\text { schädel }\end{array}$ & - \\
\hline
\end{tabular}




\begin{tabular}{|l|l|l|}
\hline 4.) & Poröse AL am Alveolarknochen & Mandibula \\
\hline 5.) & Poröse AL an Langknochenschäften & $\begin{array}{l}\text { Humerus, Radii, Ulnae, Femura, } \\
\text { Tibiae }\end{array}$ \\
\hline 6.) & Poröse AL am Schulterblatt & - \\
\hline 7.) & Poröse AL am Darmbein & - \\
\hline B & Nebenmerkmale & $\begin{array}{l}\text { Poröse OB/AL am harten Gaumen } \\
\text { (Stomatitis) }\end{array}$ \\
\hline 1.) & Poröse Auskleidung der Alveolen & Mandibula \\
\hline 2.) & Pymmetrische poröse AL am & - \\
\hline 3.) & $\begin{array}{l}\text { Syneren Schädeldach, flächig/in den } \\
\text { Impressiones digitatae }\end{array}$ & \\
\hline 4.) & Poröse OB der Langknochenschäfte & - \\
\hline 5.) & $\begin{array}{l}\text { Poröse Außenfläche des Großen } \\
\text { Keilbeinflügels }\end{array}$ & - \\
\hline C & Weitere Auffälligkeiten & - \\
\hline
\end{tabular}

Tab. 36: Merkmalstabelle zur Diagnose von Skorbut (nach SCHULTZ 1994), angewandt auf Skelet 437 aus dem Gräberfeld Bácsalmás-Óalmás

$\mathrm{AL}=$ Auflagerung, $\mathrm{OB}=$ Oberfläche, - = Merkmal liegt nicht vor

Es lassen sich ein Neben- und drei Kardinalmerkmale für Skorbut nachweisen, die teilweise auch mikroskopisch bestätigt werden können.

Natürlich wird die Diagnose abgeschwächt durch das Fehlen vieler Knochen, allerdings lässt die Tatsache, dass alle erhaltenen Teile Auflagerungen aufweisen, vermuten, dass die nicht erhaltenen Knochen ein ähnliches Bild bieten würden.
Bácsalmás-Óalmás
Grab 439
$1-1,5 \mathrm{~J}$
$\mathbf{m}=\mathbf{w}$

\section{Makroskopischer Befund}

Bei dem Individuum aus Grab 439 der Grabung Bácsalmás-Óalmás handelt es sich um ein Kleinkind von 12 bis 18 Monaten.

In der makroskopischen Untersuchung lassen sich feinporöse Auflagerungen an allen erhaltenen Knochen feststellen. Sie sind flach und gut integriert, allerdings an vielen Stellen durch Erosion schlecht beurteilbar.

An der oberen Extremität finden sich Auflagerungen in der Fossa supraspinata sowie an der Spina scapulae der rechten Scapula, an der Diaphyse und der distalen Metaphyse des linken Humerus (Bildtafel 18, Bild 2 und 3), an der proximalen Diaphyse ventral der Radii sowie am Schaft beider Ulnae und an der proximalen Metaphyse der linken Ulna.

Auch an den Claviculae und den Rippen (Bildtafel 18, Bild 4) lassen sich Auflagerungen nachweisen. 
An der unteren Extremität erkennt man Auflagerungen am Os ilium, an den Femurschäften, am mittleren Diaphysendrittel der rechten Tibia sowie an den proximalen Schäften der Fibulae.

Vom Schädel sind nur ein Frontalfragment mit Teilen der beiden Orbitadächer und ein kleines Stück des Os occipitale erhalten.

An den Orbitadächern erkennt man Cribra Grad 1, in der Fossa cranii anterior scheinen sich feinporöse Auflagerungen in den Impressiones digitatae zu befinden.

Am Os occipitale lassen sich außen flache, gut integrierte Auflagerungen nachweisen.

\section{Mikroskopischer Befund}

Es wurden Schliffe des rechten Femurs, des linken Humerus, der linken Tibia und der rechten Ulna angefertigt.

Am Femur lassen sich schwach ausgeprägte Auflagerungen vom Typ I nachweisen, die allein jedoch nicht diagnosesichernd wären, sondern nur im Verbund mit den übrigen Befunden.

Im Präparat des Humerus sind Auflagerungen vom Typ I zu erkennen, die sich teilweise sehr schwer vom physiologischen appositionellen Knochenwachstum abgrenzen lassen. Darüber hinaus fallen Umbauprozesse in der Spongiosa auf, die sich durch unregelmäßige große Resorptionshöhlen äußern. Die ehemalige Corticalis ist teilweise sehr schwer abzugrenzen.

Wie beim Humerus fällt auch im Tibia-Präparat eine unruhige Spongiosastruktur auf, die Bälkchen sind schräg angeordnet und es finden sich unregelmäßige Gefäßforamina. Vor allem im dicken Schliff sind jedoch Auflagerungen vom Typ I klar zu erkennen und auch eine Abgrenzung zur ehemaligen Corticalis ist möglich.

Eindeutig diagnose sichernd ist das Präparat der rechten Ulna. Hier lassen sich Auflagerungen vom Typ I durch das Wachsen auf der obersten Corticalis-Schicht sehr gut vom appositionellen Wachstum unterscheiden, bei dem das Wachstum eher um die Gefäßforamina der äußeren Schichten herum erfolgen würde. Auch hier finden in der Spongiosa aktive Umbauprozesse statt, es lassen sich sogar mehrere Howship'sche Lakunen nachweisen (Bildtafel 18, Bild 5, 6 und 7).

\section{Gesamtbeurteilung}

\begin{tabular}{|l|l|l|}
\hline A & Kardinalmerkmale \\
\hline 1.) & Poröse AL am äußeren Schädeldach & Occipitale \\
\hline 2.) & $\begin{array}{l}\text { Poröse AL am Orbitadach (Cribra } \\
\text { orbitalia) }\end{array}$ & Cribra Grad 1 beidseits \\
\hline 3.) & $\begin{array}{l}\text { Poröse AL am äußeren Gesichts- } \\
\text { schädel }\end{array}$ & - \\
\hline 4.) & Poröse AL am Alveolarknochen & - \\
\hline 5.) & Poröse AL an Langknochenschäften & $\begin{array}{l}\text { Humerus, Radii, Ulnae, Femura, Tibia, } \\
\text { Fibulae }\end{array}$ \\
\hline
\end{tabular}




\begin{tabular}{|c|c|c|}
\hline 6.) & Poröse AL am Schulterblatt & rechts \\
\hline 7.) & Poröse AL am Darmbein & beidseits \\
\hline B & Nebenmerkmale & \\
\hline 1.) & $\begin{array}{l}\text { Poröse OB/AL am harten Gaumen } \\
\text { (Stomatitis) }\end{array}$ & - \\
\hline 2.) & Poröse Auskleidung der Alveolen & - \\
\hline 3.) & $\begin{array}{l}\text { Symmetrische poröse AL am } \\
\text { inneren Schädeldach, flächig/in den } \\
\text { Impressiones digitatae }\end{array}$ & Os frontale \\
\hline 4.) & Poröse OB der Langknochenschäfte & alle \\
\hline 5.) & $\begin{array}{l}\text { Poröse Außenfläche des Großen } \\
\text { Keilbeinflügels }\end{array}$ & - \\
\hline $\mathrm{C}$ & Weitere Auffälligkeiten & Poröse AL an Rippen und Claviculae \\
\hline
\end{tabular}

Tab. 37: Merkmalstabelle zur Diagnose von Skorbut (nach SCHULTZ 1994), angewandt auf Skelet 439 aus dem Gräberfeld Bácsalmás-Óalmás

$\mathrm{AL}=$ Auflagerung, $\mathrm{OB}=$ Oberfläche, $-=$ Merkmal liegt nicht vor

Es lassen sich fünf Kardinal- und zwei Nebenmerkmale für Skorbut nachweisen, die auch mikroskopisch gesichert werden können. Damit erscheint die Diagnose Skorbut sehr wahrscheinlich, auch wenn, wie beim zuvor beschriebenen Individuum diskutiert, das Fehlen großer Teile des Skelets, vor allem des Schädels, natürlich zu Fehldiagnosen führen kann. Da jedoch alle vorhandenen Knochen Auflagerungen aufweisen, ist ein generalisiertes Geschehen anzunehmen. Der hohe Integrationsgrad der Auflagerungen spricht dafür, dass das Kind die Erkrankung eine Weile überlebt hat.
Bácsalmás-Óalmás
Grab 451
$2,5-3 \mathbf{J}$
$\mathbf{m}=\mathbf{w}$

Makroskopischer Befund

Bei dem Individuum aus Grab 451 der Grabung Bácsalmás-Óalmás handelt es sich um ein Kleinkind von 2,5 bis 3 Jahren.

Bei der makroskopischen Untersuchung des Postcraniums lassen sich sehr diskrete Auflagerungen nachweisen. Sie sind feinporös, flach und gut integriert.

An der rechten Scapula finden sich Auflagerungen in der Fossa supraspinata, an der linken in der Fossa infraspinata.

An den Humeri kann man sie distal an den dorsalen Diaphysen nachweisen, rechts auch an der distalen Metaphyse.

Auch am ventralen Schaft und der proximalen Metaphyse des linken Radius, den ventralen Diaphysen beider Ulnae sowie der proximalen Metaphyse der linken Ulna sind Auflagerungen zu erkennen. 
An den Femura finden sich lediglich kleinflächige Auflagerungen an den Ventralseiten der Schäfte, ebenso an der proximalen Diaphyse der rechten Tibia. Die Ventralseite der linken Tibia ist ebenso wie die Medialkante der rechten Fibula auf ganzer Schaftlänge mit Auflagerungen bedeckt.

Darüber hinaus finden sich diskrete Auflagerungen an beiden Claviculae, an den Ventral- und teilweise auch Dorsalseiten der Rippen beider Seiten sowie an den Darm- und Schambeinen beidseits.

Wesentlich deutlicher ausgeprägt sind die Auflagerungen am Schädel. Sie kleiden die Schädelinnenseite von Os frontale und parietale flächig aus (Bildtafel 19, Bild 1) und bedecken die Außenflächen der Squamae temporales und der Alae majores ossis sphenoidalis (Bildtafel 19, Bild 2 und 3). Auch am linken Os parietale entlang der Sutura lambdoidea finden sich Auflagerungen an der Außenseite.

Der knöcherne Gaumen ist ebenso wie die Innen- und Außenseite der Mandibulae mit feinporösen Auflagerungen bedeckt, desgleichen der Alveolarbereich aller Kiefer.

An den Orbitadächern finden sich Cribra Grad 3 (Bildtafel 18, Bild 8).

\section{Mikroskopischer Befund}

Es wurden Schliffe der linken Orbita, des linken Os parietale sowie der rechten Tibia angefertigt.

Am Orbitadach ist die der Orbitahöhle zugewandte Seite mit Auflagerungen vom Typ I bedeckt, sehr gut sind die für Skorbut typischen backsteinförmigen Mosaikstrukturen aus Knochenbrücken zu erkennen (Bildtafel 19, Bild 4 und 5).

Hinweise auf eine Druckatrophie der Lamina externa, wie sie bei einer Anämie zu erwarten wäre, ergeben sich nicht. Im Bereich der Diploë scheint es Blutungen, vermutlich durch rupturierte Diploë-Gefäße, gegeben zu haben, der Knochen erscheint unruhig und frisch umgebaut, es handelt sich um Faserknochen. Außerdem findet man eingebrochene Knochenbälkchen, was auf den Druck durch ein Hämatom zurückzuführen ist.

In den Präparaten des Os parietale (Bildtafel 19, Bild 6, 7 und 8) erkennt man an der Lamina externa kompakte Auflagerungen vom Typ I. Dabei sieht man folgende Dreischichtung: außen Auflagerungen, darunter Faserknochen, dann Lamellenknochen. Der ursprüngliche Verlauf der Lamina externa ist noch gut zu erkennen.

An der Lamina interna fallen Reste von Auflagerungen und verhältnismäßig tiefe Impressiones digitatae auf, die z.B. durch intracraniale Blutergüsse mit konsekutiver Erhöhung des intracranialen Drucks entstanden sein könnten. Außerdem ist in einem Präparat 
der Sulcus sinus sagittalis, der mit Auflagerungen vom Typ I ausgefüllt ist, zu erkennen (Bildtafel 19, Bild 7).

An der Tibia lassen sich diskrete Auflagerungen vom Typ I nachweisen, die bereits sehr gut integriert sind.

\section{Gesamtbeurteilung}

\begin{tabular}{|c|c|c|}
\hline $\mathbf{A}$ & \multicolumn{2}{|l|}{ Kardinalmerkmale } \\
\hline 1.) & Poröse AL am äußeren Schädeldach & Os parietale, Os temporale \\
\hline 2.) & $\begin{array}{l}\text { Poröse AL am Orbitadach (Cribra } \\
\text { orbitalia) }\end{array}$ & Cribra Grad 3 beidseits \\
\hline 3.) & $\begin{array}{l}\text { Poröse AL am äußeren Gesichts- } \\
\text { schädel }\end{array}$ & Os sphenoidale \\
\hline 4.) & Poröse AL am Alveolarknochen & Mandibula, Maxilla \\
\hline 5.) & Poröse AL an Langknochenschäften & $\begin{array}{l}\text { Humeri, Radius, Ulnae, Femura, } \\
\text { Tibiae, Fibulae }\end{array}$ \\
\hline 6.) & Poröse AL am Schulterblatt & beidseits \\
\hline 7.) & Poröse AL am Darmbein & beidseits \\
\hline B & Nebenmerkmale & \\
\hline 1.) & $\begin{array}{l}\text { Poröse OB/AL am harten Gaumen } \\
\text { (Stomatitis) }\end{array}$ & $\mathrm{AL}$ \\
\hline 2.) & Poröse Auskleidung der Alveolen & - \\
\hline 3.) & $\begin{array}{l}\text { Symmetrische poröse } \mathrm{AL} \text { am } \\
\text { inneren Schädeldach, flächig/in den } \\
\text { Impressiones digitatae }\end{array}$ & Frontale, Parietale \\
\hline 4.) & Poröse OB der Langknochenschäfte & Alle \\
\hline 5.) & $\begin{array}{l}\text { Poröse Außenfläche des Großen } \\
\text { Keilbeinflügels }\end{array}$ & AL beidseits \\
\hline $\mathrm{C}$ & Weitere Auffälligkeiten & $\begin{array}{l}\text { AL der Rippen, Claviculae, } \\
\text { Schambeine }\end{array}$ \\
\hline
\end{tabular}

Tab. 38: Merkmalstabelle zur Diagnose von Skorbut (nach SCHULTZ 1994), angewandt auf Skelet 451 aus dem Gräberfeld Bácsalmás-Óalmás

$\mathrm{AL}=$ Auflagerung, $\mathrm{OB}=$ Oberfläche, $-=$ Merkmal liegt nicht vor

Es lassen sich alle Kardinal- und vier Nebenmerkmale für Skorbut nachweisen, die auch lichtmikroskopisch dargestellt werden können.

Damit bestätigt sich die Diagnose Skorbut.

\subsection{Untersuchungsbefunde des einzelnen Individuums Kiszombor}
Kiszombor
Grab 299
Sign. 299
$6 \mathbf{J}+/-12 \mathbf{M}$
$\mathbf{m}=\mathbf{w}$

Makroskopischer Befund

Hinter der Bezeichnung Kiszombor, Signatur 299, Grab 299 verbirgt sich der Schädel eines ca. 6jährigen Kindes. 
Schon bei oberflächlicher Betrachtung fallen gewaltige spongiöse Hyperostosen auf den Tubera parietalia auf (Bildtafel 20, Bild 1). Sie haben einen Durchmesser von ca. $10 \mathrm{~cm}$ und in der Mitte eine Dicke von ca. 0,5 cm. Zum Rand hin werden die Neubildungen flacher und gehen in eine feinporöse Oberfläche über. Von ringsum sprießen Gefäßimpressionen in die Hyperostosen ein. Auch auf dem Os frontale finden sich sternförmige Gefäßimpressionen.

Auffällig ist, dass die Sutura sagittale nahezu vollständig verstrichen ist, obwohl das Kind sich gerade erst im Zahnwechsel befand, also zum Todeszeitpunkt vermutlich ca. 6 Jahre alt war.

Sehr diskrete feinporöse Auflagerungen mit schwach profiliertem Rand liegen an den Alae majores ossis sphenoidalis.

An den Orbitadächern finden sich Cribra Grad 2-3, auch diese umgeben von deutlichen Gefäßimpressionen.

Das Palatum durum ist von feinporöser Beschaffenheit, ebenso die Alveolarfortsätze. Dies lässt sich jedoch durch den Zahnwechsel ausreichend erklären. Darüber hinaus fallen an den Mandibulae kleinflächige feinporöse Auflagerungen an der Innenseite der Incisura mandibulae und außen mittig auf dem Ramus mandibulae auf.

\section{Mikroskopischer Befund}

Es wurde ein Schliff aus dem Bereich der Hyperostosis rechts entnommen. Dabei zeigte sich ein massiver Bürstensaum im Sinne einer Auflagerung vom Typ II. Es sollte also von einer Anämie ausgegangen werden (Bildtafel 20, Bild 2).

\section{Gesamtbeurteilung}

\begin{tabular}{|l|l|l|}
\hline A & Kardinalmerkmale \\
\hline 1.) & Poröse AL am äußeren Schädeldach & Parietale \\
\hline 2.) & $\begin{array}{l}\text { Poröse AL am Orbitadach (Cribra } \\
\text { orbitalia) }\end{array}$ & Cribra Grad 2-3 beidseits \\
\hline 3.$)$ & $\begin{array}{l}\text { Poröse AL am äußeren Gesichts- } \\
\text { schädel }\end{array}$ & Sphenoidale \\
\hline 4.) & Poröse AL am Alveolarknochen & - \\
\hline 5.) & Poröse AL an Langknochenschäften & - \\
\hline 6.) & Poröse AL am Schulterblatt & - \\
\hline 7.) & Poröse AL am Darmbein & - \\
\hline B & Nebenmerkmale & $\begin{array}{l}\text { Poröse OB/AL am harten Gaumen } \\
\text { (Stomatitis) }\end{array}$ \\
\hline 1.) OB \\
\hline 2.) & Poröse Auskleidung der Alveolen & - \\
\hline 3.) & $\begin{array}{l}\text { Symmetrische poröse AL am } \\
\text { inneren Schädeldach, flächig/in den } \\
\text { Impressiones digitatae }\end{array}$ \\
\hline 4.) & Poröse OB der Langknochenschäfte & - \\
\hline
\end{tabular}




\begin{tabular}{|l|l|l|}
\hline 5.) & $\begin{array}{l}\text { Poröse Außenfläche des Großen } \\
\text { Keilbeinflügels }\end{array}$ & AL beidseits \\
\hline C & Weitere Auffälligkeiten & Massive Gefäßimpressionen \\
\hline
\end{tabular}

Tab. 39: Merkmalstabelle zur Diagnose von Skorbut (nach SCHULTZ 1994), angewandt auf das einzelne Skelet Kiszombor 299

$\mathrm{AL}=$ Auflagerung, $\mathrm{OB}=$ Oberfläche, - = Merkmal liegt nicht vor

Es lassen sich drei Kardinal- und zwei Nebenmerkmale für Skorbut nachweisen. Dennoch erscheint die Diagnose unwahrscheinlich, da sowohl der makroskopische Aspekt als auch der mikroskopische Befund dem typischen Bild eines stark ausgeprägten anämischen Bürstenschädels entspricht.

\title{
3.4 Untersuchungsbefunde der einzelnen Individuen des Gräberfelds Sükösd-Ságod
}

\author{
Sükösd-Ságod \\ Grab 134 \\ ca. 4 Jahre \\ $\mathbf{m}=\mathbf{w}$

\section{Makroskopischer Befund}

Bei dem Individuum aus Grab 134 der Grabung Sükösd-Ságod handelt es sich um ein Kind von etwa vier Jahren. Es ist nur der Schädel erhalten. Bei der makroskopischen Untersuchung fallen massive hämorrhagische Auflagerungen an der Lamina interna von Os frontale, Os parietale (Bildtafel 20, Bild 7) und Os occipitale auf. Sie kleiden die Impressiones digitatae und die venösen Sulci des Os occipitale (Bildtafel 20, Bild 6) und teilweise auch des Os temporale aus, konfluieren jedoch auch flächig.

Ähnliche Auflagerungen finden sich außen an der Squama ossis temporalis rechts und an den Alae majores ossis sphenoidalis. Die Alae minores ossis sphenoidalis sind ebenfalls befallen.

Auch die Gesichtsfläche der Maxilla ist mit Auflagerungen bedeckt (Bildtafel 20, Bild 5), ebenso das Palatinum und der Alveolarfortsatz.

An der Mandibula finden sich Auflagerungen außen am Mentum und am Ramus sowie innen an der Incisura mandibulae (Bildtafel 20, Bild 4). Der Alveolarfortsatz weist nur um einen einzigen Molaren herum Auflagerungen auf.

An den Orbitadächern finden sich Cribra Grad 3 (Bildtafel 20, Bild 3).

\section{Mikroskopischer Befund}

Es wurden Schliffe vom linken Os frontale, dem linken Os occipitale, beiden Orbitae und dem linken Os parietale angefertigt.

Am Os frontale erkennt man an der Lamina externa Auflagerungen vom Typ I (Bildtafel 20, Bild 8), bei denen es sich wohl um ein mehrphasiges Geschehen handelt, wobei die ältere, 
also tiefere Schicht diploëisiert erscheint. Insgesamt fällt auf, dass die Diploë an den Stellen mit Auflagerungen verdickt ist, was auch eine physiologische Reaktion sein kann.

Am Os occipitale finden sich massive hämorrhagische Auflagerungen vom Typ I an der Lamina interna. In der Durchsicht sind einige Einbrüche in die Diploë zu erkennen, was auf eine Hirndrucksymptomatik zurückzuführen ist. Dies führte auch zu einer gewissen Sklerosierung und Verdichtung der Diploë. Die Impressiones digitatae sind mit Auflagerungen vom Typ I ausgefüllt, ebenso der angeschnittene Sulcus, in welchem sich die Auflagerungen sogar mehrphasig darstellen (Bildtafel 21, Bild 3, 4 und 5).

Das Präparat der linken Orbita ist aufgrund diagenetischer Veränderungen kaum beurteilbar.

An der rechten Orbita lassen sich Auflagerungen vom Typ I nachweisen. In der Diploë fallen große Gefäßforamina und Blutmarkräume auf (Bildtafel 21, Bild 2).

Am Os parietale ist eine meningeale Reaktion hämorrhagischer Art an der Lamina interna zu erkennen. Die Impressiones digitatae sind mit Auflagerungen vom Typ I verfüllt. Gut zu erkennen ist die Dreischichtung von Auflagerungen innen, daran grenzend unreifer Faserknochen und schließlich besser organisierter Knochen außen (Bildtafel 21, Bild 1).

\section{Gesamtbeurteilung}

\begin{tabular}{|c|c|c|}
\hline $\mathbf{A}$ & \multicolumn{2}{|l|}{ Kardinalmerkmale } \\
\hline 1.) & Poröse AL am äußeren Schädeldach & Temporale, Frontale \\
\hline 2.) & $\begin{array}{l}\text { Poröse AL am Orbitadach (Cribra } \\
\text { orbitalia) }\end{array}$ & Cribrum Grad 3 beidseits \\
\hline 3.) & $\begin{array}{l}\text { Poröse AL am äußeren Gesichts- } \\
\text { schädel }\end{array}$ & Maxilla, Sphenoidale \\
\hline 4.) & Poröse AL am Alveolarknochen & Maxilla, (Mandibula) \\
\hline 5.) & Poröse AL an Langknochenschäften & - \\
\hline 6.) & Poröse AL am Schulterblatt & - \\
\hline 7.) & Poröse AL am Darmbein & - \\
\hline B & Nebenmerkmale & \\
\hline 1.) & $\begin{array}{l}\text { Poröse OB/AL am harten Gaumen } \\
\text { (Stomatitis) }\end{array}$ & $\mathrm{AL}$ \\
\hline 2.) & Poröse Auskleidung der Alveolen & - \\
\hline 3.) & $\begin{array}{l}\text { Symmetrische poröse AL am } \\
\text { inneren Schädeldach, flächig/in den } \\
\text { Impressiones digitatae }\end{array}$ & $\begin{array}{l}\text { Frontale, Parietale, Temporale, } \\
\text { Occipitale }\end{array}$ \\
\hline 4.) & Poröse OB der Langknochenschäfte & - \\
\hline 5.) & $\begin{array}{l}\text { Poröse Außenfläche des Großen } \\
\text { Keilbeinflügels }\end{array}$ & AL beidseits \\
\hline $\mathrm{C}$ & Weitere Auffälligkeiten & - \\
\hline
\end{tabular}

Tab. 40: Merkmalstabelle zur Diagnose von Skorbut (nach SCHULTZ 1994), angewandt auf Skelet 134 aus dem Gräberfeld Sükösd-Ságod

$\mathrm{AL}=$ Auflagerung, $\mathrm{OB}=$ Oberfläche, $-=$ Merkmal liegt nicht vor 
Es lassen sich vier Kardinal- und drei Nebenmerkmale für Skorbut nachweisen, die auch lichtmikroskopisch gesichert sind. Damit ist eine Skorbuterkrankung sehr wahrscheinlich.

Allerdings muss man natürlich beachten, dass die Sicherheit der Diagnose durch das vollständige Fehlen des Postcraniums eingeschränkt wird.

\section{Sükösd-Ságod}

Grab 288

Neonatus

$\mathbf{m}=\mathbf{w}$

\section{Makroskopischer Befund}

Bei dem Individuum aus Grab 288 der Grabung Sükösd-Ságod handelt es sich um ein Kind, das vermutlich kurz nach der Geburt gestorben ist.

Bei der makroskopischen Untersuchung fallen diskrete feinporöse Auflagerungen auf, die gut integriert zu sein scheinen.

Sie befinden sich an der linken Scapula in den Fossae infraspinata und subscapularis, an den Humeri an den Diaphysen und distalen Metaphysen (Bildtafel 21, Bild 6), ebenso am rechten Radius dorsal und über die gesamte ventrale Schaftlänge der linken Ulna.

Auch einige Rippen weisen an der Innen- und Außenseite Auflagerungen auf.

An der unteren Extremität finden sich Auflagerungen an Diaphyse und distaler Metaphyse des rechten Femurs, an den Diaphysen beider Tibiae und an der distalen Hälfte der linken Fibula. Auch an der Außenseite des linken Os ilium lassen sich feinporöse Auflagerungen nachweisen.

Am Os parietale und in der Fossa cranii anterior sind die Impressiones digitatae mit feinporösen Auflagerungen verfüllt, an der Lamina interna des Os frontale konfluieren sie. Am Os occipitale erkennt man nur zwei kleinflächige Auflagerungen in den Sulci der venösen Sinus.

An der Schädelaußenseite finden sich diskrete Auflagerungen an der Glabella und an den Ossa zygomatica sowie je zwei kleine Flecken am Os occipitale und am rechten Os parietale. Von der Mandibula sind nur erodierte Fragmente erhalten. Diese scheinen jedoch von feinporösen Auflagerungen bedeckt zu sein.

An den Orbitadächern finden sich Cribra Grad 2-3 (Bildtafel 21, Bild 7).

\section{Mikroskopischer Befund}

Es wurden Schliffe von einem Occipitalfragment, dem linken Os parietale und der linken Tibia angefertigt.

Am Os occipitale sind schwache Auflagerungen vom Typ I auszumachen, die sich allerdings schlecht gegen das physiologische appositionelle Knochenwachstum im Säuglingsalter abgrenzen lassen. 
Das Präparat des Os parietale zeigt keine Pathologien.

Auch an der Tibia fällt es schwer, Auflagerungen vom Typ I vom normalen Schalenknochen abzugrenzen (Bildtafel 21, Bild 8).

Gesamtbeurteilung

\begin{tabular}{|c|c|c|}
\hline $\mathbf{A}$ & \multicolumn{2}{|l|}{ Kardinalmerkmale } \\
\hline 1.) & Poröse AL am äußeren Schädeldach & Frontale, Parietale, Occipitale \\
\hline 2.) & $\begin{array}{l}\text { Poröse AL am Orbitadach (Cribra } \\
\text { orbitalia) }\end{array}$ & Cribra Grad 2-3 \\
\hline 3.) & $\begin{array}{l}\text { Poröse AL am äußeren Gesichts- } \\
\text { schädel }\end{array}$ & Os zygomaticum \\
\hline 4.) & Poröse AL am Alveolarknochen & - \\
\hline 5.) & Poröse AL an Langknochenschäften & $\begin{array}{l}\text { Humeri, Radius, Ulna, Femur, Tibiae, } \\
\text { Fibula }\end{array}$ \\
\hline 6.) & Poröse AL am Schulterblatt & $\begin{array}{l}\text { Fossae infraspinata und subscapularis } \\
\text { links }\end{array}$ \\
\hline 7.) & Poröse AL am Darmbein & links \\
\hline B & Nebenmerkmale & \\
\hline 1.) & $\begin{array}{l}\text { Poröse OB/AL am harten Gaumen } \\
\text { (Stomatitis) }\end{array}$ & - \\
\hline 2.) & Poröse Auskleidung der Alveolen & - \\
\hline 3.) & $\begin{array}{l}\text { Symmetrische poröse AL am } \\
\text { inneren Schädeldach, flächig/in den } \\
\text { Impressiones digitatae }\end{array}$ & Frontale, Parietale, Occipitale \\
\hline 4.) & Poröse OB der Langknochenschäfte & Alle \\
\hline 5.) & $\begin{array}{l}\text { Poröse Außenfläche des Großen } \\
\text { Keilbeinflügels }\end{array}$ & - \\
\hline $\mathrm{C}$ & Weitere Auffälligkeiten & $\begin{array}{l}\text { AL an Innen- und Außenseiten einiger } \\
\text { Rippen }\end{array}$ \\
\hline
\end{tabular}

Tab. 41: Merkmalstabelle zur Diagnose von Skorbut (nach SCHULTZ 1994), angewandt auf Skelet 288 aus dem Gräberfeld Sükösd-Ságod

$\mathrm{AL}=$ Auflagerung, $\mathrm{OB}=$ Oberfläche, - = Merkmal liegt nicht vor

Es lassen sich sechs Kardinal- und zwei Nebenmerkmale für Skorbut nachweisen, dies macht die Diagnose Skorbut wahrscheinlich. Allerdings ist die Histologie nicht eindeutig beweisend, da die Abgrenzung zum physiologischen appositionellen Wachstum schwierig ist.

Auch das geringe Alter des Kindes könnte an der Diagnose zweifeln lassen, da es eigentlich noch durch die Mutter mit Vitamin C versorgt werden sollte. Wie bereits bei dem Individuum aus Grab 436 der Population Bácsalmás-Óalmás diskutiert, gibt es jedoch auch hierfür Erklärungsmöglichkeiten. 


\section{Erklärungen zu Bildtafel 1}

Bild 1: Sk 1272, Barbing. Kind, ca. 12 Jahre alt. Makroskopische Vergleichsaufnahme beider Humeri. Deutlich erkennt man die Deformierung des rechten Humerus.

$\rightarrow$ ausgeprägte Osteomyelitis

Bild 2: Sk 1272, Barbing. Kind, ca. 12 Jahre alt. Makroskopische Detailaufnahme des rechten Humerus. Zu sehen ist die vermutlich entzündlich bedingte Veränderung des distalen Gelenkbereichs.

Bild 3: Sk 1272, Barbing. Kind, ca. 12 Jahre alt. Lichtmikroskopische Darstellung (Schliffdicke $50 \mu \mathrm{m}$, polarisiertes Durchlicht mit Hilfsobjekt Rot 1. Ordnung (Quarz) als Kompensator, Vergrößerung 100-fach): Das Präparat aus dem linken Humerus zeigt eine Auflagerung vom Typ I (hämorrhagisch). Mittig ist ein Gefäßforamen zu erkennen. $\rightarrow$ Organisiertes subperiostales Hämatom mit Knochenneubildung im Rahmen von Skorbut

Bild 4: Sk 1279, Barbing. Kind, ca. 3 Jahre alt. Makroskopische Aufnahme der linken Tibia. Deutlich zu sehen sind die feinporösen Auflagerungen am Schaft. $\rightarrow$ Hämorrhagisches Geschehen im Rahmen von Skorbut

Bild 5: Sk 1279, Barbing. Kind, ca. 3 Jahre alt. Röntgen-Aufnahme der linken Tibia. In Bereich der Schaftmitte sind medial Auflagerungen zu erkennen (grüne Pfeile). Distal ist eine Harris-Linie abgebildet (weiße Pfeile). $\rightarrow$ Organisiertes subperiostales Hämatom im Rahmen von Skorbut und Verdacht auf vorangegangene Krankheits- oder Mangelzustände in der Wachstumsphase

Bild 6: Sk 1279, Barbing. Kind, ca. 3 Jahre alt. Lichtmikroskopische Darstellung (Schliffdicke 50 um, einfaches Durchlicht, Vergrößerung 16-fach): Es handelt sich um eine Übersichtsaufnahme der linken Tibia. Im Bereich der Corticalis erkennt man diskrete Auflagerungen (Pfeile) vom Typ 1 (hämorrhagisch), differentialdiagnostisch muss an physiologisches appositionelles Knochenwachstum gedacht werden. Auffällig ist die spongiös aufgelockerte Struktur des Knochens mit zahlreichen Resorptionshöhlen (Sterne).

Bild 7: Sk 1279, Barbing. Kind, ca. 3 Jahre alt. Lichtmikroskopische Darstellung (Schliffdicke 50 um, einfaches Durchlicht, Vergrößerung 400-fach): Detailaufnahme einer der bei Bild 6 beschriebenen Resorptionshöhlen. Es sind drei Howship'sche Lakunen zu erkennen (Pfeile). $\rightarrow$ sehr aktiver Resorptionsprozess

Bild 8: Sk 1281, Barbing. Männlich, 25 - 35 Jahre alt. Makroskopische Detailaufnahme der Auflagerungen an der linken Ulna. Der Schnittrand an der rechten Bildkante markiert den Bereich, aus dem die Probe zur Anfertigung der histologischen Präparate (siehe auch Bild 1 und 2 auf Bildtafel 2) entnommen wurde. $\rightarrow$ Subperiostale Einblutung mit nachfolgender Knochenneubildung im Rahmen einer systemischen Skorbuterkrankung 


\section{Bildtafel 1}
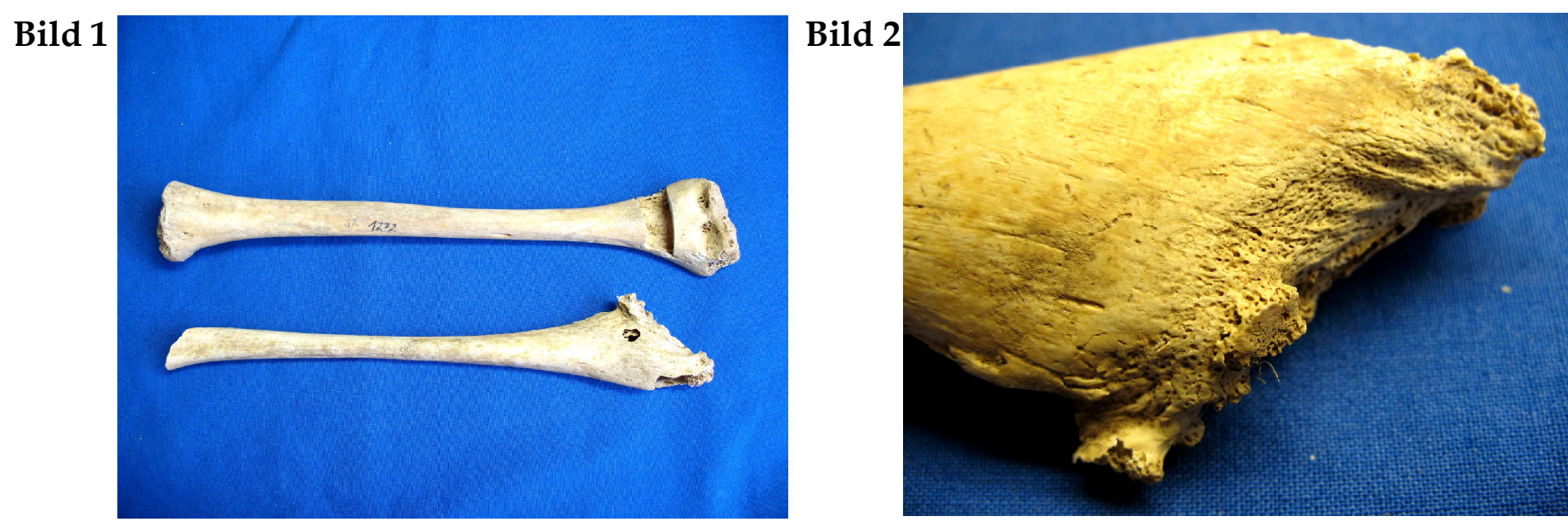

Bild 3

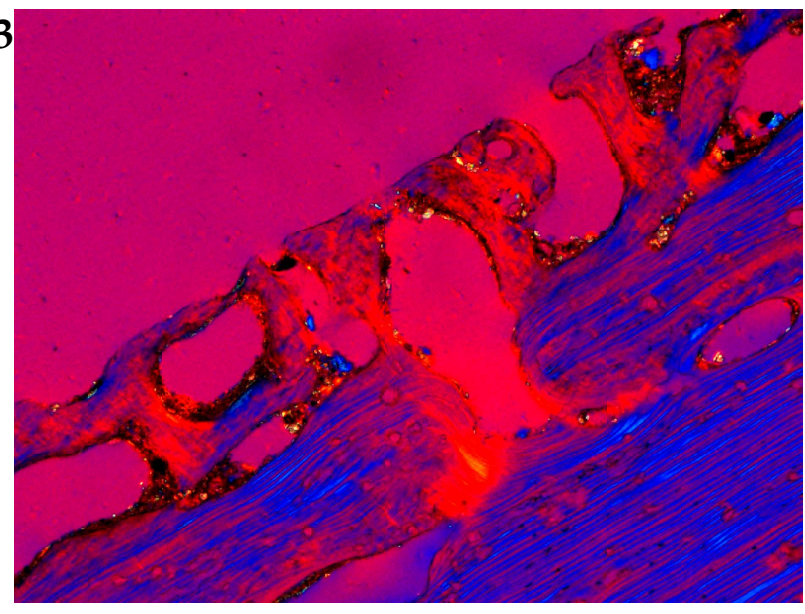

Bild 4

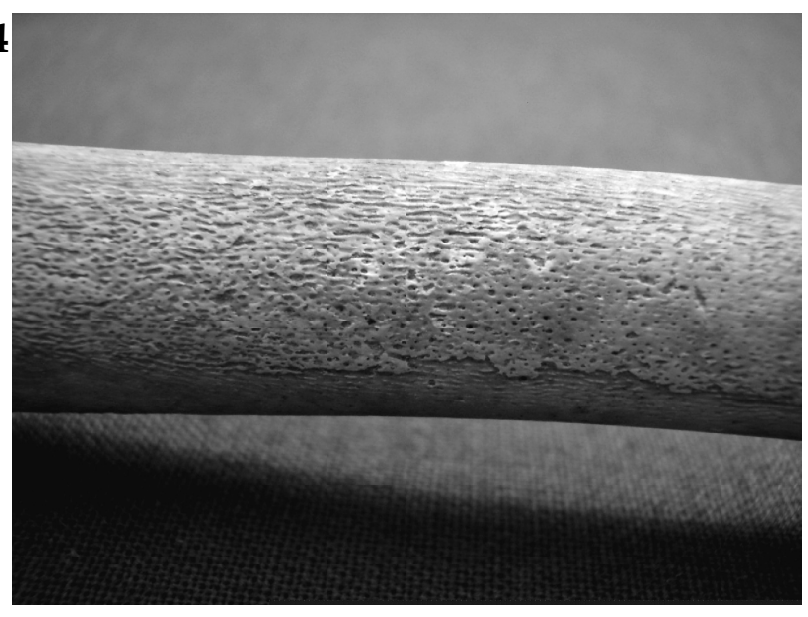

Bild 5

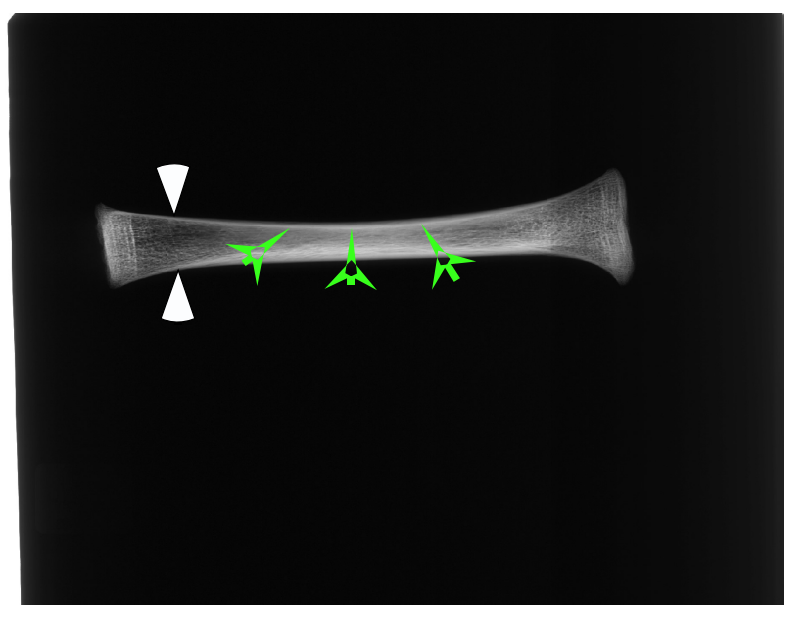

Bild 7

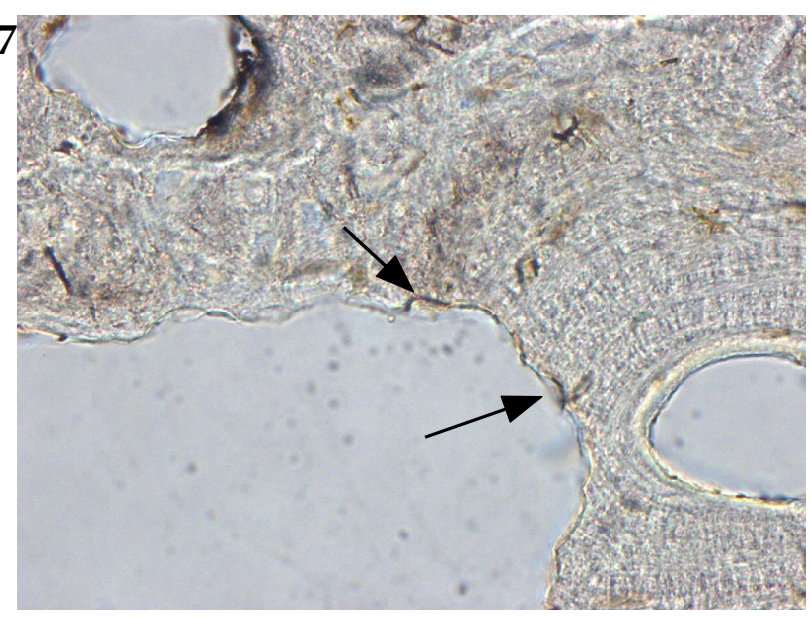

Bild 6

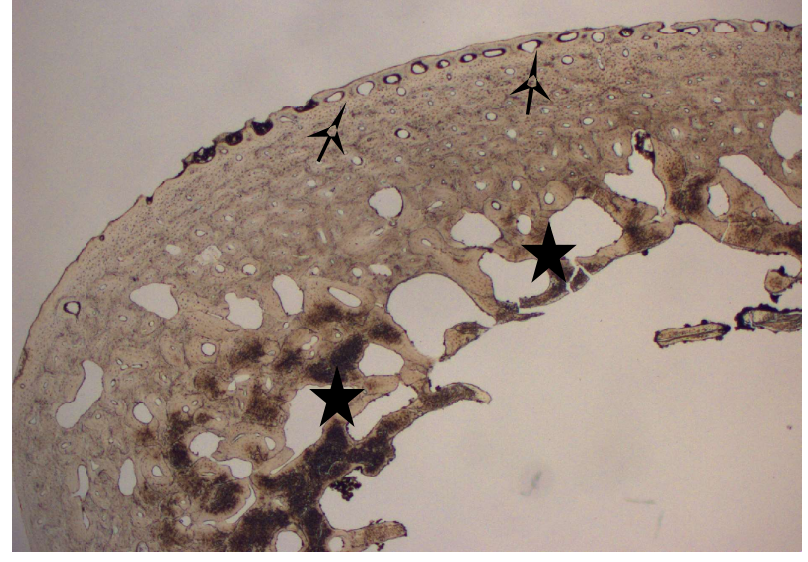

Bild 8

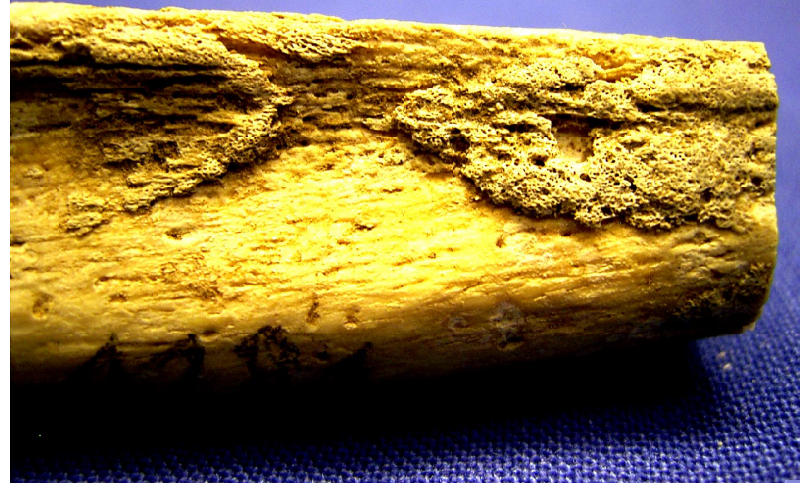




\section{Erklärungen zu Bildtafel 2}

Bild 1: Sk 1281, Barbing. Männlich, 25 - 35 Jahre alt. Lichtmikroskopische Darstellung (Schliffdicke 50 $\mu \mathrm{m}$, polarisiertes Durchlicht mit Hilfsobjekt Rot 1. Ordnung (Quarz) als Kompensator, Vergrößerung 16-fach): Das Präparat aus der linken Ulna zeigt mehrlagige Auflagerungen vom Typ I (hämorrhagisch), im darunter liegenden Lamellenknochen fallen Gefäßeinbrüche (Pfeile) auf $\rightarrow$ faserknöchern organisiertes, ehemaliges Hämatom im Rahmen von Skorbut mit Druckatrophie der darunter liegenden Knochensubstanz

Bild 2: Sk 1281, Barbing. Männlich, 25 - 35 Jahre alt. Lichtmikroskopische Darstellung (Schliffdicke 70 $\mu \mathrm{m}$, polarisiertes Durchlicht mit Hilfsobjekt Rot 1. Ordnung (Quarz) als Kompensator, Vergrößerung 25-fach): Das Präparat aus der linken Ulna zeigt eine Vergrößerung der in Bild 1 gezeigten Auflagerung vom Typ 1 (hämorrhagisch), darunter ist normaler Lamellenknochen $\mathrm{zu}$ erkennen. $\rightarrow$ faserknöchern organisiertes, ehemaliges Hämatom im Rahmen von Skorbut

Bild 3: Sk 1290, Barbing. Kind, ca. 4 Jahre alt. Makroskopische Aufnahme der sechsten Rippe links. Deutlich sind die feinporösen Auflagerungen zu erkennen. $\rightarrow$ Organisiertes subperiostales Hämatom im Rahmen von Skorbut

Bild 4: Sk 1290, Barbing. Kind, ca. 4 Jahre alt. Makroskopische Aufnahme der Schädelinnenseite des linken Os parietale. Die Impressiones digitatae sind stark ausgeprägt, mit feinporösen Auflagerungen ausgekleidet und mit feinen Gefäßimpressionen durchzogen. $\rightarrow$ Hämorrhagisches Geschehen mit Hirndrucksteigerung im Rahmen von Skorbut

Bild 5: Sk 1290, Barbing. Kind, ca. 4 Jahre alt. Lichtmikroskopische Darstellung (Schliffdicke 50 $\mu$ m, einfaches Durchlicht, Vergrößerung 25-fach): Es handelt sich um ein Präparat aus der 6. Rippe links. Rechts unten im Bild ist die der Pleura zugewandte Kante der Rippe zu sehen, darüber liegen mehrlagige Auflagerungen vom Typ 1 (hämorrhagisch). Es lassen sich drei Krankheitsschübe abgrenzen (Zahlen), wobei der letzte (3) die heftigste Ausprägung zeigt. $\rightarrow$ Schubweise auftretende subperiostale Hämorrhagien mit nachfolgender Knochenneubildung in Folge schweren chronischen Vitamin-C-Mangels

Bild 6: Sk 1290, Barbing. Kind, ca. 4 Jahre alt. Lichtmikroskopische Darstellung (Schliffdicke 50 $\mu \mathrm{m}$, polarisiertes Durchlicht mit Hilfsobjekt Rot 1. Ordnung (Quarz) als Kompensator, Vergrößerung 25-fach): Es handelt sich um das unter Bild 5 beschriebene Präparat, zur Verdeutlichung der unterschiedlichen Strukturen der verschiedenen Knochenschichten wurde hier polarisiertes Durchlicht verwendet.

Bild 7: Sk 1290, Barbing. Kind, ca. 4 Jahre alt. Röntgenbild der 6. Rippe links. Durch die Auflagerungen (grüner Pfeil) entsteht das typische Bild einer Doppelkontur (pinke Pfeile) an der pleuralen Seite.

Bild 8: Sk 1293, Barbing. Weiblich, 25 - 30 Jahre alt. Makroskopische Übersichtsaufnahme der rechten Mandibula. Man beachte die feinporösen Auflagerungen an der Mandibula im Bereich der Höhlung unterhalb des zweiten Prämoralen rechts (siehe auch Bild 1 auf Bildtafel 3) $\rightarrow$ tiefer Zahnabszess mit Affektion des darunter liegenden Knochens 


\section{Bildtafel 2}

Bild 1

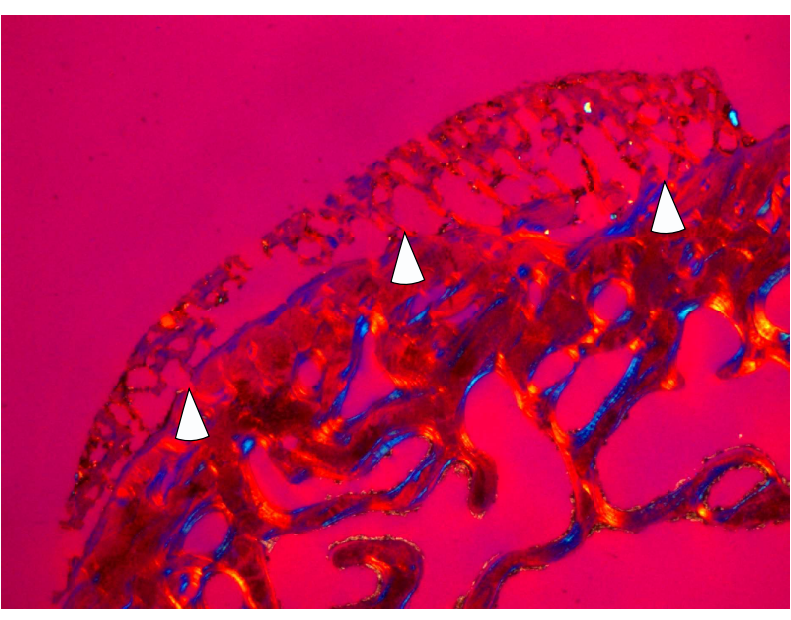

Bild 3

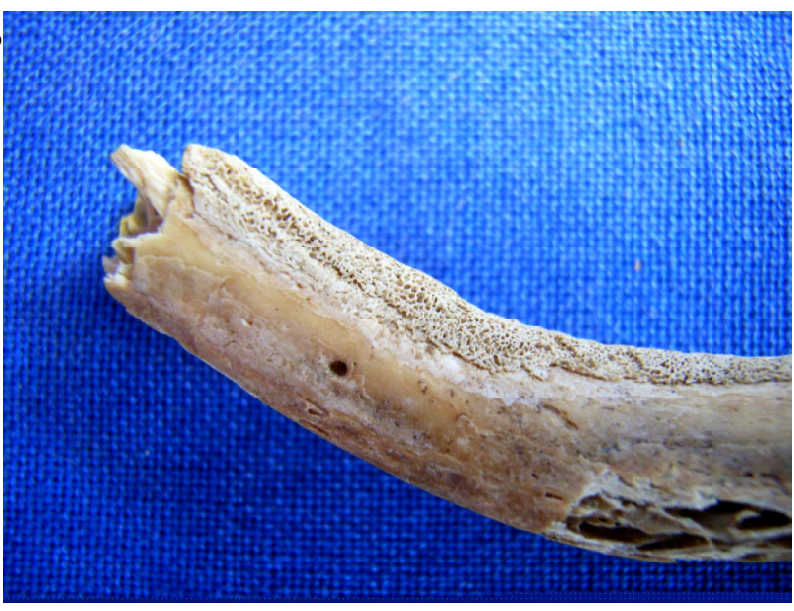

Bild 5

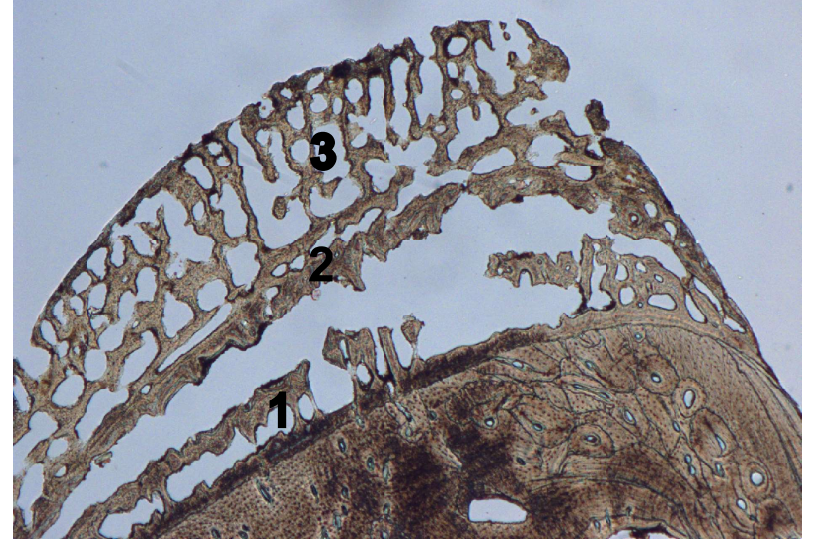

Bild 7

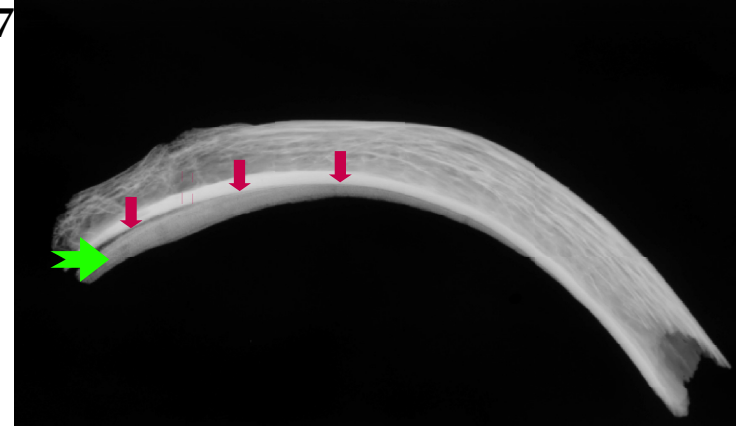

Bild 2

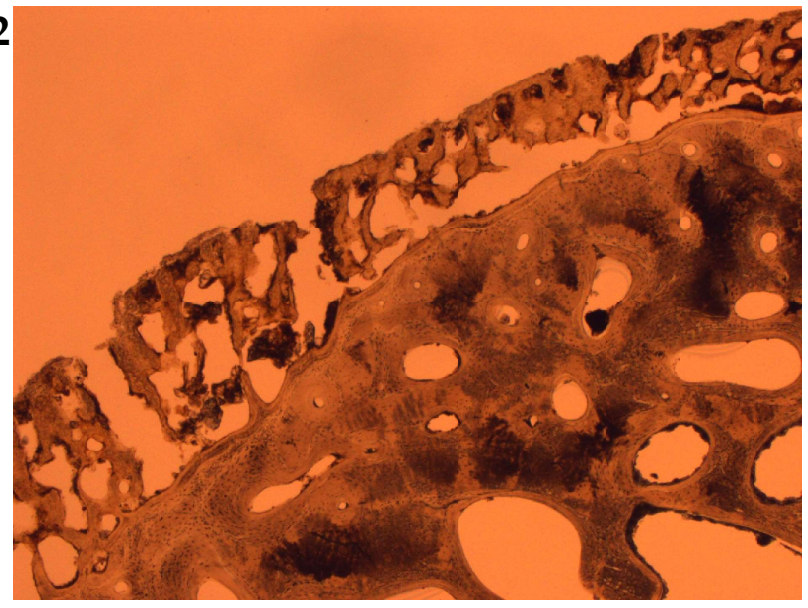

Bild 4

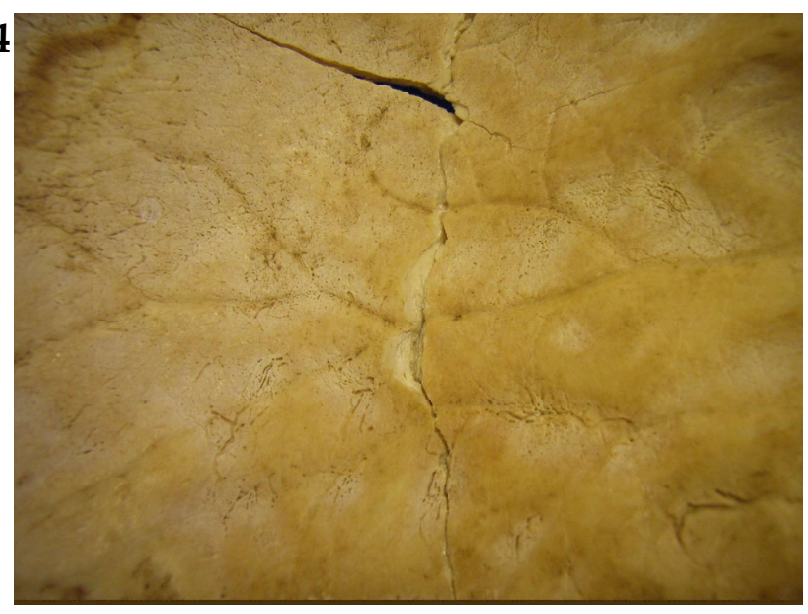

Bild 6

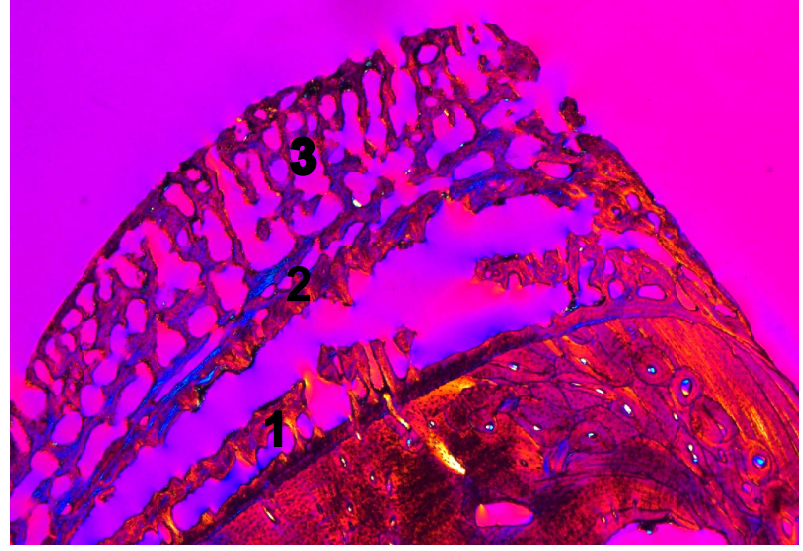

Bild 8

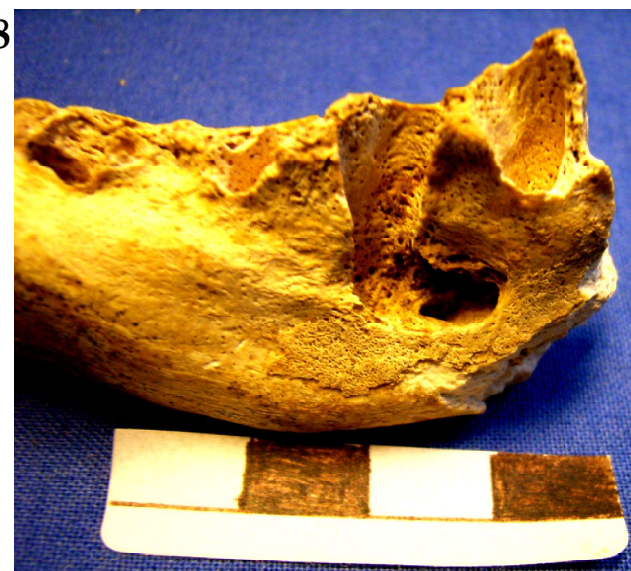




\section{Erklärungen zu Bildtafel 3}

Bild 1: Sk 1293, Barbing. Weiblich, 25 - 30 Jahre alt. Detailaufnahme der feinporösen Auflagerungen an der Mandibula im Bereich einer Höhlung unterhalb des zweiten Prämoralen rechts (siehe auch Bildtafel 2, Bild 8) $\rightarrow$ tiefer Zahnabszess mit Affektion des darunter liegenden Knochens

Bild 2: Sk 1293, Barbing. Weiblich, 25 - 30 Jahre alt. Röntgenbild der Mandibula. Im Bereich der in den vorangegangenen Bildern gezeigten Abszesshöhle sind flache feinporöse Auflagerungen zu erkennen (Pfeile). Die darunter liegende Spongiosa ist entzündlich verändert. $\rightarrow$ tiefer Zahnabszess mit Affektion des darunter liegenden Knochens

Bild 3: Sk 1293, Barbing. Weiblich, 25 - 30 Jahre alt. Lichtmikroskopische Darstellung (Schliffdicke $50 \mu \mathrm{m}$, einfaches Durchlicht, Vergrößerung 16-fach): Schnitt durch den in den vorangegangenen Bildern gezeigten Abszess in der rechten Mandibula. Obwohl der Erhaltungszustand des Knochens nicht besonders gut ist, sind deutliche Auflagerungen vom Typ I (hämorrhagisch) zu erkennen. Rechts unten im Bild ist der mit Dreck verfüllte Canalis mandibularis angeschnitten. Die darum liegende Spongiosa erscheint entzündlich verändert. $\rightarrow$ tiefer Zahnabszess mit Affektion des darunter liegenden Knochens

Bild 4: Sk 1293, Barbing. Weiblich, 25 - 30 Jahre alt. Lichtmikroskopische Darstellung (Schliffdicke $50 \mu \mathrm{m}$, polarisiertes Durchlicht mit Hilfsobjekt Rot 1. Ordnung (Quarz) als Kompensator, Vergrößerung 16-fach): Es handelt sich um ein Präparat aus der linken Tibia. Zu sehen ist eine zarte Auflagerung vom Typ I (hämorrhagisch). $\rightarrow$ Organisiertes subperiostales Hämatom infolge chronischen Vitamin-C-Mangels

Bild 5: Sk 1297, Barbing. Kind, ca. 4 Jahre alt. Makroskopische Detailaufnahme der rechten Mandibula. Es sind gut integrierte feinporöse Auflagerungen im Alveolarbereich zu sehen.

Subperiostales Hämatom mit konsekutiver Knochenneubildung im fortgeschrittenen Heilungsstadium, vermutlich im Rahmen von Skorbut

Bild 6: Sk 1298, Barbing. Kind, ca. 3 - 4 Jahre alt. Röntgenbild der rechten Tibia. Am Schaft sind medial dem ehemaligen Periost aufgesetzte Auflagerungen (grüne Pfeile) zu erkennen. Außerdem sind proximal und distal je eine Harrislinie zu erkennen (weiße Pfeile). $\rightarrow$ Knochenneubildung nach subperiostaler Einblutung im Rahmen von Skorbut, vermutlich auch in der Vergangenheit schon Mangelernährung oder Erkrankungen, die zu vorübergehenden Wachstumsstopps führten

Bild 7: Sk 1299, Barbing. Kind, ca. 0,5 - 1,5 Jahre alt. Makroskopische Detailaufnahme der linken Mandibula: In den Alveolen sind feinporöse Auskleidungen zu erkennen. $\rightarrow$ Knochenneubildungen infolge von Zahnfleischblutungen im Rahmen von Skorbut

Bild 8: Sk 1299, Barbing. Kind, ca. 0,5 - 1,5 Jahre alt. Makroskopische Aufnahme der Außenseite des Os occipitale. Es sind feinporöse Auflagerungen $\mathrm{zu}$ sehen. $\rightarrow$ Knochenneubildungen infolge subpericranialer Blutungen im Rahmen von Skorbut 


\section{Bildtafel 3}

Bild 1

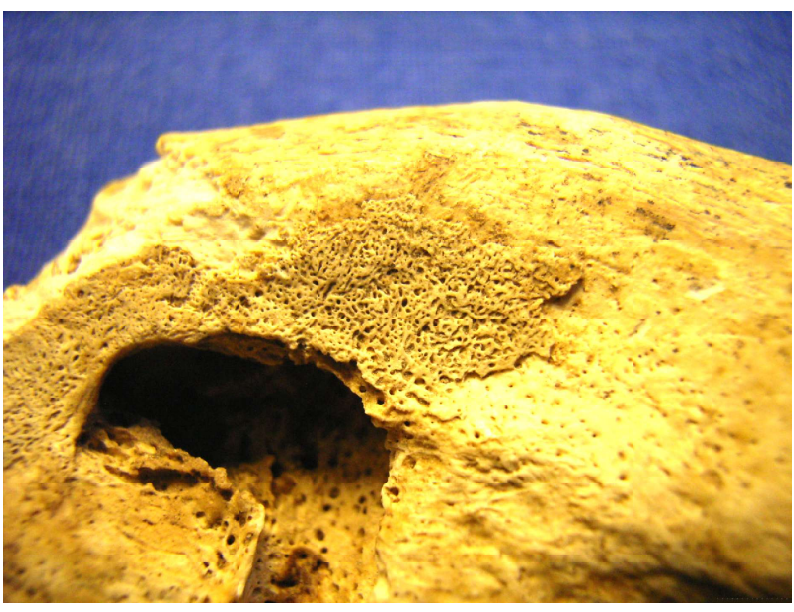

Bild 3

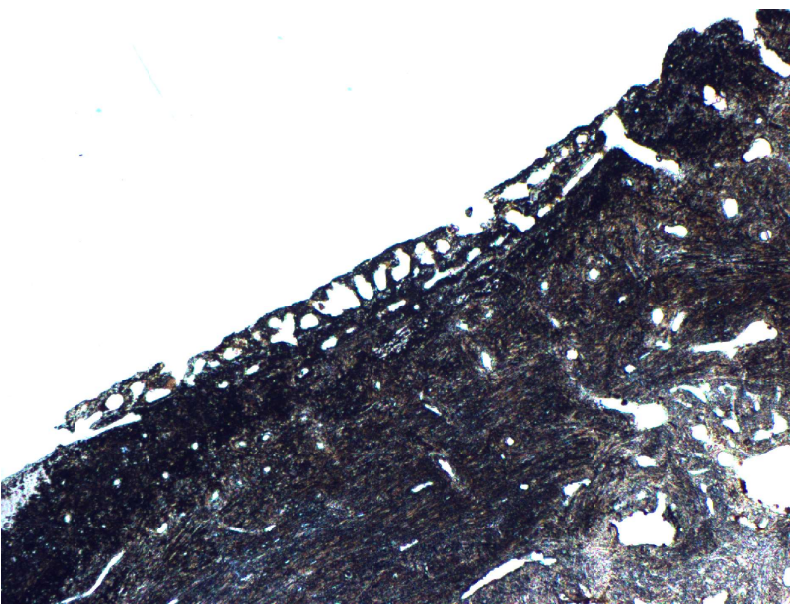

Bild 5

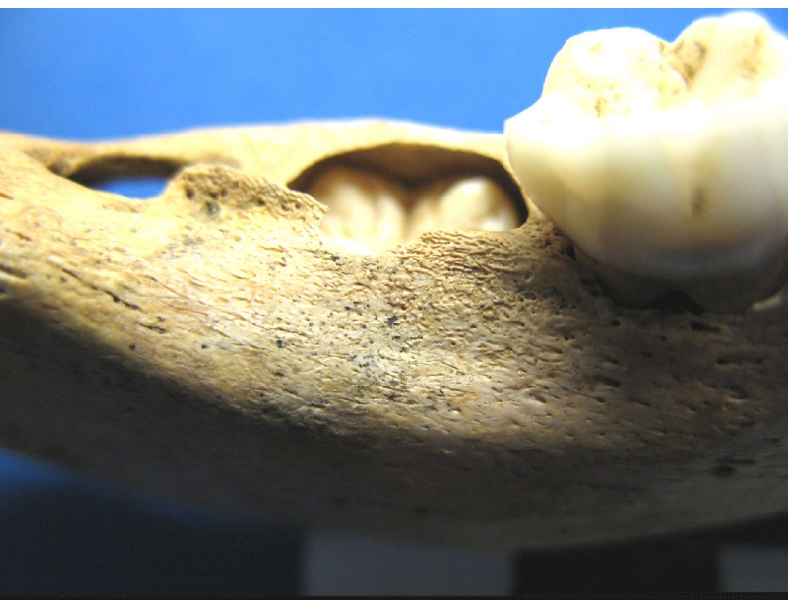

Bild 7

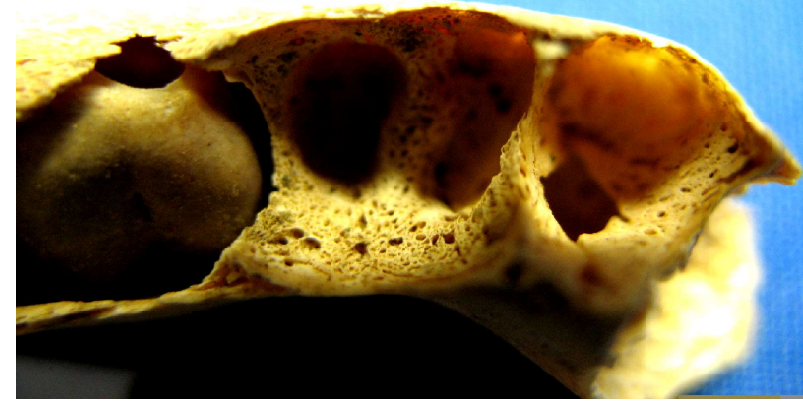

Bild 2

Bild 4

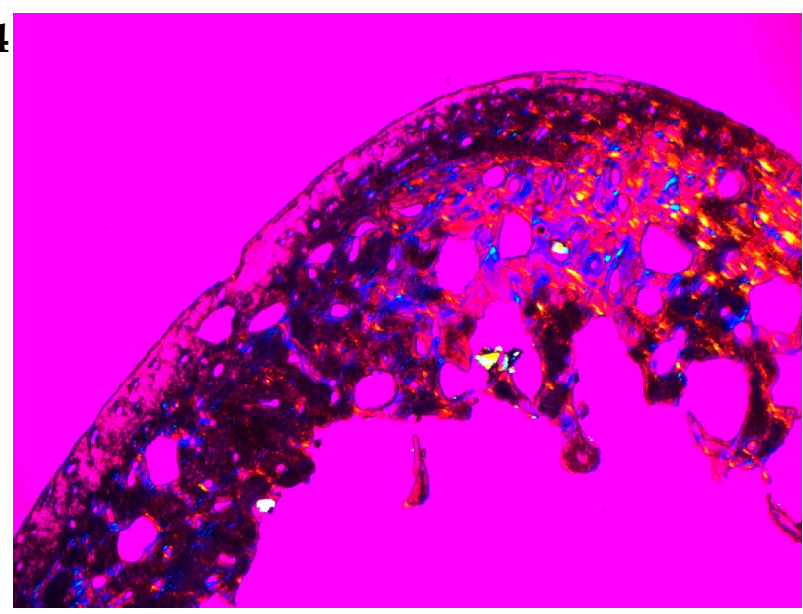

Bild 6

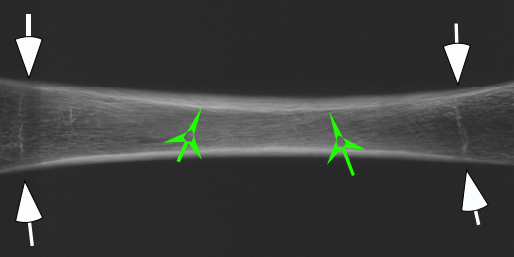

Bild 8

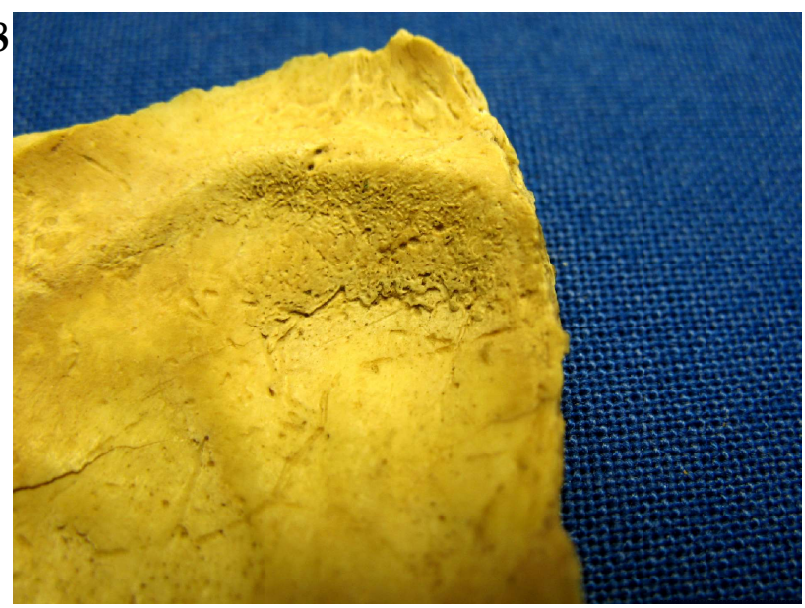




\section{Erklärungen zu Bildtafel 4}

Bild 1: Sk 1299, Barbing. Kind, ca. 0,5 - 1,5 Jahre alt. Makroskopische Detailaufnahme des rechten Orbitadaches. Es liegen feinporöse Auflagerungen vor. $\rightarrow$ Organisiertes subperiostales Hämatom im Rahmen von Skorbut

Bild 2: Sk 1299, Barbing. Kind, ca. 0,5 - 1,5 Jahre alt. Makroskopische Detailaufnahme der Außenseite des Os parietale. Es sind feinporöse Auflagerungen im Bereich der Sutura sagittalis zu erkennen. $\rightarrow$ Knochenneubildungen infolge subpericranialer Blutungen im Rahmen von Skorbut

Bild 3: Sk 1300, Barbing. Kind, 4 - 5 Jahre alt. Makroskopische Detailaufnahme der rechten Tibia. An der medialen Schaftseite liegen feinporöse Auflagerungen vor. $\rightarrow$ Knochenneubildungen infolge eines ausgedehnten subperiostalen Hämatoms unter chronischem Vitamin-C-Mangel

Bild 4: Sk 1301, Barbing. Kind, < 3 Jahre alt. Makroskopische Aufnahme des linken Os frontale. An der Lamina interna sind ausgedehnte feinporöse Auflagerungen zu sehen (siehe auch die folgenden Bilder). $\rightarrow$ Knochenneubildungen infolge subpericranialer Blutungen im Rahmen von Skorbut

Bild 5: Sk 1301, Barbing. Kind, < 3 Jahre alt. Weitere makroskopische Aufnahme des linken Os frontale. Auch hier liegen an verschiedenen Bereichen der Lamina interna, unter anderem neben der Crista frontalis, flächige feinporöse Auflagerungen vor. $\rightarrow$ Knochenneubildungen infolge subpericranialer Blutungen im Rahmen von Skorbut

Bild 6: Sk 1301, Barbing. Kind, < 3 Jahre alt. Makroskopische Aufnahme des rechten Os frontale. An der Lamina interna sind, insbesondere im Bereich der Sutura coronoidea, ähnliche Auflagerungen zu sehen, wie in den vorangegangen Bildern des linken Os frontale gezeigt. $\rightarrow$ Knochenneubildungen infolge subpericranialer Blutungen im Rahmen von Skorbut

Bild 7: Sk 1301, Barbing. Kind, < 3 Jahre alt. Makroskopische Aufnahme des linken Os frontale. Auch an der Schädelaußenseite sind ausgedehnte feinporöse Auflagerungen zu sehen, die den Veränderungen an der Lamina interna in den vorangegangenen Bildern entsprechen.

Knochenneubildungen infolge subpericranialer Blutungen im Rahmen von Skorbut

Bild 8: Sk 1301, Barbing. Kind, < 3 Jahre alt. Makroskopische Detailaufnahme des linken Orbitadaches: Es sind deutliche feinporöse Auflagerungen zu erkennen $\rightarrow$ Cribra orbitalia Grad (1-) 2 infolge chronischen Vitamin-C-Mangels 


\section{Bildtafel 4}

Bild 1

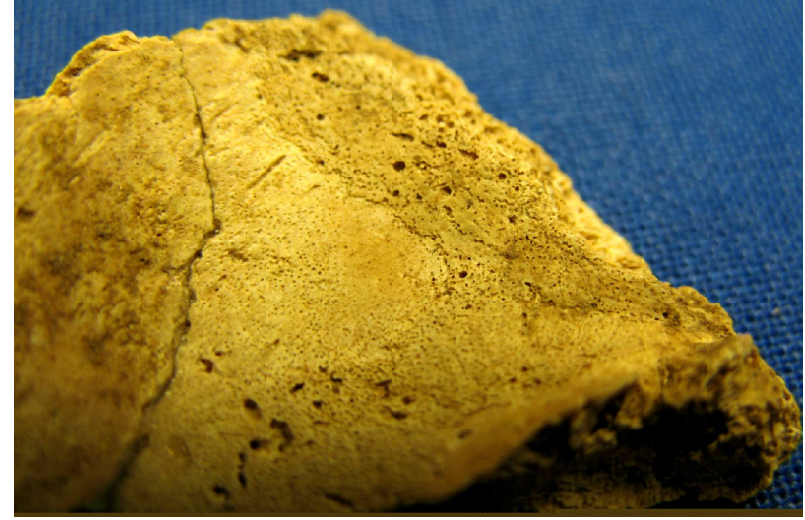

Bild 3

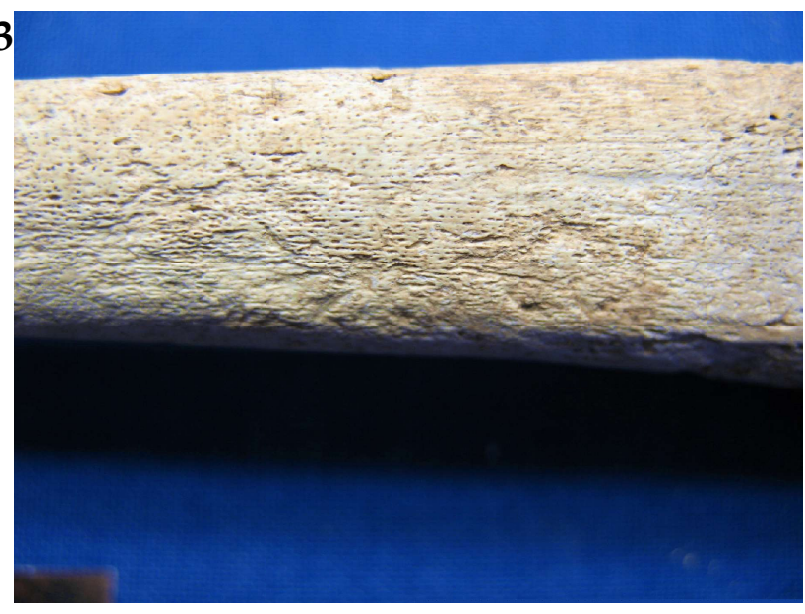

Bild 5

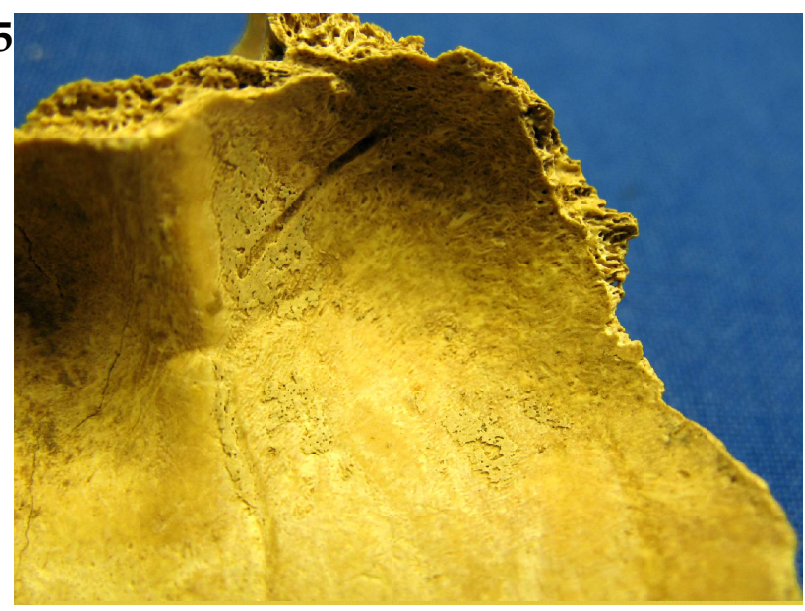

Bild 7

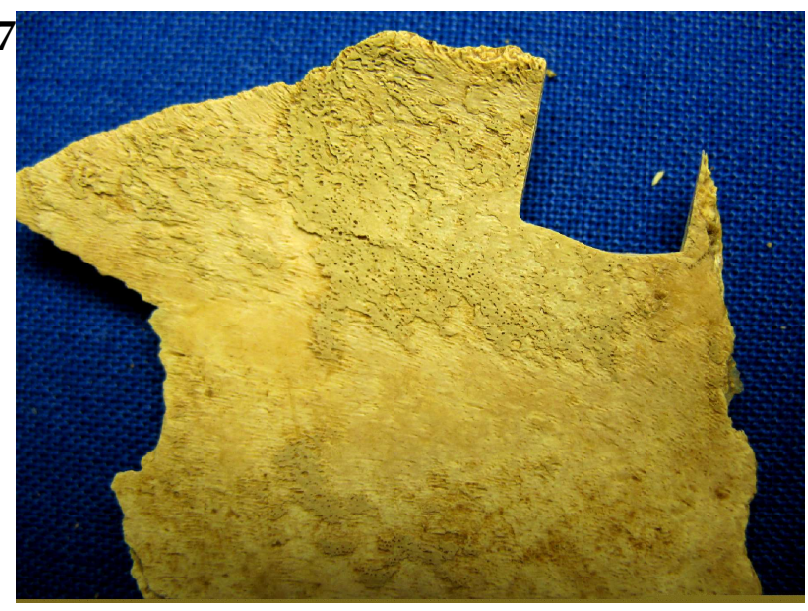

Bild 2

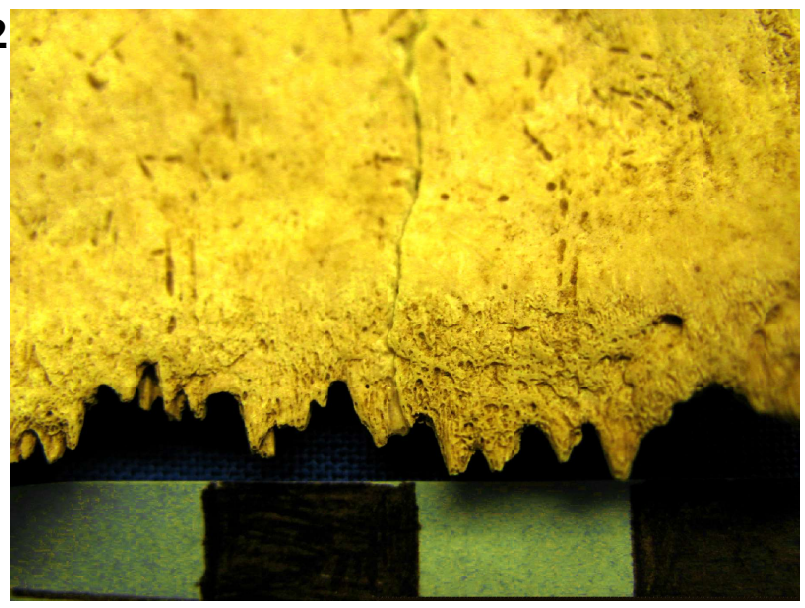

Bild 4

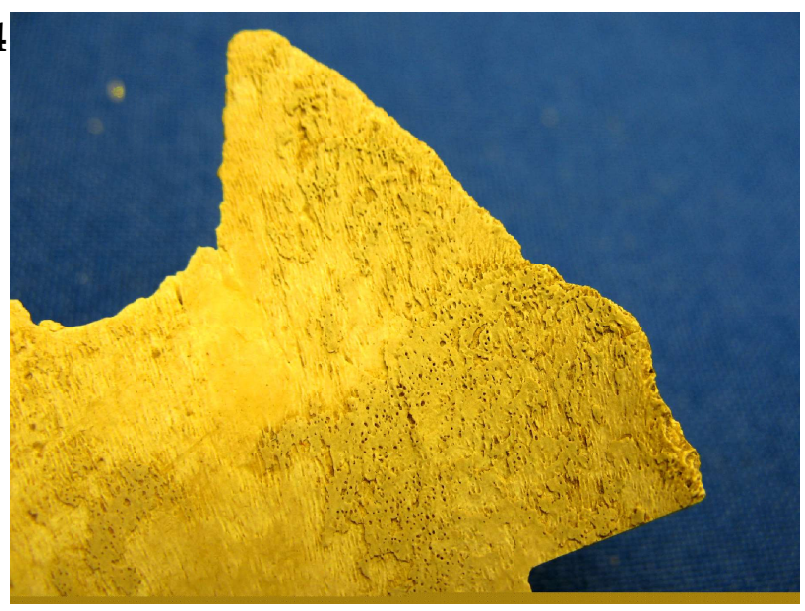

Bild 6

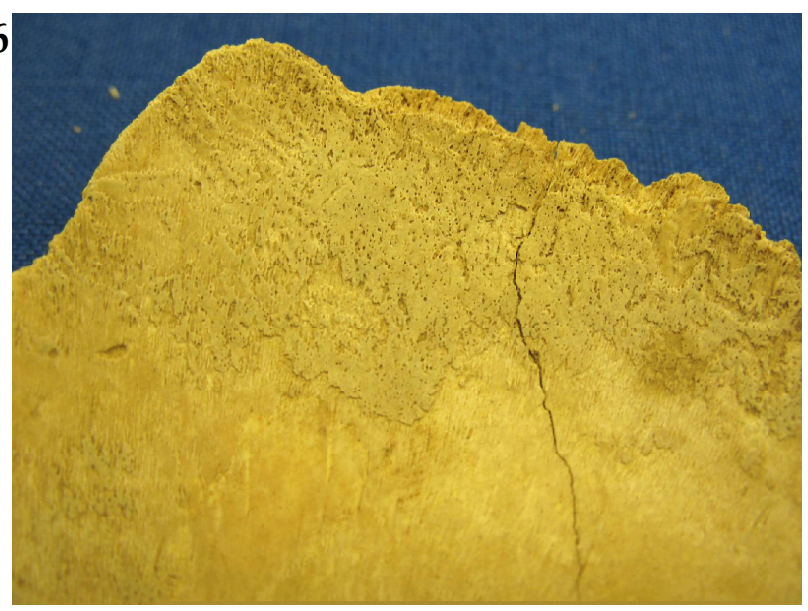

Bild 8

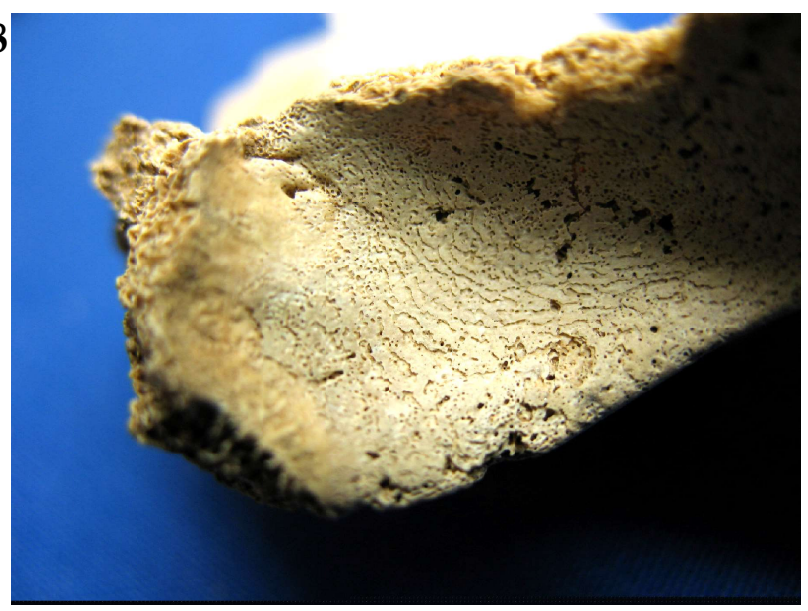




\section{Erklärungen zu Bildtafel 5}

Bild 1: Sk 1301, Barbing. Kind, < 3 Jahre alt. Makroskopische Aufnahme der Innenseite des Os occipitale. Im Sulcus sinus occipitalis sind ähnliche feinporöse Auflagerungen wie bei den vorangegangenen Bildern zu sehen (vgl. Bild $4-8$ auf Bildtafel 4) $\rightarrow$ Knochenneubildungen infolge subpericranialer Blutungen im Rahmen von Skorbut

Bild 2: Sk 1301, Barbing. Kind, < 3 Jahre alt. Lichtmikroskopische Darstellung (Schliffdicke 70 $\mu$ m, polarisiertes Durchlicht mit Hilfsobjekt Rot 1. Ordnung (Quarz) als Kompensator, Vergrößerung 100-fach): Das Präparat stammt aus dem linken Os parietale, das die gleichen Auflagerungen aufweist, wie auf den vorangegangenen Bildern zu sehen. Es zeigt deutliche Auflagerungen vom Typ I (hämorrhagisch) an der Lamina interna. Im polarisierten Licht lässt sich die neu gebildete Substanz gut von der ehemaligen Generallamelle (Pfeile) abgrenzen. $\rightarrow$ faserknöchern organisiertes Hämatom im Rahmen von Skorbut

Bild 3: Sk 1301, Barbing. Kind, < 3 Jahre alt. Lichtmikroskopische Darstellung (Schliffdicke 50 $\mu$ m, polarisiertes Durchlicht mit Hilfsobjekt Rot 1. Ordnung (Quarz) als Kompensator, Vergrößerung 25-fach): Es handelt es sich um den dünneren Schliff des unter Bild 2 beschriebenen Präparats. Auch hier lassen sich die mehrlagigen Auflagerungen (zwischen den weißen Pfeilen) gut von der ursprünglichen Knochensubstanz abgrenzen. In der Diploë fällt ein gut erhaltenes Gefäßforamen auf (schwarzer Pfeil).

Bild 4: Sk 1334, Barbing. Geschlecht unklar, ca. 23 - 28 Jahre alt. Makroskopische Aufnahme der rechten Tibia. Es fallen großflächige feinporöse Auflagerungen auf, die deutlich erhaben sind und feine Wülste bilden (siehe auch nächste Abbildung). $\rightarrow$ Periostitis im Rahmen einer unspezifischen Osteomyelitis

Bild 5: Sk 1334, Barbing. Geschlecht unklar, ca. 23 - 28 Jahre alt. Lichtmikroskopische Darstellung (Schliffdicke 50um, einfaches Durchlicht, Vergrößerung 16-fach): Es handelt sich um ein Präparat aus der rechten Tibia. Zu sehen sind deutliche Auflagerungen (Sterne) vom Typ IV (unspezifische Entzündung) mit Auflösung der Grenzlamelle (grüner Pfeil) zum darunter liegenden Knochen. Im ursprünglichen Knochen sind Spaltbildungen zu beobachten (rosa Pfeil). $\rightarrow$ Periostitis im Rahmen einer unspezifischen Osteomyelitis

Bild 6: Sk 1340, Barbing. Männlich, 25 - 45 Jahre alt. Makroskopische Detailaufnahme des Os occipitale. An der Lamina interna, insbesondere im Bereich der Sulci der venösen Sinus, finden sich flache, gut integrierte, feinporöse Auflagerungen. Außerdem fallen vermehrte Gefäßimpressionen auf. $\rightarrow$ hämorrhagische Knochenneubildung im fortgeschrittenen Heilungsstadium, vermutlich nach einer einige Monate zurückliegenden Skorbuterkrankung

Bild 7: Sk 1356, Barbing. Weiblich, 60 - 80 Jahre alt. Lichtmikroskopische Darstellung (Schliffdicke 70 $\mu$ m, polarisiertes Durchlicht mit Hilfsobjekt Rot 1. Ordnung (Quarz) als Kompensator, Vergrößerung 16-fach): Das Präparat stammt aus dem linken Os frontale. Der Bildausschnitt zeigt die Lamina externa (links) und die darunter liegende Diploë. Dort fällt eine Erweiterung der Markräume auf. Im Gegensatz dazu sind die hier nicht angeschnittenen Markräume an der Lamina interna (siehe Bild 8) verengt. $\rightarrow$ starke Umbauvorgänge in der Diploë, vereinbar mit dem Bild einer Leukämie

Bild 8: Sk 1356, Barbing. Weiblich, 60 - 80 Jahre alt. Lichtmikroskopische Darstellung (Schliffdicke 70um, einfaches Durchlicht, Vergrößerung 16-fach): Es handelt sich um dasselbe Präparat wie im vorangegangenen Bild. Der Bildausschnitt hier zeigt die Lamina interna (rechts) und die darunter liegende Diploë. Hier fällt eine Verengung der Markräume auf. Im Gegensatz dazu sind die hier nicht angeschnittenen Markräume an der Lamina externa (siehe Bild 7) erweitert. $\rightarrow$ starke Umbauvorgänge in der Diploë, vereinbar mit dem Bild einer Leukämie 


\section{Bildtafel 5}
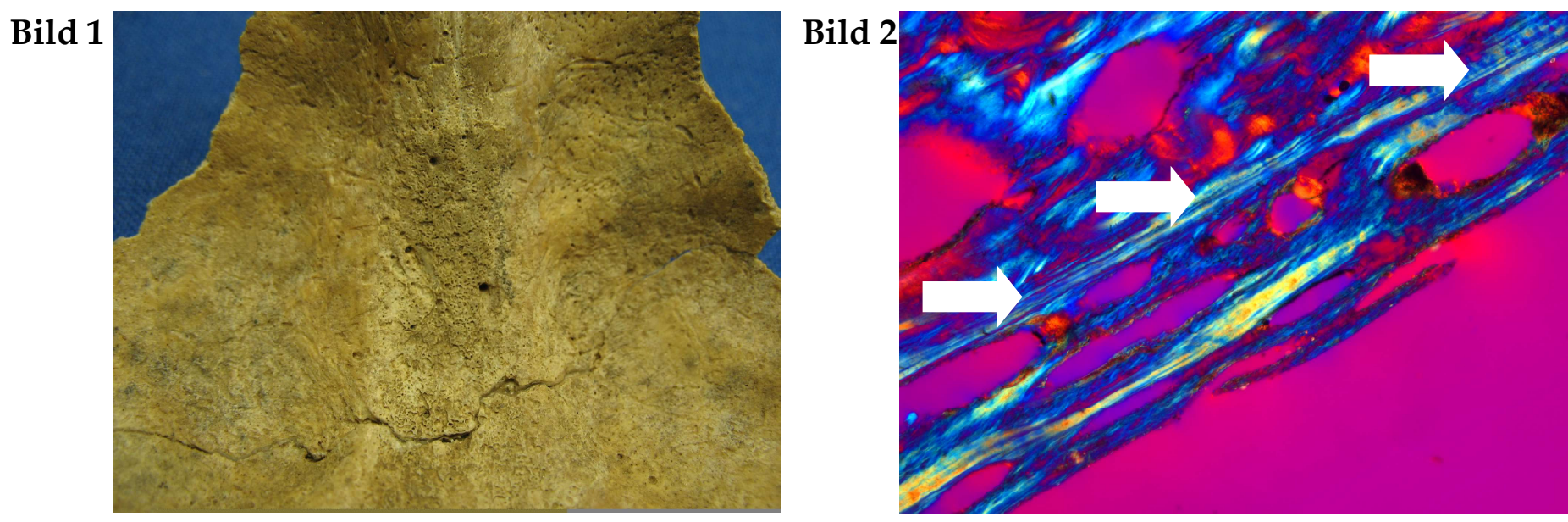

Bild 3

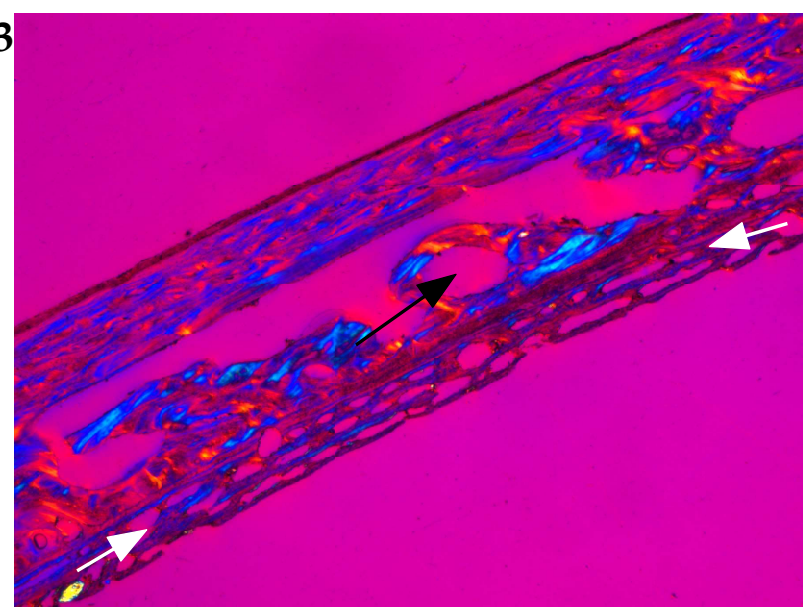

Bild 5

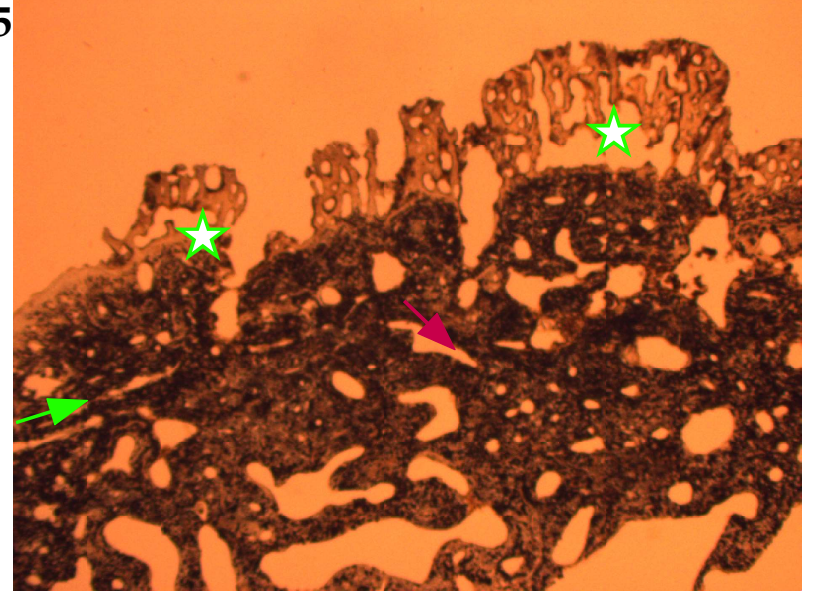

Bild 4

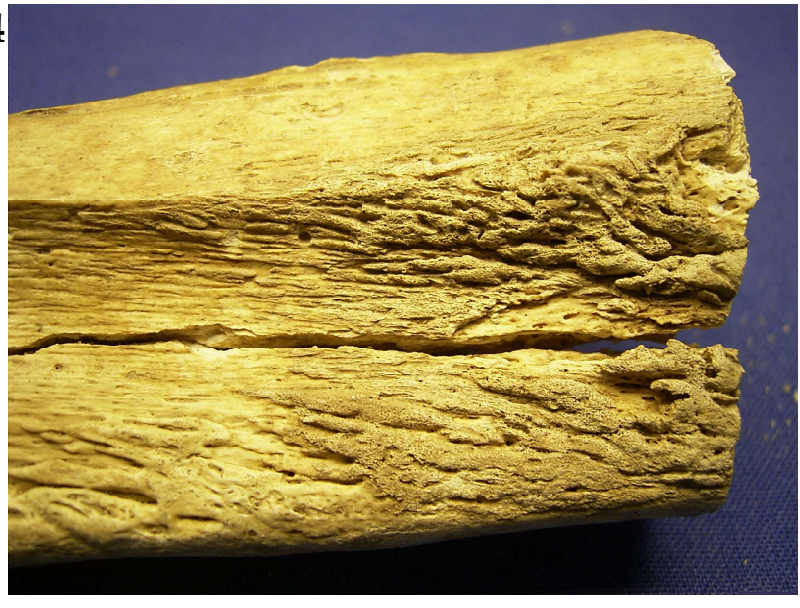

Bild 6

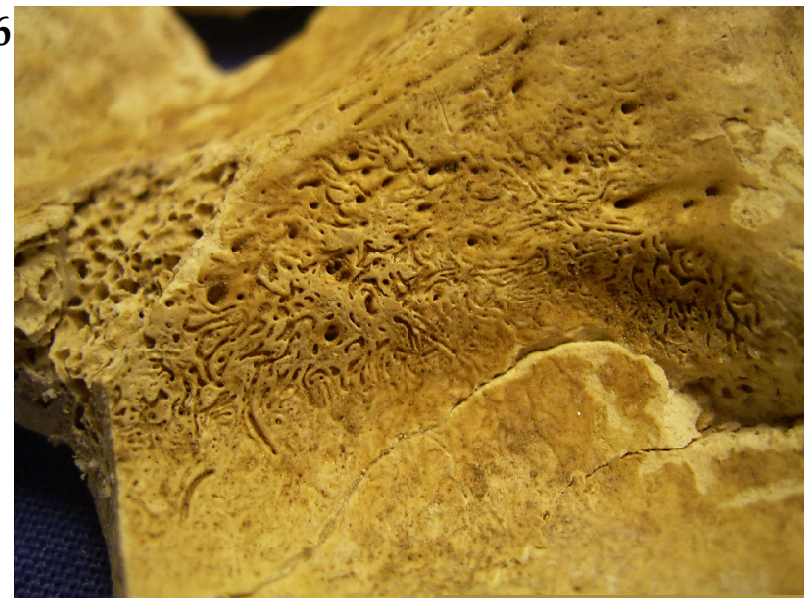

Bild 7

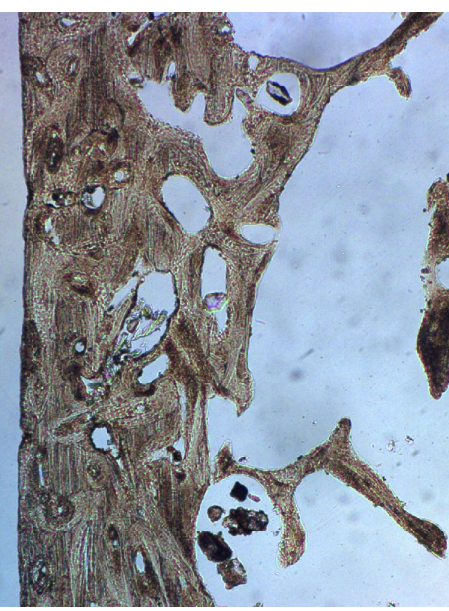

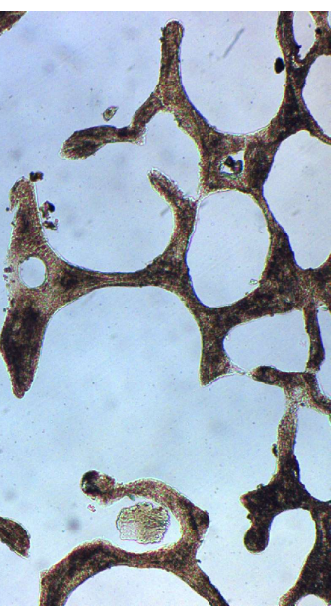

Bild 8

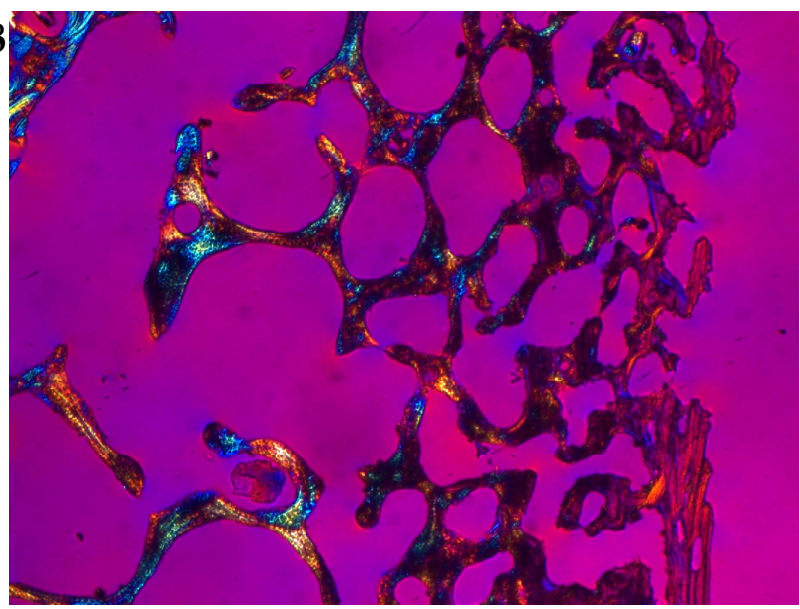




\section{Erklärungen zu Bildtafel 6}

Bild 1: Sk 1356, Barbing. Weiblich, 60 - 80 Jahre alt. Röntgenbild der rechten Tibia. Am distalen Ende im Bereich der Syndesmose ist eine kleine, gut integrierte Auflagerung zu erkennen (Pfeil). Knochenneubildung in Folge eines subperiostalen Hämatoms im fortgeschrittenen Heilungsstadium Bild 2: Sk 1363, Barbing. Kind, ca. 7 Jahre alt. Makroskopische Detailaufnahme von feinporösen Auflagerungen am dorsolateralen Schaft des linken Femurs. Der Schnittrand rechts zeigt, wo das Präparat für die Anfertigung der Dünnschliffe (siehe Bild 1 und 2 auf Bildtafel 7) entnommen wurde. $\rightarrow$ organisiertes subperiostales Hämatom im Rahmen von Skorbut

Bild 3: Sk 1363, Barbing. Kind, ca. 7 Jahre alt. Makroskopische Aufnahme der linken Squama temporalis. In den Impressiones digitatae sind feinporöse Auflagerungen zu erkennen. subpericraniale Hämatome mit konsekutiver Knochenneubildung im Rahmen von Skorbut

Bild 4: Sk 1363, Barbing. Kind, ca. 7 Jahre alt. Makroskopische Detailaufnahme der linken Squama temporalis zur Darstellung der feinporösen Auflagerungen in den Impressiones digitatae. subpericraniale Hämatome mit konsekutiver Knochenneubildung im Rahmen von Skorbut

Bild 5: Sk 1363, Barbing. Kind, ca. 7 Jahre alt. Makroskopische Aufnahme der rechten Squama temporalis. Auch hier sind scharf begrenzte feinporöse Auflagerungen zu erkennen (siehe auch die folgenden Bilder). $\rightarrow$ subpericraniale Hämatome mit konsekutiver Knochenneubildung im Rahmen von Skorbut

Bild 6: Sk 1363, Barbing. Kind, ca. 7 Jahre alt. Makroskopische Detailaufnahme der rechten Squama temporalis zur Darstellung der im vorangegangenen Bild gezeigten feinporösen Auflagerungen. subpericraniale Hämatome mit konsekutiver Knochenneubildung im Rahmen von Skorbut

Bild 7: Sk 1363, Barbing. Kind, ca. 7 Jahre alt. Makroskopische Aufnahme der rechten Tibia. Am anterolateralen Schaft finden sich flächige feinporöse Auflagerungen. $\rightarrow$ organisierte subperiostale Hämatome infolge chronischen Vitamin-C-Mangels 


\section{Bildtafel 6}

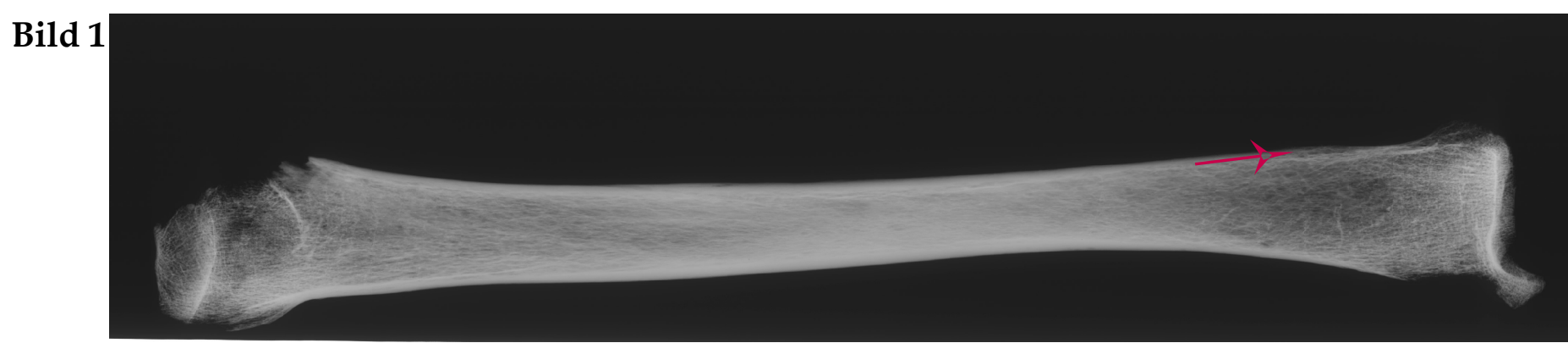

Bild 2

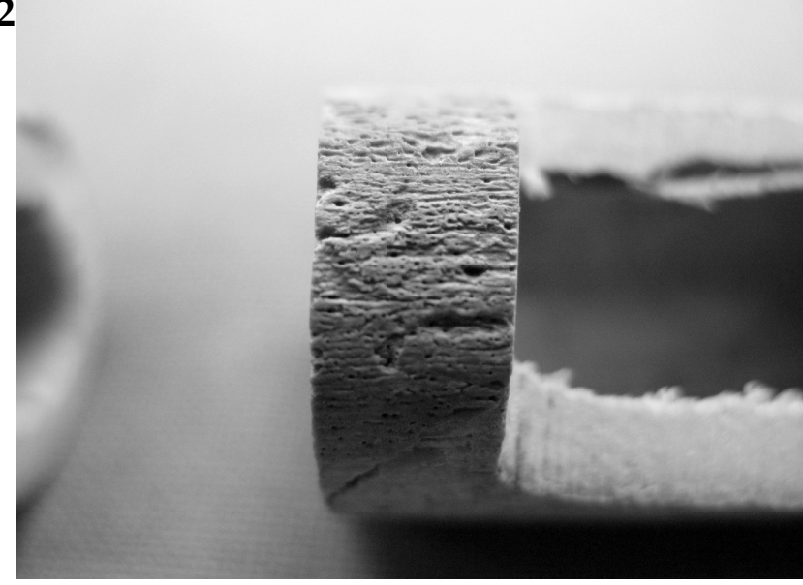

Bild 4

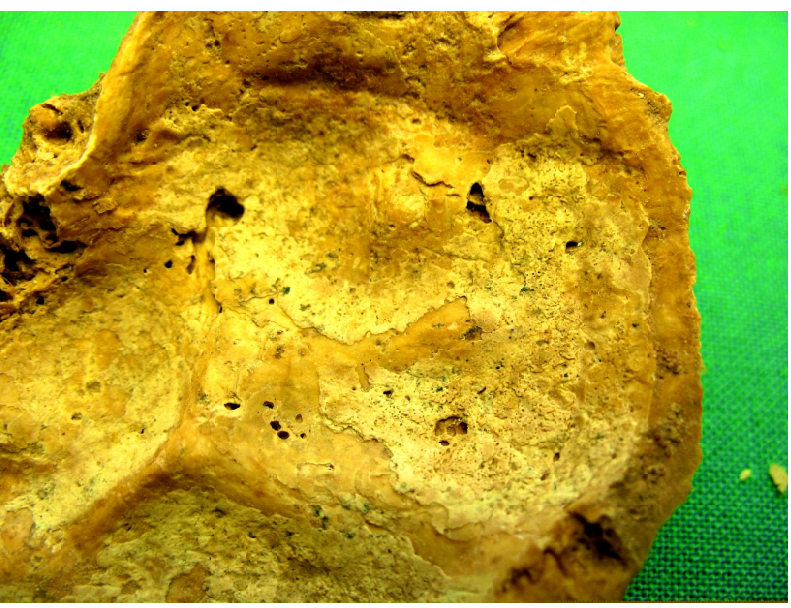

Bild 6

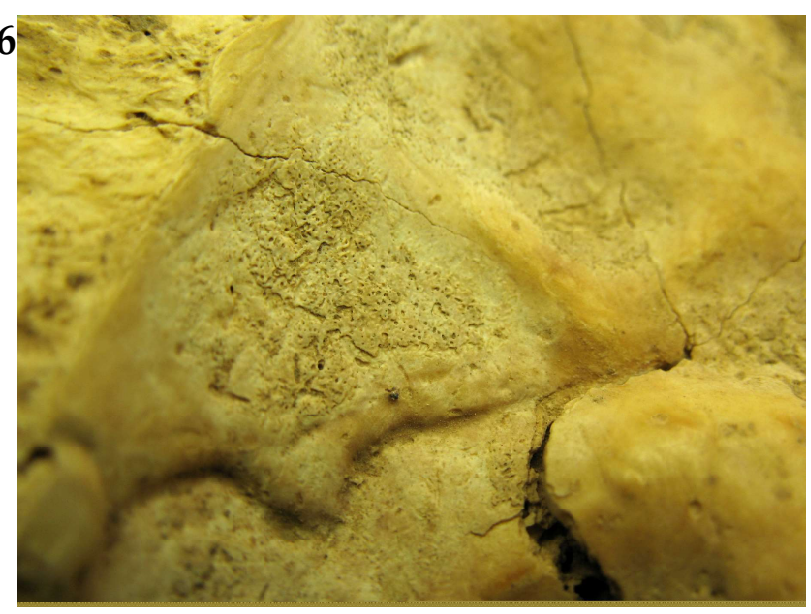

Bild 3

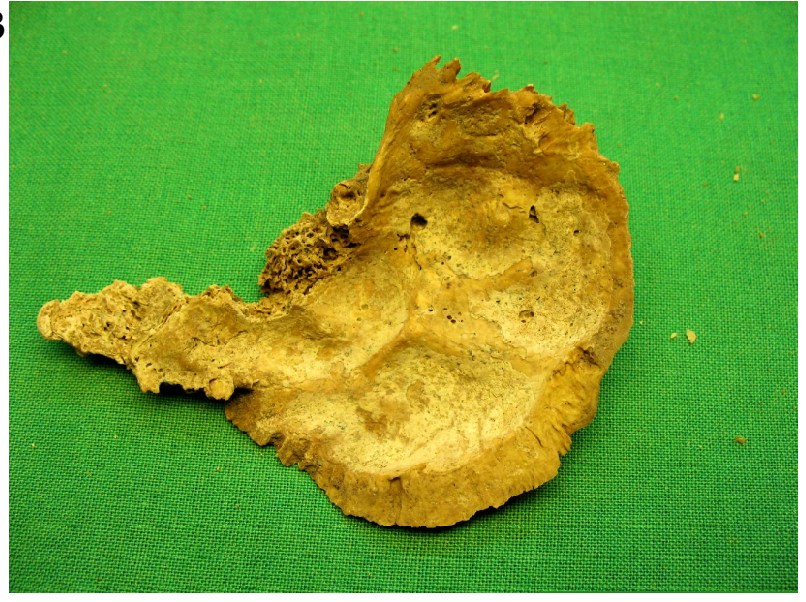

Bild 5

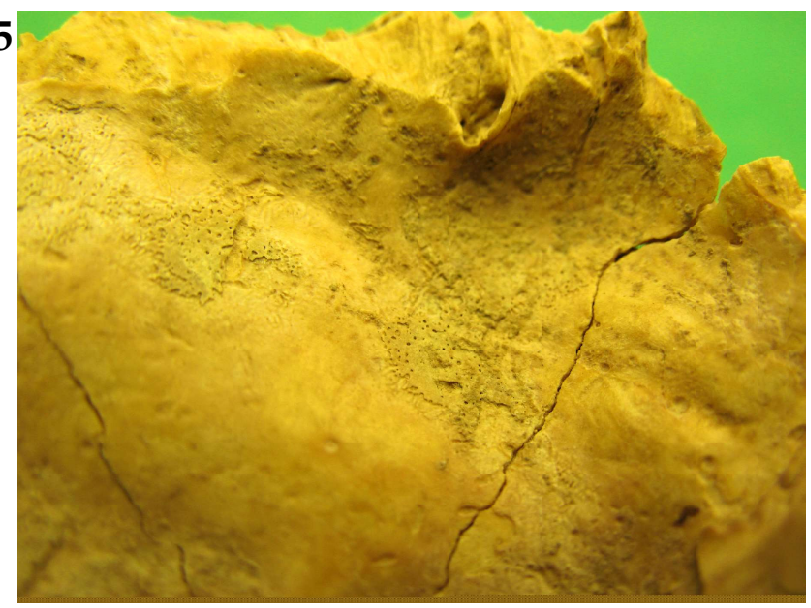

Bild 7

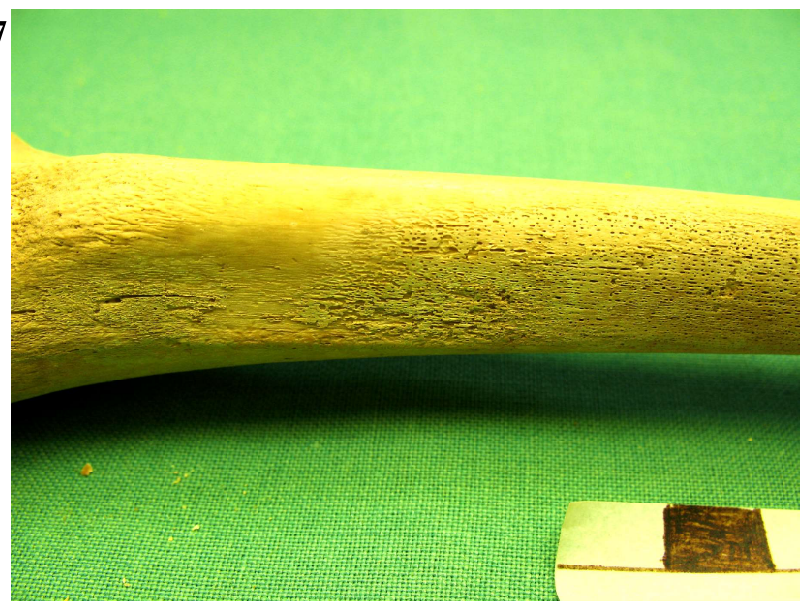




\section{Erklärungen zu Bildtafel 7}

Bild 1: Sk 1363, Barbing. Kind, ca. 7 Jahre alt. Lichtmikroskopische Darstellung (Schliffdicke 50um, einfaches Durchlicht, Vergrößerung 16-fach): Das Präparat stammt aus dem linken Femur und zeigt Auflagerungen (Pfeil) vom Typ I (hämorrhagisch), die stellenweise zweilagig sind. $\rightarrow$ Knochenneubildung infolge subperiostaler Hämorrhagien bei Skorbut, in zwei Krankheitsphasen entstanden, wobei die äußere, frische Auflagerung sich auf die bereits integrierte ältere Neubildung aufsetzte

Bild 2: Sk 1363, Barbing. Kind, ca. 7 Jahre alt. Lichtmikroskopische Darstellung (Schliffdicke 70 $\mathrm{m}$, einfaches Durchlicht, Vergrößerung 100-fach): Das Präparat stammt aus dem linken Femur. Es handelt sich um eine Vergrößerung der Auflagerungen vom Typ I (hämorrhagisch), die an dieser Stelle zweilagig (Zahlen) sind. $\rightarrow$ Knochenneubildung infolge subperiostaler Hämorrhagien bei Skorbut, in zwei Krankheitsphasen entstanden, wobei die äußere, frische Auflagerung (2) sich auf die bereits integrierte ältere Neubildung (1) aufsetzte

Bild 3: Sk 1365, Barbing. Männlich, > 65 Jahre alt. Makroskopische Detailaufnahme des rechten Calcaneus. An der Lateralseite befinden sich feinporöse Auflagerungen mit profiliertem Rand von mehreren Zentimetern Ausdehnung. $\rightarrow$ Organisiertes subperiostales Hämatom, vermutlich als Folge eines Traumas (siehe auch Bild 4)

Bild 4: Sk 1365, Barbing. Männlich, > 65 Jahre alt. Weitere makroskopische Detailaufnahme der bei Bild 3 beschriebenen Auflagerungen am rechten Calcaneus. $\rightarrow$ Organisiertes subperiostales Hämatom, vermutlich traumatisch bedingt (siehe auch Bild 3)

Bild 5: Sk 1365, Barbing. Männlich, > 65 Jahre alt. Makroskopische Detailaufnahme der rechten Tibia. Dieses Bild wurde als Beispiel für eine durch Gefäßimpression entstandene so genannte „Schnürfurche“ (Pfeil) ausgewählt.

Bild 6: Sk 1368, Barbing. Kind, ca. 4 Jahre alt. Makroskopische Detailaufnahme der linken Maxilla. $\mathrm{Zu}$ sehen sind flache feinporöse Auflagerungen. $\rightarrow$ Stomatitis im Rahmen von Skorbut

Bild 7: Sk 1371, Barbing. Kind, ca. 1 Jahr alt. Makroskopische Darstellung des rechten Femurs, Ventralansicht. An der medialen Schaftseite finden sich stark erodierte (in der Übersicht nicht zu erkennende) feinporöse Auflagerungen. Auffällig ist die Verbreiterung der distalen Metaphysenplatten (siehe auch Bild 8). $\rightarrow$ Veränderungen im Rahmen einer Skorbuterkrankung

Bild 8: Sk 1371, Barbing. Kind, ca. 1 Jahr alt. Makroskopische Darstellung des rechten Femurs, Dorsalansicht. Auffällig ist die Verbreiterung der distalen Metaphysenplatten (siehe auch Bild 7). Veränderungen im Rahmen einer Skorbuterkrankung 


\section{Bildtafel 7}

Bild 1

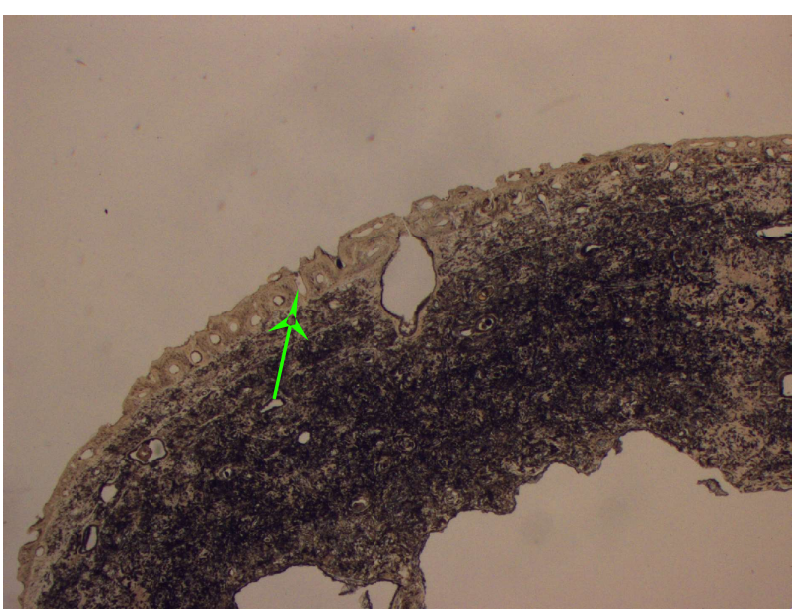

Bild 3

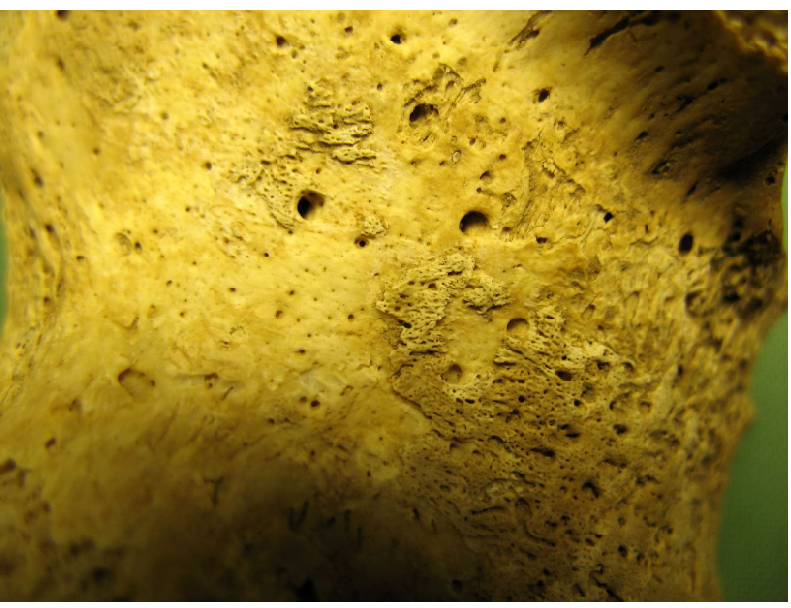

Bild 5

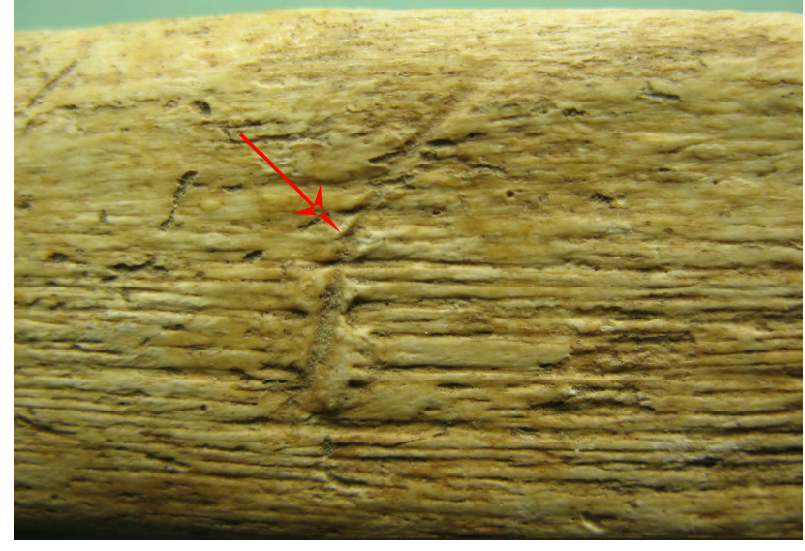

Bild 7

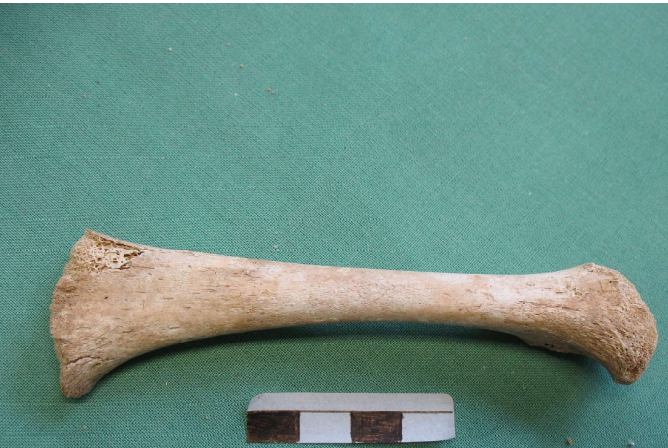

Bild 2

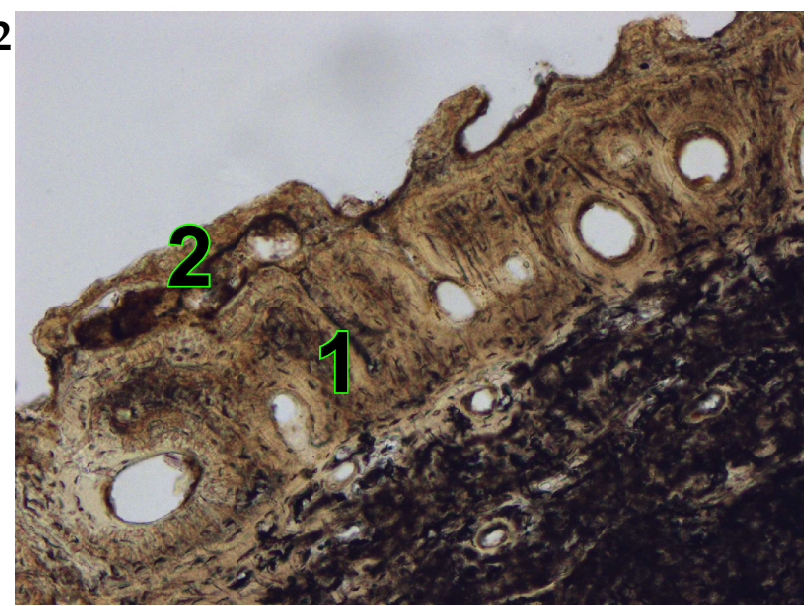

Bild 4

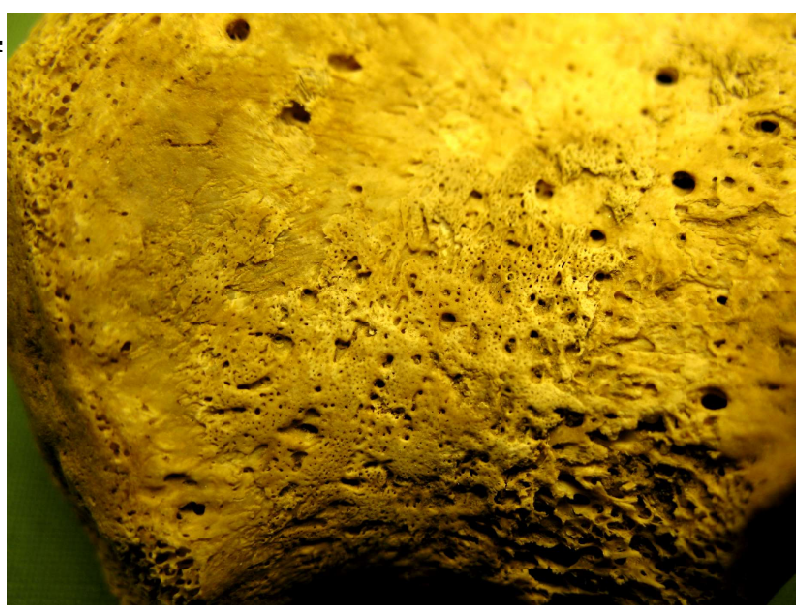

Bild 6

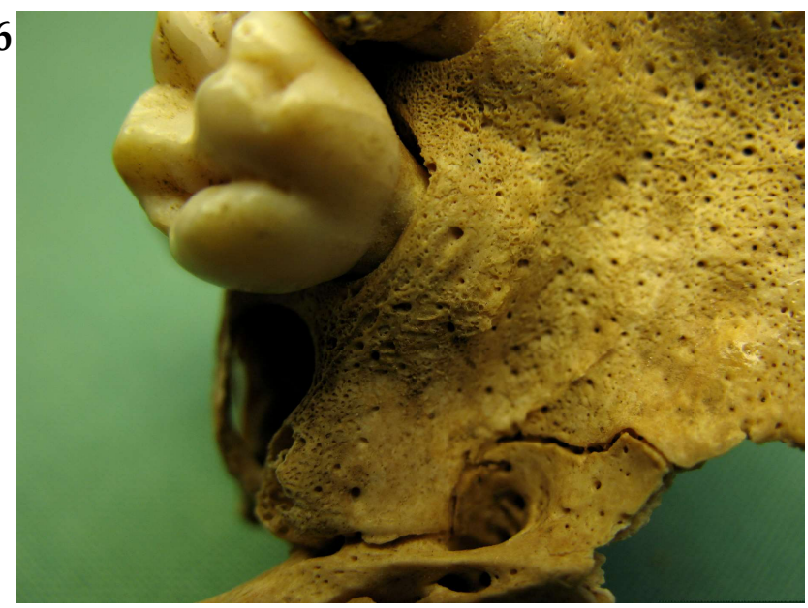

Bild 8

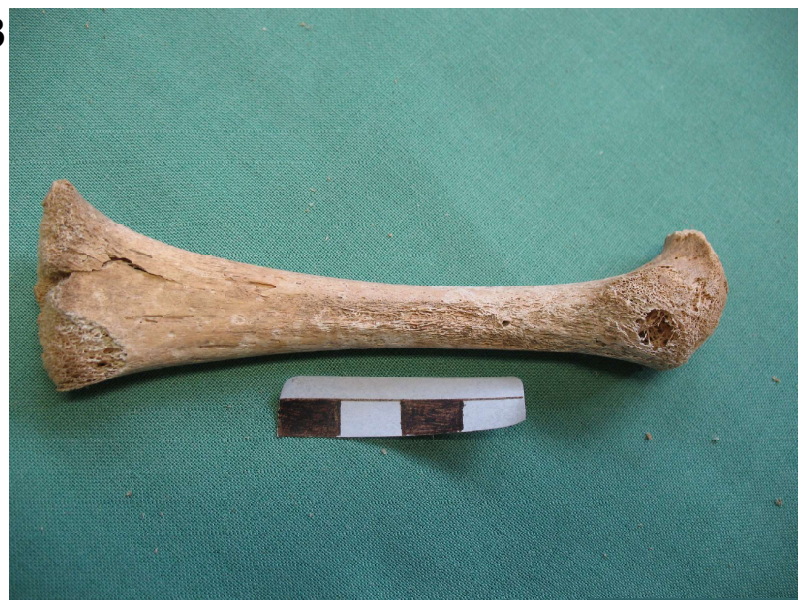




\section{Erklärungen zu Bildtafel 8}

Bild 1: Sk 1372, Barbing. Kind, ca. 2 Jahre alt. Makroskopische Detailaufnahme des Os occipitale. In den Sulci der venösen Sinus finden sich feinporöse Auflagerungen. $\rightarrow$ Knochenneubildungen infolge skorbutischer Hämorrhagien

Bild 2: Sk 1377, Barbing. Weiblich, 17 -23 Jahre alt. Makroskopische Detailaufnahme der linken Tibia, Ansicht von ventral. Die feinporösen Oberflächenveränderungen bedecken den Schaft vor allem ventral, reichen aber bis dorsal. Zusätzlich zur Porösität weisen sie ein wellenförmig gesträhntes Relief auf (siehe auch Bild 3). $\rightarrow$ Verdacht auf Periostitis im Rahmen von Lepra

Bild 3: Sk 1377, Barbing. Weiblich, 17 -23 Jahre alt. Lichtmikroskopische Darstellung (Schliffdicke $70 \mu \mathrm{m}$, polarisiertes Durchlicht, Vergrößerung 25-fach): Das Präparat stammt aus dem linken Femur. Es gibt keinen Anhalt für einen hämorrhagischen Prozess. Die Corticalis ist sehr stark ausgeprägt, es fallen tiefe Gefäßimpressionen (Pfeil) und annähernd polsterförmige Auflagerungen (Sterne) vom Typ V (lepromatös) auf. $\rightarrow$ Verdacht auf lepromatöse Periostitis

Bild 4: Sk 1380, Barbing. Weiblich, 23 - 25 Jahre alt. Röntgenbild der fünften Rippe rechts (siehe auch Bild 5). Die Pfeile markieren Auflagerungen, die über das ehemalige Periost gewachsen sind. $\rightarrow$ Hämorrhagische Pleuritis mit konsekutiver Knochenneubildung im Rahmen von Skorbut

Bild 5: Sk 1380, Barbing. Weiblich, 23 - 25 Jahre alt. Lichtmikroskopische Darstellung (Schliffdicke $70 \mu \mathrm{m}$, polarisiertes Durchlicht mit Hilfsobjekt Rot 1. Ordnung (Quarz) als Kompensator, Vergrößerung 16-fach): Es handelt sich um ein Präparat aus der fünften Rippe rechts (siehe auch Bild 4). Es fallen massive Auflagerungen (Pfeile) vom Typ I (hämorrhagisch) auf. $\rightarrow$ Hämorrhagische Pleuritis mit konsekutiver Knochenneubildung im Rahmen von Skorbut

Bild 6: Sk 1380, Barbing. Weiblich, 23 - 25 Jahre alt. Lichtmikroskopische Darstellung (Schliffdicke $50 \mu \mathrm{m}$, einfaches Durchlicht, Vergrößerung 16-fach): Es handelt sich um ein Präparat aus der linken Fibula. Der Erhaltungszustand ist mäßig. Dennoch sind Auflagerungen (Pfeile) vom Typ I (hämorrhagisch) zu erkennen. $\rightarrow$ subperiostale Blutungen mit konsekutiver Knochenauflagerung im Rahmen von Skorbut

Bild 7: : Sk 1380, Barbing. Weiblich, 23 - 25 Jahre alt. Lichtmikroskopische Darstellung (Schliffdicke $70 \mu \mathrm{m}$, einfaches Durchlicht, Vergrößerung 25-fach): Es handelt es sich um ein Präparat aus der linken Tibia des in den vorangegangenen Bildern dargestellten Individuums. Hier fallen kurze polsterartige Neubildungen (Sterne) vom Typ V (lepromatös) auf, die unter Erhalt der Generallamelle (Pfeil) angelagert sind. Der Organisationsgrad ist fortgeschritten. $\rightarrow$ Verdacht auf Lepra (zusätzlich zu der in den vorangegangenen Bildern dokumentierten Skorbuterkrankung)

Bild 8: Sk 1383, Barbing. Männlich, 40 - 59 Jahre alt. Makroskopische Detailaufnahme der linken Scapula. In der Fossa subscapularis finden sich feinporöse Auflagerungen mit profiliertem Rand (siehe auch Bildtafel 9, Bild 1). $\rightarrow$ Subperiostale Einblutung mit nachfolgender Knochenneubildung im Rahmen einer Skorbuterkrankung 


\section{Bildtafel 8}

Bild 1

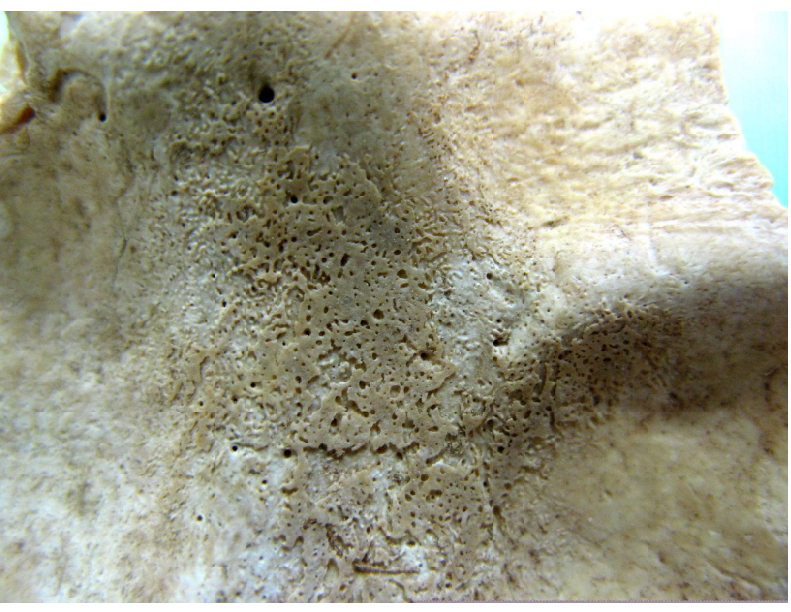

Bild 3

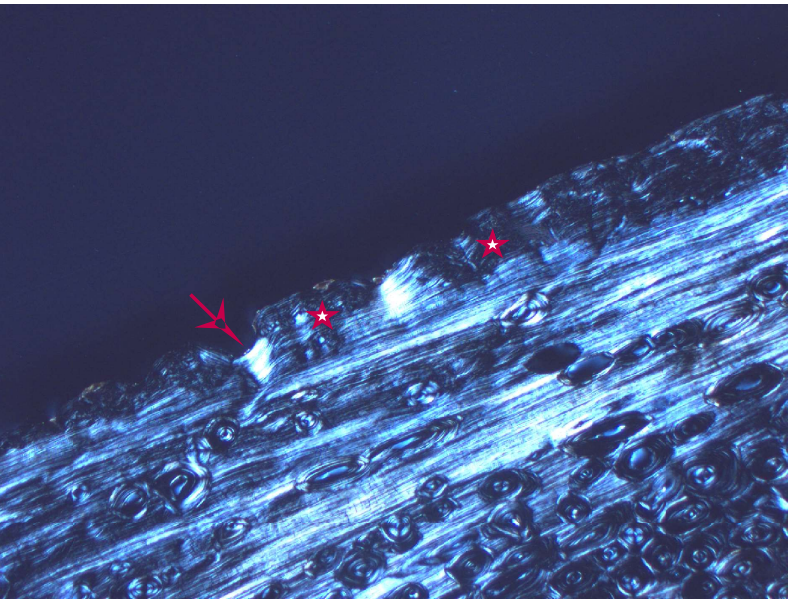

Bild 5

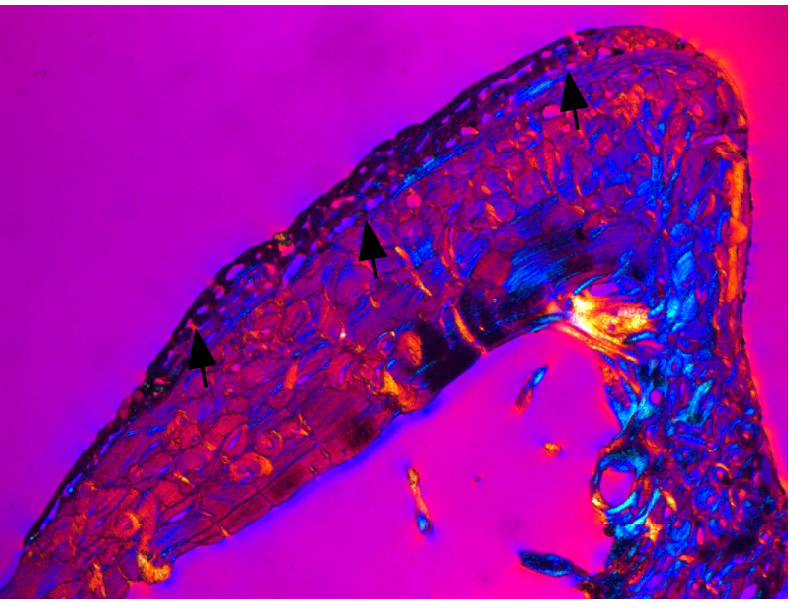

\section{Bild 7}

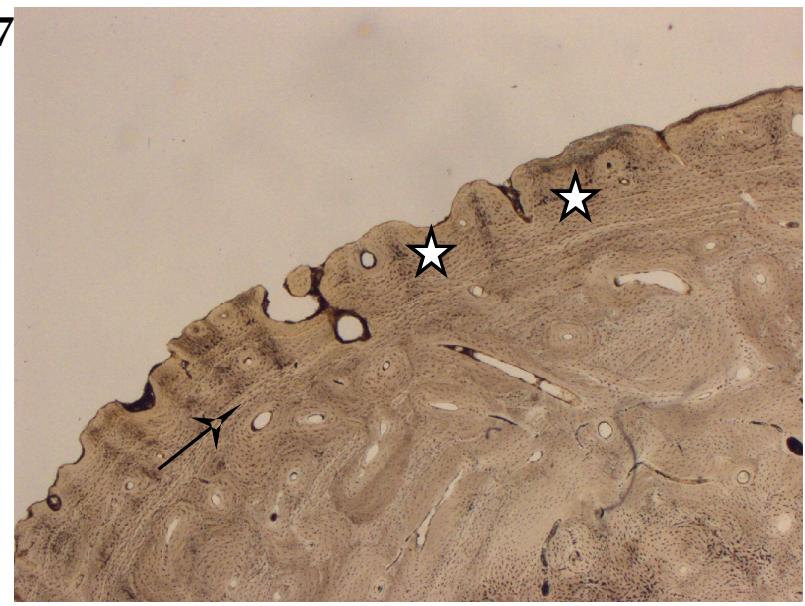

Bild 2

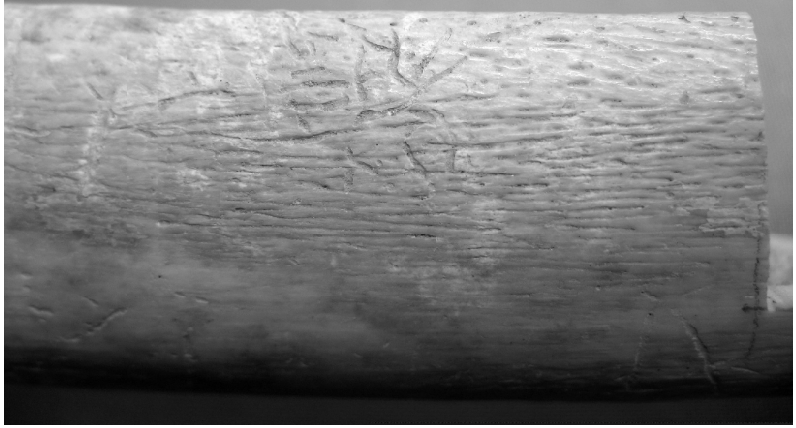

Bild 4

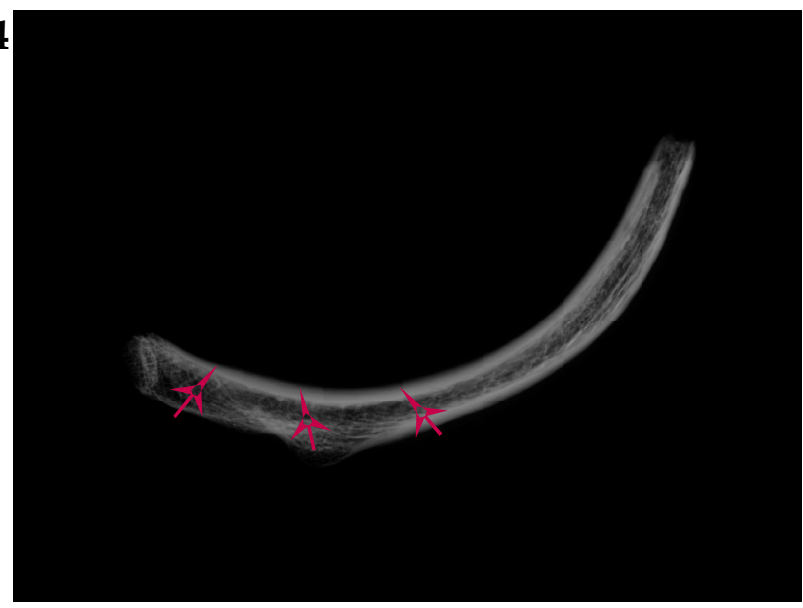

Bild 6

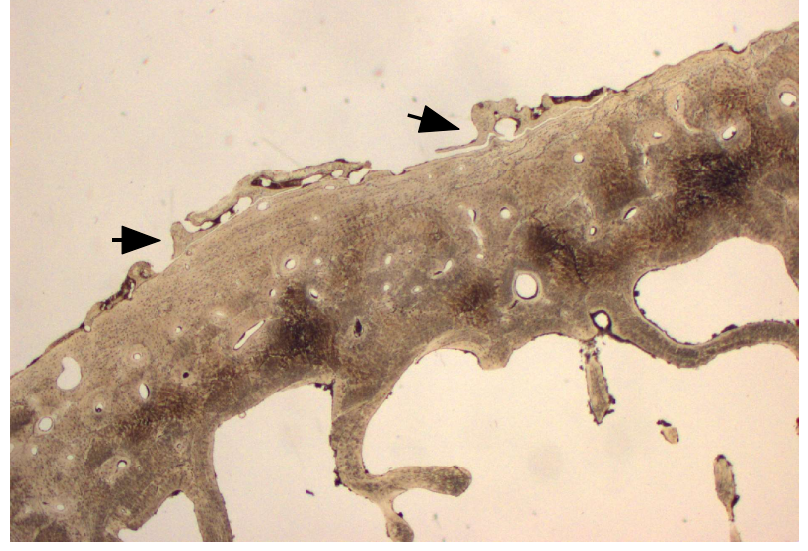

Bild 8

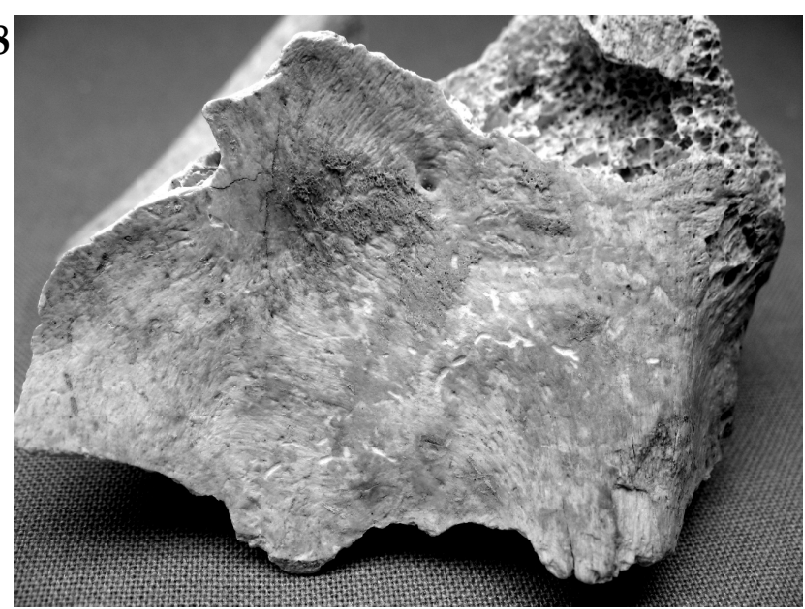




\section{Erklärungen zu Bildtafel 9}

Bild 1: Sk 1383, Barbing. Männlich, 40 - 59 Jahre alt. Makroskopische Detailaufnahme der feinporösen Auflagerungen in der Fossa subscapularis der linken Scapula (siehe auch Bildtafel 8,

Bild 8) $\rightarrow$ Subperiostale Einblutung mit nachfolgender Knochenneubildung im Rahmen einer Skorbuterkrankung

Bild 2: Sk 1383, Barbing. Männlich, 40 - 59 Jahre alt. Makroskopische Darstellung der linken Tibia. Es finden sich deutliche mehrlagige feinporöse Auflagerungen an der lateralen Schaftseite (siehe auch nächste Bilder). $\rightarrow$ organisierte subperiostale Hämatome im Rahmen von Skorbut

Bild 3: Sk 1383, Barbing. Männlich, 40 - 59 Jahre alt. Makroskopische Detailaufnahme der mehrlagigen feinporösen Auflagerungen an der linken Tibia $\rightarrow$ organisierte subperiostale Hämatome im Rahmen von Skorbut

Bild 4: Sk 1383, Barbing. Männlich, 40 - 59 Jahre alt. Weitere makroskopische Detailaufnahme der mehrlagigen feinporösen Auflagerungen an der linken Tibia, distaler Schaft $\rightarrow$ organisierte subperiostale Hämatome im Rahmen von Skorbut

Bild 5: Sk 1383, Barbing. Männlich, 40 - 59 Jahre alt. Makroskopische Darstellung der rechten Tibia. Wie an der linken Tibia finden sich auch hier deutliche mehrlagige feinporöse Auflagerungen an der lateralen Schaftseite (siehe auch nächste Bilder). $\rightarrow$ organisierte subperiostale Hämatome im Rahmen von Skorbut

Bild 6: Sk 1383, Barbing. Männlich, 40 - 59 Jahre alt. Makroskopische Detailaufnahme der beim vorangegangenen Bild beschriebenen Auflagerungen an der rechten Tibia $\rightarrow$ organisierte subperiostale Hämatome im Rahmen von Skorbut

Bild 7: Sk 1385, Barbing. Juvenil, 14 - 16 Jahre alt. Makroskopische Detailaufnahme des rechten Os ilii. Es fällt ein großes Foramen nutritivum auf, das von massiven feinporösen Auflagerungen umgeben ist. Vermutlich ist das hier austretende Gefäß oder einer seiner Äste rupturiert und führte zu der subperiostalen Einblutung, welche die Auflagerungen bewirkte. $\rightarrow$ Knochenneubildungen infolge von subperiostalen Blutungen im Rahmen von Skorbut

Bild 8: Sk 1385, Barbing. Juvenil, 14 - 16 Jahre alt. Makroskopische Detailaufnahme des linken Femurs. Auch hier sind mehrlagige feinporöse Auflagerungen zu sehen (siehe auch vorangegangenes Bild sowie Bild 1 und 2 auf Bildtafel 10). $\rightarrow$ Knochenneubildungen infolge subperiostaler Hämatome im Rahmen von Skorbut 


\section{Bildtafel 9}
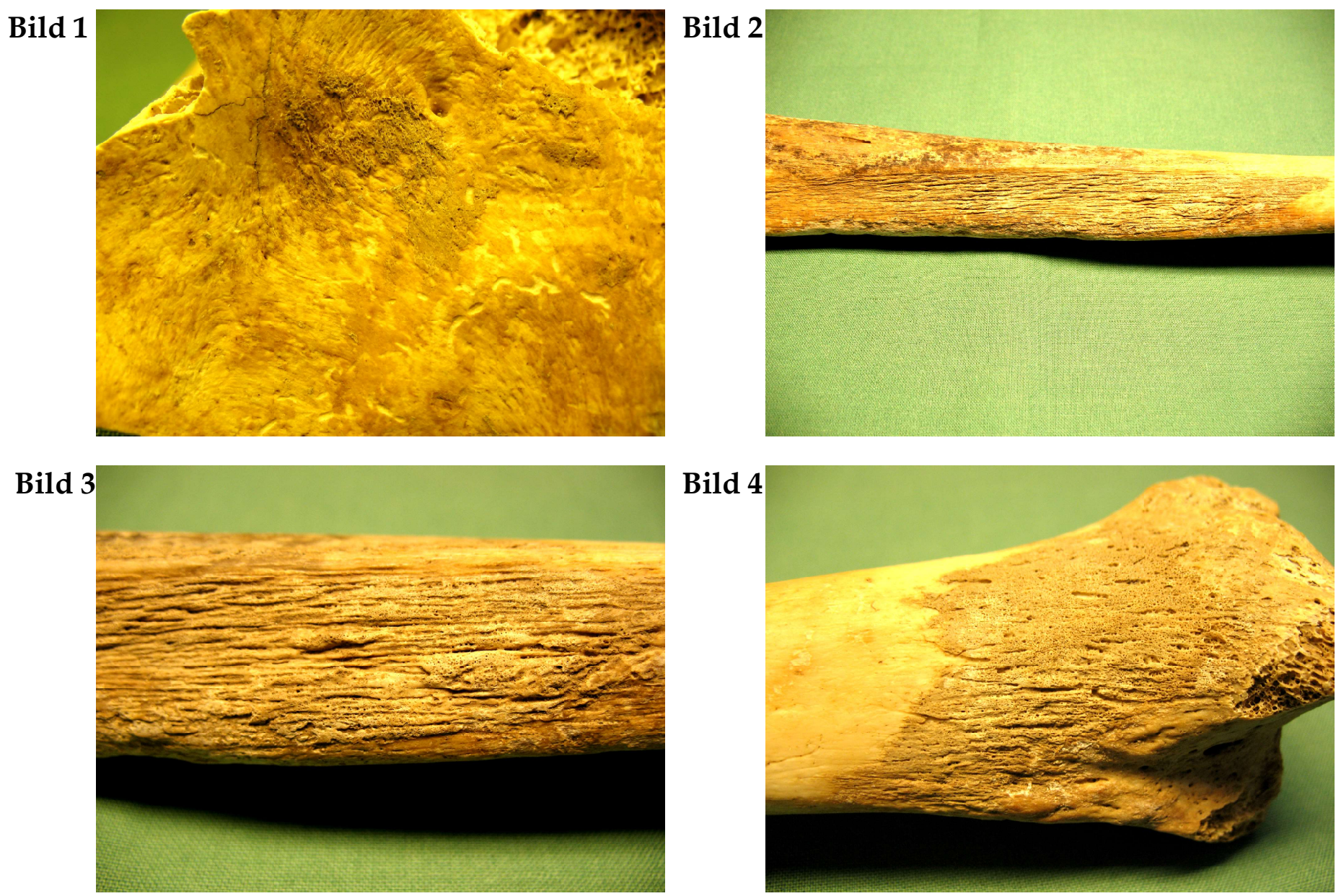

Bild 5

Bild 6
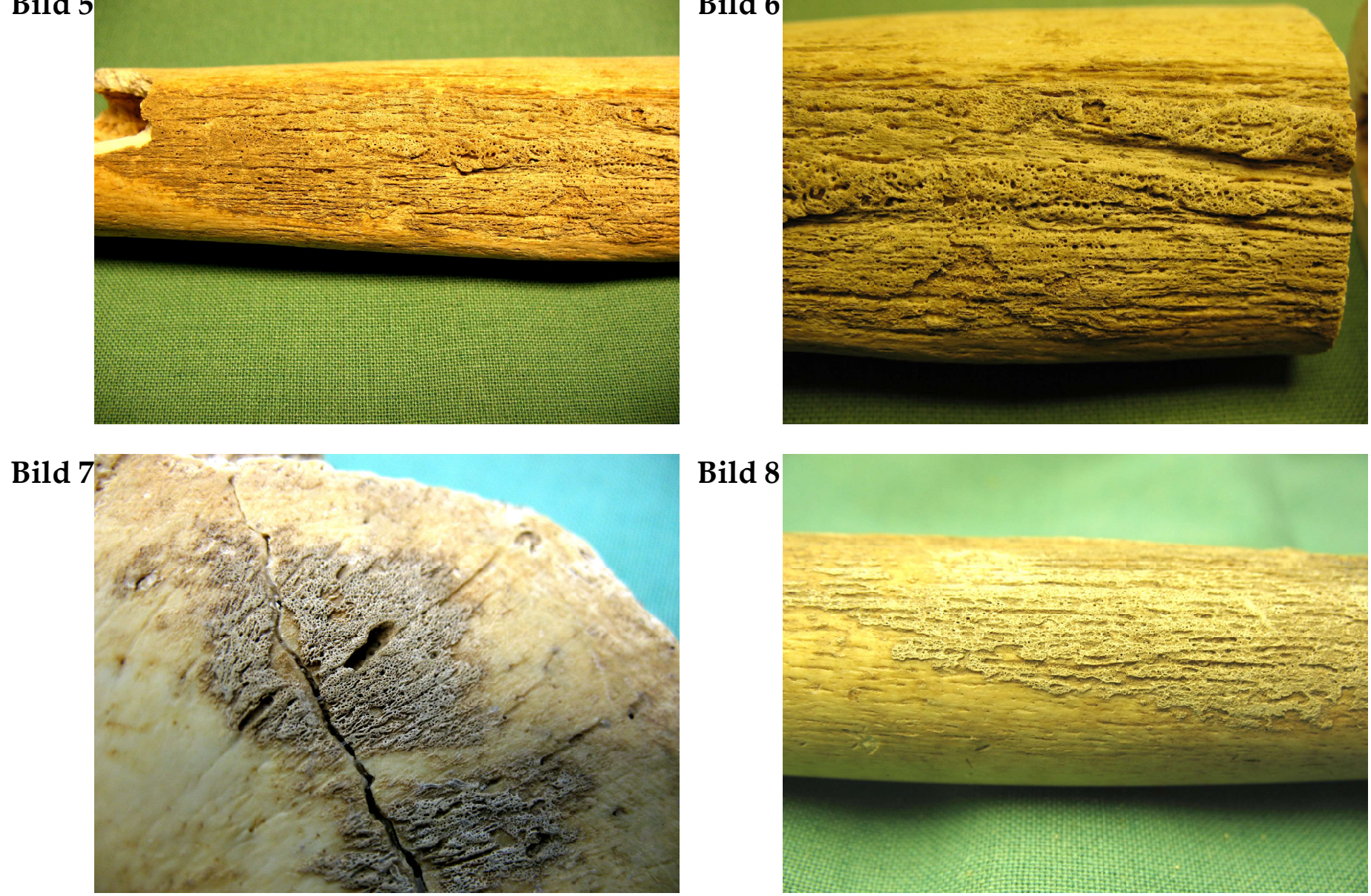

Bild 8

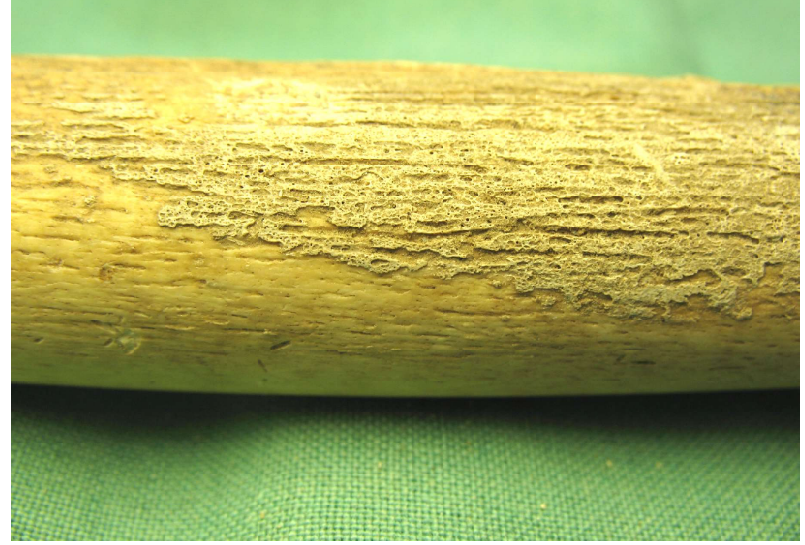




\section{Erklärungen zu Bildtafel 10}

Bild 1: Sk 1385, Barbing. Juvenil, 14 - 16 Jahre alt. Weitere makroskopische Detailaufnahme der feinporösen Auflagerungen am linken Femur (siehe auch Bild 8 auf Bildtafel 9) $\rightarrow$ organisiertes subperiostales Hämatom im Rahmen von Skorbut

Bild 2: Sk 1385, Barbing. Juvenil, 14 - 16 Jahre alt. Makroskopische Detailaufnahme der rechten Fibula. Hier finden sich wie an Femur und Os ilium feinporöse Auflagerungen, die allerdings großenteils erodiert sind. $\rightarrow$ subperiostale Hämorrhagien mit konsekutiver Knochenneubildung bei chronischem Vitamin-C-Mangel

Bild 3: Sk 1386, Barbing. Kind, ca. 18 Monate alt. Makroskopische Detailaufnahme der Alveolen der Maxilla. Es finden sich feinporöse Alveolarauskleidungen. $\rightarrow$ Knochenneubildungen infolge skorbutischer Zahnfleischblutungen

Bild 4: Sk 1386, Barbing. Kind, ca. 18 Monate alt. Makroskopische Detailaufnahme des Os occipitale. In den Sulci der venösen Sinus sind deutlich feinporöse Auflagerungen zu erkennen. Auch in den Impressiones digitatae finden sich feinporöse Auflagerungen, sie sind von pinselstrichartig verzweigten Gefäßimpressionen durchzogen (siehe auch folgende Bilder) $\rightarrow$ subpericraniale Blutungen mit Knochenneubildungen im Rahmen von Skorbut

Bild 5: Sk 1386, Barbing. Kind, ca. 18 Monate alt. Weitere makroskopische Detailaufnahme der unter Bild 4 beschriebenen Veränderungen am Os occipitale.

Bild 6: Sk 1386, Barbing. Kind, ca. 18 Monate alt. Makroskopische Darstellung des linken Os parietale. Ähnlich wie am Os occipitale (siehe vorangegangene Bilder) finden sich auch hier feinporöse Auflagerungen insbesondere in den Impressiones digitatae, teilweise aber auch konfluierend, und verstärkte Gefäßimpressionen $\rightarrow$ Hämorrhagisches Geschehen im Rahmen von Skorbut

Bild 7: Sk 1386, Barbing. Kind, ca. 18 Monate alt. Makroskopische Detailaufnahme eines weiteren Fragments des Os parietale (siehe auch Bild 6). Deutlich sind hier Auflagerungen in Form zapfenförmiger Platten zwischen kräftigen Gefäßimpressionen zu erkennen. $\rightarrow$ Hämorrhagisches Geschehen im Rahmen von Skorbut

Bild 8 Sk 1386, Barbing. Kind, ca. 18 Monate alt. Makroskopische Aufnahme der Außenseite der linken Ala major ossis sphenoidalis. Die Oberfläche ist großenteils feinporös gestaltet, vermutlich hatten die in der Bildmitte zu sehenden Auflagerungen ursprünglich eine größere Ausdehnung. $\rightarrow$ Hämorrhagisches Geschehen im Rahmen von Skorbut 


\section{Bildtafel 10}

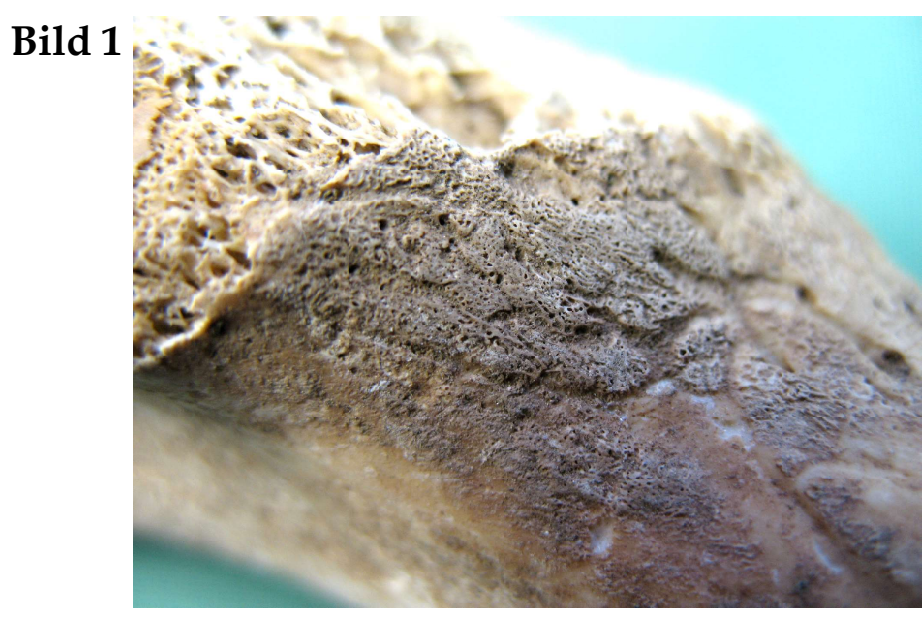

Bild 2

Bild 3

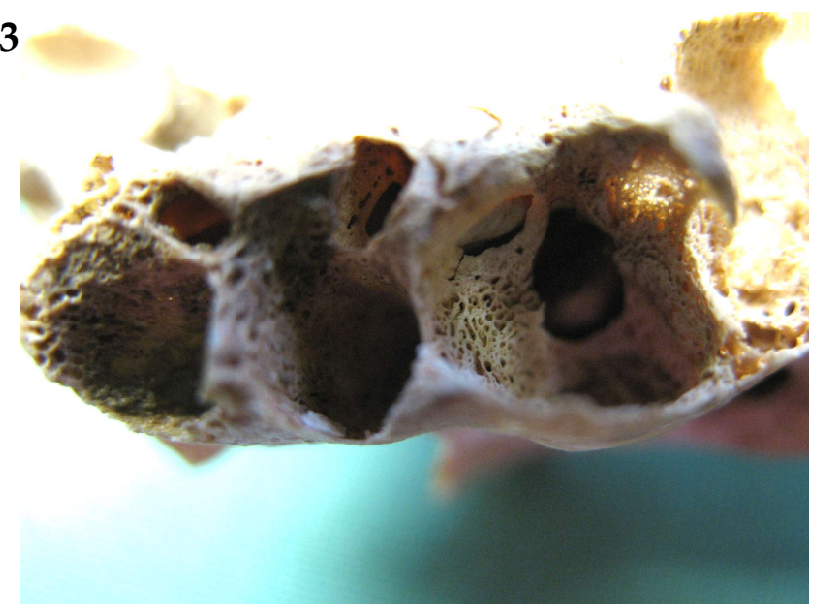

Bild 5

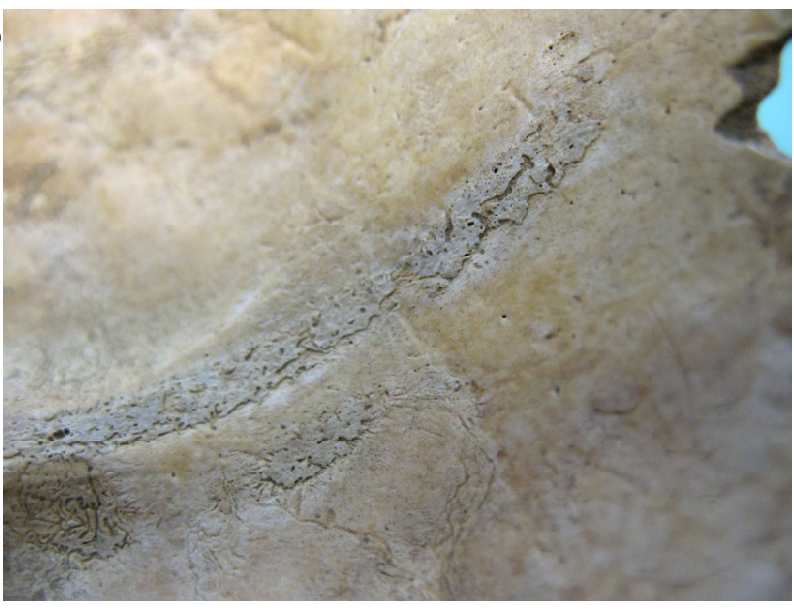

Bild 7

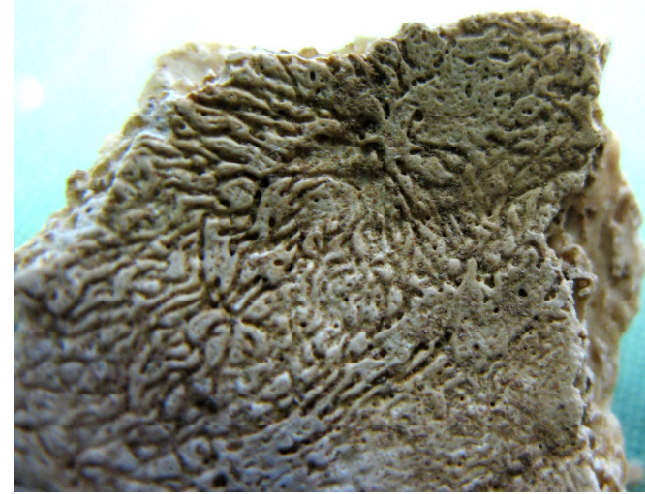

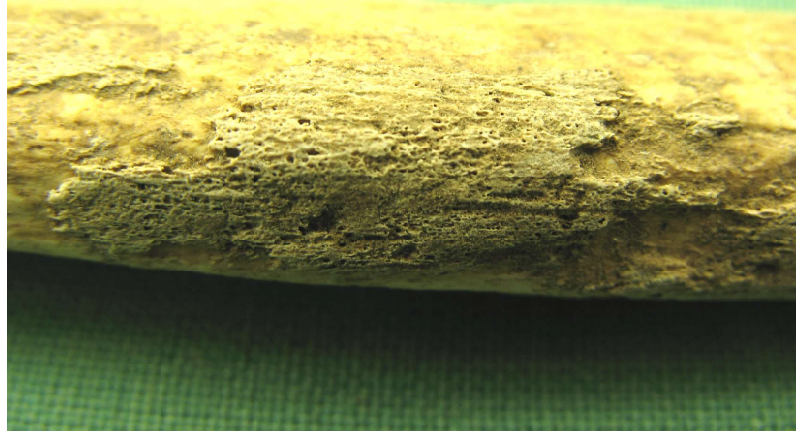

Bild 4

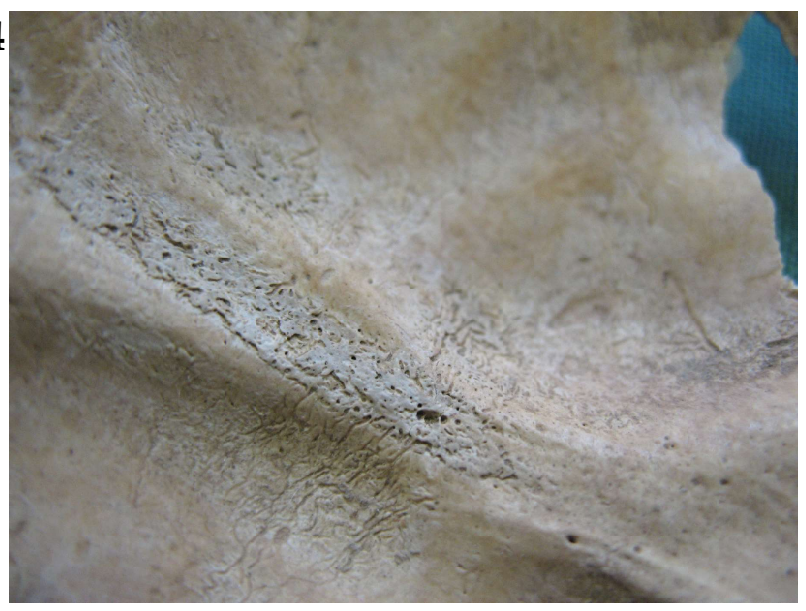

Bild 6

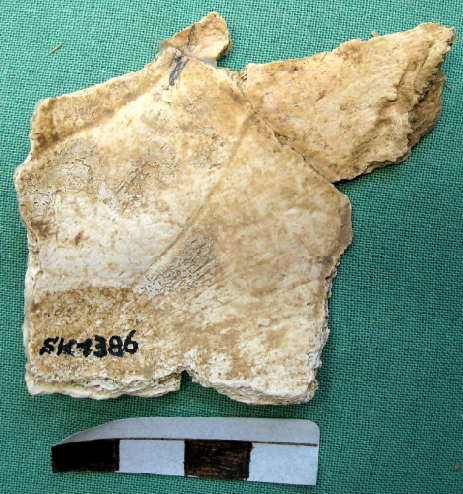

Bild 8

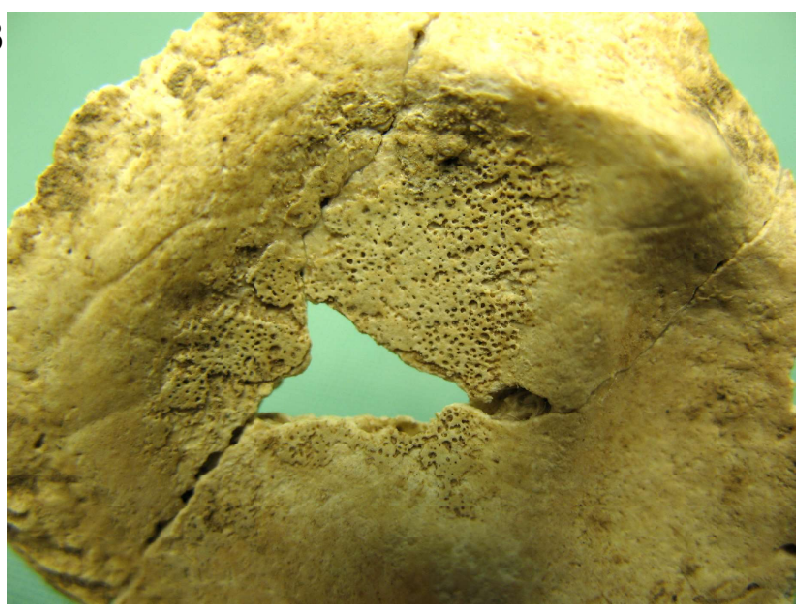




\section{Erklärungen zu Bildtafel 11}

Bild 1: Bácsalmás-Óalmás, Grab 285. Kind, 6 - 7 Jahre alt. Makroskopische Detailaufnahme der rechten Incisura mandibulae. Es sind von pinselstrichartigen Gefäßnetzen durchzogene feinporöse Auflagerungen $\mathrm{zu}$ sehen. $\rightarrow$ Skorbutische subperiostale Blutungen mit konsekutiver Knochenneubildung

Bild 2: Bácsalmás-Óalmás, Grab 285. Kind, $6-7$ Jahre alt. Lichtmikroskopische Darstellung (Schliffdicke 50 $\mathrm{m}$, polarisiertes Durchlicht mit Hilfsobjekt Rot 1. Ordnung (Quarz) als Kompensator, Vergrößerung 16-fach): Es handelt sich um eine Übersichtsaufnahme eines Präparats aus dem linken Os frontale. An der Lamina interna (unten) findet sich eine Auflagerung (Pfeile) vom Typ I (hämorrhagisch), der Umbau zu Lamellenknochen hat bereits begonnen (Sterne). $\rightarrow$ durch Skorbut bedingte Hämorrhagie mit Knochenneubildung im fortgeschrittenen Heilungsstadium

Bild 3: Bácsalmás-Óalmás, Grab 285. Kind, 6 - 7 Jahre alt. Lichtmikroskopische Darstellung

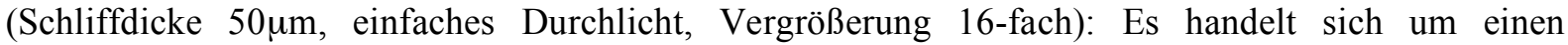
Dünnschliff der linken Orbita. Am linken Bildrand ist der Überaugenwulst zu sehen. Es sind mehrere Gefäßimpressionen (Pfeile) und Gefäßdurchtritte mit Eröffnungen (Sterne) zu erkennen. $\rightarrow$ Vermehrte Gefäßimpressionen verursachen den makroskopischen Aspekt eines Cribrum orbitale, eine mögliche Ursache könnte eine beginnende Anämie sein.

Bild 4: Bácsalmás-Óalmás, Grab 381. Kind, 1,5 - 2 Jahre alt. Makroskopische Aufnahme des linken Femurs. Im Bereich der Metaphysen befinden sich feinporöse Auflagerungen mit profiliertem Rand.

Hämorrhagisches Geschehen im Rahmen von Skorbut mit folgender Knochenneubildung (siehe auch nächste Bilder)

Bild 5: Bácsalmás-Óalmás, Grab 381. Kind, 1,5 - 2 Jahre alt. Makroskopische Aufnahme der Dorsalseite des rechten Femurs. Hier finden sich ähnliche Auflagerungen wie beim in Bild 4 gezeigten Gegenstück. $\rightarrow$ organisiertes subperiostales Hämatom im Rahmen von Skorbut

Bild 6: Bácsalmás-Óalmás, Grab 381. Kind, 1,5 - 2 Jahre alt. Makroskopische Detailaufnahme der in Bild 5 gezeigten Auflagerungen an der Dorsalseite des rechten Femurs $\rightarrow$ organisiertes subperiostales Hämatom im Rahmen von Skorbut

Bild 7: Bácsalmás-Óalmás, Grab 381. Kind, 1,5 - 2 Jahre alt. Makroskopische Detailaufnahme der Fossa olecrani des linken Humerus. Auch hier finden sich feinporöse Auflagerungen mit profiliertem Rand. $\rightarrow$ skorbutische subperiostale Einblutung mit konsekutivem Knochenwachstum

Bild 8: Bácsalmás-Óalmás, Grab 381. Kind, 1,5 - 2 Jahre alt. Makroskopische Detailaufnahme der 1. Rippe rechts. Hier sind ähnliche Auflagerungen wie bei den Femura und dem Humerus gezeigt (siehe vorige Bilder). $\rightarrow$ organisiertes subperiostales Hämatom im Rahmen von Skorbut 


\section{Bildtafel 11}

Bild 1

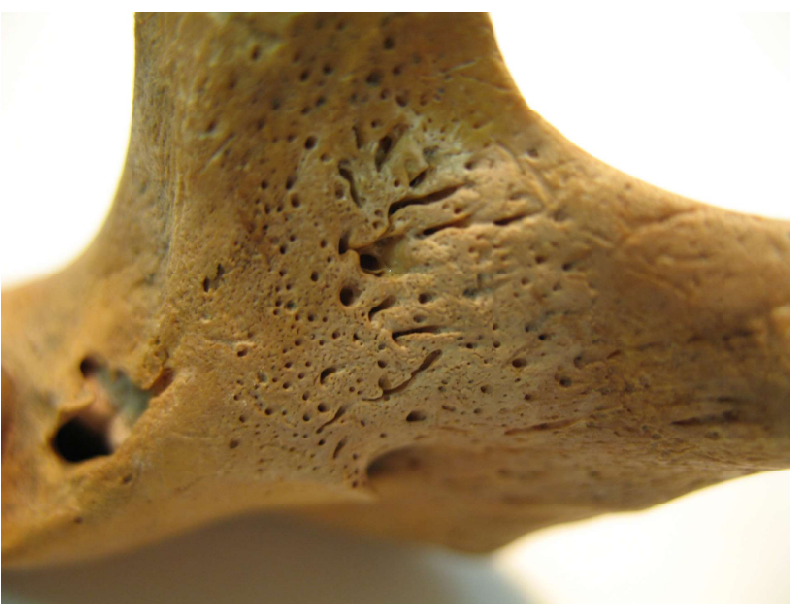

Bild 3

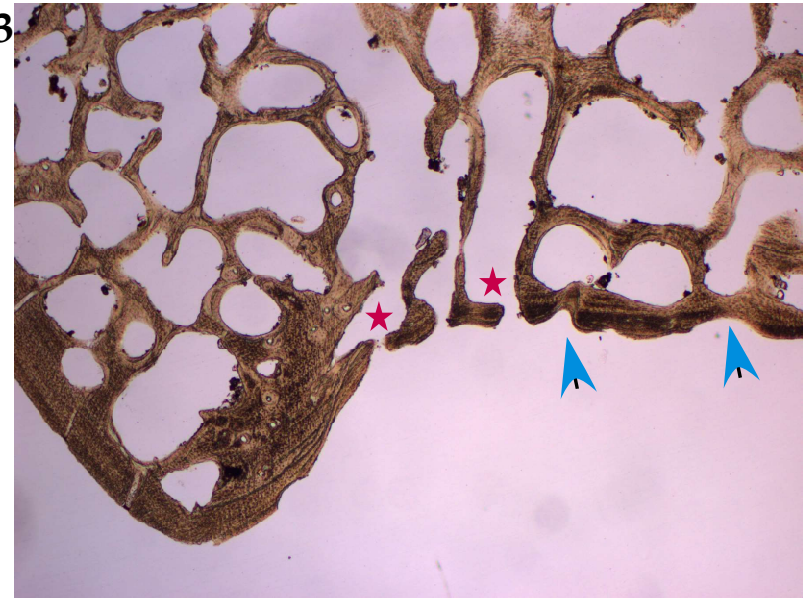

Bild 5

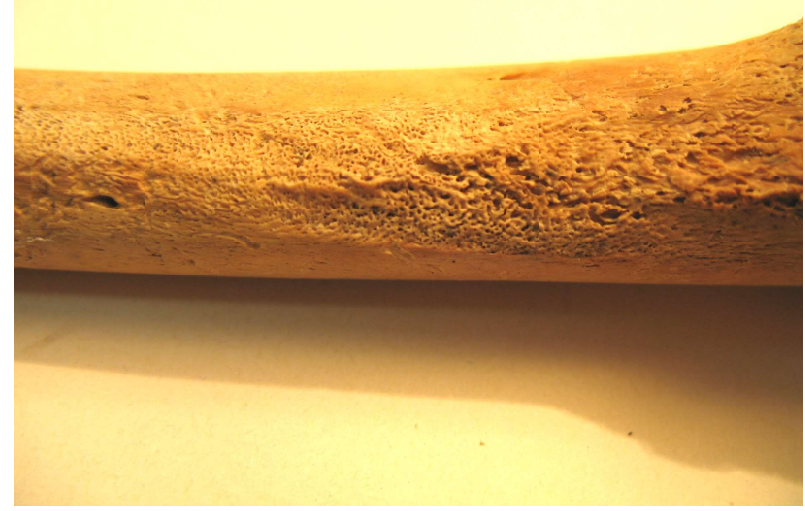

Bild 7

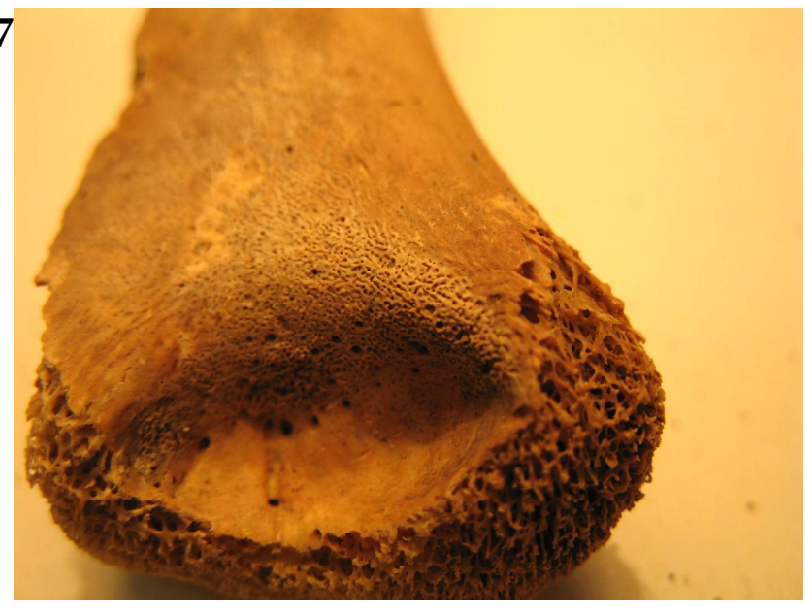

Bild 2

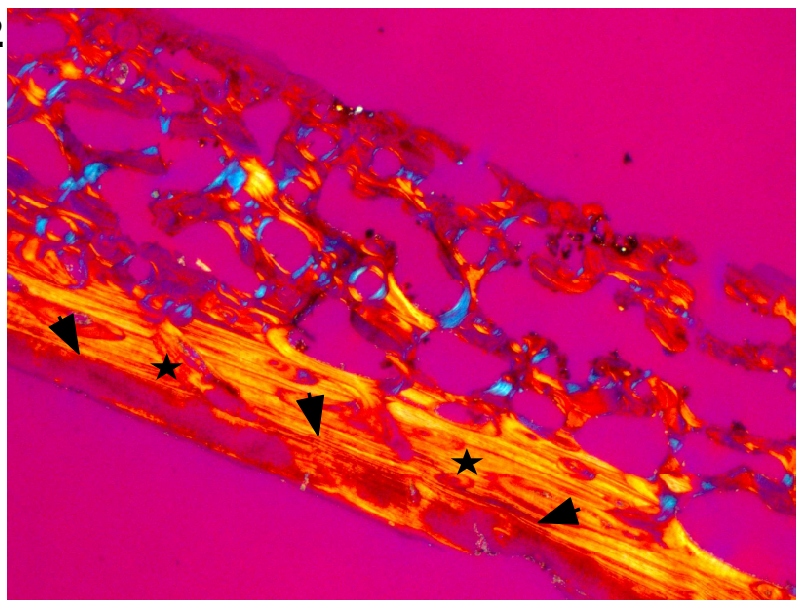

Bild 4

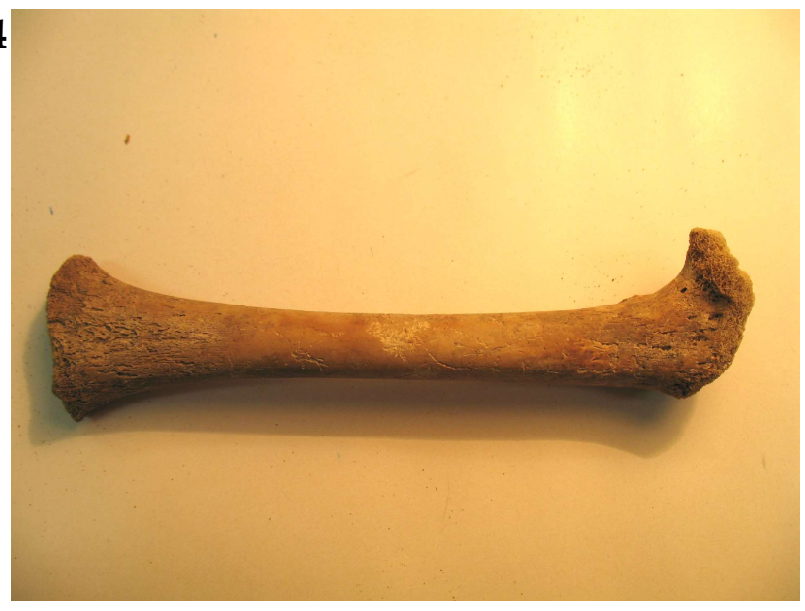

Bild 6

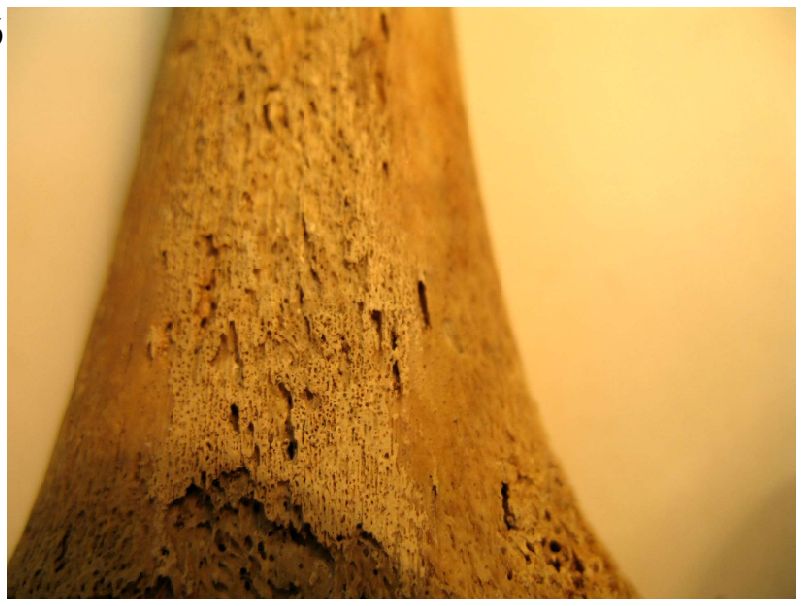

Bild 8

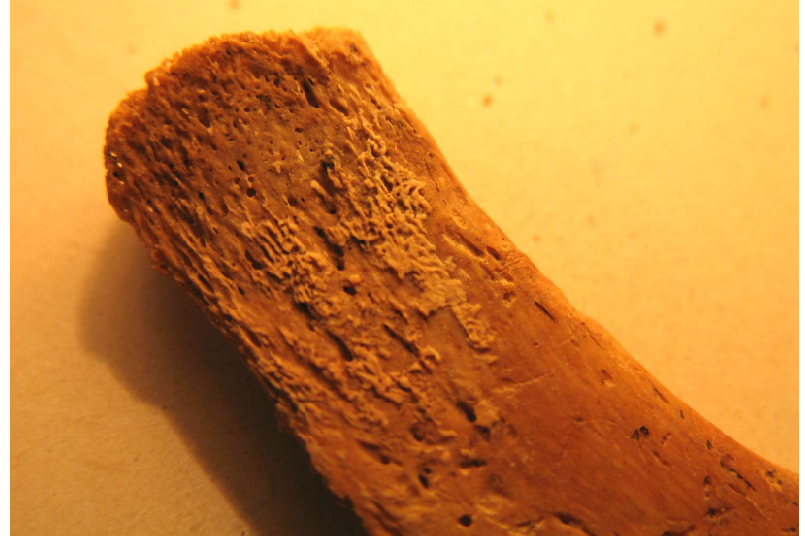




\section{Erklärungen zu Bildtafel 12}

Bild 1: Bácsalmás-Óalmás, Grab 381. Kind, 1,5 - 2 Jahre alt. Makroskopische Detailaufnahme der 2. Rippe links. Zu sehen sind ebensolche feinporösen Auflagerungen wie an den anderen Knochen dieses Individuums (siehe Bildtafel 11, Bild $4-8$ ) $\rightarrow$ Knochenneubildungen infolge von skorbutischen Hämorrhagien

Bild 2: Bácsalmás-Óalmás, Grab 381. Kind, 1,5 - 2 Jahre alt. Makroskopische Aufnahme der linken Tibia. Auch hier sind die zuvor beschriebenen Auflagerungen (siehe vorherige Bilder) zu sehen $\rightarrow$ Knochenauflagerungen infolge von skorbutischen Hämorrhagien

Bild 3: Bácsalmás-Óalmás, Grab 381. Kind, 1,5 - 2 Jahre alt. Weitere makroskopische Detailaufnahme der zuvor beschriebenen Auflagerungen (siehe vorheriges Bild) an der linken Tibia.

$\rightarrow$ Knochenauflagerungen infolge von skorbutischen Hämorrhagien

Bild 4: Bácsalmás-Óalmás, Grab 381. Kind, 1,5 - 2 Jahre alt. Makroskopische Detailaufnahme der rechten Ulna. Die Extremitas proximalis ist annähernd vollständig mit feinporösen Auflagerungen bedeckt. $\rightarrow$ subperiostale Einblutungen mit Knochenwachstum im Rahmen von Skorbut

Bild 5: Bácsalmás-Óalmás, Grab 381. Kind, 1,5 - 2 Jahre alt. Makroskopische Aufnahme des Os frontale. An Glabella und Margines supraorbitales sind deutliche feinporöse Auflagerungen zu sehen, die denen am Postcranium (siehe vorherige Bilder) entsprechen. $\rightarrow$ Subpericraniale Blutung mit konsekutiver Knochenneubildung im Rahmen von Skorbut (lichtmikroskopische Darstellung siehe Bildtafel 14, Bild 2 und 3)

Bild 6: Bácsalmás-Óalmás, Grab 381. Kind, 1,5 - 2 Jahre alt. Makroskopische Aufnahme des Os frontale. Auch an der Lamina interna, insbesondere in den Impressiones digitatae, aber auch flächig konfluierend, finden sich die zuvor besprochenen feinporösen Auflagerungen (siehe vorherige Bilder).

$\rightarrow$ Subpericraniale Blutung mit konsekutiver Knochenneubildung im Rahmen von Skorbut (lichtmikroskopische Darstellung siehe Bildtafel 14, Bild 2 und 3)

Bild 7: Bácsalmás-Óalmás, Grab 381. Kind, 1,5 - 2 Jahre alt. Makroskopische Aufnahme des rechten Os zygomaticum. Es sind wiederum feinporöse Auflagerungen (siehe vorige Bilder) zu sehen. Knochenneubildungen infolge subpericranialer Blutungen im Rahmen von Skorbut

Bild 8: Bácsalmás-Óalmás, Grab 381. Kind, 1,5 - 2 Jahre alt. Makroskopische Aufnahme der linken Ala major ossis sphenoidalis. Auch diese ist mit feinporösen Auflagerungen (siehe vorige Bilder) bedeckt. $\rightarrow$ Hämorrhagisches Geschehen im Rahmen von Skorbut mit konsekutiver Knochenneubildung (lichtmikroskopische Darstellung siehe Bildtafel 13, Bild 7). 


\section{Bildtafel 12}

Bild 1

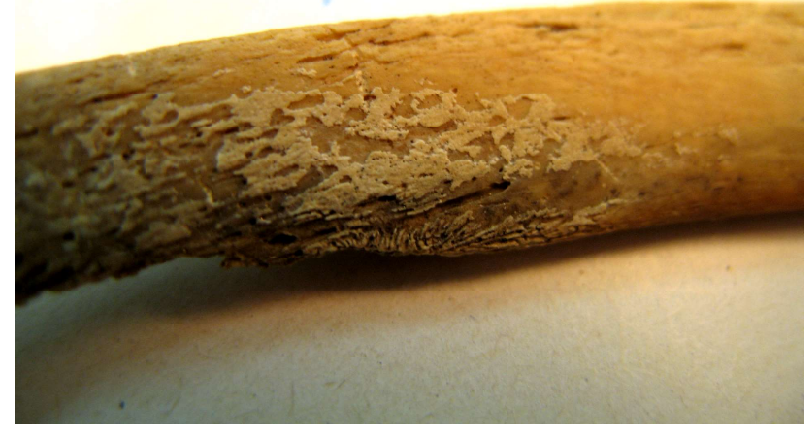

Bild 3

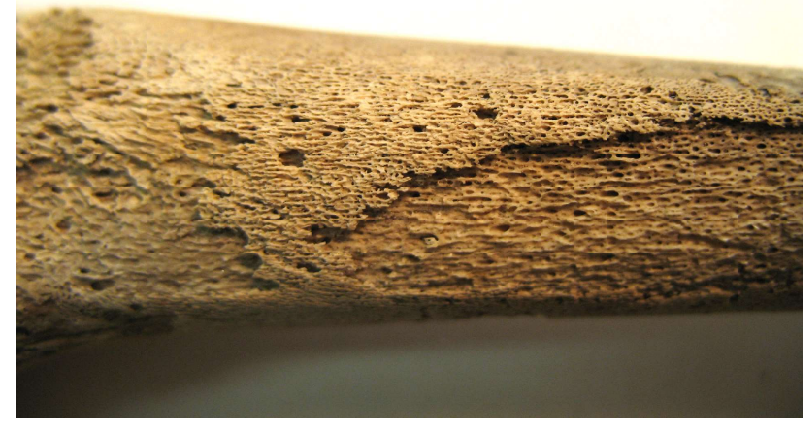

Bild 5

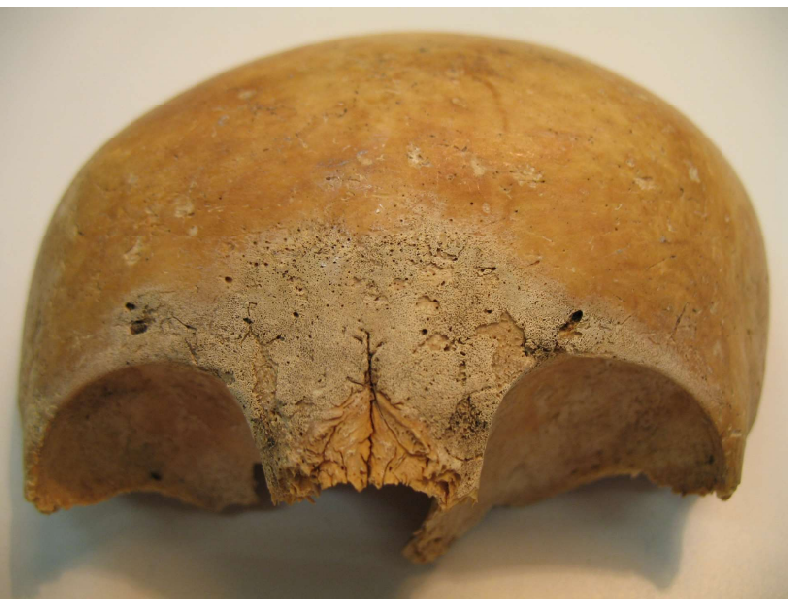

\section{Bild 7}

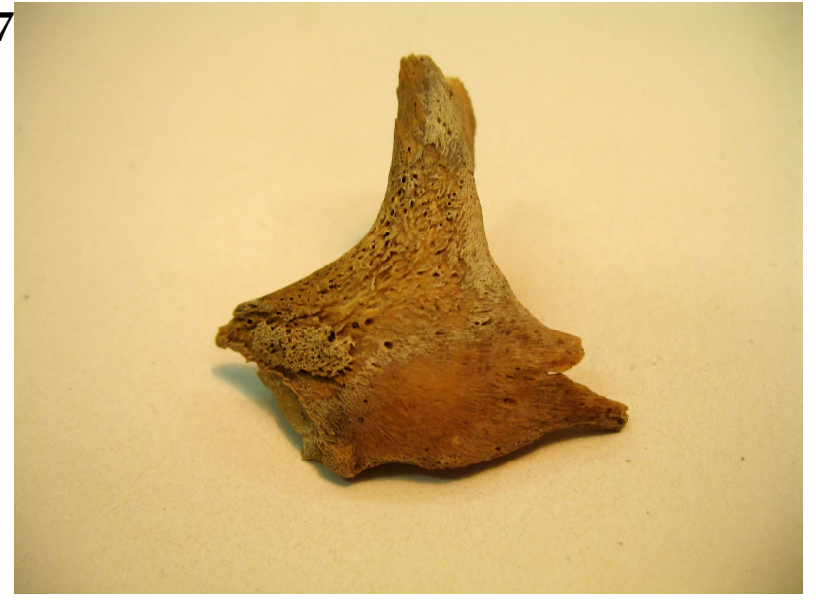

Bild 2

Bild 4

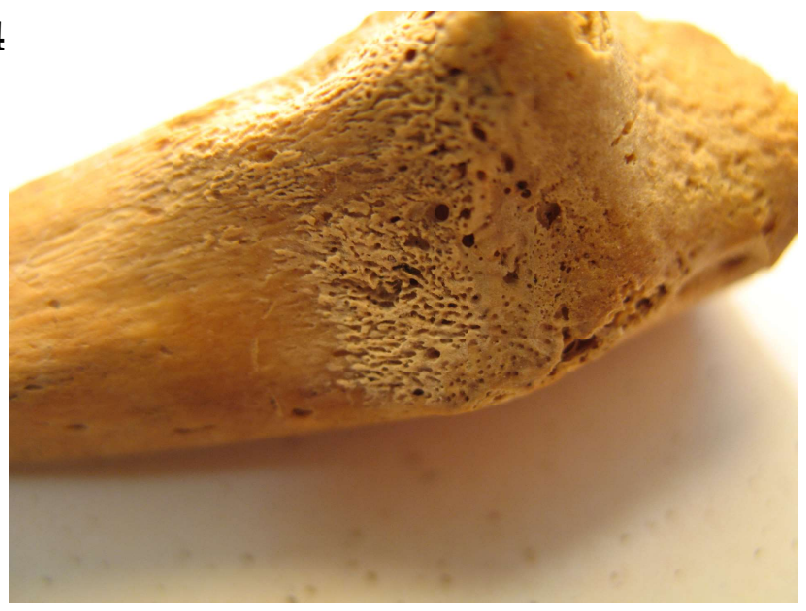

Bild 6

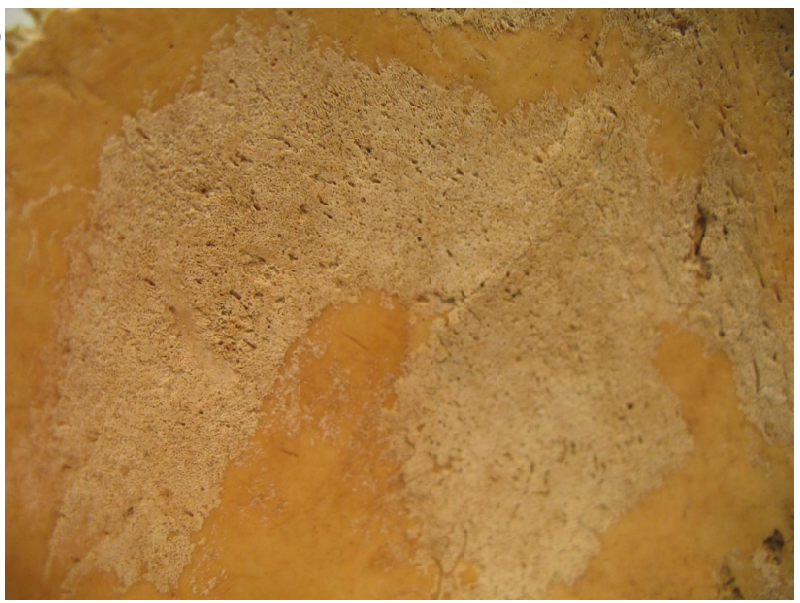

Bild 8

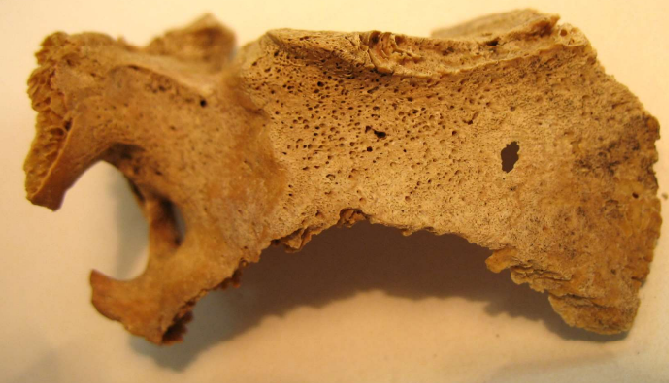




\section{Erklärungen zu Bildtafel 13}

Bild 1: Bácsalmás-Óalmás, Grab 381. Kind, 1,5 - 2 Jahre alt. Makroskopische Aufnahme des linken Os parietale. An der Lamina externa finden sich in Suturnähe flächige feinporöse Auflagerungen (vgl. Bildtafel 11, Bild $4-8$, alle Bilder Bildtafel 12). $\rightarrow$ Knochenneubildungen infolge skorbutischer Hämorrhagien

Bild 2: Bácsalmás-Óalmás, Grab 381. Kind, 1,5 - 2 Jahre alt. Makroskopische Aufnahme des linken Os parietale. Die feinporösen Auflagerungen (siehe auch vorige Bilder) sind hier vorrangig in den Impressiones digitatae, konfluieren aber darüber hinaus. $\rightarrow$ subpericraniale Blutungen im Rahmen von Skorbut mit konsekutiver Knochenneubildung

Bild 3: Bácsalmás-Óalmás, Grab 381. Kind, 1,5 - 2 Jahre alt. Makroskopische Aufnahme der Mandibula. Sowohl an den Rami als auch am Corpus und insbesondere perialveolär finden sich besagte feinporöse Auflagerungen (siehe vorige Bilder). $\rightarrow$ Hämorrhagisches Geschehen im Rahmen von Skorbut mit konsekutiver Knochenneubildung (siehe auch folgende Bilder sowie zur lichtmikroskopischen Darstellung Bildtafel 14, Bild 1)

Bild 4: Bácsalmás-Óalmás, Grab 381. Kind, 1,5 - 2 Jahre alt. Makroskopische Detailaufnahme des Alveolarbereichs der Mandibula. In den Alveolen sind feinporöse Auskleidungen zu finden (vgl. voriges Bild sowie Bild 5 und 6). $\rightarrow$ Knochenneubildung infolge skorbutischen Zahnfleischblutens

Bild 5: Bácsalmás-Óalmás, Grab 381. Kind, 1,5 - 2 Jahre alt. Weitere makroskopische Detailaufnahme des Alveolarbereichs der Mandibula zur Darstellung der Alveolarauskleidungen (vgl. Bild 3, 4 und 6) $\rightarrow$ Knochenneubildung infolge skorbutischen Zahnfleischblutens

Bild 6: Bácsalmás-Óalmás, Grab 381. Kind, 1,5 - 2 Jahre alt. Weitere makroskopische Detailaufnahme des Alveolarbereichs der Mandibula zur Darstellung der Alveolarauskleidungen (vgl. Bild 4, 5 und 6) $\rightarrow$ Knochenneubildung infolge skorbutischen Zahnfleischblutens

Bild 7: : Bácsalmás-Óalmás, Grab 381. Kind, 1,5 - 2 Jahre alt. Lichtmikroskopische Darstellung (Schliffdicke 70 $\mu \mathrm{m}$, einfaches Durchlicht, Vergrößerung 25-fach): Es handelt es sich um ein Präparat aus der linken Ala major ossis sphenoidale. Der Erhaltungszustand ist mäßig, dennoch sind an der Lamina externa (oben) Reste von Auflagerungen (Pfeile) vom Typ I (hämorrhagisch) zu sehen $\rightarrow$ Knochenauflagerungen infolge skorbutischer subpericranialer Blutungen (makroskopische Darstellung siehe Bildtafel 12, Bild 8)

Bild 8: Bácsalmás-Óalmás, Grab 381. Kind, 1,5 - 2 Jahre alt. Lichtmikroskopische Darstellung (Schliffdicke 50 $\mathrm{mm}$, polarisiertes Durchlicht, Vergrößerung 16-fach): Es handelt sich um eine Übersichtsaufnahme der linken Orbita. Am rechten Bildrand befindet sich der Überaugenwulst. Links davon ist eine flache Auflagerung (Pfeile) vom Typ I (hämorrhagisch) zu sehen (vgl. auch andere Bilder auf Bildtafeln 11, 12, 13 und 14) $\rightarrow$ Subperiostale Einblutung mit nachfolgender Knochenneubildung im Rahmen einer Skorbuterkrankung 


\section{Bildtafel 13}
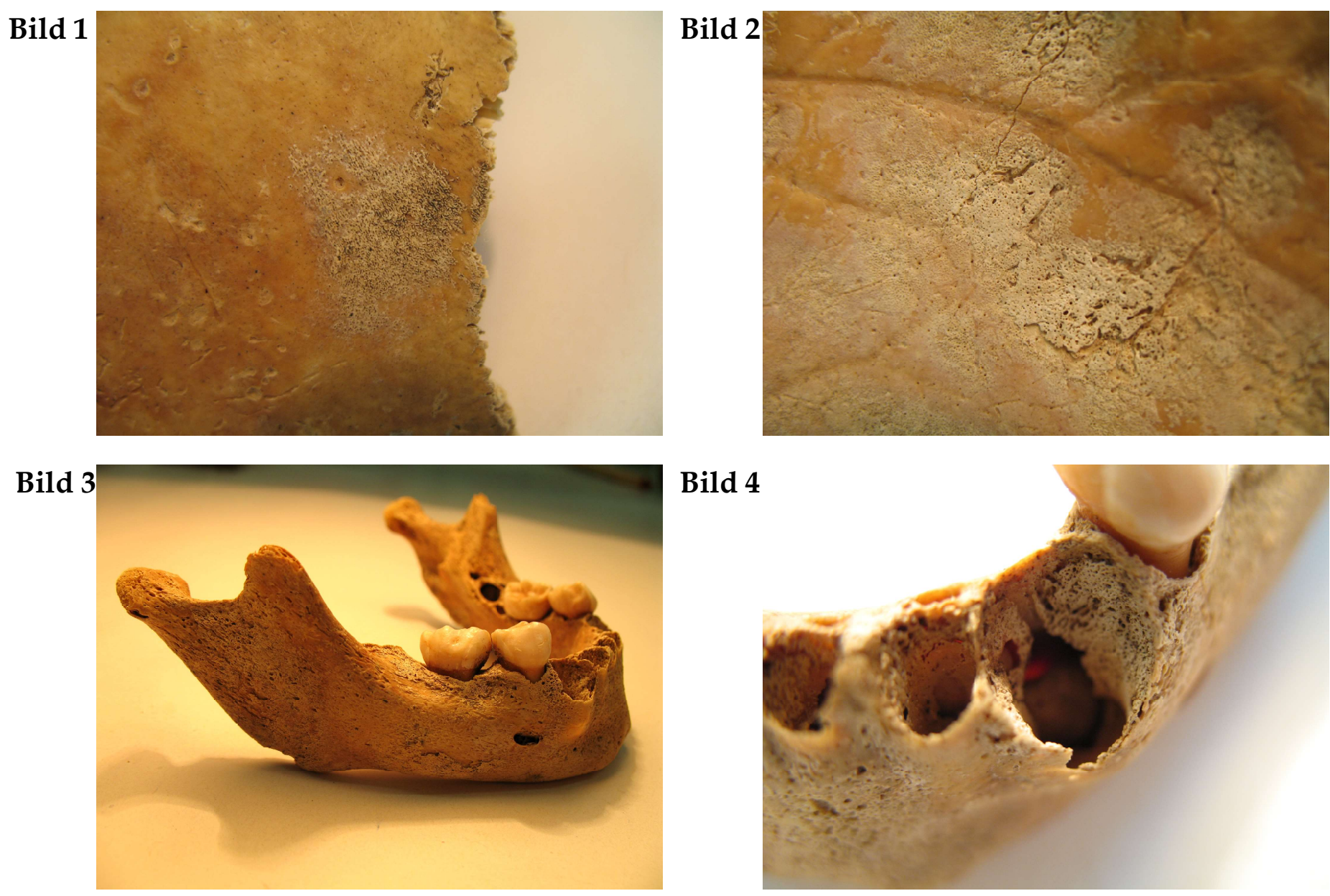

Bild 4
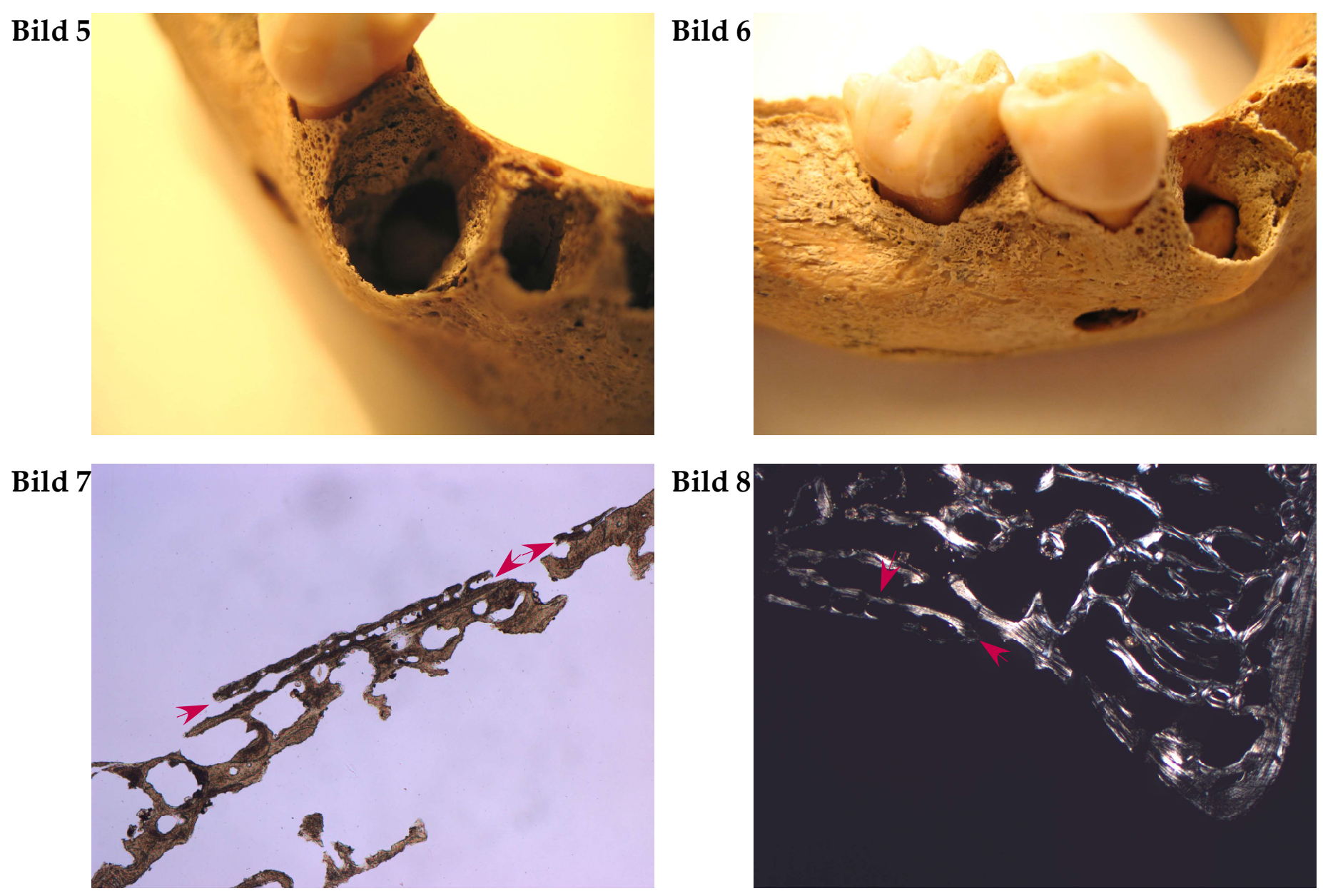


\section{Erklärungen zu Bildtafel 14}

Bild 1 Bácsalmás-Óalmás, Grab 381. Kind, 1,5 - 2 Jahre alt. Lichtmikroskopische Darstellung (Schliffdicke $70 \mu \mathrm{m}$, polarisiertes Durchlicht mit Hilfsobjekt Rot 1. Ordnung (Quarz) als Kompensator, Vergrößerung 25fach): Das Präparat aus der Mandibula zeigt massive Auflagerungen vom Typ I. Es handelt sich um ein dreiphasiges Geschehen. Außen liegen zwei Schichten von kurz vor dem Tode entstandenen Auflagerungen (3, 2). Darunter befindet sich eine ältere Auflagerung, die bereits sehr gut organisiert und kompakt ist (1). Unterhalb dieser Auflagerung fallen ausgeprägte Resorptionshölen (weiße Sterne) auf. Unter den Auflagerungen ist die ehemalige Lamina externa (Pfeil) zu erkennen, auch darunter viele Resorptionshöhlen (schwarze Sterne) $\rightarrow$ über längeren Zeitraum verlaufende Skorbuterkrankung, die in Phasen zu subperiostalen Hämorrhagien mit nachfolgender Knochenneubildung führte, wobei der letzte Schub erst kurz vor dem Tod des Kindes erfolgte (weitere Bilder des Individuums siehe unten und Bildtafeln 11, 12 und 13)

Bild 2: Bácsalmás-Óalmás, Grab 381. Kind, 1,5 - 2 Jahre alt. Lichtmikroskopische Darstellung (Schliffdicke $70 \mu \mathrm{m}$, polarisiertes Durchlicht mit Hilfsobjekt Rot 1. Ordnung (Quarz) als Kompensator, Vergrößerung 16fach): Es handelt sich um eine Übersichtsaufnahme des linken Os frontale. An der Lamina interna sind Auflagerungen (Pfeil) vom Typ 1 (hämorrhagisch) $\mathrm{zu}$ erkennen $\rightarrow$ organisiertes epidurales Hämatom im Rahmen von Skorbut (siehe auch Bild 1 und 3 sowie Bildtafeln 11, 12 und 13)

Bild 3: Bácsalmás-Óalmás, Grab 381. Kind, 1,5 - 2 Jahre alt. Lichtmikroskopische Darstellung (Schliffdicke $70 \mu \mathrm{m}$, polarisiertes Durchlicht mit Hilfsobjekt Rot 1. Ordnung (Quarz) als Kompensator, Vergrößerung 25fach): Es handelt sich um eine Vergrößerung des Präparats aus dem linken Os frontale (siehe Bild 2). Dargestellt ist ein Gefäßforamen, in dem eine Howship'sche Lakune (Pfeil) zu erkennen ist.

Bild 4: Bácsalmás-Óalmás, Grab 436. Kind, 0 - 0,5 Jahre alt. Makroskopische Aufnahme des linken Os ilii. An der Dorsalseite finden sich flächige feinporöse Auflagerungen $\rightarrow$ Hämorrhagisches Geschehen im Rahmen von Skorbut mit Knochenneubildung durch Periostreizung (siehe auch folgende Bilder, lichtmikroskopische Darstellung siehe Bildtafel 15, Bild 6 und 7)

Bild 5: Bácsalmás-Óalmás, Grab 436. Kind, 0 - 0,5 Jahre alt. Makroskopische Aufnahme des linken Humerusschafts. Auch hier finden sich massive feinporöse Auflagerungen (siehe auch Bild 4, 6 und folgende). $\rightarrow$ subperiostale Hämorrhagien mit nachfolgender Knochenneubildung infolge schweren chronischen VitaminC- Mangels

Bild 6: Bácsalmás-Óalmás, Grab 436. Kind, 0 - 0,5 Jahre alt. Weitere makroskopische Aufnahme der feinporösen Auflagerungen des linken Humerusschafts (siehe auch Bild 5) $\rightarrow$ subperiostale Hämorrhagien mit nachfolgender Knochenneubildung infolge schweren chronischen Vitamin-C- Mangels

Bild 7: Bácsalmás-Óalmás, Grab 436. Kind, $0-0,5$ Jahre alt. Makroskopische Aufnahme des rechten Humerusschafts. Auch hier finden sich massive feinporöse Auflagerungen (siehe auch vorangegangene und folgende Bilder). $\rightarrow$ subperiostale Hämorrhagien mit nachfolgender Knochenneubildung im Rahmen von Skorbut

Bild 8: Bácsalmás-Óalmás, Grab 436. Kind, 0 - 0,5 Jahre alt. Makroskopische Aufnahme der linken Scapula. In der Fossa infraspinata sind feinporöse Auflagerungen mit profiliertem Rand zu erkennen. $\rightarrow$ organisiertes subperiostales Hämatom im Rahmen von Skorbut (siehe auch vorangegangene und folgende Bilder, lichtmikroskopische Darstellung siehe Bildtafel 16, Bild 7) 


\section{Bildtafel 14}

Bild 1

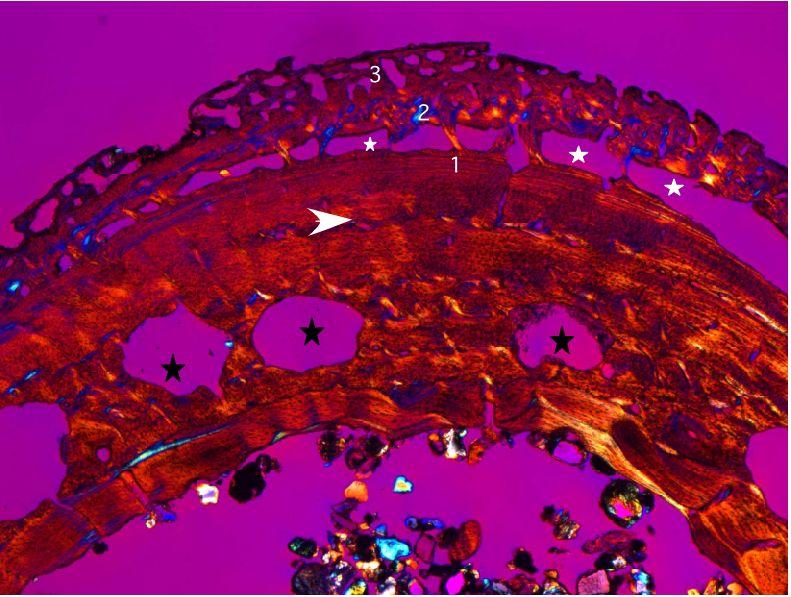

Bild 3

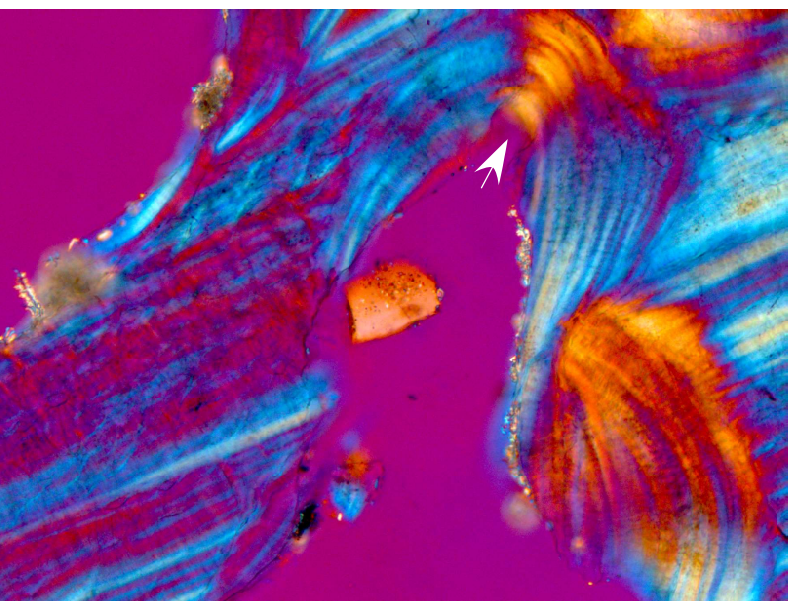

Bild 5

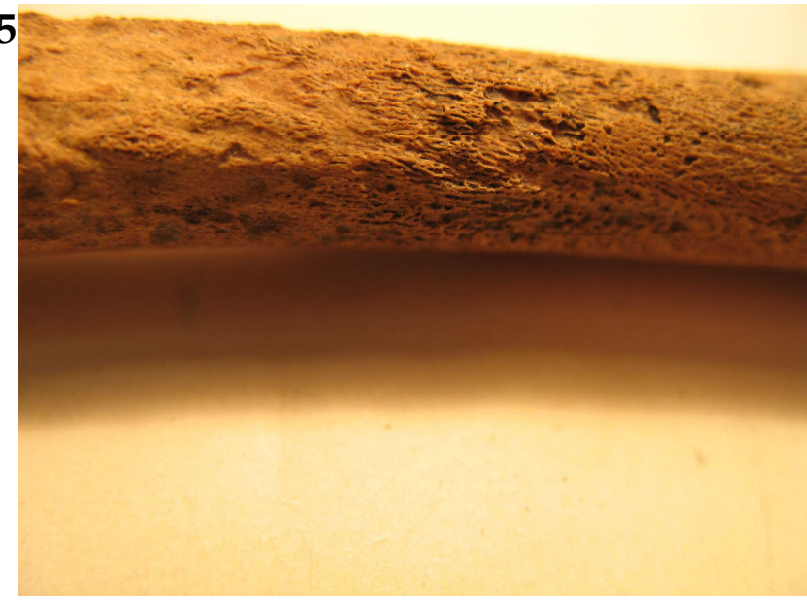

Bild 7

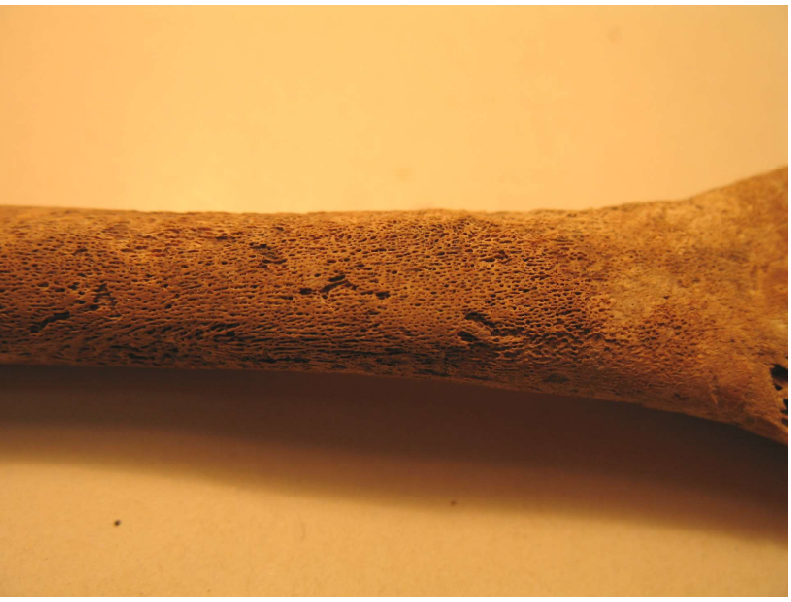

Bild 2

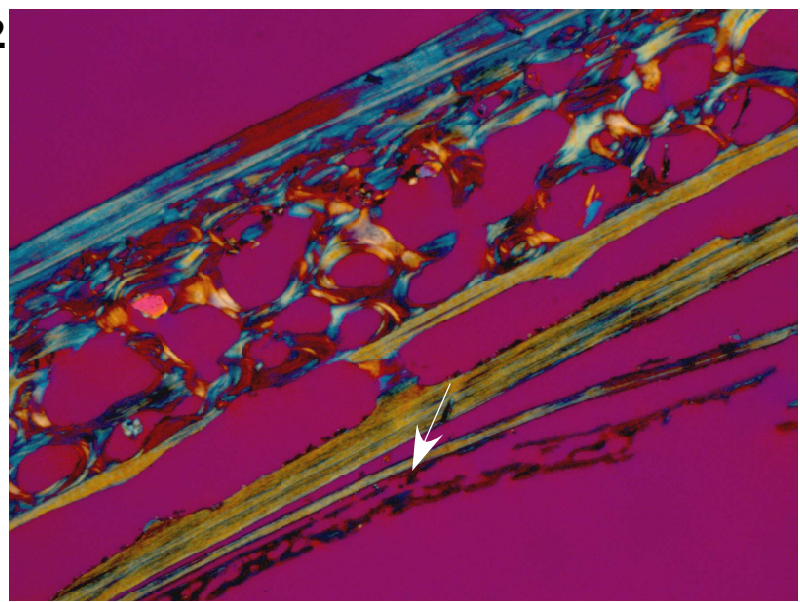

Bild 4

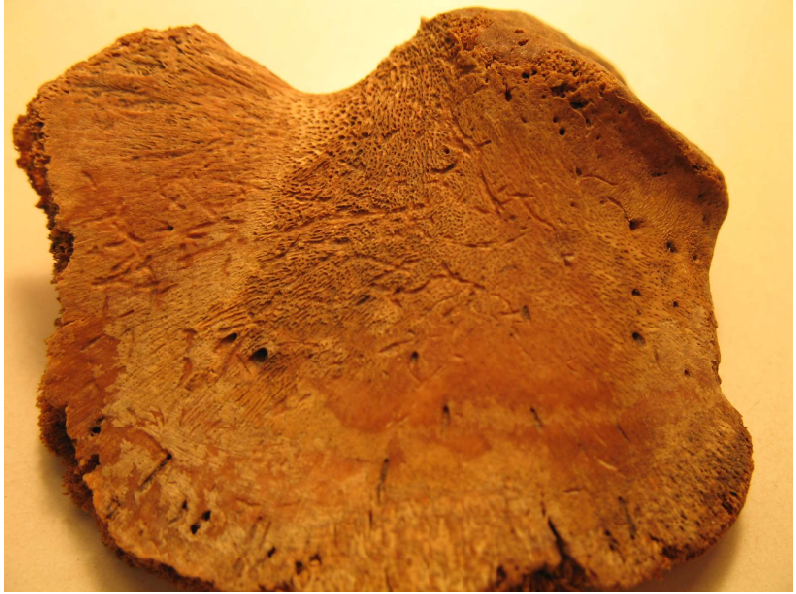

Bild 6

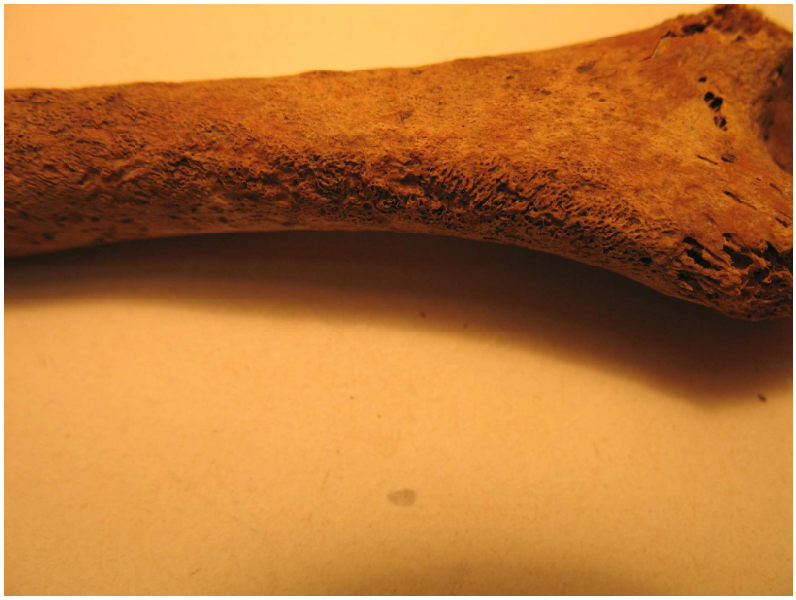

Bild 8

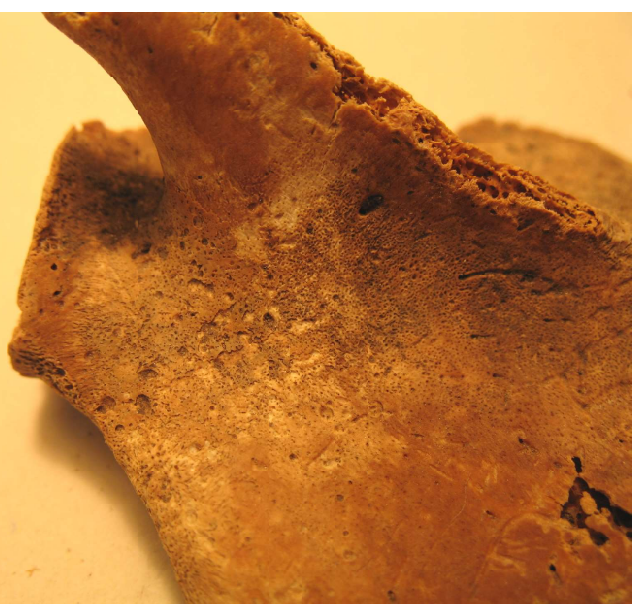




\section{Erklärungen zu Bildtafel 15}

Bild 1 Bácsalmás-Óalmás, Grab 436. Kind, 0 - 0,5 Jahre alt. Makroskopische Aufnahme der rechten Tibia. Der Schaft ist mit feinporösen Auflagerungen bedeckt und nach ventral konvex verbogen. $\rightarrow$ hämorrhagisches Geschehen mit Knochenneubildung bei chronischem Vitamin-C-Mangel (siehe Bildtafel 14 und folgende Bilder)

Bild 2: Bácsalmás-Óalmás, Grab 436. Kind, 0 - 0,5 Jahre alt. Makroskopische Detailaufnahme des linken Os frontale. Die Glabella ist mit hämorrhagischen Auflagerungen bedeckt. $\rightarrow$ organisiertes subpericraniales Hämatom im Rahmen von Skorbut (siehe auch vorangegangene und folgende Bilder, lichtmikroskopische Darstellung siehe Bildtafel 15, Bild 8 sowie Bildtafel 16, Bild 1 und 2)

Bild 3: Bácsalmás-Óalmás, Grab 436. Kind, 0 - 0,5 Jahre alt. Makroskopische Aufnahme des linken Os sphenoidale. Auf dem Dorsum sellae und den Alae minores finden sich feinporöse Auflagerungen. $\rightarrow$ subpericraniale Blutungen mit konsekutiver Knochenneubildung im Rahmen von Skorbut

Bild 4: Bácsalmás-Óalmás, Grab 436. Kind, 0 - 0,5 Jahre alt. Makroskopische Detailaufnahme des linken Os sphenoidale. Auch an der Facies cerebralis der Ala major ossis sphenoidalis sind feinporöse Auflagerungen zu erkennen $\rightarrow$ Hämorrhagisches Geschehen im Rahmen von Skorbut mit Knochenneubildung durch Periostreizung (siehe auch vorangegangene und folgende Bilder, lichtmikroskopische Darstellung siehe Bildtafel 16, Bild 3)

Bild 5: Bácsalmás-Óalmás, Grab 436. Kind, 0 - 0,5 Jahre alt. Weitere makroskopische Detailaufnahme des linken Os sphenoidale. Auch an der Facies temporalis der Ala major ossis sphenoidalis sind feinporöse Auflagerungen zu erkennen. $\rightarrow$ Hämorrhagisches Geschehen im Rahmen von Skorbut mit Knochenneubildung durch Periostreizung (siehe auch vorangegangene und folgende Bilder)

Bild 6: Bácsalmás-Óalmás, Grab 436. Kind, 0 - 0,5 Jahre alt. Lichtmikroskopische Darstellung (Schliffdicke 50 $\mu$ m, einfaches Durchlicht, Vergrößerung 16-fach): Es handelt sich um eine Übersichtsaufnahme des linken Os ilii. Es sind fragliche Auflagerungen (Pfeile) vom Typ I (hämorrhagisch) zu erkennen, die nicht eindeutig vom normalen appositionellen Wachstum abzugrenzen sind. Außerdem fällt eine streifenförmige Anordnung der Knochenbälkchen in der Substantia compacta auf, die sich am ehesten durch Druckbelastung bei Bewegung erklären lässt. Dies überrascht angesichts des geringen Alters des Kindes. $\rightarrow$ fragliche Knochenneubildung infolge subperiostaler Hämorrhagien bei Skorbut (siehe auch vorangegangene makroskopische Bilder, insbesondere Bildtafel 14, Bild 4, sowie folgende mikroskopische Darstellungen desselben Individuums)

Bild 7: Bácsalmás-Óalmás, Grab 436. Kind, 0 - 0,5 Jahre alt. Lichtmikroskopische Darstellung (Schliffdicke 50um, einfaches Durchlicht, Vergrößerung 25-fach): Es handelt sich um eine Detailaufnahme des unter Bild 6 beschriebenen Präparats aus dem Os ilii. Hier sind die Auflagerungen (Pfeile) vom Typ I (hämorrhagisch) besser zu erkennen. Es sind drei Gefäßforamina sowie die typische Deckplatte erhalten. $\rightarrow$ subperiostale Hämorrhagien mit nachfolgender Knochenneubildung im Rahmen von Skorbut

Bild 8: Bácsalmás-Óalmás, Grab 436. Kind, 0 - 0,5 Jahre alt. Lichtmikroskopische Darstellung (Schliffdicke 70 $\mu$ m, kleiner Schliff, polarisiertes Durchlicht mit Hilfsobjekt Rot 1. Ordnung (Quarz) als Kompensator, Vergrößerung 16-fach): In der Übersicht dieses Präparats aus dem Os frontale zeigen sich an der Lamina externa (oben) deutliche, aber teilweise zerstörte Auflagerungen (schwarze Pfeile), vermutlich vom Typ I (hämorrhagisch). Auch an der Lamina interna (unten) lassen sich Auflagerungen (weiße Pfeile) vom Typ I erkennen, diese sind flacher und kompakter als die an der Außenseite. Die insgesamt schwach ausgeprägte Knochenstruktur und die faserknöcherne Organisation lassen sich durch das geringe Alter des Kindes erklären. $\rightarrow$ organisierte epidurale Blutungen im Rahmen von Skorbut (siehe auch vorangegangene Bilder und Bildtafel 16) 


\section{Bildtafel 15}

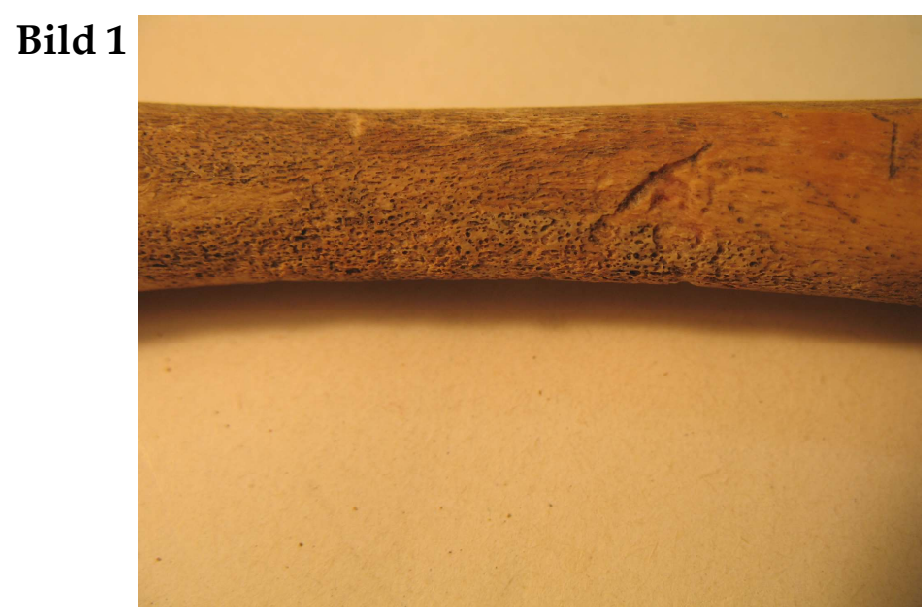

Bild 2

\section{Bild 3}

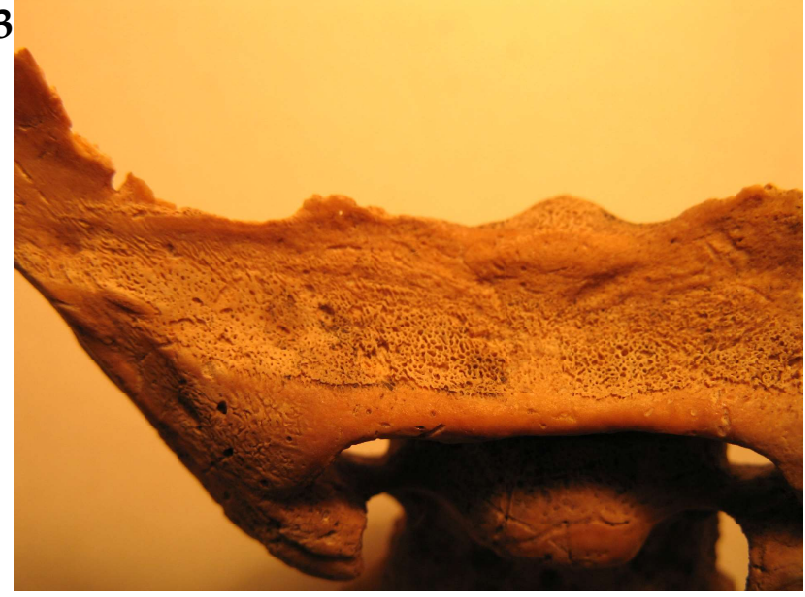

Bild 5

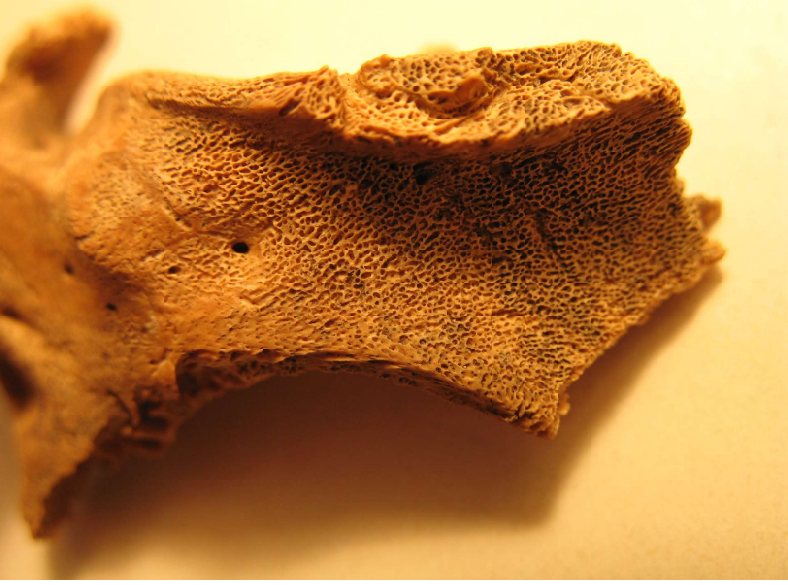

\section{Bild 7}

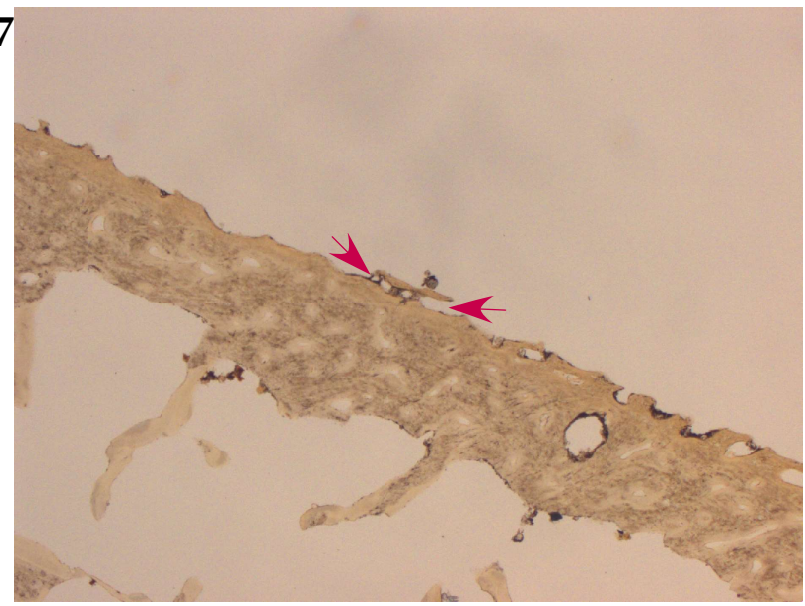

Bild 4

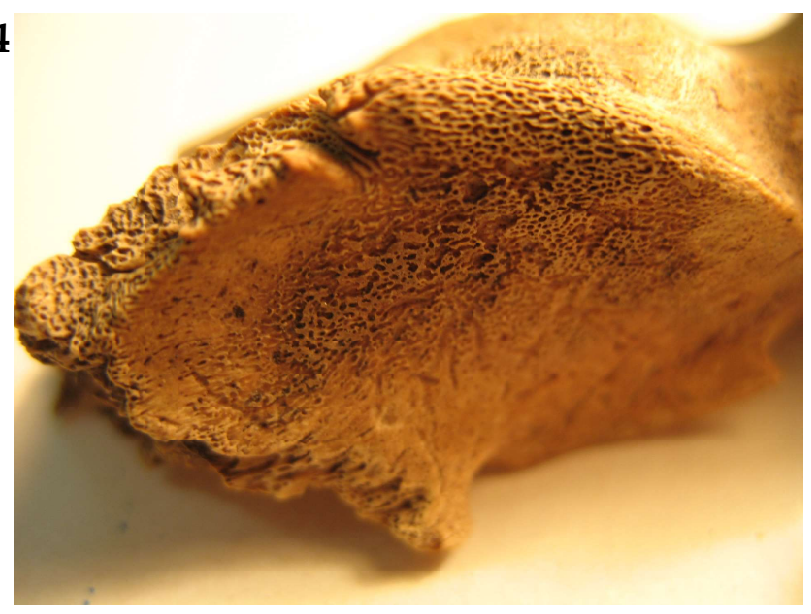

Bild 6

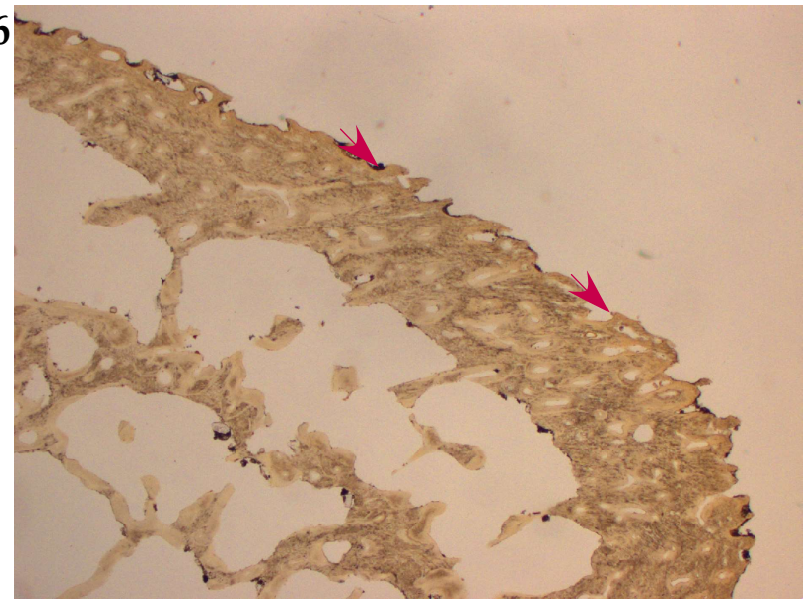

Bild 8

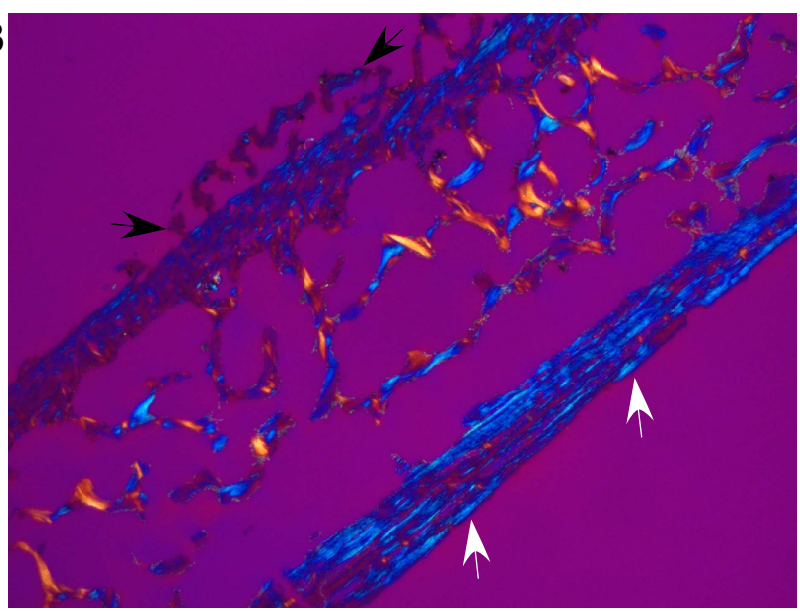




\section{Erklärungen zu Bildtafel 16}

Bild 1: Bácsalmás-Óalmás, Grab 436. Kind, 0 - 0,5 Jahre alt. Lichtmikroskopische Darstellung (Schliffdicke 70 $\mu$ m, kleiner Schliff, polarisiertes Durchlicht mit Hilfsobjekt Rot 1. Ordnung (Quarz) als Kompensator, Vergrößerung 25-fach): Detailaufnahme der bei Bildtafel 15, Bild 8 beschriebenen Auflagerungen (Pfeile) vom Typ I (hämorrhagisch) an der Lamina interna (unten). Die ursprüngliche Lamina interna lässt sich nicht eindeutig abgrenzen. Die feine Streifung des Faserknochens ist vermutlich durch Mikrokanälchen verursacht, in denen die Fortsätze der Osteozyten lagen. $\rightarrow$ organisierte epidurale Blutungen im Rahmen von Skorbut (siehe auch folgende Bilder und Bildtafel 15)

Bild 2: Bácsalmás-Óalmás, Grab 436. Kind, 0 - 0,5 Jahre alt. Lichtmikroskopische Darstellung (Schliffdicke 70 $\mu$ m, kleiner Schliff, polarisiertes Durchlicht mit Hilfsobjekt Rot 1. Ordnung (Quarz) als Kompensator, Vergrößerung 16-fach): Es handelt sich um eine weitere Übersichtsaufnahme des Präparats von Bild 1. Es zeigt Auflagerungen (Pfeil) vom Typ I (hämorrhagisch) an der Lamina interna (unten) im Suturbereich.

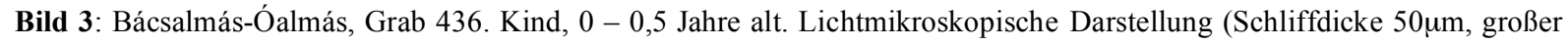
Schliff, einfaches Durchlicht, Vergrößerung 16-fach): Es handelt sich um ein weiteres Präparat aus dem rechten Os frontale (siehe auch Bildtafel 15, Bild 8). An der Lamina interna (unten) sind deutliche Auflagerungen (Pfeile) vom Typ I (hämorrhagisch) zu erkennen, die teilweise mehrlagig erscheinen. Die Lamina externa (oben) ist hier unauffällig. $\rightarrow$ organisierte epdurale Blutungen im Rahmen von Skorbut

Bild 4: Bácsalmás-Óalmás, Grab 436. Kind, 0 - 0,5 Jahre alt. Lichtmikroskopische Darstellung (Schliffdicke 70 4 m, einfaches Durchlicht, Vergrößerung 25-fach): Es handelt sich um ein Präparat aus der linken Ala major ossis sphenoidalis (makroskopische Darstellung siehe Bildtafel 15, Bild 3, 4 und 5). An der Lamina interna (oben) finden sich Reste einer Auflagerung (Pfeile) vom Typ I (hämorrhagisch), diese scheinen auch im Bereich der Sutur (links) vorzuliegen. $\rightarrow$ organisiertes subpericraniales Hämatom im Rahmen von Skorbut (siehe auch vorangegangene und folgende Bilder)

Bild 5: Bácsalmás-Óalmás, Grab 436. Kind, 0 - 0,5 Jahre alt. Lichtmikroskopische Darstellung (Schliffdicke 70um, polarisiertes Durchlicht mit Hilfsobjekt Rot 1. Ordnung (Quarz) als Kompensator, Vergrößerung 100-fach): Es handelt sich um ein Präparat aus der rechten Clavicula. Dargestellt sind Auflagerungen (Pfeile) vom Typ I (hämorrhagisch). $\rightarrow$ subperiostale Blutungen mit konsekutiver Knochenneubildung im Rahmen von Skorbut

Bild 6: Bácsalmás-Óalmás, Grab 436. Kind, 0 - 0,5 Jahre alt. Lichtmikroskopische Darstellung (Schliffdicke 70 $\mu \mathrm{m}$, polarisiertes Durchlicht mit Hilfsobjekt Rot 1. Ordnung (Quarz) als Kompensator, Vergrößerung 25-fach): Es handelt sich um ein Präparat aus dem rechten Os occipitale. Es sind Auflagerungen (Pfeil) vom Typ I an der Lamina interna zu sehen, insbesondere im Bereich der Sutura lambdoidea. $\rightarrow$ Hämorrhagisches Geschehen im Rahmen von Skorbut mit Knochenneubildung durch Periostreizung (siehe auch vorangegangene und folgende Bilder)

Bild 7: Bácsalmás-Óalmás, Grab 436. Kind, 0 - 0,5 Jahre alt. Lichtmikroskopische Darstellung (Schliffdicke 70um, polarisiertes Durchlicht mit Hilfsobjekt Rot 1. Ordnung (Quarz) als Kompensator, Vergrößerung 25-fach): Es handelt sich um ein Präparat aus der linken Scapula. Dargestellt sind Auflagerungen (Pfeil) vom Typ I (hämorrhagisch), die sich an der Außenseite befinden. Wie auch bei einigen anderen Präparaten dieses Individuums erscheinen die Trabekel der Substantia spongiosa sehr zart. Dies lässt sich auf das geringe Alter des Kindes zurückführen. $\rightarrow$ subperiostale Hämorrhagien mit nachfolgender Knochenneubildung im Rahmen von Skorbut (siehe auch vorige und folgende Bilder)

Bild 8: Bácsalmás-Óalmás, Grab 436. Kind, 0 - 0,5 Jahre alt. Lichtmikroskopische Darstellung (Schliffdicke 50 $\mu$, kleiner Schliff, polarisiertes Durchlicht mit Hilfsobjekt Rot 1. Ordnung (Quarz) als Kompensator, Vergrößerung 100-fach) der rechten Tibia. Es handelt sich um eine Detailaufnahme von Auflagerungen (1) vom Typ I (hämorrhagisch). Darunter finden sich regelmäßig angeordnete Gefäßforamina im Faserknochen (2), ganz unten ist Lamellenknochen (3) zu sehen. $\rightarrow$ organisiertes subperiostales Hämatom im Rahmen von Skorbut (siehe auch vorige Bilder, insbesondere Bildtafel 15, Bild 1, und Bildtafel 17) 


\section{Bildtafel 16}
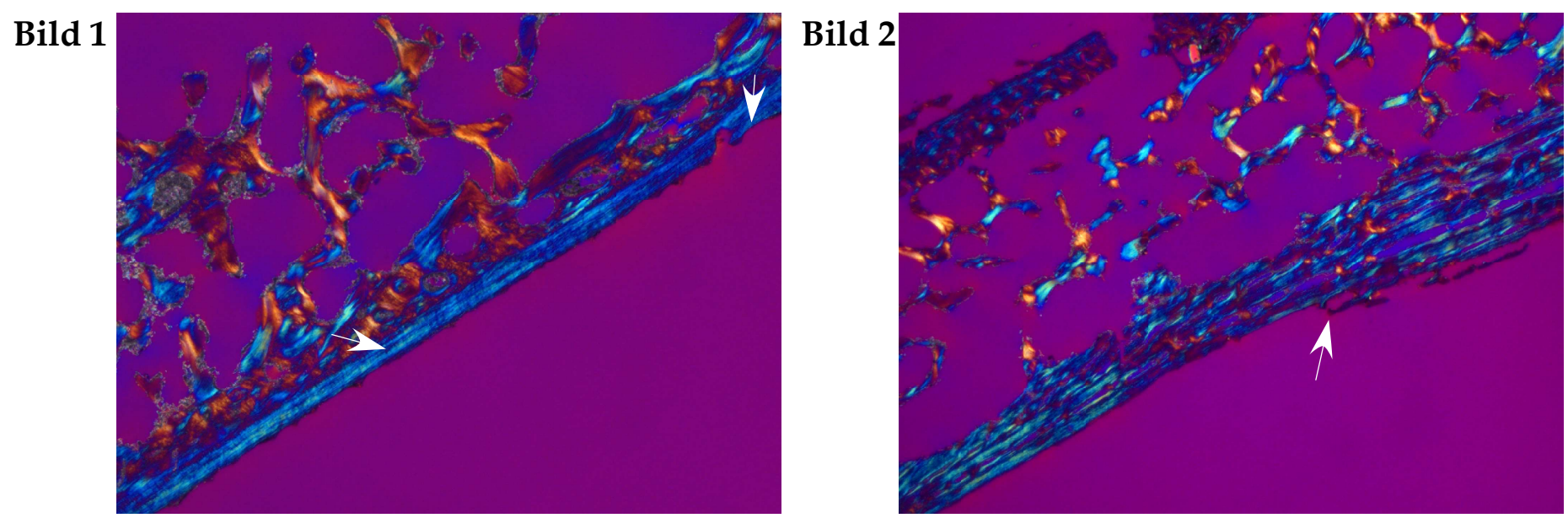

Bild 3

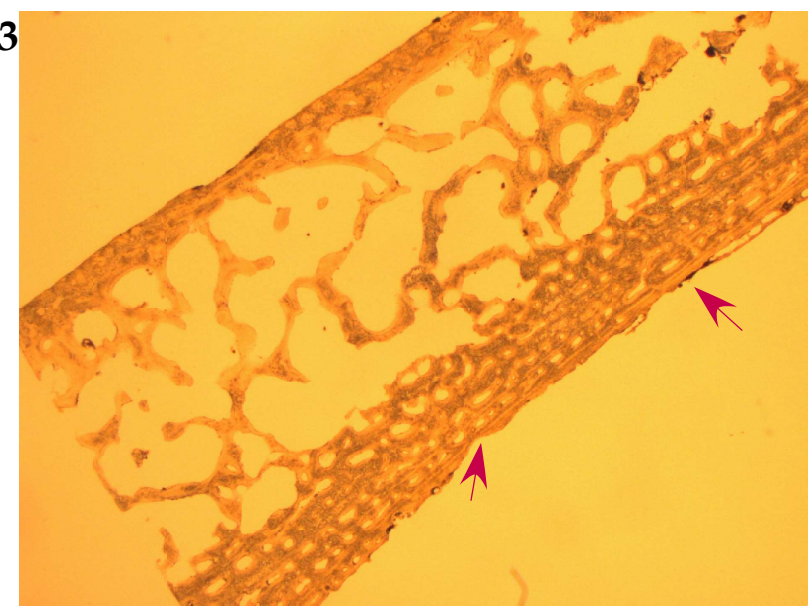

Bild 5

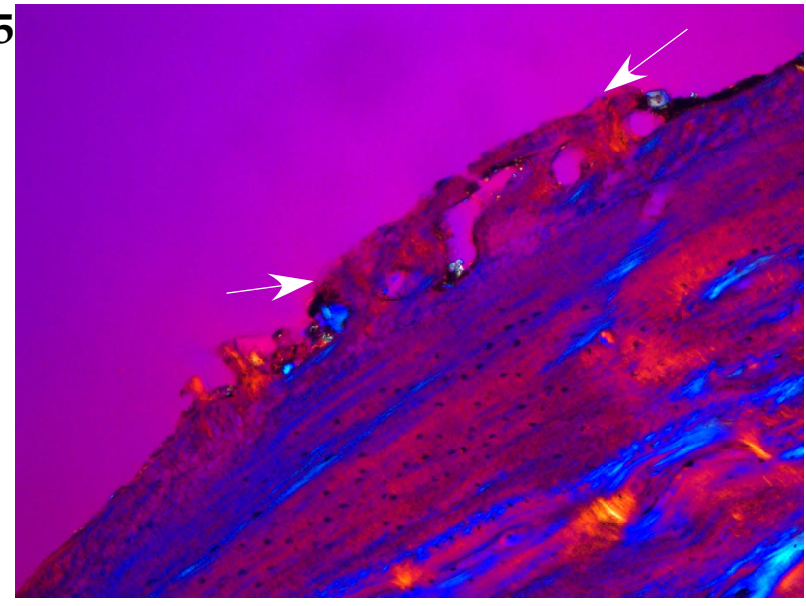

Bild 7

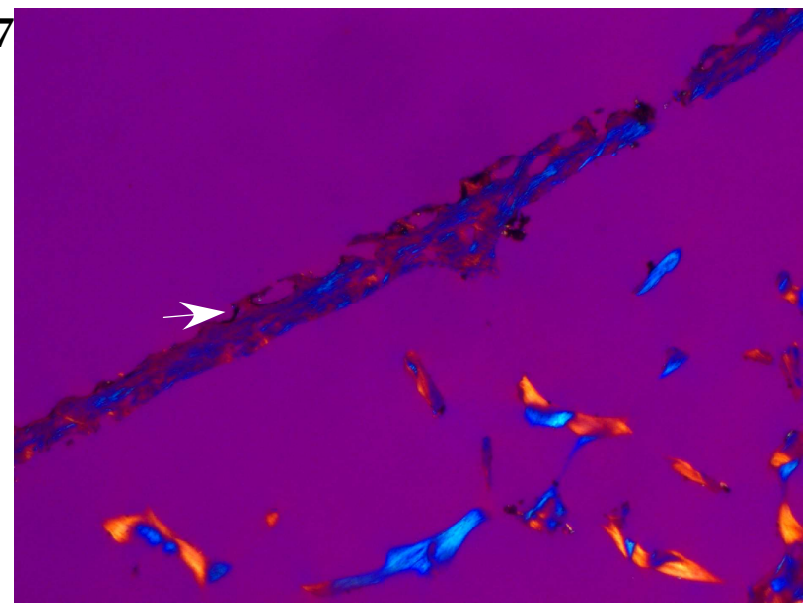

Bild 4

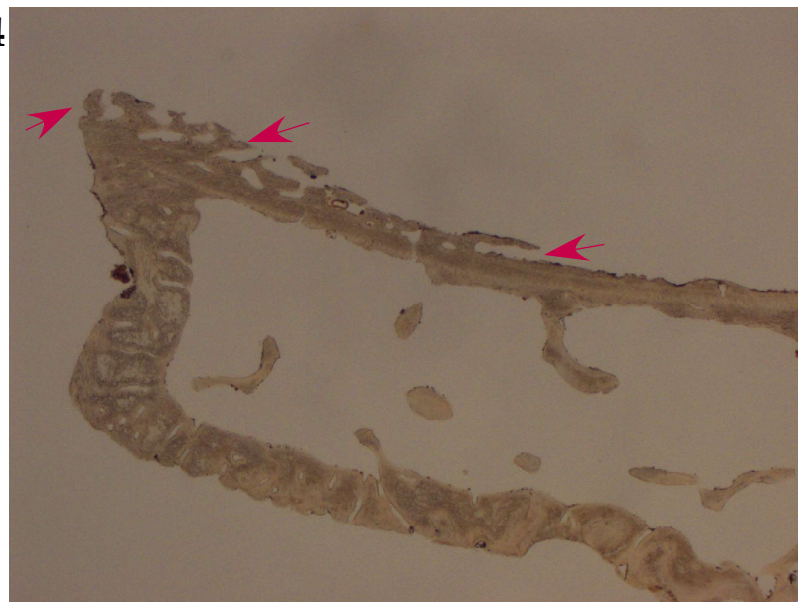

Bild 6

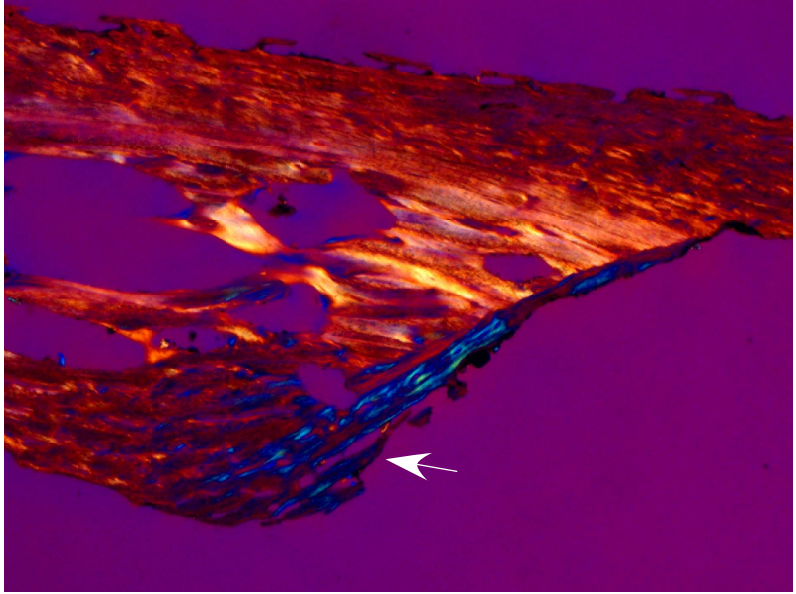

Bild 8

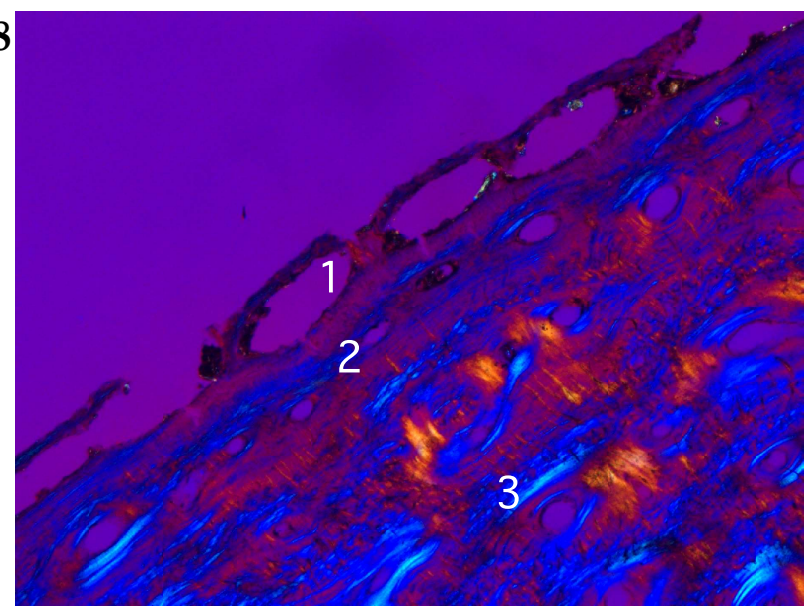




\section{Erklärungen zu Bildtafel 17}

Bild 1 Bácsalmás-Óalmás, Grab 436. Kind, 0 - 0,5 Jahre alt. Lichtmikroskopische Darstellung (Schliffdicke $70 \mu \mathrm{m}$, polarisiertes Durchlicht mit Hilfsobjekt Rot 1. Ordnung (Quarz) als Kompensator, Vergrößerung 25fach): Es handelt sich um den dicken Schliff aus der rechten Tibia (dünner Schliff siehe Bildtafel 16, Bild 8). Dargestellt ist eine Auflagerung (Pfeile) vom Typ I (hämorrhagisch). $\rightarrow$ hämorrhagisches Geschehen mit Knochenneubildung im Rahmen von Skorbut (siehe auch folgendes Bild und Bildtafel 16)

Bild 2: Bácsalmás-Óalmás, Grab 436. Kind, 0 - 0,5 Jahre alt. Lichtmikroskopische Darstellung (Schliffdicke $50 \mu \mathrm{m}$, polarisiertes Durchlicht mit Hilfsobjekt Rot 1. Ordnung (Quarz) als Kompensator, Vergrößerung 16fach): Das Präparat stammt aus dem linken Femur. Es zeigt den physologischen Schalenknochen eines Säuglings. Der Faserknochen ist unreif, im Bereich der Corticalis sind neue Knochenlagen schalenförmig angeordnet. Die äußerste Schicht wirkt unruhiger, dies ist zum Teil durch Erosion bedingt, es könnte sich jedoch auch um sehr diskrete Auflagerungen handeln. $\rightarrow$ physiologisches appositionelles Wachstum beim Säugling ohne sicheren Hinweis auf ein hämorrhagisches Geschehen (siehe auch vorangegangene Bilder)

Bild 3: Bácsalmás-Óalmás, Grab 437. Kind, 1 - 1,5 Jahre alt. Makroskopische Darstellung des linken Humerus. Am distalen Schaft befinden sich feinporöse Auflagerungen. $\rightarrow$ organisiertes subperiostales Hämatom unter chronischem Vitamin-C-Mangel (siehe auch folgende Bilder)

Bild 4: Bácsalmás-Óalmás, Grab 437. Kind, 1 - 1,5 Jahre alt. Makroskopische Aufnahme der fragmentierten rechten Mandibula. Auch hier finden sich massive feinporöse Auflagerungen $\rightarrow$ Hämorrhagisches Geschehen im Rahmen von Skorbut mit Knochenneubildung durch Periostreizung (siehe auch voriges und folgende Bilder)

Bild 5: Bácsalmás-Óalmás, Grab 437. Kind, 1 - 1,5 Jahre alt. Makroskopische Aufnahme des einzigen erhaltenen Occipitalfragments. Auch hier finden sich feinporöse Auflagerungen, die allerdings teilweise erodiert sind (siehe auch Bild 4 und folgende). $\rightarrow$ subperiostale Hämorrhagien mit nachfolgender Knochenneubildung in Folge schweren chronischen Vitamin-C-Mangels

Bild 6: Bácsalmás-Óalmás, Grab 437. Kind, 1 - 1,5 Jahre alt. Makroskopische Aufnahme der feinporösen Auflagerungen an der rechten Tibia. $\rightarrow$ subperiostale Blutungen mit nachfolgender Knochenneubildung im Rahmen von Skorbut (siehe auch vorangegangene und folgende Bilder )

Bild 7: Bácsalmás-Óalmás, Grab 437. Kind, 1 - 1,5 Jahre alt. Lichtmikroskopische Darstellung (Schliffdicke $70 \mu \mathrm{m}$, einfaches Durchlicht, Vergrößerung 16-fach): Es handelt sich um eine Übersichtsaufnahme eines Präparats aus dem linken Femur. Es fallen teilweise mehrlagige Auflagerungen (rosa Pfeile) vom Typ I (hämorrhagisch) auf. Die Linea aspera ist sehr ausgeprägt. Der Verlauf der Sehnenfasern lässt sich teilweise noch erkennen (grüne Pfeile). Dadurch entsteht der Eindruck, dass der Knochen unter Einwirkung eines kräftigen Muskelzugs gestanden hat. Vermutlich war das Kind schon in der Lage zu laufen. $\rightarrow$ subperiostale Hämorrhagien mit nachfolgender Knochenneubildung im Rahmen von Skorbut

Bild 8: Bácsalmás-Óalmás, Grab 437. Kind, 1 - 1,5 Jahre alt. Lichtmikroskopische Darstellung (Schliffdicke $70 \mu \mathrm{m}$, polarisiertes Durchlicht mit Hilfsobjekt Rot 1. Ordnung (Quarz) als Kompensator, Vergrößerung 25fach): Es handelt sich um ein Präparat aus der rechten Mandibula. Man erkennt zarte, gut organisierte Auflagerungen (Pfeil) vom Typ I (hämorrhagisch) an der Lamina externa, die im polarisierten Durchlicht als blaue Linie (Sterne) gut abgrenzbar ist. $\rightarrow$ organisiertes subperiostales Hämatom im Rahmen von Skorbut (siehe auch vorige Bilder und Bildtafel 18, Bild 1) 


\section{Bildtafel 17}

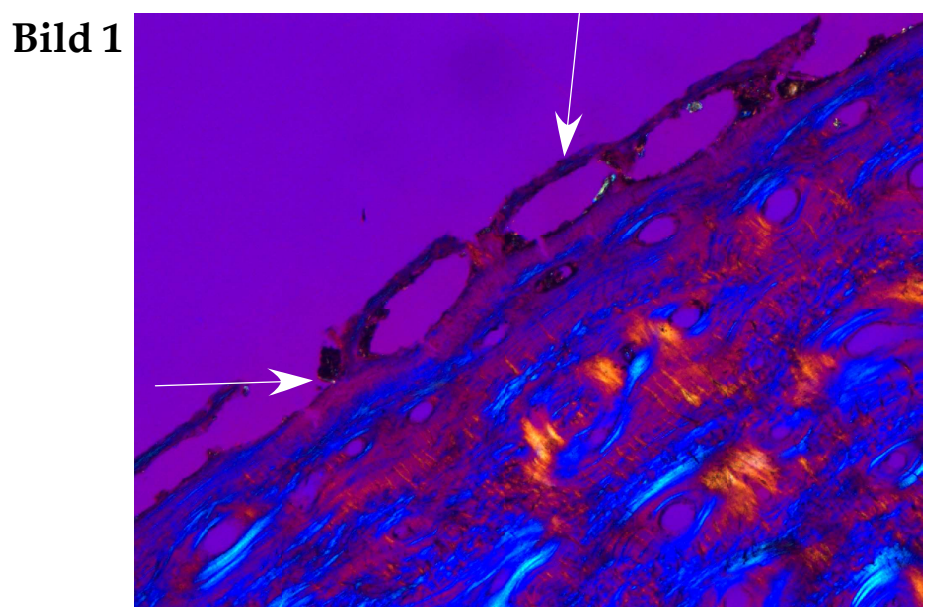

Bild 3

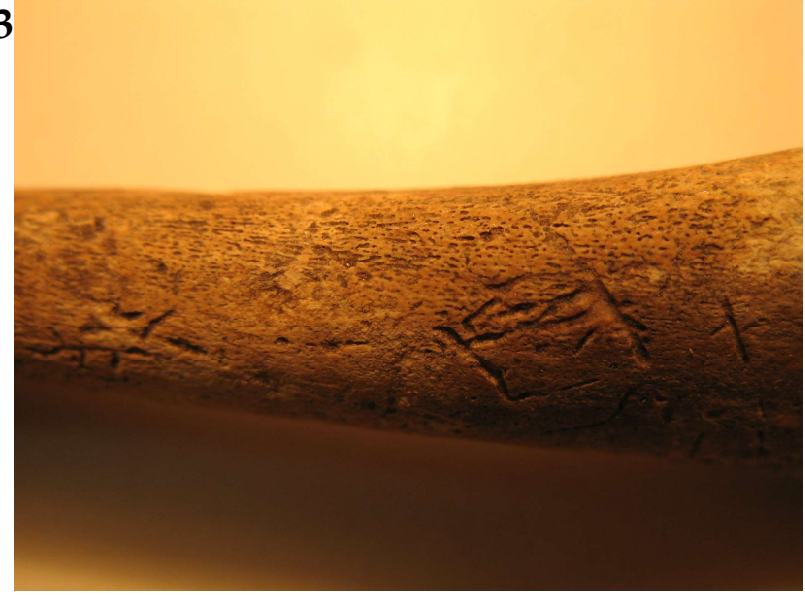

Bild 5

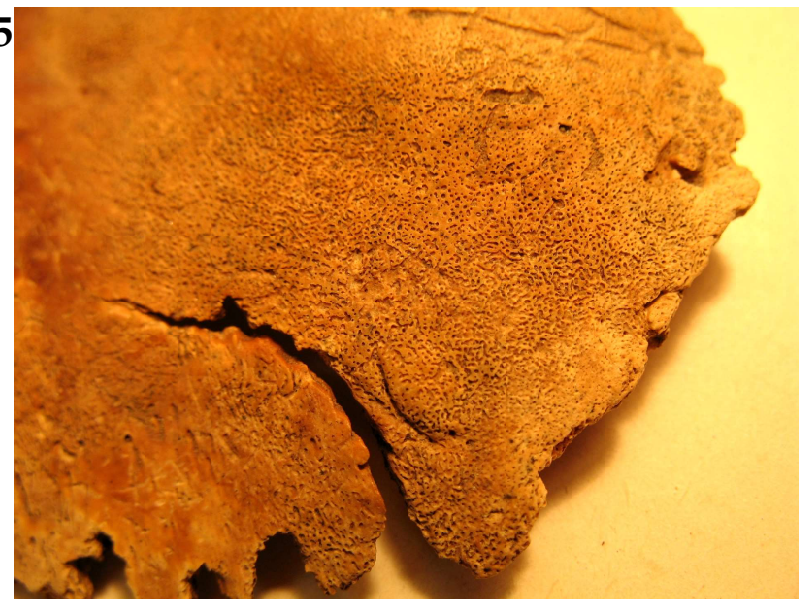

\section{Bild 7}

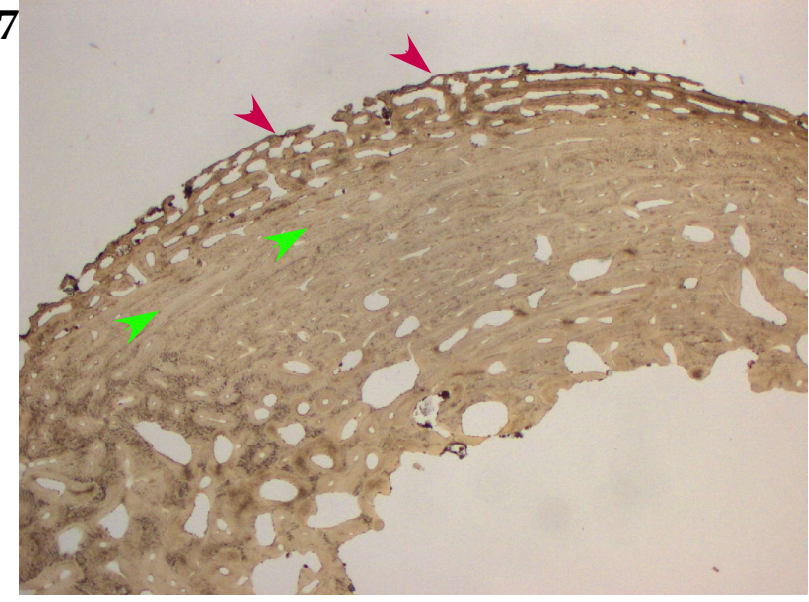

Bild 2

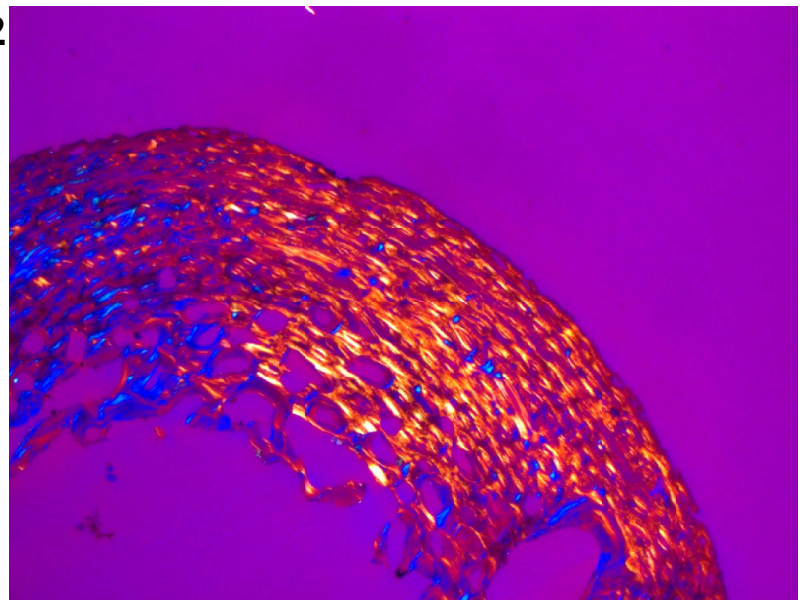

Bild 4

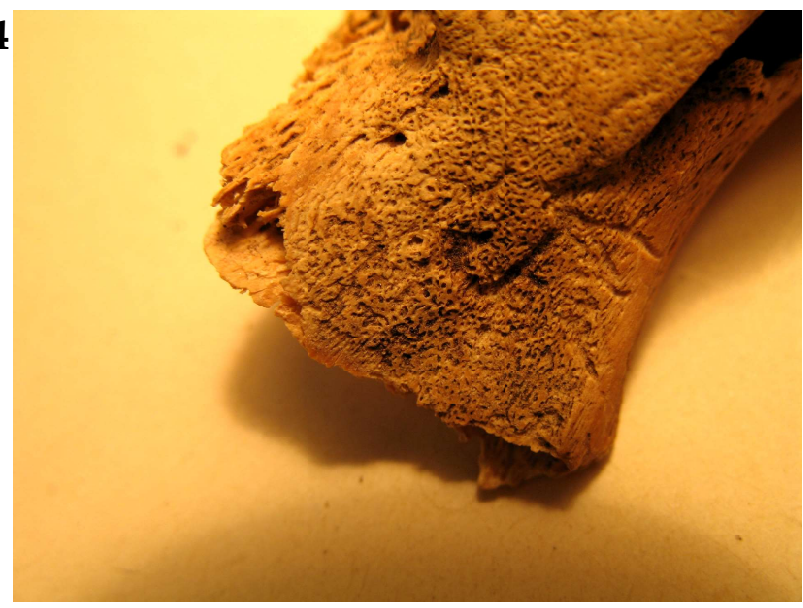

Bild 6

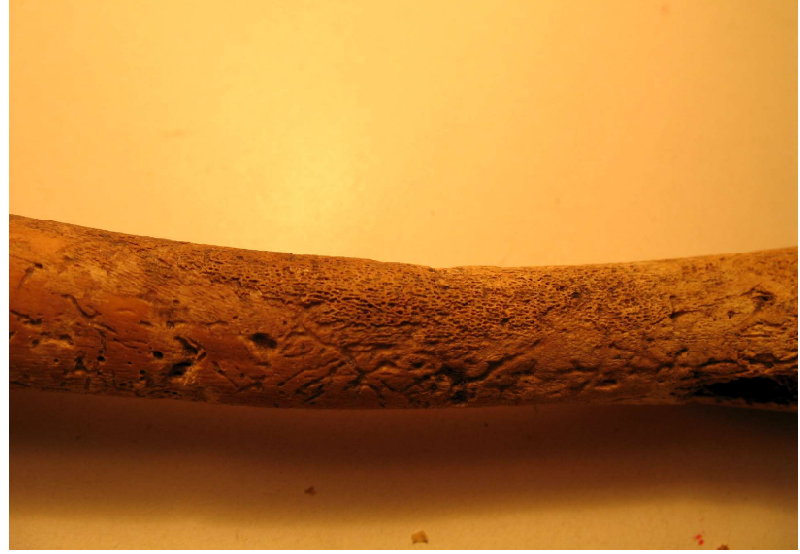

Bild 8

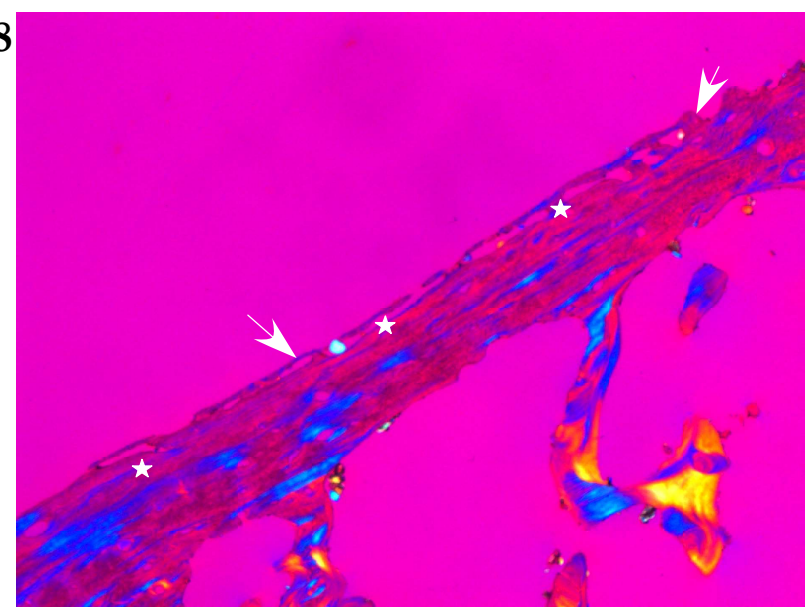




\section{Erklärungen zu Bildtafel 18}

Bild 1 Bácsalmás-Óalmás, Grab 437. Kind, 1 - 1,5 Jahre alt. Lichtmikroskopische Darstellung (Schliffdicke $70 \mu \mathrm{m}$, polarisiertes Durchlicht mit Hilfsobjekt Rot 1. Ordnung (Quarz) als Kompensator, Vergrößerung 25fach): Es handelt sich um eine Detailaufnahme des Präparats aus der rechten Mandibula (siehe Bildtafel 17, Bild 8). Hier ist die Lamina externa (Pfeil) unterhalb der Auflagerung vom Typ I (hämorrhagisch) deutlich zu erkennen. $\rightarrow$ hämorrhagisches Geschehen mit Knochenneubildung im Rahmen von Skorbut

Bild 2: Bácsalmás-Óalmás, Grab 439. Kind, 1 - 1,5 Jahre alt. Makroskopische Aufnahme des linken Humerus: Der Schaft ist von feinporösen Auflagerungen bedeckt. $\rightarrow$ organisiertes subperiostales Hämatom im Rahmen von Skorbut (siehe auch folgende Bilder)

Bild 3: Bácsalmás-Óalmás, Grab 439. Kind, 1 - 1,5 Jahre alt. Makroskopische Darstellung der rechten Tibia. Am distalen Schaft befinden sich feinporöse Auflagerungen. $\rightarrow$ organisiertes subperiostales Hämatom unter chronischem Vitamin-C-Mangel (siehe auch voriges und folgende Bilder)

Bild 4: Bácsalmás-Óalmás, Grab 439. Kind, 1 - 1,5 Jahre alt. Makroskopische Aufnahme eines Rippenfragments. Auch hier finden sich feinporöse Auflagerungen $\rightarrow$ Hämorrhagische Pleuritis im Rahmen von Skorbut mit Knochenneubildung (siehe auch voriges und folgende Bilder)

Bild 5: Bácsalmás-Óalmás, Grab 439. Kind, 1 - 1,5 Jahre alt. Lichtmikroskopische Darstellung (Schliffdicke $50 \mu \mathrm{m}$, einfaches Durchlicht, Vergrößerung 16-fach): Das Präparat aus der rechten Ulna zeigt in der Übersichtsaufnahme eine Auflagerung vom Typ I (hämorrhagisch). Die Corticalis (Sterne) darunter ist diagenetisch verfärbt, was den falschen Eindruck erweckt, die Auflagerung sei mehrlagig. $\rightarrow$ organisiertes subperiostales Hämatom im Rahmen von Skorbut (siehe auch andere Bilder dieses Individuums)

Bild 6: Bácsalmás-Óalmás, Grab 439. Kind, 1 - 1,5 Jahre alt. Lichtmikroskopische Darstellung (Schliffdicke

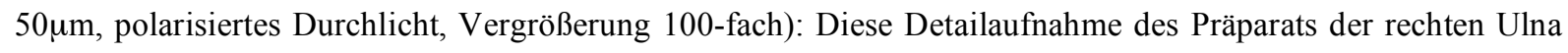
(siehe voriges Bild) zeigt das Herauswachsen der Auflagerung aus der Corticalis (Sterne). Das ermöglicht eine Abgrenzung gegen die Differentialdiagnose appositionelles Wachstum, in dem Fall würde sich die neue Knochensubstanz stärker um bereits bestehende Gefäßforamina anordnen. Außerdem fällt eine fachwerkartige Anordnung der Trabekel in der Substantia spongiosa auf. $\rightarrow$ subperiostale Blutungen mit nachfolgender Knochenneubildung im Rahmen von Skorbut (siehe auch folgendes Bild)

Bild 7: Bácsalmás-Óalmás, Grab 439. Kind, 1 - 1,5 Jahre alt. Lichtmikroskopische Darstellung (Schliffdicke 50 $\mathrm{m}$, polarisiertes Durchlicht mit Hilfsobjekt Rot 1. Ordnung (Quarz) als Kompensator, Vergrößerung 100fach): Es handelt sich um eine weitere Detailaufnahme des Ulna-Präparats (siehe vorige Bilder). Dargestellt ist eine Resorptionshöhle. Auf zwölf, drei und sechs Uhr sind Howship'sche Lakunen (Pfeile) zu sehen. $\rightarrow$ aktiver resorbierender Prozess

Bild 8: Bácsalmás-Óalmás, Grab 451. Kind, 2,5 - 3 Jahre alt. Makroskopische Detailaufnahme des Os frontale. Am Dach der linken Orbita sind massive, gut integrierte, feinporöse Auflagerungen zu erkennen. $\rightarrow$ Cribra orbitalia Grad 3 im Rahmen von Skorbut im beginnenden Ausheilungsstadium (weitere Bilder des Individuums siehe Bildtafel 19, lichtmikroskopische Darstellung der Orbita Bildtafel 19, Bild 4 und 5) 


\section{Bildtafel 18}

Bild 1

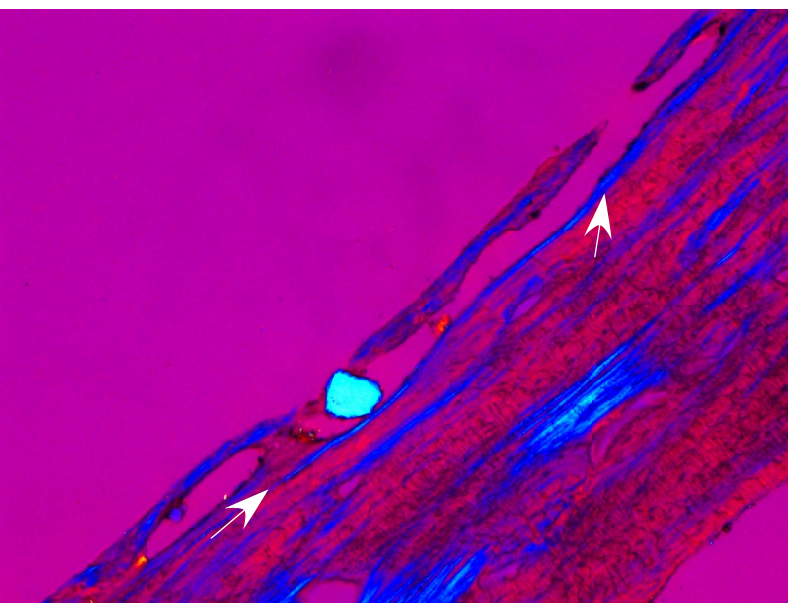

Bild 3

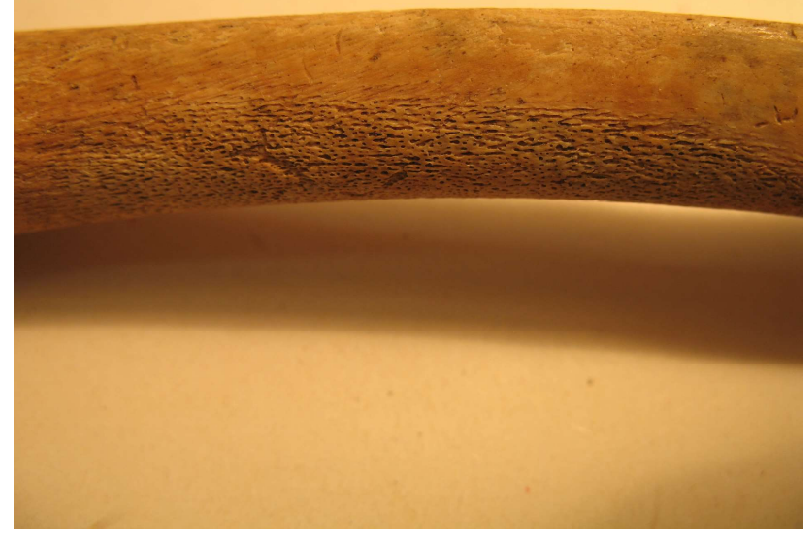

Bild 5

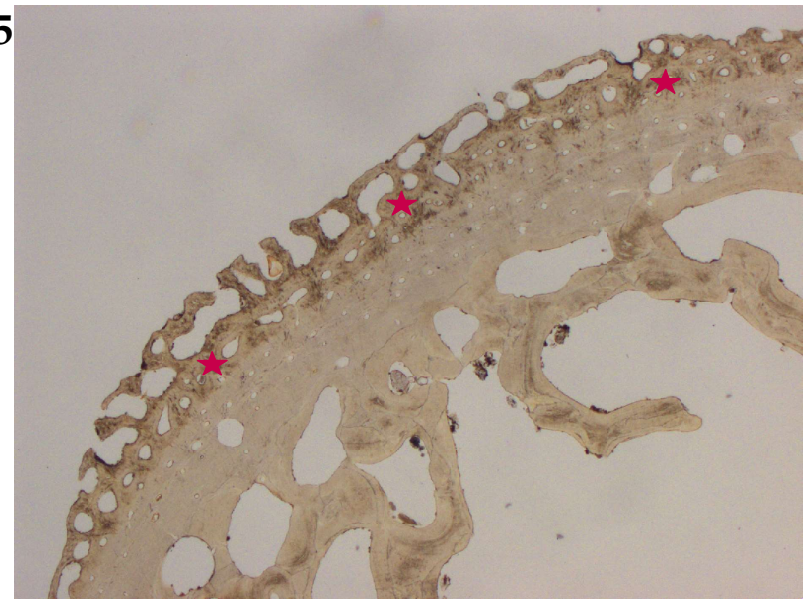

Bild 7

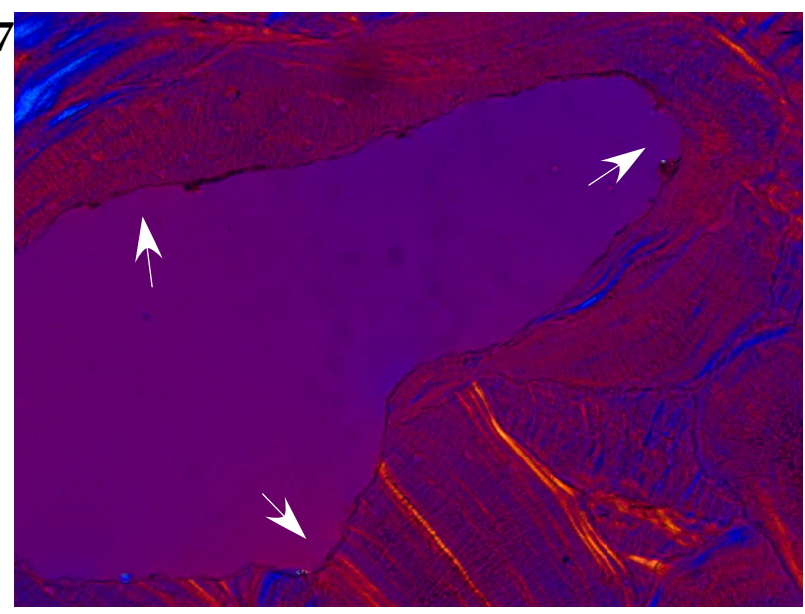

Bild 2

Bild 4

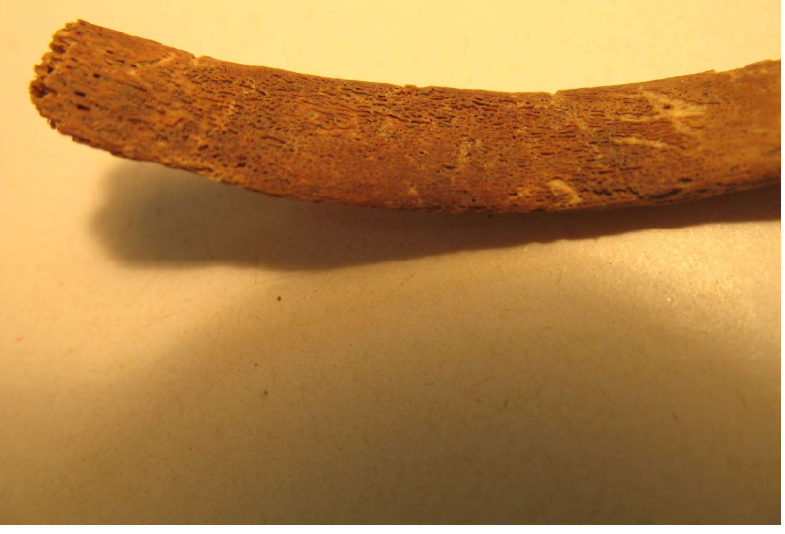

Bild 6

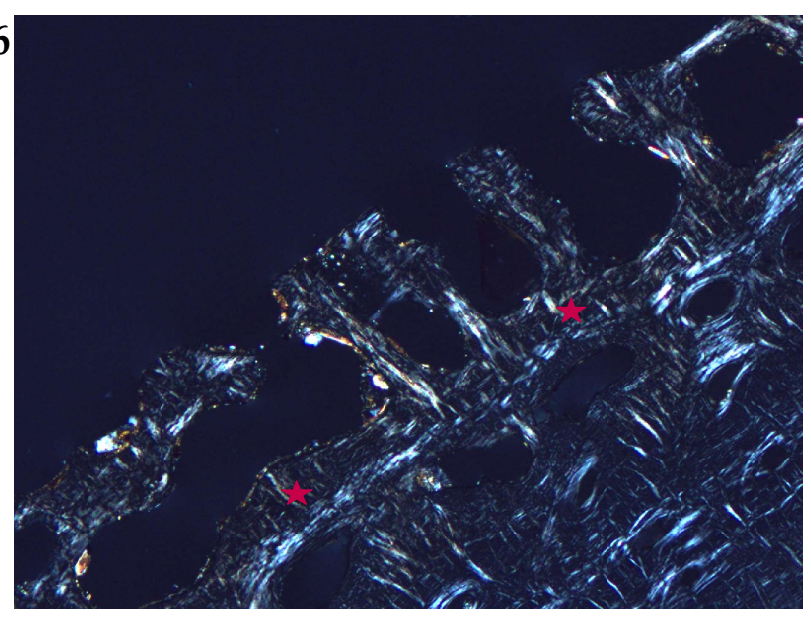

Bild 8

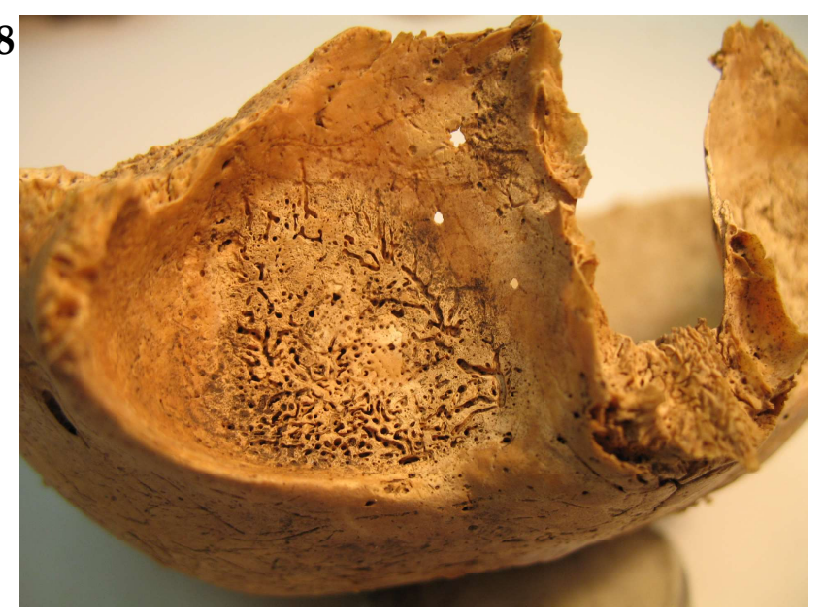




\section{Erklärungen zu Bildtafel 19}

Bild 1 Bácsalmás-Óalmás, Grab 451. Kind, 2,5 - 3 Jahre alt. Makroskopische Aufnahme des linken Os parietale: An der Lamina interna sind flächige feinporöse Auflagerungen zu erkennen. $\rightarrow$ hämorrhagisches Geschehen mit Knochenneubildung bei chronischem Vitamin-C-Mangel (siehe auch Bildtafel 18, Bild 8, und folgende Bilder)

Bild 2: Bácsalmás-Óalmás, Grab 451. Kind, 2,5 - 3 Jahre alt. Makroskopische Detailaufnahme der rechten Ala major ossis sphenoidalis: An der Facies orbitalis ist eine feinporöse Auflagerung mit deutlicher Gefäßzeichnung zu sehen. $\rightarrow$ organisiertes subpericraniales Hämatom im Rahmen von Skorbut (siehe auch weitere Bilder)

Bild 3: Bácsalmás-Óalmás, Grab 451. Kind, 2,5 - 3 Jahre alt. Weitere makroskopische Detailaufnahme der rechten Ala major ossis sphenoidalis: Auch die Facies temporalis ist mit feinporösen Auflagerungen bedeckt. $\rightarrow$ subpericraniale Blutungen mit konsekutiver Knochenneubildung im Rahmen von Skorbut (siehe vorige und folgende Bilder)

Bild 4: Bácsalmás-Óalmás, Grab 451. Kind, 2,5 - 3 Jahre alt. Lichtmikroskopische Darstellung (Schliffdicke 70 $\mu \mathrm{m}$, polarisiertes Durchlicht, Vergrößerung 25-fach) des Orbitadachs: Detailaufnahme einer Auflagerung vom Typ I (hämorrhagisch), deren Deckplatte durch Erosion großenteils zerstört ist. Neubildung (Pfeile) und ursprüngliche Knochensubstanz (Sterne) lassen sich gut abgrenzen. $\rightarrow$ Cribra orbitalia im Rahmen von Skorbut (siehe auch vorangegangene und folgende Bilder, makroskopische Darstellung Bildtafel 18, Bild 8)

Bild 5: Bácsalmás-Óalmás, Grab 451. Kind, 2,5 - 3 Jahre alt. Lichtmikroskopische Darstellung (Schliffdicke 70um, polarisiertes Durchlicht mit Hilfsobjekt Rot 1. Ordnung (Quarz) als Kompensator, Vergrößerung 25-fach): Es handelt sich um eine Detailaufnahme des Orbita-Präparats (siehe Bild 4). Dargestellt ist der Randbereich der Auflagerung (Pfeile) vom Typ I (hämorrhagisch). Am rechten Bildrand sind eingebrochene Knochenbälkchen (Sterne) zu sehen, was wohl auf den Druck durch das Hämatom zurückzuführen ist. $\rightarrow$ Cribra orbitalia im Rahmen von Skorbut (makroskopische Darstellung siehe Bildtafel 18, Bild 8)

Bild 6: Bácsalmás-Óalmás, Grab 451. Kind, 2,5 - 3 Jahre alt. Lichtmikroskopische Darstellung (Schliffdicke 70um, einfaches Durchlicht, Vergrößerung 16-fach): Es handelt sich um eine Übersichtsaufnahme eines Präparats des linken Os parietale. An der Lamina externa (oben) befinden sich kompakte Auflagerungen (rosa Pfeile) vom Typ I (hämorrhagisch). An der Lamina interna (unten) fallen tiefe Impressiones digitatae (grüne Pfeile) auf, die im Rahmen intracranialer Druckerhöhung, beispielsweise durch Blutergüsse, entstanden sein könnten. $\rightarrow$ Knochenneubildung infolge subpericranialer Hämorrhagien bei Skorbut (siehe auch folgende Bilder und Bild 1)

Bild 7: Bácsalmás-Óalmás, Grab 451. Kind, 2,5 - 3 Jahre alt. Lichtmikroskopische Darstellung (Schliffdicke 70um, polarisiertes Durchlicht mit Hilfsobjekt Rot 1. Ordnung (Quarz) als Kompensator, Vergrößerung 16-fach): Es handelt sich um eine weitere Übersichtsaufnahme des Os parietale (siehe Bild 6). Hier ist an der Lamina interna der Sulcus sinus parietale (Pfeile) angeschnitten, der mit Auflagerungen (Sterne) vom Typ I (hämorrhagisch) gefüllt ist. $\rightarrow$ subperiostale Hämorrhagien mit nachfolgender Knochenneubildung im Rahmen von Skorbut (siehe auch Bild 8)

Bild 8: Bácsalmás-Óalmás, Grab 451. Kind, 2,5 - 3 Jahre alt. Lichtmikroskopische Darstellung (Schliffdicke 70um, einfaches Durchlicht, Vergrößerung 100-fach): Es handelt sich um eine Detailaufnahme der Auflagerungen vom Typ I (hämorrhagisch) an der Lamina externa des Os parietale (siehe Bild 6). Hier ist die Schichtung der Knochenstrukturen gut zu erkennen: Außen Auflagerung (AL), darunter die ehemalige Lamina externa (Sterne), dann Faserknochen, ganz unten Lamellenknochen (nur angeschnitten) $\rightarrow$ organisierte subpericraniale Blutungen im Rahmen von Skorbut 


\section{Bildtafel 19}
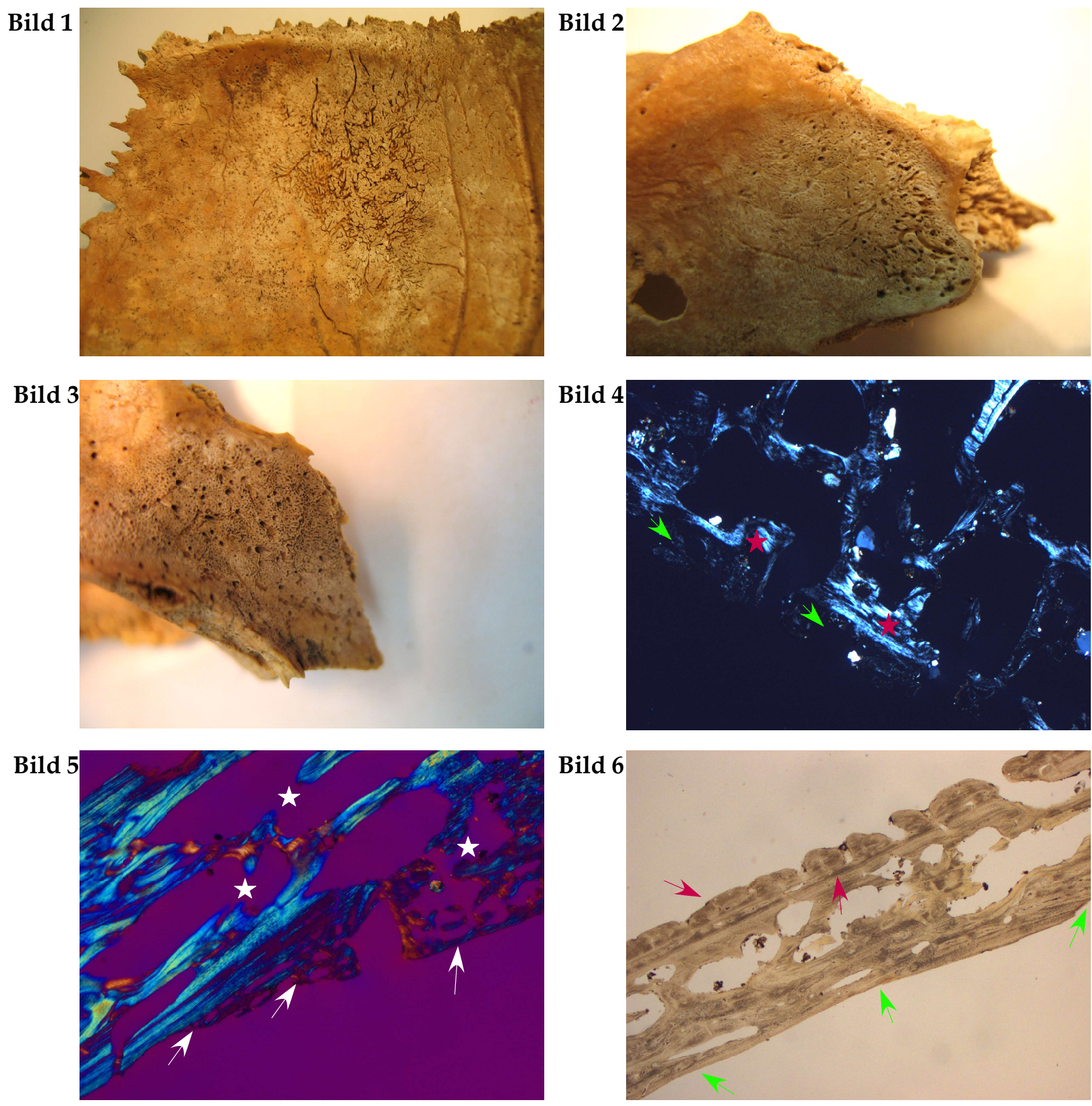

Bild 6
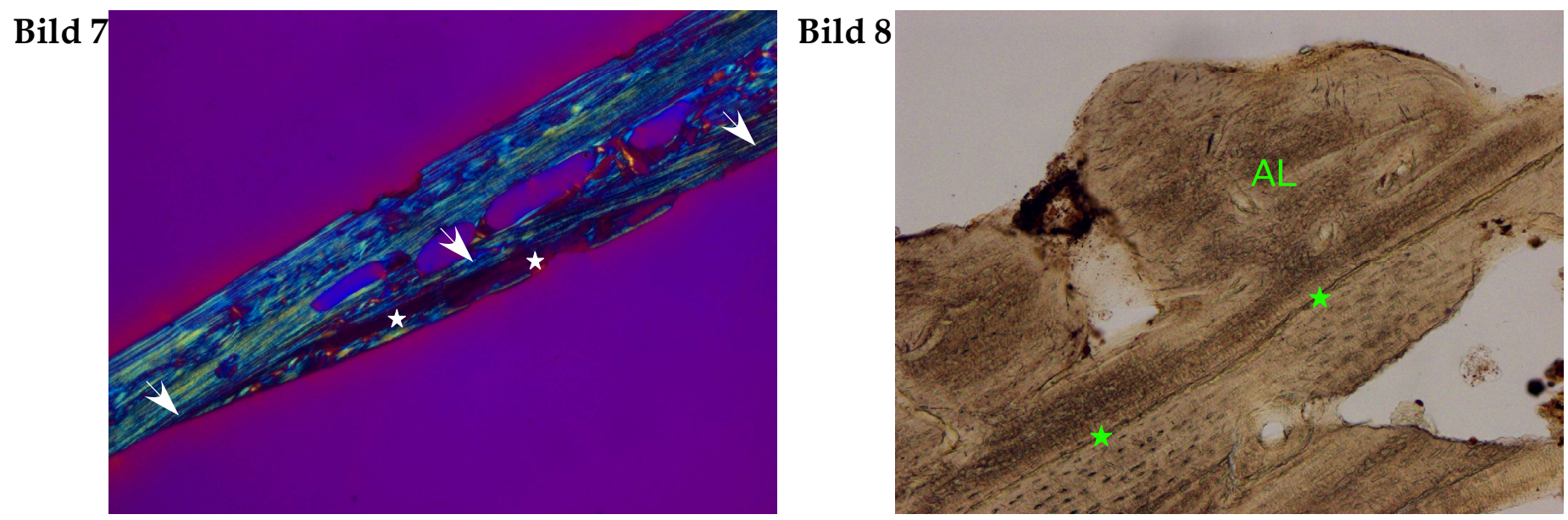


\section{Erklärungen zu Bildtafel 20}

Bild 1 Kiszombor 299, Kind, ca. 6 Jahre alt. Makroskopische Aufnahme des Schädels: Es fallen gewaltige spongiöse Hyperostosen an den Tubera parietalia auf. $\rightarrow$ „Bürstenschädel“ im Rahmen einer schweren Anämie

Bild 2: Kiszombor 299, Kind, ca. 6 Jahre alt. Lichtmikroskopische Darstellung (Schliffdicke 70 4 m, polarisiertes Durchlicht mit Hilfsobjekt Rot 1. Ordnung (Quarz) als Kompensator, Vergrößerung 16fach): Es handelt sich um ein Präparat aus dem rechten Os parietale (siehe Bild 1). Die linke Bildseite wird eingenommen von einem Bürstensaum an der ehemaligen Lamina externa (Stern), entsprechend dem Bild eine Auflagerung vom Typ II (anämisch). $\rightarrow$ „Bürstenschädel“ im Rahmen einer schweren Anämie

Bild 3: Sükösd-Ságod, 134, Kind, ca. 4 Jahre alt. Makroskopische Aufnahme des linken Orbitadachs: Es sind massive feinporöse Auflagerungen mit deutlicher Gefäßzeichnung zu erkennen $\rightarrow$ Cribra orbitalia Grad 3 im Rahmen von Skorbut (siehe auch folgende Bilder)

Bild 4: Sükösd-Ságod, 134, Kind, ca. 4 Jahre alt. Makroskopische Detailaufnahme des linken Ramus mandibulae. Im Bereich der Icisurae mandibulae sind feinporöse Auflagerungen zu sehen.

Hämorrhagisches Geschehen im Rahmen von Skorbut mit Knochenneubildung durch Periostreizung (siehe auch andere Bilder dieses Individuums)

Bild 5: Sükösd-Ságod, 134, Kind, ca. 4 Jahre alt. Makroskopische Detailaufnahme der linken Maxilla. An der anterolateralen Fläche befinden sich feinporöse Auflagerungen. $\rightarrow$ Knochenneubildung infolge subperiostaler Blutungen bei Skorbut (siehe auch andere Bilder des Individuums)

Bild 6: Sükösd-Ságod, 134, Kind, ca. 4 Jahre alt. Makroskopische Detailaufnahme des Os occipitale. An der Lamina interna finden sich im Sulcus sinus occipitalis feinporöse Auflagerungen mit deutlicher Gefäßzeichnung. $\rightarrow$ subpericraniale Blutungen mit nachfolgender Knochenneubildung im Rahmen von Skorbut (siehe weitere Bilder des Individuums)

Bild 7 Sükösd-Ságod, 134, Kind, ca. 4 Jahre alt. Makroskopische Aufnahme der Lamina interna des linken Os parietale. Die Impressiones digitatae sind mit feinporösen Auflagerungen verfüllt. $\rightarrow$ subpericraniale Hämorrhagien mit nachfolgender Knochenneubildung im Rahmen von Skorbut

Bild 8: Sükösd-Ságod, 134, Kind, ca. 4 Jahre alt. Lichtmikroskopische Darstellung (Schliffdicke $70 \mu \mathrm{m}$, polarisiertes Durchlicht mit Hilfsobjekt Rot 1. Ordnung (Quarz) als Kompensator, Vergrößerung 25-fach): Es handelt sich um eine Detailaufnahme von Auflagerungen vom Typ I (hämorrhagisch) an der Lamina externa (Sterne) des linken Os frontale. Die Auflagerung besteht aus zwei Lagen (1, 2), wobei die tiefere Schicht (2) diploëisiert erscheint. $\rightarrow$ organisierte subpericraniale Blutungen im Rahmen von Skorbut (siehe auch vorangegangene Bilder und Bildtafel 21) 


\section{Bildtafel 20}

Bild 1

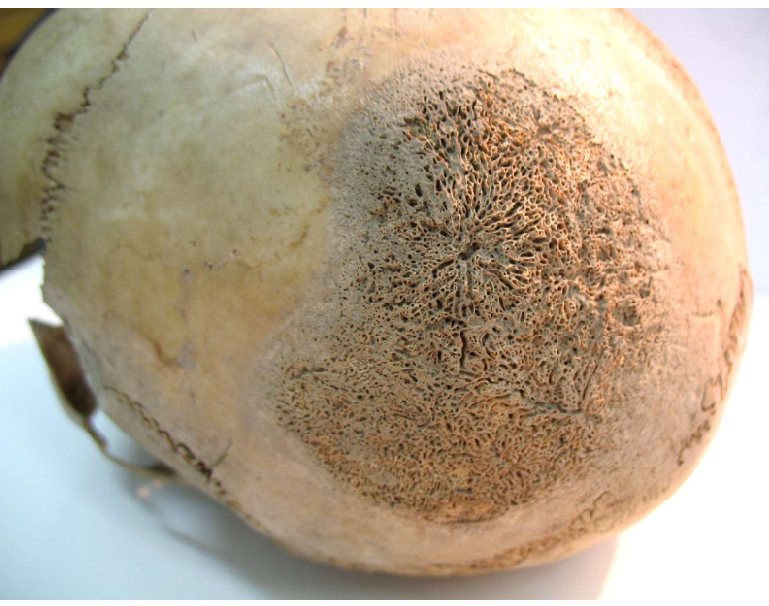

Bild 3

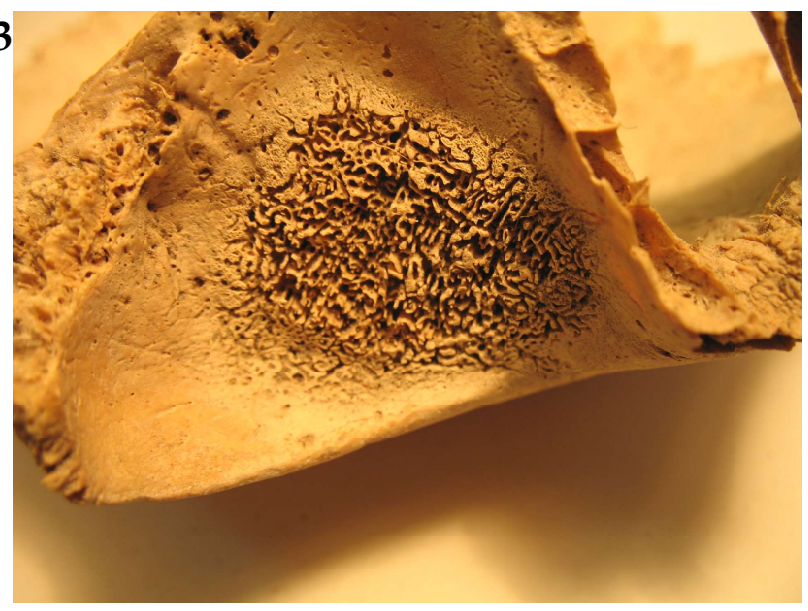

Bild 5

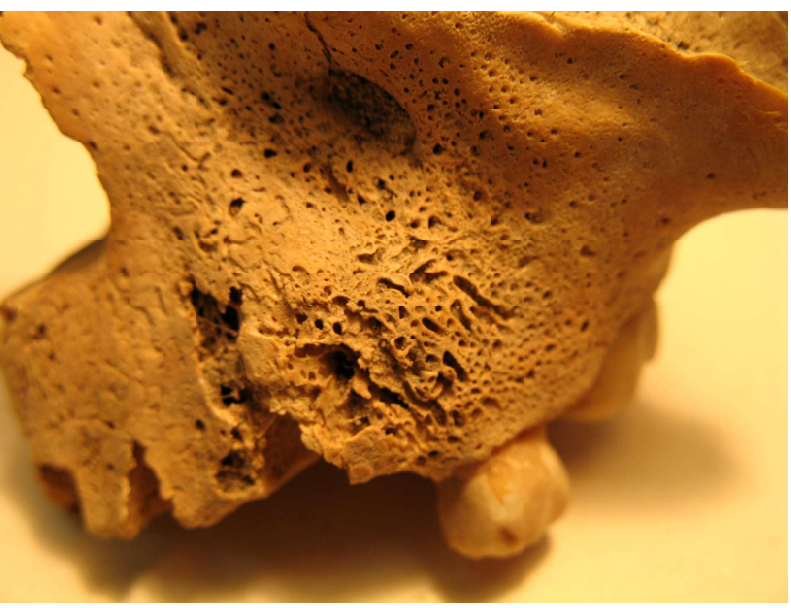

Bild 7

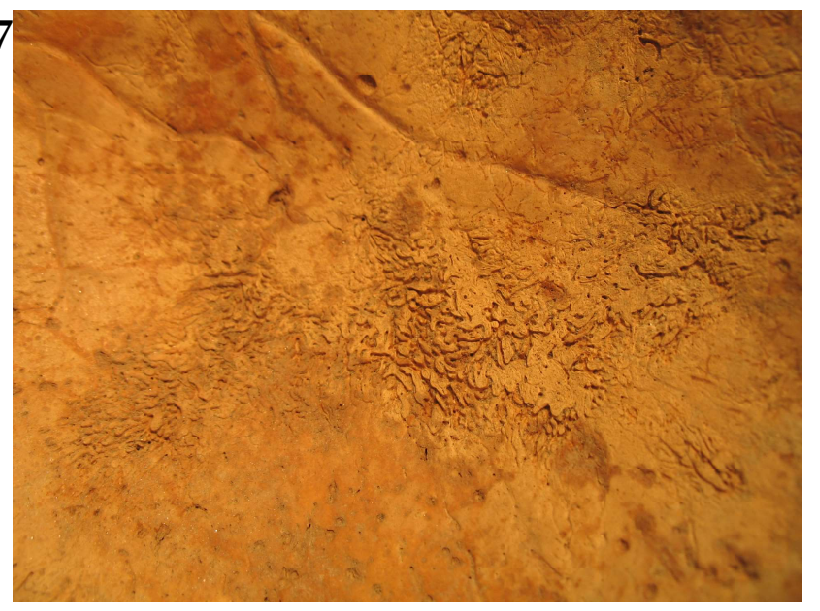

Bild 2

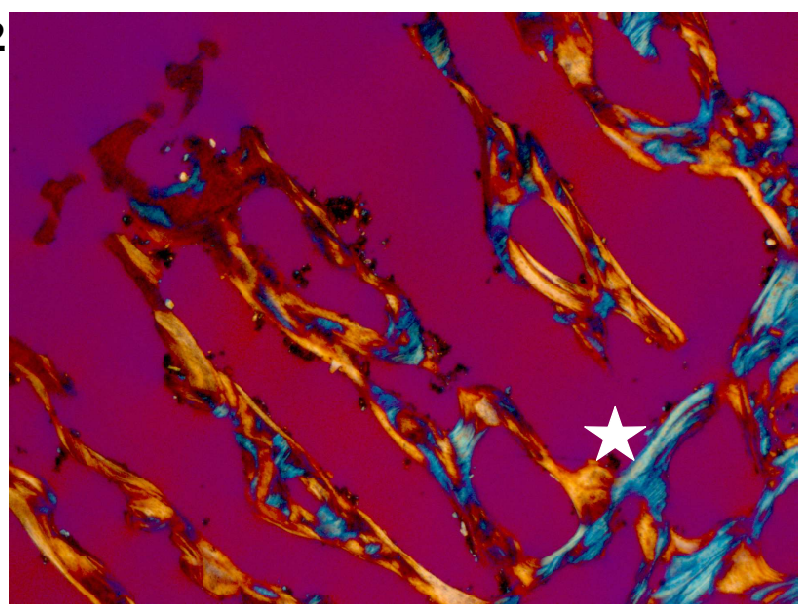

Bild 4

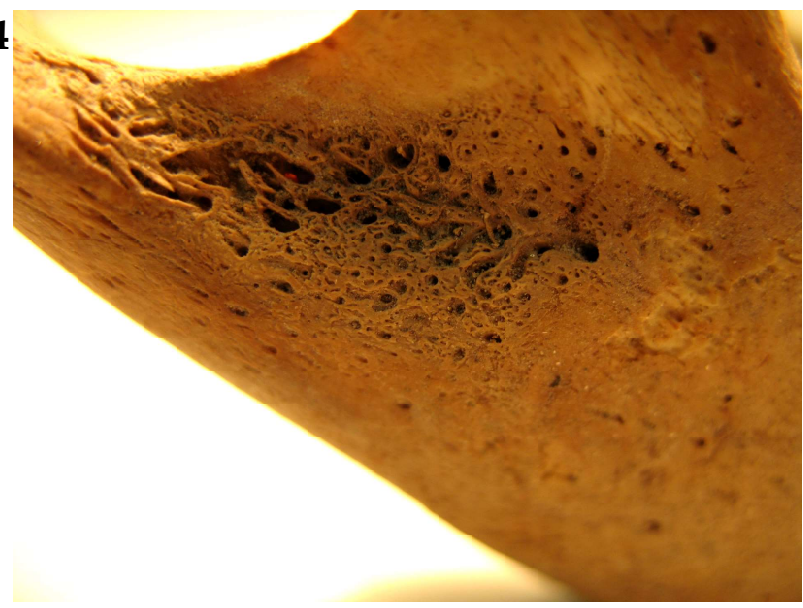

Bild 6

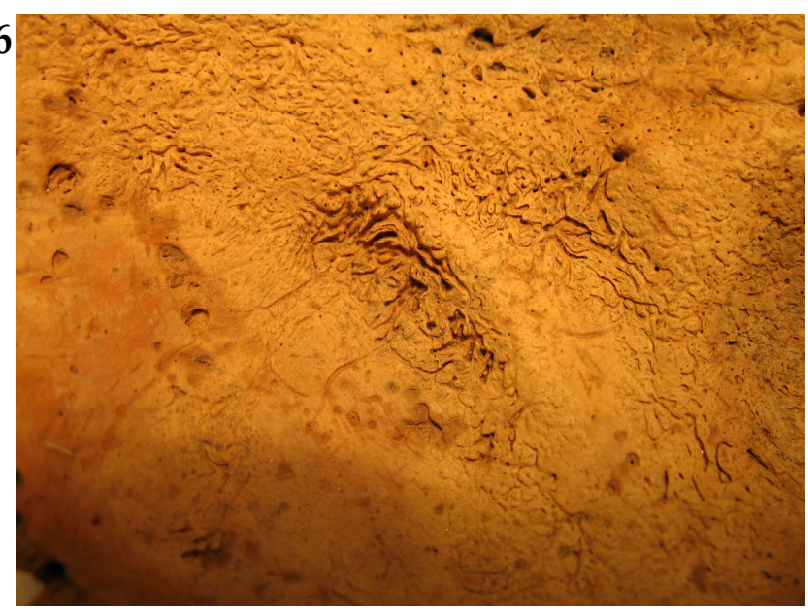

Bild 8

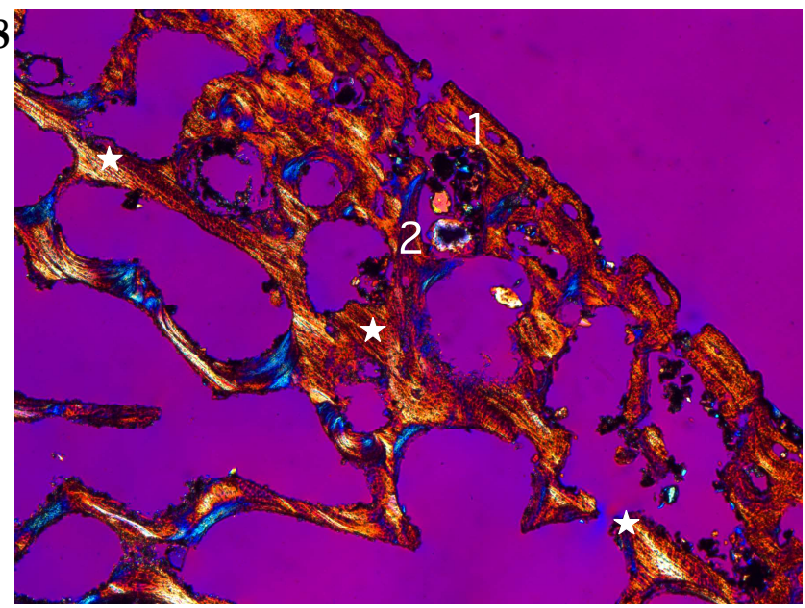




\section{Erklärungen zu Bildtafel 21}

Bild 1 Sükösd-Ságod, 134, Kind, ca. 4 Jahre alt. Lichtmikroskopische Darstellung (Schliffdicke 50um, einfaches Durchlicht, Vergrößerung 16-fach): Es handelt sich um ein Präparat des linken Os parietale, an dessen Lamina interna (unten) Auflagerungen vom Typ I (hämorrhagisch) liegen. Gut zu erkennen ist die Dreischichtung des Knochens: innen Auflagerung (1), daran grenzen unreifer Faserknochen (2) und schließlich besser organisierter Knochen außen (3). $\rightarrow$ organisiertes epidurales Hämatom im Rahmen von Skorbut (siehe folgende Bilder und Bildtafel 20)

Bild 2: Sükösd-Ságod, 134, Kind, ca. 4 Jahre alt. Lichtmikroskopische Darstellung (Schliffdicke 50um, polarisiertes Durchlicht, Vergrößerung 16-fach): Es handelt sich um ein Präparat des rechten Orbitadachs. Dargestellt ist der Bereich des Stirnrandes. Hier scheint es zu einer Dopplung der äußeren Lamelle durch Einlagerung größerer Blutmarkräume (Pfeile) zu kommen. Außerdem sind Auflagerungen (Sterne) vom Typ I (hämorrhagisch) zu erkennen. $\rightarrow$ Cribra orbitalia im Rahmen von Skorbut (siehe auch andere Bilder des Individuums)

Bild 3: Sükösd-Ságod, 134, Kind, ca. 4 Jahre alt. Lichtmikroskopische Darstellung (Schliffdicke 50um, einfaches Durchlicht, Vergrößerung 16-fach): Das Präparat des linken Os occipitale zeigt den Sulcus sinus occipitalis, der mit mehrlagigen Auflagerungen (Sterne) verfüllt ist. Die ehemalige Lamina interna (Pfeile) ist gut zu erkennen. $\rightarrow$ hämorrhagisches Geschehen mit Knochenneubildung im Rahmen von Skorbut (siehe auch andere Bilder des Individuums)

Bild 4: Sükösd-Ságod, 134, Kind, ca. 4 Jahre alt. Lichtmikroskopische Darstellung (Schliffdicke 50um, polarisiertes Durchlicht mit Hilfsobjekt Rot 1. Ordnung (Quarz) als Kompensator, Vergrößerung 16-fach): Es handelt sich um das in Bild 3 beschriebene Präparat, diesmal im polarisierten Durchlicht, was eine bessere Abgrenzung der Auflagerung (Sterne) zur ursprünglichen Knochensubstanz und der Lamina interna (Pfeile) ermöglicht. $\rightarrow$ Hämorrhagisches Geschehen im Rahmen von Skorbut mit Knochenneubildung durch Periostreizung (siehe auch andere Bilder dieses Individuums)

Bild 5: Sükösd-Ságod, 134, Kind, ca. 4 Jahre alt. Lichtmikroskopische Darstellung (Schliffdicke 50 $\mu$ m, einfaches Durchlicht, Vergrößerung 100-fach): Es handelt sich um eine Detailaufnahme des linken Os occipitale. Wie auch in Bild 4 und 5 zu erkennen, ist die Diploë stark verdichtet und sklerosiert. Dies lässt sich auf eine Hirndrucksteigerung, beispielsweise durch endocraniale Blutungen, zurückführen. $\rightarrow$ skorbutische intracraniale Blutungen mit Knochenneubildung und Hirndrucksteigerung

Bild 6: Sükösd-Ságod, 288, Neonatus. Makroskopische Detailaufnahme des linken Humerus. An der Diaphyse und distalen Metaphyse sind diskrete feinporöse Auflagerungen zu erkennen, die gut integriert sind. $\rightarrow$ organisiertes subperiostales Hämatom im Rahmen von Skorbut (siehe auch folgende Bilder)

Bild 7 : Sükösd-Ságod, 288, Neonatus. Die makroskopische Detailaufnahme des rechten Orbitadachs zeigt feinporöse Auflagerungen mit deutlicher Gefäßzeichnung. $\rightarrow$ Cribra orbitalia Grad $2-3$ im Rahmen von Skorbut (siehe auch andere Bilder des Individuums)

Bild 8: : Sükösd-Ságod, 288, Neonatus. Lichtmikroskopische Darstellung (Schliffdicke 70um, einfaches Durchlicht, Vergrößerung 16-fach): Das Präparat stammt von der linken Tibia. Man sieht den typischen Schalenknochen eines sehr kleinen Kindes mit wenig Spongiosa. Es besteht der Verdacht auf Auflagerungen (grüne Pfeile) vom Typ I (hämorrhagisch). Deutlich zu erkennen sind Impressionen von Periostgefäßen (rosa Pfeile). $\rightarrow$ Verdacht auf organisiertes subperiostales Hämatom im Rahmen von Skorbut (siehe auch andere Bilder des Individuums) 


\section{Bildtafel 21}
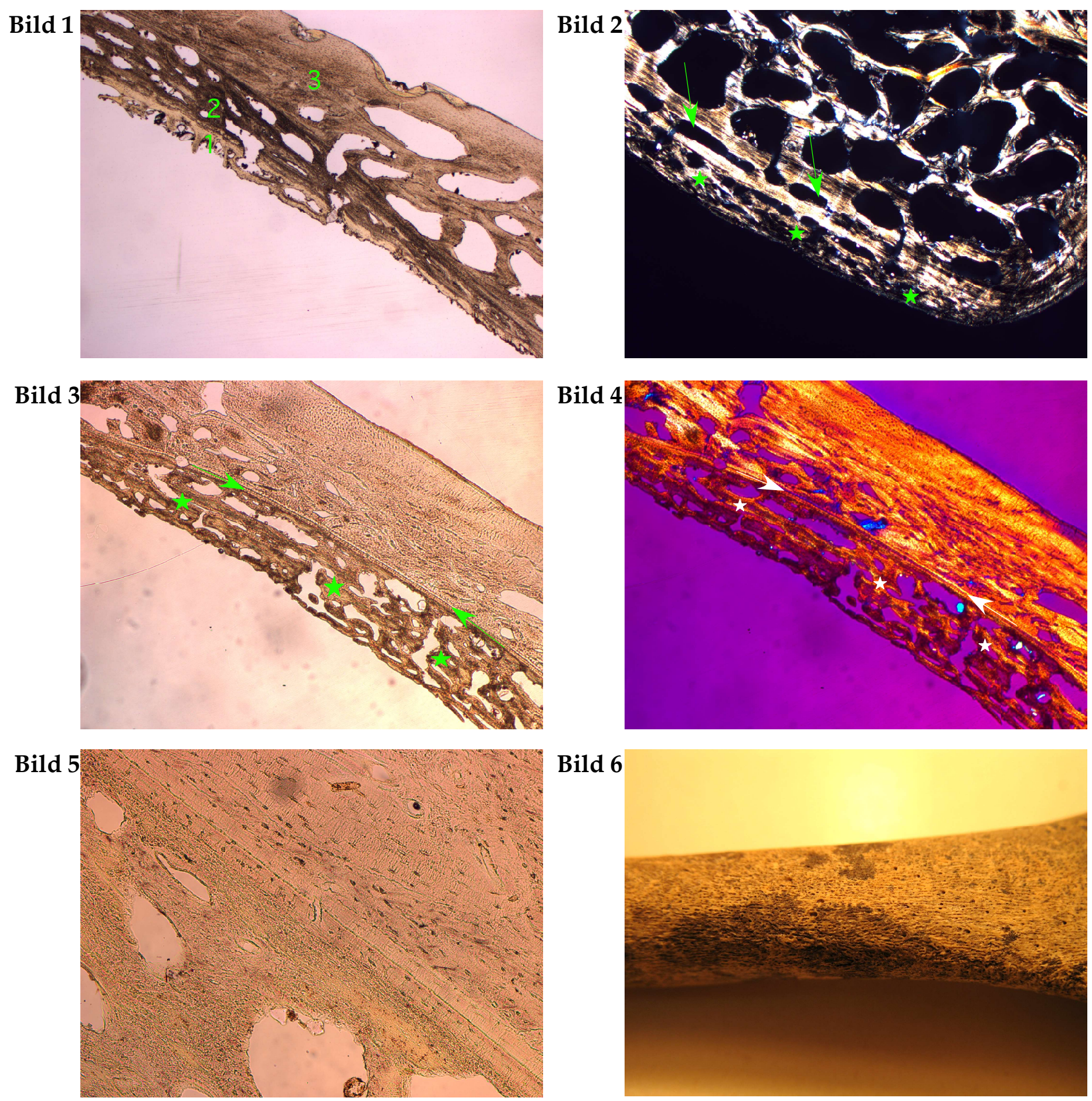

Bild 6
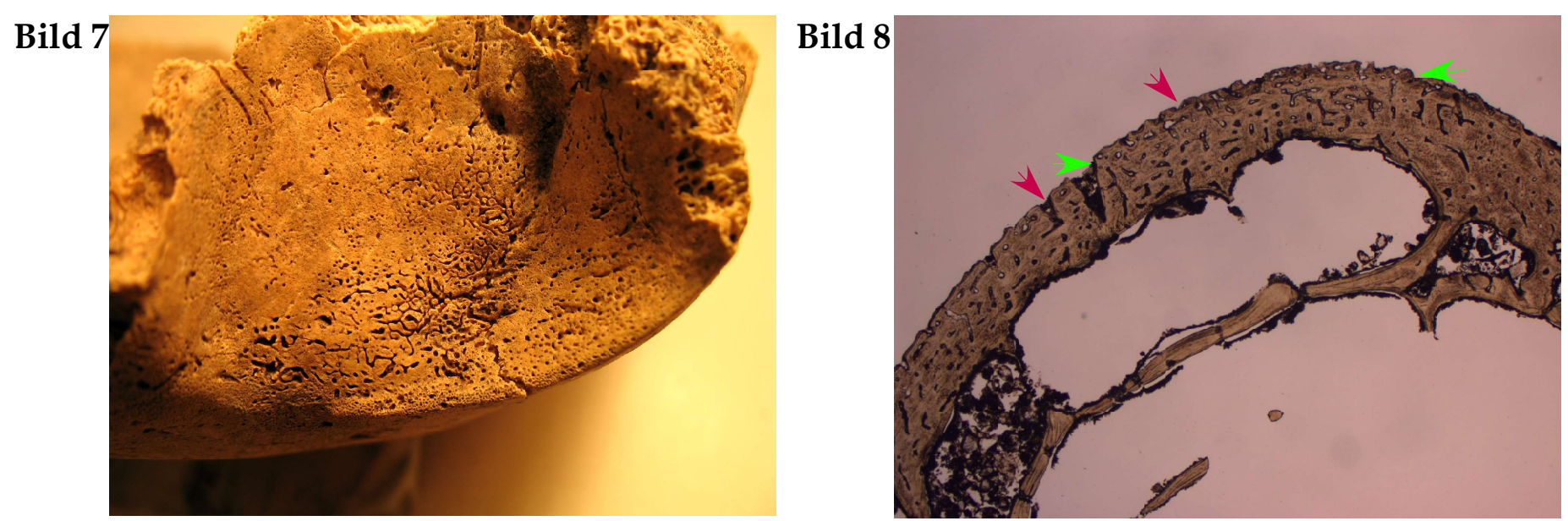


\section{$\underline{\text { Häufigkeit der unterschiedlichen Diagnosen }}$}

\subsection{Häufigkeit der verschiedenen Diagnosen bei den Skeleten des Gräberfelds Barbing-} Kreuzhof

Es wurden 134 Skelete des Gräberfelds Barbing auf Hinweise für Skorbut, also vor allem feinporöse Oberflächenveränderungen oder Auflagerungen, untersucht.

Wurde eine solche Läsion an den Langknochen, dem Schädel, den Schulterblättern, den Rippen oder dem Beckenknochen gefunden, wurde das Skelet weitergehenden Untersuchungen unterzogen.

Insgesamt wurden 27 Skelete in die engere Untersuchung eingeschlossen, darunter 16 Kinder zwischen dem Säuglingsalter und ca. 15 Jahren und elf adulte Individuen zwischen etwa 25 und bis zu 80 Jahren.

Nach dem in Kapitel 2.2 j) Material und Methoden - Diagnosestellung vorgestellten Bewertungsschema werden folgende Gruppen unterschieden:

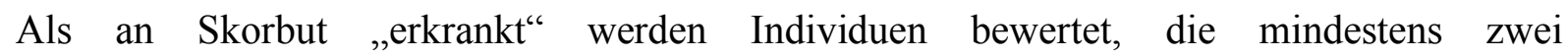
Kardinalmerkmale oder ein Kardinalmerkmal und zwei Nebenmerkmale oder drei Nebenmerkmale für Skorbut aufweisen.

Zusätzlich wird im Folgenden unterschieden, ob die Diagnose lichtmikroskopisch gesichert ist oder nicht.

Mit „Verdacht auf Skorbut“ (Abkürzung: V. a. Skorbut) werden Individuen bezeichnet, die nur ein Kardinalmerkmal oder ein Kardinalmerkmal und ein Nebenmerkmal oder zwei Nebenmerkmale für Skorbut zeigen.

Weist ein Individuum weniger Merkmale auf, wird eine Skorbuterkrankung ausgeschlossen.

Nach dieser Einteilung ergeben sich für die 27 Barbinger Skelete folgende Befunde:

Neunzehn von 27 Individuen (70\%) ${ }^{72}$ sind krank, bei sechs (22\%) Individuen liegt eine mikroskopische Sicherung vor, während bei 13 (48\%) Individuen nur makroskopische Befunde zugrunde liegen.

Von den 19 kranken Individuen sind 15 Kinder (93\% aller Kinder) und vier Erwachsene (36\% aller Erwachsenen), unter den mikroskopisch gesicherten Fällen sind vier Kinder (25\% aller Kinder) und zwei Erwachsene (18\% aller Erwachsenen), bei zehn (62\% aller Kinder) Kindern und zwei Erwachsenen (18\% aller Erwachsenen) gibt es nur makroskopische Befunde.

Vier von 27 Individuen (15\%) werden mit „Verdacht auf Skorbut“ bewertet, darunter ein Kind (6\% aller Kinder) und drei Erwachsene (27\% aller Erwachsenen).

\footnotetext{
${ }^{72}$ Bei den Prozentzahlen wurde aufgrund der kleinen Fallzahlen auf Nachkommastellen verzichtet und auf volle Zahlen gerundet.
} 
Bei zwei von 27 Individuen (7\%) kann keine Diagnose angegeben werden, Skorbut ist ausgeschlossen. Beides sind adulte Individuen (18\% aller Erwachsenen).

Bei zwei adulten Individuen (7\% aller Individuen, 18\% aller Erwachsenen) wird die Diagnose Lepra gestellt, wobei eines vermutlich zusätzlich an Skorbut erkrankt ist.

Bei einem adulten Individuum (3\% aller Individuen, 9\% aller Erwachsenen) ist die wahrscheinlichste Diagnose Leukämie (vgl. Kapitel 3.1, Sk 1356).

Bezogen auf die Gesamtpopulation von Barbing sind also 19 von 134 (14\%) Individuen sicher an Skorbut erkrankt, bei weiteren vier (ca. 3\%) besteht der Verdacht auf Skorbut, das macht zusammen 23 von 134 Individuen (17\%), bei denen von einem manifesten chronischen Vitamin-C-Mangel ausgegangen werden muss.

\begin{tabular}{|l|l|l|l|l|l|}
\hline & Skorbut & V. a. Skorbut & gesund & V. a. Lepra & V.a. Leukämie \\
\hline Gesamt (n=27) & $19(70 \%)$ & $4(15 \%)$ & $2(7 \%)$ & $2(7 \%)$ & $1(4 \%)$ \\
\hline Adulte (n=11) & $4(36 \%)$ & $3(27 \%)$ & $2(18 \%)$ & $2(18 \%)$ & $1(9 \%)$ \\
\hline Kinder (n=16) & $15(94 \%)$ & $1(6 \%)$ & 0 & 0 & 0 \\
\hline
\end{tabular}

Tab. 42: Häufigkeit der Diagnosen bei den 27 ausgewählten Barbinger Skeleten. Man beachte, dass ein Individuum (Sk 1380) vermutlich sowohl an Lepra als auch an Skorbut erkrankt ist und daher zweimal in der Berechnung erscheint.

V. a. $=$ Verdacht auf

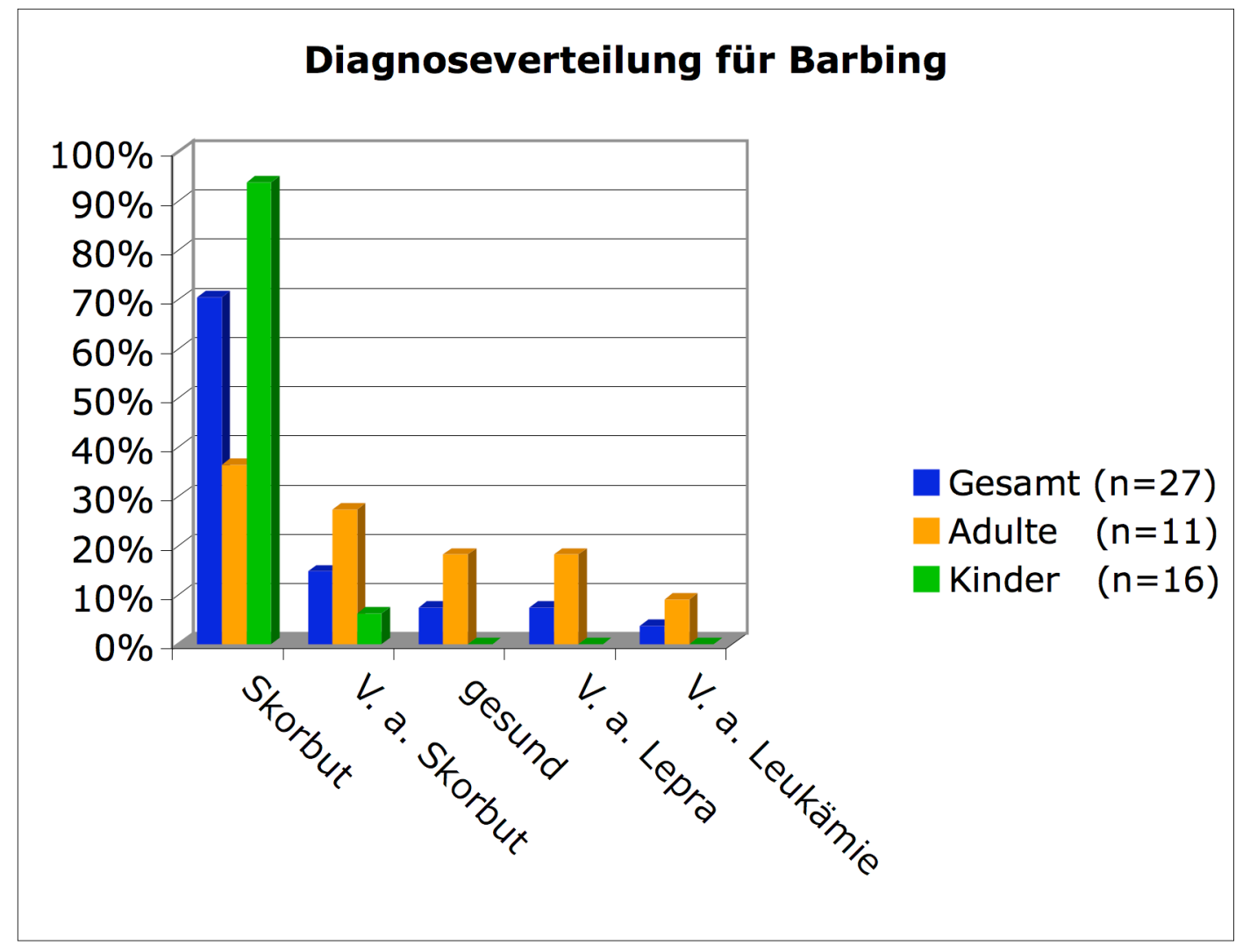

Abb. 9: Häufigkeit der unterschiedlichen Diagnosen der 27 ausgewählten Barbinger Skelete.

Man beachte, dass ein Individuum (Sk 1380) vermutlich sowohl an Lepra als auch an Skorbut erkrankt ist und daher zweimal in der Berechnung erscheint.

V. a. = Verdacht auf 
Wie in Kapitel 2.2 e) Material und Methoden - Geschlechtsbestimmung erläutert, wurde bei Kindern keine Geschlechtsbestimmung durchgeführt. Für die erwachsenen Individuen der Barbinger Auswahl ergibt sich folgende Geschlechtsverteilung: Von elf Erwachsenen sind sechs männlich, vier weiblich und bei einem ist keine Zuordnung möglich.

Unter den vier an Skorbut erkrankten adulten Individuen sind drei Männer und eine Frau, bei den drei unter Verdacht auf Skorbut stehenden sind ein Mann, eine Frau und ein Individuum mit unbestimmbarem Geschlecht.

Die beiden Individuen ohne Diagnose sind männlich; die vermutlich an Leukämie und Lepra erkrankten sind weiblich.

\begin{tabular}{|l|l|l|l|l|l|}
\hline & Skorbut & V. a. Skorbut & gesund & V. a. Lepra & V. a. Leukämie \\
\hline Adulte (n=11) & 4 & 3 & 2 & 2 & 1 \\
\hline Männer (n=6) & 3 & 1 & 2 & 0 & 0 \\
\hline Frauen (n=4 & 1 & 1 & 0 & 2 & 1 \\
\hline unklar (n=1) & 0 & 1 & 0 & 0 & 0 \\
\hline
\end{tabular}

Tab. 43: Häufigkeit der Diagnosen in Bezug auf das Geschlecht für die elf adulten Individuen der Barbinger Auswahl

Man beachte, dass ein Individuum (Sk 1380) vermutlich sowohl an Lepra als auch an Skorbut erkrankt ist und daher zweimal in der Berechnung erscheint.

V. a. = Verdacht auf

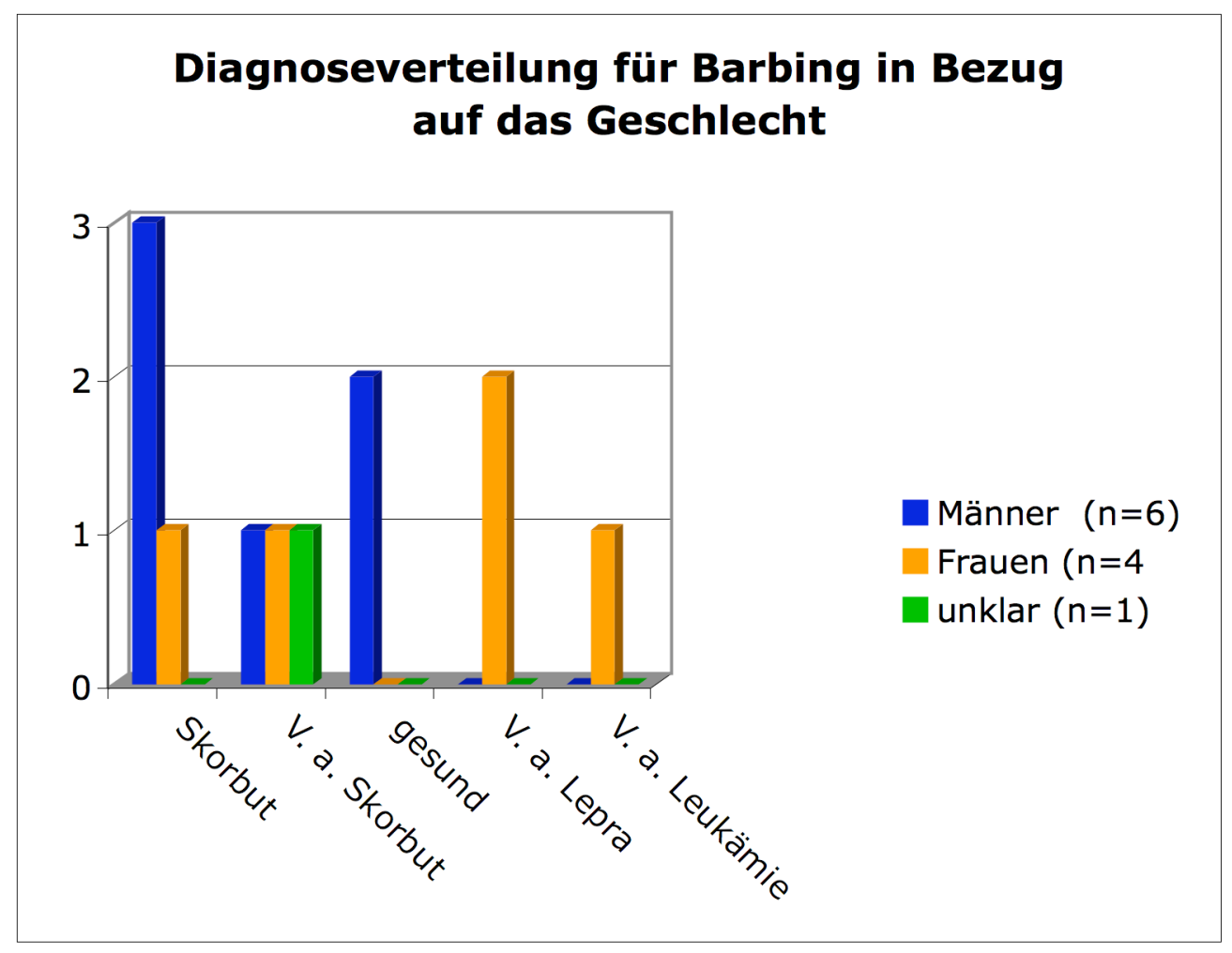

Abb. 10: Häufigkeit der Diagnosen in Bezug auf das Geschlecht für die elf adulten Individuen der Barbinger Auswahl

Man beachte, dass ein Individuum (Sk 1380) vermutlich sowohl an Lepra als auch an Skorbut erkrankt ist und daher zweimal in der Berechnung erscheint. V. a. = Verdacht auf 


\subsection{Häufigkeit der unterschiedlichen Diagnosen bei den Skeleten aus Ungarn}

Wie im Kapitel 2.1 Material erläutert, wurden neun ungarische Skelete aus unterschiedlichen Grabungen auf Skorbut untersucht, die zuvor von ungarischen Kollegen ${ }^{73}$ als verdächtig auf Skorbut ausgewählt worden waren.

Es handelt sich dabei ausschließlich um Kinderskelete. Bei acht von neun Skeleten (89\%) lautet die Hauptdiagnose Skorbut, bei einem (11\%) Anämie. Alle Diagnosen sind lichtmikroskopisch gesichert.

\begin{tabular}{|l|l|l|}
\hline & Skorbut & Anämie \\
\hline Gesamt (n=9) & 8 & 1 \\
\hline
\end{tabular}

Tab. 44: Übersicht über die bei den ungarischen Skeleten gefundenen Diagnosen

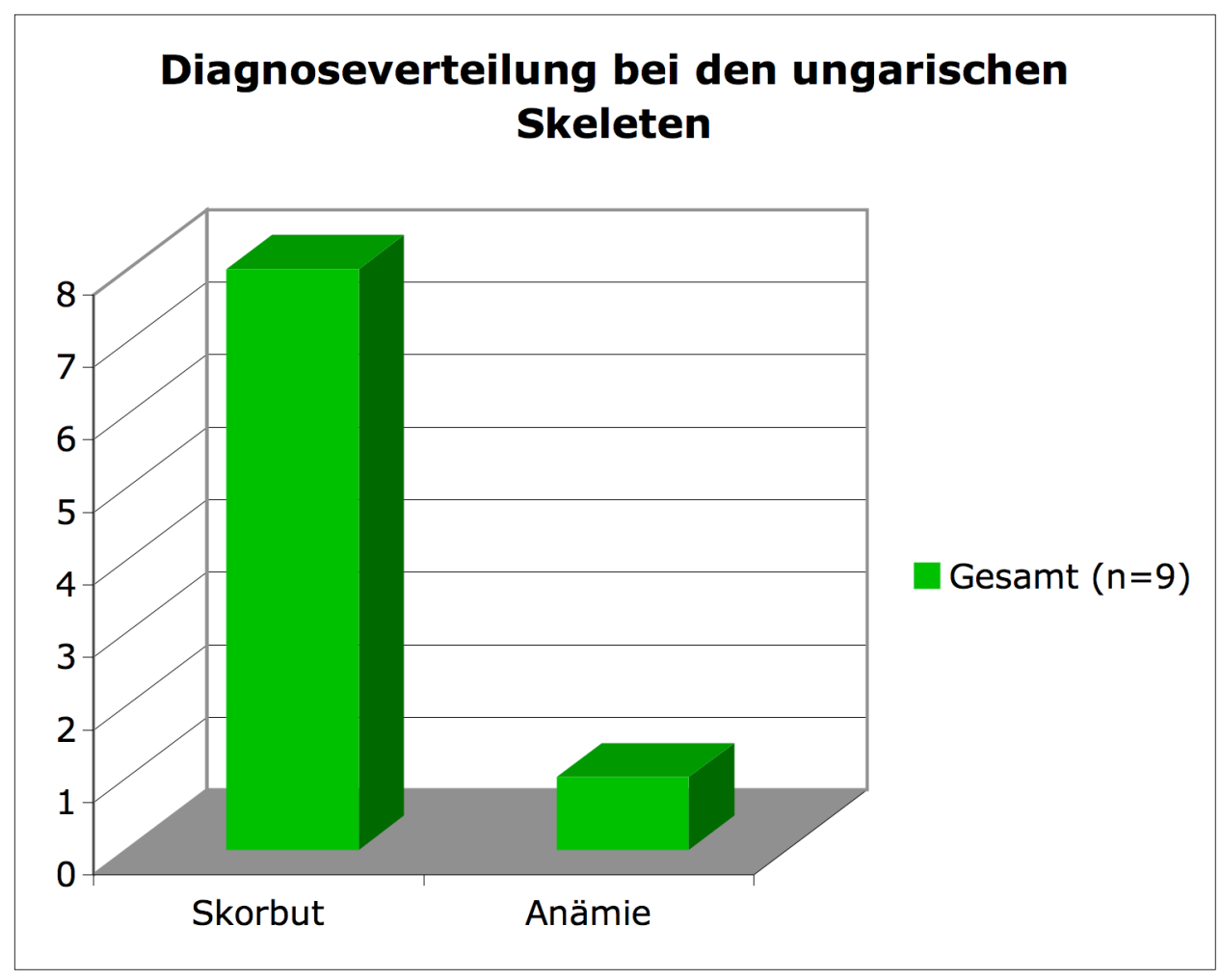

Abb. 11: Übersicht über die bei den ungarischen Skeleten gefundenen Diagnosen

\footnotetext{
${ }^{73}$ Marcsik A, Lovász G, Molnár E
} 


\subsection{Häufigkeit der unterschiedlichen Diagnosen bei allen untersuchten Skeleten}

Fasst man die Untersuchung der ungarischen und der Barbinger Skelete zusammen, so kommt man auf eine Gesamtzahl von 36 Skeleten. Darunter sind 25 Kinder und elf Erwachsene.

Die Diagnose Skorbut wird für 27 der 36 Individuen (75\%) gestellt, davon sind vier Individuen adult (36\% aller Erwachsenen) und 23 Kinder (92\% aller Kinder).

Verdacht auf Skorbut besteht bei vier der 36 Individuen (11\%), darunter sind drei Erwachsene (27\% aller Erwachsenen) und ein Kind (4\% aller Kinder).

Bei zwei adulten Individuen kann keine Diagnose gestellt werden (6\% aller bzw. 18\% der adulten Individuen).

Zwei adulte Individuen sind vermutlich an Lepra erkrankt (6\% aller bzw. 18\% der adulten Individuen), eins davon außerdem an Skorbut.

Bei einem adulten Individuum (3\% aller bzw. 9\% der adulten Individuen) lautet die Verdachtsdiagnose Leukämie.

Anämie wird bei einem Kind (3\% aller bzw. 4\% der Kinder) diagnostiziert.

\begin{tabular}{|l|l|l|l|l|l|l|}
\hline & Skorbut & V. a. Skorbut & gesund & V. a. Lepra & V. a. Leukämie & Anämie \\
\hline Gesamt (n=36) & $27(75 \%)$ & $4(11 \%)$ & $2(6 \%)$ & $2(6 \%)$ & $1(3 \%)$ & $1(3 \%)$ \\
\hline Adulte (n=11) & $4(36 \%)$ & $3(27 \%)$ & $2(18 \%)$ & $2(18 \%)$ & $1(9 \%)$ & 0 \\
\hline Kinder (n=25) & $23(92 \%)$ & $1(4 \%)$ & 0 & 0 & 0 & $1(4 \%)$ \\
\hline
\end{tabular}

Tab. 45: Häufigkeit der unterschiedlichen Diagnosen bei allen untersuchten Skeleten. Man beachte, dass ein Individuum (Sk 1380) vermutlich sowohl an Lepra als auch an Skorbut erkrankt ist und daher zweimal in der Berechnung erscheint.

V. a. = Verdacht auf 


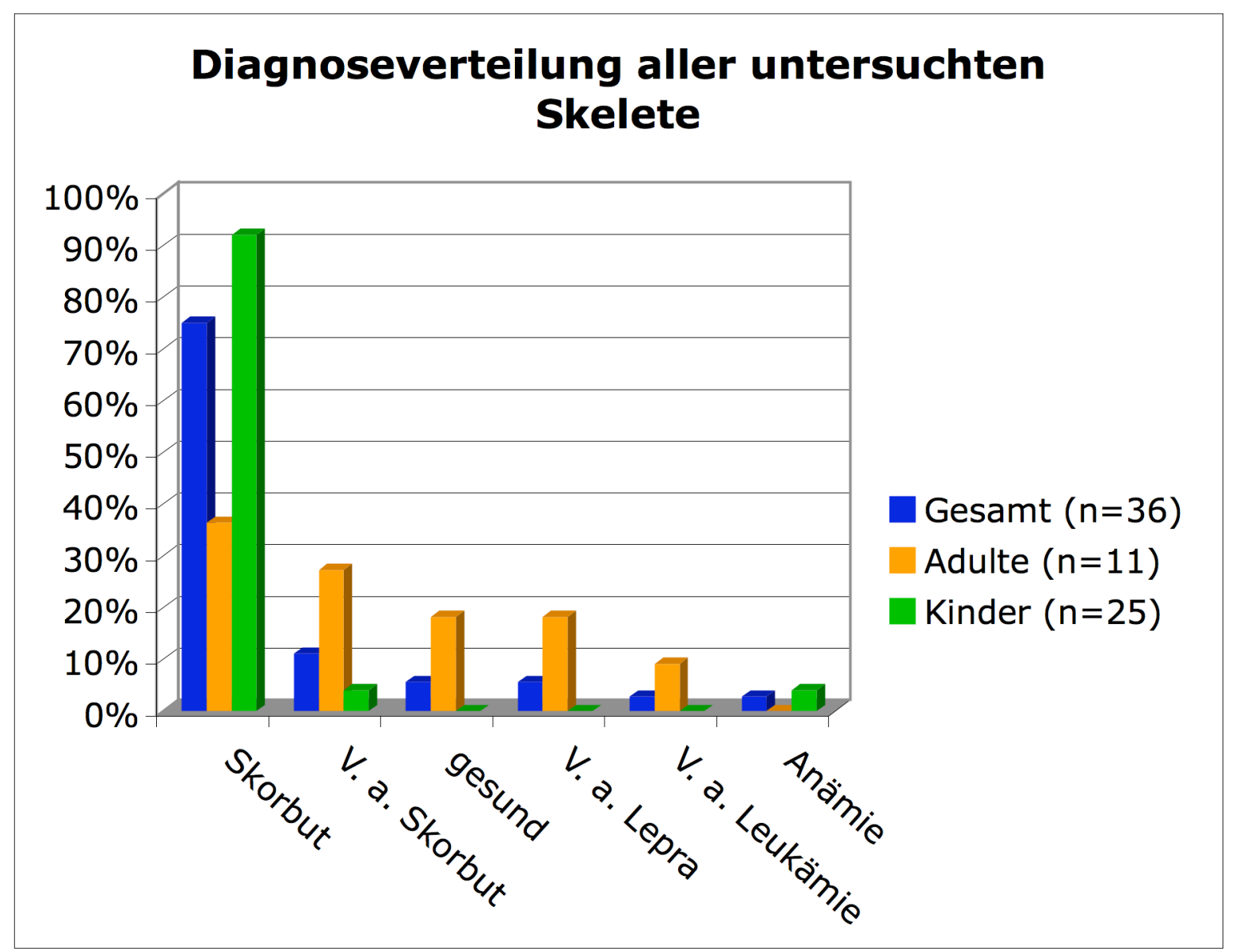

Abb. 12: Häufikeit der unterschiedlichen Diagnosen bei allen untersuchten Skeleten. Man beachte, dass ein Individuum (Sk 1380) vermutlich sowohl an Lepra als auch an Skorbut erkrankt ist und daher zweimal in der Berechnung erscheint.

V. a. $=$ Verdacht auf 


\section{Häufigkeit und Verteilung der einzelnen Kardinalmerkmale für Skorbut}

Ziel dieser Arbeit ist es, Untersuchungskriterien für die Diagnostik von Skorbut an historischen Skeleten zu entwickeln. Daher wird im Folgenden die Häufigkeit des Auftretens der unterschiedlichen Merkmale herausgearbeitet, um möglicherweise eine Aussage über die Wichtigkeit des einzelnen Merkmals für die Diagnosestellung treffen zu können. Dabei werden alle untersuchten Skelete der verschiedenen Populationen gemeinsam betrachtet.

\subsection{Poröse Auflagerungen am Schädeldach (Kardinalmerkmal 1)}

Von den 36 untersuchten Skeleten weisen insgesamt 14 Individuen (39\%) feinporöse Auflagerungen am Schädeldach auf, davon sind 13 (36\%) an Skorbut erkrankt, eins (3\%) an Anämie. Bei den Merkmalsträgern handelt es sich ausschließlich um Kinder (56\% aller 25 Kinder).

Bei acht von 36 Individuen (22\%) ist das Schädeldach nicht beurteilbar, darunter fünf (14\%) mit Diagnose Skorbut, bei zweien (6\%) besteht Verdacht auf Skorbut. Unter den Individuen ohne befundbares Schädeldach sind drei Kinder (12\% aller Kinder), davon zwei ( $8 \%$ aller Kinder) mit Skorbut und eins (4\% aller Kinder) mit Verdacht auf Skorbut.

Bei den übrigen 14 Individuen (39\%), davon acht Kinder (32\% aller Kinder), sind die Schädeldächer unauffällig. Darunter sind neun Individuen (25\%) mit Diagnose Skorbut, auch die acht Kinder (32\% aller Kinder), bei zwei weiteren Individuen (6\%) besteht Verdacht auf Skorbut.

\begin{tabular}{|l|l|l|l|}
\hline & Merkmal positiv & Nicht beurteilbar & Knochen unauffällig \\
\hline Skorbut & $13(36 \%)$ & $5(14 \%)$ & $9(25 \%)$ \\
\hline V. a. Skorbut & 0 & $2(6 \%)$ & $2(6 \%)$ \\
\hline Sonstiges & $1(3 \%)$ & $1(3 \%)$ & $3(8 \%)$ \\
\hline
\end{tabular}

Tab. 46: Häufigkeit poröser Auflagerungen am äußeren Schädeldach bei Individuen mit Skorbut, Verdacht auf Skorbut und Individuen mit anderen Diagnosen. Dieses Merkmal wurde nur bei Kindern gefunden. Individuum Sk 1380, das vermutlich sowohl an Lepra als auch an Skorbut erkrankt ist, wird hier in der Gruppe der Skorbutkranken geführt. Die Abweichung von 100\% ergibt sich durch Auf- bzw. Abrundung. V. a. = Verdacht auf 


\section{Häufigkeit poröser Auflagerungen am äußeren Schädeldach aller Skelete $(n=36)$}

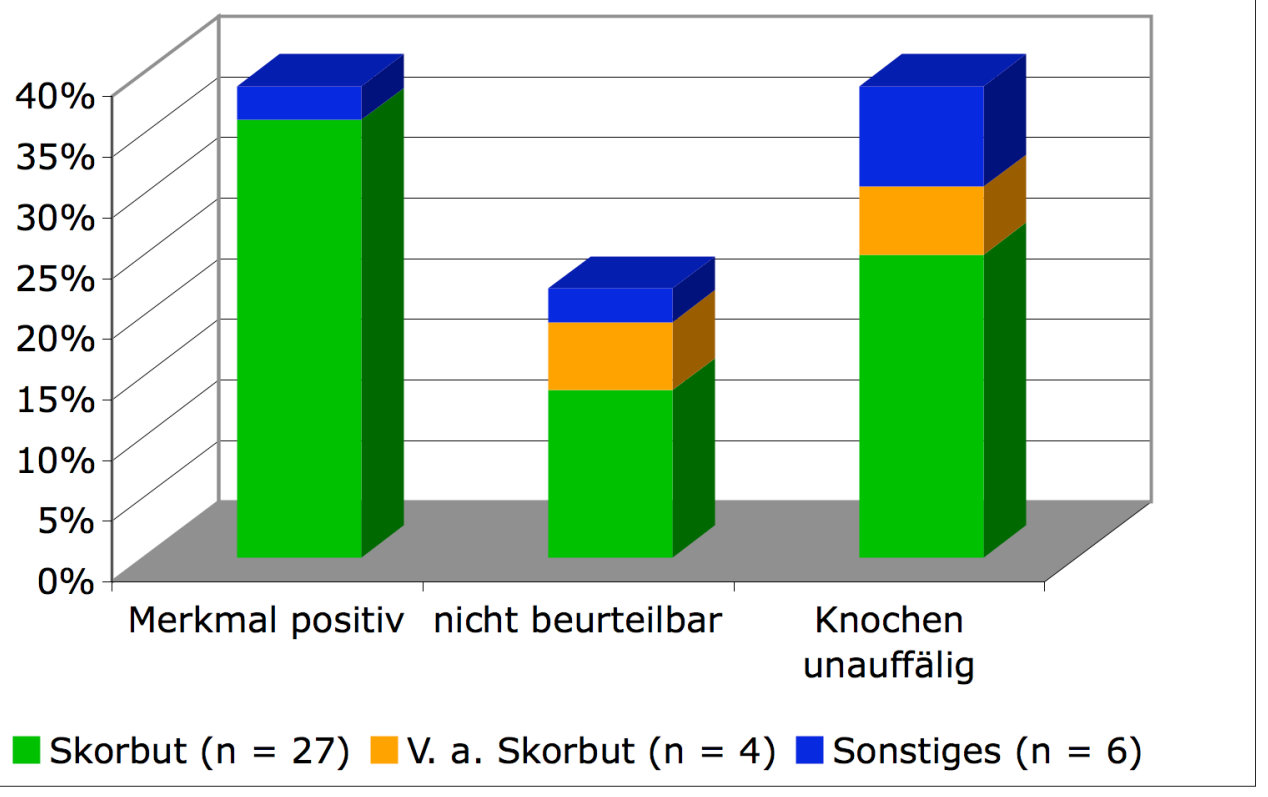

Abb. 13: Häufigkeit poröser Auflagerungen am äußeren Schädeldach bei Individuen mit Skorbut, Verdacht auf Skorbut und Individuen mit anderen Diagnosen

Individuum Sk 1380, das vermutlich sowohl an Lepra als auch an Skorbut erkrankt ist, wird hier in der Gruppe der Skorbutkranken geführt.

V. a. $=$ Verdacht auf

Betrachtet man die Gesamtzahl aller Skorbutfälle, 27 Individuen, so weisen 14 von 27 Erkrankten (52\% der Skorbutkranken) das Kardinalmerkmal poröse Auflagerung am äußeren Schädeldach auf, darunter ausschließlich Kinder. Bei fünf (19\% der Skorbutkranken) an Skorbut erkrankten Individuen ist das Schädeldach nicht beurteilbar, bei neun Individuen (33\% der Skorbutkranken) ist es unauffällig.

\begin{tabular}{|l|l|l|l|}
\hline & Merkmal positiv & Nicht beurteilbar & Knochen unauffällig \\
\hline Skorbutkranke $(\mathbf{n}=\mathbf{2 7})$ & $13(48 \%)$ & $5(19 \%)$ & $9(33 \%)$ \\
\hline
\end{tabular}

Tab. 47: Häufigkeit poröser Auflagerungen bei den an Skorbut erkrankten Individuen. Dieses Merkmal wurde nur bei Kindern gefunden (Prozentzahlen beziehen sich auf Gesamtzahl der Skorbutfälle). 


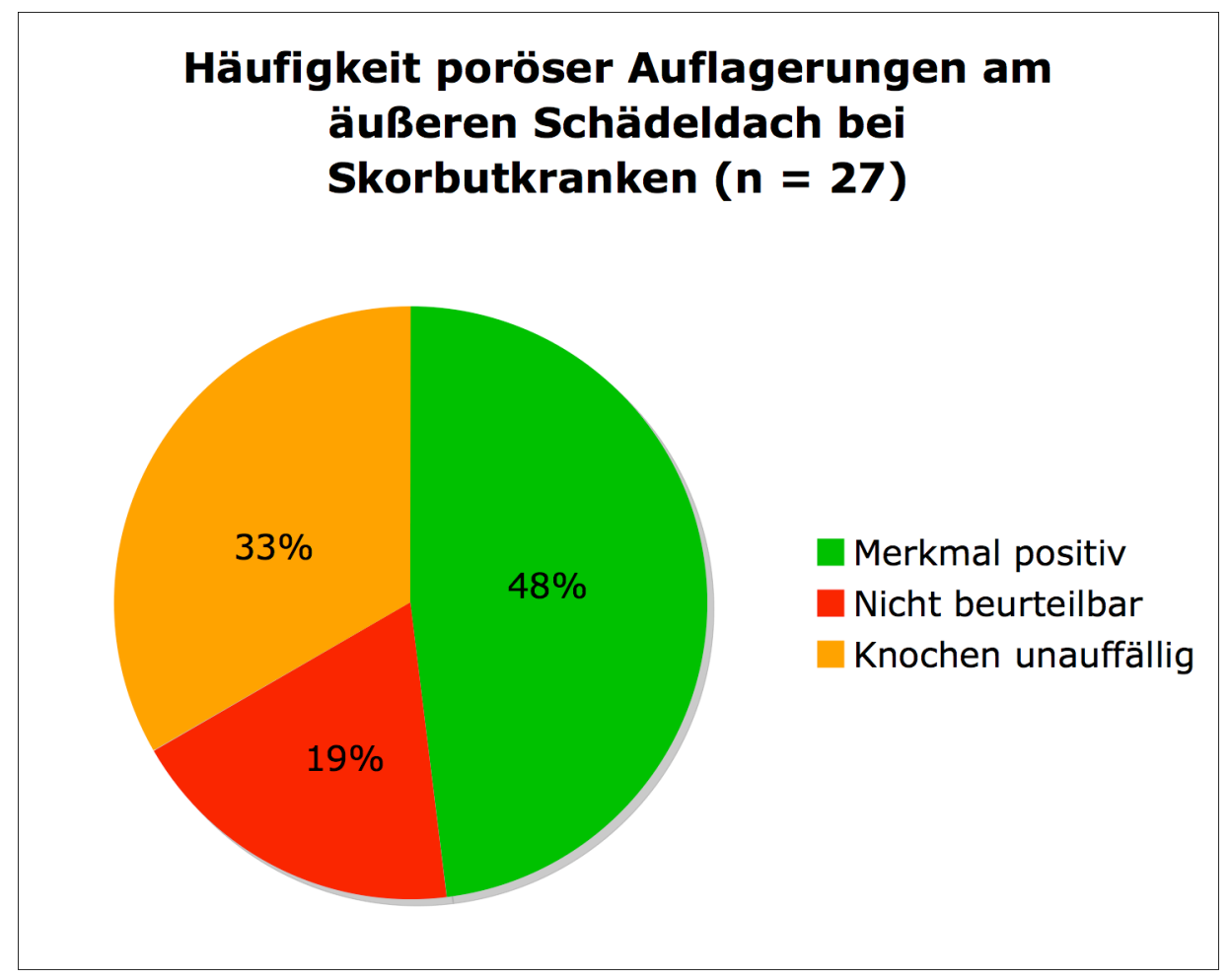

Abb. 14: Häufigkeit poröser Auflagerungen bei den an Skorbut erkrankten Individuen. Dieses Merkmal wurde nur bei Kindern gefunden.

Bezogen auf die an Skorbut erkrankten Kinder (23), weisen 13 Individuen (57\% aller skorbutkranken Kinder) poröse Auflagerungen am äußeren Schädeldach auf, bei zwei Kindern ( $9 \%$ aller skorbutkranken Kinder) kann das Schädeldach nicht beurteilt werden, bei acht Kindern (35\% aller skorbutkranken Kinder) ist es unauffällig.

\begin{tabular}{|l|l|l|l|}
\hline & Merkmal positiv & Nicht beurteilbar & Knochen unauffällig \\
\hline Skorbutkranke Kinder $(\mathbf{n}=\mathbf{2 3})$ & $13(57 \%)$ & $2(9 \%)$ & $8(35 \%)$ \\
\hline
\end{tabular}

Tab. 48: Häufigkeit poröser Auflagerungen am äußeren Schädeldach bei skorbutkranken Kindern (Prozentzahlen beziehen sich auf Gesamtzahl der infantilen Skorbutfälle, die Abweichung von 100\% ergibt sich durch Aufbzw. Abrundung)

Von den vier an Skorbut erkrankten Erwachsenen zeigt kein Individuum poröse Auflagerungen am äußeren Schädeldach, bei je zwei Individuen (je 50\% der skorbutkranken Erwachsenen) ist das Schädeldach nicht beurteilbar bzw. unauffällig.

\begin{tabular}{|l|l|l|l|}
\hline & Merkmal positiv & Nicht beurteilbar & Knochen unauffällig \\
\hline Skorbutkranke Erwachsene $(n=4)$ & 0 & $2(50 \%)$ & $2(50 \%)$ \\
\hline
\end{tabular}

Tab. 49: Häufigkeit poröser Auflagerungen am äußeren Schädeldach bei skorbutkranken Erwachsenen (Prozentzahlen beziehen sich auf Gesamtzahl der adulten Skorbutfälle) 


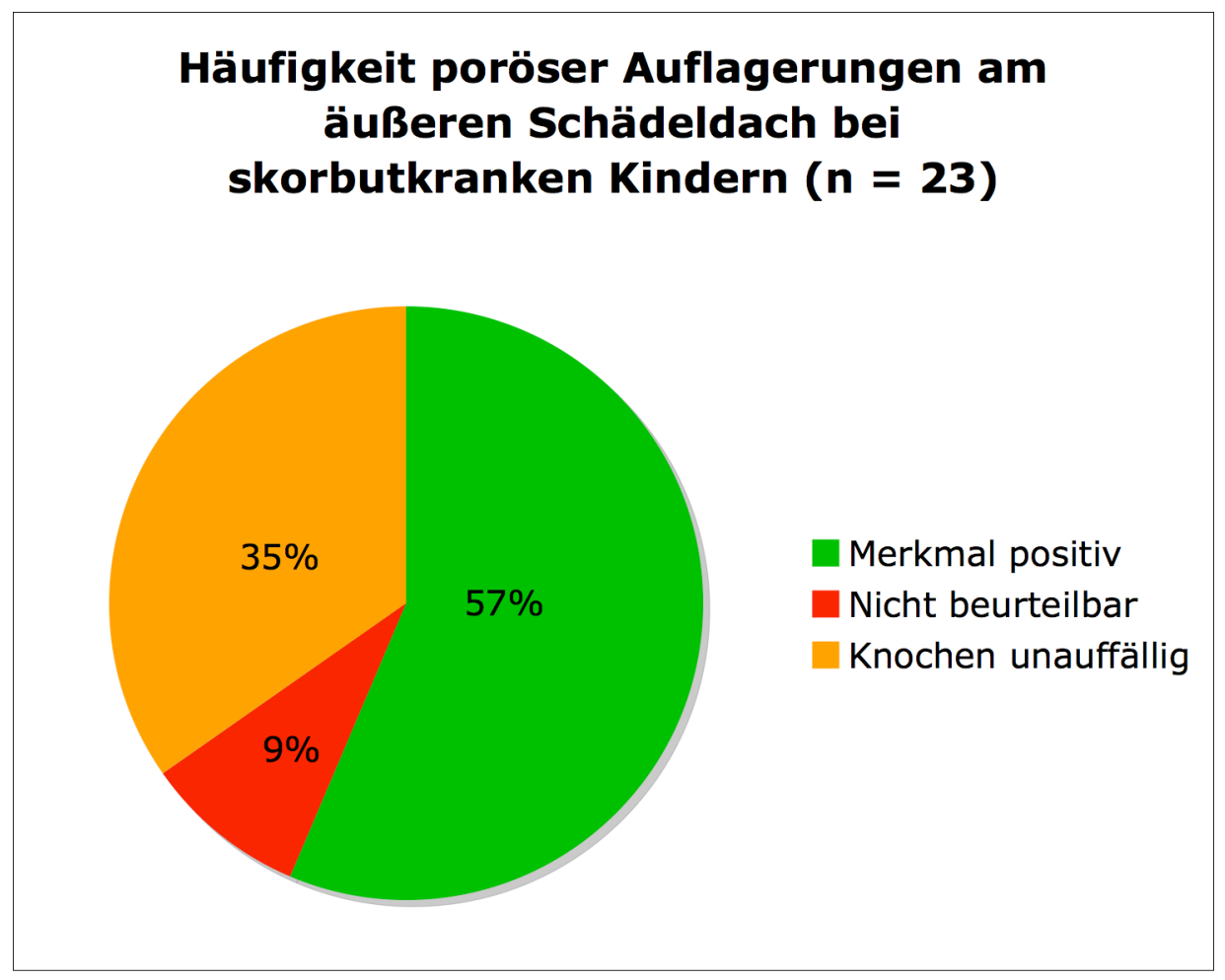

Abb. 15: Häufigkeit poröser Auflagerungen am äußeren Schädeldach bei skorbutkranken Kindern (die Abweichung von 100\% ergibt sich durch Auf- bzw. Abrundung)

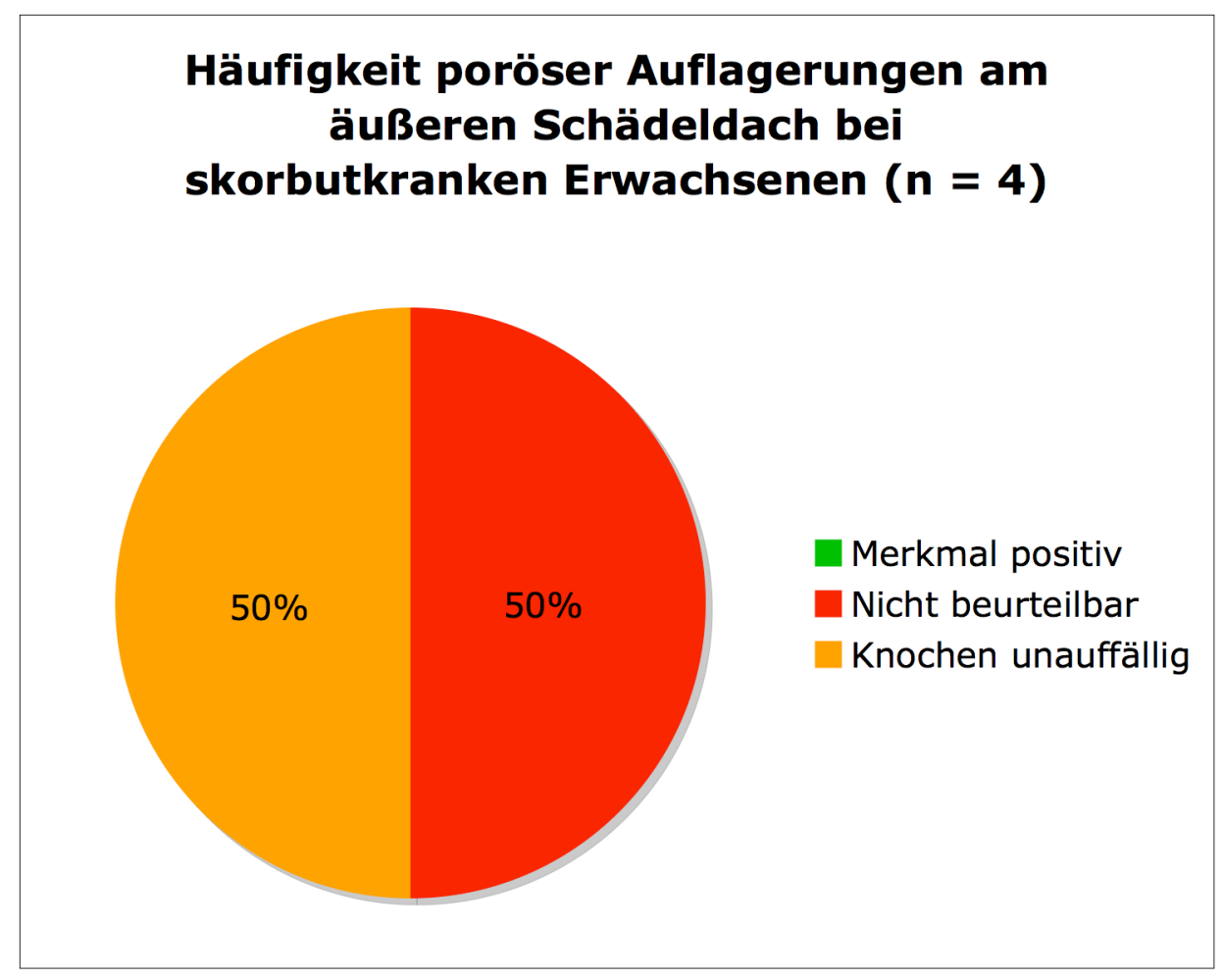

Abb. 16: Häufigkeit poröser Auflagerungen am äußeren Schädeldach bei skorbutkranken Erwachsenen 


\subsection{Poröse Auflagerungen am Orbitadach/Cribra orbitalia (Kardinalmerkmal 2)}

Von den 36 untersuchten Individuen weisen 15 (42\%) Cribra orbitalia auf, davon 14 Kinder (39\%). Dreizehn der Individuen mit Cribra orbitalia sind an Skorbut erkrankt (36\% von 36), bei einem weiteren (3\%) besteht Verdacht auf Skorbut, eins hat Anämie (3\%).

Bei neun Individuen (25\%) sind die Orbitadächer nicht beurteilbar, auch bei acht Skorbutkranken (22\%).

Zwölf Individuen (33\%) zeigen unauffällige Orbitadächer, darunter sechs mit Skorbut (16\%) und drei (8\%) mit Verdacht auf Skorbut.

\begin{tabular}{|l|l|l|l|}
\hline & Merkmal positiv & Nicht beurteilbar & Knochen unauffällig \\
\hline Skorbut & $13(36 \%)$ & $8(22 \%)$ & $6(17 \%)$ \\
\hline V. a. Skorbut & $1(3 \%)$ & 0 & $3(8 \%)$ \\
\hline Sonstiges & $1(3 \%)$ & $1(3 \%)$ & $3(8 \%)$ \\
\hline
\end{tabular}

Tab. 50: Häufigkeit von Cribra orbitalia bei Individuen mit Skorbut, Verdacht auf Skorbut und Individuen mit anderen Diagnosen

Individuum Sk 1380, das vermutlich sowohl an Lepra als auch an Skorbut erkrankt ist, wird hier in der Gruppe der Skorbutkranken geführt.

V. a. = Verdacht auf

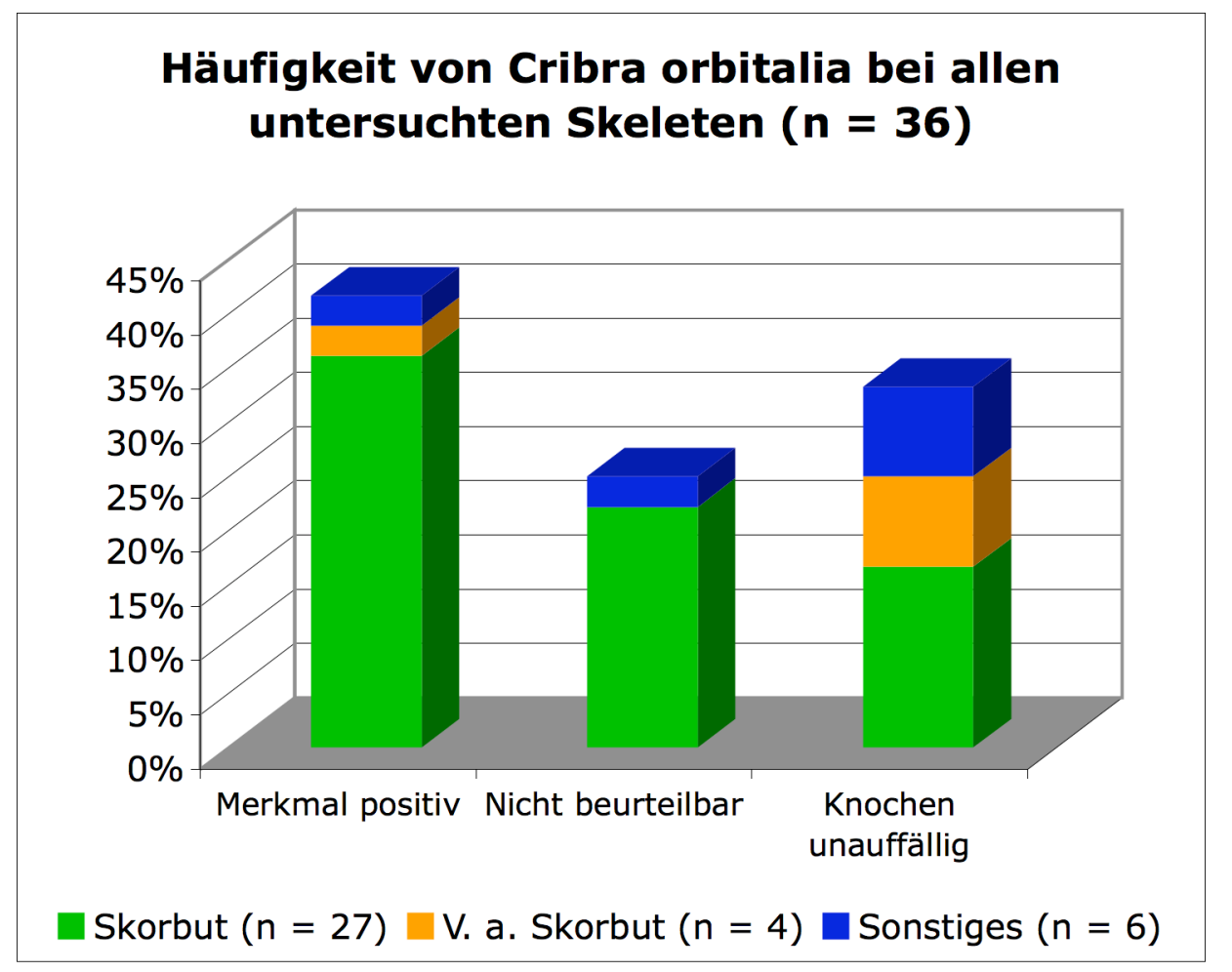

Abb. 17: Häufigkeit von Cribra orbitalia bei Individuen mit Skorbut, Verdacht auf Skorbut und Individuen mit anderen Diagnosen

Individuum Sk 1380, das vermutlich sowohl an Lepra als auch an Skorbut erkrankt ist, wird hier in der Gruppe der Skorbutkranken geführt.

V. a. = Verdacht auf 
Von 25 untersuchten Kindern zeigen 14 (56\% aller Kinder) Cribra orbitalia, davon 13 (52\% aller Kinder) mit Skorbut. Bei sieben Kindern (28\% der Kinder), die alle Skorbut hatten, sind die Orbitadächer nicht befundbar. Vier Kinder (16\% der Kinder) haben unauffällige Orbitadächer, drei (12\% aller Kinder) davon sind skorbutkrank, bei einem (4\% der Kinder) besteht der Verdacht einer Skorbuterkrankung.

\begin{tabular}{|l|l|l|l|}
\hline & Merkmal positiv & Nicht beurteilbar & Knochen unauffällig \\
\hline Skorbut & $13(52 \%)$ & $6(24 \%)$ & $4(16 \%)$ \\
\hline V. a. Skorbut & 0 & 0 & $1(4 \%)$ \\
\hline Sonstiges & $1(4 \%)$ & 0 & 0 \\
\hline
\end{tabular}

Tab. 51: Häufigkeit von Cribra orbitalia bei Kindern mit Skorbut, V. a. Skorbut und Kindern mit anderen Diagnosen (Prozentzahlen beziehen sich auf Gesamtzahl der Kinder)

V. a. = Verdacht auf

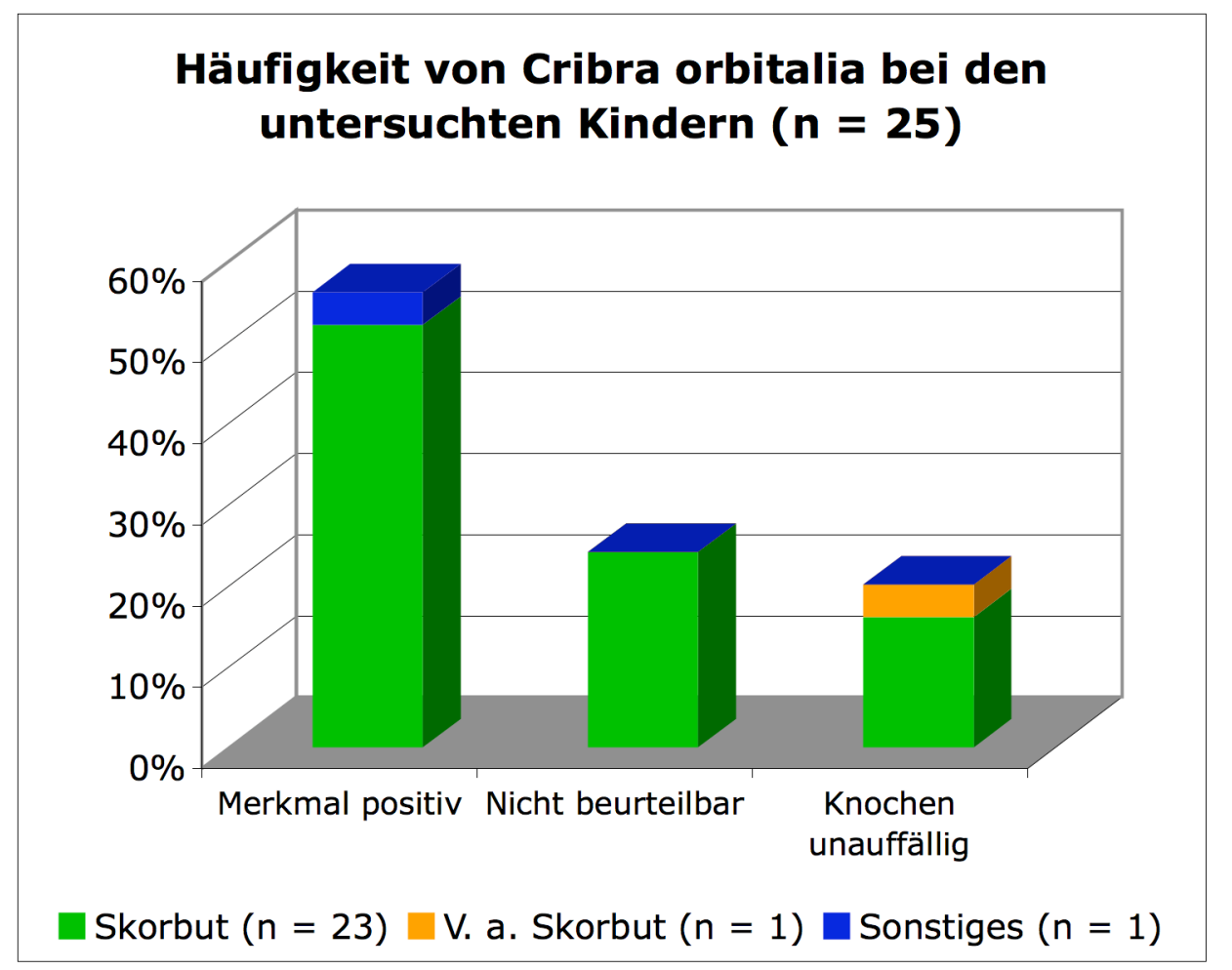

Abb. 18: Häufigkeit von Cribra orbitalia bei Kindern mit Skorbut, Verdacht auf Skorbut und Kindern mit anderen Diagnosen (Prozentzahlen beziehen sich auf Gesamtzahl der Kinder)

V. a. $=$ Verdacht auf

Von den elf untersuchten Erwachsenen hat nur ein Individuum (9\% der Erwachsenen) Cribra orbitalia, bei diesem besteht Verdacht auf Skorbut. Bei drei Erwachsenen $(27 \%$ der Erwachsenen) sind die Orbitadächer nicht befundbar, davon zwei (18\% der Erwachsenen) mit Skorbut. Sieben Erwachsene (64\%) haben unauffällige Orbitadächer, darunter zwei (18\% der Erwachsenen) mit Skorbut. 


\begin{tabular}{|l|l|l|l|}
\hline & Merkmal positiv & Nicht beurteilbar & Knochen unauffällig \\
\hline Skorbut & 0 & $2(18 \%)$ & $2(18 \%)$ \\
\hline V. a. Skorbut & $1(9 \%)$ & 0 & $2(18 \%)$ \\
\hline Sonstiges & 0 & $1(9 \%)$ & $3(27 \%)$ \\
\hline
\end{tabular}

Tab. 52: Häufigkeit von Cribra orbitalia bei Erwachsenen mit Skorbut, Verdacht auf Skorbut und Erwachsenen mit anderen Diagnosen (Prozentzahlen beziehen sich auf Gesamtzahl der Erwachsenen, die Abweichung von $100 \%$ ergibt sich durch Auf- bzw. Abrundung)

Individuum Sk 1380, das vermutlich sowohl an Lepra als auch an Skorbut erkrankt ist, wird hier in der Gruppe der Skorbutkranken geführt.

V. a. $=$ Verdacht auf

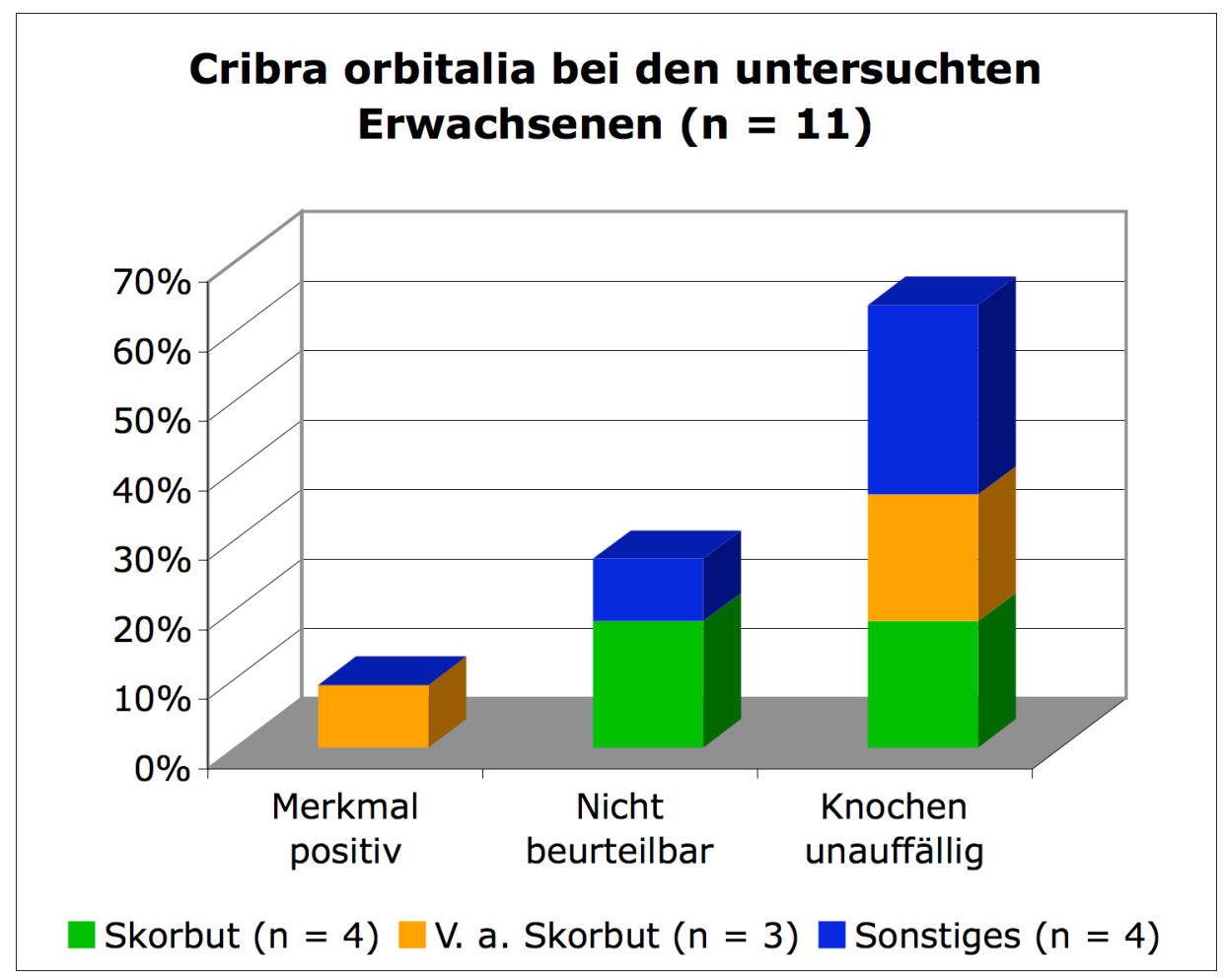

Abb. 19: Häufigkeit von Cribra orbitalia bei Erwachsenen mit Skorbut, Verdacht auf Skorbut und Erwachsenen mit anderen Diagnosen (Prozentzahlen beziehen sich auf Gesamtzahl der Erwachsenen)

Individuum Sk 1380, das vermutlich sowohl an Lepra als auch an Skorbut erkrankt ist, wird hier in der Gruppe der Skorbutkranken geführt.

V. a. = Verdacht auf

Betrachtet man alle an Skorbut erkrankten Individuen (27), so weisen 13 (48\% der Skorbutkranken) Cribra orbitalia auf, bei fünf (18\%) lassen sich die Orbitadächer nicht befunden, neun Individuen (33\%) haben unauffällige Orbitae.

\begin{tabular}{|l|l|l|l|}
\hline & Merkmal positiv & Nicht beurteilbar & Knochen unauffällig \\
\hline Skorbutkranke (n=27) & $13(48 \%)$ & $8(30 \%)$ & $6(22 \%)$ \\
\hline
\end{tabular}

Tab. 53: Häufigkeit von Cribra orbitalia bei den untersuchten Skorbutkranken (Prozentzahlen beziehen sich auf Gesamtzahl der Skorbutfälle) 


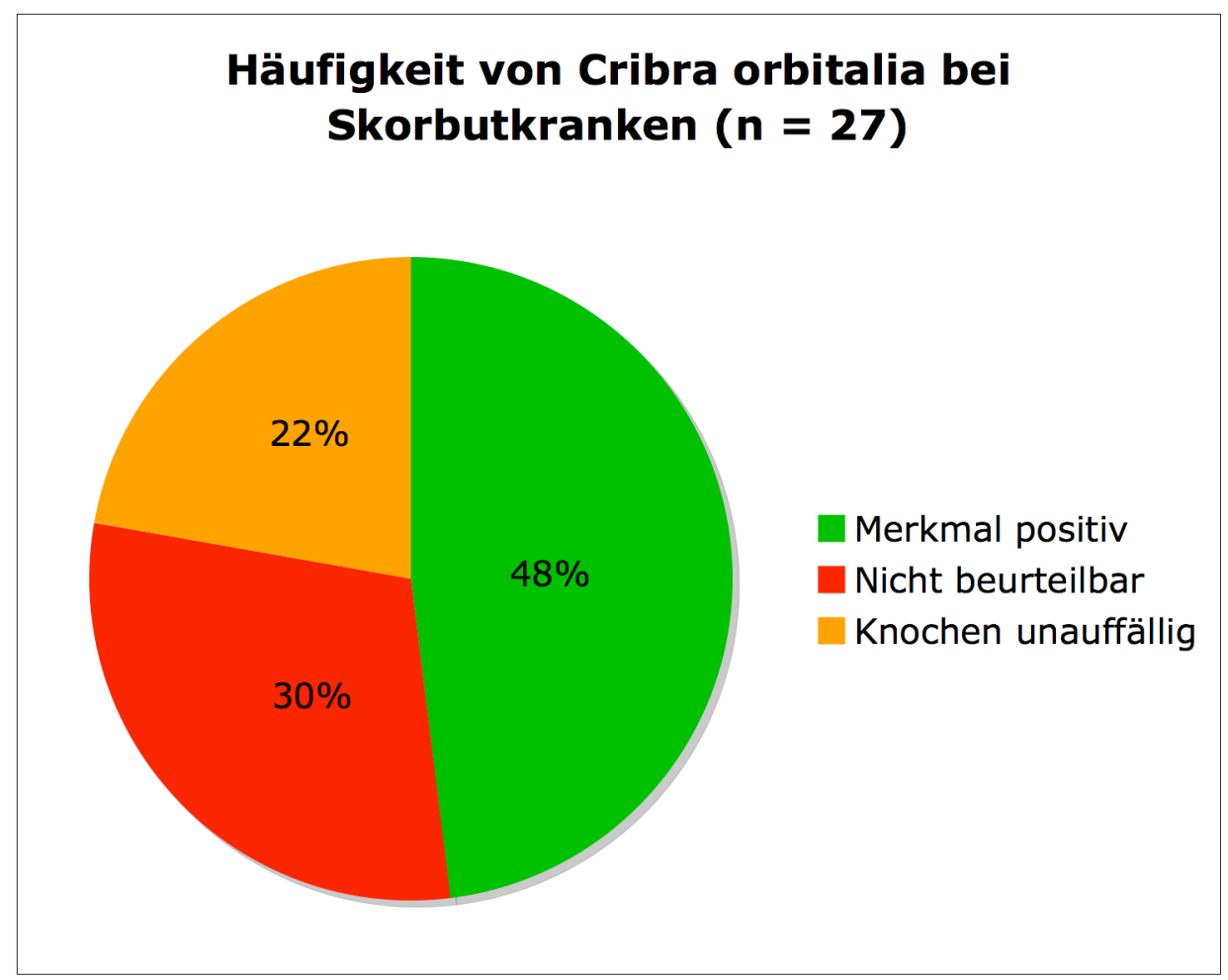

Abb. 20: Häufigkeit von Cribra orbitalia bei den untersuchten Skorbutkranken (Prozentzahlen beziehen sich auf Gesamtzahl der Skorbutfälle)

Unter den skorbutkranken Kindern (23) sind 13 (57\% der skorbutkranken Kinder) mit Cribra orbitalia, sechs (26\% der skorbutkranken Kinder) ohne befundbare Orbitae und vier $(17 \%)$ mit unauffälligen Orbitadächern.

\begin{tabular}{|l|l|l|l|}
\hline & Merkmal positiv & Nicht beurteilbar & Knochen unauffällig \\
\hline Skorbutkranke Kinder $(\mathbf{n}=\mathbf{2 3})$ & $13(57 \%)$ & $6(26 \%)$ & $4(17 \%)$ \\
\hline
\end{tabular}

Tab. 54: Häufigkeit von Cribra orbitalia bei den untersuchten skorbutkranken Kindern (Prozentzahlen beziehen sich auf Gesamtzahl der infantilen Skorbutfälle)

Von den vier an Skorbut erkrankten Erwachsenen weist kein Individuum Cribra orbitalia, je zwei (je 50\% der skorbutkranken Erwachsenen) haben unauffällige bzw. nicht befundbare Orbitadächer.

\begin{tabular}{|l|l|l|l|}
\hline & Merkmal positiv & Nicht beurteilbar & Knochen unauffällig \\
\hline Skorbutkranke Erwachsene $(\mathbf{n}=4)$ & 0 & $2(50 \%)$ & $2(50 \%)$ \\
\hline
\end{tabular}

Tab. 55: Häufigkeit von Cribra orbitalia bei skorbutkranken Erwachsenen (Prozentzahlen beziehen sich auf Gesamtzahl der adulten Skorbutfälle) 


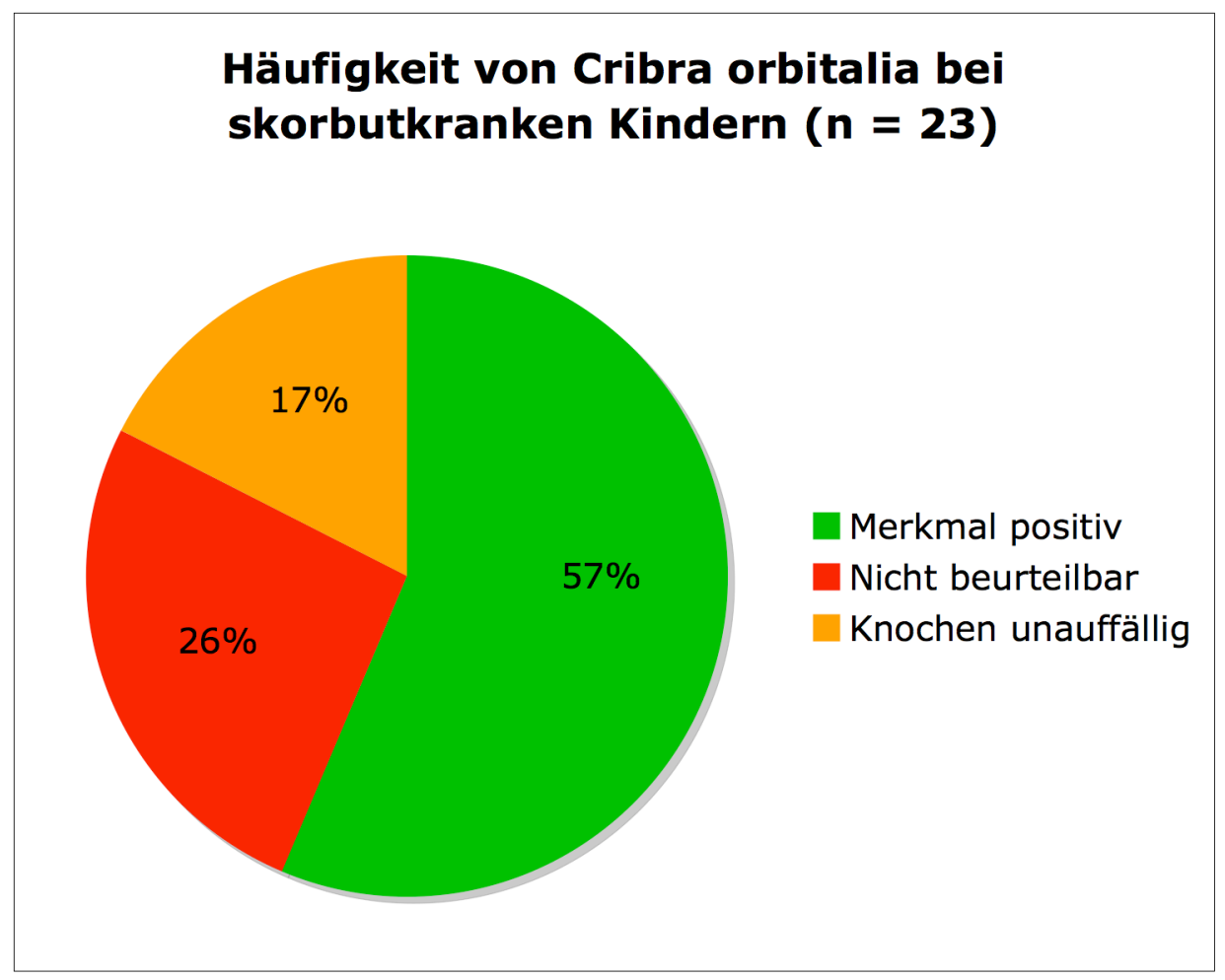

Abb. 21: Häufigkeit von Cribra orbitalia bei den untersuchten skorbutkranken Kindern (Prozentzahlen beziehen sich auf Gesamtzahl der infantilen Skorbutfälle)

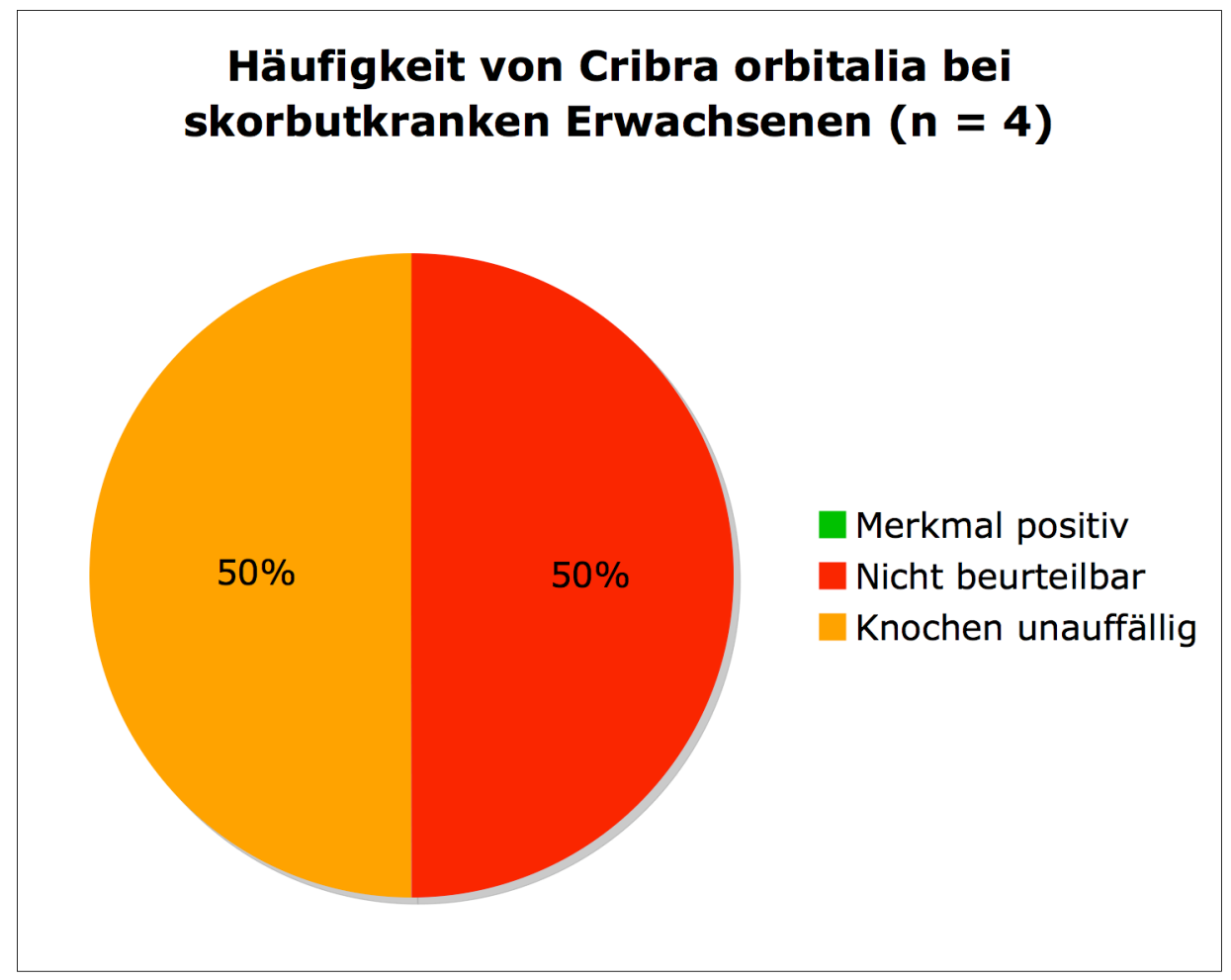

Abb. 22: Häufigkeit von Cribra orbitalia bei skorbutkranken Erwachsenen (Prozentzahlen beziehen sich auf Gesamtzahl der adulten Skorbutfälle) 


\subsection{Poröse Auflagerungen am äußeren Gesichtsschädel (Kardinalmerkmal 3)}

Bei $14(39 \%)$ von 36 untersuchten Individuen liegen poröse Auflagerungen am äußeren Gesichtsschädel vor, dabei handelt es sich ausschließlich um Kinder. Dreizehn (36\%) von ihnen sind an Skorbut erkrankt.

Dreizehn (36\%) der Individuen haben keinen befundbaren Gesichtsschädel, darunter zehn (28\%) Skorbutkranke.

Zehn (28\%) der Individuen haben unauffällige Gesichtsschädel, darunter auch fünf (14\%) mit Skorbut.

\begin{tabular}{|l|l|l|l|}
\hline & Merkmal positiv & Nicht beurteilbar & Knochen unauffällig \\
\hline Skorbut & $13(36 \%)$ & $10(28 \%)$ & $4(11 \%)$ \\
\hline V. a. Skorbut & 0 & $2(6 \%)$ & $2(6 \%)$ \\
\hline Sonstiges & $1(3 \%)$ & $1(3 \%)$ & $3(8 \%)$ \\
\hline
\end{tabular}

Tab. 56: Häufigkeit poröser Auflagerungen am äußeren Gesichtsschädel bei Individuen mit Skorbut, Verdacht auf Skorbut und Individuen mit anderen Diagnosen

Individuum Sk 1380, das vermutlich sowohl an Lepra als auch an Skorbut erkrankt ist, wird hier in der Gruppe der Skorbutkranken geführt. Die Abweichung von 100\% ergibt sich durch Auf- bzw. Abrundung.

V. a. $=$ Verdacht auf

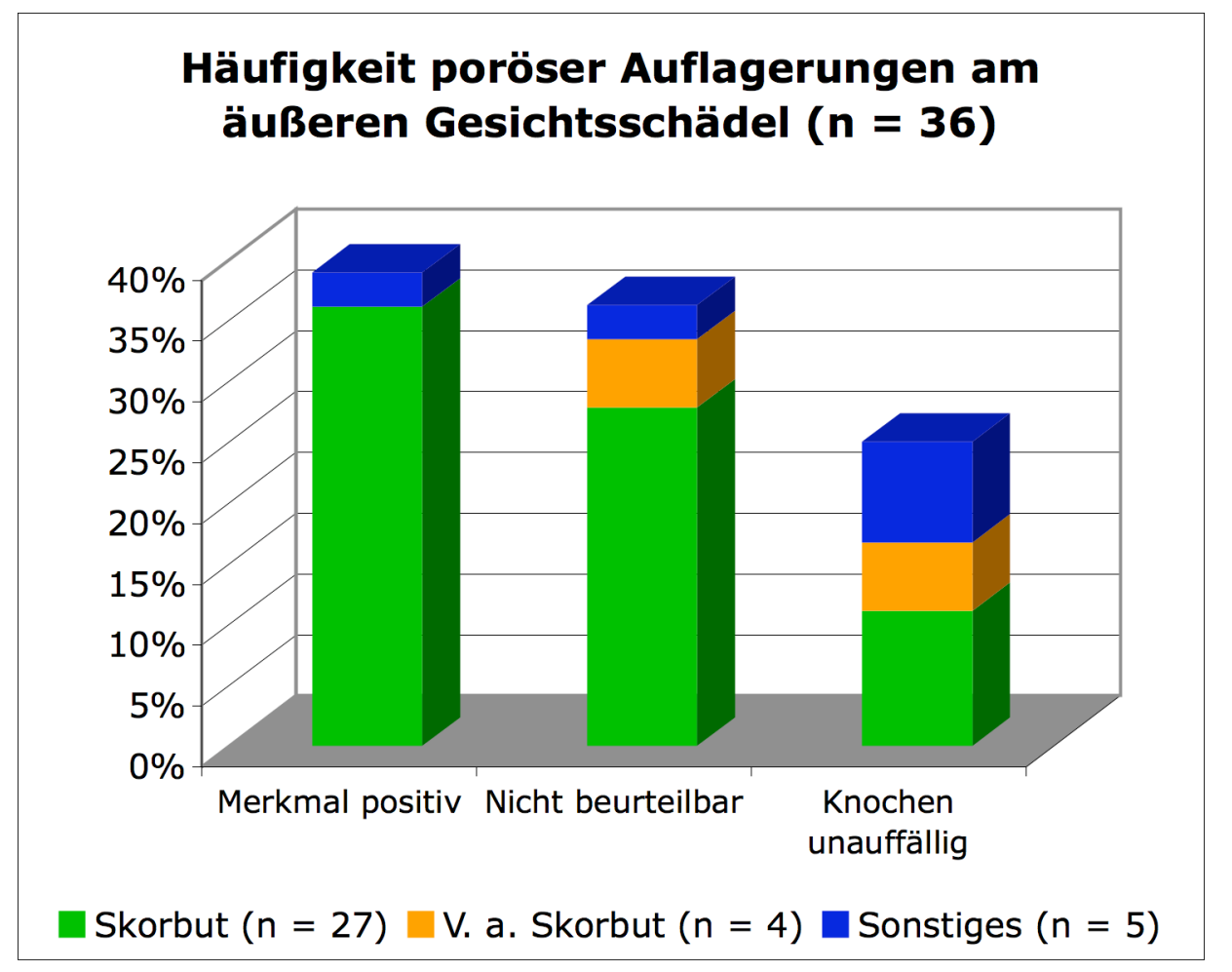

Abb. 23: Häufigkeit poröser Auflagerungen am äußeren Gesichtsschädel bei Individuen mit Skorbut, Verdacht auf Skorbut und Individuen mit anderen Diagnosen

Individuum Sk 1380, das vermutlich sowohl an Lepra als auch an Skorbut erkrankt ist, wird hier in der Gruppe der Skorbutkranken geführt.

V. a. $=$ Verdacht auf 
Betrachtet man nur die 25 untersuchten Kinder, so zeigen 14 (56\% aller Kinder) poröse Auflagerungen am äußeren Gesichtsschädel, davon 13 (52\% aller Kinder) mit Skorbut.

Neun Kinder (36\%) haben keinen befundbaren Gesichtsschädel, darunter auch acht (32\%) mit sicherem und eines (4\%) mit Verdacht auf Skorbut.

Bei drei Kindern (12\% aller Kinder) sind die Gesichtsschädel unauffällig, obwohl alle drei an Skorbut erkrankt sind.

\begin{tabular}{|l|l|l|l|}
\hline & Merkmal positiv & Nicht beurteilbar & Knochen unauffällig \\
\hline Skorbut & $13(52 \%)$ & $8(32 \%)$ & $2(8 \%)$ \\
\hline V. a. Skorbut & 0 & $1(4 \%)$ & 0 \\
\hline Sonstiges & $1(4 \%)$ & 0 & 0 \\
\hline
\end{tabular}

Tab. 57: Häufigkeit poröser Auflagerungen am äußeren Gesichtsschädel bei Kindern mit Skorbut, Verdacht auf Skorbut und Kindern mit anderen Diagnosen V. a. = Verdacht auf

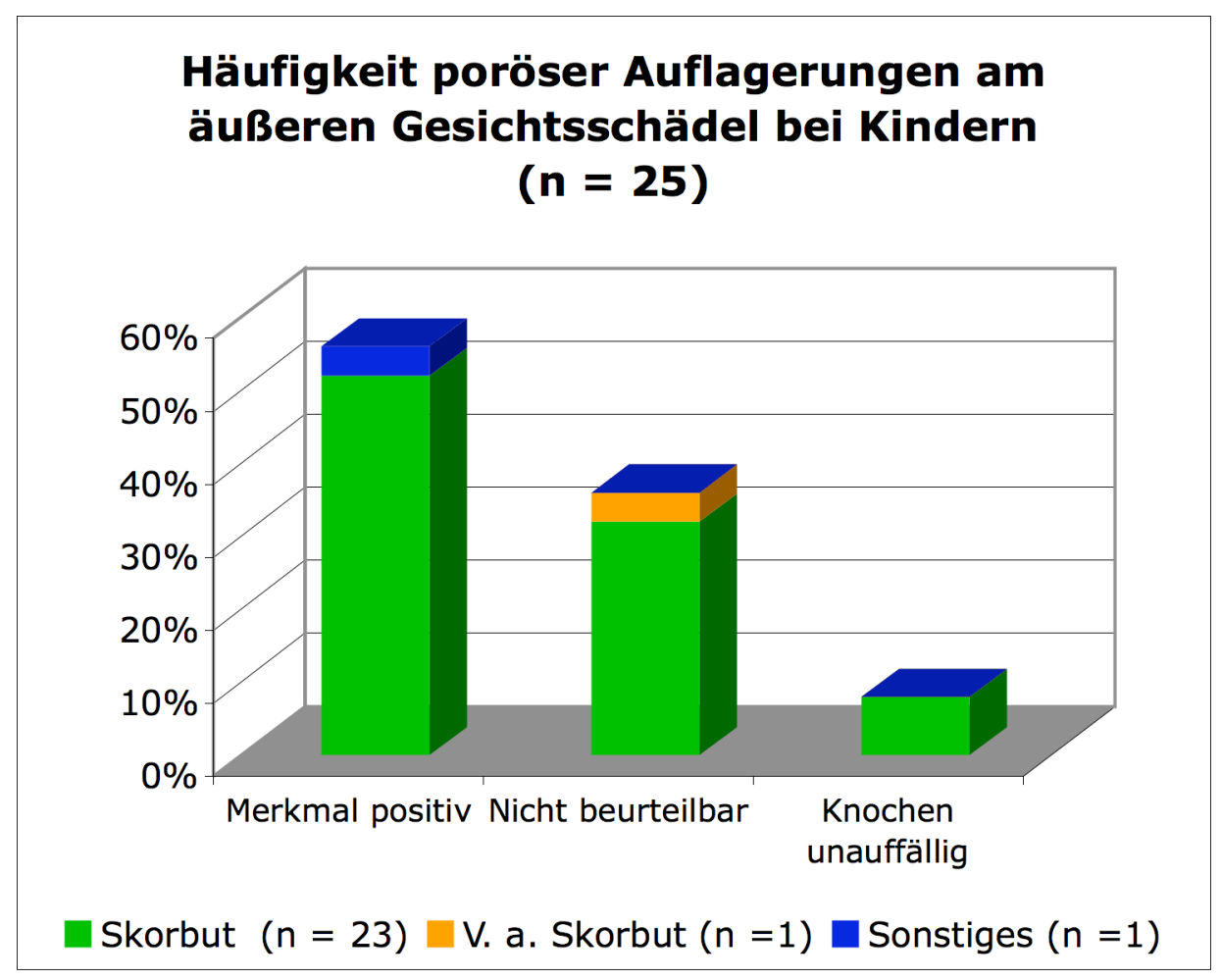

Abb. 24: Häufigkeit poröser Auflagerungen am äußeren Gesichtsschädel bei Kindern mit Skorbut, Verdacht auf Skorbut und Kindern mit anderen Diagnosen

V. a. = Verdacht auf

Keines der elf untersuchten adulten Individuen weist poröse Auflagerungen am äußeren Schädeldach auf. Bei vier (36\% aller Erwachsenen) ist der Gesichtsschädel nicht beurteilbar, darunter zwei (18\%) mit Skorbut und eins (9\%) mit Verdacht auf Skorbut. 


\begin{tabular}{|l|l|l|l|}
\hline & Merkmal positiv & Nicht beurteilbar & Knochen unauffällig \\
\hline Skorbut & 0 & $2(18 \%)$ & $2(18 \%)$ \\
\hline V. a. Skorbut & 0 & $1(9 \%)$ & $2(18 \%)$ \\
\hline sonstiges & 0 & $1(9 \%)$ & $3(27 \%)$ \\
\hline
\end{tabular}

Tab. 58: Häufigkeit poröser Auflagerungen am äußeren Gesichtsschädel bei Erwachsenen mit Skorbut, Verdacht auf Skorbut und Individuen mit anderen Diagnosen

Individuum (Sk 1380), das vermutlich sowohl an Lepra als auch an Skorbut erkrankt ist, wird hier in der Gruppe der Skorbutkranken geführt. Die Abweichung von 100\% ergibt sich durch Auf- bzw. Abrundung.

V. a. = Verdacht auf

\section{Häufigkeit poröser Auflagerungen am äußeren Gesichtsschädel von Erwachsenen $(\mathbf{n}=11)$}

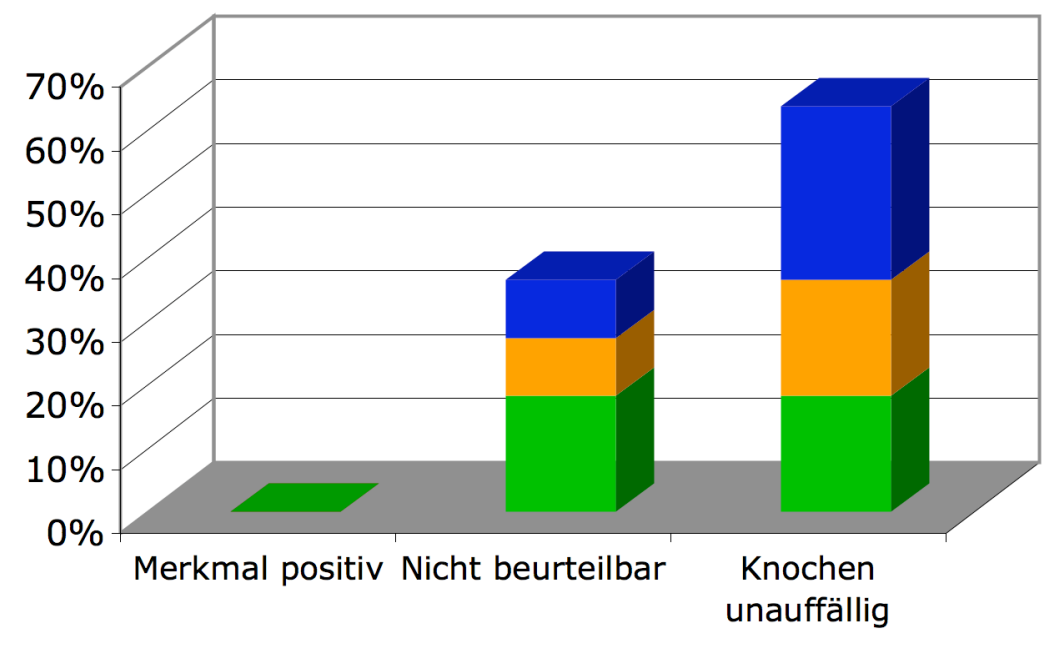

Skorbut $(n=4) \square$ V. a. Skorbut $(n=3) \square$ Sonstiges $(n=4)$

Abb. 25: Häufigkeit poröser Auflagerungen am äußeren Gesichtsschädel bei Erwachsenen mit Skorbut, V.a. Skorbut und Individuen mit anderen Diagnosen

Individuum (Sk 1380), das vermutlich sowohl an Lepra als auch an Skorbut erkrankt ist, wird hier in der Gruppe der Skorbutkranken geführt.

V. a. $=$ Verdacht auf

Bei den insgesamt 27 skorbutkranken Individuen finden sich in 13 Fällen (48\% aller Skorbutkranken) feinporöse Auflagerungen am äußeren Gesichtsschädel, bei zehn Individuen (37\% aller Skorbutkranken) ist der äußere Gesichtsschädel nicht beurteilbar, fünf Individuen (18\% aller Skorbutkranken) haben unauffällige Gesichtsschädel.

\begin{tabular}{|l|l|l|l|}
\hline & Merkmal positiv & Nicht beurteilbar & Knochen unauffällig \\
\hline Skorbutkranke (n=27) & $13(48 \%)$ & $10(37 \%)$ & $4(15 \%)$ \\
\hline
\end{tabular}

Tab. 59: Häufigkeit poröser Auflagerungen am äußeren Gesichtsschädel bei den untersuchten Skorbutkranken (Prozentzahlen beziehen sich auf Gesamtzahl der Skorbutfälle) 


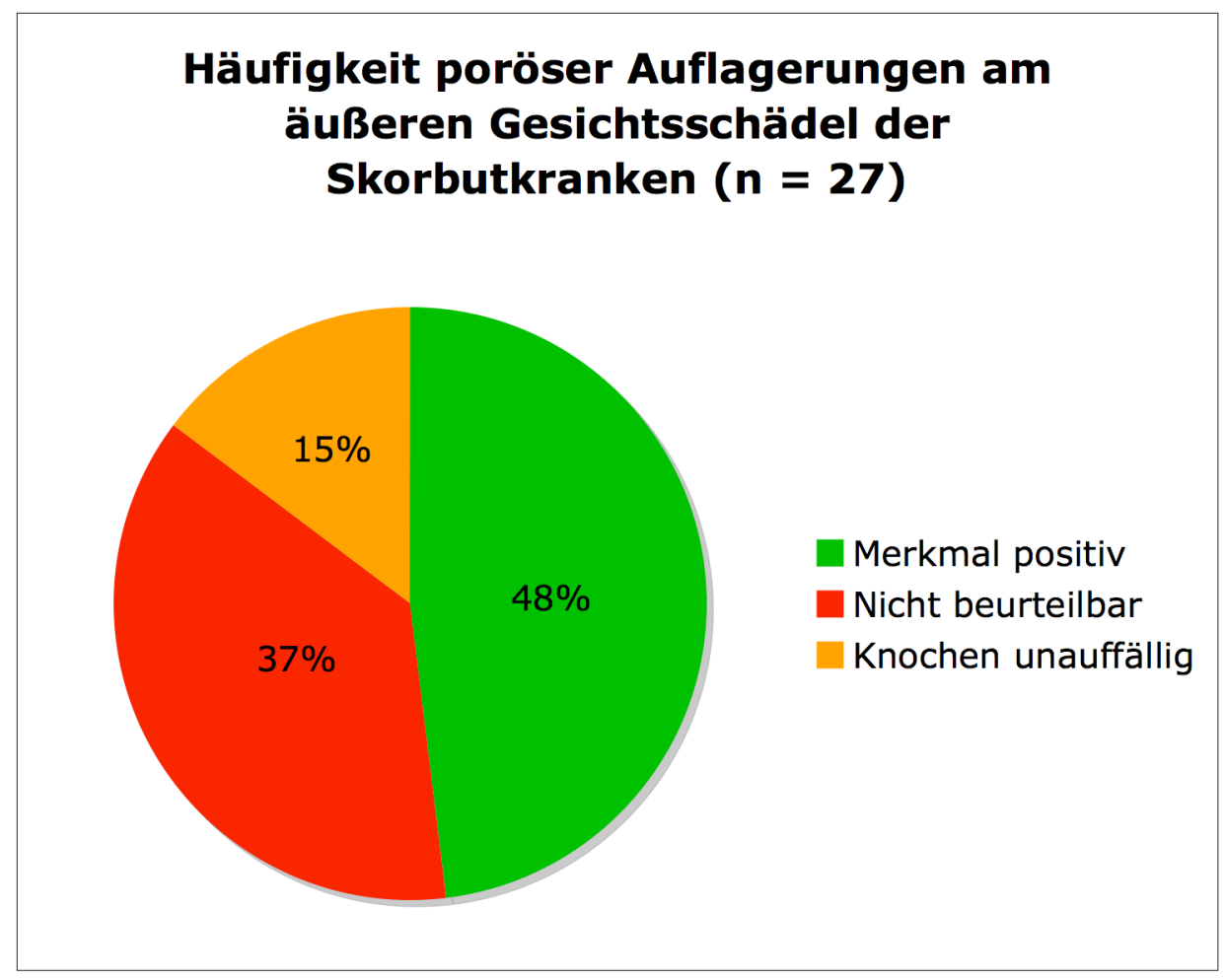

Abb. 26: Häufigkeit poröser Auflagerungen am äußeren Gesichtsschädel bei den untersuchten Skorbutkranken (Prozentzahlen beziehen sich auf Gesamtzahl der Skorbutfälle)

Von den 23 an Skorbut erkrankten Kindern haben 13 (57\% der skorbutkranken Kinder) poröse Auflagerungen am äußeren Gesichtsschädel, bei acht Kindern $(35 \%$ der skorbutkranken Kinder) ist der äußere Gesichtsschädel nicht beurteilbar, bei zwei Kindern (9\%) ist er unauffällig.

\begin{tabular}{|l|l|l|l|}
\hline & Merkmal positiv & Nicht beurteilbar & Knochen unauffällig \\
\hline Skorbutkranke Kinder $(\mathbf{n}=\mathbf{2 3})$ & $13(57 \%)$ & $8(35 \%)$ & $2(9 \%)$ \\
\hline
\end{tabular}

Tab. 60: Häufigkeit poröser Auflagerungen am äußeren Gesichtsschädel bei den untersuchten skorbutkranken Kindern (Prozentzahlen beziehen sich auf Gesamtzahl der skorbutkranken Kinder)

Keines der vier an Skorbut erkrankten adulten Individuen hat poröse Auflagerungen am äußeren Gesichtsschädel, bei je zweien (50\% der adulten Skorbutfälle) ist der Gesichtsschädel unauffällig bzw. nicht befundbar.

\begin{tabular}{|l|l|l|l|}
\hline & Merkmal positiv & Nicht beurteilbar & Knochen unauffällig \\
\hline Skorbutkranke Erwachsene $(\mathbf{n}=4)$ & 0 & $2(50 \%)$ & $2(50 \%)$ \\
\hline
\end{tabular}

Tab. 61: Häufigkeit poröser Auflagerungen am äußeren Gesichtsschädel bei den untersuchten skorbutkranken Erwachsenen (Prozentzahlen beziehen sich auf Gesamtzahl der skorbutkranken Erwachsenen) 


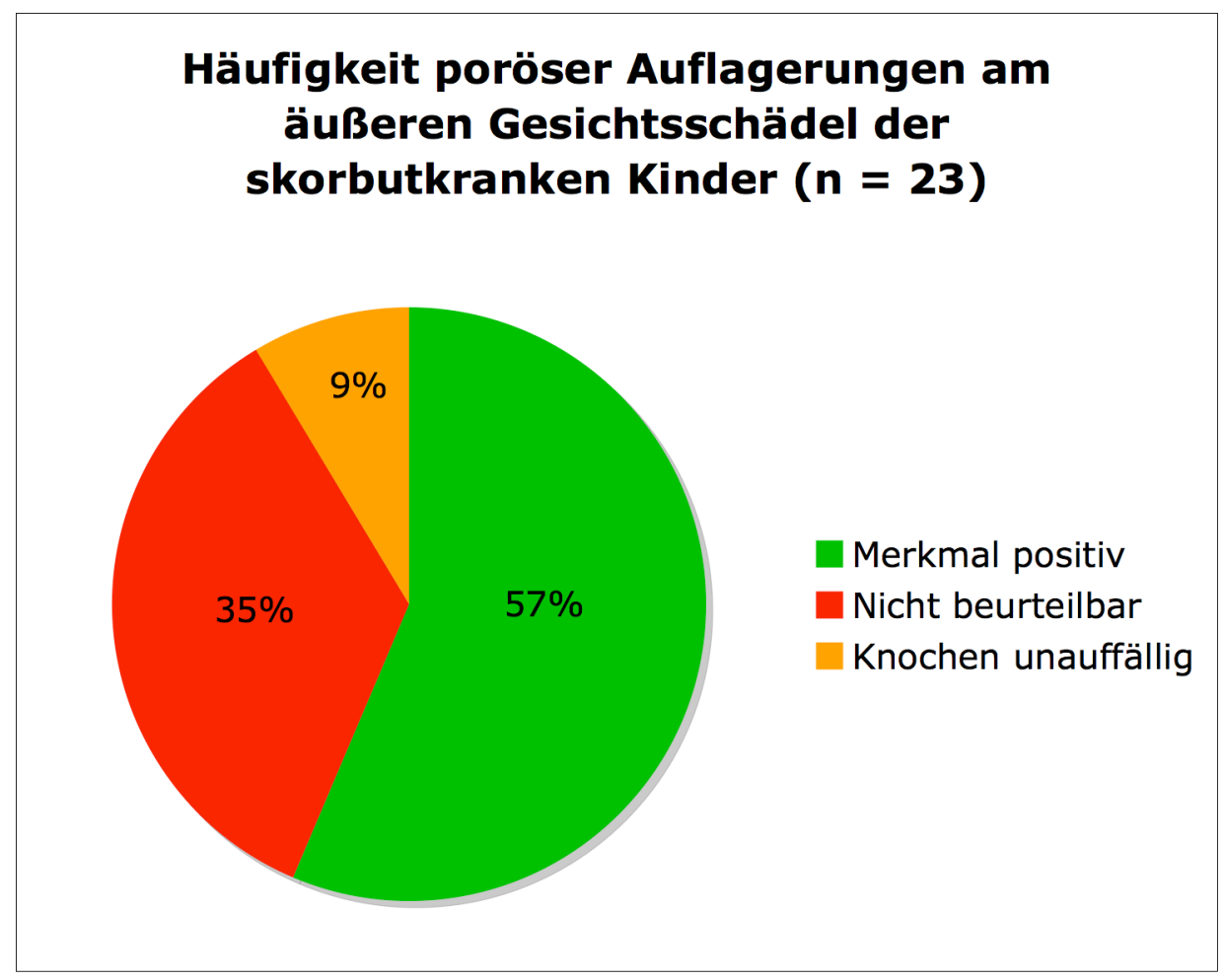

Abb. 27: Häufigkeit poröser Auflagerungen am äußeren Gesichtsschädel bei den untersuchten skorbutkranken Kindern (Prozentzahlen beziehen sich auf Gesamtzahl der skorbutkranken Kinder)

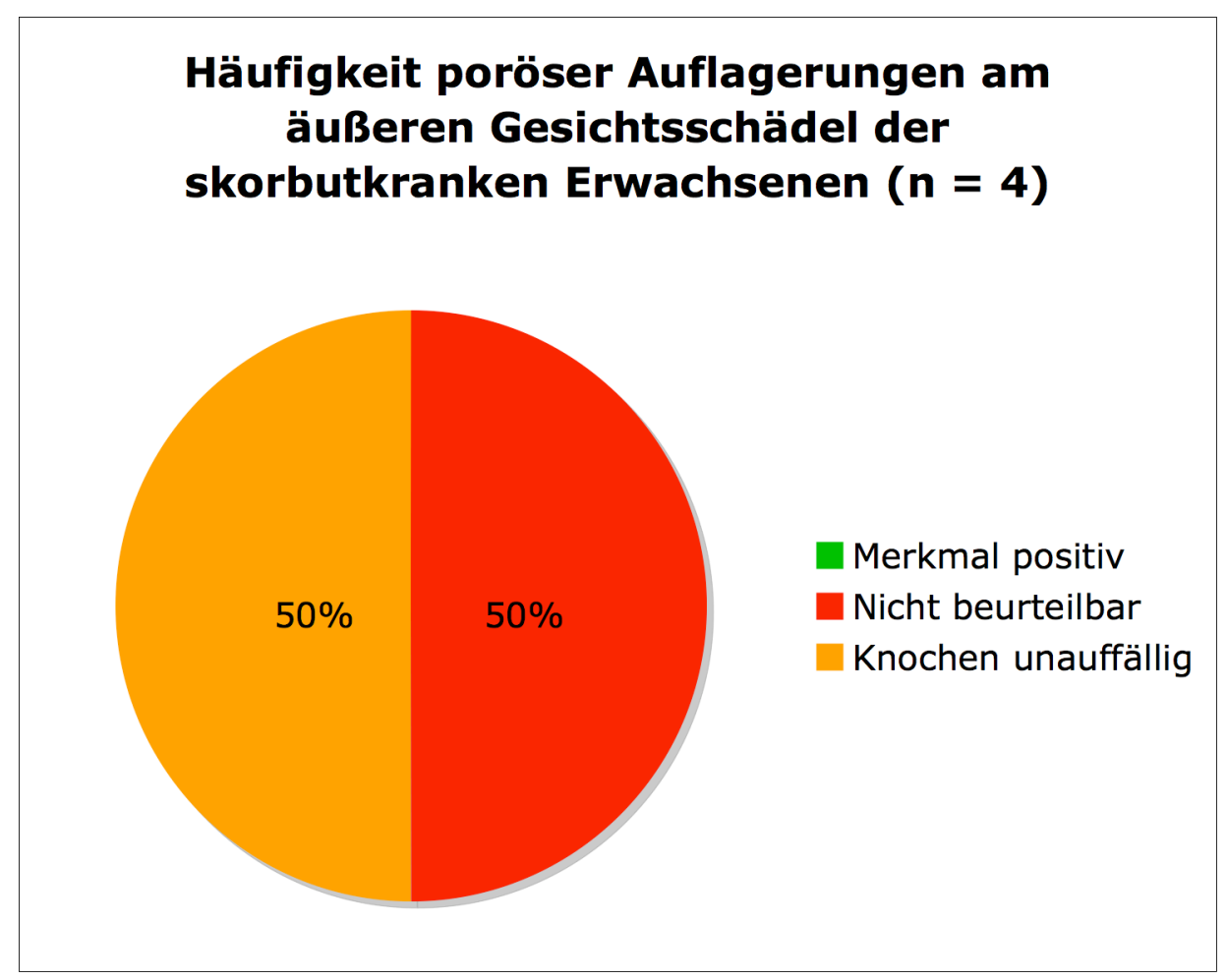

Abb. 28: Häufigkeit poöser Auflagerungen am äußeren Gesichtsschädel bei den untersuchten skorbutkranken Erwachsenen (Prozentzahlen beziehen sich auf Gesamtzahl der skorbutkranken Erwachsenen) 


\subsection{Poröse Auflagerungen am Alveolarknochen (Kardinalmerkmal 4)}

Bei 17 (47\%) der 36 untersuchten Individuen liegen poröse Auflagerungen am Alveolarknochen vor, davon sind 16 (44\%) an Skorbut erkrankt.

In zwölf Fällen (33\%) ist der Alveolarknochen nicht befundbar, unter anderem bei neun Skorbutfällen (25\%) und zwei Individuen (6\%) mit Verdacht auf Skorbut.

Sieben Individuen (19\%) haben unauffällige Alveolarknochen, darunter je zwei (6\%) Skorbutkranke und Individuen mit Verdacht auf Skorbut.

\begin{tabular}{|c|c|c|c|}
\hline & Merkmal positiv & Nicht beurteilbar & Knochen unauffällig \\
\hline Skorbut & $16(44 \%)$ & $9(25 \%)$ & $2(6 \%)$ \\
\hline V. a. Skorbut & 0 & $2(6 \%)$ & $2(6 \%)$ \\
\hline Sonstiges & $1(3 \%)$ & $1(3 \%)$ & $3(8 \%)$ \\
\hline
\end{tabular}

Tab. 62: Häufigkeit poröser Auflagerungen am Alveolarknochen bei Individuen mit Skorbut, Verdacht auf Skorbut und Individuen mit anderen Diagnosen

Individuum Sk 1380, das vermutlich sowohl an Lepra als auch an Skorbut erkrankt ist, wird hier in der Gruppe der Skorbutkranken geführt. Die Abweichung von 100\% ergibt sich durch Auf- bzw. Abrundung.

V. a. = Verdacht auf

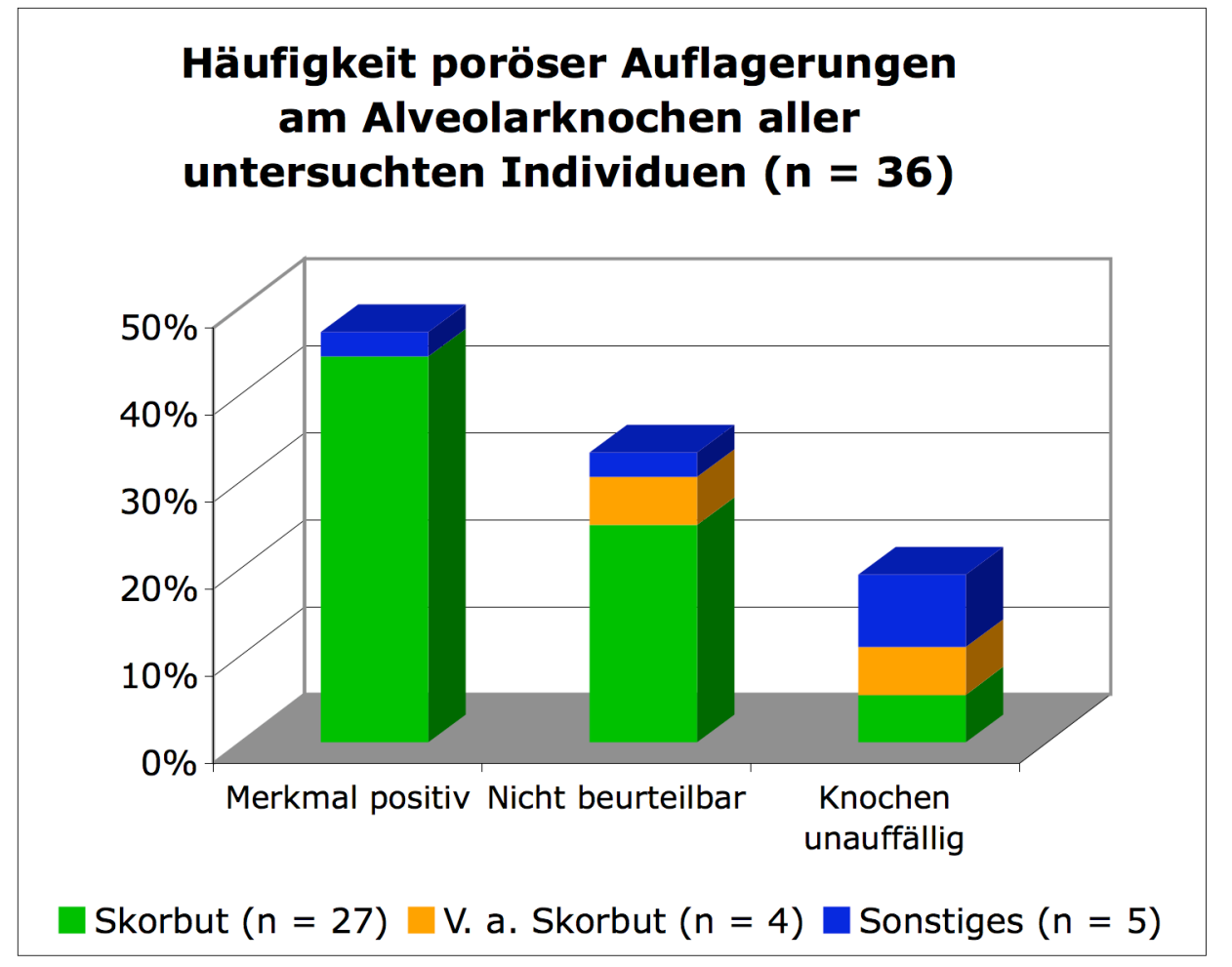

Abb. 29: Häufigkeit poröser Auflagerungen am Alveolarknochen bei Individuen mit Skorbut, Verdacht auf Skorbut und Individuen mit anderen Diagnosen

Individuum Sk 1380, das vermutlich sowohl an Lepra als auch an Skorbut erkrankt ist, wird hier in der Gruppe der Skorbutkranken geführt.

V. a. = Verdacht auf

Unter den 25 untersuchten Kindern sind 15 (60\% aller Kinder) mit porösen Auflagerungen am Alveolarknochen, darunter 14 (56\% aller Kinder) mit Skorbut. 
Bei neun Kindern (36\% aller Kinder) ist keine Befundung der Alveolarknochen möglich, davon haben acht (32\% aller Kinder) Skorbut, bei einem (4\% aller Kinder) besteht der Verdacht auf Skorbut.

Zwei Kinder (8\% aller Kinder) haben unauffällige Alveolarknochen, darunter ein (4\% aller Kinder) Skorbutfall.

\begin{tabular}{|l|l|l|l|}
\hline & Merkmal positiv & Nicht beurteilbar & Knochen unauffällig \\
\hline Skorbut & $14(56 \%)$ & $8(32 \%)$ & $1(4 \%)$ \\
\hline V. a. Skorbut & 0 & $1(4 \%)$ & 0 \\
\hline Sonstiges & 0 & 0 & $1(4 \%)$ \\
\hline
\end{tabular}

Tab. 63: Häufigkeit poröser Auflagerungen am Alveolarknochen bei Kindern mit Skorbut, Verdacht auf Skorbut und Kindern mit anderen Diagnosen

V. a. = Verdacht auf

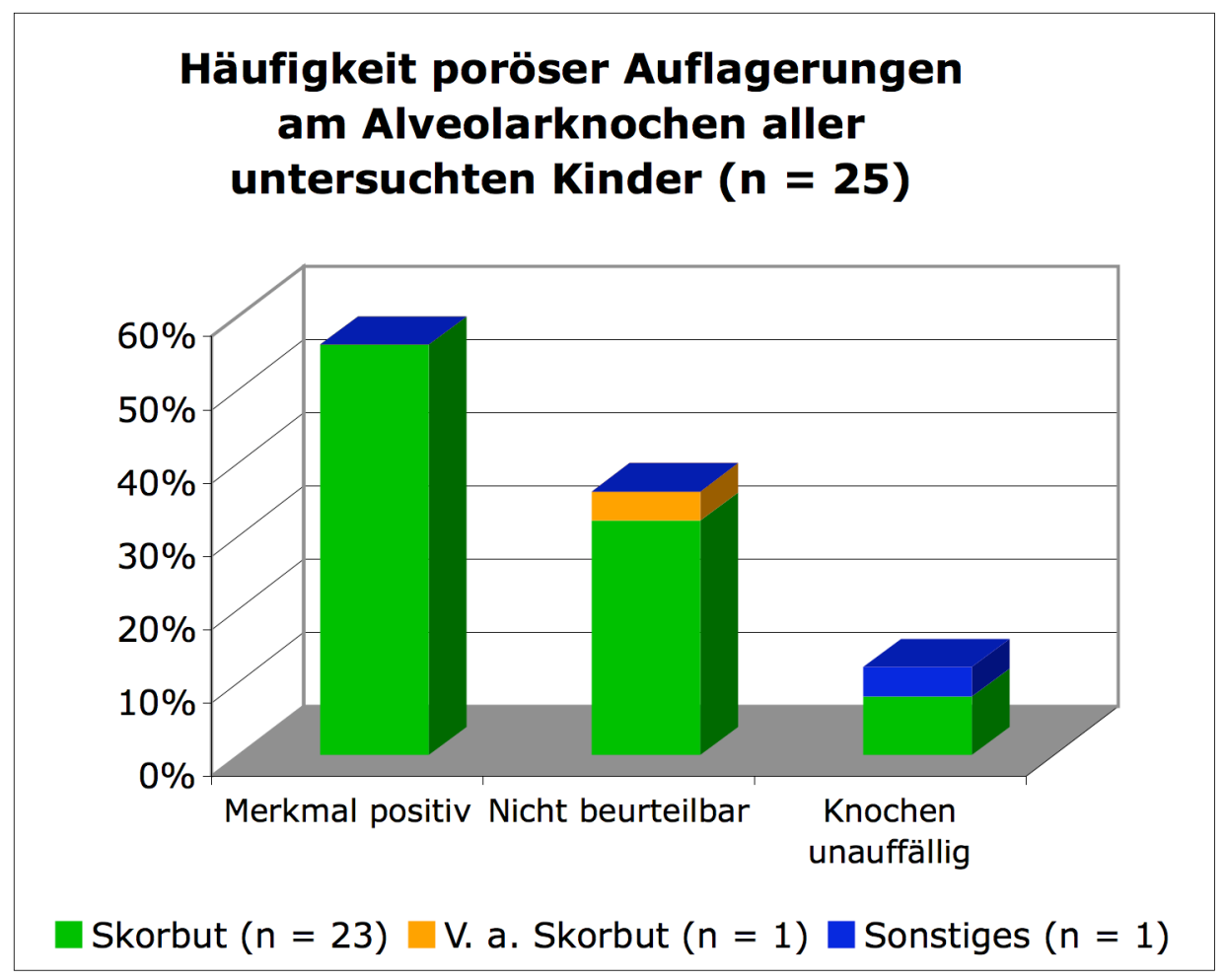

Abb. 30: Häufigkeit poröser Auflagerungen am Alveolarknochen bei Kindern mit Skorbut, Verdacht auf Skorbut und Kindern mit anderen Diagnosen

V. a. = Verdacht auf

Von elf untersuchten Erwachsenen haben drei $(27 \%$ aller Erwachsenen) poröse Auflagerungen am Alveolarknochen, darunter zwei (18\% aller Erwachsenen) mit Skorbut.

In drei Fällen (27\% aller Erwachsenen) ist der Alveolarknochen nicht beurteilbar, darunter je ein Individuum (9\% aller Erwachsenen) mit Skorbut und Verdacht auf Skorbut.

Fünf Erwachsene (46\% aller Erwachsenen) zeigen unauffällige Alveolarknochen, darunter ein Skorbutfall (9\% aller Erwachsenen) und zwei (18\% aller Erwachsenen) Individuen mit Verdacht auf Skorbut. 


\begin{tabular}{|l|l|l|l|}
\hline & Merkmal positiv & Nicht beurteilbar & Knochen unauffällig \\
\hline Skorbut & $2(18 \%)$ & $1(9 \%)$ & $1(9 \%)$ \\
\hline V. a. Skorbut & 0 & $1(9 \%)$ & $2(18 \%)$ \\
\hline Sonstiges & $1(9) \%$ & $1(9 \%)$ & $2(18 \%)$ \\
\hline
\end{tabular}

Tab. 64: Häufigkeit poröser Auflagerungen am Alveolarknochen bei Erwachsenen mit Skorbut, Verdacht auf Skorbut und Erwachsenen mit anderen Diagnosen Individuum Sk 1380, das vermutlich sowohl an Lepra als auch an Skorbut erkrankt ist, wird hier in der Gruppe der Skorbutkranken geführt. Die Abweichung von 100\% ergibt sich durch Auf- bzw. Abrundung.

V. a. = Verdacht auf

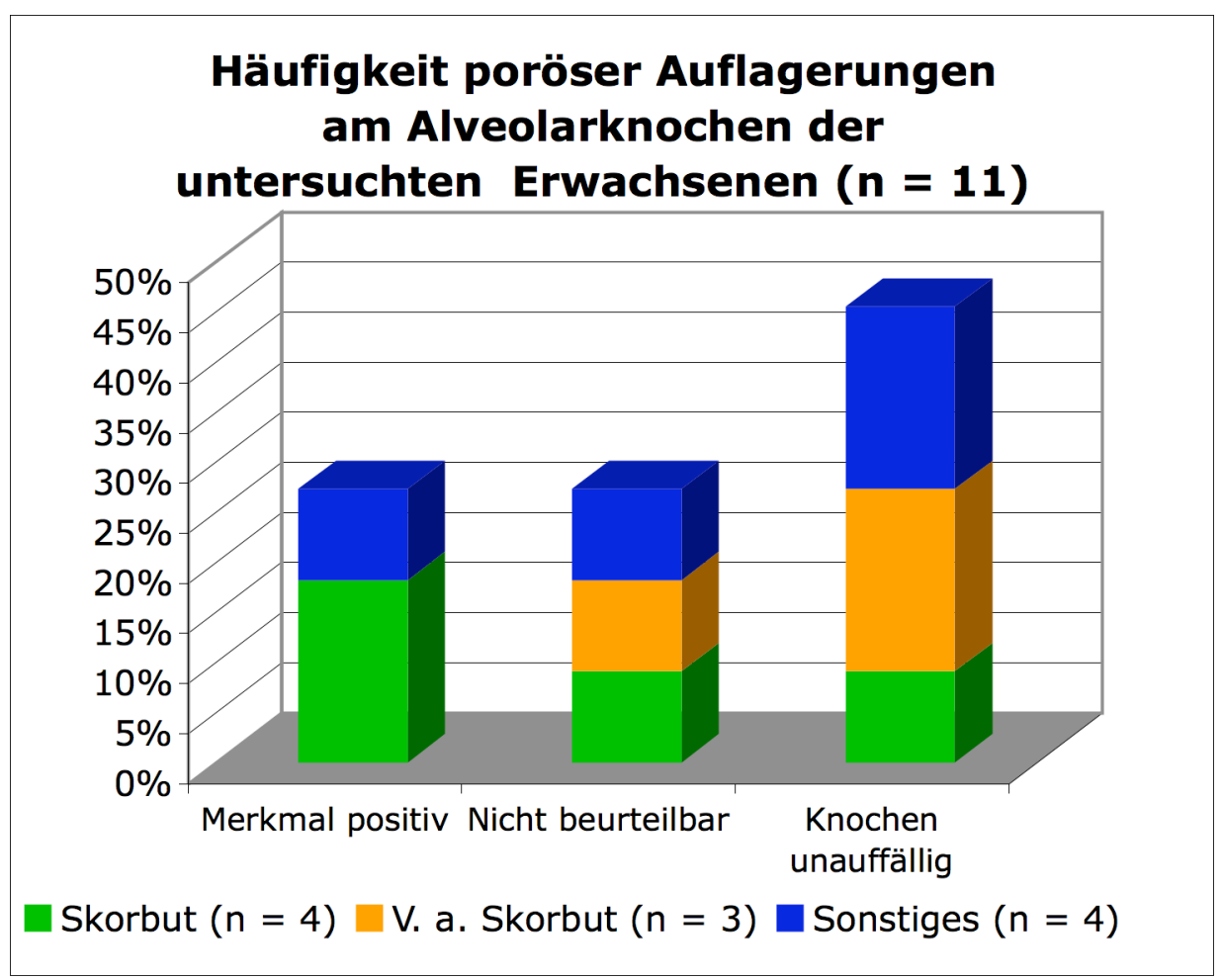

Abb. 31: Häufigkeit von porösen Auflagerungen am Alveolarknochen bei Erwachsenen mit Skorbut, wahrscheinlichem Skorbut und Erwachsenen mit anderen Diagnosen

Individuum Sk 1380, das vermutlich sowohl an Lepra als auch an Skorbut erkrankt ist, wird hier in der Gruppe der Skorbutkranken geführt.

V. a. $=$ Verdacht auf

Unter den 27 untersuchten Skorbutfällen sind 16 (59\% aller Skorbutfälle) mit porösen Auflagerungen am Alveolarknochen, bei neun (33\% aller Skorbutfälle) ist keine Beurteilung möglich, zwei Individuen (7\% aller Skorbutfälle) haben unauffällige Alveolarknochen.

\begin{tabular}{|l|l|l|l|}
\hline & Merkmal positiv & Nicht beurteilbar & Knochen unauffällig \\
\hline Skorbutkranke $(\mathbf{n}=\mathbf{2 7})$ & $16(59 \%)$ & $9(33 \%)$ & $2(7 \%)$ \\
\hline
\end{tabular}

Tab. 65: Häufigkeit poröser Auflagerungen am Alveolarknochen der untersuchten Skorbutkranken (Prozentzahlen beziehen sich auf Gesamtzahl der Skorbutfälle, die Abweichung von 100\% ergibt sich durch Auf- bzw. Abrundung) 


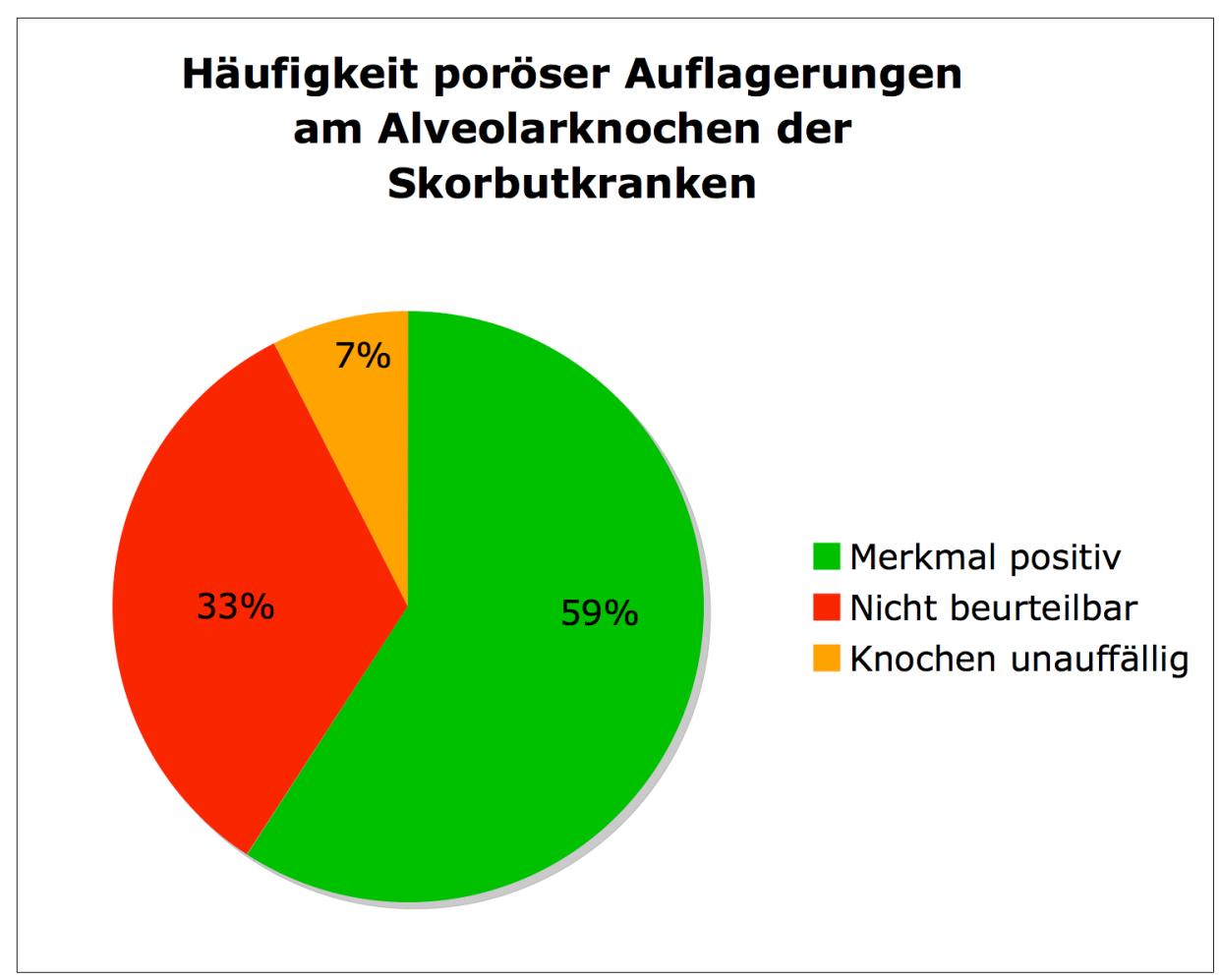

Abb. 32: Häufigkeit poröser Auflagerungen am Alveolarknochen der untersuchten Skorbutkranken (Prozentzahlen beziehen sich auf Gesamtzahl der Skorbutfälle, die Abweichung von 100\% ergibt sich durch Auf- bzw. Abrundung)

Unter den 23 skorbutkranken Kindern sind 14 (61\% aller skorbutkranken Kinder) mit porösen Auflagerungen an den Alveolarknochen, eins (4\% aller skorbutkranken Kinder) mit unauffälligen und acht (35\% aller skorbutkranken Kinder) mit nicht beurteilbaren Alveolarknochen.

\begin{tabular}{|l|l|l|l|}
\hline & Merkmal positiv & Nicht beurteilbar & Knochen unauffällig \\
\hline Skorbutkranke Kinder $(\mathbf{n}=\mathbf{2 3})$ & $14(61 \%)$ & $8(35 \%)$ & $1(4 \%)$ \\
\hline
\end{tabular}

Tab. 66: Häufigkeit poröser Auflagerungen am Alveolarknochen der untersuchten skorbutkranken Kindern (Prozentzahlen beziehen sich auf Gesamtzahl der infantilen Skorbutfälle)

Bei den vier skorbutkranken Erwachsenen finden sich in zwei Fällen (50\% der adulten Skorbutfälle) poröse Auflagerungen am Alveolarknochen, je ein Individuum $(25 \%$ der adulten Skorbutfälle) haben unauffällige bzw. nicht befundbare Alveolarknochen.

\begin{tabular}{|l|l|l|l|}
\hline & Merkmal positiv & Nicht beurteilbar & Knochen unauffällig \\
\hline Skorbutkranke Erwachsene $(n=4)$ & $2(50 \%)$ & $1(25 \%)$ & $1(25 \%)$ \\
\hline
\end{tabular}

Tab. 67: Häufigkeit poröser Auflagerungen am Alveolarknochen der untersuchten skorbutkranken Erwachsenen (Prozentzahlen beziehen sich auf Gesamtzahl der adulten Skorbutfälle) 


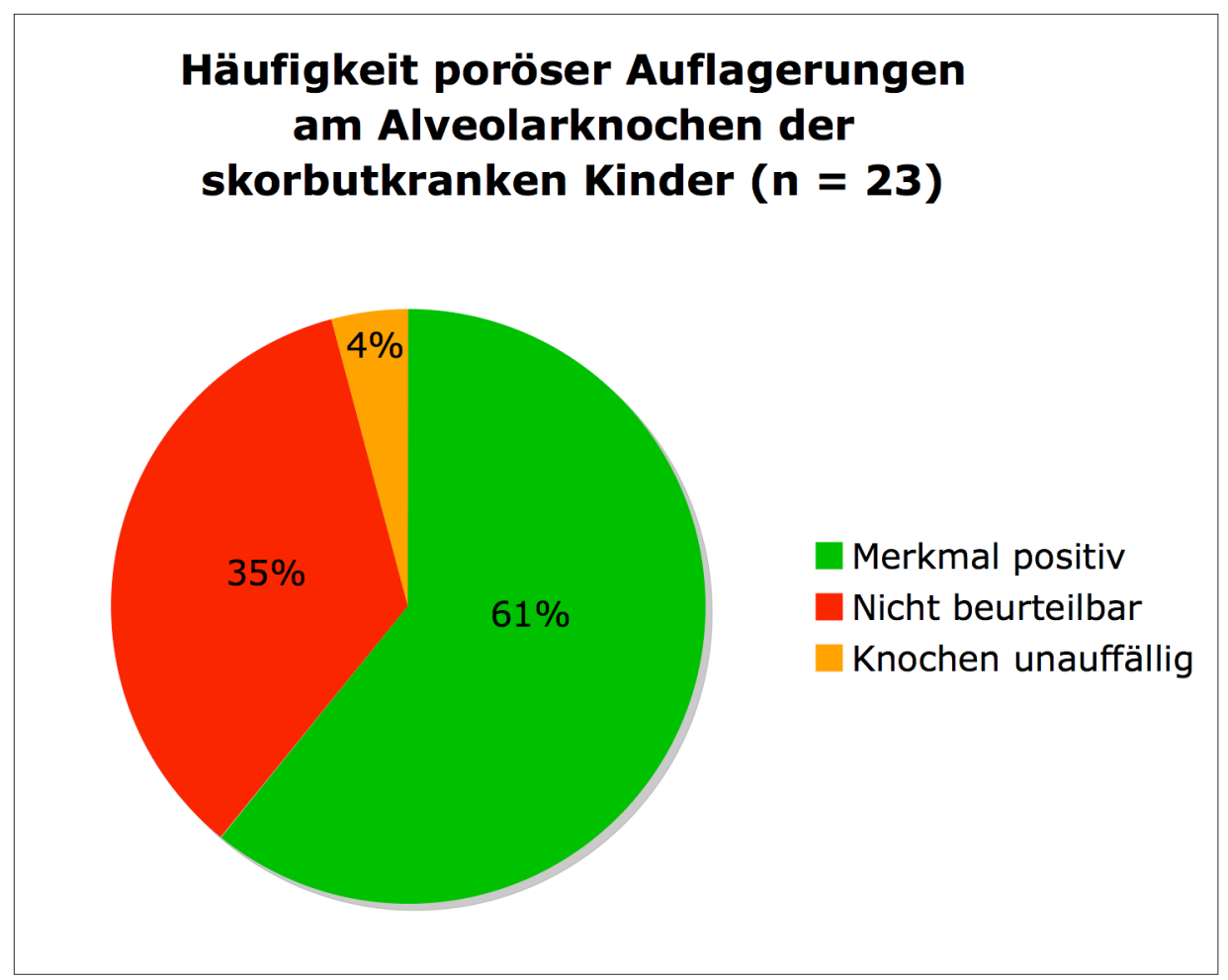

Abb. 33: Häufigkeit poröser Auflagerungen am Alveolarknochen der untersuchten skorbutkranken Kinder (Prozentzahlen beziehen sich auf Gesamtzahl der infantilen Skorbutfälle)

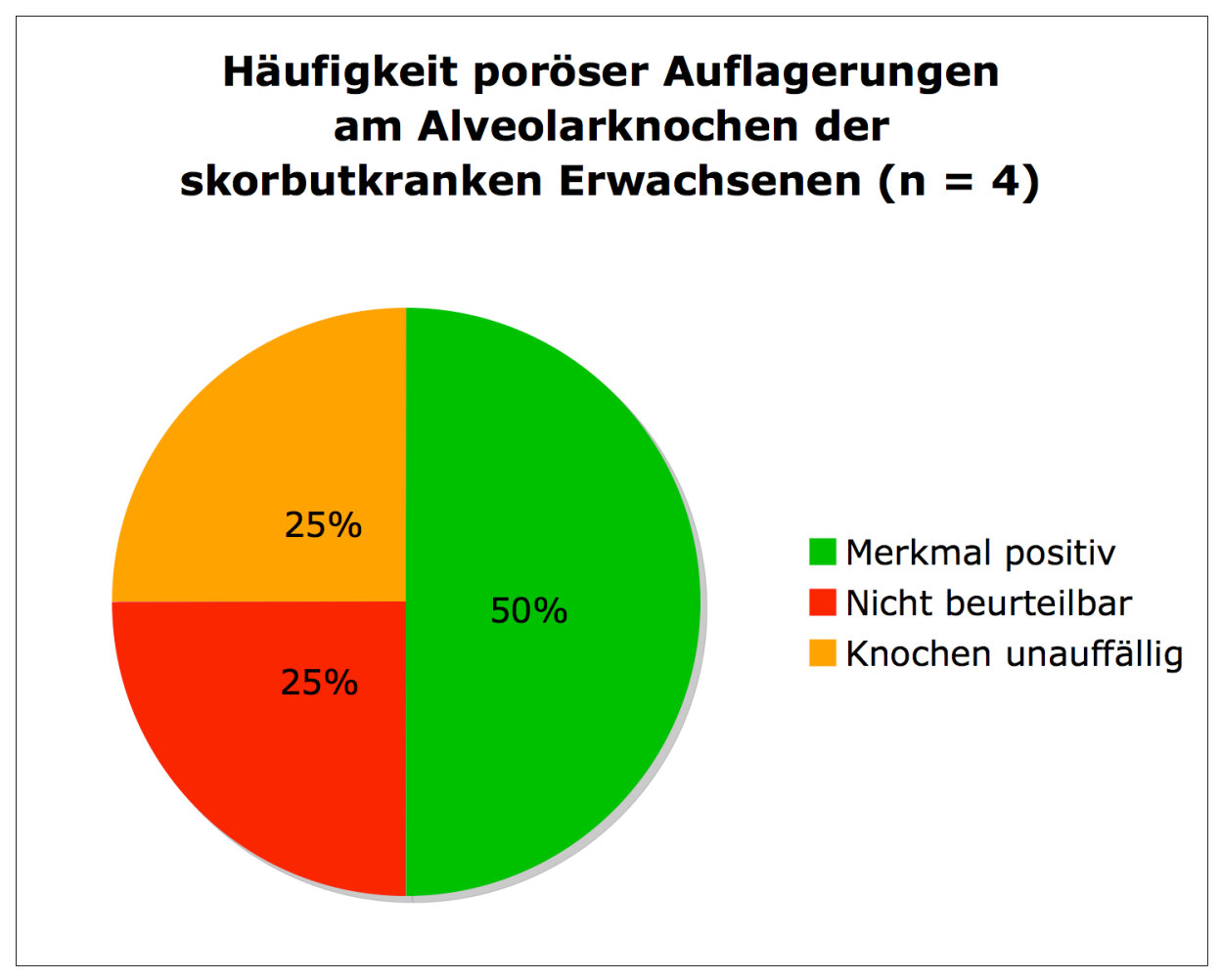

Abb. 34: Häufigkeit poröser Auflagerungen am Alveolarknochen der untersuchten skorbutkranken Erwachsenen (Prozentzahlen beziehen sich auf Gesamtzahl der adulten Skorbutfälle) 


\subsection{Poröse Auflagerungen an Langknochen (Kardinalmerkmal 5)}

Bei 27 (75\%) von 36 untersuchten Individuen liegen poröse Auflagerungen an Langknochen vor, $21(58 \%)$ von ihnen sind an Skorbut erkrankt. Drei (8\%) der untersuchten Individuen haben keine befundbaren Langknochen, darunter zwei (6\%) Skorbutkranke.

Sechs (17\%) der Individuen haben „unauffällige“ Langknochen, darunter auch vier (11\%) mit Skorbut. Allerdings ist hierbei zu beachten, dass in die Gruppe „unauffällig“ auch diejenigen Knochen fallen, die eine poröse Oberfläche, aber keine Auflagerungen haben. Diese werden gesondert unter Kapitel 3.18 Poröse Oberflächen an Langknochen (Nebenmerkmal 4) behandelt.

\begin{tabular}{|l|l|l|l|}
\hline & Merkmal positiv & Nicht beurteilbar & Knochen unauffällig \\
\hline Skorbut & $21(58 \%)$ & $2(6 \%)$ & $4(11 \%)$ \\
\hline V. a. Skorbut & $4(11 \%)$ & 0 & 0 \\
\hline Sonstiges & $2(6 \%)$ & $1(3 \%)$ & $2(6 \%)$ \\
\hline
\end{tabular}

Tab. 68: Häufigkeit poröser Auflagerungen an den Langknochen bei Individuen mit Skorbut, Verdacht auf Skorbut und Individuen mit anderen Diagnosen

Individuum Sk 1380, das vermutlich sowohl an Lepra als auch an Skorbut erkrankt ist, wird hier in der Gruppe der Skorbutkranken geführt. Unter „unauffällig“ fallen hier auch Langknochen mit poröser Oberfläche (vgl. Nebenmerkmal 4). Die Abweichung von 100\% ergibt sich durch Auf- bzw. Abrundung. V. a. = Verdacht auf

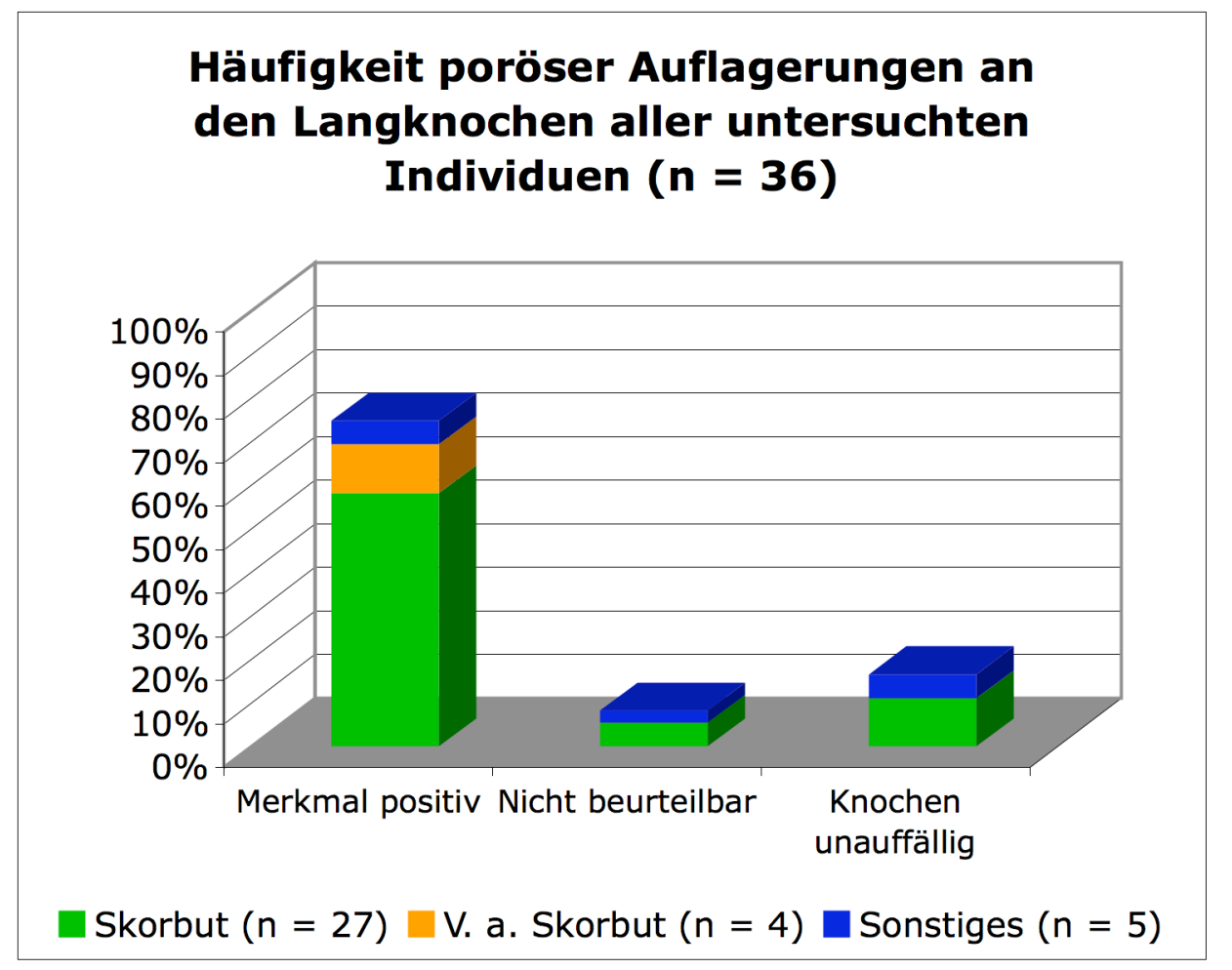

Abb. 35: Häufigkeit poröser Auflagerungen an Langknochen bei Individuen mit Skorbut, Verdacht auf Skorbut und Individuen mit anderen Diagnosen

Individuum Sk 1380, das vermutlich sowohl an Lepra als auch an Skorbut erkrankt ist, wird hier in der Gruppe der Skorbutkranken geführt. Unter „,unauffällig“ fallen hier auch Langknochen mit poröser Oberfläche (vgl. Nebenmerkmal 4). V. a. = Verdacht auf 
Betrachtet man nur die 25 untersuchten Kinder, so zeigen 18 (72\% aller Kinder) poröse Auflagerungen an den Langknochen, davon 17 (68\% aller Kinder) mit Skorbut.

Drei Kinder (12\% aller Kinder) haben keine befundbaren Langknochen, darunter auch zwei (8\% aller Kinder) mit Skorbut.

Bei vier Kindern (16\% aller Kinder) sind die Langknochen unauffällig, diese sind an Skorbut erkrankt.

\begin{tabular}{|l|l|l|l|}
\hline & Merkmal positiv & Nicht beurteilbar & Knochen unauffällig \\
\hline Skorbut & $17(68 \%)$ & $2(8 \%)$ & $4(16 \%)$ \\
\hline V. a. Skorbut & $1(4 \%)$ & 0 & 0 \\
\hline sonstiges & 0 & $1(4 \%)$ & 0 \\
\hline
\end{tabular}

Tab. 69: Häufigkeit poröser Auflagerungen an Langknochen bei Kindern mit Skorbut, Verdacht auf Skorbut und Kindern mit anderen Diagnosen Unter „unauffällig“ fallen hier auch Langknochen mit poröser Oberfläche (vgl. Nebenmerkmal 4).

V. a. $=$ Verdacht auf

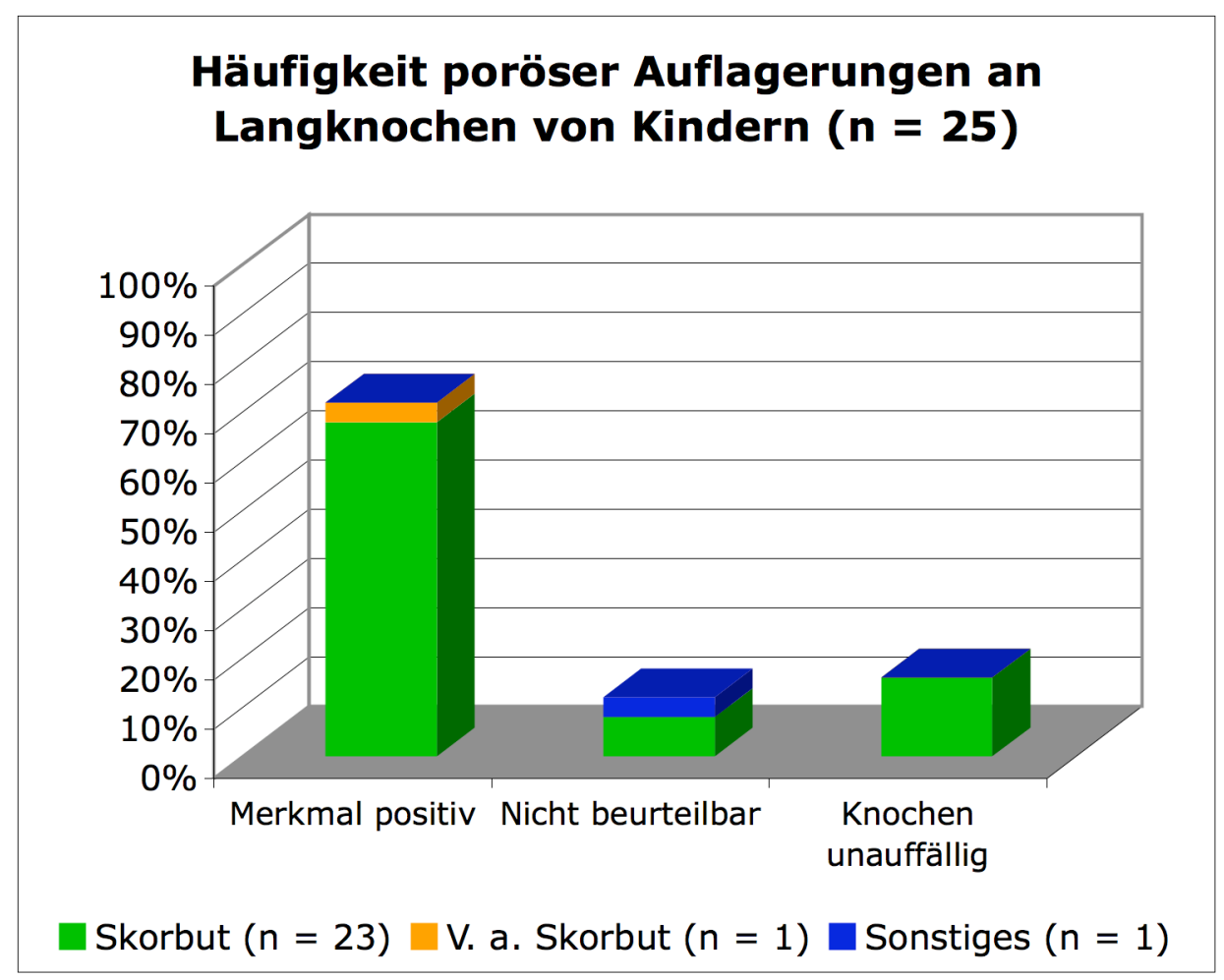

Abb. 36: Häufigkeit poröser Auflagerungen an Langknochen bei Kindern mit Skorbut, Verdacht auf Skorbut und Kindern mit anderen Diagnosen

Unter „unauffällig“ fallen hier auch Langknochen mit poröser Oberfläche (vgl. Nebenmerkmal 4).

V. a. $=$ Verdacht auf

Neun (82\% aller Erwachsenen) der elf untersuchten adulten Individuen weisen poröse Auflagerungen an den Langknochen auf, davon sind vier (36\% aller Erwachsenen) sicher und drei (27\% aller Erwachsenen) wahrscheinlich an Skorbut erkrankt. 
Bei zwei adulten Individuen (18\% aller Erwachsenen) sind die Langknochen unauffällig, diese sind vermutlich nicht an Skorbut erkrankt.

\begin{tabular}{|l|l|l|l|}
\hline & Merkmal positiv & Nicht beurteilbar & Knochen unauffällig \\
\hline Skorbut & $4(36 \%)$ & 0 & 0 \\
\hline V.a. Skorbut & $3(27 \%)$ & 0 & 0 \\
\hline Sonstiges & $2(18 \%)$ & 0 & $2(18 \%)$ \\
\hline
\end{tabular}

Tab. 70: Häufigkeit poröser Auflagerungen an den Langknochen von Erwachsenen mit Skorbut, V.a. Skorbut und Individuen mit anderen Diagnosen

Individuum Sk 1380, das vermutlich sowohl an Lepra als auch an Skorbut erkrankt ist, wird hier in der Gruppe der Skorbutkranken geführt.

Unter „unauffällig“ fallen hier auch Langknochen mit poröser Oberfläche (vgl. Nebenmerkmal 4). Die Abweichung von 100\% ergibt sich durch Auf- bzw. Abrundung.

V. a. $=$ Verdacht auf

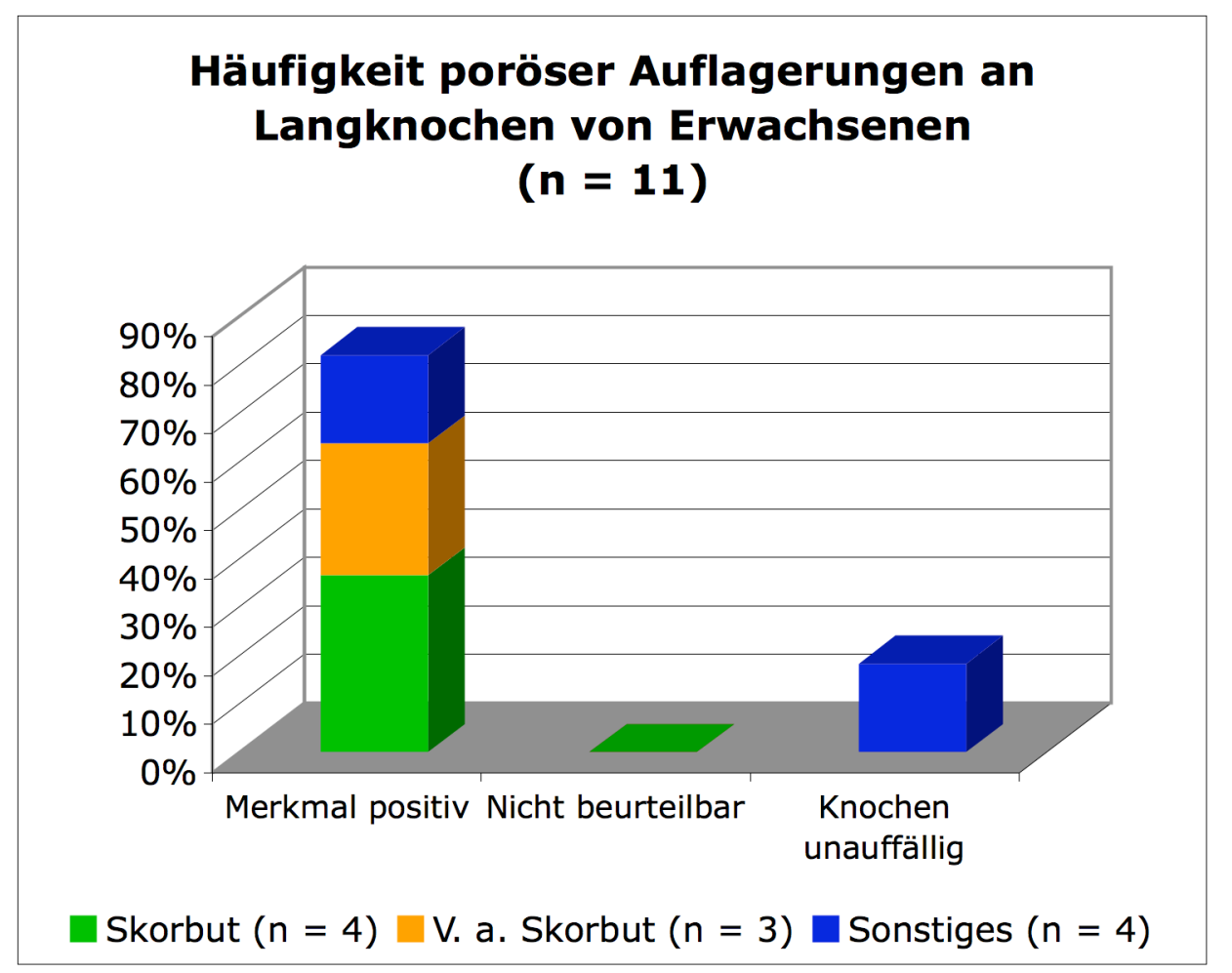

Abb. 37: Häufigkeit poröser Auflagerungen an den Langknochen von Erwachsenen mit Skorbut, V. a. Skorbut und Individuen mit anderen Diagnosen

Individuum Sk 1380, das vermutlich sowohl an Lepra als auch an Skorbut erkrankt ist, wird hier in der Gruppe der Skorbutkranken geführt.

Unter „unauffällig“ fallen hier auch Langknochen mit poröser Oberfläche (vgl. Nebenmerkmal 4).

V. a. $=$ Verdacht auf

Bei den insgesamt 27 skorbutkranken Individuen finden sich in 21 Fällen $(78 \%$ aller Skorbutkranken) feinporöse Auflagerungen an den Langknochen, bei zwei Individuen (7\% aller Skorbutkranken) sind die Langknochen nicht beurteilbar, vier Individuen $(15 \%$ aller Skorbutkranken) haben unauffällige Langknochen. 
Ein wesentlicher Unterschied im Befall der oberen oder der unteren Extremität lässt sich nicht feststellen. Bei den meisten Individuen sind alle Extremitäten gleichermaßen betroffen. In sechs Fällen sind lediglich die unteren Extremitäten betroffen, allerdings fehlt davon in vier Fällen die obere Extremität völlig. Zwei der Individuen mit betroffener unterer Extremität und fehlender oberer Extremität weisen hämorrhagische Auflagerungen an den Scapulae auf, sodass man darauf schließen kann, dass auch die oberen Extremitäten betroffen waren. Es gibt allerdings auch zwei Skelete mit erhaltenen unauffälligen Extremitäten bei Befall der unteren Extremitäten und der Scapulae.

\begin{tabular}{|l|l|l|l|}
\hline & Merkmal positiv & Nicht beurteilbar & Knochen unauffällig \\
\hline Skorbutkranke $(\mathbf{n}=\mathbf{2 7})$ & $21(78 \%)$ & $2(7 \%)$ & $4(15 \%)$ \\
\hline
\end{tabular}

Tab. 71: Häufigkeit poröser Auflagerungen an den Langknochen bei den untersuchten Skorbutkranken. Unter „unauffällig“ fallen hier auch Langknochen mit poröser Oberfläche (vgl. Nebenmerkmal 4). Prozentzahlen beziehen sich auf Gesamtzahl der Skorbutfälle.

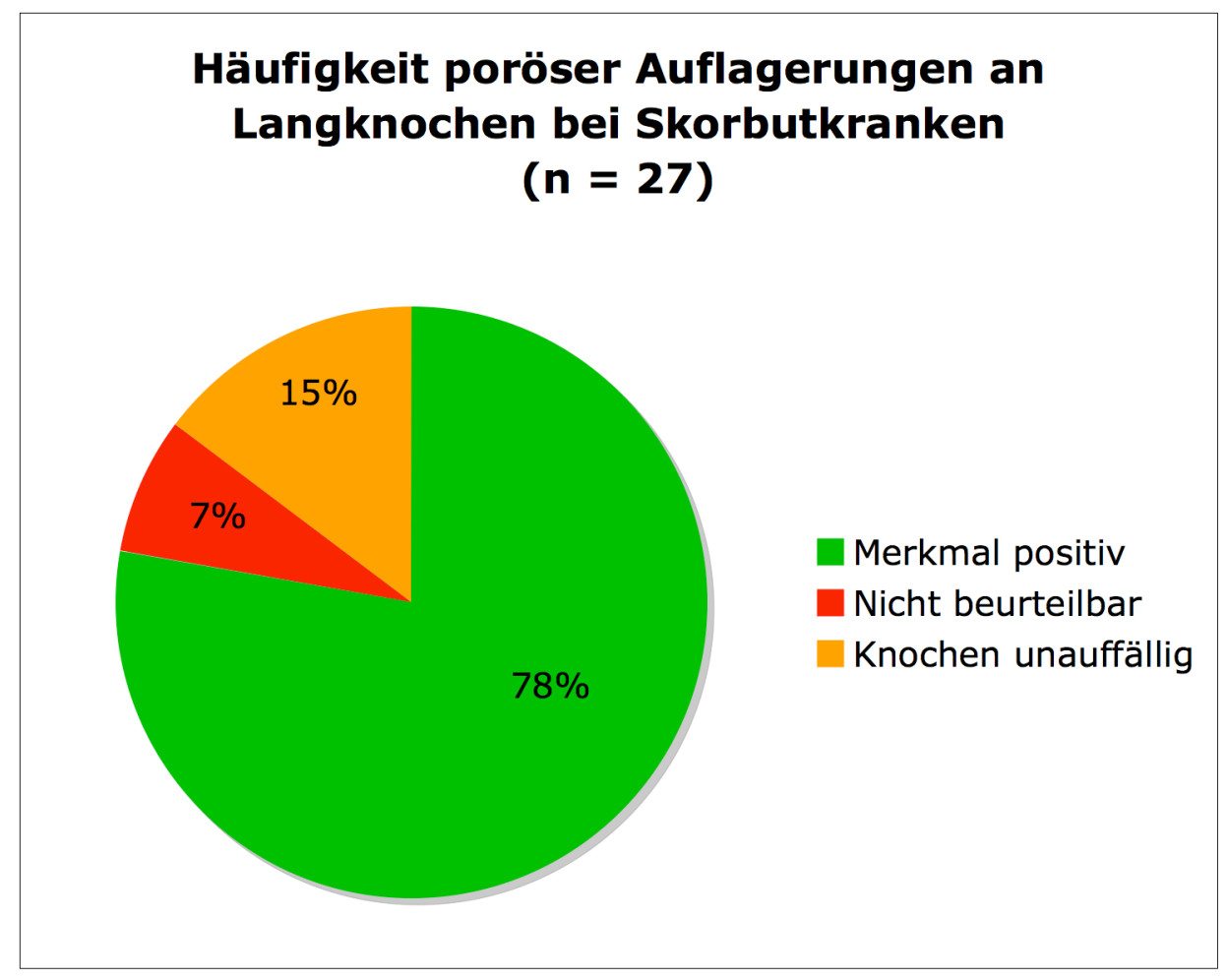

Abb. 38: Häufigkeit poröser Auflagerungen an den Langknochen bei den untersuchten Skorbutkranken. Unter ,unauffällig“ fallen hier auch Langknochen mit poröser Oberfläche (vgl. Nebenmerkmal 4). Prozentzahlen beziehen sich auf Gesamtzahl der Skorbutfälle

Von den 23 an Skorbut erkrankten Kindern haben 17 (74\% der skorbutkranken Kinder) poröse Auflagerungen an den Langknochen, bei zwei Kindern (9\% der skorbutkranken Kinder) sind die Langknochen nicht beurteilbar, bei vier Kindern (17\%) sind sie unauffällig. 


\begin{tabular}{|l|l|l|l|}
\hline & Merkmal positiv & Nicht beurteilbar & Knochen unauffällig \\
\hline Skorbutkranke Kinder $(\mathbf{n = 2 3})$ & $17(74 \%)$ & $2(9 \%)$ & $4(17 \%)$ \\
\hline
\end{tabular}

Tab. 72: Häufigkeit poröser Auflagerungen an den Langknochen der untersuchten skorbutkranken Kindern. Unter ,unauffällig“ fallen hier auch Langknochen mit poröser Oberfläche (vgl. Nebenmerkmal 4).

Prozentzahlen beziehen sich auf Gesamtzahl der skorbutkranken Kinder.

Alle vier $(100 \%)$ untersuchten adulten Skorbutfälle weisen poröse Auflagerungen an den Langknochen auf.

\begin{tabular}{|l|l|l|l|}
\hline & Merkmal positiv & Nicht beurteilbar & Knochen unauffällig \\
\hline Skorbutkranke Erwachsene $(n=4)$ & $4(100 \%)$ & 0 & 0 \\
\hline
\end{tabular}

Tab. 73: Häufigkeit poröser Auflagerungen an den Langknochen bei den untersuchten skorbutkranken Erwachsenen. Unter „,unauffällig“ fallen hier auch Langknochen mit poröser Oberfläche (vgl. Nebenmerkmal 4). Prozentzahlen beziehen sich auf Gesamtzahl der skorbutkranken Erwachsenen.

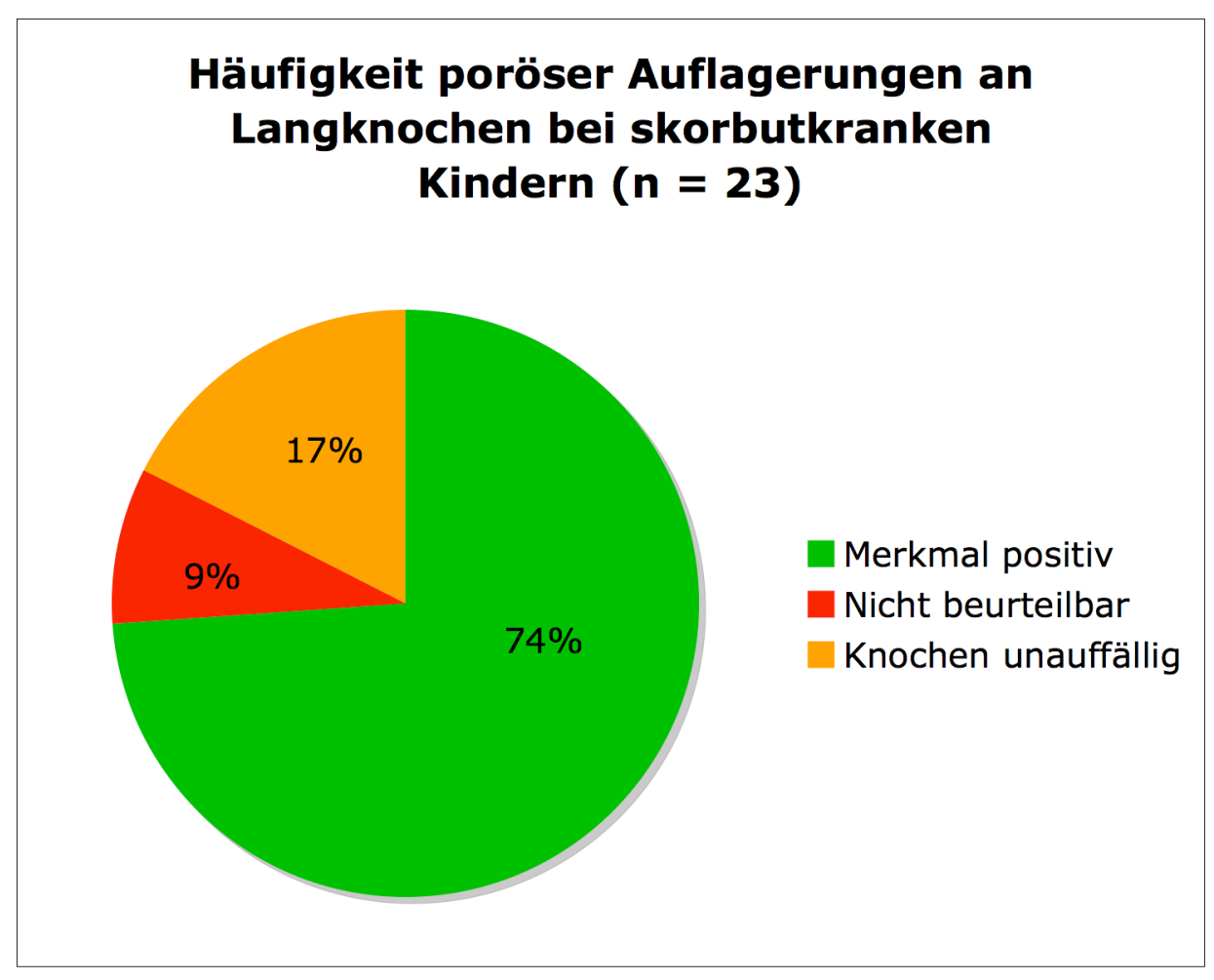

Abb. 39: Häufigkeit poröser Auflagerungen an den Langknochen von den untersuchten skorbutkranken Kindern Unter „unauffällig“ fallen hier auch Langknochen mit poröser Oberfläche (vgl. Nebenmerkmal 4).

Prozentzahlen beziehen sich auf Gesamtzahl der skorbutkranken Kinder. 


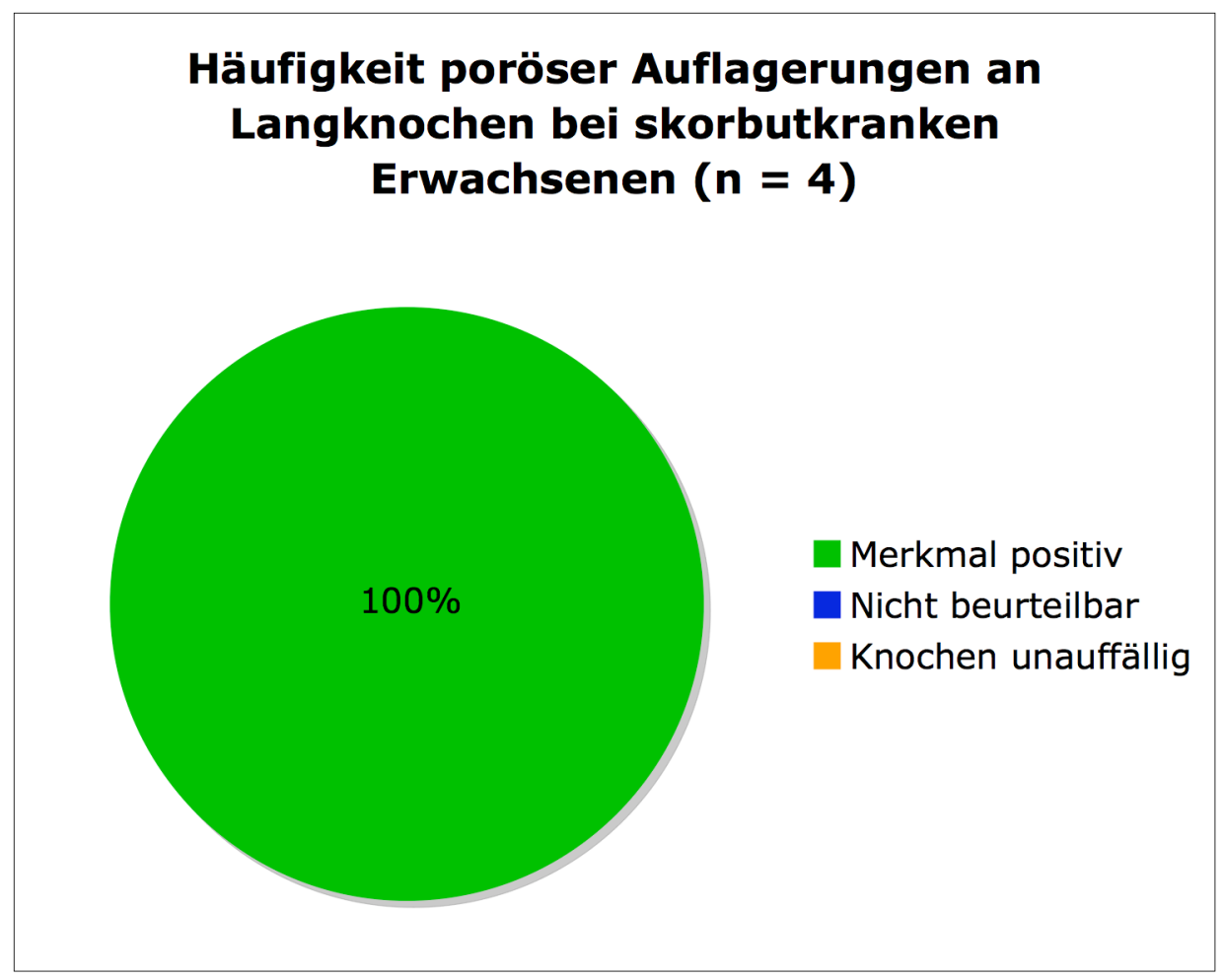

Abb. 40: Häufigkeit poröser Auflagerungen an den Langknochen bei den untersuchten skorbutkranken Erwachsenen. Unter „unauffällig“ fallen hier auch Langknochen mit poröser Oberfläche (vgl. Nebenmerkmal 4). Prozentzahlen beziehen sich auf Gesamtzahl der skorbutkranken Erwachsenen. 


\subsection{Poröse Auflagerungen am Schulterblatt (Kardinalmerkmal 6)}

Bei zehn (28\%) der 36 untersuchten Individuen liegen poröse Auflagerungen am Schulterblatt vor, diese sind alle an Skorbut erkrankt.

In 13 Fällen (36\%) sind die Schulterblätter nicht befundbar, unter anderem bei zehn Skorbutfällen (28\%) und einem Individuum (3\%) mit Verdacht auf Skorbut.

Dreizehn Individuen (36\%) haben unauffällige Schulterblätter, darunter sieben (19\%) Skorbutkranke und drei (8\%) mit Verdacht auf Skorbut.

\begin{tabular}{|l|l|l|l|}
\hline & Merkmal positiv & Nicht beurteilbar & Knochen unauffällig \\
\hline Skorbut & $10(28 \%)$ & $10(28 \%)$ & $7(19 \%)$ \\
\hline V. a. Skorbut & 0 & $1(3 \%)$ & $3(8 \%)$ \\
\hline Sonstiges & 0 & $2(6 \%)$ & $3(8 \%)$ \\
\hline
\end{tabular}

Tab. 74: Häufigkeit poröser Auflagerungen am Schulterblatt bei Individuen mit Skorbut, Verdacht auf Skorbut und Individuen mit anderen Diagnosen

Individuum Sk 1380, das vermutlich sowohl an Lepra als auch an Skorbut erkrankt ist, wird hier in der Gruppe der Skorbutkranken geführt.

V. a. = Verdacht auf

\section{Häufigkeit poröser Auflagerungen am Schulterblatt bei allen Skeleten $(n=36)$}

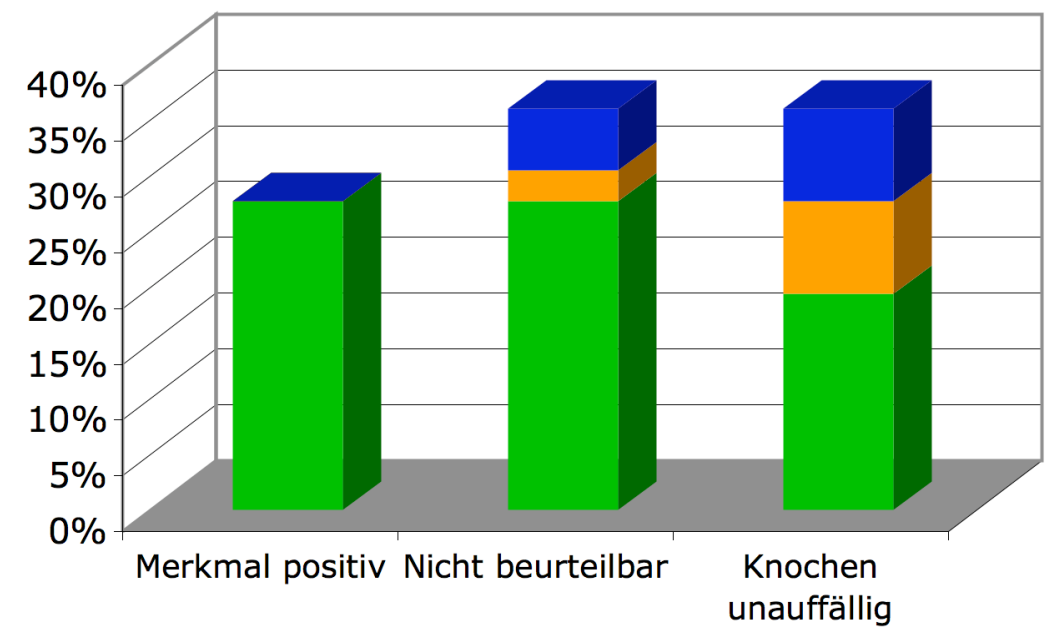

Skorbut $(n=27) \square$ V. a. Skorbut $(n=4) \square$ Sonstiges $(n=5)$

Abb. 41: Häufigkeit poröser Auflagerungen am Schulterblatt bei Individuen mit Skorbut, Verdacht auf Skorbut und Individuen mit anderen Diagnosen

Individuum Sk 1380, das vermutlich sowohl an Lepra als auch an Skorbut erkrankt ist, wird hier in der Gruppe der Skorbutkranken geführt.

V. a. $=$ Verdacht auf 
Unter den 25 untersuchten Kindern sind neun (36\% aller Kinder) mit porösen Auflagerungen am Schulterblatt, die alle an Skorbut erkrankt sind.

Bei elf Kindern (44\% aller Kinder) ist keine Befundung der Schulterblätter möglich, davon haben neun (36\% aller Kinder) sicher und ein Kind (4\% aller Kinder) wahrscheinlich Skorbut. Fünf Kinder (20\% aller Kinder) haben unauffällige Schulterblätter, alle sind Skorbutfälle.

\begin{tabular}{|l|l|l|l|}
\hline & Merkmal positiv & Nicht beurteilbar & Knochen unauffällig \\
\hline Skorbut & $9(36 \%)$ & $9(36 \%)$ & $5(20 \%)$ \\
\hline V. a. Skorbut & 0 & $1(4 \%)$ & 0 \\
\hline Sonstiges & 0 & $1(4 \%)$ & 0 \\
\hline
\end{tabular}

Tab. 75: Häufigkeit poröser Auflagerungen am Schulterblatt bei Kindern mit Skorbut, Verdacht auf Skorbut und Kindern mit anderen Diagnosen. Die Prozentzahlen beziehen sich auf die Gesamtzahl aller Kinder.

V. a. = Verdacht auf

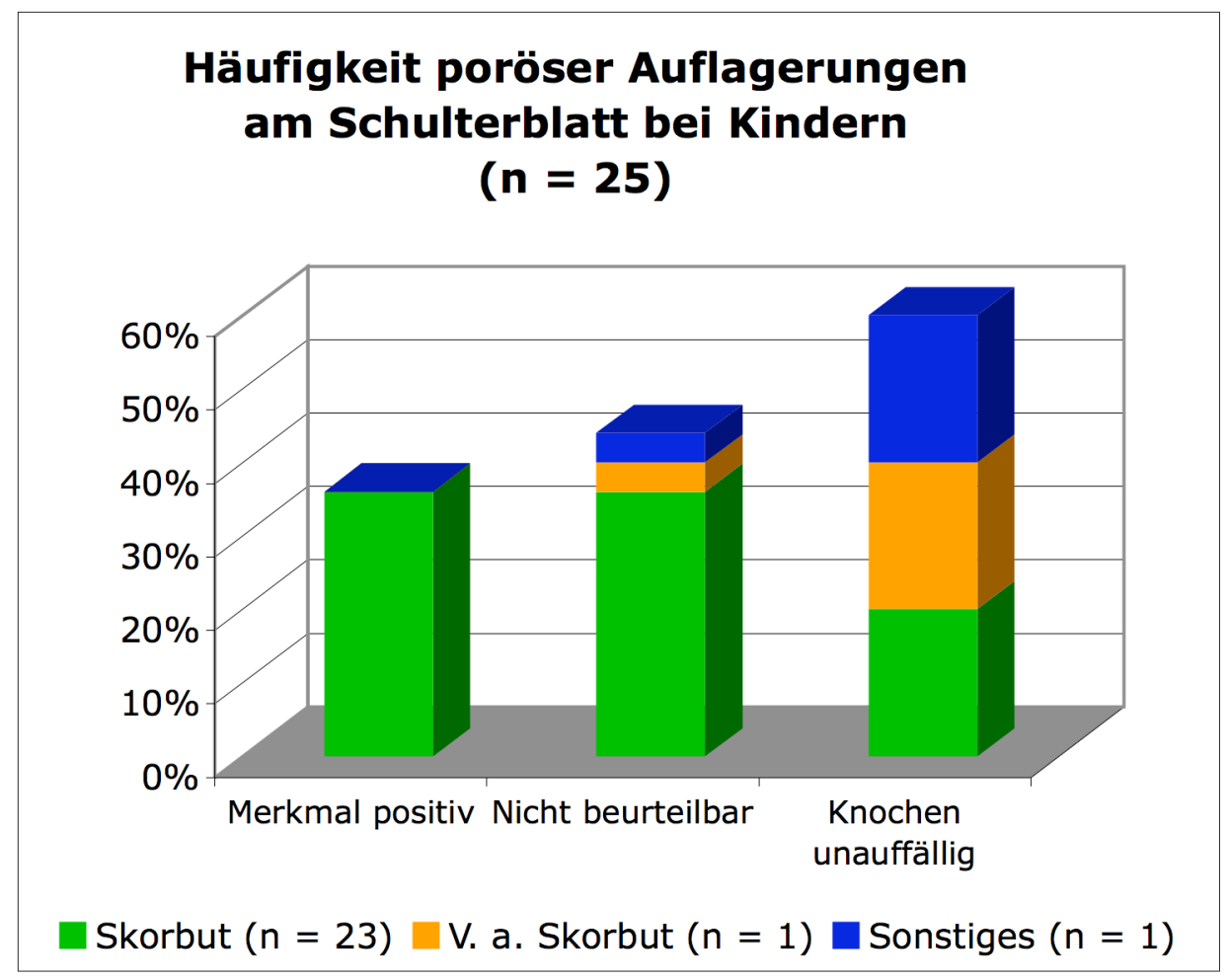

Abb. 42: Häufigkeit poröser Auflagerungen am Schulterblatt bei Kindern mit Skorbut, Verdacht auf Skorbut und Kindern mit anderen Diagnosen (Prozentzahlen beziehen sich auf die Gesamtzahl aller Kinder)

Von elf untersuchten Erwachsenen hat ein skorbutkrankes Individuum $(9 \%$ aller Erwachsenen) poröse Auflagerungen am Schulterblatt.

In zwei Fällen (18\% aller Erwachsenen) sind die Scapulae nicht beurteilbar, darunter ein Individuum ( $9 \%$ aller Erwachsenen) mit Skorbut. 
Acht Erwachsene (73\% aller Erwachsenen) zeigen unauffällige Schulterblätter, darunter zwei Skorbutfälle (18\% aller Erwachsenen) und 3 Individuen (27\% aller Erwachsenen) mit Verdacht auf Skorbut.

\begin{tabular}{|l|l|l|l|}
\hline & Merkmal positiv & Nicht beurteilbar & Knochen unauffällig \\
\hline Skorbut & $1(9 \%)$ & $1(9 \%)$ & $2(18 \%)$ \\
\hline V. a. Skorbut & 0 & 0 & $3(27 \%)$ \\
\hline Sonstiges & 0 & $1(9 \%)$ & $3(27 \%)$ \\
\hline
\end{tabular}

Tab. 76: Häufigkeit poröser Auflagerungen am Schulterblatt bei Erwachsenen mit Skorbut, Verdacht auf Skorbut und Erwachsenen mit anderen Diagnosen. Prozentzahlen beziehen sich auf Gesamtzahl aller Erwachsenen, die Abweichung von 100\% ergibt sich durch Auf- bzw. Abrundung.

Individuum Sk 1380, das vermutlich sowohl an Lepra als auch an Skorbut erkrankt ist, wird hier in der Gruppe der Skorbutkranken geführt.

V. a. = Verdacht auf

\section{Häufigkeit poröser Auflagerungen am Schulterblatt bei Erwachsenen $(\mathbf{n}=\mathbf{1 1})$}

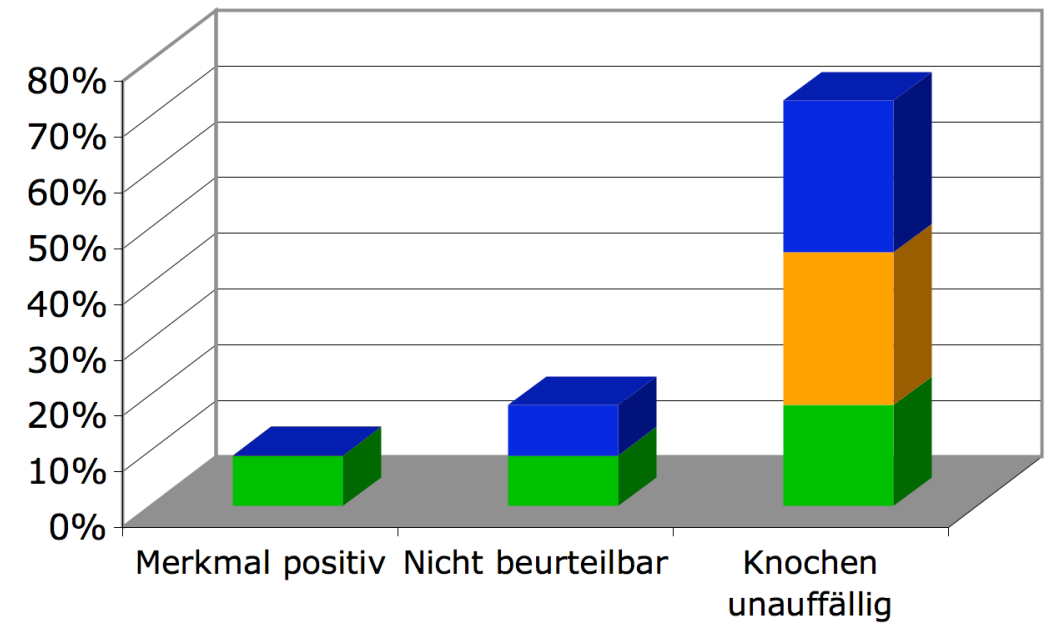

Skorbut $(n=4) \square$ V. a. Skorbut $(n=3) \square$ Sonstiges $(n=4)$

Abb. 43: Häufigkeit poröser Auflagerungen am Schulterblatt bei Erwachsenen mit Skorbut, Verdacht auf Skorbut und Erwachsenen mit anderen Diagnosen. Prozentzahlen beziehen sich auf Gesamtzahl aller Erwachsenen.

Individuum Sk 1380, das vermutlich sowohl an Lepra als auch an Skorbut erkrankt ist, wird hier in der Gruppe der Skorbutkranken geführt.

V. a. = Verdacht auf

Unter den 27 untersuchten Skorbutfällen sind zehn (37\% aller Skorbutfälle) mit porösen Auflagerungen am Schulterblatt, bei zehn (37\% aller Skorbutfälle) ist keine Beurteilung möglich, sieben Individuen (25,9\% aller Skorbutfälle) haben unauffällige Scapulae. 


\begin{tabular}{|l|l|l|l|}
\hline & Merkmal positiv & Nicht beurteilbar & Knochen unauffällig \\
\hline Skorbutkranke (n=27) & $10(37 \%)$ & $10(37 \%)$ & $7(26 \%)$ \\
\hline
\end{tabular}

Tab. 77: Häufigkeit poröser Auflagerungen am Schulterblatt bei den untersuchten Skorbutkranken. Prozentzahlen beziehen sich auf Gesamtzahl der Skorbutfälle.

\section{Häufigkeit poröser Auflagerungen am Schulterblatt bei Skorbutkranken $(n=27)$}

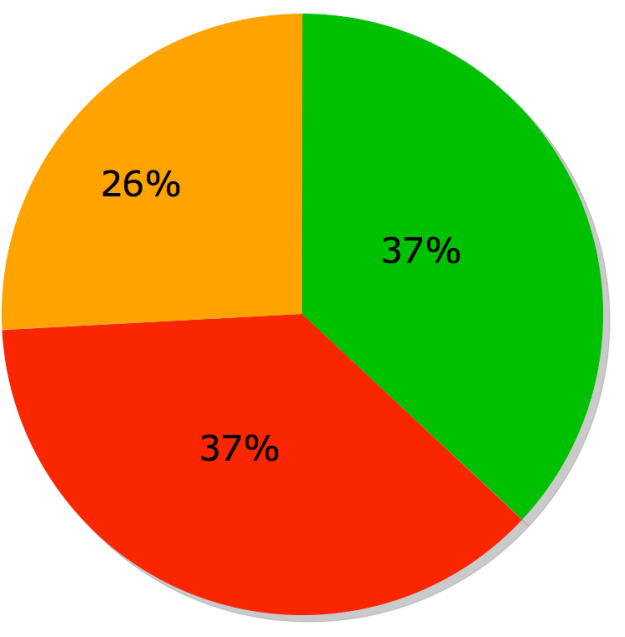

Merkmal positiv

Nicht beurteilbar

Knochen unauffällig

Abb. 44: Häufigkeit poröser Auflagerungen am Schulterblatt bei den untersuchten Skorbutkranken. Prozentzahlen beziehen sich auf Gesamtzahl der Skorbutfälle.

Unter den 23 skorbutkranken Kindern sind neun (39\% aller skorbutkranken Kinder) mit porösen Auflagerungen am Schulterblatt, fünf (22\% aller skorbutkranken Kinder) mit unauffälligen und neun (39\% aller skorbutkranken Kinder) mit nicht beurteilbaren Scapulae.

\begin{tabular}{|l|l|l|l|}
\hline & Merkmal positiv & Nicht beurteilbar & Knochen unauffällig \\
\hline Skorbutkranke Kinder $(\mathbf{n}=\mathbf{2 3})$ & $9(39 \%)$ & $9(39 \%)$ & $5(22 \%)$ \\
\hline
\end{tabular}

Tab. 78: Häufigkeit poröser Auflagerungen am Schulterblatt bei den untersuchten skorbutkranken Kindern. Prozentzahlen beziehen sich auf Gesamtzahl der infantilen Skorbutfälle.

Bei den vier skorbutkranken Erwachsenen finden sich in einem Fall (25\% der adulten Skorbutfälle) poröse Auflagerungen am Schulterblatt, ein Individuum (25\% der adulten Skorbutfälle) hat nicht befundbare Scapulae, zwei Individuen (50\% der adulten Skorbutfälle) weisen unauffällige Schulterblätter auf.

\begin{tabular}{|l|l|l|l|}
\hline & Merkmal positiv & Nicht beurteilbar & Knochen unauffällig \\
\hline Skorbutkranke Erwachsene $(\mathbf{n}=4)$ & $1(25 \%)$ & $1(25 \%)$ & $2(50 \%)$ \\
\hline
\end{tabular}

Tab. 79: Häufigkeit poröser Auflagerungen am Schulterblatt bei den untersuchten skorbutkranken Erwachsenen. Prozentzahlen beziehen sich auf Gesamtzahl der adulten Skorbutfälle. 


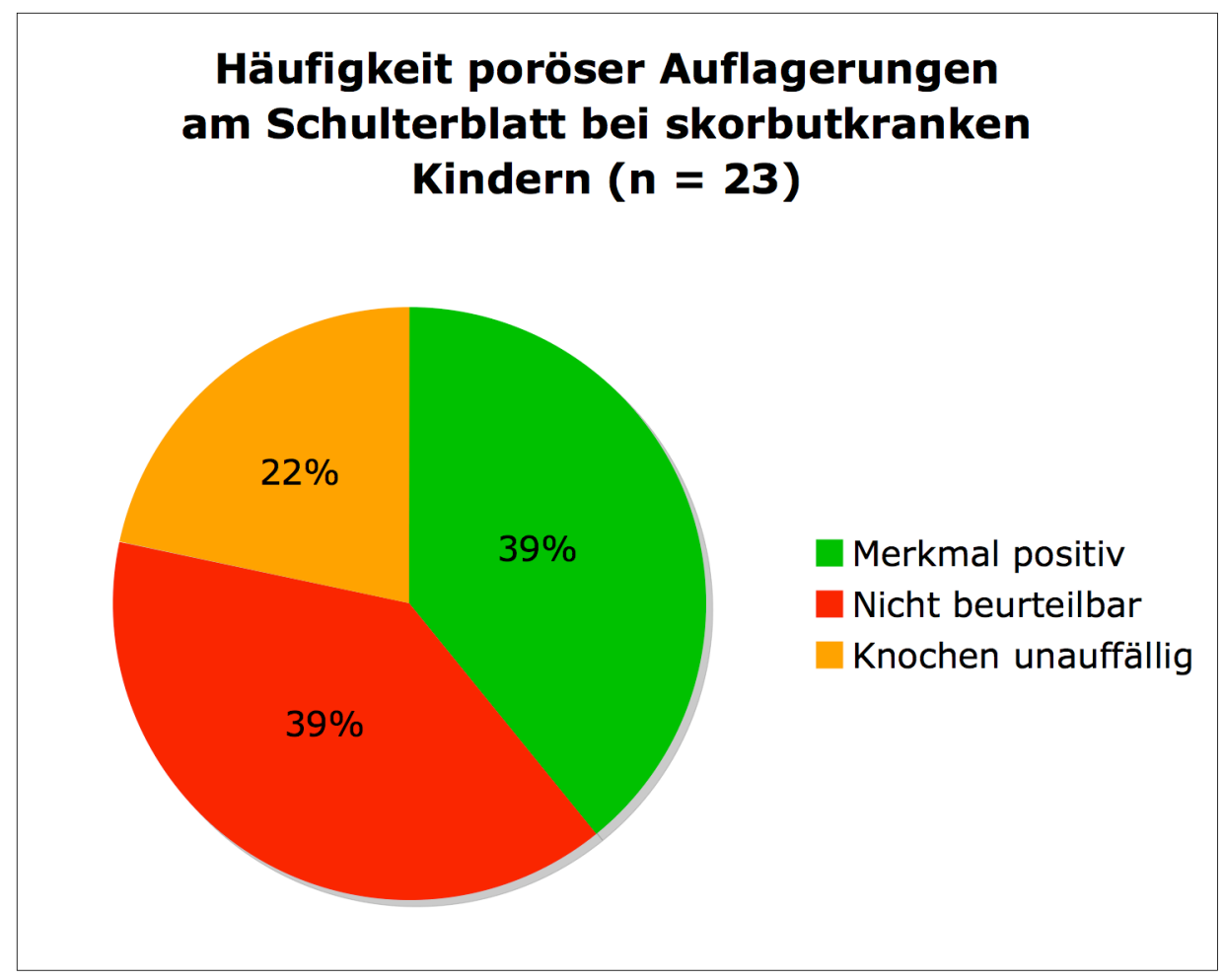

Abb. 45: Häufigkeit poröser Auflagerungen am Schulterblatt bei den untersuchten skorbutkranken Kindern. Prozentzahlen beziehen sich auf Gesamtzahl der infantilen Skorbutfälle.

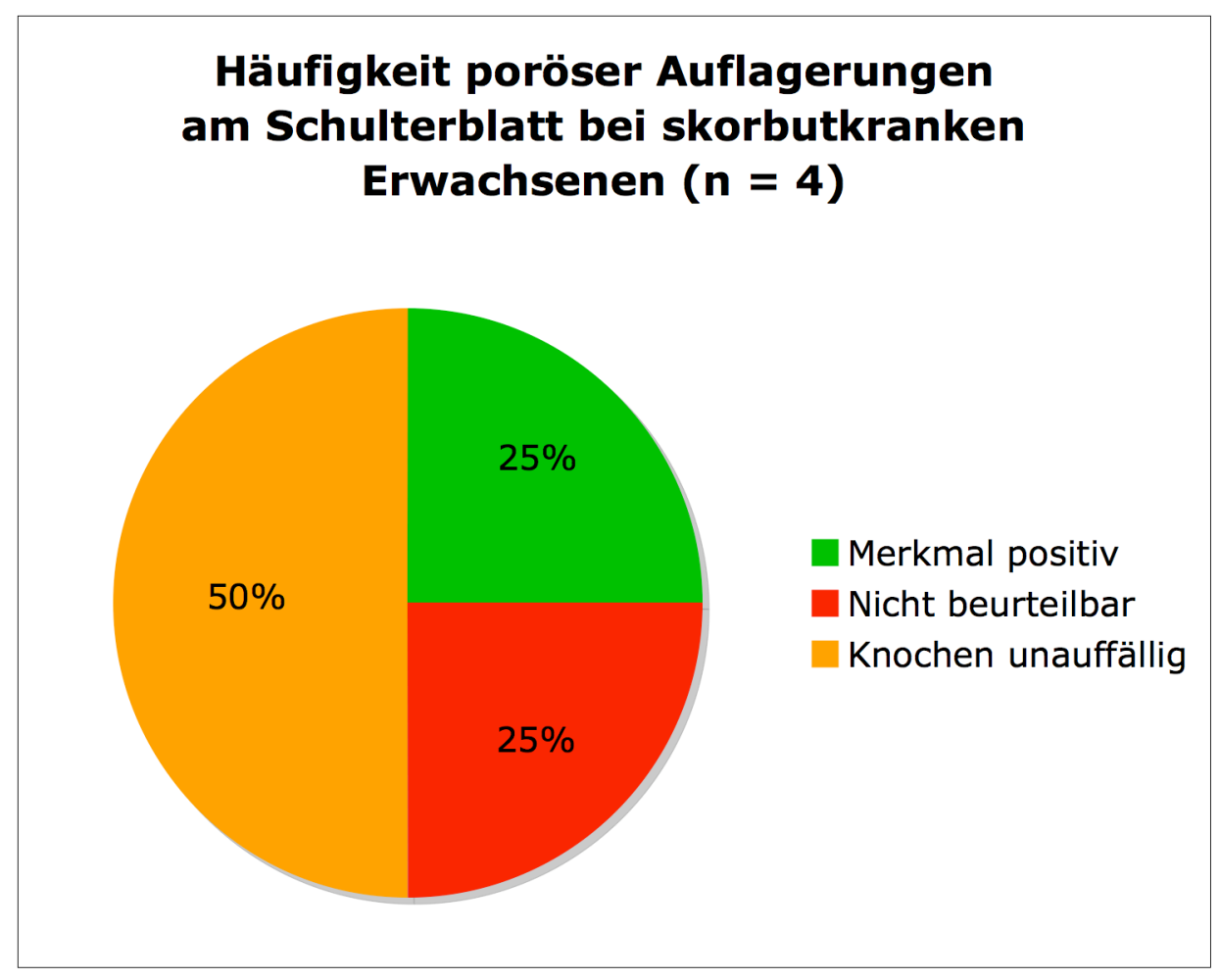

Abb. 46: Häufigkeit poröser Auflagerungen am Schulterblatt bei den untersuchten skorbutkranken Erwachsenen. Prozentzahlen beziehen sich auf Gesamtzahl der adulten Skorbutfälle. 


\section{14 Poröse Auflagerungen am Os ilii (Kardinalmerkmal 7)}

Von den 36 untersuchten Individuen weisen 10 (28\%) poröse Auflagerungen am Os ilii auf, die alle an Skorbut litten.

Bei 14 Individuen (39\%) sind die Ossa ilii nicht beurteilbar, auch bei elf Skorbutkranken (31\%) und einem (3\%) wahrscheinlich an Skorbut erkrankten Individuum.

Zwölf Individuen (33\%) zeigen unauffällige Darmbeine, darunter sechs mit Skorbut (17\%) und drei (8\%) mit wahrscheinlichem Skorbut.

\begin{tabular}{|l|l|l|l|}
\hline & Merkmal positiv & Nicht beurteilbar & Knochen unauffällig \\
\hline Skorbut & $10(28 \%)$ & $11(31 \%)$ & $6(17 \%)$ \\
\hline V. a. Skorbut & 0 & $1(3 \%)$ & $3(8 \%)$ \\
\hline Sonstiges & 0 & $2(6 \%)$ & $3(8 \%)$ \\
\hline
\end{tabular}

Tab. 80: Häufigkeit poröser Auflagerungen am Os ilii bei Individuen mit Skorbut, V. a. Skorbut und Individuen mit anderen Diagnosen

Individuum Sk 1380, das vermutlich sowohl an Lepra als auch an Skorbut erkrankt ist, wird hier in der Gruppe der Skorbutkranken geführt. Die Abweichung von 100\% ergibt sich durch Auf- bzw. Abrundung.

V. a. $=$ Verdacht auf

\section{Häufigkeit poröser Auflagerungen am Os ilii bei allen Skeleten $(n=36)$}

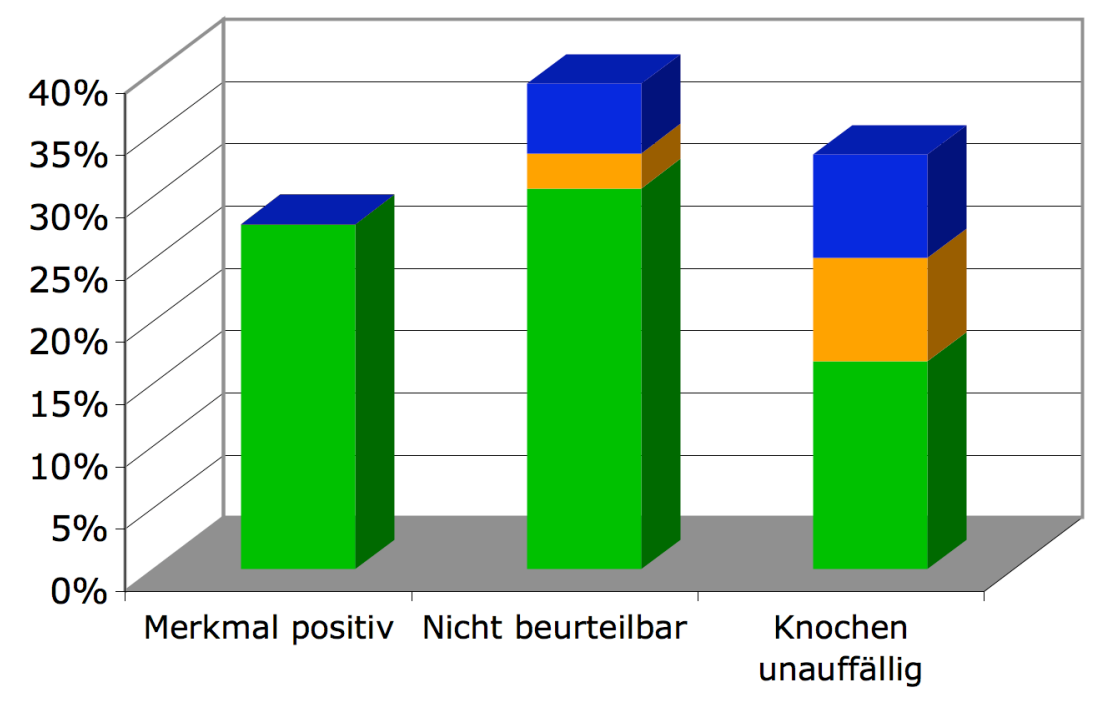

Skorbut $(n=27) \square$ V. a. Skorbut $(n=4) \square$ Sonstiges $(n=5)$

Abb. 47: Häufigkeit poröser Auflagerungen am Os ilii bei Individuen mit Skorbut, Verdacht auf Skorbut und Individuen mit anderen Diagnosen

Individuum Sk 1380, das vermutlich sowohl an Lepra als auch an Skorbut erkrankt ist, wird hier in der Gruppe der Skorbutkranken geführt.

V. a. = Verdacht auf

Von 25 untersuchten Kindern zeigen acht (32\% aller Kinder) poröse Auflagerungen am Os ilii, die alle an Skorbut erkrankt sind. Bei zwölf Kindern (48\% der Kinder) sind die 
Darmbeine nicht befundbar, darunter befinden sich zehn (40\% aller Kinder) mit Skorbut, eins (4\% aller Kinder) mit Verdacht auf Skorbut.

Fünf Kinder (20\% der Kinder), alle skorbutkrank, haben unauffällige Darmbeine.

\begin{tabular}{|l|l|l|l|}
\hline & Merkmal positiv & Nicht beurteilbar & Knochen unauffällig \\
\hline Skorbut & $8(32 \%)$ & $10(40 \%)$ & $5(20 \%)$ \\
\hline V. a. Skorbut & 0 & $1(4 \%)$ & 0 \\
\hline Sonstiges & 0 & $1(4 \%)$ & 0 \\
\hline
\end{tabular}

Tab. 81: Häufigkeit poröser Auflagerungen am Os ilii bei Kindern mit Skorbut, V. a. Skorbut und Kindern mit anderen Diagnosen. Prozentzahlen beziehen sich auf Gesamtzahl der Kinder.

V. a. $=$ Verdacht auf

\section{Häufigkeit poröser Auflagerungen am Os ilii bei Kindern $(n=25)$}

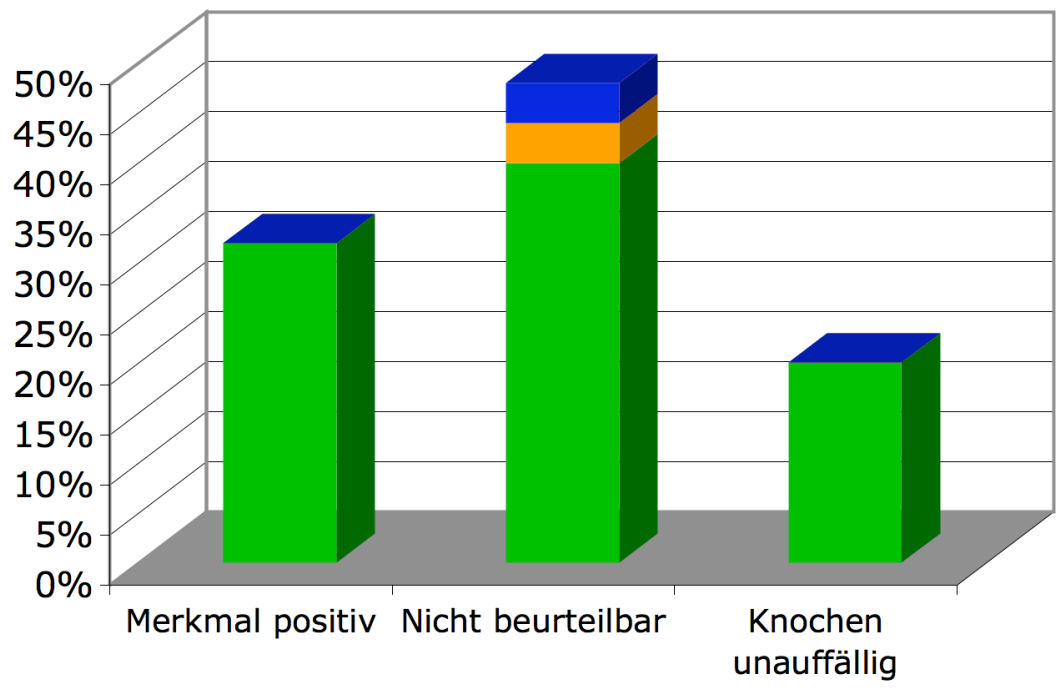

Skorbut $(n=23) \square$ V. a. Skorbut $(n=1) \square$ Sonstiges $(n=1)$

Abb. 48: Häufigkeit poröser Auflagerungen am Os ilii bei Kindern mit Skorbut, V. a. Skorbut und Kindern mit anderen Diagnosen. Prozentzahlen beziehen sich auf Gesamtzahl der Kinder V. a. = Verdacht auf

Von den elf untersuchten Erwachsenen haben nur zwei Individuen (18\% der Erwachsenen) poröse Auflagerungen am Os ilii, beides sind Skorbutfälle.

Bei zwei Erwachsenen (18\% der Erwachsenen) sind die Darmbeine nicht befundbar, davon ein Individuum (9\% der Erwachsenen) mit Verdacht auf Skorbut.

Sieben Erwachsene (64\% der Erwachsenen) haben unauffällige Ossa ilii, darunter ein Individuum (9\% der Erwachsenen) mit Skorbut und drei (27\% der Erwachsenen) mit Verdacht auf Skorbut. 


\begin{tabular}{|l|l|l|l|}
\hline & Merkmal positiv & Nicht beurteilbar & Knochen unauffällig \\
\hline Skorbut & $2(18 \%)$ & $1(9 \%)$ & $1(9 \%)$ \\
\hline V. a. Skorbut & 0 & 0 & $3(27 \%)$ \\
\hline Sonstiges & 0 & $1(9 \%)$ & $3(27 \%)$ \\
\hline
\end{tabular}

Tab. 82: Häufigkeit poröser Auflagerungen am Os ilii bei Erwachsenen mit Skorbut, V. a. Skorbut und Erwachsenen mit anderen Diagnosen. Prozentzahlen beziehen sich auf Gesamtzahl der Erwachsenen, die Abweichung von $100 \%$ ergibt sich durch Auf- bzw. Abrundung.

Individuum Sk 1380, das vermutlich sowohl an Lepra als auch an Skorbut erkrankt ist, wird hier in der Gruppe der Skorbutkranken geführt.

V.a. $=$ Verdacht auf

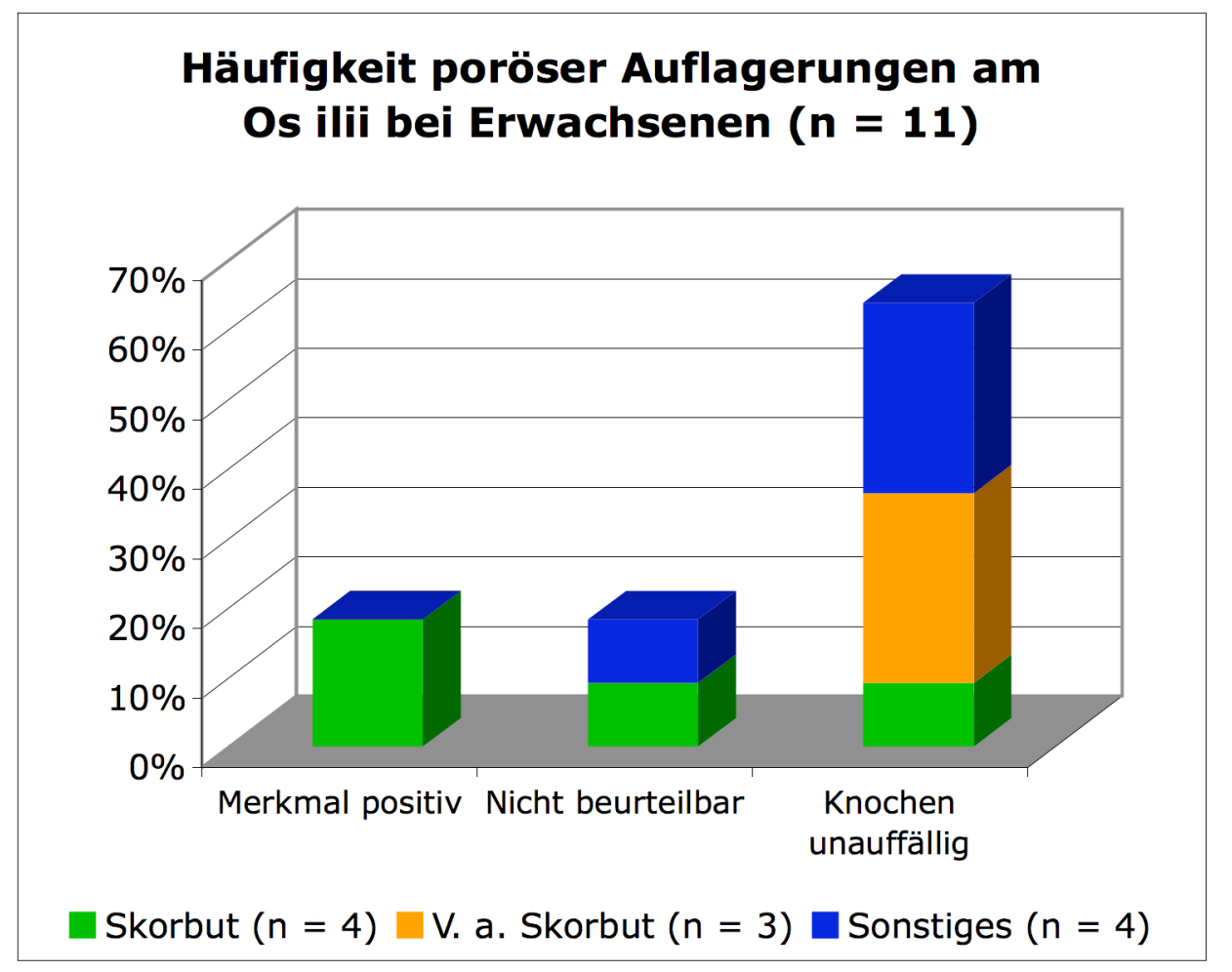

Abb. 49: Häufigkeit poröser Auflagerungen am Os ilii bei Erwachsenen mit Skorbut, Verdacht auf Skorbut und Erwachsenen mit anderen Diagnosen. Prozentzahlen beziehen sich auf Gesamtzahl der Erwachsenen.

Individuum Sk 1380, das vermutlich sowohl an Lepra als auch an Skorbut erkrankt ist, wird hier in der Gruppe der Skorbutkranken geführt.

V. a. $=$ Verdacht auf

Betrachtet man alle an Skorbut erkrankten Individuen (27), so weisen zehn $(37 \%$ der Skorbutkranken) Auflagerungen auf, bei elf Individuen (41\%) lassen sich die Ossa ilii nicht befunden, sechs (22\%) haben unauffällige Darmbeine.

\begin{tabular}{|l|l|l|l|}
\hline & Merkmal positiv & Nicht beurteilbar & Knochen unauffällig \\
\hline Skorbutkranke (n=27) & $10(37 \%)$ & $11(41 \%)$ & $6(22 \%)$ \\
\hline
\end{tabular}

Tab. 83: Häufigkeit poröser Auflagerungen am Os ilii bei den untersuchten Skorbutkranken. Prozentzahlen beziehen sich auf Gesamtzahl der Skorbutfälle. 


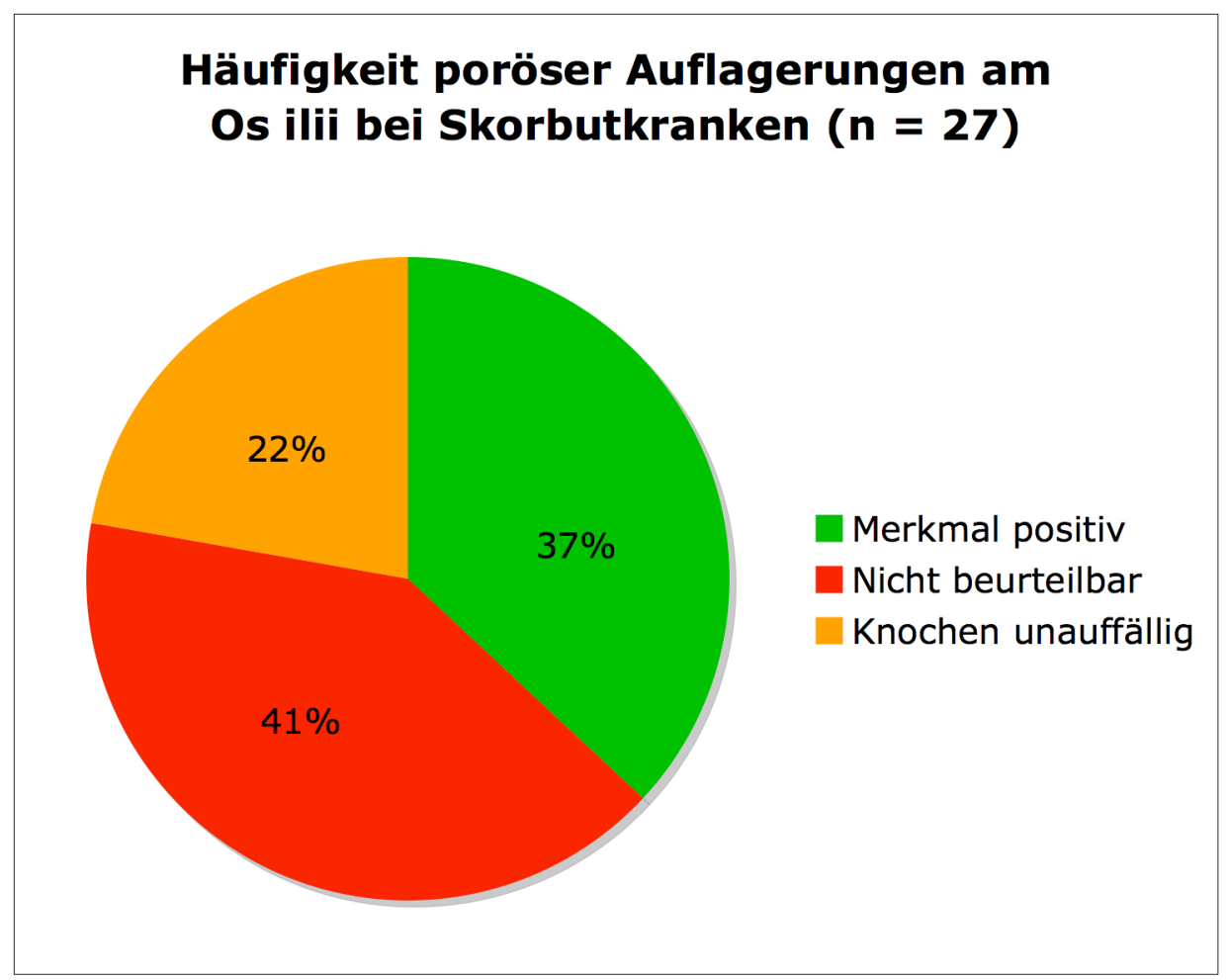

Abb. 50: Häufigkeit poröser Auflagerungen am Os ilii bei den untersuchten Skorbutkranken. Prozentzahlen beziehen sich auf Gesamtzahl der Skorbutfälle.

Unter den skorbutkranken Kindern (23) sind acht (35\% der skorbutkranken Kinder) mit porösen Auflagerungen am Os ilii, zehn (44\% der skorbutkranken Kinder) ohne befundbare und 5 (22\%) mit unauffälligen Darmbeinen.

\begin{tabular}{|l|l|l|l|}
\hline & Merkmal positiv & Nicht beurteilbar & Knochen unauffällig \\
\hline Skorbutkranke Kinder $(\mathbf{n}=\mathbf{2 3})$ & $8(35 \%)$ & $10(44 \%)$ & $5(22 \%)$ \\
\hline
\end{tabular}

Tab. 84: Häufigkeit poröser Auflagerungen am Os ilii bei den untersuchten skorbutkranken Kindern. Prozentzahlen beziehen sich auf Gesamtzahl der infantilen Skorbutfälle, die Abweichung von 100\% ergibt sich durch Auf- bzw. Abrundung.

Von den vier an Skorbut erkrankten Erwachsenen weisen zwei Individuen $(50 \%$ der skorbutkranken Erwachsenen) poröse Auflagerungen am Os ilii auf, je ein Individuum (25\% der skorbutkranken Erwachsenen) haben unauffällige bzw. nicht befundbare Darmbeine.

\begin{tabular}{|l|r|l|l|}
\hline & Merkmal positiv & Nicht beurteilbar & Knochen unauffällig \\
\hline Skorbutkranke Erwachsene $(n=4)$ & $2(50 \%)$ & $1(25 \%)$ & $1(25 \%)$ \\
\hline
\end{tabular}

Tab. 85: Häufigkeit poröser Auflagerungen am Os ilii bei skorbutkranken Erwachsenen. Prozentzahlen beziehen sich auf Gesamtzahl der adulten Skorbutfälle. 


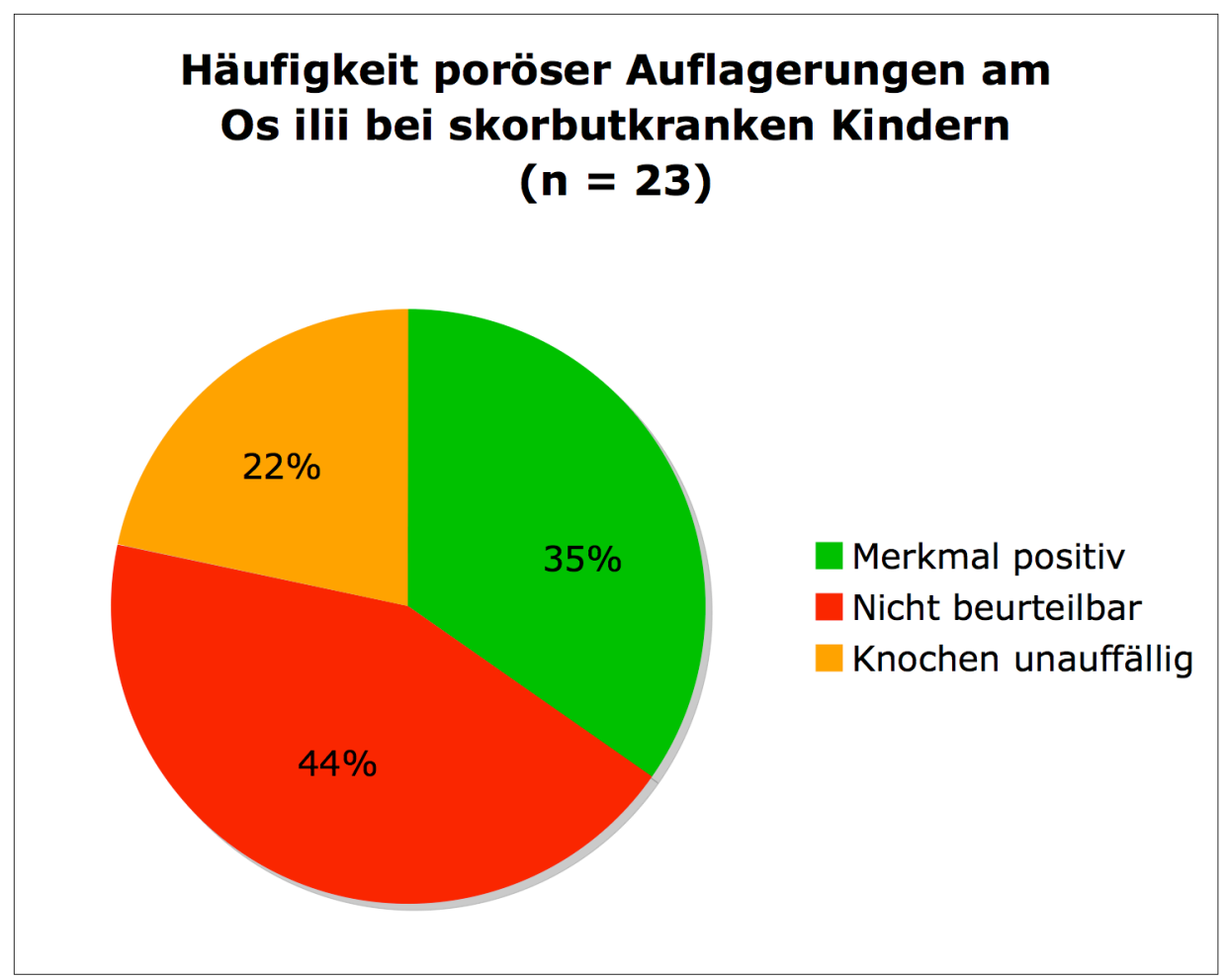

Abb. 51: Häufigkeit poröser Auflagerungen am Os ilium bei den untersuchten skorbutkranken Kindern. Prozentzahlen beziehen sich auf Gesamtzahl der infantilen Skorbutfälle, die Abweichung von 100\% ergibt sich durch Auf- bzw. Abrundung.

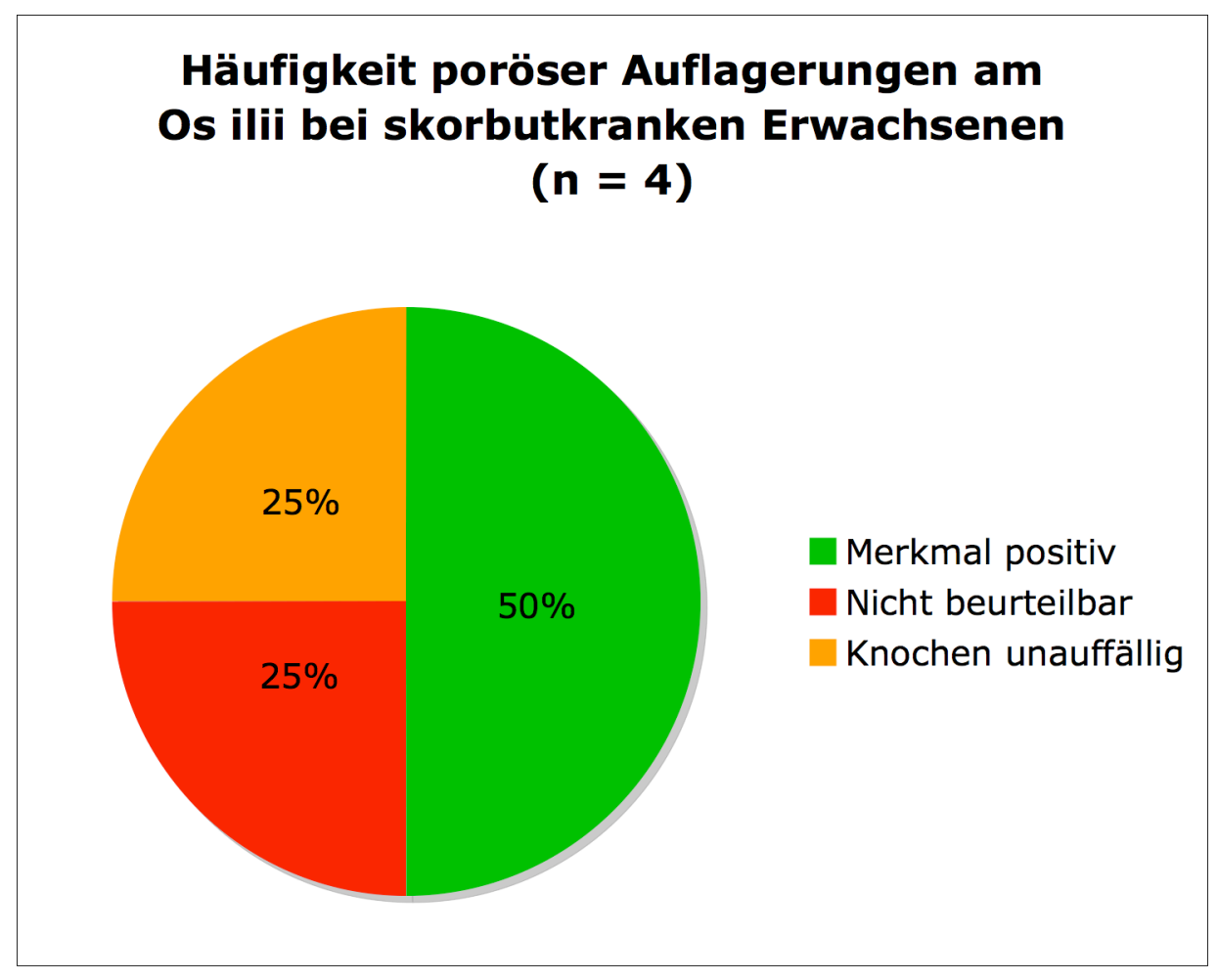

Abb. 52: Häufigkeit poröser Auflagerungen am Os ilii bei skorbutkranken Erwachsenen. Prozentzahlen beziehen sich auf Gesamtzahl der adulten Skorbutfälle. 


\section{Häufigkeit und Verteilung der einzelnen Nebenmerkmale für Skorbut}

\section{15 Poröse Oberfläche bzw. Auflagerungen am harten Gaumen/Stomatitis}

\section{(Nebenmerkmal 1)}

Wie bereits in Kapitel 2.2 Methoden erläutert, differiert der Begriff der Stomatitis in der Paläopathologie von dem im klinischen Alltag gebräuchlichen. In der Paläopathologie bezeichnet man entzündliche Prozesse an der Schleimhaut im Bereich des harten Gaumens als Stomatitis. Diese ist unspezifisch, sie kann verschiedene Ursachen haben: Mangelnde Mundhygiene und Erkrankungen der Zähne und des Zahnhalteapparats, Pilz- und Virusinfektionen. Begünstigt wird eine Stomatitis durch systemische Erkrankungen wie Skorbut, Diabetes mellitus oder Leukämie (WEIHMANN 2010).

Bei 21 (58\%) der 36 untersuchten Individuen liegt eine Stomatitis vor, davon sind 15 (42\%) an Skorbut erkrankt.

In 13 Fällen (36\%) ist der harte Gaumen nicht befundbar, unter anderem bei elf Skorbutfällen (31\%) und einem Individuum (3\%) mit Verdacht auf Skorbut.

Zwei Individuen (6\%) haben unauffällige Gaumen, darunter je ein (3\%) sicherer und ein wahrscheinlicher Skorbutkranker.

\begin{tabular}{|l|l|l|l|}
\hline & Merkmal positiv & Nicht beurteilbar & Knochen unauffällig \\
\hline Skorbut & $15(42 \%)$ & $11(31 \%)$ & $1(3 \%)$ \\
\hline V.a. Skorbut & $2(6 \%)$ & $1(3 \%)$ & $1(3 \%)$ \\
\hline Sonstiges & $4(11 \%)$ & $1(3 \%)$ & 0 \\
\hline
\end{tabular}

Tab. 86: Häufigkeit von Stomatitiden bei Individuen mit Skorbut, Verdacht auf Skorbut und Individuen mit anderen Diagnosen

Individuum Sk 1380, das vermutlich sowohl an Lepra als auch an Skorbut erkrankt ist, wird hier in der Gruppe der Skorbutkranken geführt. Die Abweichung von 100\% ergibt sich durch Auf- bzw. Abrundung.

V. a. $=$ Verdacht auf 


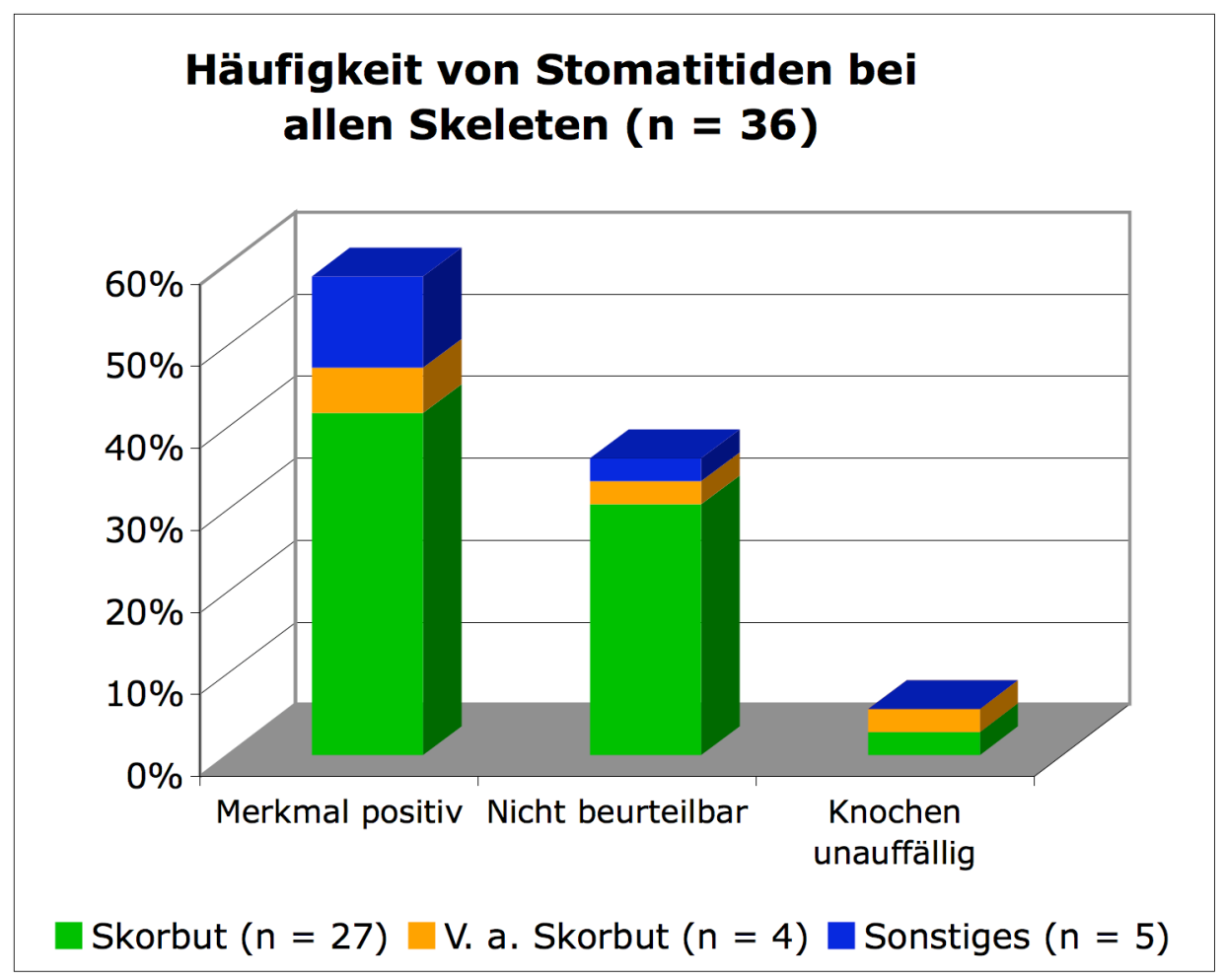

Abb. 53: Häufigkeit von Stomatitiden bei Individuen mit Skorbut, V. a. Skorbut und Individuen mit anderen Diagnosen

Individuum Sk 1380, das vermutlich sowohl an Lepra als auch an Skorbut erkrankt ist, wird hier in der Gruppe der Skorbutkranken geführt.

V. a. = Verdacht auf

Unter den 25 untersuchten Kindern sind 15 (60\% aller Kinder) mit Stomatitis, darunter 14 (56\% aller Kinder) mit Skorbut.

Bei zehn Kindern (40\% aller Kinder) ist keine Befundung des harten Gaumens möglich, davon haben neun (36\% aller Kinder) Skorbut und bei einem (4\% aller Kinder) besteht Verdacht auf Skorbut.

\begin{tabular}{|l|l|l|l|}
\hline & Merkmal positiv & Nicht beurteilbar & Knochen unauffällig \\
\hline Skorbut & $14(56 \%)$ & $9(36 \%)$ & 0 \\
\hline V. a. Skorbut & 0 & $1(4 \%)$ & 0 \\
\hline Sonstiges & $1(4 \%)$ & 0 & 0 \\
\hline
\end{tabular}

Tab. 87: Häufigkeit von Stomatitiden bei Kindern mit Skorbut, Verdacht auf Skorbut und Kindern mit anderen Diagnosen (Prozentzahlen beziehen sich auf Gesamtzahl aller Kinder)

V. a. $=$ Verdacht auf 


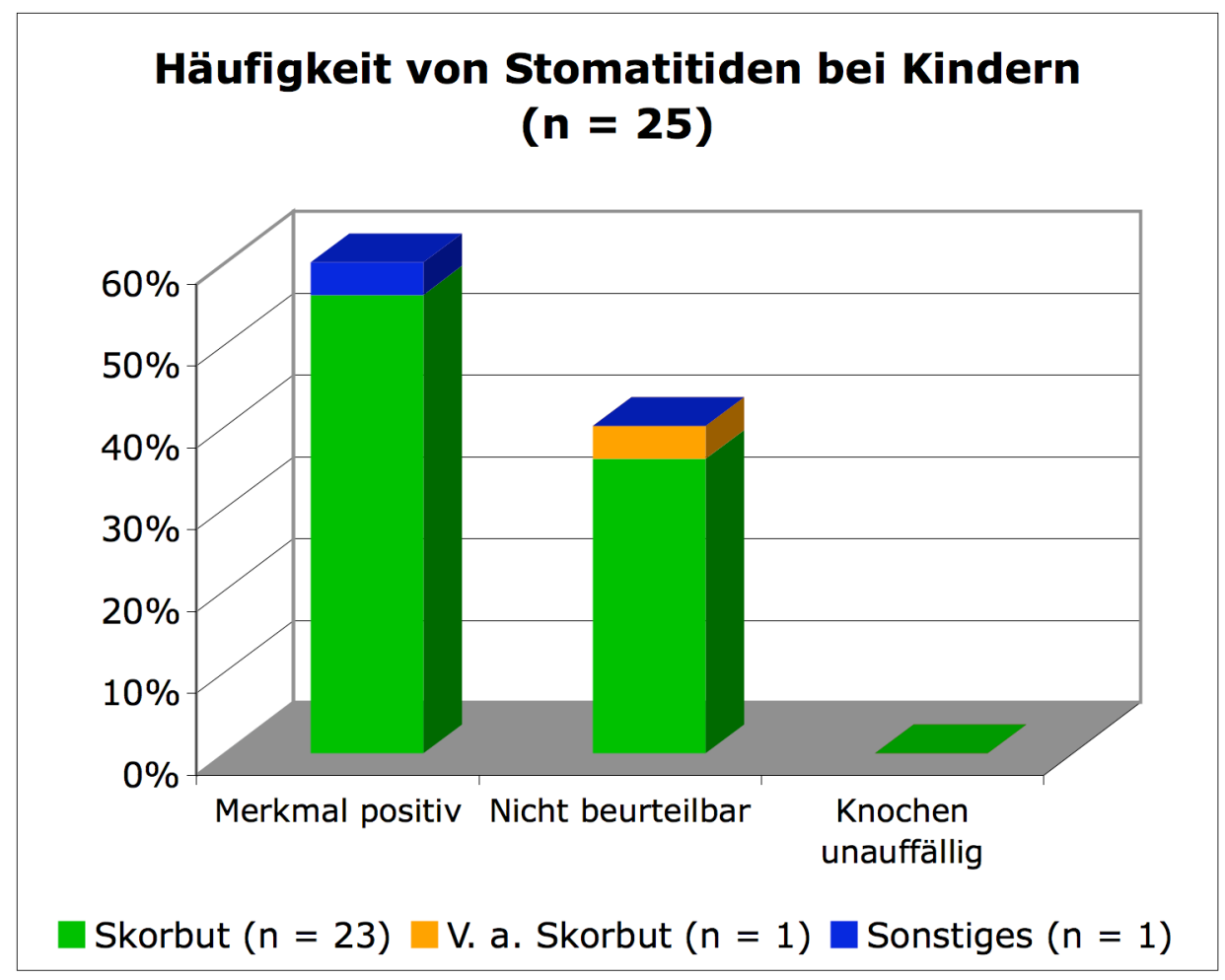

Abb. 54: Häufigkeit von Stomatitiden bei Kindern mit Skorbut, V.a. Skorbut und Kindern mit anderen Diagnosen (Prozentzahlen beziehen sich auf Gesamtzahl aller Kinder)

V. a. $=$ Verdacht auf

Von elf untersuchten Erwachsenen haben sechs (55\% aller Erwachsenen) eine Stomatitis, darunter ein Individuum ( $9 \%$ aller Erwachsenen) mit Skorbut und zwei $(18 \%$ aller Erwachsenen) mit Verdacht auf Skorbut.

In drei Fällen (27\% aller Erwachsenen) ist der harte Gaumen nicht beurteilbar, darunter zwei Individuen (18\% aller Erwachsenen) mit Skorbut.

Zwei Erwachsene (18\% aller Erwachsenen) zeigen unauffällige harte Gaumen, darunter ein Skorbutfall (9\% aller Erwachsenen) und ein (9\% aller Erwachsenen) Individuum mit Verdacht auf Skorbut.

\begin{tabular}{|l|l|l|l|}
\hline & Merkmal positiv & Nicht beurteilbar & Knochen unauffällig \\
\hline Skorbut & $1(9 \%)$ & $2(18 \%)$ & $1(9 \%)$ \\
\hline V. a. Skorbut & $2(18 \%)$ & 0 & $1(9 \%)$ \\
\hline Sonstiges & $3(27) \%$ & $1(9 \%)$ & 0 \\
\hline
\end{tabular}

Tab. 88: Häufigkeit von Stomatitiden bei Erwachsenen mit Skorbut, Verdacht auf Skorbut und Erwachsenen mit anderen Diagnosen. Prozentzahlen beziehen sich auf Gesamtzahl aller Erwachsenen, die Abweichung von 100\% ergibt sich durch Auf- bzw. Abrundung.

Individuum Sk 1380, das vermutlich sowohl an Lepra als auch an Skorbut erkrankt ist, wird hier in der Gruppe der Skorbutkranken geführt.

V. a. = Verdacht auf 


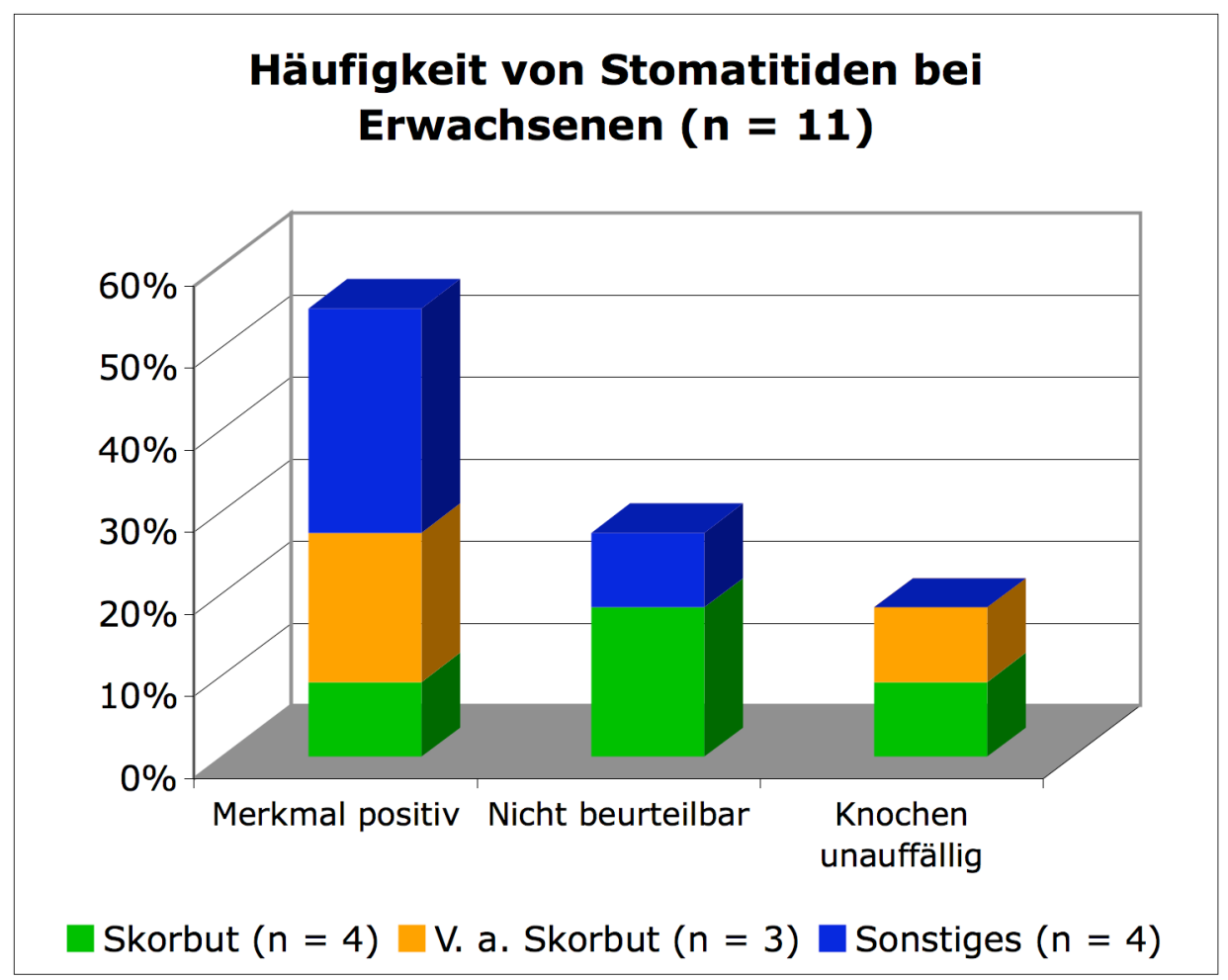

Abb. 55: Häufigkeit von Stomatitiden bei Erwachsenen mit Skorbut, V. a. Skorbut und Erwachsenen mit anderen Diagnosen. Prozentzahlen beziehen sich auf Gesamtzahl aller Erwachsenen.

Individuum Sk 1380, das vermutlich sowohl an Lepra als auch an Skorbut erkrankt ist, wird hier in der Gruppe der Skorbutkranken geführt.

V. a. = Verdacht auf

Unter den 27 untersuchten Skorbutfällen sind 15 (56\% aller Skorbutfälle) mit Stomatitis, bei elf (41\% aller Skorbutfälle) ist keine Beurteilung möglich, ein Individuum (4\% aller Skorbutfälle) hat einen unauffälligen harten Gaumen.

\begin{tabular}{|l|l|l|l|}
\hline & Merkmal positiv & Nicht beurteilbar & Knochen unauffällig \\
\hline Skorbutkranke $(\mathbf{n}=\mathbf{2 7})$ & $15(56 \%)$ & $11(41 \%)$ & $1(4 \%)$ \\
\hline
\end{tabular}

Tab. 89: Häufigkeit von Stomatitiden bei den untersuchten Skorbutkranken (Prozentzahlen beziehen sich auf Gesamtzahl der Skorbutfälle, die Abweichung von 100\% ergibt sich durch Auf- bzw. Abrundung) 


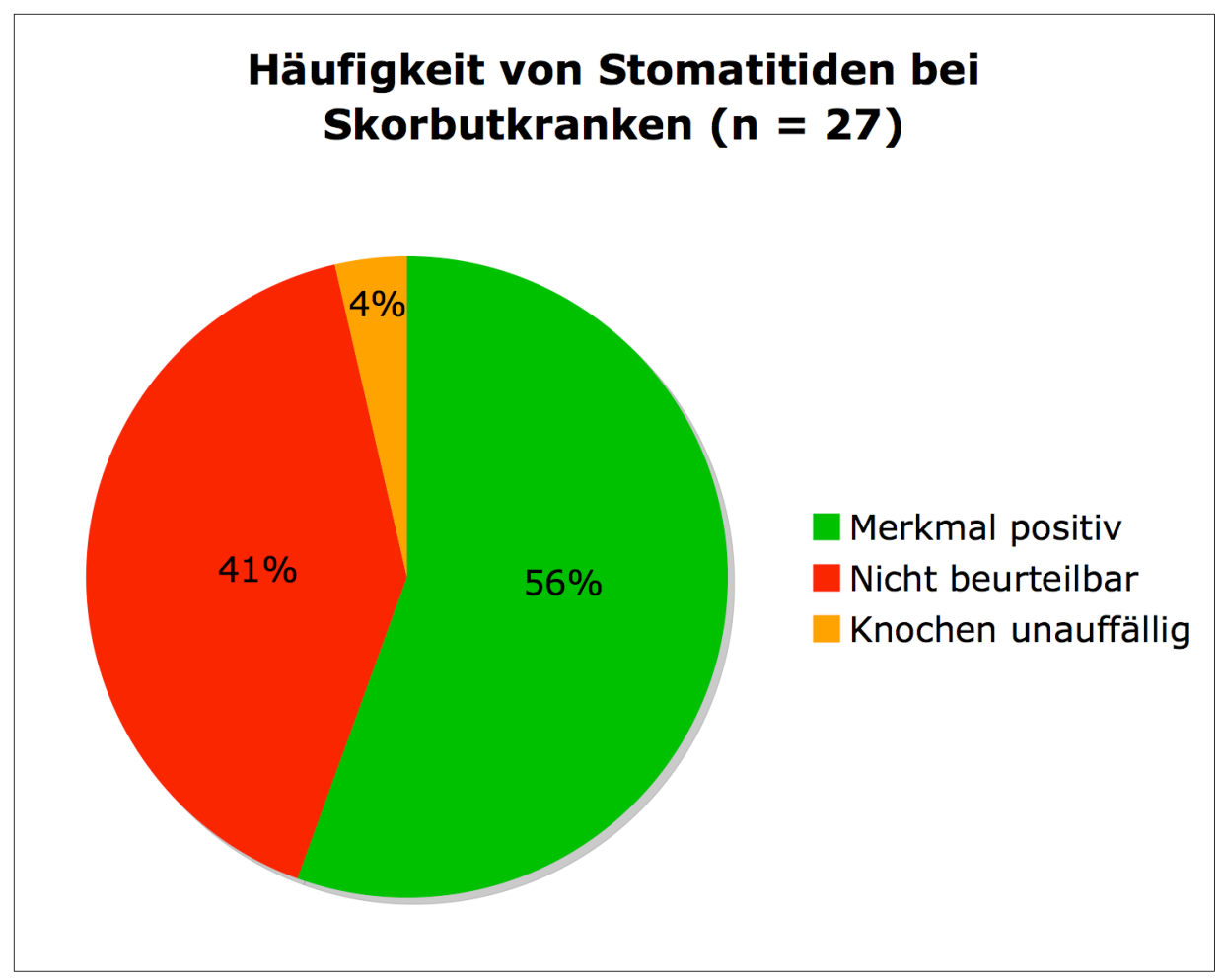

Abb. 56: Häufigkeit von Stomatitiden bei den untersuchten Skorbutkranken. Prozentzahlen beziehen sich auf Gesamtzahl der Skorbutfälle, die Abweichung von 100\% ergibt sich durch Auf- bzw. Abrundung.

Unter den 23 skorbutkranken Kindern sind 14 (61\% aller skorbutkranken Kinder) mit Stomatitis und neun (39\% aller skorbutkranken Kinder) mit nicht beurteilbaren harten Gaumen.

\begin{tabular}{|l|l|l|l|}
\hline & Merkmal positiv & Nicht beurteilbar & Knochen unauffällig \\
\hline Skorbutkranke Kinder $(\mathbf{n}=\mathbf{2 3})$ & $14(61 \%)$ & $9(39 \%)$ & 0 \\
\hline
\end{tabular}

Tab. 90: Häufigkeit von Stomatitiden bei den untersuchten skorbutkranken Kindern (Prozentzahlen beziehen sich auf Gesamtzahl der infantilen Skorbutfälle)

Bei den vier skorbutkranken Erwachsenen findet sich in einem Fall (25\% der adulten Skorbutfälle) eine Stomatitis, zwei Individuen (50\% der adulten Skorbutfälle) haben nicht befundbare und ein Individuum ( $25 \%$ der adulten Skorbutfälle) einen unauffälligen harten Gaumen.

\begin{tabular}{|l|l|l|l|}
\hline & Merkmal positiv & Nicht beurteilbar & Knochen unauffällig \\
\hline Skorbutkranke Erwachsene $(\mathbf{n}=4)$ & $1(25 \%)$ & $2(50 \%)$ & $1(25 \%)$ \\
\hline
\end{tabular}

Tab. 91: Häufigkeit von Stomatitiden bei den untersuchten skorbutkranken Erwachsenen (Prozentzahlen beziehen sich auf Gesamtzahl der adulten Skorbutfälle) 


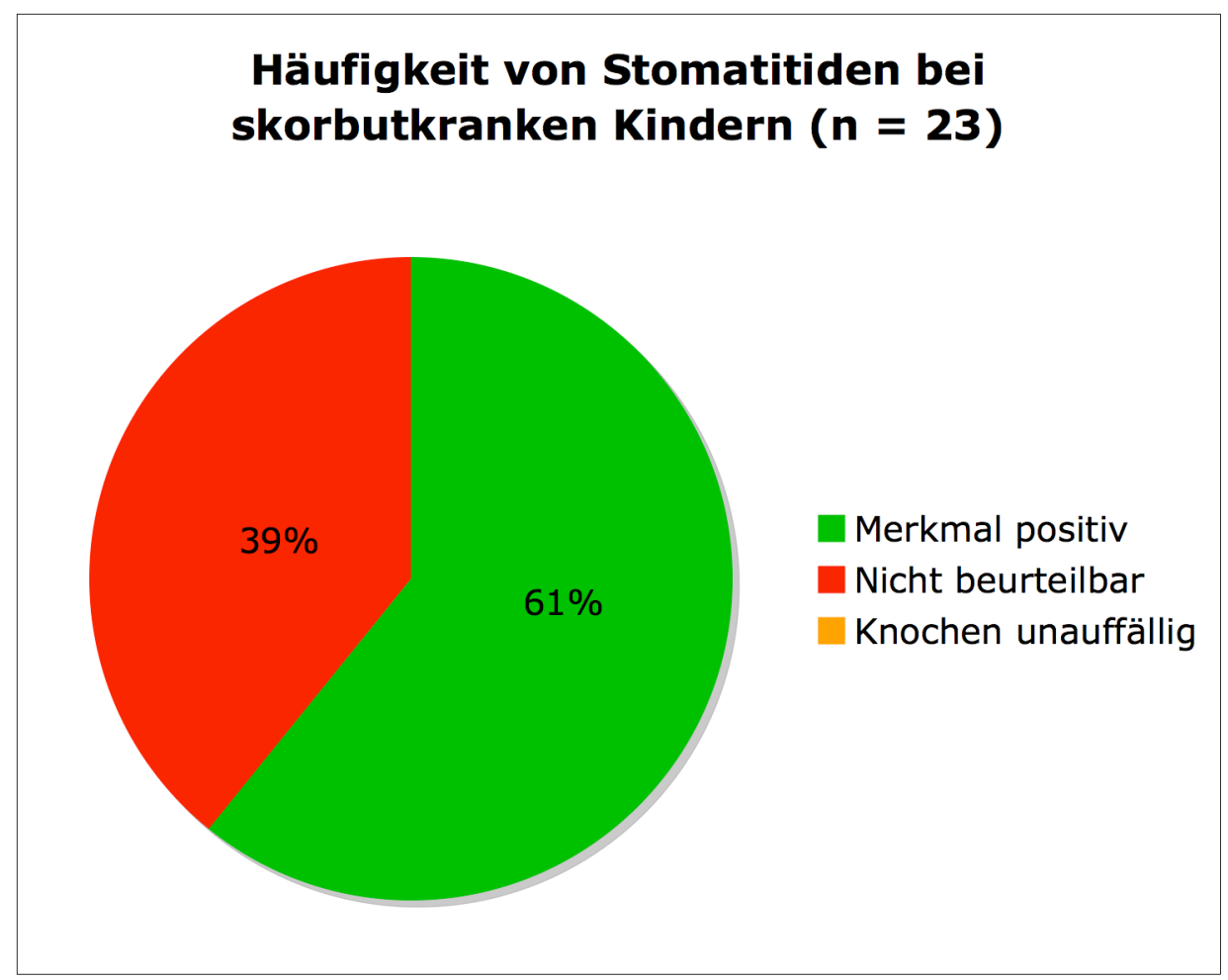

Abb. 57: Häufigkeit von Stomatitiden bei den untersuchten skorbutkranken Kindern (Prozentzahlen beziehen sich auf Gesamtzahl der infantilen Skorbutfälle)

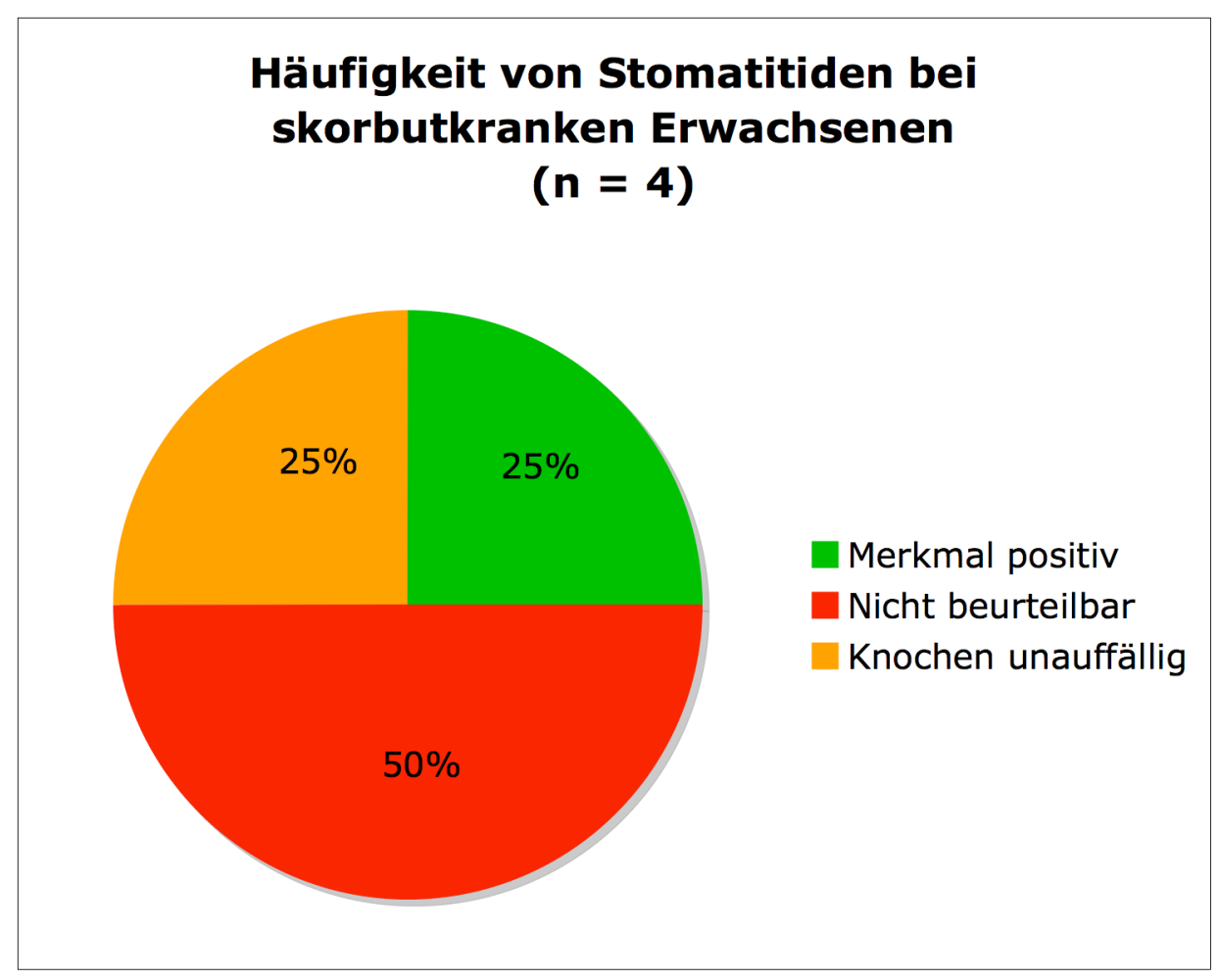

Abb. 58: Häufigkeit von Stomatitiden bei den untersuchten skorbutkranken Erwachsenen (Prozentzahlen beziehen sich auf Gesamtzahl der adulten Skorbutfälle) 


\subsection{Poröse Auskleidungen der Alveolen (Nebenmerkmal 2)}

Bei sechs (17\%) von 36 untersuchten Individuen liegen poröse Auskleidungen der Alveolen vor, fünf (14\%) von ihnen sind an Skorbut erkrankt, bei einem Individuum (3\%) besteht Verdacht auf Skorbut.

Dreizehn (36\%) der Individuen haben keinen befundbaren Processus alveolaris, darunter elf (31\%) Skorbutkranke und zwei Individuen (6\%) mit Verdacht auf Skorbut.

Siebzehn (47\%) der Individuen haben unauffällige Alveolen, darunter auch elf (31\%) mit sicherem und zwei (6\%) mit Verdacht auf Skorbut.

\begin{tabular}{|l|l|l|l|}
\hline & Merkmal positiv & Nicht beurteilbar & Knochen unauffällig \\
\hline Skorbut & $5(14 \%)$ & $11(31 \%)$ & $11(31 \%)$ \\
\hline V. a. Skorbut & $1(3 \%)$ & $1(3 \%)$ & $2(6 \%)$ \\
\hline Sonstiges & 0 & $1(3 \%)$ & $4(11 \%)$ \\
\hline
\end{tabular}

Tab. 92: Häufigkeit poröser Auskleidungen der Alveolen bei Individuen mit Skorbut, Verdacht auf Skorbut und Individuen mit anderen Diagnosen

Individuum Sk 1380, das vermutlich sowohl an Lepra als auch an Skorbut erkrankt ist, wird hier in der Gruppe der Skorbutkranken geführt. Die Abweichung von 100\% ergibt sich durch Auf- bzw. Abrundung.

V. a. $=$ Verdacht auf

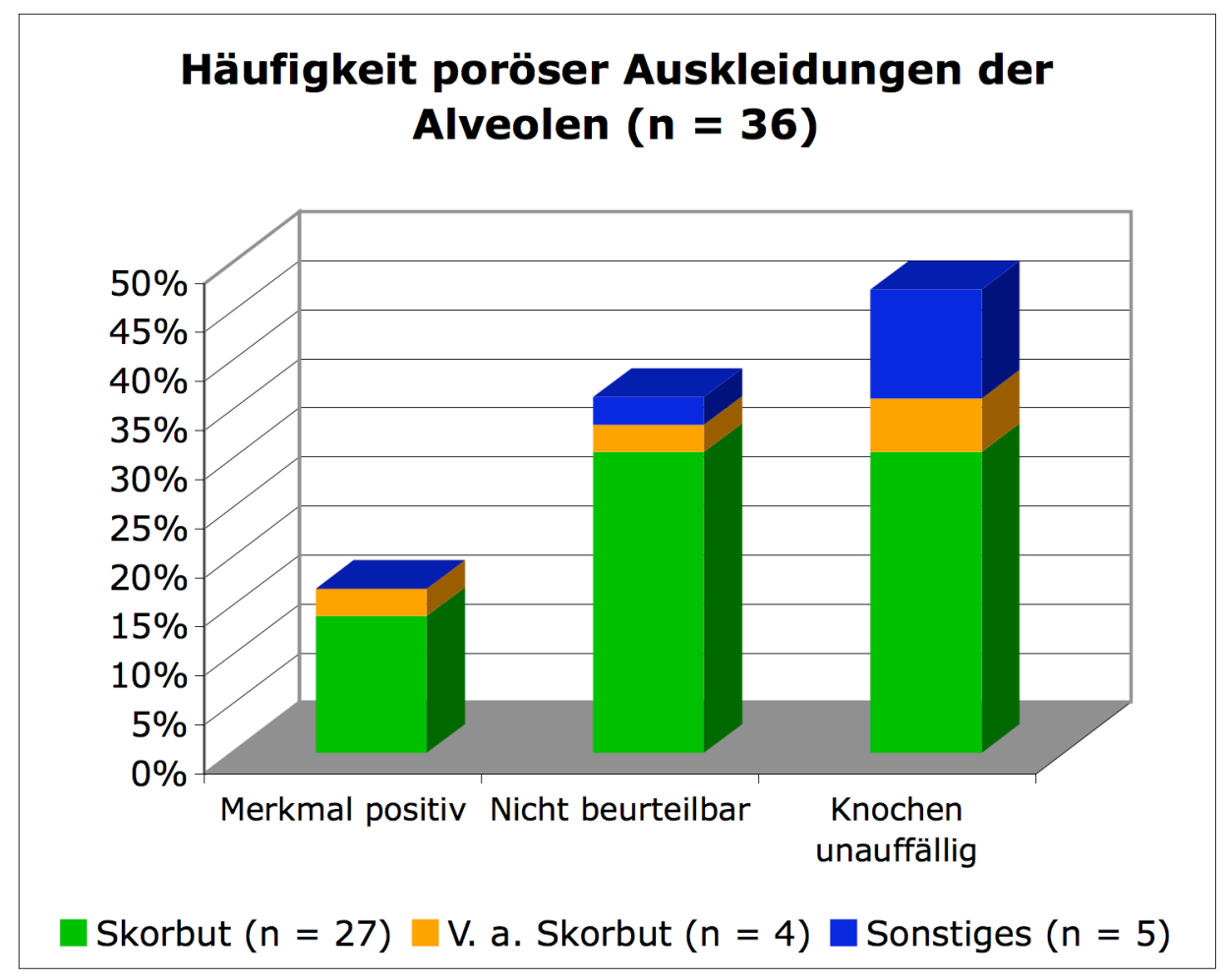

Abb. 59: Häufigkeit poröser Auskleidungen der Alveolen bei Individuen mit Skorbut, Verdacht auf Skorbut und Individuen mit anderen Diagnosen

Individuum Sk 1380, das vermutlich sowohl an Lepra als auch an Skorbut erkrankt ist, wird hier in der Gruppe der Skorbutkranken geführt.

V. a. $=$ Verdacht auf 
Betrachtet man nur die 25 untersuchten Kinder, so zeigen fünf (20\% aller Kinder) poröse Auskleidungen der Alveolen, alle mit Skorbut.

Zehn Kinder (40\%) haben keinen befundbaren Processus alveolaris, darunter auch neun (36\%) mit Skorbut und eines (4\%) mit Verdacht auf Skorbut.

Bei zehn Kindern (40\% aller Kinder) sind die Alveolarfortsätze unauffällig, obwohl neun davon (36\%) an Skorbut erkrankt sind.

\begin{tabular}{|l|l|l|l|}
\hline & Merkmal positiv & Nicht beurteilbar & Knochen unauffällig \\
\hline Skorbut & $5(20 \%)$ & $9(36 \%)$ & $9(36 \%)$ \\
\hline V. a. Skorbut & 0 & $1(4 \%)$ & 0 \\
\hline Sonstiges & 0 & 0 & $1(4 \%)$ \\
\hline
\end{tabular}

Tab. 93: Häufigkeit poröser Auskleidungen der Alveolen bei Kindern mit Skorbut, V. a. Skorbut und Kindern mit anderen Diagnosen (Prozentzahlen beziehen sich auf Gesamtzahl der Kinder) V. a. $=$ Verdacht auf

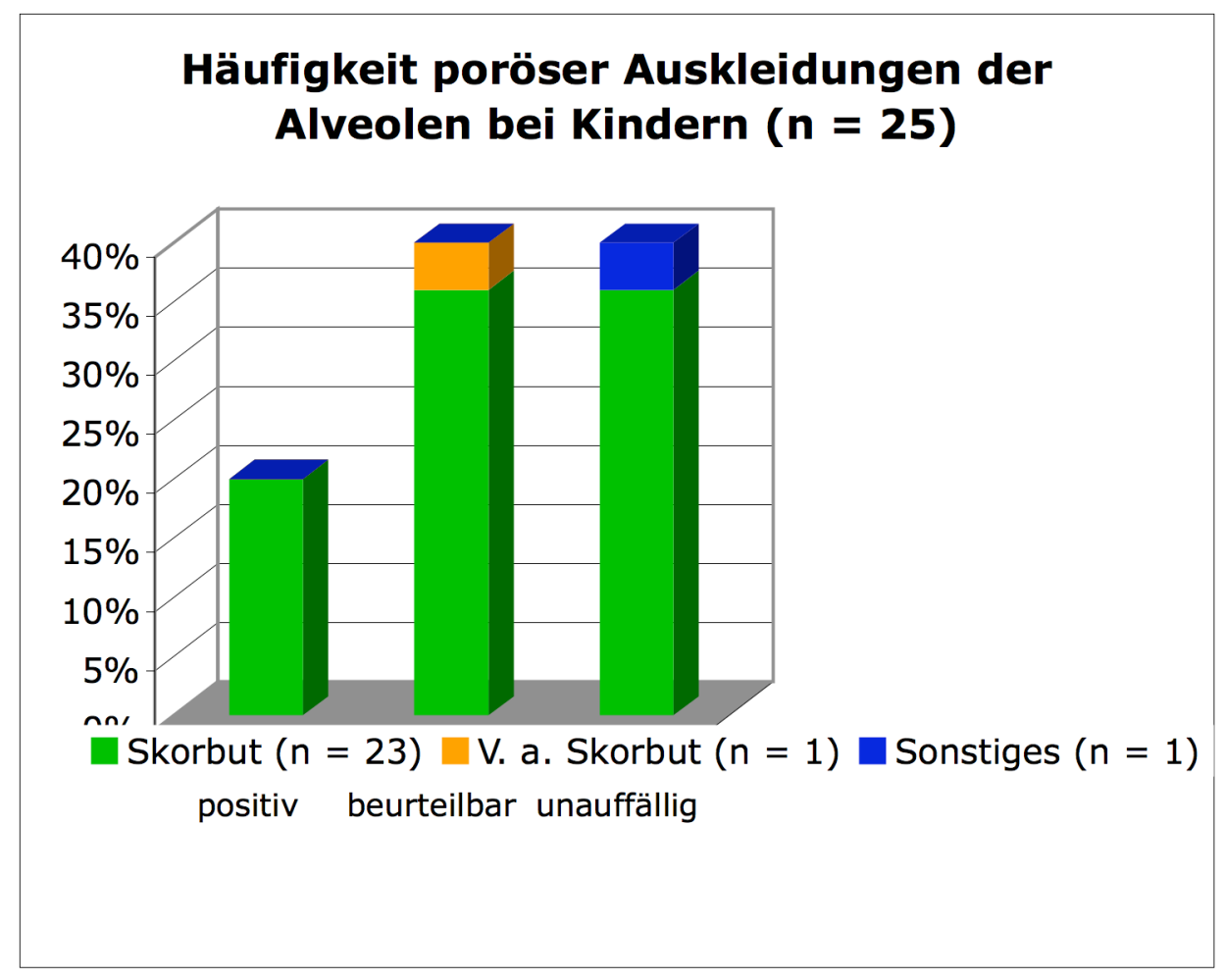

Abb. 60: Häufigkeit poröser Auskleidungen der Alveolen bei Kindern mit Skorbut, V. a. Skorbut und Kindern mit anderen Diagnosen (Prozentzahlen beziehen sich auf Gesamtzahl der Kinder)

V. a. $=$ Verdacht auf

Nur ein Individuum (9\%) der elf untersuchten Erwachsenen weist poröse Auskleidungen der Alveolen auf, bei diesem besteht Verdacht auf Skorbut. Bei drei (27\% aller Erwachsenen) ist der Alveolarfortsatz nicht beurteilbar, darunter zwei Individuen (18\%) mit Skorbut. Bei sieben Adulten (64\% aller Erwachsenen) sind die Alveolen unauffällig, darunter je zwei Individuen (18\% aller Erwachsenen) mit Skorbut bzw. Verdacht auf Skorbut. 


\begin{tabular}{|l|l|l|l|}
\hline & Merkmal positiv & Nicht beurteilbar & Knochen unauffällig \\
\hline Skorbut & 0 & $2(18 \%)$ & $2(18 \%)$ \\
\hline V. a. Skorbut & $1(9 \%)$ & 0 & $2(18 \%)$ \\
\hline Sonstiges & 0 & $1(9 \%)$ & $3(27 \%)$ \\
\hline
\end{tabular}

Tab. 94: Häufigkeit poröser Auskleidungen der Alveolen bei Erwachsenen mit Skorbut, V. a. Skorbut und Individuen mit anderen Diagnosen (Prozentzahlen beziehen sich auf Gesamtzahl aller Erwachsenen, die Abweichung von 100\% ergibt sich durch Auf- bzw. Abrundung)

Individuum Sk 1380, das vermutlich sowohl an Lepra als auch an Skorbut erkrankt ist, wird hier in der Gruppe der Skorbutkranken geführt.

V. a. $=$ Verdacht auf

\section{Häufigkeit poröser Auskleidungen der Alveolen bei Erwachsenen $(n=11)$}

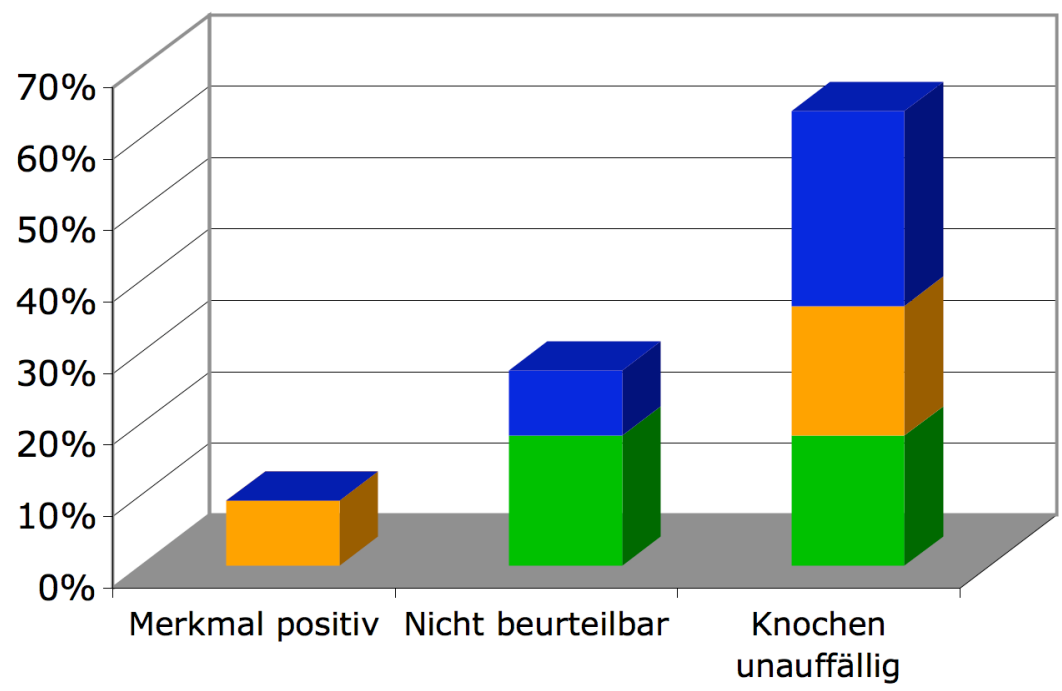

Skorbut $(n=4) \square$ V. a. Skorbut $(n=3) \square$ Sonstiges $(n=4)$

Abb. 61: Häufigkeit poröser Auskleidungen der Alveolen bei Erwachsenen mit Skorbut, V. a. Skorbut und Individuen mit anderen Diagnosen. Prozentzahlen beziehen sich auf Gesamtzahl aller Erwachsenen.

Individuum Sk 1380, das vermutlich sowohl an Lepra als auch an Skorbut erkrankt ist, wird hier in der Gruppe der Skorbutkranken geführt.

V. a. $=$ Verdacht auf

Bei den insgesamt 27 skorbutkranken Individuen finden sich in fünf Fällen $(19 \%$ aller Skorbutkranken) poröse Alveolarauskleidungen, bei elf Individuen $(41 \%$ aller Skorbutkranken) ist der Processus alveolaris nicht beurteilbar, elf Individuen (41\% aller Skorbutkranken) haben unauffällige Alveolen.

\begin{tabular}{|l|l|l|l|}
\hline & Merkmal positiv & Nicht beurteilbar & Knochen unauffällig \\
\hline Skorbutkranke $(\mathbf{n}=\mathbf{2 7})$ & $5(19 \%)$ & $11(41 \%)$ & $11(41 \%)$ \\
\hline
\end{tabular}

Tab. 95: Häufigkeit poröser Alveolarauskleidungen bei den untersuchten Skorbutkranken. Prozentzahlen beziehen sich auf Gesamtzahl der Skorbutfälle, die Abweichung von 100\% ergibt sich durch Auf- bzw. Abrundung. 


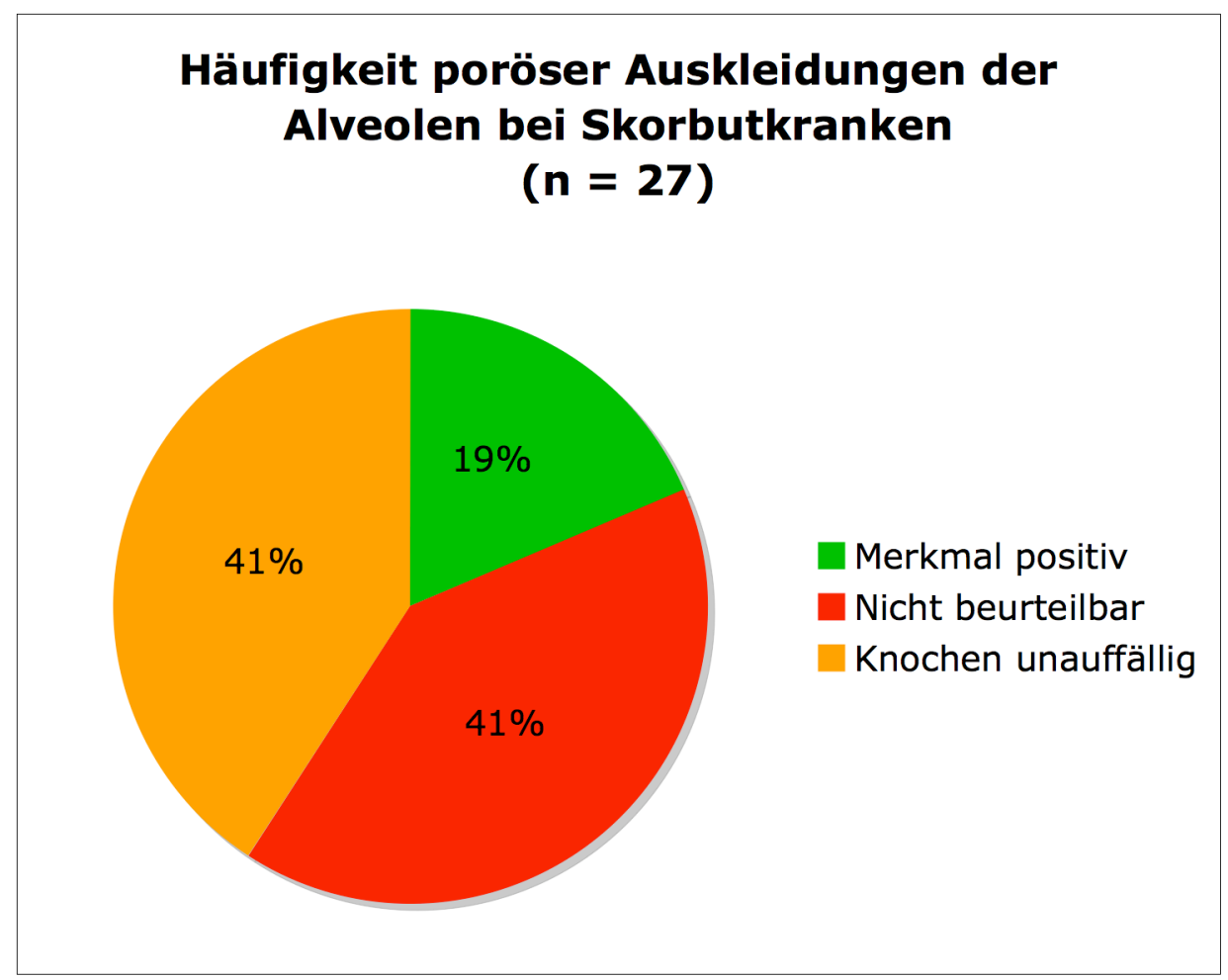

Abb. 62: Häufigkeit poröser Alveolarauskleidungen bei den untersuchten Skorbutkranken. Prozentzahlen beziehen sich auf Gesamtzahl der Skorbutfälle, die Abweichung von 100\% ergibt sich durch Auf- bzw. Abrundung.

Von den 23 an Skorbut erkrankten Kindern haben fünf (22\% der skorbutkranken Kinder) poröse Alveolarauskleidungen, bei neun Kindern (39\% der skorbutkranken Kinder) ist der Alveolarfortsatz nicht beurteilbar, bei neun Kindern (39\%) ist er unauffällig.

\begin{tabular}{|l|l|l|l|}
\hline & Merkmal positiv & Nicht beurteilbar & Knochen unauffällig \\
\hline Skorbutkranke Kinder $(\mathbf{n}=\mathbf{2 3})$ & $5(22 \%)$ & $9(39 \%)$ & $9(39 \%)$ \\
\hline
\end{tabular}

Tab. 96: Häufigkeit poröser Alveolarauskleidungen bei den untersuchten skorbutkranken Kindern (Prozentzahlen beziehen sich auf Gesamtzahl der infantilen Skorbutfälle)

Keines der vier an Skorbut erkrankten adulten Individuen hat poröse Alveolarauskleidungen, bei je zwei Individuen (50\% der adulten Skorbutfälle) ist der Alveolarfortsatz unauffällig bzw. nicht befundbar.

\begin{tabular}{|l|l|l|l|}
\hline & Merkmal positiv & Nicht beurteilbar & Knochen unauffällig \\
\hline Skorbutkranke Erwachsene $(n=4)$ & 0 & $2(50 \%)$ & $2(50 \%)$ \\
\hline
\end{tabular}

Tab. 97: Häufigkeit poröser Alveolarauskleidungen bei den untersuchten skorbutkranken Erwachsenen (Prozentzahlen beziehen sich auf Gesamtzahl der adulten Skorbutfälle) 


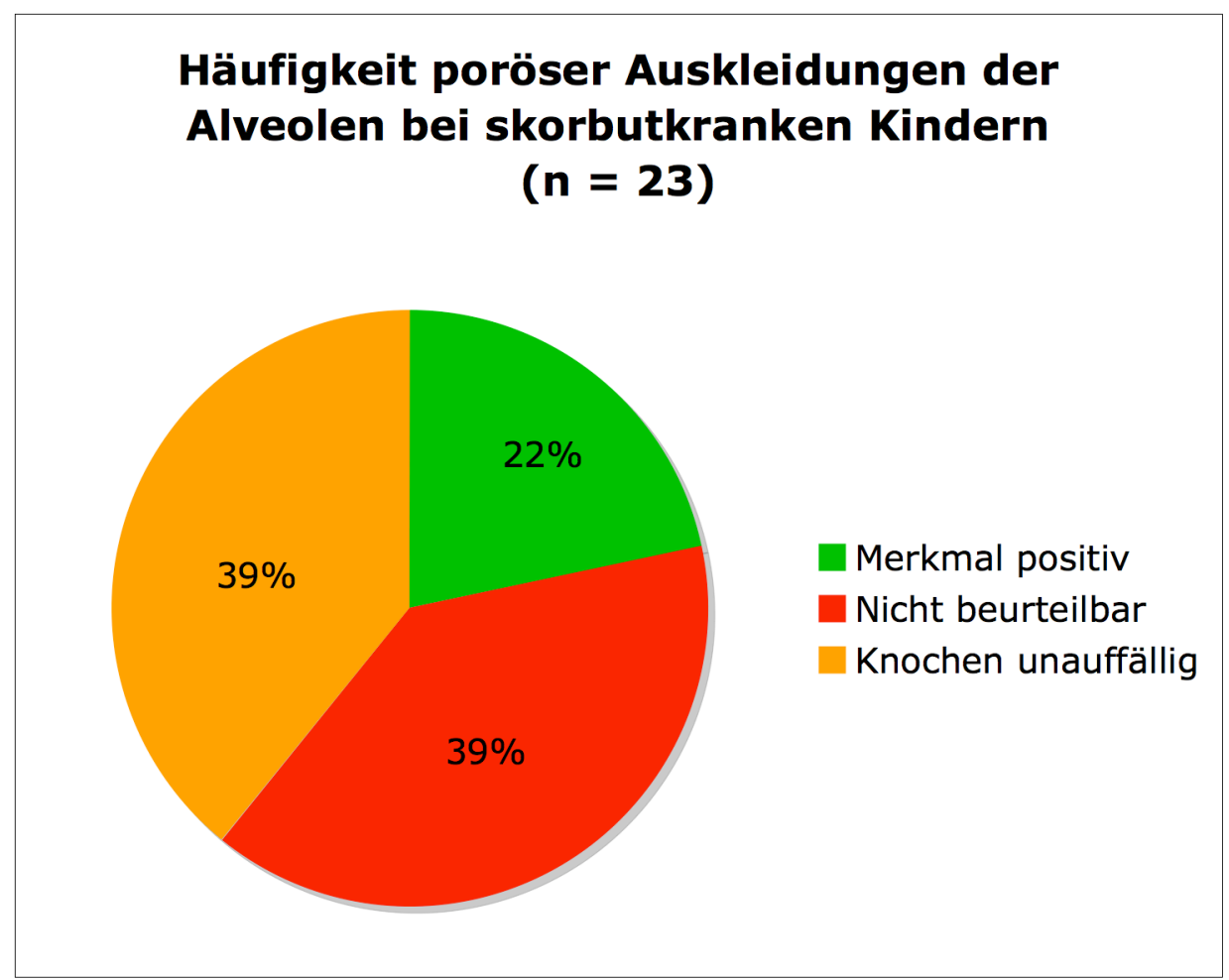

Abb. 63: Häufigkeit poröser Alveolarauskleidungen bei den untersuchten skorbutkranken Kindern (Prozentzahlen beziehen sich auf Gesamtzahl der infantilen Skorbutfälle)

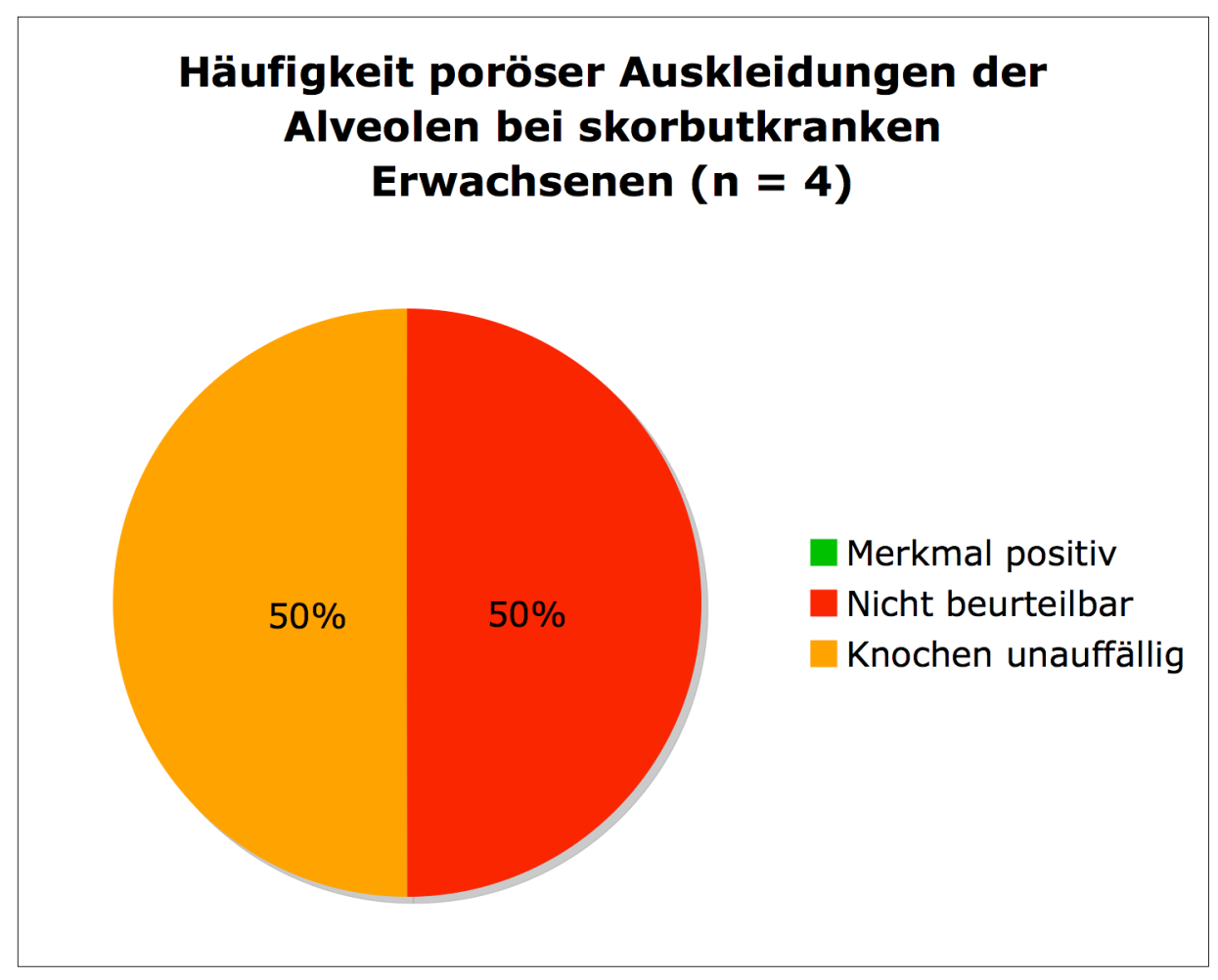

Abb. 64: Häufigkeit poröser Alveolarauskleidungen bei den untersuchten skorbutkranken Erwachsenen (Prozentzahlen beziehen sich auf Gesamtzahl der adulten Skorbutfälle) 


\subsection{Symmetrische poröse Auflagerungen in den Impressiones digitatae oder flächig am inneren Schädeldach (Nebenmerkmal 3)}

Von den 36 untersuchten Individuen weisen 18 (50\%) poröse Auflagerungen am inneren Schädeldach auf, von denen 17 (47\%) sicher und ein Individuum (3\%) wahrscheinlich an Skorbut erkrankt sind.

Bei acht Individuen (22\%) ist das innere Schädeldach nicht befundbar, auch bei sieben Skorbutkranken (19\%).

Zehn Individuen (28\%) zeigen unauffällige Schädelinnenseiten, darunter je drei (8\%) mit Skorbut und wahrscheinlichem Skorbut.

\begin{tabular}{|l|l|l|l|}
\hline & Merkmal positiv & Nicht beurteilbar & Knochen unauffällig \\
\hline Skorbut & $17(47 \%)$ & $7(19 \%)$ & $3(8 \%)$ \\
\hline V. a. Skorbut & $1(3 \%)$ & 0 & $3(8 \%)$ \\
\hline Sonstiges & 0 & $1(3 \%)$ & $4(11 \%)$ \\
\hline
\end{tabular}

Tab. 98: Häufigkeit poröser Auflagerungen am inneren Schädeldach bei Individuen mit Skorbut, V. a. Skorbut und Individuen mit anderen Diagnosen

Individuum Sk 1380, das vermutlich sowohl an Lepra als auch an Skorbut erkrankt ist, wird hier in der Gruppe der Skorbutkranken geführt. Die Abweichung von 100\% ergibt sich durch Auf- bzw. Abrundung.

V. a. = Verdacht auf

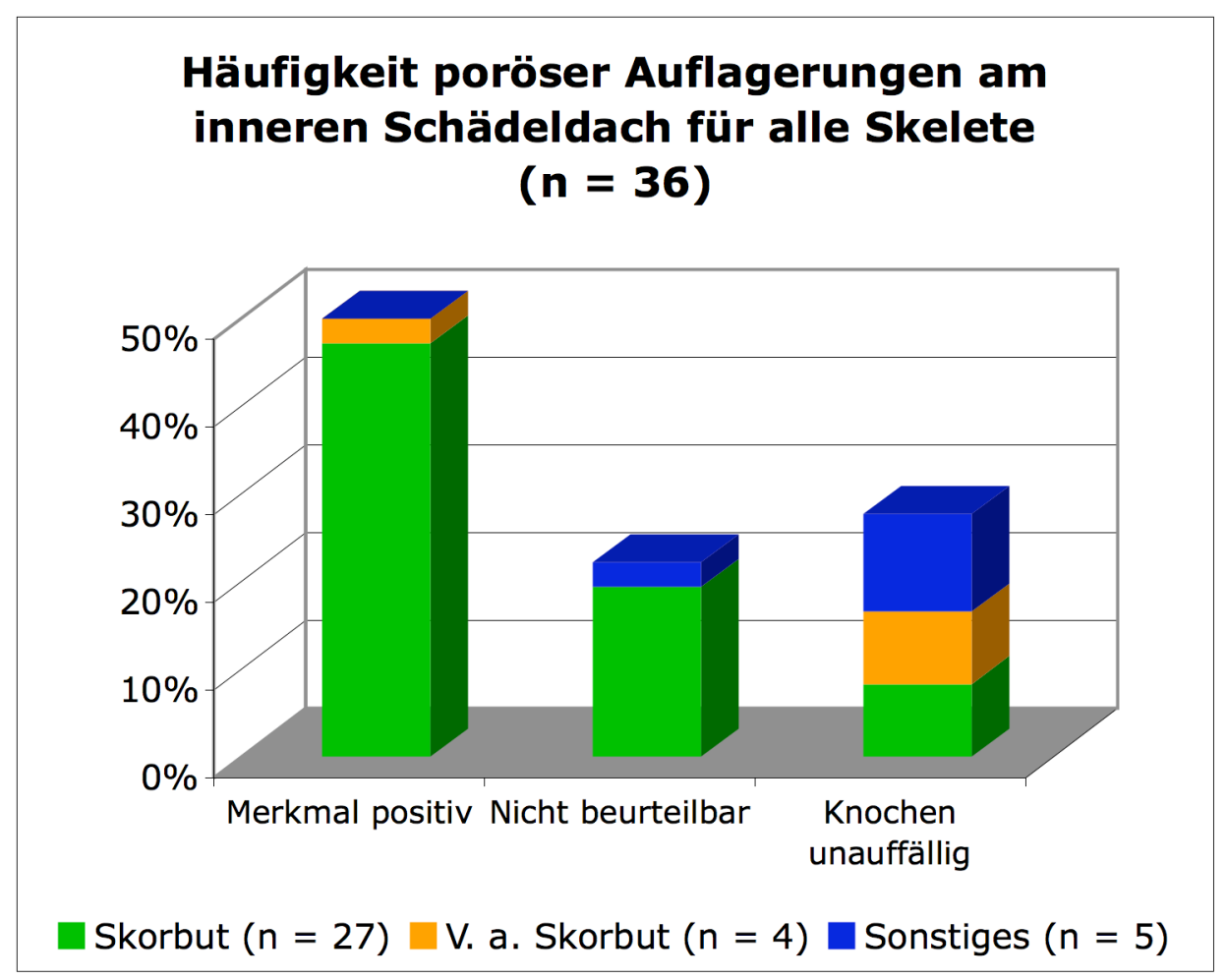

Abb. 65: Häufigkeit poröser Auflagerungen am inneren Schädeldach bei Individuen mit Skorbut, Verdacht auf Skorbut und Individuen mit anderen Diagnosen

Individuum Sk 1380, das vermutlich sowohl an Lepra als auch an Skorbut erkrankt ist, wird hier in der Gruppe der Skorbutkranken geführt.

V. a. = Verdacht auf 
Von 25 untersuchten Kindern zeigen 16 (64\% aller Kinder), die alle an Skorbut erkrankt sind, poröse Auflagerungen am inneren Schädeldach. Bei vier Kindern (16\% der Kinder) sind die Schädeldächer nicht befundbar, auch diese sind alle skorbutkrank.

Fünf Kinder (20\% der Kinder), darunter drei (12\% aller Kinder) Skorbutkranke und eins (4\% aller Kinder) mit Verdacht auf Skorbut, haben unauffällige innere Schädeldächer.

\begin{tabular}{|l|l|l|l|}
\hline & Merkmal positiv & Nicht beurteilbar & Knochen unauffällig \\
\hline Skorbut & $16(64 \%)$ & $4(16 \%)$ & $3(12 \%)$ \\
\hline V. a. Skorbut & 0 & 0 & $1(4 \%)$ \\
\hline Sonstiges & 0 & 0 & $1(4 \%)$ \\
\hline
\end{tabular}

Tab. 99: Häufigkeit poröser Auflagerungen am inneren Schädeldach bei Kindern mit Skorbut, V. a. Skorbut und Kindern mit anderen Diagnosen (Prozentzahlen beziehen sich auf Gesamtzahl der Kinder)

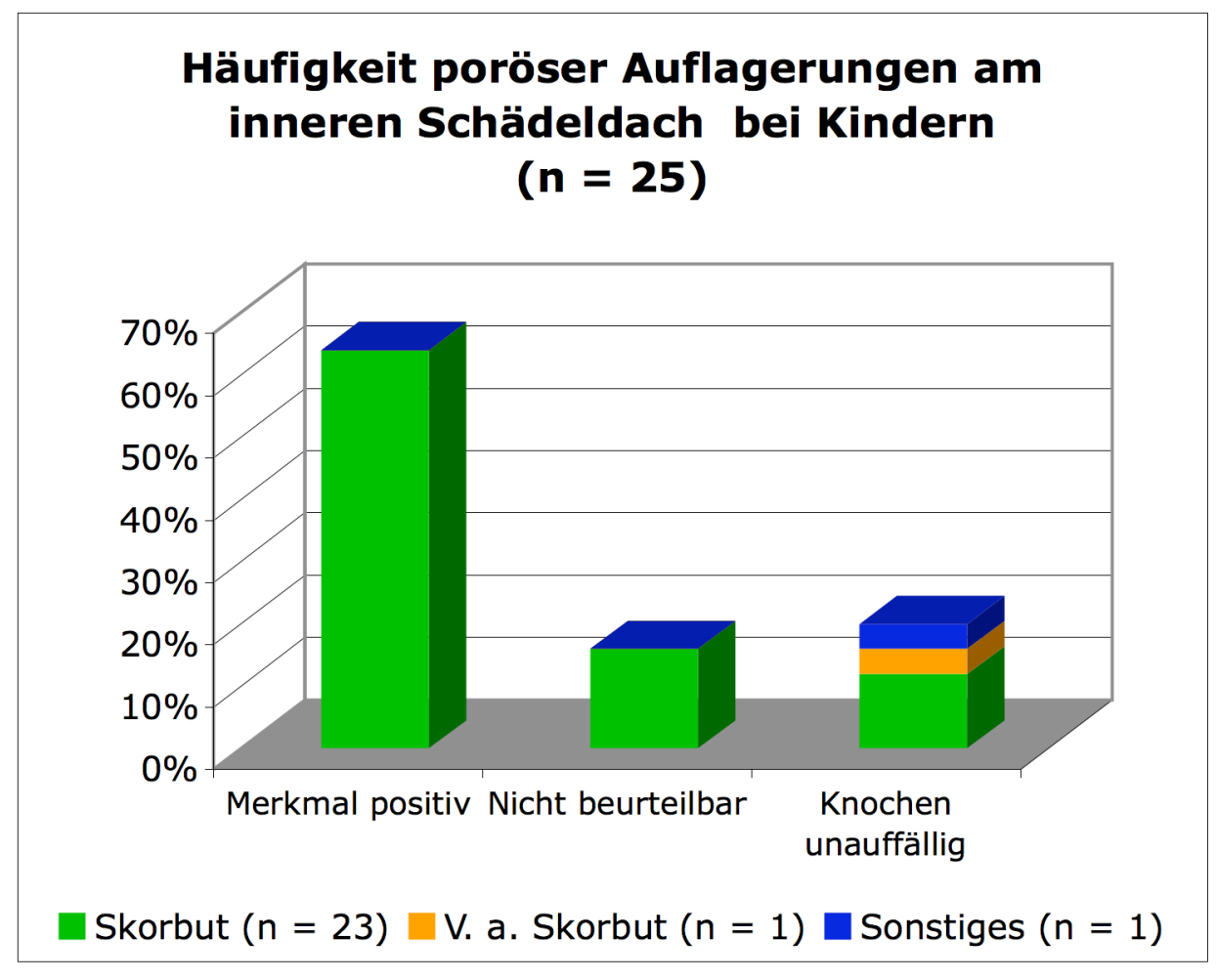

Abb. 66: Häufigkeit poröser Auflagerungen am inneren Schädeldach bei Kindern mit Skorbut, V. a. Skorbut und Kindern mit anderen Diagnosen (Prozentzahlen beziehen sich auf Gesamtzahl der Kinder)

Von den elf untersuchten Erwachsenen haben nur zwei Individuen (18\% der Erwachsenen) poröse Auflagerungen am inneren Schädeldach, davon je ein Individuum $(9 \%$ der Erwachsenen) mit Diagnose Skorbut und Verdacht auf Skorbut.

Bei vier Erwachsenen (36\% der Erwachsenen) sind die Schädeldächer nicht befundbar, davon drei Individuen (27\% der Erwachsenen) mit Skorbut.

Fünf Erwachsene (46\% der Erwachsenen) haben unauffällige Schädeldächer, darunter zwei Individuen (18\% der Erwachsenen) mit Verdacht auf Skorbut. 


\begin{tabular}{|l|l|l|l|}
\hline & Merkmal positiv & Nicht beurteilbar & Knochen unauffällig \\
\hline Skorbut & $1(9 \%)$ & $3(27 \%)$ & 0 \\
\hline V. a. Skorbut & $1(9 \%)$ & 0 & $2(18 \%)$ \\
\hline Sonstiges & 0 & $1(9 \%)$ & $3(27 \%)$ \\
\hline
\end{tabular}

Tab. 100: Häufigkeit poröser Auflagerungen am inneren Schädeldach bei Erwachsenen mit Skorbut, Verdacht auf Skorbut und Erwachsenen mit anderen Diagnosen. Prozentzahlen beziehen sich auf Gesamtzahl der Erwachsenen, die Abweichung von 100\% ergibt sich durch Auf- bzw. Abrundung.

Individuum Sk 1380, das vermutlich sowohl an Lepra als auch an Skorbut erkrankt ist, wird hier in der Gruppe der Skorbutkranken geführt.

V. a. $=$ Verdacht auf

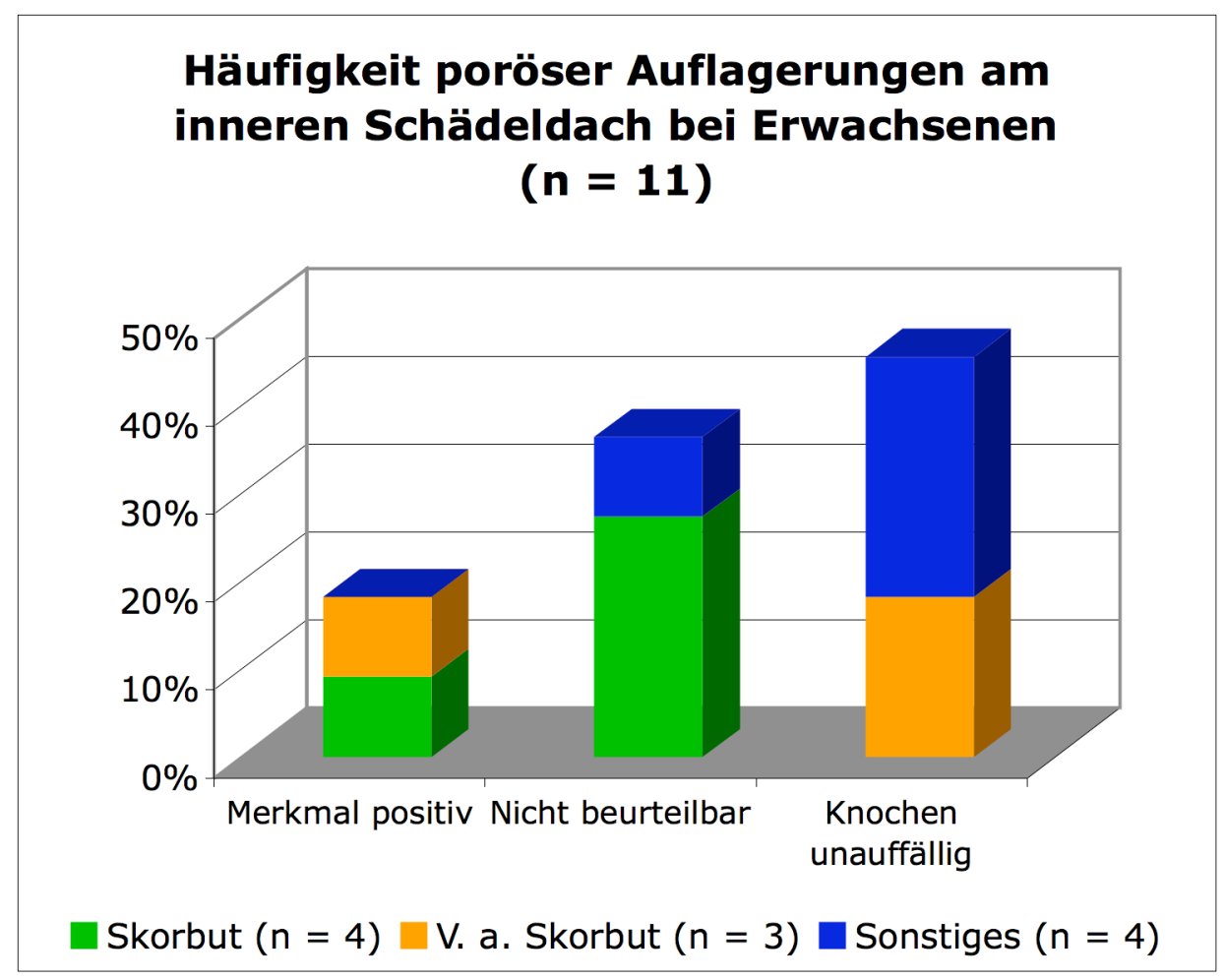

Abb. 67: Häufigkeit poröser Auflagerungen am inneren Schädeldach bei Erwachsenen mit Skorbut, Verdacht auf Skorbut und Erwachsenen mit anderen Diagnosen (Prozentzahlen beziehen sich auf Gesamtzahl der Erwachsenen)

Individuum Sk 1380, das vermutlich sowohl an Lepra als auch an Skorbut erkrankt ist, wird hier in der Gruppe der Skorbutkranken geführt.

V. a. = Verdacht auf

Betrachtet man alle an Skorbut erkrankten Individuen (27), so weisen 17 (63\% der Skorbutkranken) poröse Auflagerungen an der Schädelinnenseite auf, bei sieben Individuen (26\%) lassen sich die Schädeldächer nicht befunden, drei (11\%) haben ein unauffälliges inneres Schädeldach.

\begin{tabular}{|l|l|l|l|}
\hline & Merkmal positiv & Nicht beurteilbar & Knochen unauffällig \\
\hline Skorbutkranke $(\mathbf{n}=\mathbf{2 7})$ & $17(63 \%)$ & $7(26 \%)$ & $3(11 \%)$ \\
\hline
\end{tabular}

Tab. 101: Häufigkeit poröser Auflagerungen am inneren Schädeldach bei den untersuchten Skorbutkranken (Prozentzahlen beziehen sich auf Gesamtzahl der Skorbutfälle) 


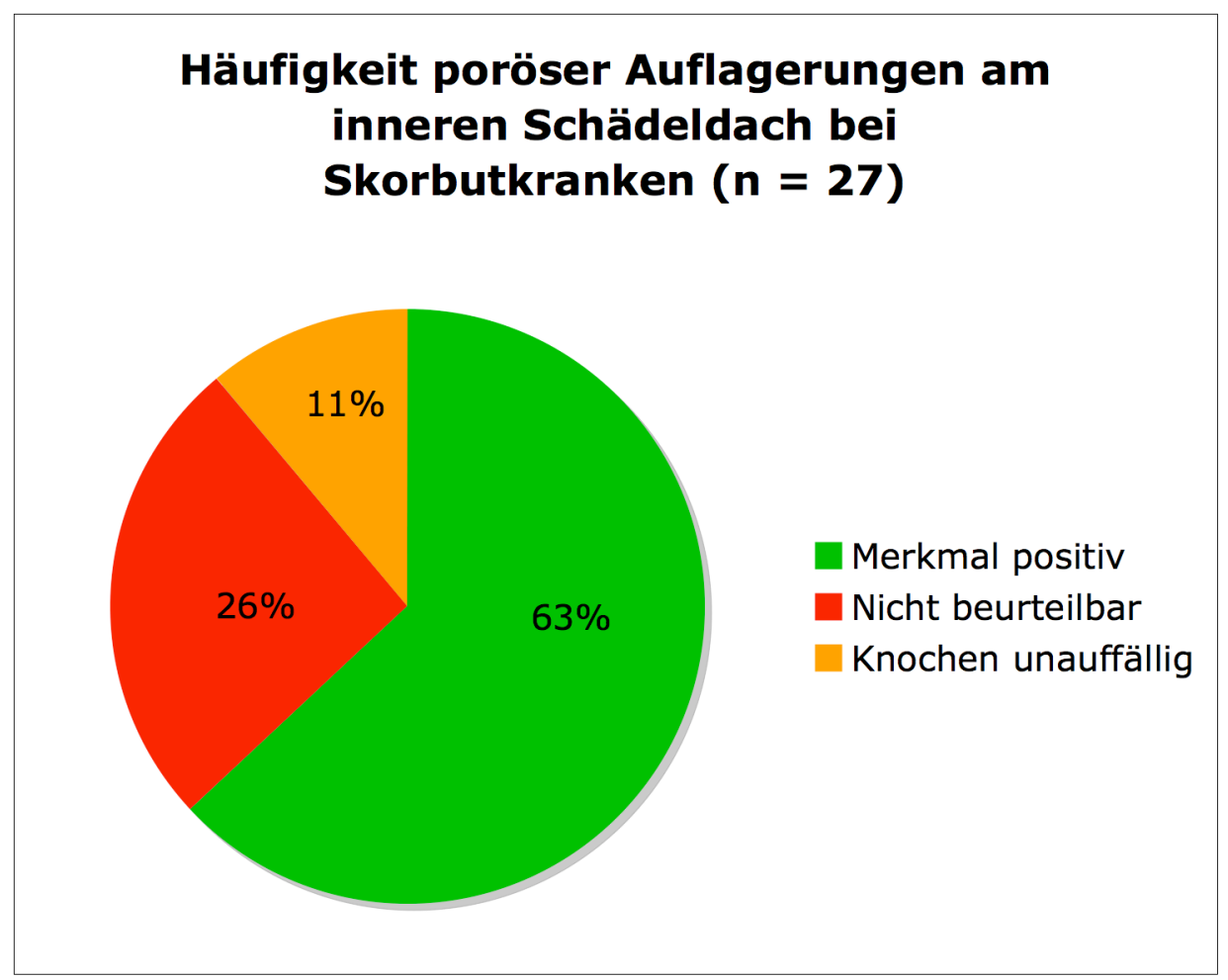

Abb. 68: Häufigkeit poröser Auflagerungen am inneren Schädeldach bei den untersuchten Skorbutkranken (Prozentzahlen beziehen sich auf Gesamtzahl der Skorbutfälle)

Unter den skorbutkranken Kindern (23) sind 16 (70\% der skorbutkranken Kinder) mit porösen Auflagerungen am inneren Schädeldach, vier (17\% der skorbutkranken Kinder) ohne befundbare und drei $(13 \%$ der skorbutkranken Kinder $)$ mit unauffälligen inneren Schädeldächern.

\begin{tabular}{|l|l|l|l|}
\hline & Merkmal positiv & Nicht beurteilbar & Knochen unauffällig \\
\hline Skorbutkranke Kinder $(\mathbf{n}=\mathbf{2 3})$ & $16(70 \%)$ & $4(17 \%)$ & $3(13 \%)$ \\
\hline
\end{tabular}

Tab. 102: Häufigkeit poröser Auflagerungen am inneren Schädeldach bei den untersuchten skorbutkranken Kindern (Prozentzahlen beziehen sich auf Gesamtzahl der infantilen Skorbutfälle)

Von den vier an Skorbut erkrankten Erwachsenen weist ein Individuum $(25 \%$ der skorbutkranken Erwachsenen) poröse Auflagerungen am inneren Schädeldach auf, drei Individuen (75\% der skorbutkranken Erwachsenen) haben kein befundbares Schädeldach.

\begin{tabular}{|l|l|l|l|}
\hline & Merkmal positiv & Nicht beurteilbar & Knochen unauffällig \\
\hline Skorbutkranke Erwachsene $(\mathbf{n}=4)$ & $1(25 \%)$ & $3(75 \%)$ & 0 \\
\hline
\end{tabular}

Tab. 103: Häufigkeit poröser Auflagerungen am inneren Schädeldach bei skorbutkranken Erwachsenen (Prozentzahlen beziehen sich auf Gesamtzahl der adulten Skorbutfälle). 


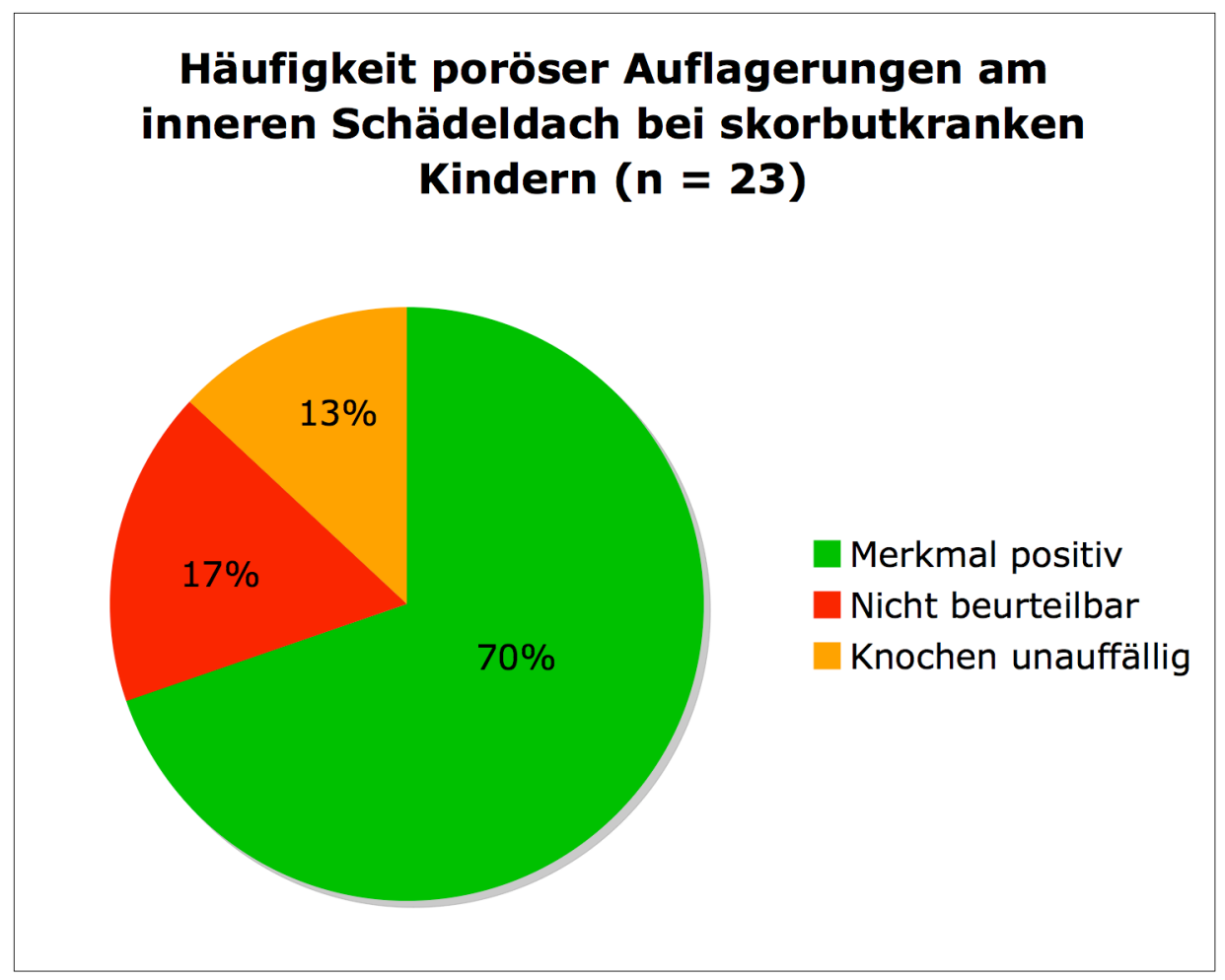

Abb. 69: Häufigkeit poröser Auflagerungen am inneren Schädeldach bei den untersuchten skorbutkranken Kindern (Prozentzahlen beziehen sich auf Gesamtzahl der infantilen Skorbutfälle)

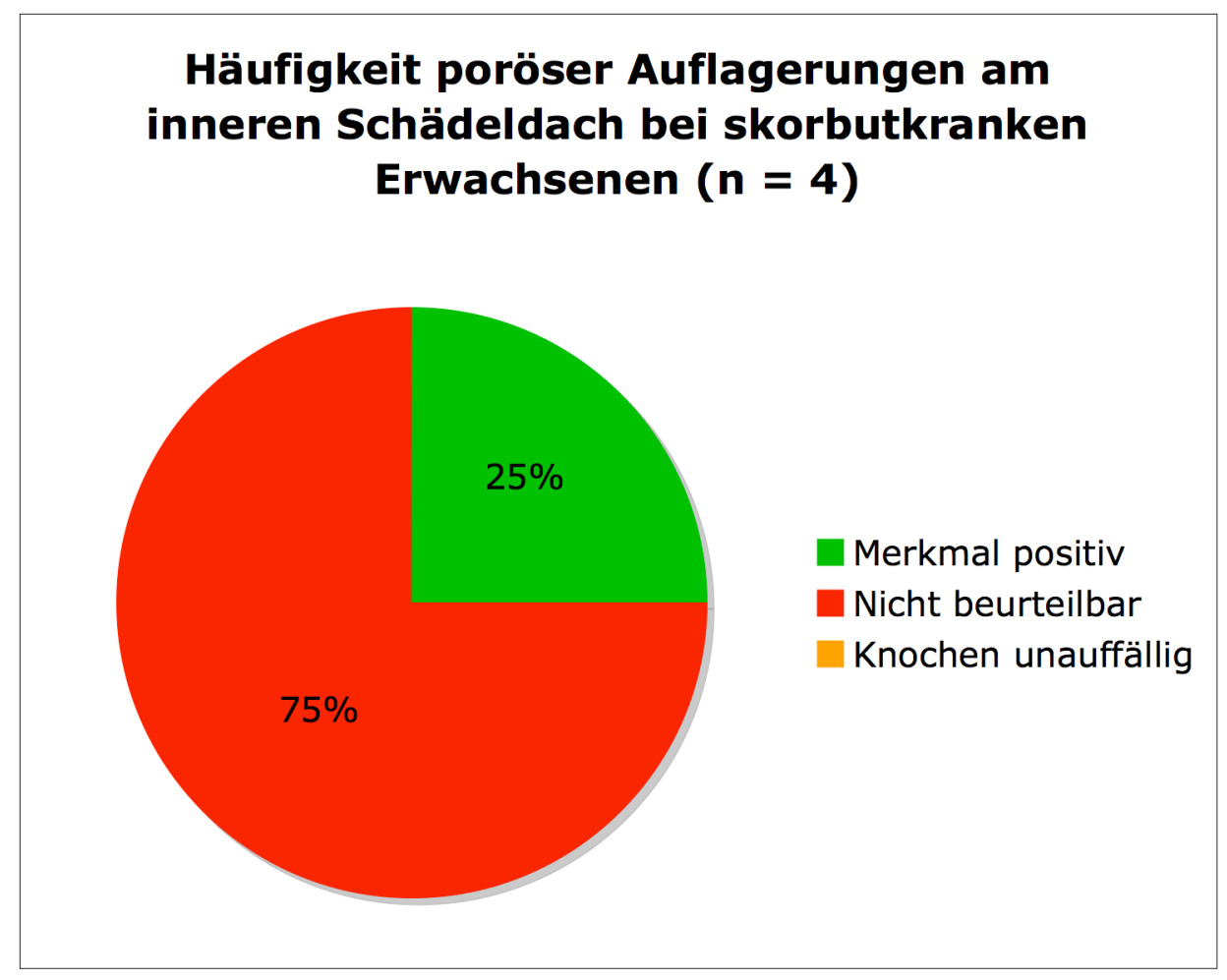

Abb. 70: Häufigkeit poröser Auflagerungen am inneren Schädeldach bei skorbutkranken Erwachsenen (Prozentzahlen beziehen sich auf Gesamtzahl der adulten Skorbutfälle) 


\subsection{Poröse Oberflächen an Langknochen (Nebenmerkmal 4)}

Bei 19 (53\%) von 36 untersuchten Individuen liegen poröse Oberflächen an Langknochen vor, $15(42 \%)$ von ihnen sind sicher, ein Individuum $(3 \%)$ wahrscheinlich an Skorbut erkrankt.

Vier (11\%) der Individuen haben keine ausreichend befundbaren Langknochen, darunter drei (8\%) Skorbutkranke.

Dreizehn (36\%) der Individuen haben keine porösen Oberflächen an den Langknochen, darunter auch neun (25\%) mit Skorbut. Dabei gilt allerdings zu beachten, dass jene Individuen, die poröse Auflagerungen an den Langknochen aufweisen, auch unter die Gruppe „unauffällig“ fallen, da sie das Kriterium „poröse Oberfläche“ im engeren Sinne nicht erfüllen.

\begin{tabular}{|l|l|l|l|}
\hline & Merkmal positiv & Nicht beurteilbar & Nicht porös \\
\hline Skorbut & $15(42 \%)$ & $3(8 \%)$ & $9(25 \%)$ \\
\hline V. a. Skorbut & $1(3 \%)$ & 0 & $3(8 \%)$ \\
\hline Sonstiges & $3(8 \%)$ & $1(3 \%)$ & $1(3 \%)$ \\
\hline
\end{tabular}

Tab. 104: Häufigkeit poröser Oberflächen an den Langknochen bei Individuen mit Skorbut, Verdacht auf Skorbut und Individuen mit anderen Diagnosen

Unter „,nicht porös“ fallen auch Individuen mit Auflagerungen an den Langknochen (vgl. Kardinalmerkmal 5). Individuum Sk 1380, das vermutlich sowohl an Lepra als auch an Skorbut erkrankt ist, wird hier in der Gruppe der Skorbutkranken geführt. V. a. = Verdacht auf

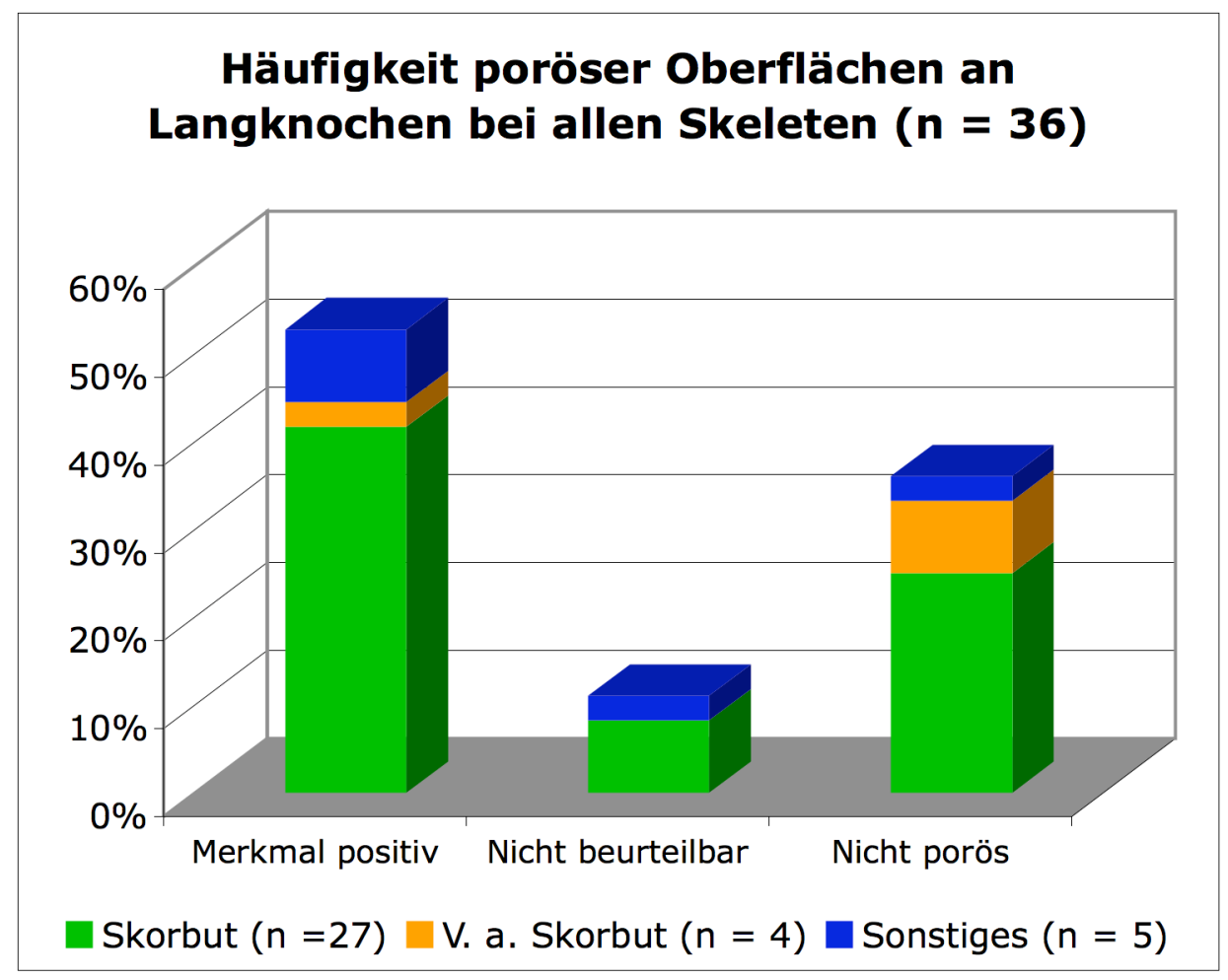

Abb. 71: Häufigkeit poröser Oberflächen an den Langknochen bei Individuen mit Skorbut, Verdacht auf Skorbut und Individuen mit anderen Diagnosen

Unter „nicht porös“ fallen auch Individuen mit Auflagerungen an den Langknochen (vgl. Kardinalmerkmal 5).

Individuum Sk 1380, das vermutlich sowohl an Lepra als auch an Skorbut erkrankt ist, wird hier in der Gruppe der Skorbutkranken geführt. V. a. = Verdacht auf 
Betrachtet man nur die 25 untersuchten Kinder, so zeigen 13 (52\% aller Kinder) poröse Oberflächen an den Langknochen, davon zwölf (48\% aller Kinder) mit Skorbut und ein Kind (4\% aller Kinder) mit Verdacht auf Skorbut.

Vier Kinder (16\%) haben keine ausreichend befundbaren Langknochen, darunter auch drei (12\%) mit Skorbut.

Bei acht Kindern (32\% aller Kinder), die alle an Skorbut erkrankt sind, liegt keine poröse Oberfläche der Langknochen vor.

\begin{tabular}{|l|l|l|l|}
\hline & Merkmal positiv & Nicht beurteilbar & Nicht porös \\
\hline Skorbut & $12(48 \%)$ & $3(12 \%)$ & $8(32 \%)$ \\
\hline V. a. Skorbut & $1(4 \%)$ & 0 & 0 \\
\hline Sonstiges & 0 & $1(4 \%)$ & 0 \\
\hline
\end{tabular}

Tab. 105: Häufigkeit poröser Oberflächen an Langknochen bei Kindern mit Skorbut, Verdacht auf Skorbut und Kindern mit anderen Diagnosen (Prozentzahlen beziehen sich auf Gesamtzahl aller Kinder)

Unter „nicht porös“ fallen auch Individuen mit Auflagerungen an den Langknochen (vgl. Kardinalmerkmal 5). V. a. = Verdacht auf

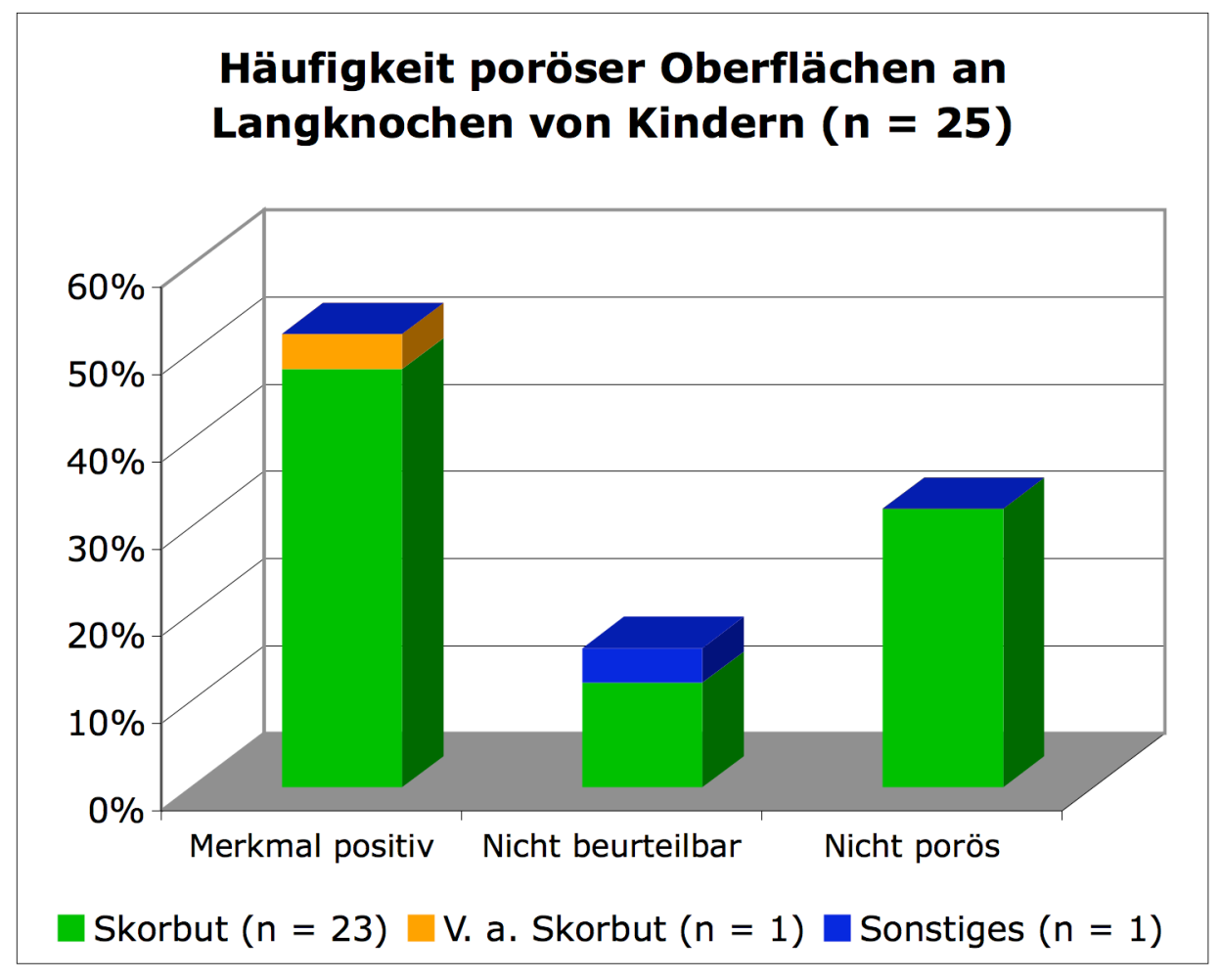

Abb. 72: Häufigkeit poröser Oberflächen an Langknochen bei Kindern mit Skorbut, Verdacht auf Skorbut und Kindern mit anderen Diagnosen

Unter „,nicht porös“ fallen auch Individuen mit Auflagerungen an den Langknochen (vgl. Kardinalmerkmal 5). Individuum Sk 1380, das vermutlich sowohl an Lepra als auch an Skorbut erkrankt ist, wird hier in der Gruppe der Skorbutkranken geführt.

V. a. $=$ Verdacht auf

Sechs (55\% aller Erwachsenen) der elf untersuchten adulten Individuen weisen poröse Oberflächen an den Langknochen auf, davon sind drei (27\% aller Erwachsenen) an Skorbut erkrankt. 
Bei fünf adulten Individuen (46\% aller Erwachsenen) sind die Langknochen nicht porös, davon sind eins (9\%) sicher und drei (27\%) vermutlich an Skorbut erkrankt.

\begin{tabular}{|l|l|l|l|}
\hline & Merkmal positiv & Nicht beurteilbar & Nicht porös \\
\hline Skorbut & $3(27 \%)$ & 0 & $1(9, \%)$ \\
\hline V. a. Skorbut & 0 & 0 & $3(27 \%)$ \\
\hline Sonstiges & $3(27 \%)$ & 0 & $1(9 \%)$ \\
\hline
\end{tabular}

Tab. 106: Häufigkeit poröser Oberflächen an den Langknochen von Erwachsenen mit Skorbut, Verdacht auf Skorbut und Individuen mit anderen Diagnosen

Unter „,nicht porös“ fallen auch Individuen mit Auflagerungen an den Langknochen (vgl. Kardinalmerkmal 5). Individuum Sk 1380, das vermutlich sowohl an Lepra als auch an Skorbut erkrankt ist, wird hier in der Gruppe der Skorbutkranken geführt. Die Abweichung von 100\% ergibt sich durch Auf- bzw. Abrundung.

V. a. = Verdacht auf

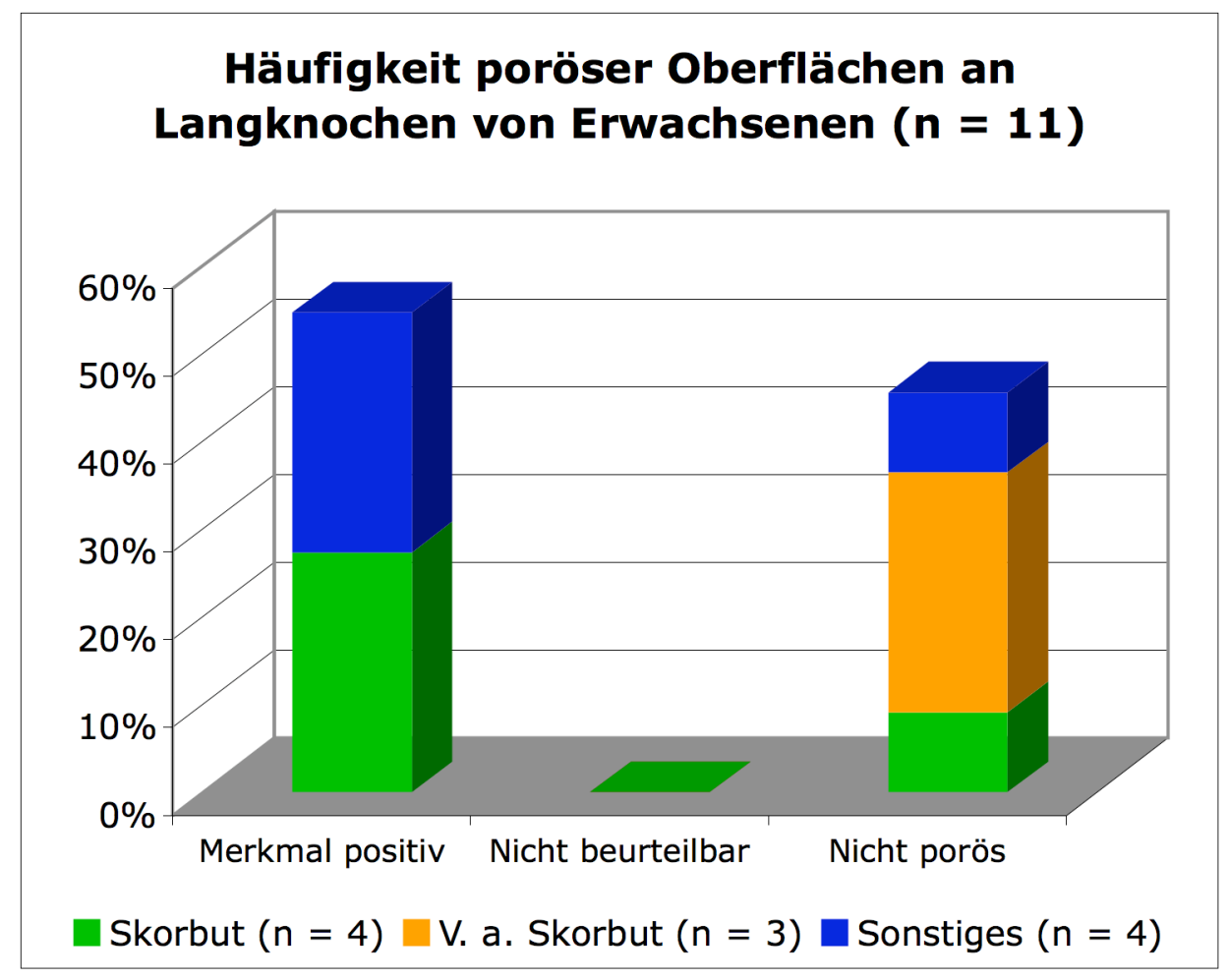

Abb. 73: Häufigkeit poröser Oberflächen an den Langknochen von Erwachsenen mit Skorbut, Verdacht auf Skorbut und Individuen mit anderen Diagnosen

Unter „,nicht porös“ fallen auch Individuen mit Auflagerungen an den Langknochen (vgl. Kardinalmerkmal 5).

Individuum Sk 1380, das vermutlich sowohl an Lepra als auch an Skorbut erkrankt ist, wird hier in der Gruppe der Skorbutkranken geführt.

V. a. $=$ Verdacht auf

Bei den insgesamt 27 skorbutkranken Individuen finden sich in 15 Fällen $(56 \%$ aller Skorbutkranken) feinporöse Oberflächen an den Langknochen, bei drei Individuen (11\% aller Skorbutkranken) sind die Langknochen nicht ausreichend beurteilbar, neun Individuen (33\% aller Skorbutkranken) haben keine porösen Langknochen. 


\begin{tabular}{|l|l|l|l|}
\hline & Merkmal positiv & Nicht beurteilbar & Nicht porös \\
\hline Skorbutkranke (n=27) & $15(56 \%)$ & $3(11 \%)$ & $9(33 \%)$ \\
\hline
\end{tabular}

Tab. 107: Häufigkeit poröser Oberflächen an den Langknochen bei den untersuchten Skorbutkranken (Prozentzahlen beziehen sich auf Gesamtzahl der Skorbutfälle)

Unter „nicht porös“ fallen auch Individuen mit Auflagerungen an den Langknochen (vgl. Kardinalmerkmal 5).

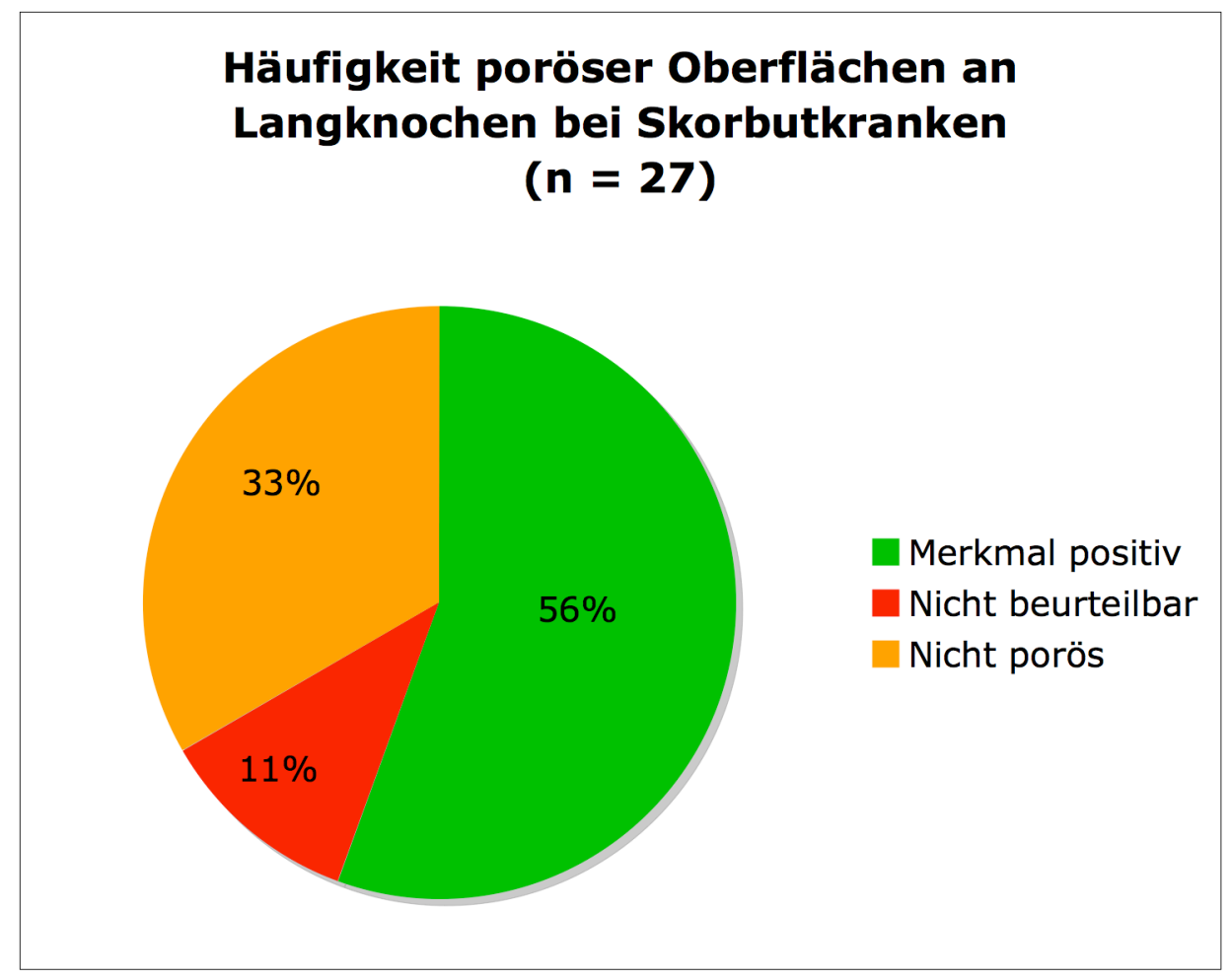

Abb. 74: Häufigkeit poröser Oberflächen an den Langknochen bei den untersuchten Skorbutkranken (Prozentzahlen beziehen sich auf Gesamtzahl der Skorbutfälle)

Unter „nicht porös“ fallen auch Individuen mit Auflagerungen an den Langknochen (vgl. Kardinalmerkmal 5).

Von den 23 an Skorbut erkrankten Kindern haben zwölf (52\% der skorbutkranken Kinder) poröse Oberflächen an den Langknochen, bei drei Kindern (13\% der skorbutkranken Kinder) sind die Langknochen nicht ausreichend beurteilbar, bei acht Kindern (35\%) liegt keine Porosität vor.

\begin{tabular}{|l|l|l|l|}
\hline & Merkmal positiv & Nicht beurteilbar & Nicht porös \\
\hline Skorbutkranke Kinder $(\mathbf{n}=\mathbf{2 3})$ & $12(52 \%)$ & $3(13 \%)$ & $8(35 \%)$ \\
\hline
\end{tabular}

Tab. 108: Häufigkeit poröser Oberflächen an den Langknochen von den untersuchten skorbutkranken Kindern (Prozentzahlen beziehen sich auf Gesamtzahl der skorbutkranken Kinder)

Unter „nicht porös“ fallen auch Individuen mit Auflagerungen an den Langknochen (vgl. Kardinalmerkmal 5).

Von 4 untersuchten adulten Skorbutfällen haben 3 (75\% der adulten Skorbutkranken) poröse Langknochenoberflächen.

\begin{tabular}{|l|l|l|l|}
\hline & Merkmal positiv & Nicht beurteilbar & Nicht porös \\
\hline Skorbutkranke Erwachsene $(\mathrm{n}=4)$ & $3(75 \%)$ & 0 & $1(25 \%)$ \\
\hline
\end{tabular}

Tab. 109: Häufigkeit poröser Oberflächen an den Langknochen bei den untersuchten skorbutkranken Erwachsenen (Prozentzahlen beziehen sich auf Gesamtzahl der skorbutkranken Erwachsenen)

Unter „nicht porös“ fallen auch Individuen mit Auflagerungen an den Langknochen (vgl. Kardinalmerkmal 5). 


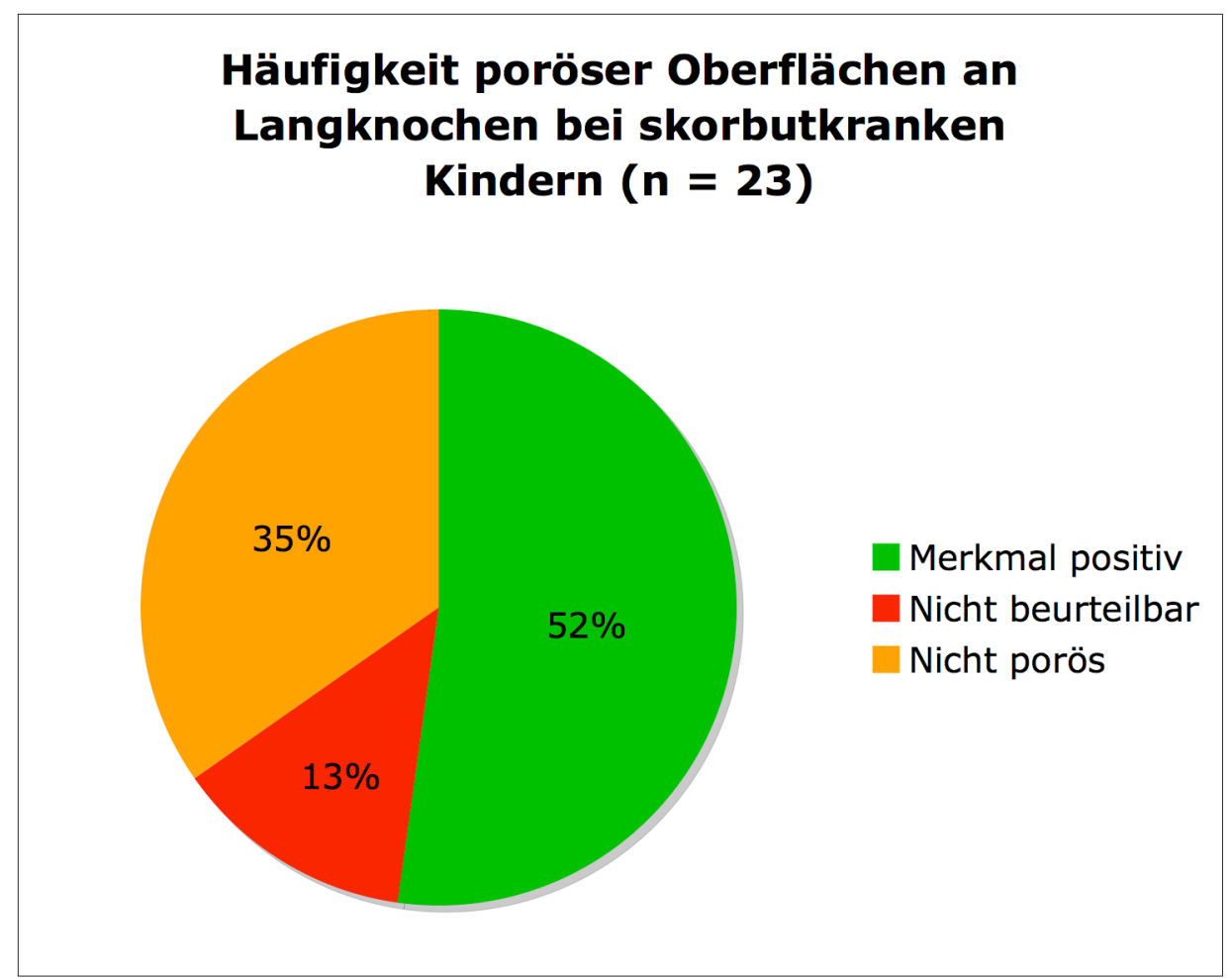

Abb. 75: Häufigkeit poröser Oberflächen an den Langknochen von den untersuchten skorbutkranken Kindern (Prozentzahlen beziehen sich auf Gesamtzahl der skorbutkranken Kinder)

Unter ,nicht porös“ fallen auch Individuen mit Auflagerungen an den Langknochen (vgl. Kardinalmerkmal 5).

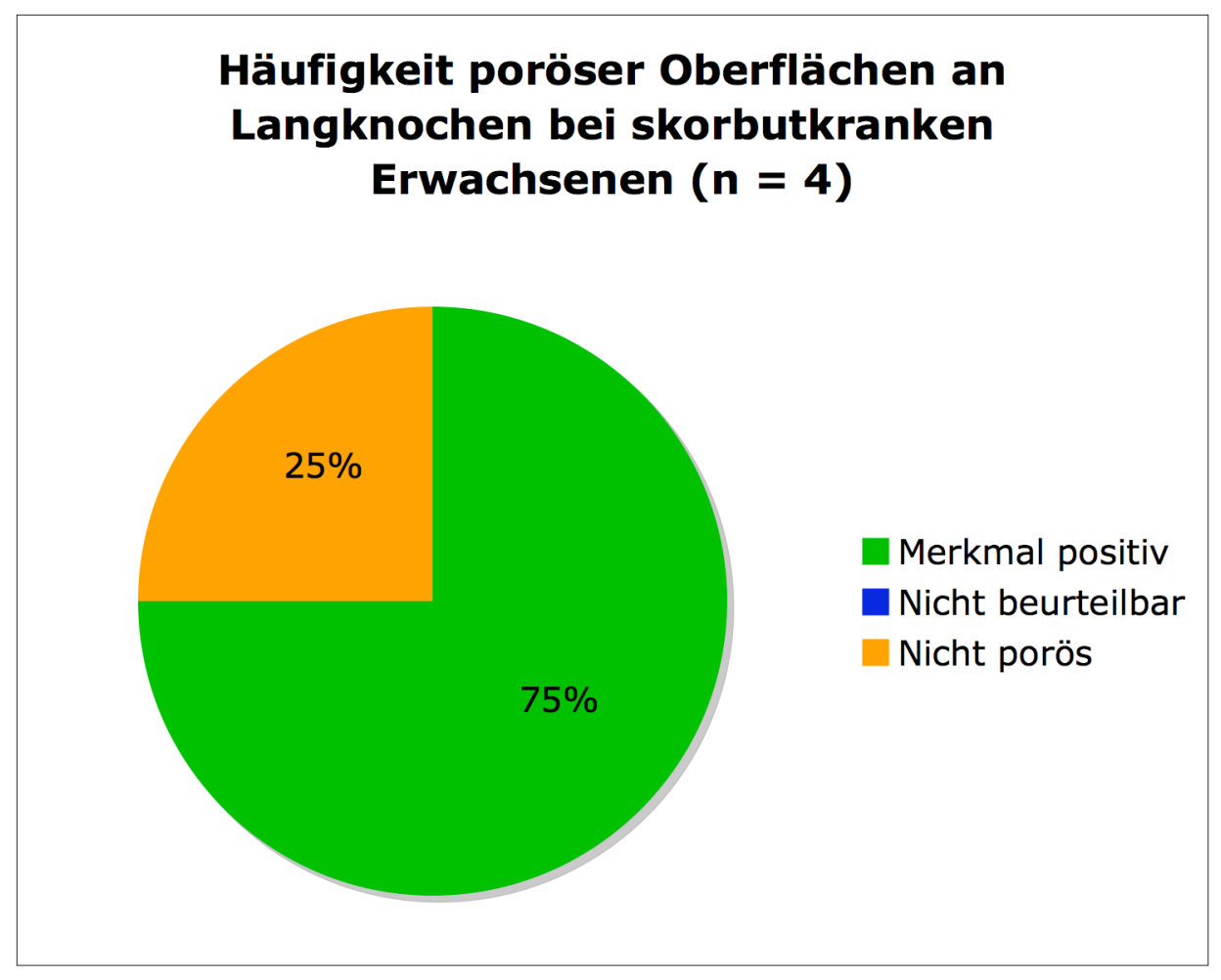

Abb. 76: Häufigkeit poröser Oberflächen an den Langknochen bei den untersuchten skorbutkranken Erwachsenen (Prozentzahlen beziehen sich auf Gesamtzahl der skorbutkranken Erwachsenen).

Unter ,nicht porös“ fallen auch Individuen mit Auflagerungen an den Langknochen (vgl. Kardinalmerkmal 5). 


\subsection{Poröse Außenfläche der Ala major ossis sphenoidalis / der Squama temporalis}

\section{(Nebenmerkmal 5)}

Wenn im Folgenden von der Außenfläche der Ala major ossis sphenoidalis die Rede ist, so bezieht sich dies stets auch auf die angrenzende Squama temporalis, soweit diese erhalten ist. Bei elf (31\%) der 36 untersuchten Individuen liegen poröse Außenflächen der Ala major ossis sphenoidalis vor, davon sind zehn (28\%) an Skorbut erkrankt.

In 20 Fällen (56\%) sind die Großen Keilbeinflügel nicht befundbar, unter anderem bei 16 Skorbutfällen (44\%) und zwei Individuen (6\%) mit Verdacht auf Skorbut.

Fünf Individuen (14\%) haben unauffällige Keilbeine, darunter ein (3\%) Skorbutfall und zwei Individuen (6\%) mit Verdacht auf Skorbut.

\begin{tabular}{|l|l|l|l|}
\hline & Merkmal positiv & Nicht beurteilbar & Knochen unauffällig \\
\hline Skorbut & $10(28 \%)$ & $16(44 \%)$ & $1(3 \%)$ \\
\hline V. a. Skorbut & 0 & $2(6 \%)$ & $2(6 \%)$ \\
\hline Sonstiges & $1(3 \%)$ & $2(6 \%)$ & $2(6 \%)$ \\
\hline
\end{tabular}

Tab. 110: Häufigkeit poröser Außenflächen der Ala major ossis sphenoidalis/der Squama temporalis bei Individuen mit Skorbut, Verdacht auf Skorbut und Individuen mit anderen Diagnosen

Individuum Sk 1380, das vermutlich sowohl an Lepra als auch an Skorbut erkrankt ist, wird hier in der Gruppe der Skorbutkranken geführt. Die Abweichung von 100\% ergibt sich durch Auf- bzw. Abrundung.

V. a. $=$ Verdacht auf

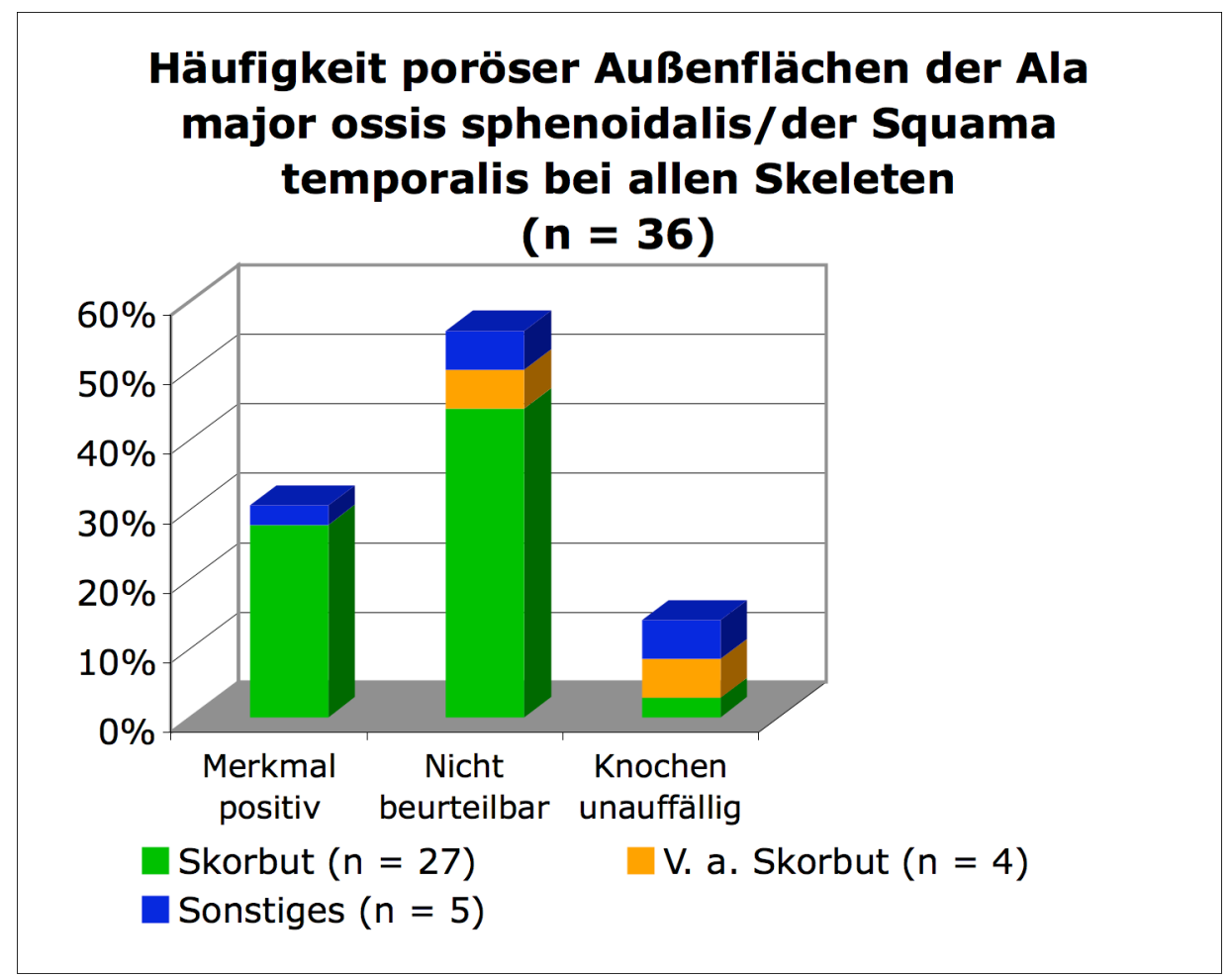

Abb. 77: Häufigkeit poröser Außenflächen der Ala major ossis sphenoidalis/der Squama temporalis bei Individuen mit Skorbut, Verdacht auf Skorbut und Individuen mit anderen Diagnosen Individuum Sk 1380, das vermutlich sowohl an Lepra als auch an Skorbut erkrankt ist, wird hier in der Gruppe der Skorbutkranken geführt.

V. a. $=$ Verdacht auf 
Unter den 25 untersuchten Kindern sind elf (44\% aller Kinder) mit porösen Außenflächen des Keilbeinflügels, von denen zehn (40\% aller Kinder) an Skorbut erkrankt sind.

Bei 13 Kindern (52\% aller Kinder) ist keine Befundung der Alae majores möglich, davon haben zwölf (48\% aller Kinder) sicher und ein Kind (4\% aller Kinder) wahrscheinlich Skorbut.

Ein Kind (4\% aller Kinder) hat unauffällige Keilbeinflügel und Skorbut.

\begin{tabular}{|l|l|l|l|}
\hline & Merkmal positiv & Nicht beurteilbar & Knochen unauffällig \\
\hline Skorbut & $10(40 \%)$ & $12(48 \%)$ & $1(4 \%)$ \\
\hline V. a. Skorbut & 0 & $1(4 \%)$ & 0 \\
\hline Sonstiges & $1(4 \%)$ & 0 & 0 \\
\hline
\end{tabular}

Tab. 111: Häufigkeit poröser Außenflächen der Ala major ossis sphenoidalis/der Squama temporalis bei Kindern mit Skorbut, Verdacht auf Skorbut und Individuen mit anderen Diagnosen (Prozentzahlen beziehen sich auf Gesamtzahl aller Kinder)

V. a. = Verdacht auf

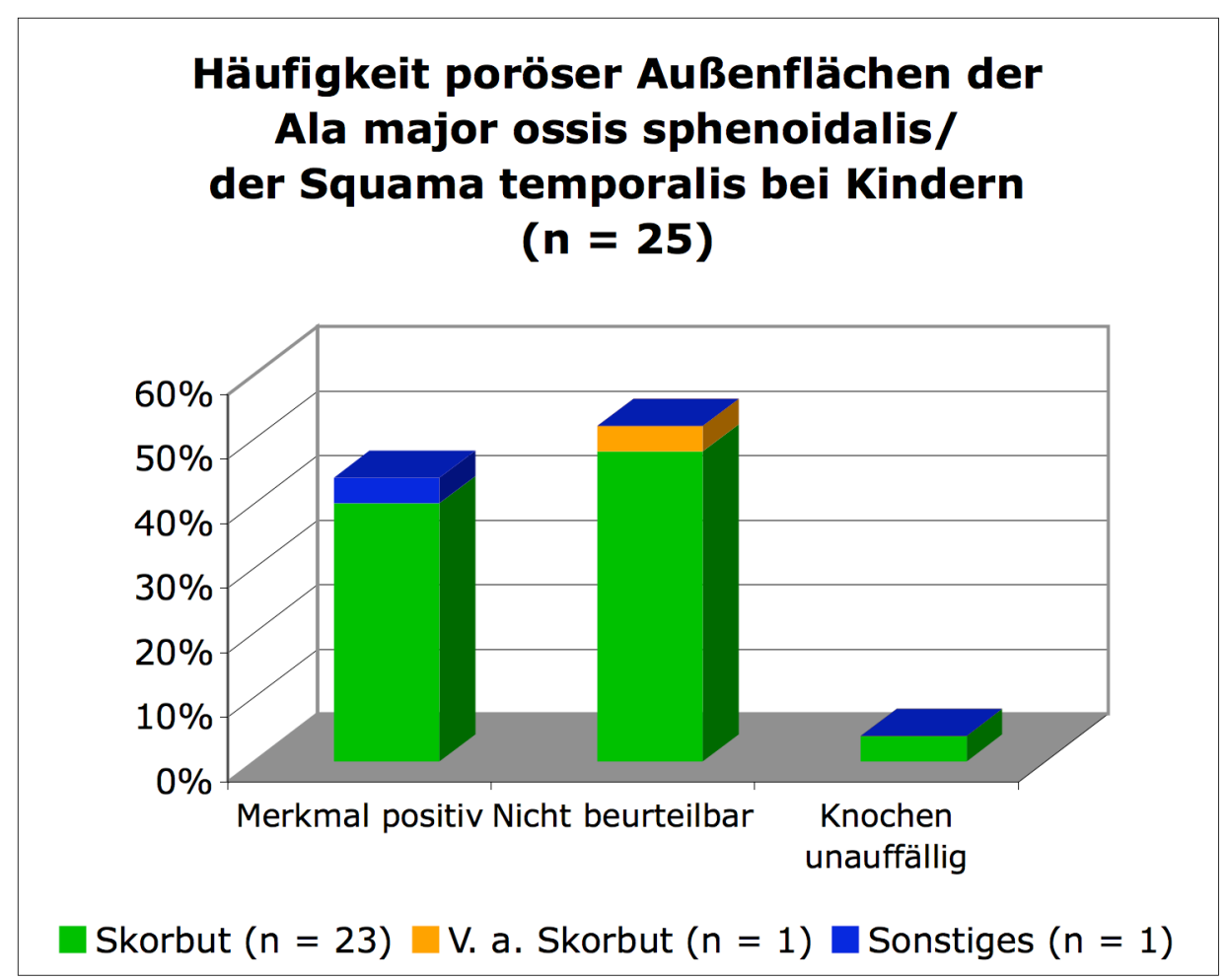

Abb. 78: Häufigkeit poröser Außenflächen der Ala major ossis sphenoidalis/der Squama temporalis bei Kindern mit Skorbut, V. a. Skorbut und Individuen mit anderen Diagnosen (Prozentzahlen beziehen sich auf Gesamtzahl aller Kinder)

V. a. $=$ Verdacht auf

Von elf untersuchten Erwachsenen hat kein Individuum eine poröse Ala major ossis sphenoidalis.

In sieben Fällen (64\% aller Erwachsenen) sind die Keilbeine nicht beurteilbar, darunter vier Individuen (36\% aller Erwachsenen) mit Skorbut und ein Individuum (9\% aller Erwachsenen) mit Verdacht auf Skorbut. 
Vier Erwachsene (36\% aller Erwachsenen) zeigen unauffällige Keilbeinflügel, darunter zwei Individuen (18\% aller Erwachsenen) mit Verdacht auf Skorbut.

\begin{tabular}{|l|l|l|l|}
\hline & Merkmal positiv & Nicht beurteilbar & Knochen unauffällig \\
\hline Skorbut & 0 & $4(36 \%)$ & 0 \\
\hline V. a. Skorbut & 0 & $1(9 \%)$ & $2(18 \%)$ \\
\hline Sonstiges & 0 & $2(18 \%)$ & $2(18 \%)$ \\
\hline
\end{tabular}

Tab. 112: Häufigkeit poröser Außenflächen der Ala major ossis sphenoidalis/der Squama temporalis bei Erwachsenen mit Skorbut, Verdacht auf Skorbut und Individuen mit anderen Diagnosen. Prozentzahlen beziehen sich auf Gesamtzahl aller Erwachsenen, die Abweichung von 100\% ergibt sich durch Auf- bzw. Abrundung. Individuum Sk 1380, das vermutlich sowohl an Lepra als auch an Skorbut erkrankt ist, wird hier in der Gruppe der Skorbutkranken geführt.

V. a. = Verdacht auf

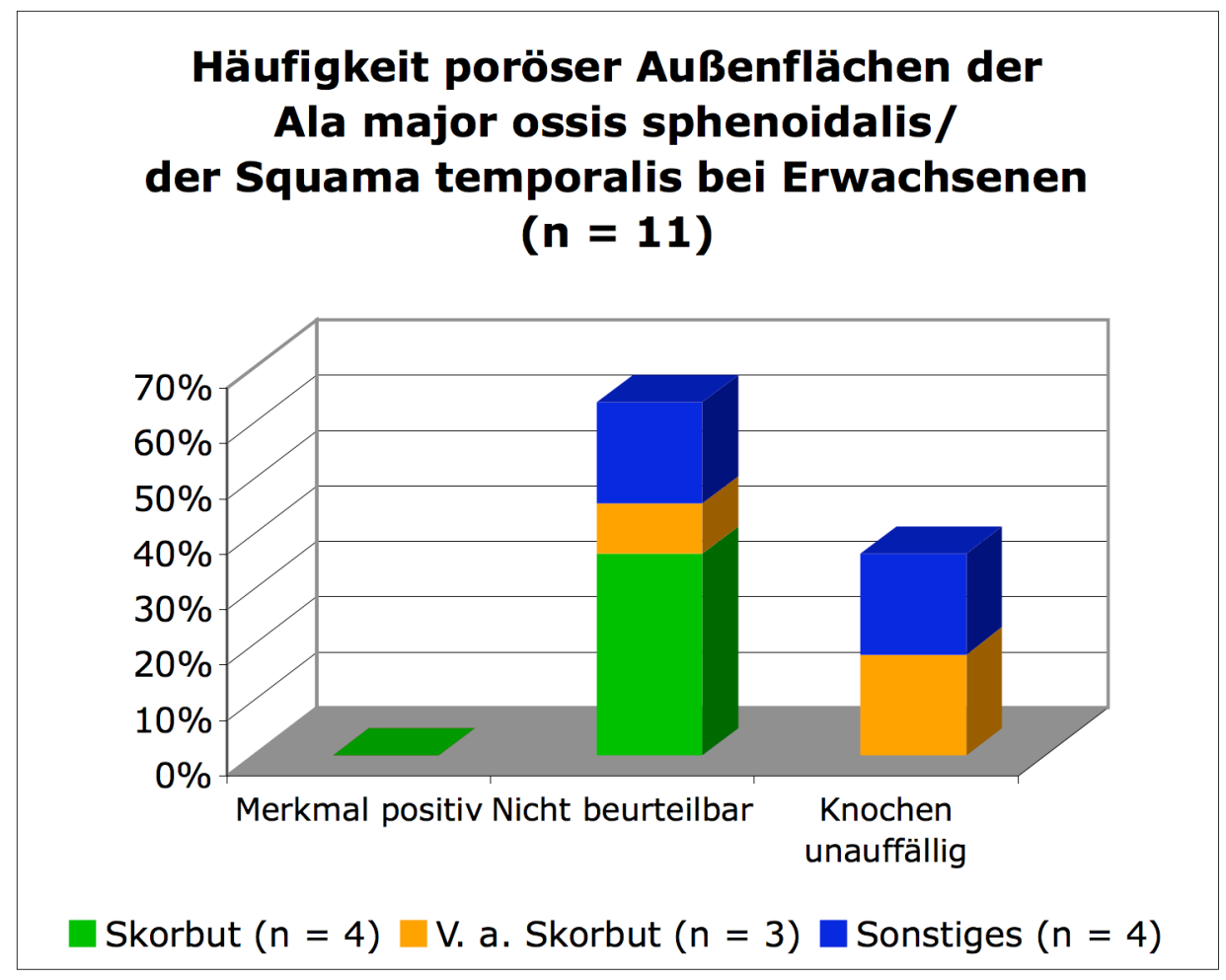

Abb. 79: Häufigkeit poröser Außenflächen der Ala major ossis sphenoidalis/der Squama temporalis bei Erwachsenen mit Skorbut, Verdacht auf Skorbut und Individuen mit anderen Diagnosen (Prozentzahlen beziehen sich auf Gesamtzahl aller Erwachsenen)

Individuum Sk 1380, das vermutlich sowohl an Lepra als auch an Skorbut erkrankt ist, wird hier in der Gruppe der Skorbutkranken geführt.

V. a. = Verdacht auf

Unter den 27 untersuchten Skorbutfällen sind zehn (37\% aller Skorbutfälle) mit poröser Außenfläche der Ala major ossis sphenoidalis, bei 16 (59\% aller Skorbutfälle) ist keine Beurteilung möglich, ein Individuum (4\% aller Skorbutfälle) hat unauffällige Keilbeinflügel.

\begin{tabular}{|l|l|l|l|}
\hline & Merkmal positiv & Nicht beurteilbar & Knochen unauffällig \\
\hline Skorbutkranke $(\mathbf{n}=\mathbf{2 7})$ & $10(37 \%)$ & $16(59 \%)$ & $1(4 \%)$ \\
\hline
\end{tabular}

Tab. 113: Häufigkeit poröser Außenflächen der Ala major ossis sphenoidalis/der Squama temporalis bei den untersuchten Skorbutkranken (Prozentzahlen beziehen sich auf Gesamtzahl der Skorbutfälle) 


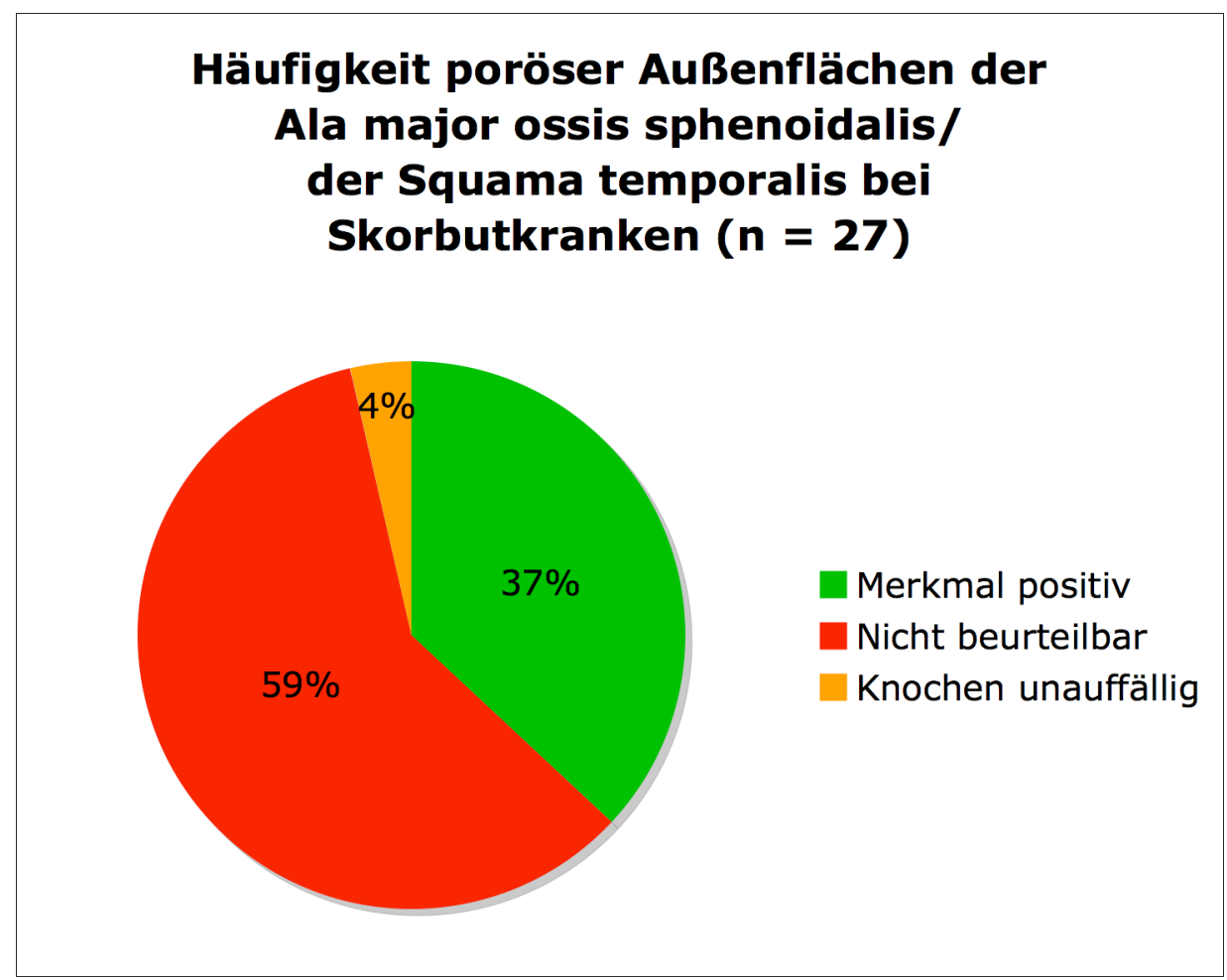

Abb. 80: Häufigkeit poröser Außenflächen der Ala major ossis sphenoidalis/der Squama temporalis bei den untersuchten Skorbutkranken (Prozentzahlen beziehen sich auf Gesamtzahl der Skorbutfälle)

Unter den 23 skorbutkranken Kindern sind zehn (44\% aller skorbutkranken Kinder) mit porösen Außenflächen der Großen Keilbeinflügel, zwölf (52\% aller skorbutkranken Kinder) mit nicht befundbaren und ein Kind (4\% aller skorbutkranken Kinder) mit unauffälligen Alae majores ossis sphenoidalis.

\begin{tabular}{|l|l|l|l|}
\hline & Merkmal positiv & Nicht beurteilbar & Knochen unauffällig \\
\hline Skorbutkranke Kinder $(\mathbf{n}=\mathbf{2 3})$ & $10(44 \%)$ & $12(52 \%)$ & $1(4 \%)$ \\
\hline
\end{tabular}

Tab. 114: Häufigkeit poröser Außenflächen der Ala major ossis sphenoidalis/der Squama temporalis bei den untersuchten skorbutkranken Kindern (Prozentzahlen beziehen sich auf Gesamtzahl der infantilen Skorbutfälle)

Bei keinem der vier adulten Skorbutfälle sind die Alae majores ossis sphenoidalis befundbar.

\begin{tabular}{|l|l|l|l|}
\hline & Merkmal positiv & Nicht beurteilbar & Knochen unauffällig \\
\hline Skorbutkranke Erwachsene $(n=4)$ & 0 & $4(100 \%)$ & 0 \\
\hline
\end{tabular}

Tab. 115: Häufigkeit poröser Außenflächen der Ala major ossis sphenoidalis/der Squama temporalis bei den untersuchten skorbutkranken Erwachsenen (Prozentzahlen beziehen sich auf Gesamtzahl der adulten Skorbutfälle) 


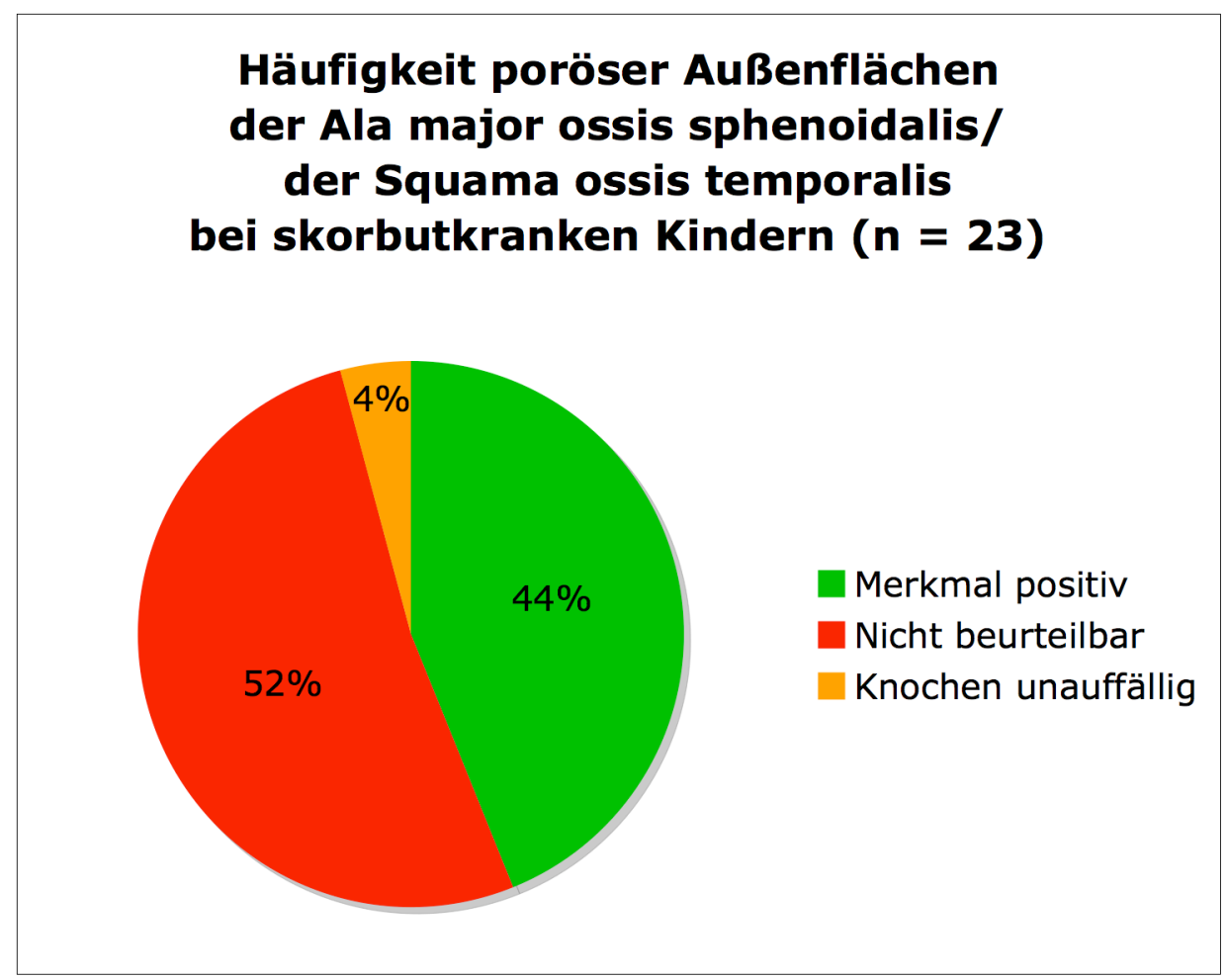

Abb. 81: Häufigkeit poröser Außenflächen der Ala major ossis sphenoidalis/der Squama temporalis bei den untersuchten skorbutkranken Kindern (Prozentzahlen beziehen sich auf Gesamtzahl der infantilen Skorbutfälle)

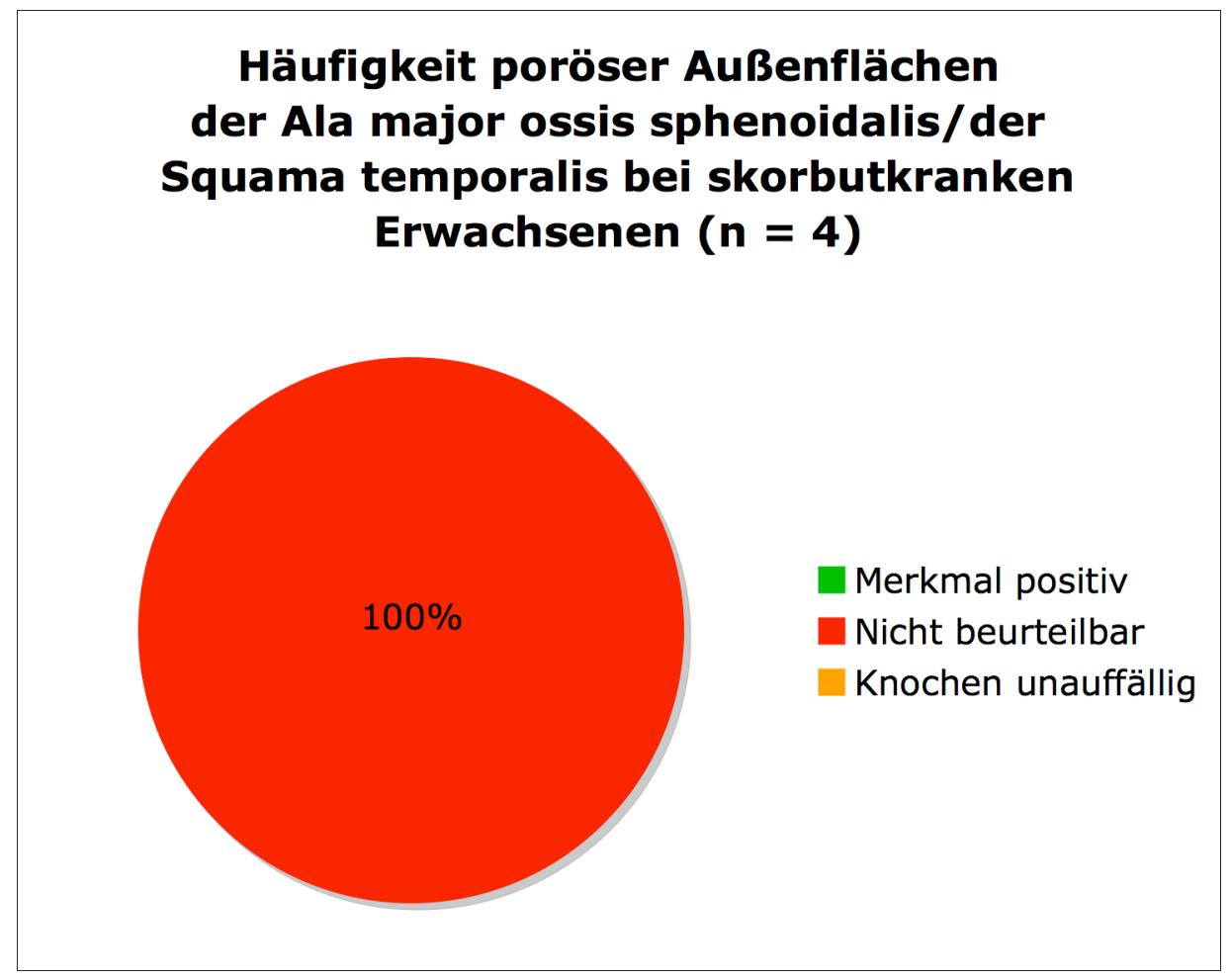

Abb. 82: Häufigkeit poröser Außenflächen der Ala major ossis sphenoidalis/der Squama temporalis bei den untersuchten skorbutkranken Erwachsenen (Prozentzahlen beziehen sich auf Gesamtzahl der adulten Skorbutfälle) 


\section{Zusammenfassung: Häufigkeit der unterschiedlichen für Skorbut kennzeichnenden}

\section{Merkmale bei den untersuchten Skeleten}

In der von SCHULTZ (1994) entwickelten Tabelle mit Merkmalen zur Diagnostik von Skorbut finden sich sieben Kardinal- und fünf Nebenmerkmale (siehe Kapitel 2.2 j) Diagnosestellung).

Auf diese wurden die 36 Individuen dieser Studie untersucht. Im Folgenden soll die Verteilung der Merkmale zusammengefasst werden. Dabei werden zwei Schwerpunkte gesetzt:

Zunächst wird überprüft, wie häufig die unterschiedlichen Merkmale bei den untersuchten Skeleten zu finden sind. Dabei wird unterschieden, in wie vielen Fällen das Individuum tatsächlich an Skorbut erkrankt ist und in wie vielen das Merkmal positiv ist, obwohl kein Skorbut vorliegt, und somit zur falsch positiven Diagnose Skorbut führen könnte.

Im nächsten Schritt wird untersucht, wie viele der skorbutkranken Individuen ein Merkmal aufweisen und bei wie vielen ein Merkmal nicht ausgeprägt ist. Damit lässt sich abschätzen, wie hoch der Ausprägungsgrad eines Merkmals ist.

Selbstverständlich wird dabei berücksichtig, ob ein Merkmal möglicherweise gar nicht nachweisbar sein kann, weil die entsprechende Struktur nicht ausreichend erhalten ist.

Da es bei Erwachsenen und Kindern unterschiedliche Erscheinungsformen von Skorbut gibt und außerdem die gefundene Zahl von adulten Skorbutfällen (4) sehr klein ist, werden Erwachsene und Kinder stets auch getrennt betrachtet.

\subsection{Zusammenfassung: Kardinalmerkmale für Skorbut}

Von den sieben Kardinalmerkmalen ist in der vorliegenden Studie Kardinalmerkmal 5 (poröse Auflagerungen auf den Langknochenschäften) am häufigsten zu finden. Insgesamt weisen 23 von 36 Individuen (64\%) Auflagerungen an den Langknochen auf. Davon sind 21 (59\%) an Skorbut erkrankt, nur zwei (6\%) Individuen haben andere Diagnosen. Damit ist die Spezifität dieses Merkmals hoch.

Unter den untersuchten Kindern weisen 17 von 25 (68\%) dieses Merkmal auf, die auch alle an Skorbut erkrankt sind, bei den adulten Individuen sind es vier von elf (36\%), und zwar alle adulten Skorbutfälle.

Betrachtet man nur die Skorbutfälle, so haben 21 von 27 (78\%) poröse Auflagerungen an den Langknochen, vier (15\%) haben keine Auflagerungen, können allerdings durch eine poröse Oberfläche der Langknochen (vgl. Nebenmerkmal 4) auffallen. 
Bei den infantilen Skorbutfällen zeigen 17 von 23 (74\%) das Kardinalmerkmal 5, unauffällig sind vier (17\%).

Die adulten Skorbutfälle (4) weisen alle Auflagerungen an den Langknochen auf.

Das nächsthäufige Kardinalmerkmal sind die porösen Auflagerungen am Alveolarknochen (Kardinalmerkmal 4) mit 17 von 36 (47\%) positiven Fällen. Unter diesen ist nur ein Individuum (3\%), das nicht an Skorbut erkrankt ist. Somit erscheint die Spezifität auch hier hoch.

Vierzehn von 25 (56\% aller bzw. 61\% der skorbutkranken Kinder) untersuchten Kindern haben poröse Auflagerungen an den Alveolarknochen; sie sind alle an Skorbut erkrankt.

Bei den adulten Individuen weisen drei von elf (27\%) das Merkmal auf, davon zwei (18\%) Skorbutkranke.

Von allen Skorbutkranken (27) haben 16 (60\%) Auflagerungen auf den Alveolarknochen, nur zwei (7\%) haben unauffällige Alveolarknochen. Damit scheint der Ausprägungsgrad dieses Merkmals hoch zu sein.

Bei den erkrankten Kindern weisen 14 von 23 (61\%) das Merkmal auf, eins (4\%) nicht.

Unter den vier adulten Skorbutfällen finden sich in zwei Fällen (50\%) Auflagerungen im Alveolarbereich, ein Individuum (25\%) zeigt keine Veränderungen.

Poröse Auflagerungen am äußeren Schädeldach (Kardinalmerkmal 1), am Orbitadach (Kardinalmerkmal 2) und am äußeren Gesichtsschädel (Kardinalmerkmal 3) finden sich gleich häufig. Alle drei Merkmale sind 14 mal (39\% aller Individuen) positiv, darunter je 13 (36\%) Skorbutfälle und nur ein Individuum (3\%) ohne Skorbut, was für eine hohe Spezifität spricht.

Alle drei Merkmale finden sich ausschließlich bei Kindern.

Bei den untersuchten Skorbutkranken haben 13 von 27 (48\%) poröse Auflagerungen am äußeren Schädeldach, bei neun (33\%) Individuen ist das Schädeldach unauffällig, die Sensitivität ist also vergleichsweise niedrig.

Bei den skorbutkranken Kindern ist Kardinalmerkmal 1 bei 13 von 23 (57\%) positiv, bei acht (35\%) negativ.

Bei keinem der vier adulten Skorbutfälle finden sich Auflagerungen am äußeren Schädeldach, in 50\% (zwei Individuen) ist das Schädeldach unauffällig.

Cribra orbitalia finden sich bei $13(48 \%)$ der 27 Skorbutfälle, bei sechs (22\%) ist dieses Merkmal negativ.

Von den skorbutkranken Kindern weisen 13 von 23 (56\%) Cribra orbitalia auf, vier (17\%) nicht. 
Bei keinem der vier adulten Skorbutfälle finden sich Cribra orbitalia, in 50\% (zwei Individuen) sind die Orbitadächer unauffällig.

Poröse Auflagerungen am Gesichtsschädel haben 13 (48\%) der 27 Skorbutfälle, bei fünf (19\%) fehlt dieses Merkmal.

Bei den 23 skorbutkranken Kindern ist die Verteilung Merkmal positiv versus Merkmal negativ 13 (48\%) zu drei (13\%), bei den übrigen Kindern war keine Befundung möglich.

Auflagerungen am Gesichtsschädel finden sich bei keinem adulten Skorbutfall, bei zwei von vier (50\%) ist der Gesichtsschädel nicht erhalten.

Ebenfalls gleich häufig finden sich poröse Auflagerungen an Scapula (Kardinalmerkmal 6) und Os ilii (Kardinalmerkmal 7). Beide sind bei 10 der 36 (28\%) Individuen zu finden, und zwar ausschließlich bei Skorbutkranken, was eine hohe Spezifität vermuten lässt.

Darunter sind bei Kardinalmerkmal 6 neun von 25 Kindern (36\%), bei Merkmal 7 acht Kinder (32\%).

Der Anteil von Skorbutkranken mit porösen Auflagerungen am Schulterblatt ist zehn von 27 (37\%), negativ ist dieses Merkmal bei sieben skorbutkranken Individuen (26\%).

Unter den 23 skorbutkranken Kindern sind neun (39\%) positiv für Kardinalmerkmal 6, fünf (22\%) negativ.

Von den vier adulten Skorbutfällen hat ein Individuum (25\%) Auflagerungen an der Scapula, bei zwei Individuen (50\%) sind die Schulterblätter unauffällig.

Poröse Auflagerungen am Os ilii haben zehn (37\%) der 27 Skorbutkranken, sechs (22\%) haben unauffällige Beckenknochen.

Unter den 23 skorbutkranken Kindern ist Kardinalmerkmal 7 bei acht (35\%) positiv, bei fünf (22\%) negativ.

Zwei (50\%) der adulten Skorbutkranken haben Auflagerungen am Os ilii, einer (25\%) hat unauffällige Beckenknochen. 


\begin{tabular}{|r|l|l|l|l|l|r|}
\hline & \multicolumn{2}{|l|}{ Alle Individuen (n= 36) } & \multicolumn{2}{l|}{ Kinder (n= 25) } & \multicolumn{2}{l|}{ Erwachsene (n= 11) } \\
\hline & Skorbut & kein Skorbut & Skorbut & kein Skorbut & Skorbut & kein Skorbut \\
\hline $\mathbf{1}$ & $13(36 \%)$ & $1(3 \%)$ & $13(52 \%)$ & $1(4 \%)$ & 0 & 0 \\
\hline $\mathbf{2}$ & $13(36 \%)$ & $1(3 \%)$ & $13(52 \%)$ & $1(4 \%)$ & 0 & 0 \\
\hline $\mathbf{3}$ & $13(36 \%)$ & $1(3 \%)$ & $13(52 \%)$ & $1(4 \%)$ & 0 & 0 \\
\hline $\mathbf{4}$ & $16(44 \%)$ & $1(3 \%)$ & $14(56 \%)$ & 0 & $2(18 \%)$ & $1(9 \%)$ \\
\hline $\mathbf{5}$ & $21(58 \%)$ & $2(6 \%)$ & $17(68 \%)$ & 0 & $4(36 \%)$ & $2(18 \%)$ \\
\hline $\mathbf{6}$ & $10(28 \%)$ & 0 & $9(36 \%)$ & 0 & $1(9 \%)$ & 0 \\
\hline $\mathbf{7}$ & $10(28 \%)$ & 0 & $8(32 \%)$ & 0 & $2(18 \%)$ & 0 \\
\hline
\end{tabular}

Tab. 116: Übersicht über die Häufigkeit aller Kardinalmerkmale und den jeweiligen Anteil von Skorbutkranken und nicht an Skorbut erkrankten Individuen. Die Prozentzahlen der ersten beiden Spalten beziehen sich auf die Gesamtzahl aller Individuen, die übrigen auf die Gesamtzahl aller Kinder bzw. aller Erwachsenen.

Individuum Sk 1380, das vermutlich sowohl an Lepra als auch an Skorbut erkrankt ist, wird hier in der Gruppe der Skorbutkranken geführt.

Legende der Kardinalmerkmale: 1 = poröse Auflagerungen am äußeren Schädeldach, 2 = Cribra orbitalia, 3 = poröse Auflagerungen am äußeren Gesichtsschädel, 4 = poröse Auflagerungen am Alveolarknochen, 5 = poröse Auflagerungen auf Langknochenschäften, 6 = poröse Auflagerungen am Schulterblatt,

$7=$ poröse Auflagerungen am Darmbein

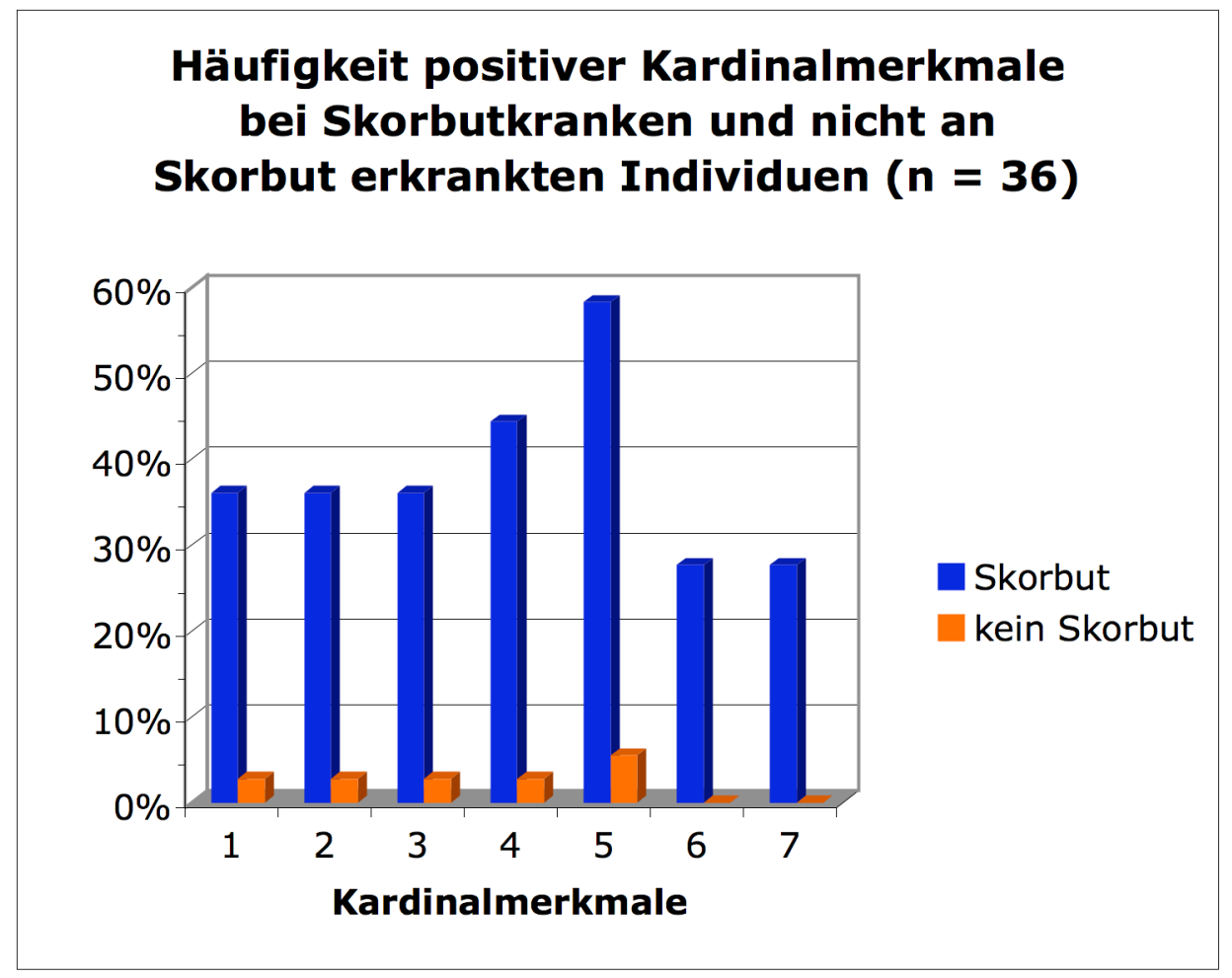

Abb. 83: Übersicht über die Häufigkeit aller Kardinalmerkmale und den jeweiligen Anteil von Skorbutkranken und nicht an Skorbut erkrankten Individuen (bei allen Erwachsenen und Kindern). Individuum Sk 1380, das vermutlich sowohl an Lepra als auch an Skorbut erkrankt ist, wird hier in der Gruppe der Skorbutkranken geführt.

Legende der Kardinalmerkmale: 1 = poröse Auflagerungen am äußeren Schädeldach, 2 = Cribra orbitalia, 3 = poröse Auflagerungen am äußeren Gesichtsschädel, 4 = poröse Auflagerungen am Alveolarknochen, $5=$ poröse Auflagerungen auf Langknochenschäften, $6=$ poröse Auflagerungen am Schulterblatt, $7=$ poröse Auflagerungen am Darmbein 


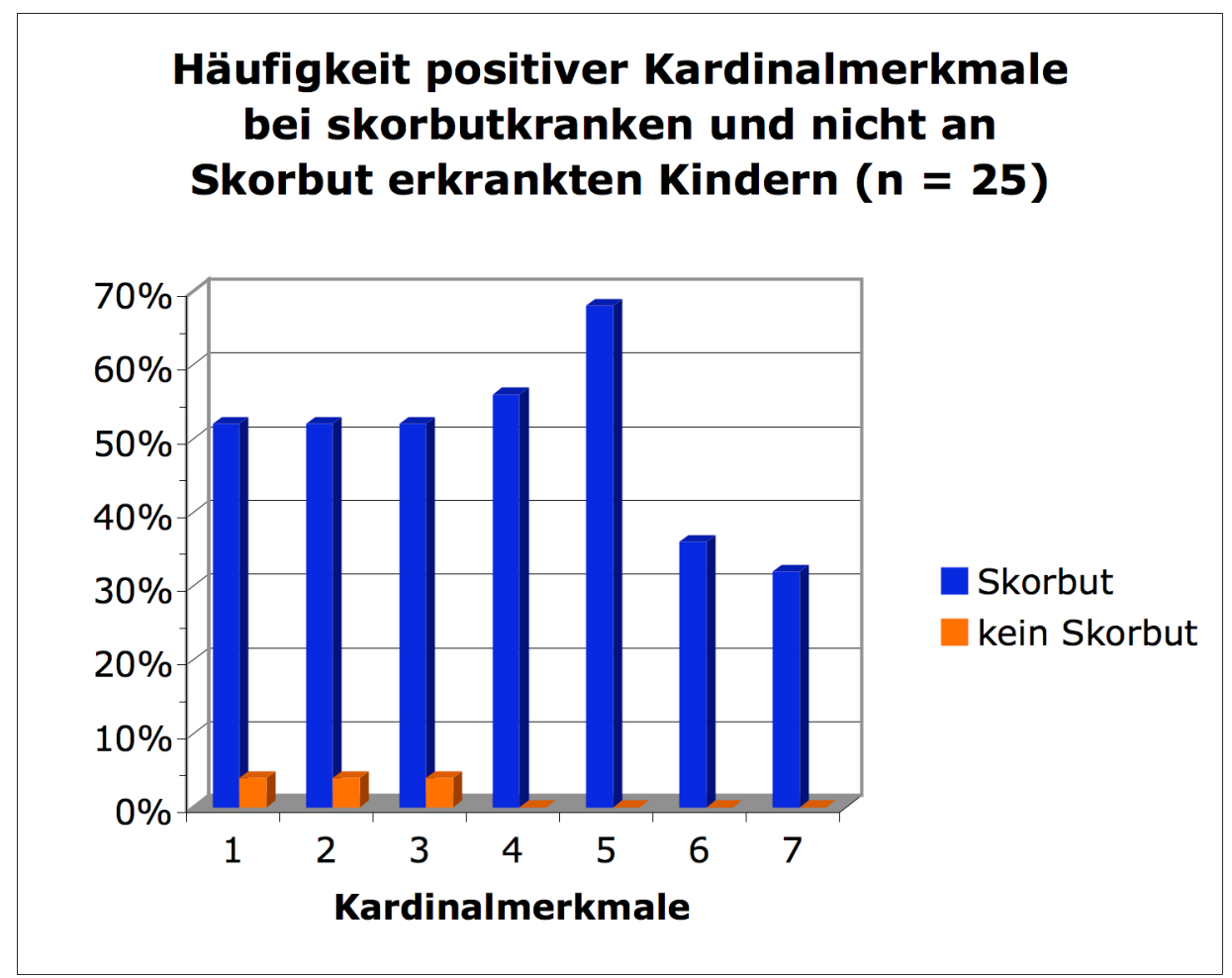

Abb. 84: Übersicht über die Häufigkeit aller Kardinalmerkmale und den jeweiligen Anteil von skorbutkranken und nicht an Skorbut erkrankten Kindern. Legende der Kardinalmerkmale: 1 = poröse Auflagerungen am äußeren Schädeldach, 2 = Cribra orbitalia, 3 = poröse Auflagerungen am äußeren Gesichtsschädel,

4 = poröse Auflagerungen am Alveolarknochen, 5 = poröse Auflagerungen auf Langknochenschäften,

$6=$ poröse Auflagerungen am Schulterblatt, 7 = poröse Auflagerungen am Darmbein

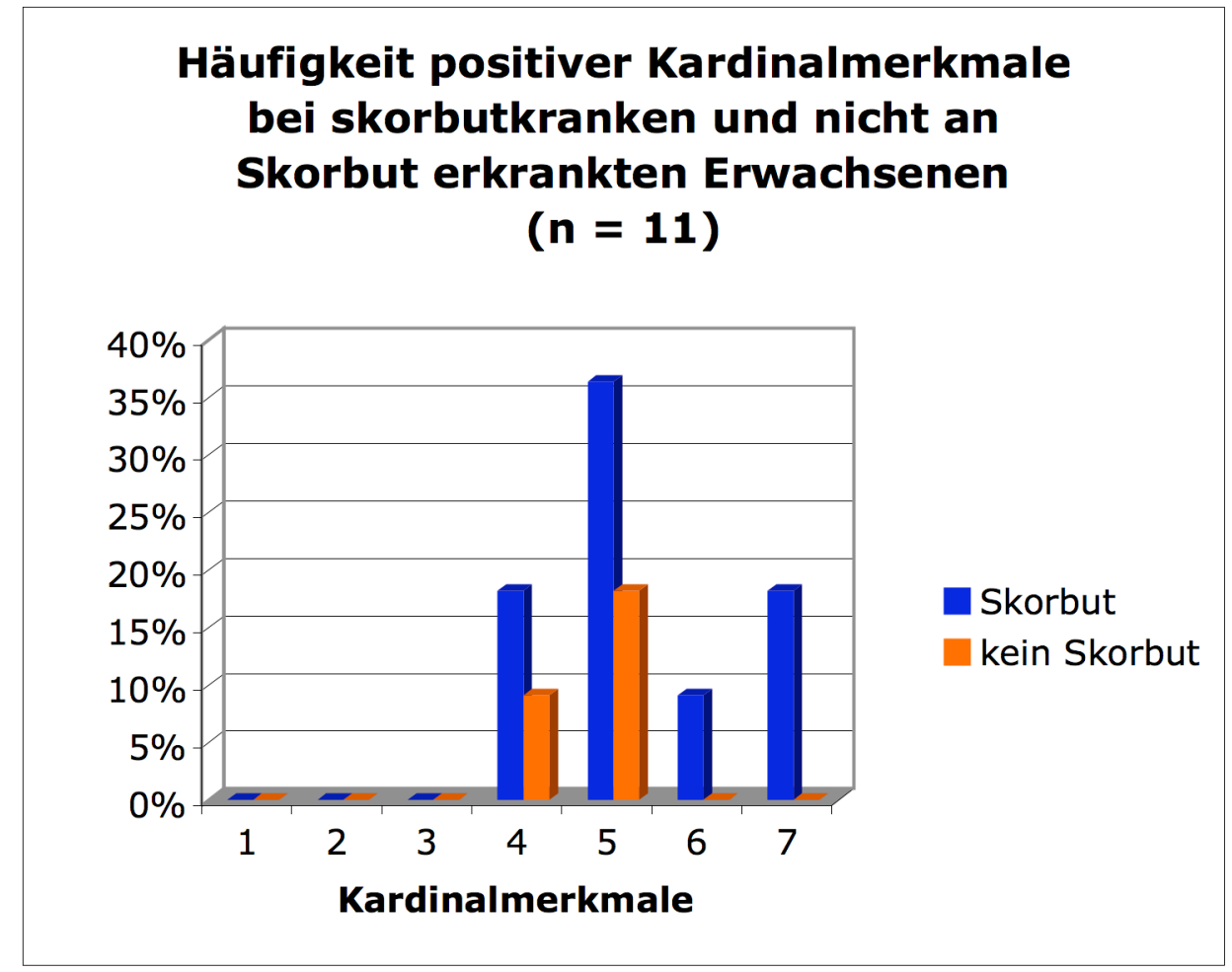

Abb. 85: Übersicht über die Häufigkeit aller Kardinalmerkmale und den jeweiligen Anteil von skorbutkranken und nicht an Skorbut erkrankten Erwachsenen. Individuum Sk 1380, das vermutlich sowohl an Lepra als auch an Skorbut erkrankt ist, wird hier in der Gruppe der Skorbutkranken geführt.

Legende der Kardinalmerkmale: 1 = poröse Auflagerungen am äußeren Schädeldach, 2 = Cribra orbitalia, 3 = poröse Auflagerungen am äußeren Gesichtsschädel, 4 = poröse Auflagerungen am Alveolarknochen, 5 = poröse Auflagerungen auf Langknochenschäften, $6=$ poröse Auflagerungen am Schulterblatt,

$7=$ poröse Auflagerungen am Darmbein 


\begin{tabular}{|r|r|r|r|r|r|r|}
\hline & \multicolumn{2}{|l|}{ Skorbutkranke (n=27) } & \multicolumn{2}{l|}{ Skorbutkranke Kinder (n=23) } & \multicolumn{2}{l|}{ Skorbutkranke Erwachsene (n= 4) } \\
\hline & Merkmal positiv & Merkmal negativ & Merkmal positiv & Merkmal negativ & Merkmal positiv & Merkmal negativ \\
\hline $\mathbf{1}$ & $13(48 \%)$ & $9(33 \%)$ & $13(57 \%)$ & $8(35 \%)$ & 0 & $2(50 \%)$ \\
\hline $\mathbf{2}$ & $13(48 \%)$ & $6(22 \%)$ & $13(57 \%)$ & $4(17 \%)$ & 0 & $2(50 \%)$ \\
\hline $\mathbf{3}$ & $13(48 \%)$ & $5(19 \%)$ & $13(57 \%)$ & $3(13 \%)$ & 0 & $2(50 \%)$ \\
\hline $\mathbf{4}$ & $16(59 \%)$ & $2(7 \%)$ & $14(61 \%)$ & $1(4 \%)$ & $2(50 \%)$ & $1(25 \%)$ \\
\hline $\mathbf{5}$ & $21(77 \%)$ & $4(15 \%)$ & $17(74 \%)$ & $4(17 \%)$ & $4(100 \%)$ & 0 \\
\hline $\mathbf{6}$ & $10(37 \%)$ & $7(26 \%)$ & $9(39 \%)$ & $5(22 \%)$ & $1(25 \%)$ & $2(50 \%)$ \\
\hline $\mathbf{7}$ & $10(37 \%)$ & $6(22 \%)$ & $8(35 \%)$ & $5(22 \%)$ & $2(50 \%)$ & $1(25 \%)$ \\
\hline
\end{tabular}

Tab. 117: Übersicht über den Anteil der positiven und negativen Merkmalsausprägungen bei den untersuchten Skorbutkranken. Die Prozentzahlen der ersten beiden Spalten beziehen sich auf die Gesamtzahl der Skorbutkranken, die übrigen auf die infantilen bzw. adulten Skorbutfälle.

Legende der Kardinalmerkmale: 1 = poröse Auflagerungen am äußeren Schädeldach, 2 = Cribra orbitalia, 3 = poröse Auflagerungen am äußeren Gesichtsschädel, 4 = poröse Auflagerungen am Alveolarknochen, 5 = poröse Auflagerungen auf Langknochenschäften, 6 = poröse Auflagerungen am Schulterblatt,

$7=$ poröse Auflagerungen am Darmbein

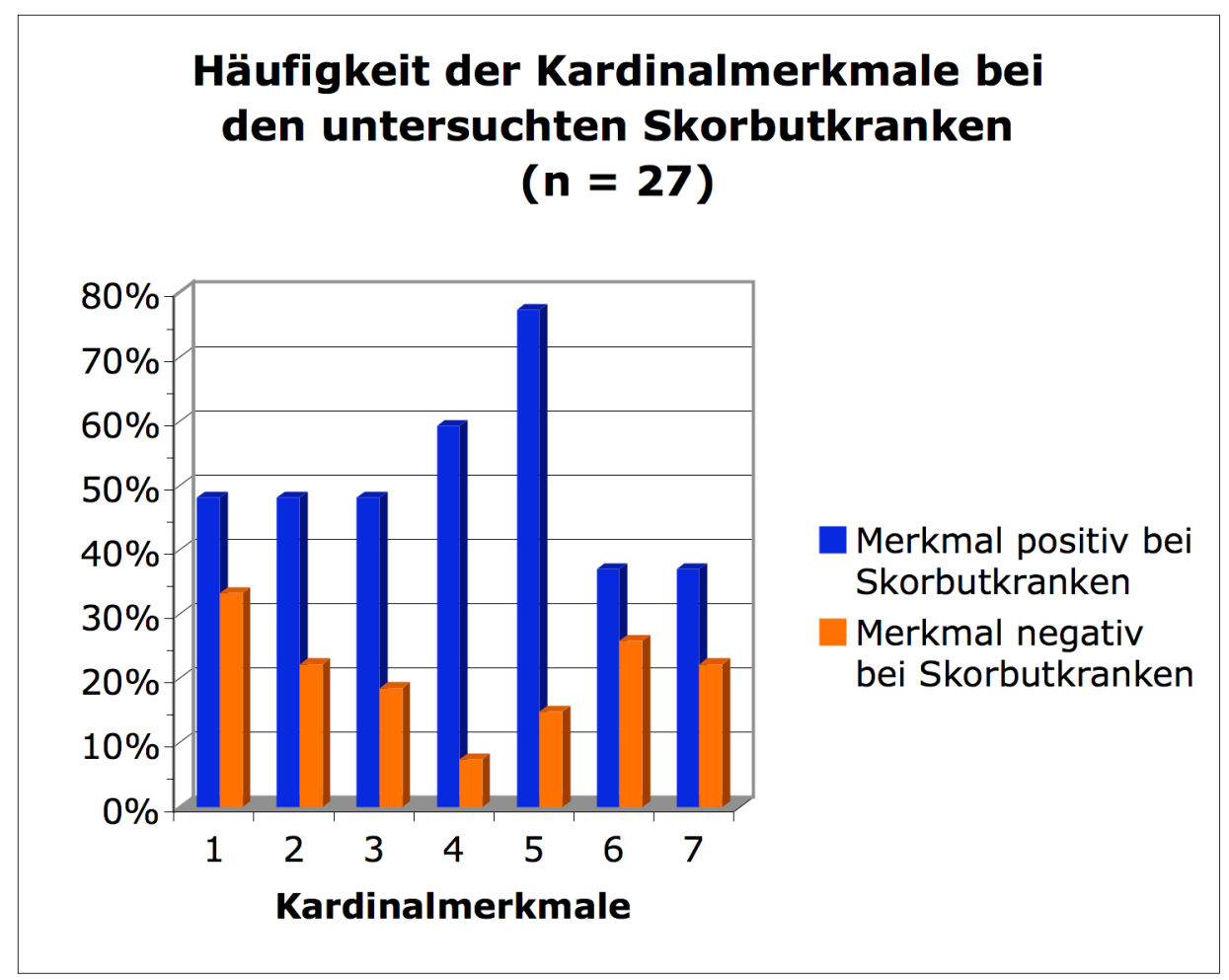

Abb. 86: Übersicht über den Anteil der positiven und negativen Merkmalsausprägungen bei den untersuchten Skorbutkranken (Erwachsene und Kinder)

Legende der Kardinalmerkmale: 1 = poröse Auflagerungen am äußeren Schädeldach, 2 = Cribra orbitalia, 3 = poröse Auflagerungen am äußeren Gesichtsschädel, 4 = poröse Auflagerungen am Alveolarknochen, 5 = poröse Auflagerungen auf Langknochenschäften, 6 = poröse Auflagerungen am Schulterblatt,

$7=$ poröse Auflagerungen am Darmbein 


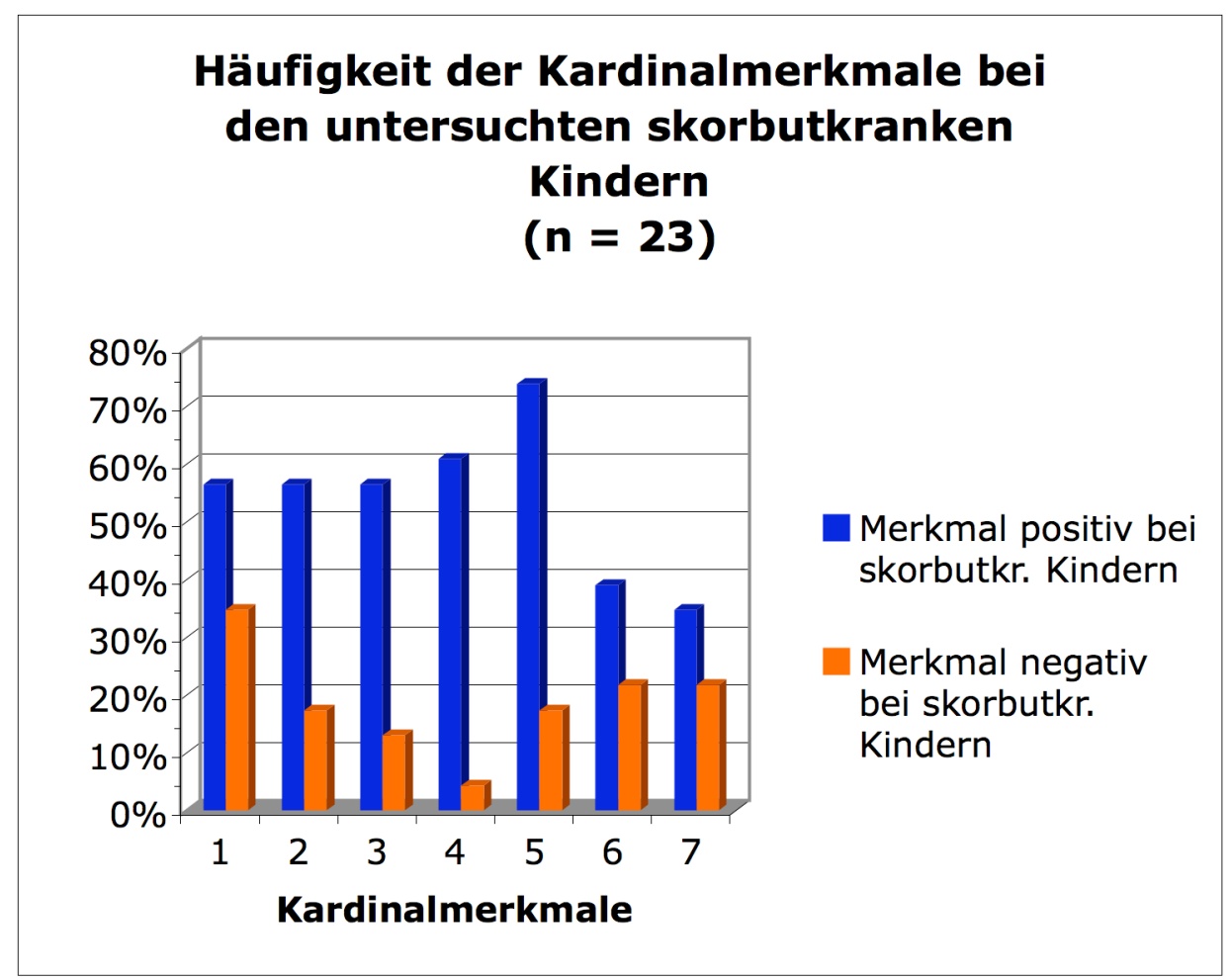

Abb. 87: Übersicht über den Anteil der positiven und negativen Merkmalsausprägungen bei den untersuchten skorbutkranken Kindern

Legende der Kardinalmerkmale: 1 = poröse Auflagerungen am äußeren Schädeldach, 2 = Cribra orbitalia, 3 = poröse Auflagerungen am äußeren Gesichtsschädel, 4 = poröse Auflagerungen am Alveolarknochen, 5 = poröse Auflagerungen auf Langknochenschäften, 6 = poröse Auflagerungen am Schulterblatt, $7=$ poröse Auflagerungen am Darmbein

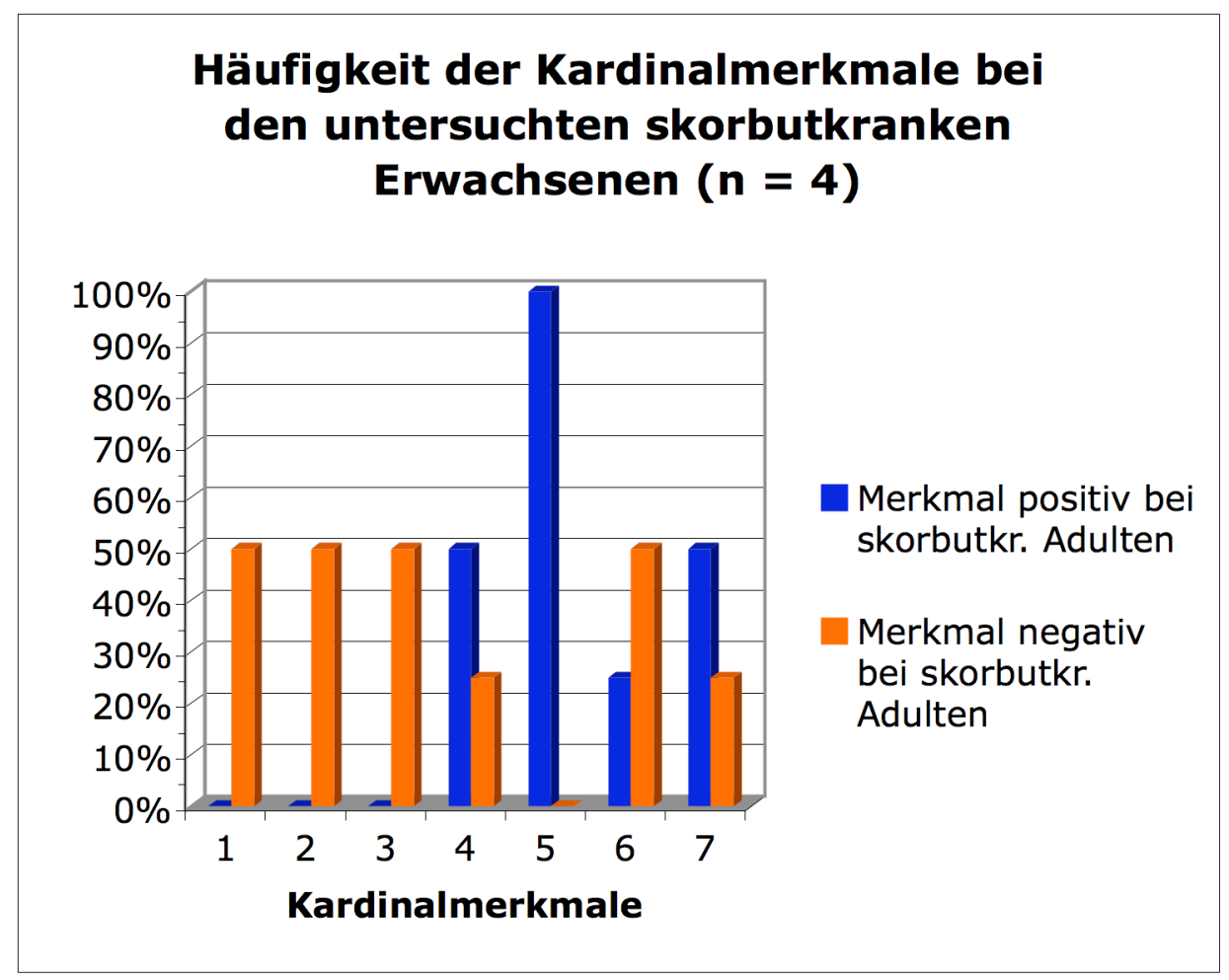

Abb. 88: Übersicht über den Anteil der positiven und negativen Merkmalsausprägungen bei den untersuchten skorbutkranken Erwachsenen

Legende der Kardinalmerkmale: 1 = poröse Auflagerungen am äußeren Schädeldach, 2 = Cribra orbitalia, 3 = poröse Auflagerungen am äußeren Gesichtsschädel, 4 = poröse Auflagerungen am Alveolarknochen, $5=$ poröse Auflagerungen auf Langknochenschäften, 6 = poröse Auflagerungen am Schulterblatt,

$7=$ poröse Auflagerungen am Darmbein 


\subsection{Zusammenfassung: Nebenmerkmale für Skorbut}

Von den Nebenmerkmalen ist die Stomatitis (Nebenmerkmal 1) am häufigsten, die bei 19 von 36 Individuen (53\%) vorliegt, darunter sind aber auch vier (11\%), die nicht an Skorbut erkrankt sind.

Unter den 25 Kindern sind 15 (60\%) mit Stomatitis, davon sind 14 (56\%) skorbutkrank.

Nur vier der elf Erwachsenen (36\%) haben Veränderungen am harten Gaumen, darunter ein Skorbutfall (9\%).

Betrachtet man die Skorbutfälle, so haben 15 von 27 (56\%) eine Stomatitis, nur bei einem Individuum (4\%) ist das Merkmal negativ, also ist die Merkmalsausprägung bei Skorbut recht hoch. Von den skorbutkranken Kindern haben 14 von 23 (61\%) Veränderungen am harten Gaumen, bei keinem ist das Merkmal Stomatitis negativ.

Unter den skorbutkranken Erwachsenen finden sich je ein Individuum (je 25\%) mit und ohne Ausprägung des Merkmals.

Das nächsthäufige Nebenmerkmal mit 18 von 36 (50\%) betroffenen Individuen sind poröse Langknochenoberflächen (Nebenmerkmal 4). Davon sind 15 (42\%) Individuen an Skorbut erkrankt, drei (8\%) nicht.

Von den 12 merkmalspositiven Kindern (48\% aller Kinder) haben alle Skorbut.

Bei den Erwachsenen sind je drei (27\%) der Individuen mit porösen Langknochenoberflächen skorbutkrank und nicht skorbutkrank.

Die Merkmalsausprägung scheint nicht sehr hoch, unter den Skorbutkranken finden sich 15 (56\%) mit porösen Langknochenoberflächen und neun (33\%) ohne. Allerdings muss man dabei berücksichtigen, dass in die Gruppe der Individuen ohne poröse Oberfläche auch diejenigen fallen, die poröse Auflagerungen an den Langknochen aufweisen (vgl. Nebenmerkmal 4 in der vorangegangen Merkmalsbesprechung).

Bei zwölf von 23 skorbutkranken Kindern (52\%) ist Nebenmerkmal 4 positiv und bei sieben (30\%) negativ.

Von den vier adulten Skorbutfällen zeigen drei (75\%) poröse Langknochenoberflächen, ein Individuum (25\%) nicht.

Nebenmerkmal 3 (poröse Auflagerungen am inneren Schädeldach) ist bei 17 der 36 Individuen (47\%) positiv, dabei handelt es sich ausschließlich um Skorbutfälle, die Spezifität ist also hoch.

Sechzehn (64\%) der 25 untersuchten Kinder und eins von elf adulten Individuen (9\%) zeigen dieses Merkmal. 
Von 27 Skorbutkranken haben 17 (63\%) Auflagerungen am inneren Schädeldach, bei drei kranken Individuen (11,1\%) ist das innere Schädeldach unauffällig.

Bei den 23 skorbutkranken Kindern ist das Verhältnis positiv zu negativ $16(70 \%)$ zu drei $(13 \%)$.

Zwei von vier (50\%) adulten Skorbutkranken haben Auflagerungen am inneren Schädeldach, ein krankes Individuum (25\%) nicht.

Vergleichsweise selten finden sich poröse Oberflächen an den Alae majores ossis sphenoidalis bzw. der Squama temporalis (Nebenmerkmal 5), nämlich in elf von 36 Fällen (31\%), dabei handelt es sich ausschließlich um Kinder. Davon sind 10 kindliche Individuen (28\% aller Individuen bzw. 40\% der Kinder) skorbutkrank und eins (3\% aller Individuen bzw. $4 \%$ der Kinder) nicht.

Von allen Skorbutkranken weisen zehn (37\% der 27 Skorbutkranken bzw. 44\% der 23 skorbutkranken Kinder) Porositäten am Keilbeinflügel auf, bei einem $(3 \%$ der 27 Skorbutkranken bzw. 4\% der 23 skorbutkranken Kinder) ist die Ala major ossis sphenoidalis unauffällig.

Bei keinem adulten Skorbutfall sind die Keilbeinflügel beurteilbar.

Am seltensten finden sich poröse Auskleidungen der Alveolen (Nebenmerkmal 2), nämlich nur in fünf von 36 Fällen (14\%), die alle an Skorbut erkrankt sind. Das Merkmal zeigt sich nur bei Kindern (20\% der 25 Kinder).

Bei den Skorbutkranken sind fünf von 27 (19\%) positiv, elf (41\%) negativ für das Merkmal.

Die Merkmalsausprägung für poröse Alveolarauskleidungen ist also sehr gering.

Von den 23 skorbutkranken Kindern haben fünf (22\%) Auskleidungen in den Alveolen, bei neun $(39 \%)$ sind diese unauffällig.

Keines der vier adulten skorbutkranken Individuen weist dieses Merkmal auf, bei zwei Individuen (50\%) ist der Alveolarfortsatz unauffällig.

\begin{tabular}{|l|l|l|l|l|l|l|}
\hline & \multicolumn{3}{|l|}{ Alle Individuen $(\mathbf{n}=\mathbf{3 6})$} & \multicolumn{2}{l|}{ Kinder $(\mathbf{n}=\mathbf{2 5})$} & \multicolumn{2}{l|}{ Erwachsene (n= 11) } \\
\hline & Skorbut & kein Skorbut & Skorbut & kein Skorbut & Skorbut & kein Skorbut \\
\hline $\mathbf{1}$ & $15(42 \%)$ & $4(11 \%)$ & $14(56 \%)$ & $1(4 \%)$ & $1(9 \%)$ & $3(27 \%)$ \\
\hline $\mathbf{2}$ & $5(14 \%)$ & 0 & $5(20 \%)$ & 0 & 0 & 0 \\
\hline $\mathbf{3}$ & $17(47 \%)$ & 0 & $16(64 \%)$ & 0 & $1(9 \%)$ & 0 \\
\hline $\mathbf{4}$ & $15(42 \%)$ & $3(8 \%)$ & $12(48 \%)$ & 0 & $3(27 \%)$ & $3(27 \%)$ \\
\hline $\mathbf{5}$ & $10(28 \%)$ & $1(3 \%)$ & $10(40 \%)$ & $1(4 \%)$ & 0 & 0 \\
\hline
\end{tabular}

Tab. 118: Übersicht über die Häufigkeit aller Nebenmerkmale und den jeweiligen Anteil von Skorbutkranken und nicht an Skorbut erkrankten Individuen. Die Prozentzahlen der ersten beiden Spalten beziehen sich auf die Gesamtzahl aller Individuen, die übrigen auf die Gesamtzahl aller Kinder bzw. aller Erwachsenen.

Individuum Sk 1380, das vermutlich sowohl an Lepra als auch an Skorbut erkrankt ist, wird hier in der Gruppe der Skorbutkranken geführt.

Legende der Nebenmerkmale: 1 = Stomatitis, 2 = poröse Alveolarauskleidungen,

$3=$ poröse Auflagerungen am inneren Schädeldach, 4 = poröse Langknochenoberflächen,

5 = poröse Außenfläche der Ala major ossis sphenoidalis/der Squama temporalis 


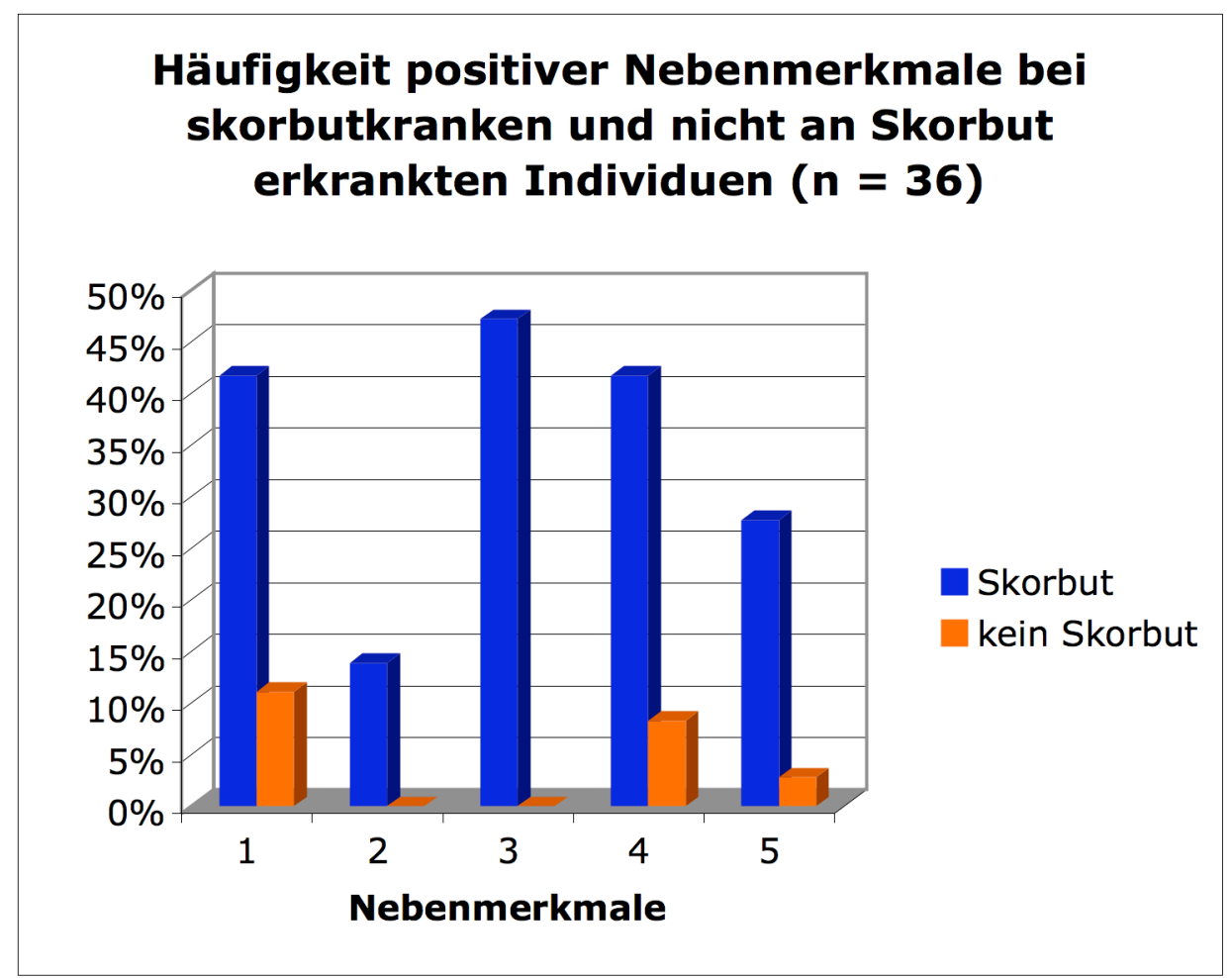

Abb. 89: Übersicht über die Häufigkeit aller Nebenmerkmale und den jeweiligen Anteil von Skorbutkranken und nicht an Skorbut erkrankten Individuen.

Individuum Sk 1380, das vermutlich sowohl an Lepra als auch an Skorbut erkrankt ist, wird hier in der Gruppe der Skorbutkranken geführt.

Legende der Nebenmerkmale: 1 = Stomatitis, 2 = poröse Alveolarauskleidungen,

3 = poröse Auflagerungen am inneren Schädeldach, 4 = poröse Langknochenoberflächen,

5 = poröse Außenfläche der Ala major ossis sphenoidalis/der Squama temporalis

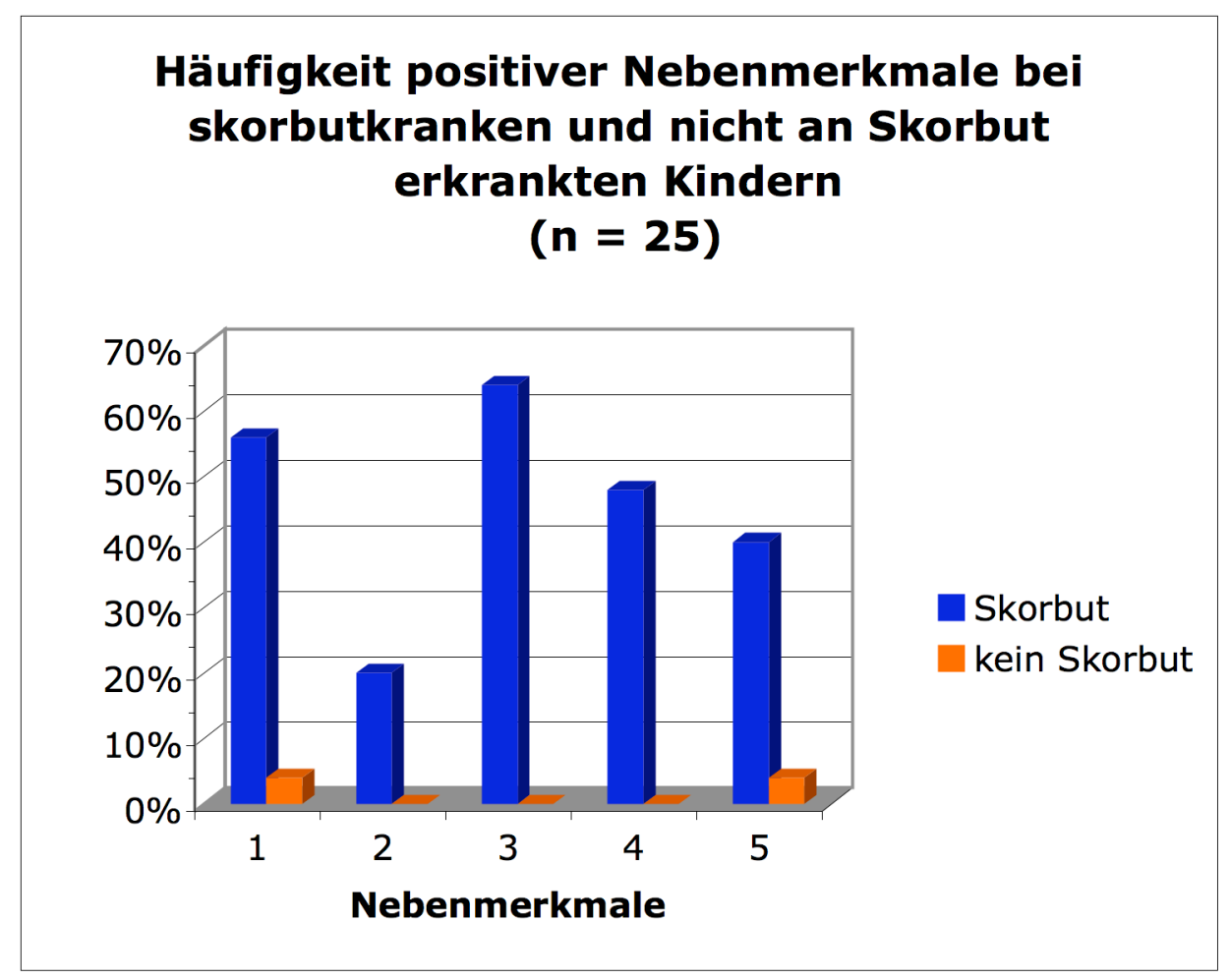

Abb. 90: Übersicht über die Häufigkeit aller Nebenmerkmale und den jeweiligen Anteil von skorbutkranken und nicht an Skorbut erkrankten Kindern.

Legende der Nebenmerkmale: 1 = Stomatitis, 2 = poröse Alveolarauskleidungen,

$3=$ poröse Auflagerungen am inneren Schädeldach, $4=$ poröse Langknochenoberflächen,

$5=$ poröse Außenfläche der Ala major ossis sphenoidalis/der Squama temporalis 


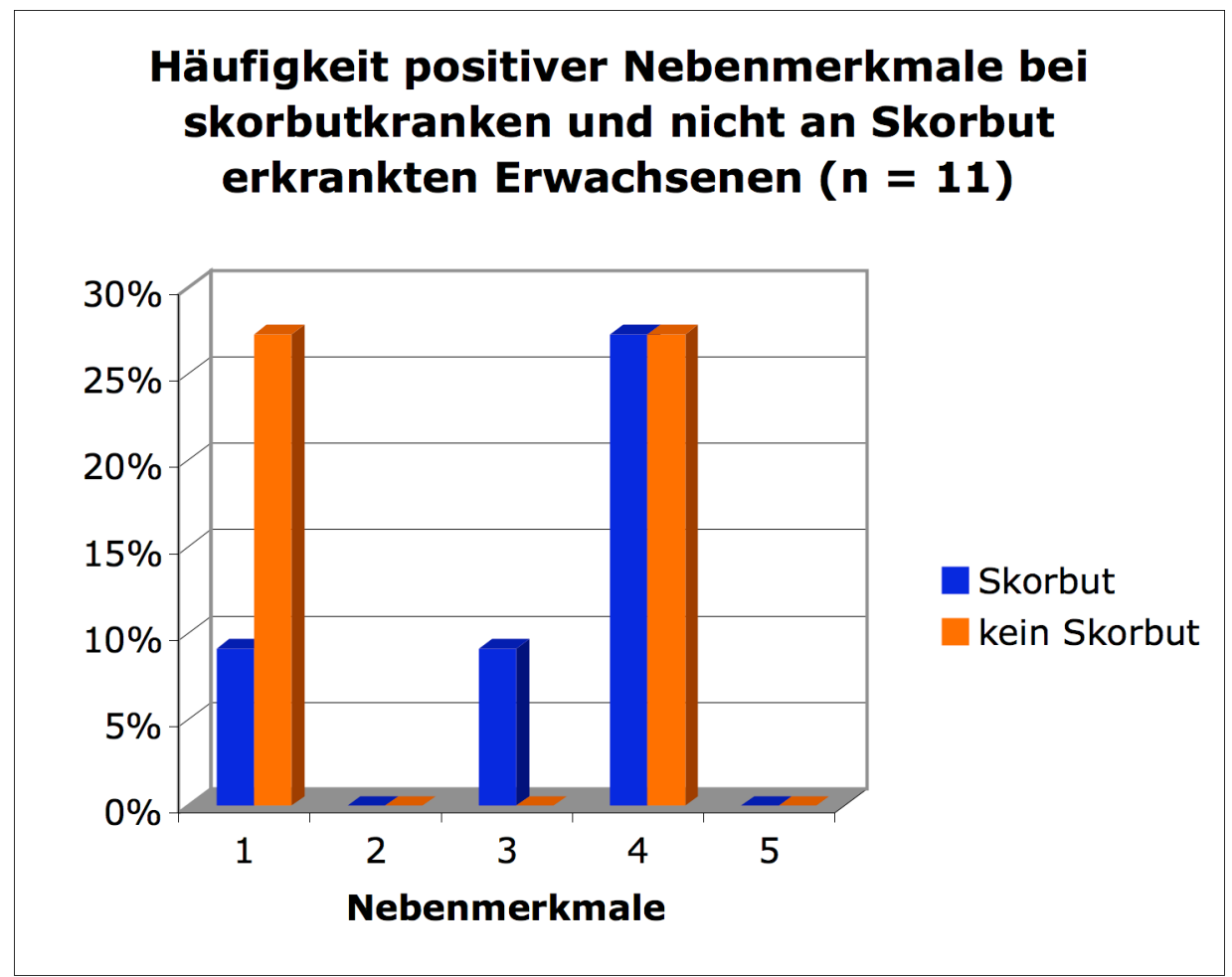

Abb. 91: Übersicht über die Häufigkeit aller Nebenmerkmale und den jeweiligen Anteil von skorbutkranken und nicht an Skorbut erkrankten Erwachsenen.

Individuum Sk 1380, das vermutlich sowohl an Lepra als auch an Skorbut erkrankt ist, wird hier in der Gruppe der Skorbutkranken geführt.

Legende der Nebenmerkmale: 1 = Stomatitis, 2 = poröse Alveolarauskleidungen,

3 = poröse Auflagerungen am inneren Schädeldach, 4 = poröse Langknochenoberflächen,

5 = poröse Außenfläche der Ala major ossis sphenoidalis/der Squama temporalis

\begin{tabular}{|l|r|r|r|r|r|r|}
\hline & \multicolumn{2}{l|}{ Skorbutkranke (n=27) } & \multicolumn{2}{l|}{ Skorbutkranke Kinder (n=23) } & \multicolumn{2}{l|}{ Skorbutkranke Erwachsene (n = 4) } \\
\hline & Merkmal positiv & \multicolumn{1}{l|}{ Merkmal negativ } & Merkmal positiv & Merkmal negativ & Merkmal positiv & Merkmal negativ \\
\hline $\mathbf{1}$ & $15(56 \%)$ & $1(4 \%)$ & $14(61 \%)$ & 0 & $1(25 \%)$ & $1(25 \%)$ \\
\hline $\mathbf{2}$ & $5(19 \%)$ & $11(41 \%)$ & $5(22 \%)$ & $9(39 \%)$ & 0 & $2(50 \%)$ \\
\hline $\mathbf{3}$ & $17(63 \%)$ & $3(11 \%)$ & $16(70 \%)$ & $3(13 \%)$ & $2(50 \%)$ & $1(25 \%)$ \\
\hline $\mathbf{4}$ & $15(56 \%)$ & $9(33 \%)$ & $12(52 \%)$ & $7(30 \%)$ & $3(75 \%)$ & $1(25 \%)$ \\
\hline $\mathbf{5}$ & $10(37 \%)$ & $1(4 \%)$ & $10(44 \%)$ & $1(4 \%)$ & 0 & 0 \\
\hline
\end{tabular}

Tab. 119: Übersicht über den Anteil der positiven und negativen Merkmalsausprägungen bei den untersuchten Skorbutkranken. Die Prozentzahlen der ersten beiden Spalten beziehen sich auf die Gesamtzahl der Skorbutkranken, die übrigen auf die infantilen bzw. adulten Skorbutfälle.

Legende der Nebenmerkmale: 1 = Stomatitis, 2 = poröse Alveolarauskleidungen,

3 = poröse Auflagerungen am inneren Schädeldach, 4 = poröse Langknochenoberflächen,

5 = poröse Außenfläche der Ala major ossis sphenoidalis/der Squama temporalis 


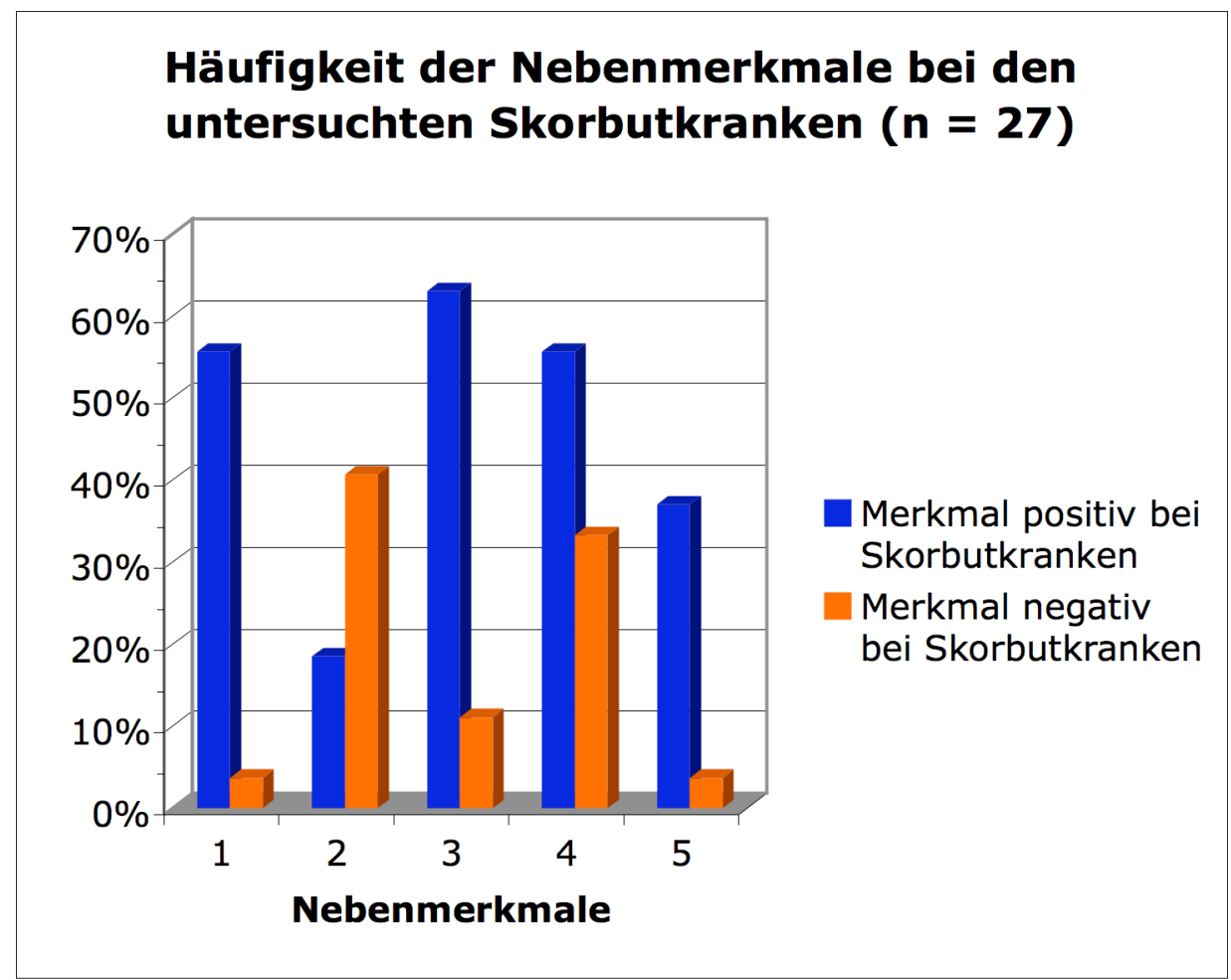

Abb. 92: Übersicht über den Anteil der positiven und negativen Merkmalsausprägungen bei den untersuchten Skorbutkranken.

Legende der Nebenmerkmale: 1 = Stomatitis, 2 = poröse Alveolarauskleidungen,

3 = poröse Auflagerungen am inneren Schädeldach, 4 = poröse Langknochenoberflächen,

$5=$ poröse Außenfläche der Ala major ossis sphenoidalis/der Squama temporalis

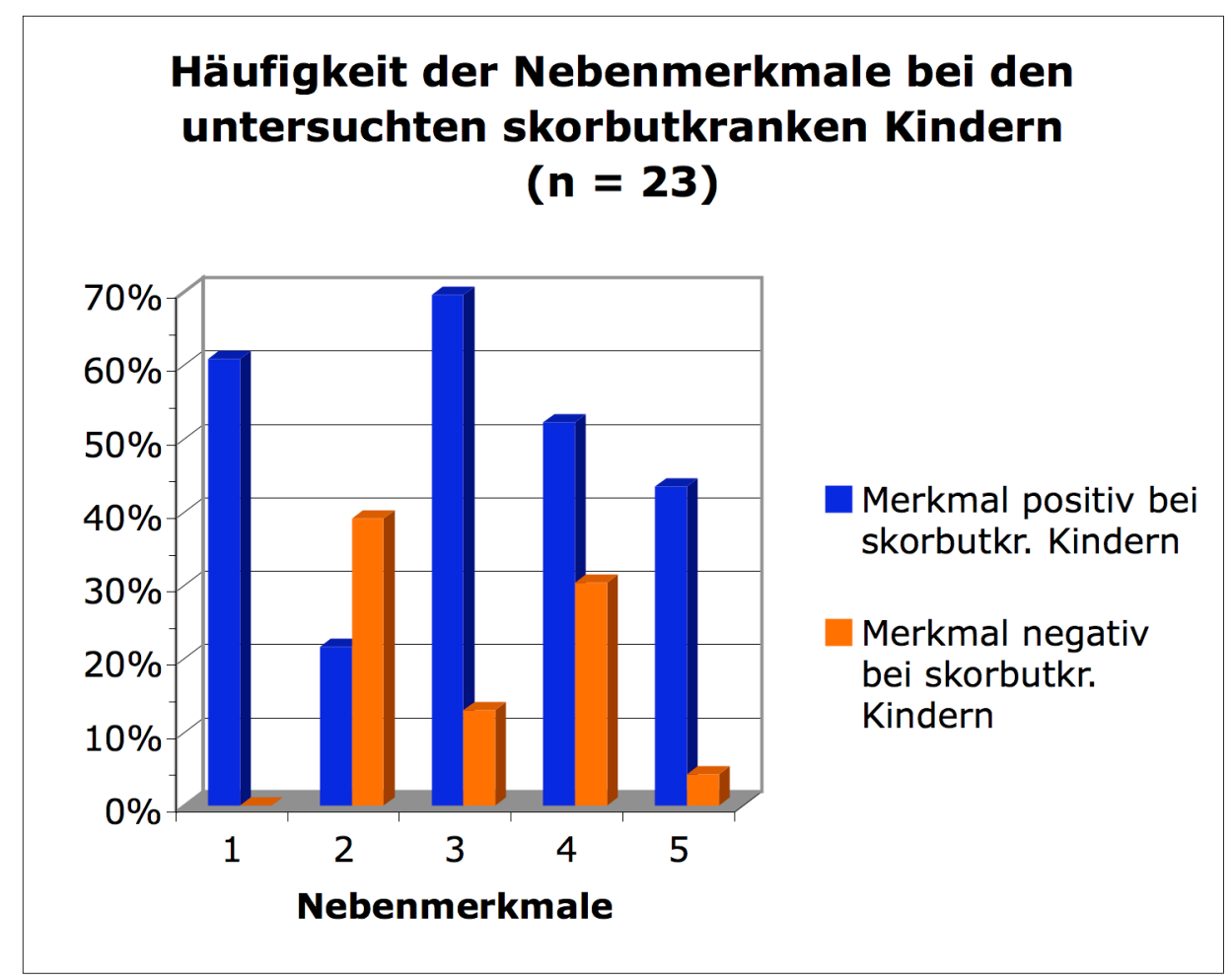

Abb. 93: Übersicht über den Anteil der positiven und negativen Merkmalsausprägungen bei den untersuchten skorbutkranken Kindern.

Legende der Nebenmerkmale: 1 = Stomatitis, 2 = poröse Alveolarauskleidungen,

3 = poröse Auflagerungen am inneren Schädeldach, 4 = poröse Langknochenoberflächen,

$5=$ poröse Außenfläche der Ala major ossis sphenoidalis/der Squama temporalis 


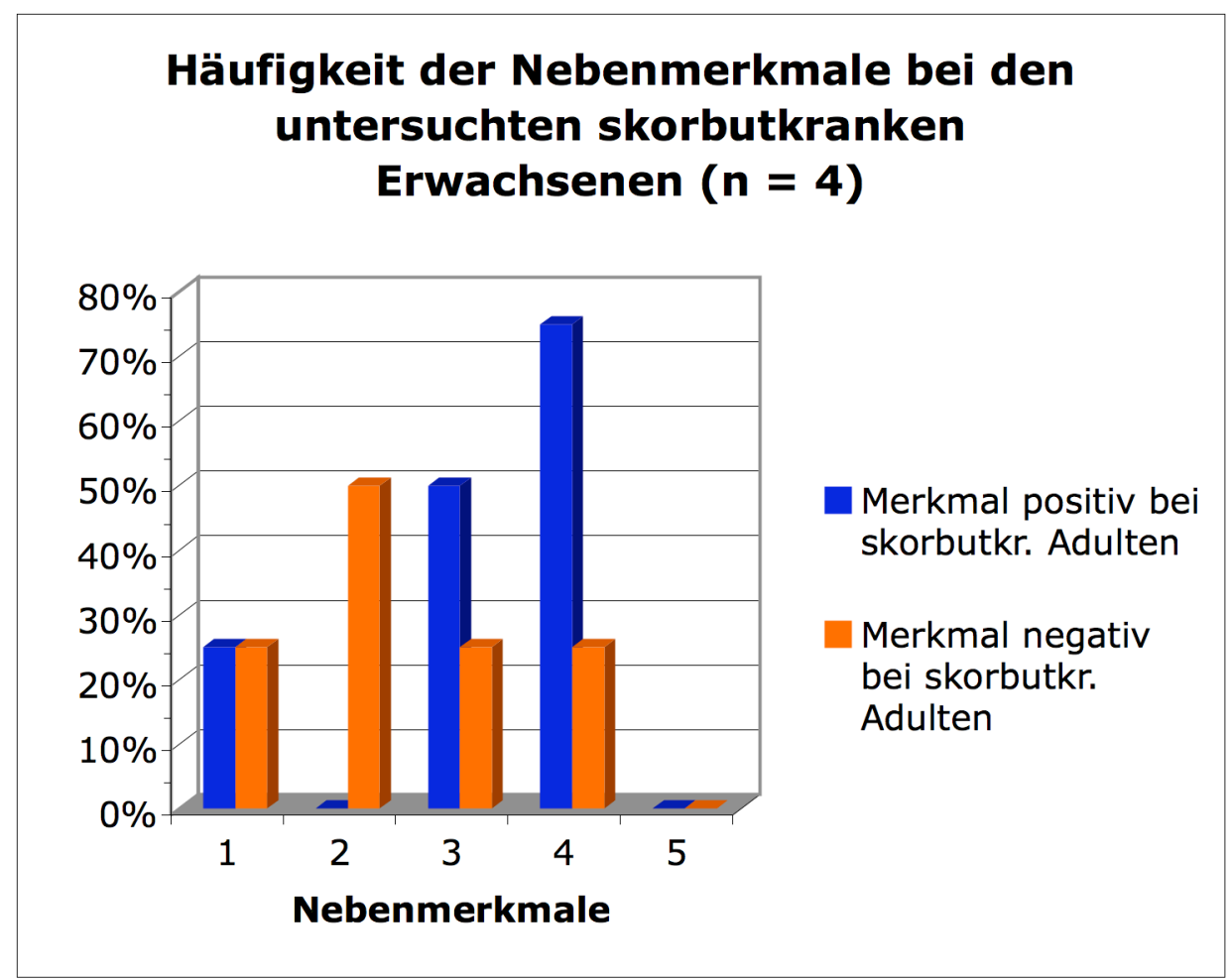

Abb. 94: Übersicht über den Anteil der positiven und negativen Merkmalsausprägungen bei den untersuchten skorbutkranken Erwachsenen.

Legende der Nebenmerkmale: 1 = Stomatitis, 2 = poröse Alveolarauskleidungen,

3 = poröse Auflagerungen am inneren Schädeldach, 4 = poröse Langknochenoberflächen,

$5=$ poröse Außenfläche der Ala major ossis sphenoidalis/der Squama temporalis 


\section{Diskussion}

\subsection{Merkmale für Skorbut am historischen Skelet}

Skorbutische Läsionen am Skelet lassen sich aufgrund ihres Entstehungsmechanismus in verschiedene Gruppen einteilen.

Ein häufig zu findendes Merkmal ist die erhöhte Porosität, die an verschiedenen Stellen des Skelets auftreten kann.

Sie ist definiert durch lokal begrenzte feine Löcher, die ohne Vergrößerung sichtbar sind, im Durchmesser typischerweise weniger als einen Millimeter betragen und die lamelläre Knochenoberfläche penetrieren. Der zugrunde liegenden Knochen kann eine Neubildung oder die ursprüngliche Knochenoberfläche sein (ORTNER et al. 1999, ORTNER et al. 2001, ORTNER und ERICKSEN 1997, SCHULTZ 1986).

Man erklärt sich diese Porosität durch eine erhöhte Vaskularisierung, die vermutlich im Rahmen einer chronischen Entzündung aufgrund der skorbutischen Hämorrhagien entsteht (BRICKLEY und IVES 2006, ORTNER und ERICKSEN 1997).

Die zweite wichtige Veränderung ist die reaktive Knochenneubildung, makroskopisch erkennbar als feinporöse Auflagerung. Diese sollte als Periostose bezeichnet werden, in Abgrenzung zu einer durch Erreger verursachten entzündlichen Reaktion des Periosts, der Periostitis. Sie wird zurückgeführt auf subperiostale Hämatome, die durch Läsionen der skorbutös geschädigten Blutgefäße entstehen. Das Abheben des Periosts von der Knochenoberfläche stellt einen Wachstumsreiz für Osteoblasten dar, es kommt zur reaktiven Knochenneubildung (AUFDERHEIDE und RODRÍGUEZ-MARTÍN 1998, BARLOW $1883^{74}$, CARLI-THIELE 1996, ORTNER et al. 2001, ORTNER und PUTSCHAR 1981). Bei dem neugebildeten Knochen handelt es sich in der Regel zuerst um Faserknochen, der im Verlauf zu Lamellenknochen umgewandelt wird, bei chronischer Entzündung scheint jedoch auch die direkte Neubildung von Lamellenknochen möglich (ORTNER und ERICKSEN 1997). Auch Einblutungen in Weichteile können zu Ossifikationen führen (SCHULTZ et al. 2007).

Vermutlich ist die reaktive Knochenneubildung nur bei stark ausgeprägtem Skorbut zu finden, wohingegen die erhöhte Porosität auch bei milderen Verläufen auftritt (ORTNER et al. 2001, BRICKLEY und IVES 2006).

\footnotetext{
${ }^{74}$ S. 186
} 
Eine dritte Manifestation des Skorbuts, die am Skelet auch lange nach klinischer Besserung nachgewiesen werden kann, ist eine Rarefikation der Knochensubstanz, die Corticalis erscheint verdünnt, das Trabekelwerk reduziert (BARLOW 1883, FRAENKEL $1908^{75}$ ).

Weitere mögliche Manifestationen am Skelet sind Epiphysendislokationen und Infraktionen. Da Heilungsvorgänge wie Callusbildung weitgehend ausbleiben, solange der Vitamin-CMangel fortbesteht, ist es schwierig, am historischen Skelet Rückschlüsse auf das Alter der Frakturen anzustellen. Die Frakturen sind also, auch wenn sie ganz frisch erscheinen, nicht unbedingt im direkten Zusammenhang mit dem Tod des Individuums zu sehen (MAAT und UYTTERSCHAUT 1984).

Es ist als Grundregel festzuhalten, dass Stellen, die besonderem mechanischen Stress ausgesetzt sind, prädestiniert für skorbutische Veränderungen sind, also Körperteile, die das Gewicht tragen, sowie Muskel- und Bandansätze (MAAT und UYTTERSCHAUT 1984, SCHULTZ 1986).

Wie bereits in der Einleitung geschildert, stellen MELIKIAN und WALDRON (2003) beim Vergleich mit den klinisch bekannten Skorbutfällen fest, dass keinerlei Übereinstimmung in Verteilung und Ausprägung der Veränderungen vorliegt. Sie kommen daher zu dem Schluss, dass die Diagnosekriterien unzulänglich sind, und bezweifeln, dass eine eindeutige Diagnosestellung Skorbut in paläopathologischen Untersuchungen überhaupt möglich ist. BRICKLEY und IVES (2006) kritisieren diesen Vergleich von klinisch stark ausgeprägten Fällen mit den untersuchten Skeleten. Sie vermuten, dass es sich bei den von ORTNER und Koautoren (ORTNER 2003, ORTNER und ERICKSEN 1997, ORTNER et al. 1999, ORTNER et al. 2001) beschriebenen und von MELIKIAN und WALDRON (2003) vielfach nachgewiesenen Veränderungen um eine schwächere Ausbildung des Skorbuts handelt, weshalb sie nicht die deutlichen Merkmale der klinisch bekannten Fälle aufweisen (BRICKLEY und IVES 2006).

Neben den bereits genannten Veränderungen kann man auch Zeichen von Sinusitiden, Mittelohrentzündungen, Meningitiden, Pleuritiden und anderen Infektionen als Hinweis auf eine mögliche Skorbuterkrankung werten, da diese für ein durch Vitamin-C-Mangel geschwächtes Immunsystem sprechen (CARLI-THIELE 1996, SCHULTZ 199076).

Im Folgenden werden die verschiedenen Lokalisationen für die beschriebenen Läsionen vom Schädel abwärts beschrieben und mit den eigenen Ergebnissen verglichen, um ein typisches Verteilungsmuster für Skorbut herauszuarbeiten.

\footnotetext{
${ }^{75}$ BARLOW 1883: S. 187, FRAENKEL 1908: S. 19f, S. 26, S. 28, S. 34

${ }^{76}$ S. $194 f$
} 


\subsection{Skorbutische Läsionen am Schädel}

\section{Schädeldach}

An der Lamina externa von Skeleten mit Verdacht auf Skorbut findet sich oft eine poröse Hyperostose, also eine Verdickung des Schädeldachs mit poröser Oberflächengestaltung. Dies ist aber kein spezifisches Merkmal für eine Skorbuterkrankung, sondern kann auch viele andere Ursachen haben, z.B. Hämatome nach Traumata, Entzündungen der Kopfhaut oder des Periosts, Anämie und Rachitis (LEWIS 2002, SCHULTZ 1986, SCHULTZ 1987, SCHULTZ 1993, SCHULTZ 2001, SCHULTZ 2011, TEMPLIN 1993).

FRAENKEL $\left(1908^{77}\right)$ beschreibt das gelegentliche Auftreten von periostalen und subperiostalen Blutungen im Bereich des Schädeldachs, namentlich an Scheitel- und Stirnbein und Schläfenschuppe. Besondere klinische Bedeutung misst er aufgrund der ungünstigen Prognose den eher seltenen subduralen Blutergüssen bei.

ORTNER et al. (2001) geben an, sowohl Zustände gesteigerter Porosität als auch reaktive Knochenneubildungen in einigen Fällen am Schädeldach gefunden zu haben, ohne näher zu bezeichnen, an welchen Stellen genau sie vorliegen. Auch SCHULTZ und Mitarbeiter assoziieren eine Periostose des Schädeldachs mit Skorbut (SCHULTZ 1987, SCHULTZ 1993, SCHULTZ 2001, SCHULTZ 2011, SCHULTZ et al. 2007, TEMPLIN 1993, WEIHMANN 2010).

Von den 36 in der vorliegenden Arbeit untersuchten Skeleten weisen 14, darunter 13 Skorbutfälle, poröse Auflagerungen am äußeren Schädeldach auf. Bei sechs Skorbutkranken ist das äußere Schädeldach unauffällig. Man kann dies also als spezifisches, aber nicht immer ausgebildetes Merkmal von Skorbut betrachten.

Auch am inneren Schädeldach werden poröse Läsionen und Auflagerungen beschrieben, vor allem im Bereich der venösen Sinus. Differentialdiagnostisch muss hier unter anderem an intracranielle Blutungen traumatischer Genese, Meningitiden, Pachymeningitiden und Meningo-Encephalopathien gedacht werden (SCHULTZ 1987, SCHULTZ 1993, SCHULTZ 2001, SCHULTZ 2011, TEMPLIN 1993, WEIHMANN 2010).

Eine Unterscheidung kann nur mikroskopisch erfolgen (siehe Kapitel 2.2i):Lichtmikroskopie

$\rightarrow$ Befundung der lichtmikroskopischen Präparate).

Ausgeprägte, oft netzartig angelegte Gefäßimpressionen an der Lamina interna können für die bindegewebige Organisation eines epiduralen Hämatoms sprechen (SCHULTZ 1987, SCHULTZ 1993, SCHULTZ 2011).

${ }^{77}$ S. 10, S. 24 
CARLI-THIELE (1996) führt epidurale Hämatome an den Ossa parieralia und frontalia ohne erkennbares Trauma und hämorrhagisch-entzündliche Prozesse an der Schädelinnenseite auf Skorbut zurück.

Intracraniale Blutungen können zu einer Erhöhung des Schädelinnendrucks führen, weshalb auch Zeichen einer Hirndrucksymptomatik, wie verstärkte Impressiones digitatae und steil stehende Orbitadächer, ein Hinweis auf Skorbut sein können (SCHULTZ 1987, SCHULTZ 1993, SCHULTZ und SCHMIDT-SCHULTZ 2013).

In der vorliegenden Studie zeigen 17 Individuen Auflagerungen am inneren Schädeldach, ausschließlich Skorbutfälle. Drei Skorbutkranke haben unauffällige innere Schädeldächer. Man kann dies also als ein recht häufig ausgeprägtes Merkmal für Skorbut mit hoher Spezifität bezeichnen.

\section{Os parietale}

BRICKLEY und IVES (2006) beobachten bei einem Kleinkind eine massive Porosität am äußeren Schädeldach des Os parietale, die auf eine schwere vaskuläre Reaktion hindeutet. Auflagerungen finden sich bei diesem Individuum nicht (BRICKLEY und IVES 2006). Auch SCHULTZ schildert eine erhöhte Porösität an der Innen- und Außenseite des Os parietale, insbesondere in Suturnähe, bei einem Kind, das auch weitere Hinweise auf eine Skorbuterkrankung zeigt (SCHULTZ 1987, SCHULTZ 1993).

BARLOW $\left(1883^{78}\right)$ dokumentiert post mortem subperiostale Hämatome, die klinisch zu Schwellungen der Parietalregion geführt hatten. Der darunter liegende Knochen erscheint porös.

\section{Os occipitale}

BRICKLEY und IVES (2006) dokumentieren mehrere Fälle von erhöhter Vaskularisierung der Lamina interna des Os occipitale. Außerdem liegen bei einem Neugeborenen an der Lamina interna Auflagerungen in Folge reaktiver Knochenneubildungen vor. Dies überrascht die Autorinnen angesichts der überwiegend resorbierenden Funktion der Dura während der Wachstumsphase. Deshalb halten sie die Unterscheidung zwischen skorbutischer Läsion und traumatischer Ursache, z. B. ein Geburtstrauma, für sehr schwierig (BRICKLEY und IVES 2006).

Poröse Läsionen treten häufig im Bereich der Lambda-Naht auf (ORTNER et al. 1999, SCHULTZ 1987, SCHULTZ 1993).

${ }^{78}$ S. 169, S. 182, S. 188 
Klinisch sind Fälle von schmerzhaften Schwellungen des Occiputs beschrieben (BARLOW $\left.1883^{79}\right)$.

\section{Fontanelle}

Barlow beschreibt auffällig große Fontanellen bei skorbutischen Kindern (BARLOW 1883 ${ }^{80}$ ). Ebenso wie der weiter unten beschriebene verzögerte Zahndurchbruch kann dies zu Altersfehlbestimmungen bei der Untersuchung historischer Skelete führen.

\section{Os sphenoidale}

Wie bereits in Kapitel 1.9 Nachweis von Skorbut an historischen Skeleten erläutert, messen ORTNER und seine unterschiedlichen Koautoren (ORTNER et al. 2001, ORTNER und ERICKSEN 1997) Veränderungen im Bereich der Ala major ossis sphenoidalis für die Diagnose Skorbut größte Bedeutung bei. Sie erklären sie durch den knochennahen Verlauf der tiefen Temporalarterien unterhalb des M. temporalis. Sei ein Individuum an Skorbut erkrankt und die Gefäße durch schadhaftes Kollagen fragil, so komme es im Rahmen des Kauakts zu Verletzungen der Gefäße durch Muskelkontraktion und damit zu Blutungen im Bereich des Periosts mit den bekannten Konsequenzen der gesteigerten Vaskularisierung oder sogar der reaktiven Knochenneubildung. Sie halten diese Veränderungen für sehr sensibel, da die annähernd einzigartige Lage der Gefäße eine Manifestation am Knochen in deutlich höherem Maße begünstige als anderswo (ORTNER et al. 2001, ORTNER und ERICKSEN 1997). Außerdem sei die Kaumuskulatur nicht von der bei Skorbut häufigen schmerzbedingten allgemeinen Schonhaltung im Sinne einer Pseudoparalyse betroffen, sondern der Appetit in der Regel nicht gemindert (ORTNER und ERICKSEN 1997, BARLOW 1883 ${ }^{81}$ ). Die Autoren gehen so weit, Veränderungen im Bereich des Os sphenoidale für pathognomonisch zu halten (ORTNER und ERICKSEN 1997), und machen sie zum Einschlusskriterium für ihre Untersuchungen (ORTNER et al. 2001). Dabei machen ORTNER et al. (2001) selbst die Einschränkung, dass ihnen auf diese Weise Skorbutfälle entgehen könnten, nennen sogar Beispiele für Fälle, die einige andere typische Zeichen für Skorbut aufwiesen, jedoch wegen fehlender Affektion des Os sphenoidale nicht in die Studie aufgenommen wurden (ORTNER et al. 2001). Streng genommen sollte man jedoch immer auch die Squama temporalis mit untersuchen, da davon auszugehen ist, dass sie aufgrund der

\footnotetext{
${ }^{79}$ S. 169

${ }^{80}$ S. 184

${ }^{81}$ S. 174
} 
Nähe und der ähnlichen anatomischen Voraussetzungen die gleichen Veränderungen aufweist wie der Große Keilbeinflügel.

BRICKLEY und IVES (2006) messen den Veränderungen im Bereich des Os sphenoidale weniger Bedeutung bei, zumal sie Skelete mit Verdacht auf Skorbut untersuchen, bei denen diese Struktur teilweise gar nicht vorhanden ist. Sie warnen davor, einem einzelnen Merkmal zu viel Gewicht zu geben, da gerade bei der Untersuchung historischer Knochen oft Teile fehlen. Die Autorinnen weisen in einem Fall Knochenneubildungen im Bereich der Alae minores und des Corpus ossis sphenoidalis nach (BRICKLEY und IVES 2006).

In der vorliegenden Studie kann die hohe Gewichtung von porösen Auflagerungen an der Ala major ossis sphenoidalis nicht bestätigt werden. Zwar findet sich das Merkmal bei elf Kindern (30,6\% aller Individuen bzw. 44\% aller Kinder), darunter aber auch bei einem Individuum, das mit großer Wahrscheinlichkeit nicht an Skorbut litt. Außerdem ist das Os sphenoidale bei einem Individuum mit Skorbut unauffällig. Bei 20 Individuen, darunter 16 sichere und zwei wahrscheinliche Skorbutfälle, sind die Alae majores nicht befundbar. Hätte man, wie Ortner und seine verschiedenen Koautoren, dieses Merkmal zum Einschlusskriterium für die Studie gemacht, so wären also mindestens 16 Skorbutfälle nicht entdeckt worden.

\section{Orbita}

Klinisch werden Ekchymosen der Augenlider, konjunktivale Blutungen und sogar eine Proptosis des Augapfels geschildert (BARLOW 1883, FRAENKEL 1908 ${ }^{82}$ ). FRAENKEL (1908) findet bei einer Autopsie eine massive blutige Infiltration des Periosts des Orbitadachs und kann mikroskopisch Knochenneubildungen nachweisen, welche seiner Beschreibung nach am ehesten einem Typ I entsprechen (FRAENKEL $1908^{83}$ ).

Seit den 1960er Jahren wurden Cribra orbitalia vor allem als Zeichen einer schweren Anämie gewertet. Doch spätestens durch die Einführung mikroskopischer Diagnostik wurde klar, dass es viele mögliche Ursachen für die Ausbildung von Cribra orbitalia gibt (SCHULTZ 1987, SCHULTZ 1993, SCHULTZ 2001, SCHULTZ 2011, WAPLER et al. 2004, WEIHMANN 2010).

In der Literatur werden häufig poröse Zustände bzw. feinporöse Auflagerungen (Cribra orbitalia) im Bereich der Orbitadächer im Zusammenhang mit Skorbut beschrieben (z.B. BRICKLEY und IVES 2006, FRAENKEL $1908^{84}$, LEWIS 2002, MAYS 2008, ORTNER et al. 1999, ORTNER et al. 2001, ORTNER und ERICKSEN 1997, ORTNER und PUTSCHAR

\footnotetext{
${ }^{82}$ BARLOW 1883: S. 170, S. 181f, FRAENKEL 1908: S. 12

${ }^{83}$ S. 24

${ }^{84}$ FRAENKEL 1908: S. 25, ORTNER/PUTSCHAR 1981: S. 272
} 
1981, SCHULTZ 1987, SCHULTZ 1993, SCHULTZ 2001, SCHULTZ 2011, TEMPLIN 1993, WEIHMANN 2010).

ORTNER (ORTNER et al. 1999, ORTNER et al. 2001) und seine verschiedenen Koautoren weisen sowohl eine erhöhte Porosität als auch reaktive Knochenneubildung an Orbitadächern nach, außerdem in einigen Fällen auch eine gesteigerte Porosität des Margo lateralis orbitae (ORTNER et al. 1999, ORTNER et al. 2001). Seltener werden auch an der endocranialen Oberfläche des Orbitadachs Auflagerungen beschrieben (ORTNER und PUTSCHAR $1981^{85}$ ). Möglicherweise reichen schon Augenbewegungen aus, um Blutungen aus skorbutischen Gefäßen zu provozieren, vor allem, wenn schon in der Entwicklungsphase im Mutterleib bzw. im Neugeborenenalter Vitamin-C-Mangel bestand (BRICKLEY und IVES 2006, ORTNER und ERICKSEN 1997). Auch kräftiges lang anhaltendes Schreien könnte zur Gefäßschädigung führen (FRAENKEL $1908^{86}$ ).

Da makroskopisch ähnliche Läsionen in der Orbita auch bei Anämie und Rachitis (BRICKLEY und IVES 2006, SCHULTZ 1987, SCHULTZ 1993, SCHULTZ 2011, SCHULTZ et al. 2007, WAPLER et al. 2004) sowie nach Hämorrhagien durch Traumata und bei entzündlichen Erkrankungen (SCHULTZ 1987, SCHULTZ 1993, SCHULTZ 2001, SCHULTZ 2011, SCHULTZ et al. 2007, WAPLER et al. 2004) auftreten können, muss eine sorgfältige Abgrenzung erfolgen, wenn möglich durch eine lichtmikroskopische Untersuchung (SCHULTZ 1987, SCHULTZ 1993, SCHULTZ 2001, SCHULTZ 2011, WAPLER et al. 2004, SCHULTZ et al. 2007). Die Differentialdiagnose mittels Lichtmikroskopie ist im Kapitel 2.2i) Lichtmikroskopie $\rightarrow$ Befundung der lichtmikroskopischen Präparate erläutert.

Es ist unwahrscheinlich, dass im Fall einer Skorbut-Erkrankung isolierte Veränderungen der Orbitae ohne Affektion anderer Schädelknochen auftreten (BRICKLEY und IVES 2006).

Wenn Hinweise auf die Ätiologie der Cribra orbitalia nicht vorliegen bzw. eine mikroskopische Diagnostik nicht möglich ist, so kann man Cribra orbitalia auch als unspezifischen Stressindikator werten (SCHULTZ und SCHMIDT-SCHULTZ 2013, SCHULTZ et al. 2007, WAPLER et al. 2004).

Alle Schädel wurden auf das Vorhandensein von Cribra orbitalia untersucht. Dabei wurde, wie im Kapitel 2.2 f) Makroskopische Untersuchung erläutert, die folgende Einteilung nach SCHULTZ (1988) verwendet:

Grad I: Leichte beginnende Veränderungen in Form einzelner, feinporöser Lochdefekte

\footnotetext{
${ }^{85}$ S. 272

${ }^{86}$ S. 12
} 
Grad II: Auffällig poröses Orbitadach; deutlich ausgeprägte Lochdefekte treten miteinander in Verbindung; geringgradige, d.h. sehr flache Vorwölbung der porösen Knochenneubildungen Grad III: Kräftiges trabekuläres Wachstum aus der Diploë des Orbitadaches in die Augenhöhle

Von den 36 in dieser Arbeit untersuchten Individuen weisen 15 (41,7\%) Cribra orbitalia auf, davon 14 Kinder (38,9\%). Dreizehn der Individuen mit Cribra orbitalia sind an Skorbut erkrankt $(36,1 \%)$, bei einem $(2,8 \%)$ besteht Verdacht auf Skorbut, eins leidet an Anämie $(2,8 \%)$.

Zwölf Individuen (33,3\%) zeigen unauffällige Orbitadächer, darunter sechs mit Skorbut $(16,7 \%)$ und drei $(8,3 \%)$ mit Verdacht auf Skorbut.

Dies zeigt, dass Cribra orbitalia ein zwar häufiges, aber nicht immer auftretendes und darüber hinaus sehr unspezifisches Zeichen für Skorbut sind.

\section{Os zygomaticum}

Am Os zygomaticum kann es durch Kontraktion des hier verlaufenden M. temporalis bzw. des am Arcus zygomaticus entspringenden M. masseter zu Blutungen kommen. Es wird eine erhöhte Porosität beobachtet (LEWIS 2002, ORTNER et al. 1999, ORTNER et al. 2001, SCHULTZ et al. 2007). Klinisch wird eine Verdickung des Os zygomaticums beschrieben (BARLOW 1883 ${ }^{87}$ ).

\section{Mandibula}

Es liegen zahlreiche klinische Berichte über eine Beteiligung der Kiefer vor. So wird von einer Schwellung der Mandibula und einem spongiösen, zu Blutungen neigenden Zahnfleisch sowie von Zahnausfall berichtet (BARLOW 1883, FRAENKEL $1908^{88}$ ). Letzterer ist das Resultat eines durch Einbau fehlerhaften Kollagens geschwächten Zahnhaltapparats und wird durch Einblutungen verstärkt.

Auf der medialen Fläche des Processus coronoideus der Mandibula werden sowohl eine gesteigerte Porosität (BRICKLEY und IVES 2006, ORTNER et al. 2001) als auch feinporöse Auflagerungen beschrieben, die auf Gefäßschädigung im Rahmen des Kauakts durch Kontraktion des hier inserierenden M. temporalis zurückgeführt werden (ORTNER 2003, ORTNER et al. 1999, ORTNER et al. 2001). In einigen Fällen sind die beschriebenen Veränderungen nicht streng auf den medialen Processus coronoideus begrenzt, was für eine

\footnotetext{
${ }^{87}$ S. 169

${ }^{88}$ BARLOW 1883: S. 169, S. 174, S. 199, FRAENKEL 1908: S. 10, S. 28
} 
Mitbeteiligung der das Foramen mandibulae durchtretenden Strukturen spricht (BRICKLEY und IVES 2006). Sollte die Porosität wachstumsbedingt sein, so würde man einen regelmäßigen Verlauf entlang des Alveolarfortsatzes erwarten (BRICKLEY und IVES 2006). Es werden auch Veränderungen im Bereich des Ramus mandibulae und im Alveolarbereich des Corpus mandibulae beschrieben (MAYS 2008, KREUTZ 1997, SCHULTZ 1987, SCHULTZ 1993, SCHULTZ et al. 2003, SCHULTZ et al. 2007, TEMPLIN 1993, WEIHMANN 2010).

SCHULTZ (1994) führt poröse Auskleidungen der Alveolen von Ober- und Unterkiefer als Nebenmerkmal für Skorbut an (SCHULTZ 1990, 1994, SCHULTZ et al. 2003, siehe auch KREUTZ 1997). Im Rahmen der vorliegenden Arbeit konnte dies fünf Mal nachgewiesen werden, und zwar ausschließlich bei Skorbutfällen. Man könnte dieses Merkmal als sehr selten, aber recht spezifisch für Skorbut werten, vermutlich kommt es nur in besonders schweren Fällen infantilen Skorbuts vor.

\section{Maxilla}

Aus der Klinik sind Schwellungen der Maxilla bekannt (BARLOW 1883 ${ }^{89}$ ), die allerdings nicht zu den häufigsten Symptomen des Skorbuts gezählt werden (FRAENKEL $1908^{90}$ ).

An der Maxilla weisen verschiedene Autoren Oberflächenveränderungen nach, die sie auf Skorbut zurückführen (BRICKLEY und IVES 2006, LEWIS 2002, ORTNER et al. 1999, ORTNER et al. 2001, ORTNER und ERICKSEN 1997, SCHULTZ et al. 2007).

Zum einen wird eine erhöhte Porosität des posterioren Anteils der Maxilla dokumentiert, was Ortner und seine Koautoren - ähnlich wie die Veränderungen am Os sphenoidale - auf die mechanische Irritation der Blutgefäße während der Kontraktion der Kaumuskulatur zurückführen (ORTNER 2003, ORTNER et al. 2001, ORTNER und ERICKSEN 1997). BRICKLEY und IVES (2006) beobachten Veränderungen auch bei Individuen, die zu jung zum Kauen waren. Sie nehmen an, dass auch das Saugen und Nuckeln von Säuglingen zur Schädigung skorbutischer Gefäße führen kann (BRICKLEY und IVES 2006).

Auch im Bereich des Foramen infraorbitale werden skorbutische Läsionen beobachtet (BRICKLEY und IVES 2006, ORTNER et al. 2001, ORTNER et al. 1999).

BRICKLEY und IVES (2006) berichten, dass die pathologische Porosität am ausgeprägtesten im Bereich des Cortex oberhalb des Alveolarfortsatzes bis zum Processus frontalis sei.

\footnotetext{
${ }^{89}$ S. 169

${ }^{90}$ S. 11
} 
Um eine pathologisch gesteigerte gegen die beim Zahndurchbruch physiologischerweise erhöhte Porosität abzugrenzen, wird verlangt, dass der poröse Bereich deutlich über den Alveolarbereich hinausreicht (BRICKLEY und IVES 2006, ORTNER et al. 1999).

Der knöcherne Gaumen ist eine weitere typische Lokalisation für Porosität (KREUTZ 1997, MAYS 2008, ORTNER et al. 2001, SCHULTZ 1987, SCHULTZ 1990, SCHULTZ 1993, TEMPLIN 1993, WEIHMANN 2010). Auch hier muss zur Abgrenzung gegen normale Porosität die Ausdehnung der Veränderungen weit über den an den Alveolarfortsatz angrenzenden Bereich und den parallel dazu verlaufenden U-förmigen Saum physiologischer Porosität hinausgehen (BRICKLEY und IVES 2006, ORTNER et al. 2001, ORTNER et al. 1999). Klinisch ist das Symptom der Stomatitis mit geschwollener, zu Blutungen neigender Mundschleimhaut und Zahnfleisch bekannt, allerdings weist BARLOW (1883 ${ }^{91}$ ) nach, dass dieses Symptom keinesfalls pathognomonisch für Skorbut sei, was seinerzeit verbreitete Meinung war, und durchaus auch fehlen kann. Sowohl BARLOW (1883) als auch FRAENKEL $1908^{92}$ machen die Beobachtung, dass Zahnfleischveränderungen nur bei Kindern auftreten, die bereits Zähne haben (BARLOW 1883, FRAENKEL 1908, siehe auch LEWIS 2002). Besonders ausgeprägt sind die Veränderungen im Bereich der Schneidezähne, vor allem, wenn diese bereits durchgebrochen sind (JAFFE 1972 $2^{93}$ ).

MELIKIAN und WALDRON (2003) weisen Porositäten der Alveolarränder so häufig nach, dass sie konstituieren, dass dies als normal, nicht als pathologisch gewertet werden müsse.

\section{Zähne}

\section{Verfärbung der Zahnwurzeln}

MAAT $(1982,2004)$ findet an den Zahnwurzeln niederländischer Seefahrer des 17. und 18. Jahrhunderts schwarze Verfärbungen, die er auf Zahnfleischblutungen zurückführt. Bei ähnlichen Befunden an den Langknochen gelingt ihm sogar der Nachweis von denaturiertem Hämoglobin (MAAT 1982, MAAT 2004). Dieser außergewöhnliche Befund ist sicher auf den besonders guten Konservierungszustand der sterblichen Überreste durch die Kälte Spitzbergens zurückzuführen. Für paläopathologische Untersuchungen an durchschnittlich erhaltenen Knochen ist dieses Kriterium vermutlich nicht geeignet.

In der vorliegenden Studie werden keine als Pathologie zu deutende Färbungen gefunden. Allerdings waren alle untersuchten Knochen schon einmal Gegenstand anderer Studien und somit vorgereinigt.

\footnotetext{
${ }^{91}$ S. 174, S. 199

${ }^{92}$ BARLOW 1883: S. 174, S. 199, FRAENKEL 1908: S. 10, S. 28

${ }^{93}$ S. 450
} 


\section{Verzögerter Zahndurchbruch}

BARLOW beschreibt in mehreren seiner Fälle einen teilweise stark verzögerten Zahndurchbruch (BARLOW 1883 ${ }^{94}$ ).

Dies kann bei der paläopathologischen Untersuchung zu Fehlern bei der Altersbestimmung führen. Daher sollten nach Möglichkeit mehrere Methoden, die unterschiedliche Altersmerkmale berücksichtigen, angewandt werden.

Im Tierversuch mit Meerschweinchen zeigt sich, dass unter Ascorbinsäuremangel die Odontoblasten die Dentinsynthese stoppen, sodass die Zähne zu wachsen aufhören, während bereits existierendes Dentin resorbiert wird. Außerdem kommt es zu Hämorrhagien und Mineralisierungen in der Pulpa (JAFFE $1972^{95}$ ).

\section{Transversale Schmelzhypoplasien}

Transversale Schmelzhypoplasien sind Schmelzbildungsstörungen der Zähne in Form von Rinnen. Es werden auch grübchenförmige Schmelzhypoplasien beschrieben (siehe auch Kapitel 1.5 Pathophysiologie des Skorbuts).

Sie sind kein spezifisches Merkmal für Skorbut, sondern können vielmehr als unspezifischer Stressindikator gewertet werden. Sie werden auf allgemeine Mangelernährung, Vitamin-DMangel, langwierige Infektionserkrankungen, (Geburts-) Traumata, Vergiftungen und Entwicklungsstörungen zurückgeführt (KREUTZ 1997, LEWIS 2002, SCHULTZ 1990, SCHULTZ und SCHMIDT-SCHULTZ 2013, SCHULTZ et al. 2003, SCHULTZ et al. 2007, TEMPLIN 1993).

Es wird vermutet, dass der durch unterschiedliche Stressfaktoren erhöhte Cortisonspiegel die Proteinsynthese hemmt und die Schmelzproduktion reduziert (LEWIS 2002). Ein gehäuftes Auftreten in einer Population, die auf Skorbut untersucht werden soll, spricht dafür, dass mangelhafte Ernährung ein Problem bei diesen Menschen war, sodass die Wahrscheinlichkeit für Mangelerkrankungen hoch erscheint. Auf der anderen Seite sollte man aus dem Fehlen transversaler Schmelzhypoplasien nicht schließen, dass die Ernährungslage unproblematisch war, denn es ist möglich, dass die Individuen unter widrigen Lebensbedingungen starben, bevor sich Schmelzhypoplasien manifestieren konnten (SCHULTZ et al. 2007). Außerdem kann auch eine verlängerte Stillzeit, wie sie gerade in ärmeren Populationen aus Mangel an Nahrung für ihre Kleinkinder, aber auch aus Gründen der Empfängnisverhütung häufiger zu finden ist, die Bildung von transversalen Schmelzhypoplasien bei Kindern verzögern. Wenn

\footnotetext{
${ }^{94}$ S. 177, S. 182

${ }^{95}$ S. 453, S. 458
} 
die Mutter nicht selbst unter einem schweren Calcium-Protein-Mangel leidet, enthält die Muttermilch genug Nähr- und Aufbaustoffe, um einen gesunden Zahnschmelz zu gewährleisten (KREUTZ 1997, SCHULTZ 1990).

Einmal entstandene Schmelzhypoplasien können nicht mehr repariert werden, sie bleiben zeitlebens bestehen (CARLI-THIELE 1996 ${ }^{96}$ ). Aufgrund ihrer Lage an der Zahnkrone kann das Alter des Individuums zum Zeitpunkt der Linienentstehung errechnet werden (SCHULTZ 1990).

\subsection{Skorbutische Läsionen am Postcranium}

\section{Langknochen}

BARLOW (1883) hält die Beteiligung der Extremitäten, insbesondere der unteren, für wegweisend in der Diagnosefindung. Klinisch fallen zumeist bilaterale Schwellungen auf, insbesondere am Oberschenkel. Sie sind gespannt und schmerzhaft, weisen aber keine Entzündungszeichen wie Überwärmung und Rötung auf (BARLOW 1883 ${ }^{97}$ ). Die Hauptursache für diese Schwellungen sind subperiostale Blutungen, darüber hinaus werden Ödeme beschrieben, markanterweise stärker am Oberschenkel als am Unterschenkel und im Knöchelbereich (BARLOW 1883 ${ }^{98}$ ). In nahezu allen beschriebenen Fällen ist die Beteiligung der unteren Extremität wesentlich ausgeprägter als die der oberen, was bei Individuen, die den aufrechten Gang schon beherrschen, auf das Körpergewicht zurückgeführt wird (BARLOW 1883, FRAENKEL 1908, JAFFE 1972, MAAT 2004, ORTNER 2003, ORTNER und PUTSCHAR $1981^{99}$ ).

Die Hämatome beginnen in der Regel auf Höhe einer frakturierten Metaphyse, können aber das Periost auf ganzer Schaftlänge abheben (LEWIS 2002, ORTNER 2003, ORTNER und PUTSCHAR $1981^{100}$ ), was - wie bereits diskutiert - zu reaktiver Knochenneubildung führt. Aufgrund der fehlerhaften Knochenbildung kommt es an den Langknochen außerdem zu einer Ausdünnung der Compacta, insbesondere im Bereich der Metaphyse, was die Brüchigkeit der Knochen erhöht (ORTNER 2003, ORTNER und PUTSCHAR $1981^{101}$ ).

\footnotetext{
${ }^{96}$ S. 168

${ }^{97}$ S. 166

${ }^{98}$ S. 166

${ }^{99}$ BARLOW 1883:, FRAENKEL 1908:, JAFFE 1972: S. 455, ORTNER/PUTSCHAR 1981:

S. 270

${ }^{100}$ S. 270

${ }^{101}$ S. 270
} 
Die Schmerzen der Extremitäten bringen die Patienten dazu, sich so wenig wie möglich zu bewegen, sie verfallen in eine so genannte Pseudo-Paralyse (BARLOW $1883^{102}$ ).

Spuren organisierter subperiostaler Hämatome an den Langknochen finden sich häufig an den hier untersuchten Skeleten: Insgesamt weisen 23 von 36 Individuen (64\%) Auflagerungen an den Langknochen auf. Davon sind 21 (58\%) an Skorbut erkrankt, nur zwei Individuen (6\%) haben andere Diagnosen. Damit kann die Spezifität dieses Merkmals als hoch gewertet werden. Unter den untersuchten Kindern weisen 17 von 25 (68\%) dieses Merkmal auf, die auch alle an Skorbut erkrankt sind; bei den adulten Individuen sind es vier von elf (36\%), und zwar alle adulten Skorbutfälle. Betrachtet man nur die Skorbutfälle, so haben 21 von 27 (78\%) poröse Auflagerungen an den Langknochen. Bei den infantilen Skorbutfällen zeigen 17 von 23 (74\%) dieses Merkmal. Die adulten Skorbutfälle weisen alle Auflagerungen an den Langknochen auf.

Dazu kommen noch jene Individuen, die eine poröse Oberfläche der Langknochen aufweisen, was auch als eine Folge der subperiostalen Hämatome gewertet wird. Achtzehn von 36 (50\%) Individuen haben poröse Langknochenoberflächen. Davon sind 15 (42\%) Individuen an Skorbut erkrankt, drei (8\%) nicht.

Aufgrund des häufigen Vorkommens dieser Veränderungen sollten poröse Auflagerungen bzw. Oberflächen als wichtiges Merkmal für Skorbut gewertet werden. Besonders interessant erscheinen diese Veränderungen für die Diagnostik von Skorbut an adulten Individuen, da sie - im Gegensatz zu den meisten anderen Merkmalen - auch hier sehr gut ausgeprägt sind.

\section{Epiphysen}

Die Epiphysen sind makroskpisch meist unauffällig und weisen keine Frakturen auf (JAFFE $\left.1972^{103}\right)$.

In der radiologischen Untersuchung kann gelegentlich auch noch nach Jahren der sogenannte Wimberger Ring sichtbar sein, verursacht durch Osteoporose der sekundären Ossifikationszentren, umgeben von vermehrt mineralisiertem Knorpel in der wachsenden Peripherie (LEWIS 2002, ORTNER 2003, ORTNER und PUTSCHAR $1981^{104}$ ).

In der vorliegenden Studie konnte diese Veränderung in keinem Fall nachgewiesen werden.

\footnotetext{
${ }^{102}$ S. 166

${ }^{103}$ S. 455

${ }^{104}$ S. 272
} 


\section{Epiphysendislokation}

Durch Einblutungen zwischen Schaft und Epiphyse und fehlerhafte Ossifikation im Bereich der Epiphysenfuge kann es bei Kindern zu Epiphysendislokationen kommen, die sich radiologisch und klinisch gelegentlich durch einen Crepitus nachweisen lassen (AUFDERHEIDE und RODRÍGUEZ-MARTÍN 1998, BARLOW 1883, FRAENKEL $1908^{105}$, LEWIS 2002). Epiphysendislokationen sind an historischen Skeleten schlecht nachzuweisen, da bei kleinen Kindern, deren Epiphysenfugen noch nicht geschlossen sind, die Knochenteile getrennt erhalten sind. Der Nachweis einer Epiphysendislokation könnte also nur erbracht werden, wenn an Epiphyse oder Schaft Spuren zu erkennen wären, die auf eine Fehlstellung der Knochenteile zueinander schließen ließen, oder bei Erwachsenen, die in ihrer Kindheit/Jugend an Skorbut litten und bei denen Epiphyse und Schaft falsch zusammenwuchsen. In letzterem Fall wäre auch ein fehlendes Längenwachstum zu erwarten. Bei den hier untersuchten Skeleten war kein Nachweis einer Epiphysendislokation möglich. BARLOW (1883) beobachtet allerdings auch, dass im Falle einer Heilung in der Regel keine Deformationen zurückbleiben (BARLOW 1883 $\left.{ }^{106}\right)$. MAAT $(1982,2004)$ beschreibt bei den kältekonservierten Skeleten niederländischer Seefahrer eine Schwarzfärbung, die er auf Blutungen zurückführt, an beiden Seiten der Epiphysenplatten der unteren Extremität bei Jugendlichen (MAAT 1982, 2004, MAAT und UYTTERSCHAUT 1984). Dies könnte auf eine Epiphysendislokation hinweisen.

\section{Infraktionen}

Durch die fehlerhafte Kollagensynthese ist die Stabilität der Knochen herabgesetzt. Dies kann zu sogenannten Infraktionen führen, also unvollständigen Frakturen mit Spaltbildungen. Diese sind in der Regel auf die Substantia corticalis beschränkt. Sie werden vor allem im Bereich der Metaphysen der unteren Extremität beobachtet und verlaufen oft longitudinal zum Schaft (AUFDERHEIDE und RODRÍGUEZ-MARTÍN 1998, FRAENKEL 1908 ${ }^{107}$, MAAT 1982, 2004). Sie sind eine mögliche Ursache für subperiostale Hämatome, da die entstandene Öffnung der Corticalis den Austritt von Blut aus dem Knochenmark ermöglicht (MAAT 1982, MAAT und UYTTERSCHAUT 1984). Vermutlich sind sie Ausdruck eines stark ausgeprägten Skorbuts (MAAT 1982).

\footnotetext{
${ }^{105}$ BARLOW 1883: S. 167, S. 180, S. 185, S. 186, FRAENKEL 1908: S. 22

${ }^{106}$ S. 168

${ }^{107}$ S. 10, S. $22 \mathrm{f}$
} 
Im Rahmen der vorliegenden Studie wurde nur bei einem Skelet eine Infraktion (Sk 1334) nachgewiesen, was allerdings darauf zurückzuführen sein kann, dass eine Abgrenzung zu postmortaler Schädigung teilweise sehr schwierig ist.

\section{Metaphysen der Langknochen}

ORTNER et al. (2001) weisen eine erhöhte Porosität im Bereich der Metaphysen von Langknochen subadulter Individuen nach. Die Grenze zwischen einer physiologischen, durch kindliches Wachstum bedingten, und einer pathologischen Porosität setzen sie bei einer Ausdehnung der Porosität von mehr als 5-10 mm. Reicht die Veränderung über diesen Abstand von der Metaphysenplatte hinaus, vermuten die Autoren eine pathologische Ursache, wahrscheinlich Skorbut (ORTNER et al. 2001).

MAAT $(1982$, 2004) weist auch an den Metaphysen der unteren Extremität die oben beschriebenen Verfärbungen sowie Infraktionen nach.

AUFDERHEIDE und RODRÍGUEZ-MARTÍN (1998) beobachten eine Verbreiterung der Metaphysen durch organisierte Hämatome an den Handgelenken, Knien und Fußgelenken von kleinen Kindern.

WEIHMANN (2010) beschreibt eine Osteophytenbildung im Bereich der Metaphysenplatte, die diese als ,gekrempelt“ erscheinen lässt.

JAFFE (1972) beschreibt eine Rarifizierung der Trabeculae und Ausdünnung der Corticalis in diesem Bereich, das Mark erscheint gelatinös und mit Detritus verfüllt (JAFFE 1972 ${ }^{108}$ ). Dies entspricht den von FRAENKEL (1908) geschilderten Veränderungen im Bereich der Trümmerfeldzone (FRAENKEL $1908^{109}$ ).

In der vorliegenden Studie konnte nur das Merkmal einer erhöhten Porosität bzw. von Auflagerungen im Metaphysenbereich nachgewiesen werden.

\section{Markblutungen}

Bei Kindern sind Einblutungen in die Markhöhle sehr selten, bei Erwachsenen häufiger beobachtet worden (JAFFE 1972 $2^{110}$ ). Bei den hier untersuchten Individuen ergab sich kein Hinweis auf Markblutungen. Allerdings wurde die Markhöhle auch nicht zu Untersuchungszwecken eröffnet.

\footnotetext{
${ }^{108}$ S. 455 f, S. 458

${ }^{109}$ S. 16

${ }^{110}$ S. 455
} 


\section{Harris-Linien}

Harris-Linien, oft auch als „Arrested Growth Lines“ (z.B. bei ORTNER und PUTSCHAR 1981) bezeichnet, gelten als unspezifischer Stressindikator (CARLI-THIELE 1996, KREUTZ 1997, SCHULTZ 1990, SCHULTZ et al. 2003, SCHULTZ et al. 2007, TEMPLIN 1993). Sie treten bei unterschiedlichen Belastungen im Kindesalter auf.

Ihr Zustandekommen erklärt man sich aus einem in einer Stressphase erfolgten Wachstumsstillstand und dem darauf wieder einsetzenden Längenwachstum des Langknochens. Im Röntgenbild stellt sich dies als strahlendichte transversale Linie dar. Vermutlich entsteht dieses Segment erhöhter Mineralisierung durch eine Verlangsamung der Teilungsrate der Knorpelzellen bei gleichzeitigem Fortbestand der Mineralisierung (LEWIS 2002). Die Chondroblasten reagieren wohl schneller auf Stress und hören auf, Knorpel zu synthetisieren. Die Osteoblasten hingegen produzieren weiterhin Osteoid, das sich als dünne Linie entlang der stagnierenden Wachstumsplatte auf die Knorpelkappe auflegt. Diese Linie allein ist noch nicht radiologisch nachweisbar. Doch wenn das normale Wachstum wieder einsetzt, erholen sich die Osteoblasten wiederum schneller als die Chondroblasten, was zur Synthese einer weiteren Osteoidschicht führt. Die beiden Schichten zusammen sind radiologisch als Harris-Linien zu erkennen (LEWIS 2002, SCHULTZ 1988, 1990, SCHULTZ et al. 2003).

Besonders häufig finden sich Harris-Linien an der Tibia, am distalen Femur, am distalen Radius, am Metacarpus und an den anterioren Rippenenden (SCHULTZ 1988).

Weibliche Individuen scheinen häufiger betroffen $\mathrm{zu}$ sein als männliche; gehäuftes Vorkommen findet sich postnatal und im Jugendalter (SCHULTZ 1988). Verschiedene Studien lassen vermuten, dass Harris-Linien nur bis zum Alter von etwa zwölf Jahren bei Mädchen und 14 Jahren bei Jungen entstehen (LEWIS 2002), möglicherweise hemmt der dann erhöhte Somatotropinspiegel ihre Ausbildung. Spätestens mit dem Schluss der Epiphysenfugen können keine Harris-Linien mehr entstehen (LEWIS 2002).

Bei manchen Individuen lassen sich mehrere Harris-Linien in einem Knochen nachweisen. Dies lässt darauf schließen, dass es einen häufigen Wechsel zwischen Stress- und Erholungsphasen gab, kontinuierlich schlechte Bedingungen würden nicht zur Ausprägung einer Harris-Linie führen, da für ihre Entstehung das in der Erholungsphase wieder einsetzende Wachstum wesentlich ist (LEWIS 2002). Im Laufe der Zeit können Harris-Linien durch subperiostalen und endostalen Umbau abgebaut werden, wobei die Wahrscheinlichkeit, abgebaut zu werden, bei sehr früh in der Kindheit entstandenen Harris-Linien geringer ist. 
Es ist unklar, ob der passagere Wachstumsstillstand $\mathrm{zu}$ einem verminderten Gesamtlängenwachstum führt, vermutlich wird er aber durch den folgenden Wachstumsschub kompensiert.

Als ursächlich für den Wachstumsstillstand werden verschiedene Faktoren angenommen. So zählen Mangelernährung und Infektionen zu den Hauptauslösern (CARLI-THIELE 1996, KREUTZ 1997, SCHULTZ 1990, SCHULTZ et al. 2003, TEMPLIN $1993^{111}$ ).

Aufgrund dieser Tatsache scheint eine Untersuchung auf Harris-Linien auch im Rahmen einer Suche nach Anzeichen für Skorbut sinnvoll. Zwar dürfen sie keinesfalls als pathognomonisch gewertet werden, sind jedoch ein guter Hinweis auf einen insgesamt geschwächten Organismus, wie es durch Vitamin-C-Mangel bedingt sein kann.

Bei den untersuchten Individuen finden sich in elf von 36 Fällen Harris-Linien.

\section{Längenwachstum}

LEWIS (2002) weist einen signifikanten Größenunterschied zwischen Kindern mit metabolischen Erkrankungen (im speziellen Skorbut und Rachitis) und gesunden Kindern nach (LEWIS 2002). Dies an historischen Skeleten zu belegen, erscheint sehr schwierig, da die Altersbestimmung bei Kindern im Wesentlichen auf der Länge der Langknochen, der Entwicklung von Ossifikationszentren, dem Schluss der Schädelnähte und dem Zahndurchbruch beruht. Sind diese Faktoren gestört, so wird das Individuum vermutlich als zu jung eingeschätzt, ohne dass diese Diskrepanz erkannt werden kann.

\section{Femur}

BARLOW (1883) beschreibt die Affektion der Oberschenkel in seinen Fällen zumeist als die heftigste. Er berichtet sowohl über Weichteilbeteiligung als auch über subperiostale Blutungen. Bei den Muskeleinblutungen findet er Blutkoagel in den tieferen Muskelschichten, in den oberflächlichen Schichten weist er Serum nach. Auch Epiphysendislokationen werden beschrieben. Es kommt auch zu spontanen Knochenbrüchen/Infrakturen ohne erkennbares Trauma, vermutlich bedingt durch die veränderte Knochenstruktur (Ausdünnung der Trabeculae). Eine Callusbildung wird nicht beobachtet (BARLOW 1883 ${ }^{112}$ ). FRAENKEL (1908) bestätigt diese Beobachtungen, hält sie allerdings für verhältnismäßig selten und weniger wichtig für die Diagnosesicherung (FRAENKEL $1908^{113}$ ).

\footnotetext{
${ }^{111}$ S. $167 f$

${ }_{112}$ S. 167 , S. 180 , S. 183 , S. 185 , S. $187 f$

${ }^{113}$ S. 10
} 
Wenn die Metaphyse des Femurs stark betroffen ist, kann es zu Einbrüchen direkt unterhalb des Femurkopfes, aber aufgrund der Trajektionskräfte oberhalb des Trochanter major, kommen, was zu einer Verkleinerung des Schenkelhalswinkels führt. Im Heilungsverlauf kommt es jedoch in der Regel nicht zu bleibenden Deformationen (ORTNER und PUTSCHAR $1981^{114}$ ).

MAAT $(1982,2004)$ weist Infraktionen und subperiostale Hämatome an den Femura nach. Außerdem beschreibt er ein Hämarthros der Hüft- und Kniegelenke. Letzteres ist eine Eigenart des Skorbuts bei Erwachsenen (JAFFE 1972 ${ }^{115}$ ), wohingegen bei der MöllerBarlow'schen Erkrankung ein Freibleiben der Gelenke auffällt (FRAENKEL $1908^{116}$ ).

\section{Tibia}

BARLOW (1883) schreibt, die Unterschenkel seien meist weniger stark befallen als die Oberschenkel, die Tibia aber in stärkerem Maße als die Fibula. Subperiostale Hämatome und auch Epiphysendislokationen der proximalen Tibia beschreibt er (BARLOW 1883 ${ }^{117}$ ). In den Untersuchungen von MAAT $(1982,2004)$ weisen die Tibiae stärkere Veränderungen auf als die Femura. Außerdem wird hier mehrfach ein Hämarthros der Fußgelenke beschrieben (MAAT 1982, 2004).

\section{Fibula}

BARLOW (1883) beschreibt auch subperiostale Blutungen an der Fibula, diese seien jedoch geringer ausgeprägt als an der Tibia (BARLOW 1883 ${ }^{118}$ ). Bei MAATs (2004) Untersuchungen dagegen sind die Fibulae die am häufigsten betroffenen Knochen.

\section{Scapula}

In den Fossa supra- und infraspinata verlaufen die Gefäße zwischen Muskeln und Knochen. Dies wird als Ursache für die recht häufig nachgewiesenen Folgen von Hämorrhagien in diesem Bereich angenommen (BRICKLEY und IVES 2006, ORTNER et al. 2001,). Hämorrhagien scheinen in der Fossa supraspinata häufiger aufzutreten als in der Fossa infraspinata (BRICKLEY und IVES 2006). Allerdings berichtet BARLOW (1883) häufiger über eine Schwellung im Bereich der Fossa infraspinata, rechts zumeist ausgeprägter als links.

\footnotetext{
${ }^{114}$ S. 272

${ }^{115}$ S. 455

${ }^{116}$ S. 9, S. 23

${ }^{117}$ S. 167, S. 180, S. 185 , S. 186

${ }^{118}$ S. 167, S. 180, S. 185
} 
Außerdem findet er auch subperiostale Blutungen auf der Ventralseite der Scapulae (BARLOW 1883 ${ }^{119}$ ). Auch FRAENKEL (1908) berichtet über das gelegentliche Auftreten von Hämatomen an den Schulterblättern (FRAENKEL 1908 ${ }^{120}$ ). MAAT (1982, 2004) beschreibt ein Hämarthros des Schultergelenks.

Bei den hier untersuchten 36 Individuen lassen sich bei zehn Individuen (neun Kinder, ein Erwachsener) feinporöse Auflagerungen als vermutliche Folge von subperiostalen Blutungen am Schulterblatt nachweisen. Dabei sind ausschließlich Individuen mit Skorbut betroffen. Dieses Merkmal kann also als zwar verhältnismäßig seltener, aber sehr sicherer Indikator für Skorbut gewertet werden.

\section{Humerus}

BARLOW (1883) weist eine Beteiligung des Humerus in einigen Fällen nach; allerdings sind die oberen Extremitäten meist schwächer befallen als die unteren. Sowohl die Ausprägung der Schwellung als auch die Ausdehnung der subperiostalen Blutungen sind geringer als an den Beinen, oft begrenzt auf die Epiphysenregion (BARLOW 1883 ${ }^{121}$ ). MAAT $(1982,2004)$ findet auch einige Fälle von Infraktionen und subperiostalen Frakturen am Humerus, diese sind jedoch - im Gegensatz zu den Befunden an der unteren Extremität - in den meisten Fällen einseitig, teilweise korrelierend mit Muskelansätzen.

\section{Radius}

Der Radius kann sowohl isoliert als auch zusammen mit der Ulna befallen sein. Klinisch besonders ausgeprägt sind Schwellungen im Bereich der Handgelenke (BARLOW $1883^{122}$ ). Dazu passen möglicherweise die Nachweise eines Hämarthros im Bereich der Ellbogen und Handgelenke (MAAT 1982, 2004).

Es werden einige - großenteils einseitige - Infraktionen und subperiostale Hämatome an Radius und Ulna gefunden (MAAT 1982, 2004).

\footnotetext{
${ }^{119}$ S. 168, S. 184, S. 188

${ }^{120}$ S. 10

${ }^{121}$ S. 168

${ }^{122}$ S. 168
} 


\section{Ulna}

Es werden isolierte Veränderungen der Ulna, aber auch eine Beteiligung beider Unterarmknochen beschrieben. Besonders betroffen ist die distale Epiphysenregion (BARLOW $1883^{123}$ ).

\section{Rippen}

In Autopsien findet BARLOW (1883) eine Infiltration der Intercostalmuskulatur durch Serum. Das Periost ist verdickt und stark vaskularisiert und durch massive subperiostale Hämatome von den Rippen getrennt. Die Rippen selbst erscheinen klein und brüchig. Die Verbindung zwischen Rippenknorpel und knöchernem Anteil ist gestört, der Knochen ist ausgedünnt, und Knorpel und Knochen sind gegeneinander verschoben, die Enden des Rippenknorpels lassen sich durch die Haut gut tasten und erkennen (BARLOW $1883^{124}$ ). FRAENKEL (1908) hält die Veränderungen der Rippen für die frühesten und oft schwersten (FRAENKEL 1908 ${ }^{125}$ ). Allerdings spielen sich die Veränderungen in erster Linie am Übergang zum Rippenknorpel ab. Da der Rippenknorpel am mazerierten Knochen nicht erhalten ist, lassen sich die Verschiebungen nicht mehr nachweisen. Hinzukommt, dass bei kleinen Kindern auch die winzigen knöchernen Rippen oft nur teilweise erhalten sind. Deshalb kann am historischen Skelet nur ein Nachweis von erhöhter Porosität bzw. Neubildungen aufgrund von subperiostalen Hämatomen oder einer blutigen Pleuritis erfolgen. Diese sind an der pleuralen Seite der Rippen stärker ausgeprägt (FRAENKEL $1908^{126}$ ).

\section{Os ilii}

Es werden subperiostale Blutungen nahe der Crista iliaca beschrieben, ebenso Einblutungen in die tiefe Muskulatur (BARLOW $1883^{127}$ ).

Bei den hier untersuchten 36 Individuen lassen sich bei zehn Individuen (acht Kinder, zwei Erwachsene) feinporöse Auflagerungen als vermutliche Folge von subperiostalen Blutungen am Os ilii nachweisen. Dabei sind ausschließlich Individuen mit Skorbut betroffen. Dieses Merkmal kann also als zwar verhältnismäßig seltener, aber sehr sicherer Indikator für Skorbut gewertet werden.

\footnotetext{
${ }^{123}$ S. 168

${ }^{124}$ S. 182 f, S. 184 , S. 188

${ }^{125}$ S. $16 f$

${ }^{126}$ S. 22

${ }^{127}$ S. 183
} 


\subsection{Bedeutung der radiologischen Untersuchung}

FRAENKEL (1908) hält den Nachweis einer Trümmerzone im epiphysennahen Bereich der Langknochen (vgl. Kapitel 2.2h) Radiologische Untersuchung) für den wichtigsten radiologischen Hinweis auf Skorbut. Am mazerierten Knochen scheint sich diese nicht darzustellen, es wurde bei keinem der in der vorliegenden Studie untersuchten Knochen ein solches Phänomen beobachtet.

Dennoch sollten radiologische Untersuchungen bei der Befundung von historischen Skeleten nicht außer Acht gelassen werden, da sie eine Darstellung von Knochenneubildungen ermöglichen, die als Reaktion auf subperiostale Hämatome entstanden sind. In der vorliegenden Arbeit konnte dies in sieben Fällen (Sk 1279, Sk 1293, Sk 1298, Sk 1301, Sk 1356, Sk 1371, Sk 1380) beobachtet werden. Dabei sollte man beachten, dass dieser Nachweis vermutlich nur während einer gewissen Zeitspanne und nicht in jedem Fall erbracht werden kann, da es nicht immer zu einer Knochenneubildung kommt bzw. diese bei Abheilung und Integration röntgenologisch nicht mehr darstellbar ist. Eine Dokumentation erhöhter Vaskularisierung, wie sie sich bei minder schwerem Befall der Knochen oder auch nach erfolgter Integration als erhöhte Porosität der Knochenoberfläche darstellt, scheint im Röntgenbild historischer Skelete nicht möglich.

Ein weiterer Grund, radiologische Untersuchungen durchzuführen, ist die Möglichkeit, Harris-Linien nachzuweisen. Eventuell können Harris-Linien auch ein Residuum der bei LEWIS (2002) beschriebenen sogenannten „Skorbut-Linie“ darstellen, da auch deren Entstehung auf einer reduzierten Chondroblastenfunktion bei fortbestehender Osteoidsynthese durch die Osteoblasten bestehe (LEWIS 2002). Im Rahmen der vorliegenden Arbeit wurden Harris-Linien in 13 Fällen nachgewiesen (Sk 1272, Sk 1279, Sk 1292, Sk 1298, Sk 1334, Sk 1342, Sk 1363, Sk 1371, Sk 1372, Sk 1377, Sk 1380, Sk 1385, Sk 1386). 


\subsection{Differentialdiagnosen}

\section{a) Wachstum}

Bei Kindern ist eine der schwierigsten Differentialdiagnosen des Skorbuts das normale Wachstum. Es ist oft schwierig, pathologische Porositäten von der während des Wachstums physiologischen Zunahme der Vaskularisierung abzugrenzen (BRICKLEY and IVES 2006, ORTNER et al. 1999, ORTNER et al. 2001). Insbesondere bei fetalen Skeleten bzw. bei Individuen, die im Laufe des ersten Lebensjahrs verstorben sind, ist es diffizil, festzustellen, ob es sich bei knöchernen Strukturen um pathologische Neubildungen oder Vaskularisierung handelt, da in diesem Lebensalter nur Geflechtknochen gebildet wird, der eine rudimentäre Struktur und hohe Porosität aufweist (ORTNER et al. 1999). ORTNER et al. (1999) machen zum Kriterium einer pathologischen Porosität, dass die einzelnen Löcher die Substantia compacta durchdringen.

Das appositionelle Wachstum führt zum Dickenzuwachs des Knochens. Dabei geht das Wachstum vom Periost aus. Zunächst wird eine Schicht radialer Knochenleisten gebildet, die der Oberfläche aufgesetzt werden. Dadurch entstehen gefäßführende Längsrillen. Im zweiten Schritt entstehen tangentiale Leisten. Die Zwischenräume zwischen den beiden Schichten bilden die Grundlage für das spätere Havers'sche System. Bei der makroskopischen Betrachtung kann es insbesondere zu Verwechslung mit pathologischen Vorgängen kommen, wenn die tangentiale Schicht noch nicht gebildet bzw. erodiert ist, da der Knochen durch die Längsleisten eine raue, gerillt erscheinende Oberfläche erhält, die mit pathologischen Porositäten verwechselt werden kann (TEMPLIN 1993).

Auch in der Röntgenuntersuchung kann das appositionelle Wachstum zu Fehldiagnosen führen, da unregelmäßiges Compactawachstum teilweise die Ausbildung von Doppelkonturen bewirkt, die mit Auflagerungen nach Periostabhebung verwechselt werden können (TEMPLIN 1993).

Lichtmikroskopisch kann es problematisch sein, physiologisches appositionelles Wachstum von pathologischen Auflagerungen zu unterscheiden. Langknochen bei Säuglingen und Kleinkindern zeigen den Bau des sogenannten „Schalenknochen“. Dabei sind die Havers'schen Kanäle noch nicht voll ausgebildet, es liegen große, unförmige Kanäle zwischen den schalenartig angelagerten Knochenschichten. Die Kollagenfasern zeigen einen unreifen Aufbau. Erst unter funktioneller Belastung entwickelt sich mit zunehmendem Alter der typische Osteonenknochen (SCHULTZ 1986). 


\section{b) Anämie}

Bei Anämie können makroskopisch schwer von skorbutischen Läsionen zu differenzierende Veränderungen, nämlich poröse Hyperostosen, am Schädeldach und an den Orbitae auftreten, und zwar sowohl gemeinsam als auch unabhängig voneinander (CARLI-THIELE $1996^{128}$, ORTNER und PUTSCHAR 1981, SCHULTZ et al. 2007, SCHULTZ 2011, TEMPLIN 1993, WAPLER et al. 2004, WEIHMANN 2010).

SCHULTZ (1994) schlägt folgende makroskopische Untersuchungskriterien vor:

Kardinalmerkmale:

1. Verdicktes Schädeldach, Außenfläche porös

2. Verdicktes Orbitadach, Außenfläche porös (entsprechend Cribra orbitalia ${ }^{\circ}$ III-IV)

3. Poröses äußeres Schädeldach

4. Poröses Orbitadach (entsprechend Cribra orbitalia ${ }^{\circ}$ I-II)

5. Spongiosaverfüllung der Langknochenschäfte

Nebenmerkmale:

1. Verdicktes Schulterblatt

2. Verdicktes Os ilii

3. Verdickte Rippen

4. Verdickte Langknochenschäfte, insb. Metaphysen

5. „Aufblähung“ (=Verdickung) der Wirbelkörper (bes. Lendenwirbelsäule und Sacrum)

Pathophysiologisch liegen hier eine Hyperplasie des Knochenmarks und eine Ausdünnung oder sogar Eröffnung der Lamina externa zugrunde (WAPLER et al. 2004, SCHULTZ 2001, 2003). Dies ermöglicht eine mikroskopische Unterscheidung zwischen Anämie und Skorbut (siehe hierzu Kapitel 2.2i) Lichtmikroskopie $\rightarrow$ Befundung der lichtmikroskopischen Präparate).

Bei Anämie findet sich, vor allem bei Kindern, eine Verdickung der Diploë, die das Schädeldach insgesamt dicker erscheinen lässt. Dabei werden die Trabekel reduziert, die verbleibenden verdickt und radial angeordnet. Die Lamina externa wird zunehmend zerstört, die Schädeloberfläche erscheint porös. Es entstehen wabenartig strukturierte subperiostale Knochenneubildungen, die in der Röntgenuntersuchung ein typisches Bild zeigen, das als „hair on end“ oder auch „Bürstenschädel“ bezeichnet wird (CARLI-THIELE 1996, ORTNER und PUTSCHAR 1981 ${ }^{129}$, SCHULTZ 1986, TEMPLIN 1993).

${ }_{128}^{128}$ ORTNER/PUTSCHAR 1981: S. 252f, CARLI-THIELE 1996: S. 201

${ }^{129}$ ORTNER/PUTSCHAR 1981: S. 252f, CARLI-THIELE 1996: S. 200 
Selten finden sich auch Veränderungen am Postcranium; diese können vor allem bei Kindern auftreten, bei denen noch alle Knochen an der Hämatopoese beteiligt sind (ORTNER und PUTSCHAR 1981 ${ }^{130}$ ). Es zeigen sich stark erweiterte Markräume und vor allem im Metaphysenbereich eine ausgedünnte Corticalis mit vergrößerten Gefäßforamina. Mechanisch nicht zwingend benötigte Trabekel werden abgebaut, die verbleibenden liegen entlang der physikalischen Belastungslinien und erscheinen verdickt. Dadurch entsteht der Eindruck eines diagonalen Kreuzgeflechts, das besonders gut an den Rippen zu beobachten ist, bei denen die Corticalis vollständig resorbiert sein kann (ORTNER und PUTSCHAR 1981 ${ }^{131}$ ).

Die distale Femurmetaphyse erscheint durch gehemmtes Remodeling oft abgeflacht und verbreitert. Man findet Harris-Linien und Frakturen, letztere eher bei adulten Individuen. Die Wirbelkörper sind porös, von geringer Höhe und abgeflacht. Durch vorzeitigen Epiphysenschluss kann es zu Fehlstellungen der Knochenteile zueinander kommen; vor allem am Humeruskopf wird fehlerhaftes Zusammenwachsen beobachtet. Das Längenwachstum kann auch insgesamt früher aufhören und so zu Kleinwuchs führen (ORTNER und PUTSCHAR $1981^{132}$ ).

Eine Differenzierung der unterschiedlichen Anämieformen (z. B. Eisenmangelanämie versus Thalassämie) allein anhand von Knochenschliffen ist nicht möglich (SCHULTZ 1990, WAPLER et al. 2004). Verschiedene Autoren versuchen zwar eine Unterscheidung der unterschiedlichen genetischen und erworbenen Variationen anhand des Verteilungsmusters der Läsionen, auch dies scheint jedoch nur in eingeschränktem Maß möglich. Beispielsweise soll bei der Eisenmangelanämie keine Manifestation am Postcranium erfolgen (ORTNER und PUTSCHAR 1981). Knochennekrosen infolge von Ischämie bei Gefäßverschluss werden nur bei Sichelzellanämie beobachtet (CARLI-THIELE 1996, ORTNER und PUTSCHAR $1981^{133}$ ). Aufgrund der vielen Ursachen für Anämie sollte von einer einseitigen Interpretation im Sinne eines Eisenmangels abgesehen werden, vielmehr kann eine Anämie als eine Art Stressindikator betrachtet werden (SCHULTZ und SCHMIDT-SCHULTZ 2013).

ORTNER et al. (1999) berichten nur von einem einzigen Fall, der sowohl deutliche Zeichen für Skorbut als auch eine für Anämie typische Knochenmarkshyperplasie des Schädeldachs aufweist. Die Autoren vermuten, dass dieses Individuum sowohl unter Anämie als auch unter Skorbut litt (ORTNER et al. 1999). ORTNER et al. (2001) halten es für eher ungewöhnlich, dass ein Skelet Zeichen für mehr als eine Mangelkrankheit gleichzeitig aufweist. Es gibt

\footnotetext{
${ }^{130}$ S. 252

${ }^{131}$ S. 253

${ }^{132}$ S. $253 \mathrm{f}$

${ }^{133}$ ORTNER/PUTSCHAR 1981: S. 251-263, CARLI-THIELE 1996: S. 196
} 
allerdings Berichte über ein gehäuftes gemeinsames Auftreten von Skorbut und Anämie (FRAENKEL $1908^{134}$, LEWIS 2002, SCHULTZ 2001, SCHULTZ und SCHMIDTSCHULTZ 2013, TEMPLIN 1993).

Bei einer umfassenden hämatologischen Untersuchung von 36 Fällen infantilen Skorbuts werden makrozytäre, normozytäre, normochrome und mikrozytäre hypochrome Anämien beobachtet. Sie scheinen allerdings nicht primär durch den Skorbut bedingt zu sein (ZUELZER et al. 1949).

Außerdem ist zu bedenken, dass nicht jede Anämie sich am Knochen manifestiert, sondern dass die Dunkelziffer der nicht mehr diagnostizierbaren schweren Anämien bei historischen Skeleten weit höher liegen könnte als angenommen (WAPLER et al. 2004).

\section{c) Meningeale Reizung}

Wie in Kapitel 1.10 Differentialdiagnosen erwähnt, können viele Formen der meningealen Reizung zu porösen Oberflächenveränderungen und Auflagerungen am inneren Schädeldach führen, die skorbutischen Läsionen sehr ähneln. Dazu gehören bakterielle und hämorrhagische Meningitiden (siehe dazu auch Kapitel 4.5 f) Unspezifische Entzündungen: Periostitis, Ostitis und Osteomyelitis), epidurale Hämatome (durch Traumata, z. B. auch Geburtstraumata), Irritationen der venösen Sinus und Neoplasien. Zur Unterscheidung ist meist eine mikroskopische Untersuchung notwendig (KREUTZ 1997, SCHULTZ 1987, SCHULTZ 1993, SCHULTZ 2011, SCHULTZ et al. 2007, TEMPLIN 1993).

\section{d) Rachitis}

Wie bereits in Kapitel 1.10 Differentialdiagnosen beschrieben, stellte die klinische Trennung von Skorbut und Rachitis lange Zeit ein Problem dar.

Rachitis ist eine Erkrankung bei Kindern, die durch Vitamin-D-Mangel hervorgerufen wird. Sie entspricht der Osteomalazie der Erwachsenen.

Die Aufnahme des Steroidderivats Vitamin D erfolgt über zwei Wege. Über die Haut wird 7Dehydrocholesterol aufgenommen und durch UV-Licht- und Wärmeeinwirkung in Vitamin $\mathrm{D}_{3}$ (Cholecalciferol) umgewandelt. Dieses kann, zusammen mit Vitamin $\mathrm{D}_{2}$ (Ergocalciferol), auch über die Nahrung aufgenommen werden. In der Leber erfolgt die Hydroxylierung zu Calcidiol (25-Hydroxycholecalciferol), welches in der Niere zu Calcitriol (1,25- 
Dihydroxycholecalciferol) umgewandelt wird. Erst die Hydroxilierungsschritte führen zur biochemischen Aktivierung des Hormons (BÄSSLER et al. 2002, LÖFFLER $2001^{135}$ ).

Werden zu wenig Vitaminvorstufen über die Haut und vor allem die Nahrung aufgenommen, entwickelt sich Rachitis. Deren Manifestation am Skeletsystem erklärt sich aus der biologischen Funktion des Vitamin D. Zum einen führt Vitamin D zu einer gesteigerten intestinalen bzw. renalen Resorption von Calcium und Phosphat. Außerdem stimuliert es auf unterschiedliche Weise sowohl die Osteoblasten- als auch die Osteoklastentätigkeit, führt also zu einem Gleichgewicht zwischen Knochenauf- und abbau und trägt somit zu einer optimalen Knochenbildung und Knochendichte bei (BÄSSLER et al. $2002^{136}$ ).

Fehlt Vitamin D, so kommt es zu einem Calcium- und Phosphatmangel, was Ossifikationsstörungen, insbesondere der wachsenden Knochen, bedingt. Die Knorpelproliferation und die Knochenmatrixsynthese durch die Osteoblasten funktionieren weitgehend normal, aber die Mineralisierung bleibt aus. Dadurch sammeln sich vor allem im Bereich der Wachstumsplatten nicht kalzifizierter Knorpel und unmineralisiertes Osteoid. Dies führt zu einer Verbreiterung und becherförmigen Einbuchtung der Metaphysen der Langknochen, besonders sind die das Körpergewicht tragenden Knochen, wie die Knieregion, betroffen (ORTNER und PUTSCHAR 1981 ${ }^{137}$, SCHULTZ und SCHMIDT-SCHULTZ 2013).

An den Rippen kommt es zu Auftreibungen der osteocartilaginösen Verbindungen, die klinisch als „Rachitischer Rosenkranz“ imponieren (TEMPLIN 1993), was zu Verwechslungen mit Skorbut führen kann. Am mazerierten Knochen lässt sich manchmal noch eine Abflachung der normalen Rippenkrümmung feststellen.

Das schnell wachsende Schädeldach des Kindes wird abgebaut und durch nicht mineralisiertes Osteoid ersetzt, in diesen Regionen erscheint das Schädeldach dünn und weich, der Fontanellenschluss ist verzögert. Man bezeichnet dieses Bild als Craniotabes. Die weiche Substanz kann zu Verformungen des Schädels, z.B. durch Liegen, führen. Am mazerierten Knochen erkennt man poröse periostale Knochenauflagerungen, insbesondere an den Tubera parietalia und frontalia, am Orbitadach und in der Supranasalregion, seltener an der Lamina interna (ORTNER und PUTSCHAR 1981'138, SCHULTZ 2011, SCHULTZ und SCHMIDT-SCHULTZ 2013, SCHULTZ et al. 2007, TEMPLIN 1993). Dabei handelt es sich

${ }^{135}$ LÖFFLER 2001: S. 494-497, BÄSSLER et al. 2002: S. 361-364

${ }^{136}$ S. $365-368$

${ }^{137}$ S. 274, S. 278

${ }^{138}$ S. 274 , S. 278 
um die Überreste des durch alte Pathologen so bezeichneten „Rachitischen Osteophyten“ (SCHULTZ und SCHMIDT-SCHULTZ 2013, TEMPLIN 1993).

Man unterscheidet zwei Formen der Rachitis: Eine poröse Form, die durch allgemeine Mangelernährung bedingt ist und eine Kombination aus Rachitis und Osteoporose darstellt. In diesem Fall ist die Knochensubstanz reduziert, die Corticalis ausgedünnt, das Trabekelwerk rarefiziert. Dies kann zu Stressfrakturen führen, insbesondere im Diaphysenbereich. Bleibende axiale Deformationen sind häufig.

Die andere ist die hyperplastische Form der Rachitis. Diese tritt bei isoliertem Vitamin-DMangel auf und ist gekennzeichnet durch massive endostale und periostale Osteoidablagerung. Die Knochen erscheinen plump, die Markräume verengt (ORTNER und PUTSCHAR $1981^{139}$ ).

Im Rahmen des Heilungsprozesses kommt es zur Mineralisierung der periostalen Auflagerungen; in diesem Fall lassen sie sich auch am mazerierten Knochen nachweisen. Dies kann zu Verwechslungen mit Skorbut führen. Allerdings haben sie ein spezifisches Verteilungsmuster, was auch makroskopisch eine Abgrenzung von Skorbut ermöglicht. So finden sich die Auflagerungen an den Rippen nur an der Außenseite und den Rändern, am Femur dorsal stärker als ventral, an der Tibia nur posterior und medial, die laterale Seite bleibt frei (ORTNER und PUTSCHAR 1981 ${ }^{140}$, SCHULTZ und SCHMIDT-SCHULTZ 2013).

Ein weiteres Kriterium für Rachitis, das auch am mazerierten Knochen gut nachzuweisen ist, sind die Deformationen der Langknochen, die teilweise auf Stressfrakturen, teilweise auf mechanische Belastung zurückzuführen sind. Bei sehr kleinen Kindern, die noch nicht laufen können, treten vor allem Verformungen an den Armknochen auf, bei Kindern, die laufen, kommt es meist zu Coxa vara, der Femur und die Unterschenkelknochen verbiegen sich.

Die Wirbelkörper können komprimiert erscheinen, das Becken ist oft abgeflacht.

Das Längenwachstum bleibt zurück, insbesondere die Femura sind verkürzt (ORTNER und PUTSCHAR $1981^{141}$ ).

Auch die Mineralisierung der Zähne ist gestört, der Zahndurchbruch ist verzögert, die Zähne weisen oft Fehlstellungen und Schmelzdefekte auf, der Kiefer kann deformiert sein (BÄSSLER et al. 2002 ${ }^{142}$ ).

\footnotetext{
${ }^{139}$ S. $274 \mathrm{f}$

${ }^{140}$ S. 275,277

${ }^{141}$ S. 257,277, S. 279

${ }^{142}$ S. 377
} 
Die Altersverteilung der Rachitis ist ähnlich wie beim infantilen Skorbut: Selten gibt es Erkrankungen vor dem vierten Lebensmonat, da die Säuglinge noch von intrauterin erworbenen Reserven zehren. Der Verteilungsgipfel liegt in der Altersgruppe zwischen sechs Monaten und zwei Jahren (ORTNER und PUTSCHAR 1981 ${ }^{143}$ ).

Die Osteomalazie des Erwachsenen, an der vor allem Frauen mit vielen Schwangerschaften und langen Stillperioden leiden, äußert sich nicht durch subperiostale Osteoidbildung, sondern durch Rarefikation der Knochensubstanz und Deformationen (ORTNER und PUTSCHAR $\left.1981^{144}\right)$. Damit ist eine Abgrenzung zum adulten Skorbut unproblematisch.

ORTNER und PUTSCHAR (1981) vermuten, dass Rachitis und Skorbut zwar häufig in Kombination auftreten, die Manifestation der einen Krankheit jedoch die Ausbildung von Symptomen am Skelet der anderen hemmt (ORTNER und PUTSCHAR $1981^{145}$ ).

Man kann davon ausgehen, dass Rachitis in historischen Populationen eine große Rolle gespielt hat. Aus dem 19. und frühen 20. Jahrhundert ist eine Prävalenz bei Kindern europäischer und nordamerikanischer Großstädte zwischen 70 und $97 \%$ bekannt (LEWIS 2002, ORTNER und PUTSCHAR 1981 ${ }^{146}$ ). Allerdings muss man bei diesen Zahlen die besonderen Umstände der Industriellen Revolution bedenken (beengte Wohnverhältnisse in großen Städten, Kinderarbeit in Fabriken und Minen mit wenig Zugang zu Sonnenlicht, kombiniert mit mangelhafter, wenig frische Zutaten enthaltender Nahrung). Zwar ist davon auszugehen, dass Rachitis auch in den vorherigen Jahrhunderten, vor allem in den Wintermonaten, vorkam, paläopathologische Berichte darüber sind jedoch selten.

\section{e) Lepra}

In zwei Fällen (Sk 1377, Sk 1380), bei denen ursprünglich der Verdacht auf Skorbut bestand, ergab sich der Verdacht auf eine Lepra-Erkrankung.

Dies mag zunächst überraschen, da Lepra heutzutage meist als Tropenkrankheit bezeichnet wird, doch es ist bekannt, dass es auch im mittelalterlichen Europa Lepra gab (BLONDIAUX et al., SCHULTZ und ROBERTS 2002). Es wird vermutet, dass sie durch römische Soldaten, Völkerwanderung und Kreuzzüge von den frühen Hauptherden Ägypten, Ostasien und Indien nach Europa eingeschleppt wurde (HAHN et al. 2000).

Die durch Mycobacterium leprae hervorgerufene granulomatöse Entzündungskrankheit hat einen oft Jahrzehnte dauernden Verlauf. Dabei werden Klinik und Verlauf von der zellulären

\footnotetext{
${ }^{143}$ S. 274

${ }^{144}$ S. $280-283$

${ }^{145}$ S. 273

${ }^{146}$ S. $273 f$
} 
Immunantwort des erkrankten Organismus bestimmt. Man unterscheidet einen tuberculoiden Typ (TT), bei dem sich nur wenige Bakterien in den Läsionen nachweisen lassen und eine starke, wenn auch ineffektive Immunantwort stattfindet, und einen lepromatösen Typ (LL), der multibakteriell ist und keine zelluläre Immunantwort aufweist. Dazwischen gibt es verschiedene Borderline-Typen, die klinisch besonders instabil sind (EDDLESTON et al. $\left.2005^{147}\right)$.

Insbesondere betrifft Lepra Haut und Schleimhäute, periphere Nerven und Weichteile. Doch auch Veränderungen am Skelet werden beobachtet und können auch an historischen Skeleten nachgewiesen werden. Sie werden im Wesentlichen auf drei Mechanismen zurückgeführt:

(1) Lepromatöse Osteomyelitis und Periostitis, (2) neurotrophe Knochen- und Gelenkläsionen und (3) Osteomyelitis und septische Arthritis durch Sekundärinfektionen (ORTNER und PUTSCHAR $1981^{148}$ ).

Die lepromatöse Osteomyelitis ist vergleichsweise selten. Sie entwickelt sich über Jahre und breitet sich kaum aus. Häufigste Lokalisation sind die Epiphysen der kleinen Knochen von Händen und Füßen. Auch am Schädeldach, an den Nasenknochen und am harten Gaumen kommt lepromatöse Osteomyelitis vor. Morphologisch zeichnet sie sich durch lokalisierte lytische Prozesse ohne begleitende Periostitis aus (ORTNER und PUTSCHAR $1981^{149}$ ).

Häufiger findet sich die lepromatöse Periostitis. Die dadurch verursachten subperiostalen Kochenneubildungen sind auch am historischen Skelet nachzuweisen und können zu Verwechslungen mit Skorbut führen. Sie zeichnen sich durch eine feine Längsstreifung aus und finden sich meist an Fibula und/oder Tibia (ORTNER und PUTSCHAR 1981 ${ }^{150}$, SCHULTZ 2011). Es handelt sich dabei um eine nicht-aggressive Form der Periostitis. Durch Neubildung von Knochensubstanz über den durch die Entzündung ausgeprägten Periostgefäßen kommt es zu den offenbar leprapezifischen polsterartigen Strukturen (SCHULTZ 2009, 2011, SCHULTZ und ROBERTS 2002).

Am häufigsten finden sich an den Knochen neurotrophe Veränderungen. Die Zerstörung der sensorischen Nerven, Veränderungen der Blutversorgung und lokaler Druck führen zu einer konzentrischen Atrophie, die sich langsam ausbreitet. Dabei bleibt die Corticalis unversehrt. An den Händen beginnt die Atrophie an den distalen Phalangen und wandert nach proximal, an den Füßen beginnt sie an den proximalen Phalangen und distalen Metatarsalen, die subluxierten distalen Zehen sind meist nicht affektiert. Häufig ist die Erstmanifestation am

\footnotetext{
${ }^{147}$ S. 442

${ }^{148}$ S. 176

${ }^{149}$ S. 177

${ }^{150}$ S. 177
} 
Kleinfinger, da der N. ulnaris besonders oft betroffen ist (ORTNER und PUTSCHAR $\left.1981^{151}\right)$.

Die durch die Nervenzerstörung bedingte Anästhesie führt auch $\mathrm{zu}$ vielen Sekundärverletzungen, insbesondere der Füße und Hände, da die betroffene Person nicht bemerkt, dass sie z.B. mit nackten Füßen auf spitze Gegenstände tritt. Dies kann zu Sekundärinfektionen, unspezifischer Osteomyelitis und schweren Mutilationen führen.

Zur makroskopischen Unterscheidung von Skorbut und Lepra am mazerierten Skelet ist vor allem das Verteilungsmuster der Läsionen interessant. So finden sich bei Lepra in der Regel nur an Fibula und Tibia subperiostale Knochenneubildungen, die außerdem oft auffällig streifig gestaltet sind. Darüber hinaus sind lytische und atrophische Prozesse an den kleinen Knochen der Extremitäten, wie sie für Lepra typisch sind, bei Skorbut nicht zu erwarten. Der Untersuchung dieser Knochen sollte also besondere Bedeutung zukommen. Das Gleiche gilt für den Gesichtsschädel, insbesondere die Nasenknochen und den harten Gaumen.

Sollte es makroskopisch dennoch Abgrenzungsprobleme geben, etwa weil entscheidende Knochen nicht erhalten oder nicht befundbar sind, so ist in der Regel die Histologie wegweisend. Siehe hierzu auch Kapitel 2.2 i) Lichtmikroskopie $\rightarrow$ Befundung der lichtmikroskopischen Präparate.

\section{f) Unspezifische Entzündungen: Periostitis, Ostitis und Osteomyelitis}

Bei Veränderungen vor allem im Bereich der Langknochen kann die Unterscheidung von Skorbut und Osteomyelitis Probleme machen (FRAENKEL $1908^{152}$ ). Letztere kann ebenfalls reaktive Knochenneubildung durch Periostabhebung hervorrufen, dies wird vor allem an den Diaphysen der unteren Extremität beobachtet (AUFDERHEIDE und RODRÍGUEZ-MARTÍN $1998^{153}$, TEMPLIN 1993).

Auch am Schädeldach und am Gesichtsschädel können entzündliche Prozesse zu Veränderungen führen, die sich makroskopisch nicht immer eindeutig von hämorrhagischen Geschehen abgrenzen lassen (SCHULTZ 1987, SCHULTZ 1993, TEMPLIN 1993, SCHULTZ 2011).

Die Unterscheidung kann am besten lichtmikroskopisch erfolgen (siehe Kapitel 2.2 i) Lichtmikroskopie $\rightarrow$ Befundung der lichtmikroskopischen Präparate).

\footnotetext{
${ }^{151}$ S. 177

${ }^{152}$ S. 32

${ }^{153}$ Seite 173
} 


\section{g) Syphilis congenita}

Besonders in der älteren Literatur wird oft die Möglichkeit der Verwechslung von Skorbut und Syphilis congenita diskutiert (BARLOW 1883, FRAENKEL 1908 ${ }^{154}$ ). Dabei wird als ein mögliches Unterscheidungskriterium das Alter bei Auftreten der Erkrankung genannt. Während die Syphilis congenita sich schon um den Zeitpunkt der Geburt manifestiert, tritt Skorbut selten vor dem vierten Lebensmonat auf (FRAENKEL $1908^{155}$ ). Allerdings ist dieses Unterscheidungskriterium sehr unzureichend, da sich die Syphilis congenita tarda auch nach dem vierten Lebensjahr manifestiert, dann jedoch in der Ausprägung eher der Tertiären Syphilis des Erwachsenen entspricht (HAHN und MIKSITS 2000). Die Infektion mit dem Erreger Treponema pallidum bei Syphilis congenita erfolgt in utero. Dies führt häufig zu Fehl- und Totgeburten. Bei starker Infektion befällt der Erreger sämtliche Organe, was zum schnellen Tod führt (HAHN und MIKSITS 2000, ORTNER und PUTSCHAR $1981^{156}$ ).

Bei Kindern, die mit Syphilis geboren werden, zeigt sich fast immer eine syphilitische Osteochondritis, die sich in allen enchondralen Wachstumszonen, vor allem aber in den besonders aktiven Metaphysen des distalen Femurs und der proximalen Tibia manifestiert. Diese lässt sich allerdings am mazerierten Knochen nicht nachweisen. Ein Hinweis darauf können bei überlebenden Kindern gehäuft auftretende transversale Frakturen im Bereich mechanisch belasteter Metaphysen sein, also an den Armen bei Krabbelkindern, bei älteren an den Beinen (ORTNER und PUTSCHAR $1981^{157}$ ).

Meist folgt auf die Osteochondritis die syphilitische Periostitis. Diese kann am historischen Skelet in Form von überwiegend symmetrischen, den Schaft umrundenden subperiostalen Knochenneubildungen an den Langknochen nachgewiesen werden. Ähnliche Veränderungen finden sich auch am Schädeldach (ORTNER und PUTSCHAR 1981 ${ }^{158}$ ).

Langfristig führt die Periostitis zu Osteosclerosis, die subperiostalen Knochenauflagerungen und die darunter liegende Corticalis verbinden sich. Dies führt zu charakteristischen Knochenverformungen, am bekanntesten die sogenannte „Säbel-Tibia“, die bei unveränderter Fibula eine Verlängerung und Konvexität des Schaftes nach vorn aufweist.

\footnotetext{
${ }^{154}$ BARLOW 1883: S. 190, FRAENKEL 1908: S. 33

${ }^{155}$ S. 33

${ }^{156}$ S. 198

${ }^{157}$ S. 198

${ }^{158}$ S. 199, 201
} 
Ein weiteres Merkmal der congenitalen Syphilis sind Daktylitiden, vor allem an den Händen, im Rahmen derer sich der Knochendurchmesser vergrößert und eine dünne knöcherne Außenhülle bildet (ORTNER und PUTSCHAR 198159).

Im Gesichtsbereich kommt es durch gestörte enchondrale Ossifikation zu der charakteristischen Sattelnase, die jedoch am historischen Skelet schlecht nachweisbar ist. Die Schneidezähne sind tonnenförmig gerundet und eingekerbt (HAHN und MIKSITS 2000, ORTNER und PUTSCHAR $1981^{160}$ ).

Die durch die Periostitis verursachten Knochenneubildungen können, je nach Ausprägung, am historischen Knochen zu Verwechslungen mit Skorbut führen, zumal das Verteilungsmuster recht ähnlich erscheint. Oftmals weisen sie eine charakteristische, baumrindenartige, strähnige Längsstreifung auf (SCHULTZ et al. 2003). Zur Unterscheidung hilfreich können Hinweise auf Daktylitiden sowie die charakteristischen Tonnenzähne und „Säbel-Tibia“ sein. Auf alle Fälle sollte eine mikroskopische Untersuchung erfolgen (SCHULTZ 2011). Abhängig vom Krankheitsstadium lassen sich hier die „Grenzstreifen“ und typische polsterförmige Knochenneubildungen nachweisen, kombiniert mit einer vom Markraum ausgehenden Resorptionszone, die zum Teil sekundär verfüllt wird (SCHULTZ et al. 2003, SCHULTZ 2011, siehe auch Kapitel 2.2 i) Lichtmikroskopie $\rightarrow$ Befundung der lichtmikroskopischen Präparate).

Eine Verwechslung zwischen venerischer Syphilis und dem Skorbut des Erwachsenen ist eher unwahrscheinlich, da die wurmfraßartigen Läsionen und Gummata der Syphilis recht spezifisch sind (ORTNER und PUTSCHAR $1981^{161}$ ).

\section{h) Infantile Corticale Hyperostose (Caffey's disease)}

Diese erstmals durch CAFFEY und SILVERMAN 1945 beschriebene Erkrankung unklarer Ätiologie führt $\mathrm{zu}$ einer Verdickung der Knochen durch exzessive subperiostale Knochenauflagerungen (CAFFEY 1946, CAFFEY und SILVERMAN 1945, JAFFE $1972^{162}$ ). Dies kann zu Verwechslungen mit Skorbut an historischen Knochen führen.

Über die Prävalenz dieser Erkrankung in historischen Populationen gibt es praktisch keine Daten. Beschreibungen datieren größtenteils aus dem 20. Jahrhundert, und es wird vermutet, dass es sich um eine relativ neue Erkrankung handelt (JAFFE 1972 ${ }^{163}$ ). Auf der anderen Seite

\footnotetext{
159 S. 201

${ }^{160}$ S. 201

${ }^{161}$ S. $182-198$

162 S. 282

${ }^{163}$ S. 282
} 
scheint Infantile Corticale Hyperostose recht häufig und auch weit verbreitet zu sein (JAFFE $1972^{164}$ ), sodass es durchaus möglich ist, dass die Erkrankung auch in früheren Jahrhunderten schon eine Rolle gespielt hat, aber nicht als eigene Entität erkannt wurde.

Darüber hinaus wird über gehäuftes Vorkommen innerhalb einzelner Familien berichtet und eine genetische Komponente diskutiert (HANSEN 1967, JAFFE 1972 ${ }^{165}$ ). Deshalb wäre es denkbar, dass gerade in historischen Populationen, die weitgehend endogam lebten, eine hohe Prävalenz herrschte.

Klinisch gibt es einige Parallelen zwischen infantilem Skorbut und Infantiler Corticaler Hyperostose: Die Erkrankung setzt meist recht abrupt ein. Zunächst zeigt sich eine Schwellung der Kiefer, insbesondere des unteren. Daneben oder alternativ kann es zu unioder bilateralen Schwellungen der Extremitäten, der Scapulae, Claviculae und Rippen kommen, dagegen sind Hand- und Fußknochen in der Regel nicht betroffen. Die Schwellungen sind hart und fest an die darunter liegende Knochenoberfläche fixiert. Anders als bei Skorbut sind sie nicht unbedingt schmerzempfindlich. Die Schwellungen können auch wandern oder sich schubweise zurück- und neubilden (CAFFEY 1946, CAFFEY und SILVERMAN 1945, HANSEN 1967, JAFFE $1972^{166}$ ).

Durch die Knochenneubildungen kann sich der Durchmesser der Langknochen verdoppeln oder sogar verdreifachen, die Metaphysen bleiben in der Regel frei. Es ist möglich, dass Unteramknochen oder Unterschenkelknochen durch Knochenbrücken miteinander verbunden werden (HANSEN 1967, JAFFE 1972 ${ }^{167}$ ).

Die Ausheilung erfolgt an zwei Stellen parallel. Zum einen wird die Schicht des neu gebildeten Knochens dünner und kompakter und beginnt sich mit der darunter liegenden Substantia compacta zu verbinden. Zum anderen wird die ursprüngliche Substantia compacta zunehmend porös und allmählich resorbiert, sodass schließlich der neu gebildete Knochen die ehemalige Substantia compacta ersetzt. Dabei erscheint die neu gebildete Substantia compacta oft ein wenig dünner als normal, die Markhöhle kann aufgeweitet sein. Auch diese Veränderungen normalisieren sich im Verlauf einiger Monate (JAFFE 1972 ${ }^{168}$ ). Man kann sich vorstellen, dass, abhängig vom Krankheitsstadium, in welchem ein Individuum verstirbt, sich ein ähnliches Bild bietet wie bei Skorbut.

\footnotetext{
${ }^{164}$ S. 282

165 S. 282

${ }^{166}$ S. $282-284$

${ }^{167}$ S. 284

${ }^{168}$ S. $284 f$
} 
Im Allgemeinen dauert die Erkrankung einige Monate an, ist aber selbst limitierend. Die Veränderungen des Skelets bilden sich zurück. Es gibt vereinzelt Berichte über einen mehrjährigen Krankheitsverlauf (CAFFEY und SILVERMAN 1945). Gelegentlich beschriebene Todesfälle werden auf Sekundärinfektionen zurückgeführt (JAFFE $1972^{169}$ ).

Ein - wenn auch ungenügendes - Unterscheidungskriterium zum Skorbut kann das Alter sein: Bei der Infantilen Corticalen Hyperostose liegt der Altersgipfel bei unter fünf Monaten, sie ist auch bei Neonaten und sogar Feten bekannt. Es scheint ein höheres Vorkommen bei Knaben zu geben (CAFFEY und SILVERMAN 1945, HANSEN 1967, JAFFE $1972^{170}$ ).

Histologische Untersuchungen im Rahmen von Autopsien ergeben abhängig vom Krankheitsstadium deutliche Entzündungszeichen sowohl im Knochen selbst als auch im Periost und in den umgebenden Weichteilen, mit gehäuftem Vorkommen von Entzündungszellen und ödematöser Aufquellung des Gewebes (JAFFE 1972 ${ }^{171}$ ). Es ist fraglich, inwieweit diese Prozesse am mazerierten Knochen nachzuweisen sind.

Höchstwahrscheinlich handelt es sich bei diesen Entzündungen und der Knochenneubildung um Autoimmunprozesse, da weder Viren noch bakterielle Erreger bisher nachgewiesen werden konnten, außerdem zeigt eine Corticosteroidtherapie gute Wirkung (HANSEN 1967, JAFFE $1972^{172}$ ).

Zusammenfassend kann gesagt werden, dass bei der Untersuchung historischer Skelete die Infantile Corticale Hyperostose als Differentialdiagnose des Skorbuts in Betracht gezogen werden sollte.

\section{i) Hypertrophe Osteoarthropathie / Osteoperiostitis ossificans toxica / Pierre-Marie-}

\section{Bamberger-Syndrom}

Eine weitere Erkrankung, die am Skelet skorbutähnliche Läsionen verursacht, ist die Hypertrophe Osteoarthropathie. Diese tritt als Begleiterkrankung bei schweren Lungenerkrankungen, wie z.B. Bronchialkarzinom, Lungenmetastasen oder massiven Infektionen, oder auch im Zusammenhang mit Herzerkrankungen auf.

Als auslösendes Agens werden periphere Hypoxie mit konsekutiver Hyperämie sowie toxische Stoffe, die durch die Primärerkrankung in den Blutkreislauf gelangen, diskutiert $\left(\right.$ JAFFE $\left.1972^{173}\right)$.

\footnotetext{
${ }^{169}$ S. 284

${ }^{170}$ S. 282

${ }^{171}$ S. 285

${ }^{172}$ S. $285 f$

${ }^{173}$ S. 291
} 
Das klinische Bild der Hypertrophen Osteoarthropathie setzt sich zusammen aus folgenden Komponenten, die sowohl in Kombination als auch einzeln auftreten können:

- Pulmonale Grunderkrankung,

- „Trommelschlägelfinger/-zehen“, verursacht in erster Linie durch Verdickung der subungualen Weichteile, wobei die Nägel rundlich vergrößert erscheinen, so genannte „Uhrglasnägel““

- Periostale Knochenneubildung (Osteophytosis) vor allem an den Röhrenknochen der Extremitäten

- Schmerzhafte Schwellung der Gelenke, bedingt durch entzündliche Veränderungen der Synovialmembran (SCHMIDT 1944, JAFFE 1972 ${ }^{174}$ ).

Für die Untersuchung historischer Skelete ausschlaggebend sind die Veränderungen an den Langknochen. Sie sind meist in der Schaftmitte am stärksten ausgeprägt und verjüngen sich zum Schaftende hin. Die Muskel- und Bandinsertionen sind kaum befallen (SCHMIDT 1944, JAFFE $1972^{175}$ ).

Histologisch zeigt sich zunächst eine Rundzellinfiltration des Stratum fibrosum des Periosts. Darauf folgt eine Proliferation des tiefer liegenden Stratum osteogenicum. Dies führt zur Auflagerung von neu gebildetem Knochen auf die ursprüngliche Corticalis, die in Schichten erfolgt. Anfangs handelt es sich um unreifen Faserknochen, dessen untere Schichten im Verlauf lamellär organisiert werden. Die ursprüngliche Corticalis wird dünner und porös, sodass die anfänglich scharfe Grenze zwischen der alten und der neu gebildeten Knochensubstanz allmählich verschwimmt. Es findet keine endostale Knochenneubildung statt, das Trabekelwerk wird nicht verdickt, sondern scheint im Gegenteil resorptiven Prozessen zu unterliegen (JAFFE $1972^{176}$ ).

Abhängig vom Stadium der Erkrankung, in dem ein Individuum verstarb, kann sich bei der Untersuchung des Skelets also durchaus ein ähnliches Bild wie bei Skorbut bieten.

Die Veränderungen finden sich an den Extremitäten, wobei Unterarm- und Unterschenkelknochen häufiger befallen sind als Oberarm und Oberschenkel. Die Metacarpal- und Metatarsalknochen sind häufiger befallen als die Phalangen, die distalen Phalangen meistens frei. Die Knochen des Rumpfes sind selten befallen, es gibt jedoch Beschreibungen von Veränderungen der Rippen, Claviculae und Wirbelsäule.

\footnotetext{
${ }^{174}$ S. 286

${ }^{175}$ S. 290

${ }^{176}$ S. $290 f$
} 
Auch die Schädelknochen sind in der Regel unverändert, allerdings lassen sich gelegentlich Knochenneubildungen an der Lamina interna und an den Nasenknochen nachweisen (JAFFE $\left.1972^{177}\right)$.

Gelenkveränderungen finden sich vor allem bei adulten Individuen mit lange bestehender Erkrankung, oft wird die Gelenkbeteiligung aber auch klinisch durch Knochenneubildung im Epiphysenbereich vorgetäuscht. Eine Ankylosierung bleibt in der Regel aus (JAFFE 1972 ${ }^{178}$ ). Treten die Veränderungen im typischen Muster auf, kann aufgrund der Verteilung der Läsionen eine Unterscheidung von Hypertropher Osteoarthropathie und Skorbut auch an historischen Skeleten erfolgen. Schwieriger wird dies, wenn Symptome einzeln auftreten oder Skeletteile fehlen.

Auf alle Fälle sollte eine gründliche Untersuchung auf mögliche Ursachen für eine Hypertrophe Osteoarthropathie erfolgen. Dazu zählen die Suche nach entzündlichen Veränderungen im Bereich der Rippen sowie Hinweise auf Tuberkulose und Metastasen oder Primärtumoren im Bereich der Knochen.

\subsection{Skorbut heute}

In der medizinischen Lehre und Berichterstattung spielt Skorbut heute nur eine untergeordnete Rolle. Durch die Kenntnis des Vitamin C und den großflächigen Einsatz des Vitamins in der Lebensmittelproduktion (unter anderem als Konservierungs- und Säuerungsmittel) und der Herstellung von Säuglingsnahrung ist ein ausgeprägter Mangel selten. Die Pathophysiologie der fehlerhaften Kollagensynthese wird im Medizinstudium theoretisch erörtert, die klinischen Folgen eines manifesten Vitamin-C-Mangels bekommt jedoch kaum ein Mediziner zu sehen.

Dennoch ist der Skorbut keinesfalls ,ausgestorben“. Immer wieder gibt es (Einzelfall-) Berichte von rezenten Skorbutfällen (beispielsweise AKIKUSA et al. 2003, MAPP und COUGHLIN 2006, WEINSTEIN et al. 2001).

In diesen taucht oft ein bestimmtes Problem auf: Der Kliniker erkennt aufgrund der Seltenheit der Erkrankung die Ursache der Symptome, die ihm präsentiert werden, nicht und diagnostiziert und therapiert vergeblich in andere Richtungen, obwohl die Therapie nach richtiger Diagnosestellung denkbar einfach wäre.

HEYMANN (2007) fasst die klinischen Symptome rezenter Skorbutfälle als Wegweiser für den Kliniker zusammen, wobei an erster Stelle die Einschränkung der Gehfähigkeit steht,

${ }_{178}^{177}$ S. 290 
gefolgt von Schmerzen der unteren Extremität und Zahnfleischbluten. Er empfiehlt die frühzeitige Untersuchung des Serumspiegels von Vitamin C in verdächtigen Fällen.

Es gibt verschiedene Risikogruppen, die besonders gefährdet sind, trotz der guten Zugänglichkeit Vitamin-C-haltiger Nahrungsmittel in Industrienationen einen manifesten Mangel zu entwickeln. Darunter fallen ältere alleinstehende Männer, die sich selbst versorgen und dabei in hohem Maß auf konservierte Nahrungsmittel zurückgreifen (HEYMANN 2007, MAPP und COUGHLIN 2006, VILTER et al. 1946, ZELLWEGER und ADOLPH 1954). Dieses Phänomen wird auch als „bachelor scurvy“ bezeichnet (VILTER et al. 1946, ZELLWEGER und ADOLPH 1954).

Ebenso gefährdet sind alkoholabhängige Personen, bei denen oft Mangelernährung, aber auch durch die körperlichen Folgen des jahrelangen Abusus bedingte Resorptionsstörungen beobachtet werden (HEYMANN 2007, HIRSCHMANN und RAUGI 1999, MAPP und COUGHLIN 2006).

Auch durch sehr einseitige Diäten, z. B. bei Magengeschwüren, ausgeprägten

Nahrungsmittelallergien oder aus weltanschaulichen Gründen, kann heutzutage ein VitaminC-Mangel entstehen (BÄSSLER et al. 2002, ZELLWEGER und ADOLPH 1954, HIRSCHMANN und RAUGI 1999).

MAPP und COUGHLIN (2006) berichten über den Fall eines jungen Mannes, der wegen Petechien und Ekchymosen beim Arzt vorstellig wurde. Nach Ausschluss anderer Ursachen für die Blutungsneigung wurde der deutlich erniedrigte Vitamin-C-Serumspiegel bestimmt. Auf Nachfrage stellte sich heraus, dass der Patient sich seit 10 Jahren frei von Obst und Gemüse ernährte. Die Substitution von Vitamin C führte zu einer Besserung der hämorrhagischen Symptome in wenigen Wochen (MAPP und COUGHLIN 2006).

Chronisch entzündliche Darmerkrankungen und Resorptionsstörungen können trotz adäquater Vitamin-C-Zufuhr durch eingeschränkte Aufnahmefähigkeit im Ileum zu Mängeln führen.

Unter Kindern findet sich Skorbut bei Fällen schwerer Vernachlässigung. Hier kann aufgrund der Hämatomneigung auch der Verdacht entstehen, das Kind werde geschlagen. Ein auffällig symmetrisches Verteilungsmuster sowie die Kombination mit den übrigen typischen Symptomen und Röntgenbefunden sollten an Skorbut denken lassen. Selbstverständlich stellt auch die Mangelernährung eine Form der Kindesmisshandlung dar, die nicht unterschätzt werden sollte (MIMASAKA et al. 2000, HEYMANN 2007). MIMASAKA et al. (2000) beschreiben einen solchen Fall. Sie untersuchten den Leichnam eines sechsjährigen Mädchens, das nach einem mehrstündigen Aufenthalt in der Badewanne tot in der verwahrlosten Wohnung seiner Eltern aufgefunden wurde. Klinisch wurde zunächst ein Tod 
durch Ertrinken vermutet. Den behandelnden Ärzten fielen jedoch zahlreiche Hämatome auf, weshalb eine gerichtsmedizinische Untersuchung erfolgte. MIMASAKA et al. (2000) beschreiben ein unterernährtes Kind mit subkutanen Einblutungen im Gesicht, an Brust, Rücken und Beinen. Außerdem fallen Zahnfleischschwellungen und -blutungen auf. An Tibiae, Femura und Humeri finden sich massive subperiostale Einblutungen, die sich durch Gewalteinwirkung nicht hinreichend erklären lassen. Röntgenuntersuchungen zeigen für Skorbut typische Befunde, wie Epiphysendislokationen, radiodense Doppelringe und „Milchglas-Atrophie“. Der Vitamin-C-Serumspiegel ist annähernd Null. Die Gerichtsmediziner stellen schließlich die Diagnose Herzkreislaufversagen durch Skorbut. Aufgrund der geringen Erfahrung mit dem Krankheitsbild von Skorbut sind zwischen dem Tod des Mädchens und der endgültigen Diagnose mehrere Monate vergangen (MIMASAKA et al. 2000).

Auch WEINSTEIN et al. (2001) berichten über einen rezenten Fall infantilen Skorbuts. Dabei handelt es sich um ein neunjähriges Mädchen mit einer allgemeinen Entwicklungsverzögerung sowie einer langjährigen Phenytoin-Therapie. Grund für die pädiatrische Vorstellung sind muskuloskeletale Schmerzen, insbesondere der unteren Extremität, unspezifische Gingivitis und arterieller Hypertonus. Unter dem Verdacht auf eine Osteomyelitis erfolgt zunächst eine antibiotische Therapie mit Clindamycin. Diese bringt keine Besserung. Untersuchungen des Skelets ergeben eine generalisierte Osteopenie, vertebrale Kompressionsfrakturen und hyperdense Linien im Bereich der linken distalen Femurmetaphyse. Ein MRT zeigt ein diffuses symmetrisches abnormes Signal an den distalen Femurmetaphysen und -epyphysen, beiden Darmbeinen, den distalen Radii und dem Weichteilbereich um die Knie. Es erfolgen eine Knochenmarkspunktion und eine Knochenbiopsie zum Ausschluss einer malignen Erkrankung. Genetische Untersuchungen der Familie ergeben keine Auffälligkeiten. Eine gründliche Diagnostik zur Abklärung der arteriellen Hypertonie bleibt ohne Ergebnisse. Eine ausführliche Anamnese ergibt eine Ernährung, die aufgrund der ausgeprägten Neigungen des Kindes ausschließlich aus gekauftem Schokoladenpudding und -kuchen, Wasser sowie gelegentlich Spaghetti, Rindfleisch und Milch besteht. Daraufhin erfolgt eine Serumuntersuchung des Ascorbinsäurespiegels. Dieser ist - trotz bereits seit einigen Wochen zugeführter Krankenhauskost - deutlich erniedrigt, ebenso der Vitamin D - und der Eisenspiegel.

Unter Vitamin-C-Substitution kommt es rasch zu einer deutlichen klinischen Besserung. Interessanterweise zeigen sich erst in einer zehn Tage nach Therapie-Beginn durchgeführten Röntgenuntersuchung die typischen Skorbutzeichen wie der „Wimberger-Ring“. Vermutlich 
hat die durch die Ascorbinsäure bedingte Steigerung des Knochenstoffwechsels zu ihrer Demaskierung geführt.

Unter Vitamin-C-Substitution kommt es auch zu einer Normalisierung des Blutdrucks. Der genaue Mechanismus ist nicht bekannt, es gibt jedoch einen tierexperimentell bestätigten Zusammenhang zwischen Vitamin-C-Spiegel und Blutdruckregulation. Möglicherweise hängt dies mit der Beteiligung des Vitamin $\mathrm{C}$ an der Katecholaminsynthese zusammen (WEINSTEIN et al. 2001).

Dieser Fall zeigt deutlich, wie wichtig es ist, frühzeitig an Skorbut zu denken. Der Patientin hätten viele aufwendige und kostspielige Untersuchungen erspart werden können (WEINSTEIN et al. 2001).

Sehr ähnlich liest sich der Fall eines neunjährigen Jungen, den AKIKUSA et al. (2003) beschreiben. Auch dieser Junge wird mit muskuloskeletalen Schmerzen, die insbesondere die Gehfähigkeit einschränken, und Zahnfleischbluten ins Krankenhaus gebracht. Die klinische Untersuchung zeigt ein großes Hämatom am rechten Oberschenkel, ohne dass ein Trauma erinnerlich ist. Außerdem fällt eine raue Haut mit lokalisierten Hyperkeratosen auf. Wie bei dem zuvor beschriebenen Fall wird auch bei diesem Jungen eine große Anzahl an Untersuchungen durchgeführt, nämlich diverse Blutanalysen, Sonographie, Röntgenuntersuchungen und eine Knochenmarkspunktion. Schließlich ergibt die Anamnese, dass der Junge sich ausschließlich von Brot, Wasser, Milch, Limonade, Müsli-Riegeln, Schokolade und anderen Süßigkeiten ernährt. Daraufhin erfolgt eine weitere Blutuntersuchung, die einen Mangel an Vitamin C, Vitamin B1 und Vitamin A aufweist. Nach vierwöchiger Substitution von Vitamin C kommt es zu einer deutlichen Besserung der Symptome und das Kind wird einer Ernährungsberatung unterzogen (AKKIKUSA et al 2003).

Eine andere Ursache für kindlichen Skorbut kann die Ernährung von Säuglingen mit selbst verarbeiteter Kuh- oder anderer Tiermilch anstelle von Säuglingsnahrung sein, wie sie gelegentlich aus weltanschaulichen Gründen erfolgt (HEYMANN 2007, WEINSTEIN et al. 2001).

Bei Erwachsenen stellt Zigarettenrauchen einen Risikofaktor für Skorbut dar, da man davon ausgeht, dass Raucher einen doppelt so hohen Turnover an Vitamin C haben wie Nichtraucher (HIRSCHMANN und RAUGI 1999).

In anderen Teilen der Erde zeigt sich ein völlig anderes Bild von Skorbut. Was in den Industrienationen als Einzellfall auftaucht, kann in Ländern, in denen die Ernährungssituation der Menschen durch Kriege, Naturkatastrophen, Dürren oder Flüchtlingsströme gestört ist, auch heute noch epidemische Ausmaße annehmen. So berichtet die WHO (WEISE PRINZO 
1999) über epidemische Ausbrüche in Somalia (1983, 1985), im Sudan (1984, 1991), in Äthiopien (1989), Nepal (1992) und Kenia (1994).

Dabei spielten unterschiedliche Faktoren eine Rolle. Bei den äthiopischen Flüchtlingen in Somalia, die 1982/83 in großer Zahl an Vitamin-C-Mangel litten, fehlte z.B. die traditionell in großen Mengen verzehrte Kamelmilch als Hauptascorbinsäurelieferant (WEISE PRINZO 1999).

Ein weiteres häufiges Problem in Flüchtlingslagern ist der fehlende Zugang zu lokalen Märkten, wo die Flüchtlinge Möglichkeiten hätten, frische, Vitamin-C-haltige Lebensmittel zu erhalten (WEISE PRINZO 1999).

Die WHO nennt einige Risikofaktoren, die Flüchtlingsgruppen besonders gefährden, an Skorbut zu erkranken. Darunter fallen große Gruppen von Flüchtlingen, die überwiegend von Lebensmittelhilfen leben, und überfüllte Camps mit hohen Infektionsraten und dadurch gesteigertem Vitamin-C-Bedarf der Erkrankten. Ein weiteres Problem stellen fehlende Möglichkeiten, frisches Obst oder Gemüse selbst anzubauen oder auf lokalen Märkten zu erhalten, dar. Besonders gefährdet sind alte Menschen sowie junge Frauen im gebärfähigen Alter, insbesondere Schwangere. Eine saisonale Häufung von Skorbutfällen wird unmittelbar nach der Trockenzeit in den afrikanischen Ländern beobachtet (WEISE PRINZO 1999).

$\mathrm{Zu}$ beachten ist, dass die Skorbuterkrankung in Flüchtlingslagern in der Regel wesentlich schwerer verläuft als unter experimentellen Bedingungen, da sie durch unzureichende Hygiene, weitere Mangelzustände und Infektionen verkompliziert wird (WEISE PRINZO 1999).

Die Prävention von Skorbut in Flüchtlingscamps ist schwierig. Die Instabilität von Vitamin C bei Lagerung, Zubereitung und Transport von Lebensmitteln stellt dabei ein großes Problem dar, insbesondere, weil in den betroffenen Regionen von einer allenfalls mangelhaften Stromversorgung mit entsprechend eingeschränkten Kühlmöglichkeiten und defizienten Transportwegen ausgegangen werden muss. Die WHO empfiehlt abhängig von der jeweiligen Situation unterschiedliche Methoden zur Vitamin-C-Versorgung (WEISE PRINZO 1999). 


\section{Zusammenfassung}

Skorbut ist eine Krankheit, die - wie kaum eine andere Erkrankung - zu allen Zeiten und überall auf der Welt vorkam und auch weiter vorkommt. Man könnte sie als „globale Volkskrankheit“ bezeichnen. Der in den letzten Jahrzehnten deutlich gestiegene Lebensstandard in der westlichen Welt mit einem beispiellosen Nahrungsmittelangebot für breite Bevölkerungsschichten lässt vergessen, welch schweres Leid bis hin zum Tod durch Mangelernährung verursacht werden kann. Vitamin-C-Mangel klingt in einer solchen Zeit nach einem harmlosen „Zipperlein“, das problemlos mit einem frei verkäuflichen VitaminPräparat kuriert werden kann. Es scheint kaum noch vorstellbar, dass noch im letzten Jahrhundert auch in Deutschland Skorbut eines der größten Gesundheitsprobleme der Bevölkerung darstellte.

Wer historische Populationen auf ihre Erkrankungen untersucht, wird jedoch kaum um diese Mangelerkrankung herumkommen.

Die vorliegende Arbeit zeigt, dass der Nachweis von Skorbut auch an sehr alten Knochen möglich ist, insbesondere gelingt die Diagnose auch an adulten Individuen, worüber bisher verhältnismäßig wenig Literatur vorliegt.

Zur Diagnostik sollte zunächst eine gründliche makroskopische Untersuchung aller vorhandenen Knochen erfolgen. Der Ausschluss von Skorbut aufgrund des Fehlens einzelner Merkmale, wie ORTNER und seine verschiedenen Koautoren (ORTNER et al. 1999, ORTNER et al. 2001, ORTNER und ERICKSEN 1997) es für die Ala major ossis sphenoidalis vorschlagen, erscheint nicht sinnvoll. Dagegen spricht die sehr variable Klinik des Skorbuts (BARLOW 1883, FRAENKEL 1908), die nicht erwarten lässt, dass sich an historischen Skeleten ein homogener Befund ergibt. Außerdem ist es schwierig, sich bei archäologischen Funden auf einzelne Knochen zu konzentrieren, da sehr häufig nur Teile des Skelets erhalten bzw. in befundbarem Zustand sind. Ziel paläopathologischer Untersuchungen sollte es aber sein, möglichst auch bei unvollständigen Skeletfunden verlässliche Diagnosen zu stellen.

Schließlich kann in dieser Arbeit eine beträchtliche Anzahl von Fällen dargestellt werden, die mit hoher Wahrscheinlichkeit an Skorbut erkrankt waren, ohne das von ORTNER (ORTNER et al. 1999, ORTNER et al. 2001, ORTNER und ERICKSEN 1997) geforderte Kriterium des Befalls der Ala major ossis sphenoidalis aufzuweisen.

Als Orientierung bei der makroskopischen Untersuchung kann die von SCHULTZ (1994) erstellte Merkmalstabelle dienen, auch wenn die rein rechnerische Auswertung vor allem bei unvollständig erhaltenen Skeleten zu Fehlinterpretationen führen kann. Sie erleichtert es, die 
einzelnen Befunde im Zusammenhang zu sehen, und hilft, eine erste Gewichtung der pathologischen Veränderungen für die Diagnose vorzunehmen. Bei der Überprüfung der verschiedenen Merkmale sollte immer auch der Erhaltungszustand berücksichtigt und eindeutig vermerkt werden, ob ein Merkmal als negativ bewertet wird, weil der Knochen unauffällig ist oder weil er fehlt. Außerdem sollte darauf geachtet werden, ob pathologische Befunde fokal oder generalisiert vorliegen.

Weiterhin sollte zwischen Kindern und Erwachsenen unterschieden werden, da die Ausprägung der Krankheitszeichen teilweise stark voneinander abweicht.

In der hier durchgeführten Studie sind Oberflächenveränderungen an den Langknochen (Kardinalmerkmal 5 und Nebenmerkmal 4) die häufigsten Krankheitszeichen bei adulten und infantilen Individuen. Beim Befall der Langknochen kann kein signifikanter Unterschied zwischen oberer und unterer Extremität festgestellt werden. Über neunzig Prozent der Skorbutfälle in dieser Arbeit weisen Oberflächenveränderungen im Bereich der Langknochen auf, bei den adulten Fällen alle. Dieses Merkmal ist also insbesondere für die Diagnostik von Skorbut an Skeleten von Erwachsenen ausschlaggebend. Da Oberflächenveränderungen der Langknochen jedoch keinesfalls pathognomonisch für Skorbut sind, muss zur Diagnosesicherung eine lichtmikroskopische Untersuchung angeschlossen werden. Sind die Veränderungen lichtmikroskopisch eindeutig hämorrhagischen Ursprungs, ist die Sensitivität und Spezifität dieses Merkmals als hoch einzustufen.

Auch das Vorkommen von porösen Auflagerungen an den Alveolarknochen ist bei den untersuchten Skorbutfällen sowohl bei adulten als auch bei infantilen Individuen hoch. In nur einem Fall ist ein nicht an Skorbut erkranktes Individuum betroffen, sodass auch die Spezifität dieses Merkmals hoch ist.

Poröse Auflagerungen am inneren Schädeldach (Nebenmerkmal 3) finden sich bei knapp der Hälfte der Skorbutfälle, bei den Kindern bei über 60 Prozent. Sie finden sich ausschließlich bei Skorbutkranken.

Eine Stomatitis (Nebenmerkmal 1) lässt sich bei mehr als 50 Prozent (bei Kindern mehr als 60 Prozent) der Skorbutfälle nachweisen, ist aber als nicht sehr spezifisch zu bewerten, weil sie auch bei nicht an Skorbut erkrankten Individuen mehrfach auftritt.

Veränderungen am äußeren Schädel werden bei Kindern häufiger beobachtet, bei Erwachsenen gar nicht. Dabei treten Auflagerungen am äußeren Schädeldach (Kardinalmerkmal 1), am Orbitadach und am Gesichtsschädel (Kardinalmerkmal 3) gleich häufig auf. Zwar sind diese Merkmale deutlich seltener als die zuerst genannten, bei mikroskopischer Abgrenzung zum anämischen Geschehen aber sehr spezifisch. 
Bei knapp einem Drittel der untersuchten Skorbutkranken sind Auflagerungen an Scapula (Kardinalmerkmal 6) und Os ilii (Kardinalmerkmal 7) nachweisbar. Diese Merkmale finden sich ausschließlich bei Individuen mit Skorbut.

Ähnlich häufig, aber nicht ausschließlich bei Skorbutkranken, treten Porösitäten der Ala major ossis sphenoidalis bzw. der Squama temporalis (Nebenmerkmal 5) auf. Die Einstufung als Nebenmerkmal ist damit gerechtfertigt.

Die geringste Merkmalsausprägung zeigen die porösen Auskleidungen der Alveolen (Nebenmerkmal 2), sie finden sich ausschließlich bei skorbutkranken Kindern.

In jedem Fall sollte, wenn möglich, eine mikroskopische Untersuchung erfolgen. Die Ergebnisse dieser Arbeit zeigen, dass makroskopisch gestellte Verdachtsdiagnosen sich erst lichtmikroskopisch bestätigen oder ausschließen lassen. Insbesondere bei der Abgrenzung von Skorbut gegenüber Anämie, Lepra und Syphilis ist die lichtmikroskopische Diagnostik hilfreich, aber auch die Unterscheidung von physiologischen Prozessen und postmortaler Beschädigung wird erleichtert. Darüber hinaus kann man anhand des mikroskopischen Befunds Aussagen über die Krankheitsdauer, einen möglicherweise schubweisen Verlauf und den Abheilungsgrad zum Todeszeitpunkt treffen. Eine lichtmikroskopische Untersuchung bietet sich auch dann an, wenn nur sehr wenige Skeletteile erhalten sind und man daher wenige Informationen über das Verteilungsmuster der Läsionen bekommt.

Auch die Röntgenuntersuchung kann hilfreich sein, da sie die gleichzeitige Betrachtung oberflächlicher wie tiefer gelegener Strukturen ermöglicht. Allerdings wurden in dieser Arbeit keine der typischen Veränderungen wie der „Wimberger Ring“ oder die „MilchglasAtrophie“ gefunden. Vermutlich hängt dies damit zusammen, dass diese Zeichen teilweise nur während bestimmter Krankheitsphasen nachweisbar sind. Außerdem sind bei den Kinderskeleten die Epiphysenplatten von den Diaphysen getrennt erhalten, der Bereich der Epiphysenfuge kann also nicht richtig befundet werden. Bei den untersuchten Skeleten ist die radiologische Untersuchung nicht beweisführend, sondern eher ergänzende Diagnostik. Dennoch ist sie sinnvoll. Zum einen gelingt in vielen Fällen der Nachweis von Harris-Linien als Stressindikator, zum anderen lassen sich auch die Auflagerungen teilweise deutlich darstellen. Abgesehen von der Bedeutung zur Diagnosesicherung sollte die Röntgenaufnahme auch zur Befunddokumentation des intakten Knochens vor der Probenentnahme für die Anfertigung von Knochendünnschliffen erfolgen.

Für zukünftige Forschung wäre es interessant, einen biochemischen Beweis der Diagnose Skorbut zu erarbeiten. So könnte der Nachweis von fehlerhaftem Kollagen in historischen Knochen den Verdacht auf Skorbut untermauern. SCHMIDT-SCHULTZ und SCHULTZ 
(2004) haben eine vielversprechende Methode zur Isolierung von Proteinen aus historischen Skeleten vorgestellt. SCHULTZ et al. (2003) gelang mittels monoklonaler Antikörper sogar der Nachweis von humanem Kollagen Typ I aus historischen Skeletfunden. Diese Untersuchungsmethode verspricht die Möglichkeit $\mathrm{zu}$ sehr präzisen Aussagen bei paläopathologischen Untersuchungen.

Auch das Vitamin C und seine verschiedenen Funktionen für den menschlichen Organismus sind keinesfalls abschließend erforscht. Immer noch gibt es viele Wirkmechanismen, die nicht genau bekannt sind, wie zum Beispiel der Zusammenhang zwischen Vitamin $\mathrm{C}$ und der Blutdruckregulation oder die Wirkung von Vitamin C auf das Immunsystem.

Letztlich ungeklärt ist auch die Frage, warum der Mensch anders als die meisten Tiere nicht im Laufe der Evolution die Fähigkeit zur Ascorbinsäuresynthese erworben bzw. warum er sie verloren hat. Offensichtlich war es in der Geschichte der Menschheit immer wieder schwierig, den dadurch bestehenden Bedarf durch externe Zufuhr zu decken.

Wie diese Arbeit deutlich macht, hat Skorbut über Jahrtausende eine der häufigsten Erkrankungen der Menschheit ausgemacht, die schweres Leid verursachte.

Kaum eine andere Krankheit konnte jedoch durch die Forschung so eindeutig identifiziert und vor allen Dingen so leicht heilbar werden, dass sie ihren Schrecken für uns heute fast verloren hat. Es ist somit ein schönes Beispiel und vielleicht auch Antrieb für andere Forscher, dass sich die intensive und teilweise aufopferungsvolle Suche nach der Ursache und Therapie einer Krankheit bezahlt macht. Dies mag auch zukünftige Wissenschaftler darin bestärken, mit ihrer Arbeit fortzufahren. 


\section{$\underline{\text { Literaturverzeichnis }}$}

Akikusa JD, Garrik D, Nash MC (2003): Scurvy: Forgotten but not gone. J Paediatr Child Health $\underline{39}, 75-77$

Aschoff L, Koch W (Hrsg.): Skorbut. Eine pathologisch-anatomische Studie. Veröffentlichungen aus dem Gebiete der Kriegs- und Konstitutionspathologie. Heft 1; Gustav Fischer, Jena 1919

Aufderheide AC, Rodríguez-Martín C: The Cambridge Encyclopedia of Human Paleopathology. Cambridge University Press, New York 1998, 310-314

Barlow T (1883): On cases described as "acute rickets" which are probably a combination of scurvy and rickets, the scurvy being an essential, and the rickets a variable, element. Medical Chirurgical Transactions $\underline{66}, 159-220$

Bässler K-H, Golly I, Loew D, Pietrzik K: Vitamin-Lexikon für Ärzte, Apotheker und Ernährungswissenschaftler. 3. Auflage; Urban \& Fischer Verlag, München 2002

Blondiaux J, Dürr J, Khouchaf L, Eisenberg L (2002): Microscopic study and X-Ray analysis of two 5th century cases of leprosy; paleoepidemiological inferences. In: The Past and Present of Leprosy. Archaeological, historical, palaeopathological and clinical approaches. Hrsg. v. : Roberts CA, Lewis ME und Manchester K. BAR International Series $\underline{1054}, 105-110$

Brickley M, Ives R (2006): Skeletal Manifestations of Infantile Scurvy. Am J Phys Anthropol 129, 163-172

Burkhardt L: Pathologische Anatomie des Schädels in seiner Beziehung zum Inhalt. Spezielle Pathologie des Schädelskelets. In: Handbuch der speziellen Pathologischen Anatomie und Histologie, Band 9, Teil 7; Springer-Verlag, Berlin u. a. 1970, 291-296

Caffey J (1946): Infantile cortical hyperostoses. J Pediatr Vol. 29, No. 5, 541-559

Caffey J: Pediatric X-Ray Diagnosis. Volume 2, 6. Auflage; Lloyd-Luke (Medical Books), London 1973, 1210-1222, 1237-1243

Caffey J, Silverman WA ( 1945): Infantile cortical hyperostoses. Preliminary report on a new syndrome. Am J Roentgenol Radium Ther Vol. 54, No. 1, 1-16

Carli-Thiele P: Spuren von Mangelerkrankungen an steinzeitlichen Kinderskeleten (Fortschritte in der Paläopathologie und Osteoarchäologie Band I). Hrsg. v. Schultz M. Verlag Erich Goltze, Göttingen 1996

Cox EV, Meynell MJ, Northam BE, Cooke WT (1967): The Anaemia of Scurvy. Am J Med 42, 220-227 
Detken S: Krankhafte Veränderungen an den menschlichen Skeleten aus dem frühmittelalterlichen Reihengräberfeld Barbing-Kreuzhof. Med. Diss. Göttingen 1991

Eddleston M, Davidson R, Wilkinson R, Pierini S: Oxford Handbook of Tropical Medicine (Practical Advice for Clinicians in the Tropics). 2. Auflage; Oxford University Press, Oxford 2005

EImadfa I, Aign W, Fritzsche D, Cremer H-D: Die große GU Nährwert-Tabelle.

Kalorien/Joule- und Nährstoffgehalte unserer Lebensmittel. Neuausgabe 1990/1991; Gräfe und Unzer, München 1989, 4-5

Ferembach D, Schwidetzky I, Stloukal M (1979): Empfehlungen für die Alters- und Geschlechtsdiagnose am Skelett. Homo 30 Anhang, 1-32

Fraenkel E: Archiv und Atlas der normalen und pathologischen Anatomie in typischen Röntgenbildern - Die Möller-Barlowsche Krankheit (Fortschritte auf dem Gebiete der Röntgenstrahlen, Ergänzungsband 18). Lucas Gräfe \& Sillem (Edmund Sillem), Hamburg 1908

Geisler H: Barbing-Kreuzhof. Eine ländliche Siedlung des frühen Mittelalters östlich von Regensburg. In: Regensburg-Kelheim-Straubing I. Zur Siedlungsgeschichte der südlichen Frankenalb, des Vorderen Bayrischen Waldes und der Donauebene; Theiss Verlag, Stuttgart 1984, 164-173

Gresky J: Ätiologie und Epidemiologie der Erkrankungen des Craniums bei der Population des bajuwarischen Gräberfeldes von Harting (Oberpfalz). Ein Beitrag zur Rekonstruktion des Gesundheitsstatus frühmittelalterlicher Populationen. Med. Diss. Göttingen 2008

Grimm J, Grimm W: Deutsches Wörterbuch. Band X, I. S. Hirzel, Leipzig 1905, 1325

Hahn H, Miksits K: Treponemen. In: Medizinische Mikrobiologie und Infektiologie. Hrsg. v. Hahn H, Falke D, Kaufman SHE, Ullmann U, 4. Auflage; Springer-Verlag, Berlin 2000, 400-410

Hahn H, Kaufmann SHE, Rodloff AC: Mykobakterien. In: Medizinische Mikrobiologie und Infektiologie. Hrsg. v. Hahn H, Falke D, Kaufmann SHE, Ullmann U, 4. Auflage; Springer-Verlag, Berlin 2000, 377-395

Hansen HG: Osteosklerose und Hyperostose. Äthiopathogenetisch ungeklärte Osteosklerosen und Hyperostosen. Infantile corticale Hyperostose (CAFFEY-SILVERMAN). In: Handbuch der Kinderheilkunde. Band VI: Erkrankungen der StützgewebeErkrankungen des Blutes-Erkrankungen der blutbildenden Organe. Hrsg. v. Opitz H und Schmid F; Springer- Verlag, Berlin u. a. 1967, 372 - 377 
Heymann WR (2007): Scurvy in Children. J Am Acad Dermatol 57, 358 - 359

Hirschmann J V, Raugi G J (1999): Adult scurvy. J Am Acad Dermatol 41, No. 6, 895 -910

Hodges RE: Vitamin C. In: Human Nutrition, A Comprehensive Treatise. Band 3B, hrsg. v. Alfin-Slater RB und Kritchevsky D; Plenum Press, New York 1980, 73-96

Hodges RE, Baker EM, Hood J, Sauberlich HE, March SC (1969): Experimental Scurvy in Man. Am J Clin Nutr Vol. 22, No. 5, 535-548

Jaffe HL: Scurvy and Certain Other Vitamin-Conditioned Disorders. In: Metabolic, Degenerative, and Inflammatory Diseases of Bones and Joints; Urban \& Schwarzenberg, München 1972, 448-478

Kluge F: Etymologisches Wörterbuch der deutschen Sprache, 22. Auflage; Walter de Gruyter, Berlin 1989, 676

Köhegyi M, Marcsik A (1971): The Avar-age Cemetry at Sükösd. Acta Antiqua et Archaeologica (Szeged) XIV, 87-94

Kreutz K: Ätiologie und Epidemiologie von Erkrankungen des Kindesalters bei der bajuwarischen Population von Straubing (Niederbayern). Beiträge zur Paläopathologie. Hrsg. v. Schultz M; Cuvillier Verlag, Göttingen 1997

Kreutzig T: Ascorbinsäure (Vitamin C) und Kollagenbiosynthese. In: Biochemie. Kurzlehrbuch zum Gegenstandskatalog 1 mit Einarbeitung der wichtigsten Prüfungsfragen, 9. Auflage, Gustav Fischer Verlag, Lübeck 1997, 305-306; 394

Kübler W: Vitamin C. In: Handbuch der Kinderheilkunde. Band IV: StoffwechselErnährung-Verdauung. Hrsg. v. Opitz H und Schmid F; Springer-Verlag, Berlin u. a. $1965,405-423$

Lang K: Biochemie der Ernährung, 4. Auflage; Dr. Dietrich Steinkopff Verlag, Darmstadt $1979,580-601$

Lewis M (2002): Urbanisation and Child Health in Medieval and Post-Medieval England. An assessment of the morbidity and mortality of non-adult skeletons from the cemetries of two urban and two rural sites in England (AD 850-1859); BAR British Series $\underline{339}$, 16$19,26-28,36,37,49,54-56$

Löffler G: Basiswissen Biochemie mit Pathobiochemie, 4. Auflage; Springer Verlag, Berlin 2001

Löffler G, Petrides PE, Heinrich PC: Biochemie und Pathobiochemie, 8. Auflage; Springer Medizin Verlag, Heidelberg 2007 
Maat GJR: Scurvy in Dutch Whalers buried at Spitsbergen. In: Proceedings of the 4th

European Meeting of the Paleopathology Association in Middelburg-Antwerpen. Hrsg. v. Hanevelt GT, Perizonius WRK und Janssens PJ; Paleopathology Association, Utrecht 1982, 82-93

Maat GJR (2004): Scurvy in Adults and Youngsters: the Dutch Experience. A Review of the History and Pathology of a Disregarded Disease. Int J Osteoarcheol 14, 77-81

Maat GJR, Uytterschaut HT: Mikroskopic Observations on Scurvy in Dutch Whalers buried at Spitsbergen. In: Proceedings of the 5th European Meeting of the Paleopathology Association in Siena. Hrsg. v. Capecchi V und Rabino Massa E; Siena University Press, Siena 1984, 211-218

Mapp SJ, Coughlin PB (2006): Scurvy in an otherwise well young man. Medical J Aust Vol. 185, No. $6,331-332$

Marcsik A, Bereczki Z (2008): Site-report Bácsalmás-Óalmás (16th-17th centuries AD), private Zusendung durch Zsolt Bereczki

Mays S (2008): A Likely Case of Scurvy from Early Bronze Age Britain. Int J Osteoarcheol $\underline{18}, 178-187$

Melikian M, Waldron T (2003): An Examination of Skulls from Two British Sites for Possible Evidence of Scurvy. Int J Osteoarcheol 13, 207-212

Mimasaka S, Funayama M, Adachi N, Nata M, Morita M (2000): A fatal case of infantile Scurvy. Int J Legal Med 114, 122-124

Molnár E (2014): informelle Mitteilung mit Informationen zum Kinderschädel aus Kiszombor

Nathan DG, Oski FA (Hrsg.): Vitamin C (Ascorbic Acid) Deficiency. In: Hematology of Infancy and Childhood, 4. Auflage; W. B. Saunders Company Harcourt Brace Jovanovich, Philadelphia 1993, 445

Ortner DJ: Metabolic Disorders. In: Identification of Pathological Conditions in Human Skeletal Remains, 2. Auflage. Hrsg. v. Ortner DJ; Academic Press, San Diego 2003, 383-393

Ortner DJ, Putschar WGJ: Identification of Pathological Conditions in Human Skeletal Remains (Smithsonian Contributions to Anthropology, Nummer 28). Smithsonian Institution Press, City of Washington 1981, 176-201, 251-258, 270-283

Ortner DJ, Ericksen MF (1997): Bone Changes in the Human Skull Probably Resulting from Scurvy in Infancy and Childhood. Int J Osteoarcheol 7, 212-220 
Ortner DJ, Kimmerle E, Diez M (1999): Skeletal evidence of scurvy in archeological skeletal samples from Peru. Am J Phys Anthropol 108, 321-331

Ortner DJ, Butler W, Cafarella J, Milligan L (2001): Evidence of Probable Scurvy in Subadults From Archeological Sites in North America. Am J Phys Anthropol 114, 343-351

Platzer W: Bewegungsapparat. In: Taschenatlas der Anatomie für Studium und Praxis, Band 1. Hrsg. v. Kahle W, Leonhardt H, Platzer W; Georg Thieme Verlag, Stuttgart 1991, Seite 109, 113, 117, 189. 199, 201, 281, 283, 287, 289, 291, 297

Probst I: Vorlesungsmitschrift der Vorlesung Biochemie für Mediziner vom 16.04.2004

Salmenperä L (1984): Vitamin C nutrition during prolonged lactation: optimal in infants while marginal in some mothers. Am J Clin Nutr $\underline{40}$, 1050-1056

Salmenperä L, Siimes A, Perheentupa J: Vitamin C, Folate, and Biotin Nutrition During Prolonged Lactation. In: Vitamin C and Minerals in Pregnancy and Lactation. Hrsg. v. Heribert Berger; Nestlé Nutrition Workshop Series, Vol. 16, Vevey/Raven Press, New York 1988, 247-259

Schlomm T: Spuren pathologischer Prozesse an den menschlichen Schädeln aus dem frühmittelalterlichen Reihengräberfeld Barbing-Kreuzhof unter besonderer Berücksichtigung der unspezifischen und tuberkulösen Entzündungen im Bereich der Meningen und der pneumatischen Schädelräume. Med. Diss. Göttingen 2000

Schmidt MB: Atrophie und Hypertrophie des Knochens einschließlich der Osteosklerose

- 3. Die sekundäre hyperplastische Periostitis (P. MARIEs Ostéoarthropathie hypertrophiante pneumique, HÖGLERs Akropachie). In: Spezielle Pathologie des Skelets und seiner Teile. Die primären Knochengeschwülste. Hrsg. v. Herzog G; Springer-Verlag, Berlin 1944, 46-57

Schmidt-Schultz TH, Schultz M (2004): Bone Protects Proteins Over Thousands of Years: Extraction, Analysis, and Interpretation of Extracellular Matrix Proteins in Archeological Skeletal Remains. Am J Phys Anthropol 123, 30-39

Schultz M: Die mikroskopische Untersuchung prähistorischer Skeletfunde. Anwendung und Aussagemöglichkeiten der differentialdiagnostischen Untersuchung in der Paläopathologie. Tagungsberichte zum Paläopathologischen Symposium in Liestal (Baselland) 21.-23. Juni 1984, Band 1, Heft 006. Hrsg. vom Amt für Museen und Archäologie BL, Liestal und dem Anthropologischen Forschungsinstitut Aesch; Aesch 1986 
Schultz M: Spuren unspezifischer Entzündungen an prähistorischen und historischen Schädeln. Ein Beitrag zur Paläopathologie. Med. Habilitationsschrift, Göttingen 1987

Schultz M: Methoden der Licht- und Elektronenmikroskopie. In: Handbuch der vergleichenden Biologie des Menschen, Band 1. Hrsg. von Knussmann R; Fischer Verlag, Stuttgart 1988 a, 698-730

Schultz M: Paläopathologische Diagnostik. In: Handbuch der vergleichenden Biologie des Menschen, Band 1. Hrsg. von Knussmann R; Fischer Verlag, Stuttgart 1988 b, 480-496

Schultz M: Erkrankungen des Kindesalters bei der frühbronzezeitlichen Population vom Ikiztepe (Türkei). Vorbericht 1988. In: Gedenkschrift für Jürgen Driehaus. Hrsg. v. Andraschko F M und Teegen W-R; Verlag Philipp von Zabern, Mainz am Rhein 1990, $83-90$

Schultz M: Spuren unspezifischer Entzündung an prähistorischen und historischen Schädeln. Ein Beitrag zur Paläopathologie; in: Anthropologische Beiträge, Band 4. Hrsg. von Kaufmann B; Anthropologisches Forschungsinstitut Aesch und Anthropologische Gesellschaft Basel, Aesch 1993

Schultz M (1994): unpublizierte Tabellen zur Diagnostik von Skorbut und Anämie, Laborskript und persönliche Mitteilung

Schultz M (2001): Paleohistopathology of Bone: A New Approach to the Study of Ancient Diseases. Yearb Phys Anthropol 44, 106-147

Schultz M : Light Microscopic Analysis in Skeletal Paleopathology. In: Identification of Pathological Conditions In Human Skeletal Remains. Second Edition. Hrsg. v. Ortner D J; Academic Press, Amsterdam u. a. 2003, 73-108

Schultz M (2009): mündliche Mitteilung

Schultz M : Light microscopic analysis of macerated pathologically changed bones. In: Hard Tissue Histology: An Anthropological Perspective. Hrsg. v. Crowder C, Stout S; CRC-Press, Boca Raton/New York/London 2011, 253-296

Schultz M, Roberts CA (2002): Diagnosis of leprosy in skeletons from an English later Medieval hospital using histological analysis. In: The Past and Present of Leprosy. Archaeological, historical, palaeopathological and clinical approaches. Hrsg. v. Roberts CA, Lewis ME und Manchester K. BAR International Series 1054, 89-104 
Schultz M, Schmidt-Schultz TH: The Role of Deficiency Diseases in Infancy and Childhood of Bronze Age Populations. In: Paleonutrition and Food Practices in the Ancient Near East: Towards a Multidisciplinary Approach, History of the Ancient Near East / Monographs. Hrsg. v. Milano L; S.A.R.G.O.N, Padova 2013, o. S.

Schultz M, Schmidt-Schultz TH, Wolf M: Paläopathologische und paläobiochemische Untersuchungen an ausgewählten Skeletfunden aus der Nekropole von Bajkara. In: Der große Kurgan von Bajkara - Bolschoi kurgan Bajkara. Hrsg. v. Parzinger H, Zajbert V, Nagler A, Plesakov A; Von Zabern Verlag, Mainz 2003, 149-194

Schultz M, Timme U, Schmidt-Schultz TH (2007): Infancy and Childhood in the PreColumbian North American Southwest - Firsts Results of the Palaeopathological Investigation of the Skeletons from the Grasshopper Pueblo, Arizona. Int $\mathrm{J}$ Osteoarcheol 17, 369-379

Stloukal M, Hanáková H (1978): Die Länge der Längsknochen altslawischer Bevölkerungen unter besonderer Berücksichtigung der Wachstumsfragen. Homo 29, 53-69

Szentpéteri J: Archaeologische Denkmaeler der Awarenzeit in Mitteleuropa. In: Varia Archaeologica Hungarica XIII/1; Budapest 2002, 333 (Auszüge zur Verfügung gestellt durch Erika Molnár, Universität Szeged)

Szilvássy J: Altersdiagnose am Skelett. In: Handbuch der vergleichenden Biologie des Menschen, Band 1. Hrsg. v. Knussmann R; Fischer Verlag, Stuttgart 1988, 421-443

Templin O: Die Kinderskelete von Bettingen im Kanton Basel-Stadt (Schweiz). Eine paläopathologische Untersuchung. Med. Diss. Göttingen 1993

Ubelaker DH: Human Skeletal Remains: Excavation, Analysis, Interpretation. Aldine Publishing Company, Chicago 1978

Vilter RW, Woolford RM und Spies TD (1946): Severe Scurvy. A clinical and hematologic Study. J Lab Clin Med Vol. 31 No.6, 609-630

Von Hagens G (1979): Impregnation of soft biological specimens with thermostetting resins and elastomers. Anat Rec 194, 247-255

Wapler U, Crubézy E, Schultz M (2004): Is Cribra Orbitalia Synonymous With Anemia? Analysis and Interpretation of Cranial Pathology in Sudan. Am J Phys Anthropol $\underline{123}$, 333-339

Weihmann U: Paläopathologische Untersuchungen an den Kinderskeleten der Mad'arovceKultur des frühbronzezeitlichen Gräberfeldes von Jelšovce, Slowakei. Med. Diss. Göttingen 2010 
Weinstein M, Babyn P, Zlotkin Stan (2001): An Orange a Day Keeps the Doctor Away: Scurvy in the Year 2000. Pediatrics, Vol. 108 No.3, 55

Weise Prinzo Z: Scurvy and its prevention and control in major emergencies, hrsg. v. d. World Health Organization, WHO/NHD/99.11, Geneva 1999

Zellweger H, Adolph WH: Vitamine und Vitaminkrankheiten. In: Handbuch der Inneren Medizin 6/2: Krankheiten aus äußeren physikalischen Ursachen. Ernährungskrankheiten. Vitamine und Vitaminkrankheiten, 4. Auflage. Hrsg. v. von Bergmann G, Frey W und Schwiegk H; Springer-Verlag, Berlin 1954, 747-760

Zuelzer WW, Hutaff L, Apt L (1949): Relationship of Anemia and Scurvy. Am J Dis Child 77, 128 
Anhang I: Beispiel eines Befundbogens für das Postcranium

Die anatomischen Zeichnungen stammen aus PLATZER 1991 (S. 109, 113, 117, 189. 199, 201).

\begin{tabular}{|c|c|}
\hline Objekt: Barbing & 05.11 .05 \\
\hline Sign.: Sh 1290 & Grab-Nr.: \\
\hline Alter: $4 y \pm 21 M$ & Geschlecht: $\quad Q=\sigma^{-1}$ \\
\hline Bemerkungen: Rippen affelatiert, & (Ha. Plunritis) \\
\hline
\end{tabular}
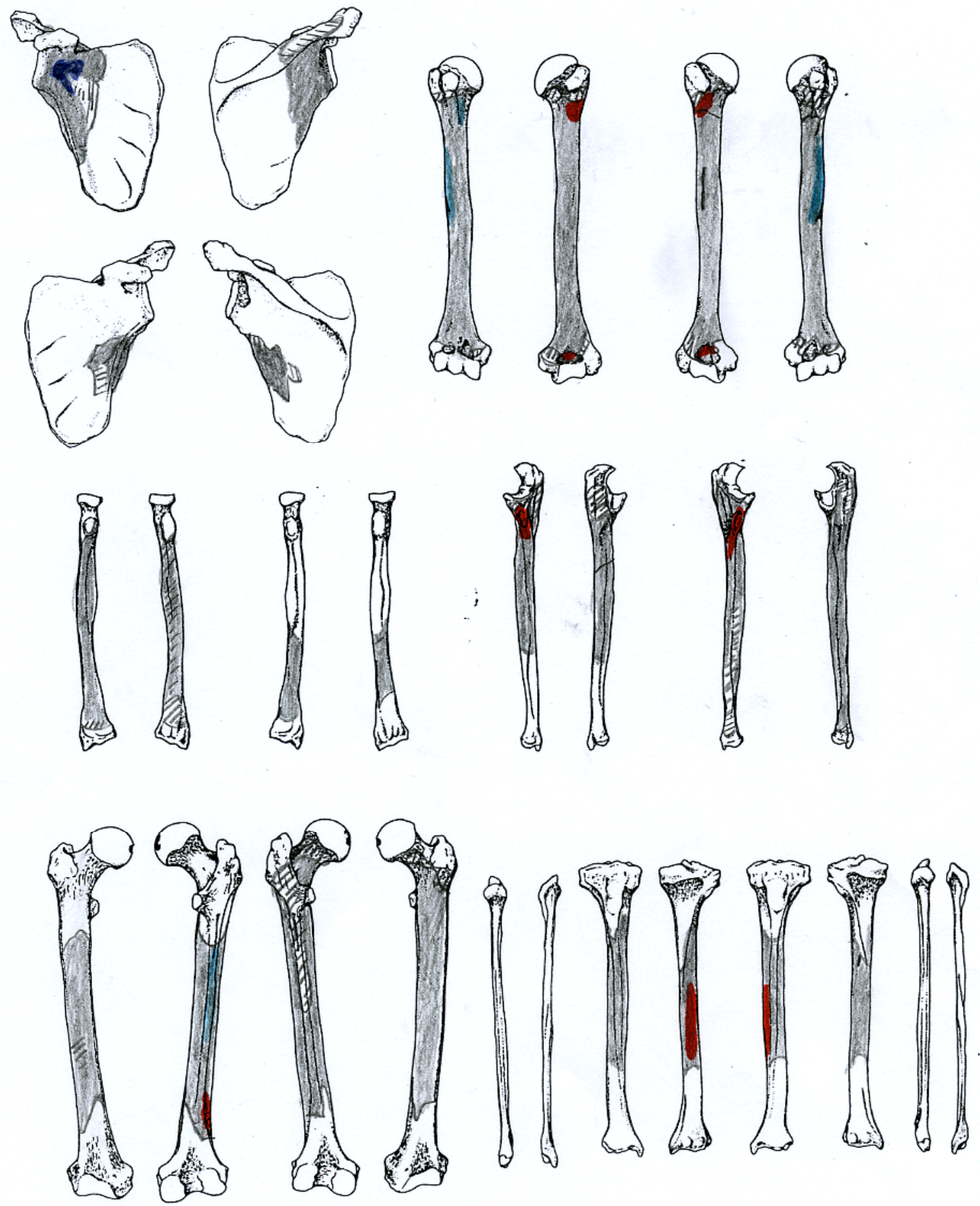


\section{Anhang II: Befundbogen für den Schädel}

Die anatomischen Zeichnungen aus PLATZER 1991 (S. 281, 283, 287, 289, 291, 297).

\begin{tabular}{|c|c|}
\hline Objekt: Barbing & Datum: 05.11 .05 \\
\hline She 1290 & Grab-Nr.: 32 \\
\hline $4 y \pm 21 M$ & Geschlecht: $\quad q=0^{-1}$ \\
\hline Bemerkungen: Va. Meningites of & Schaidelinnendrucberthónuas \\
\hline
\end{tabular}
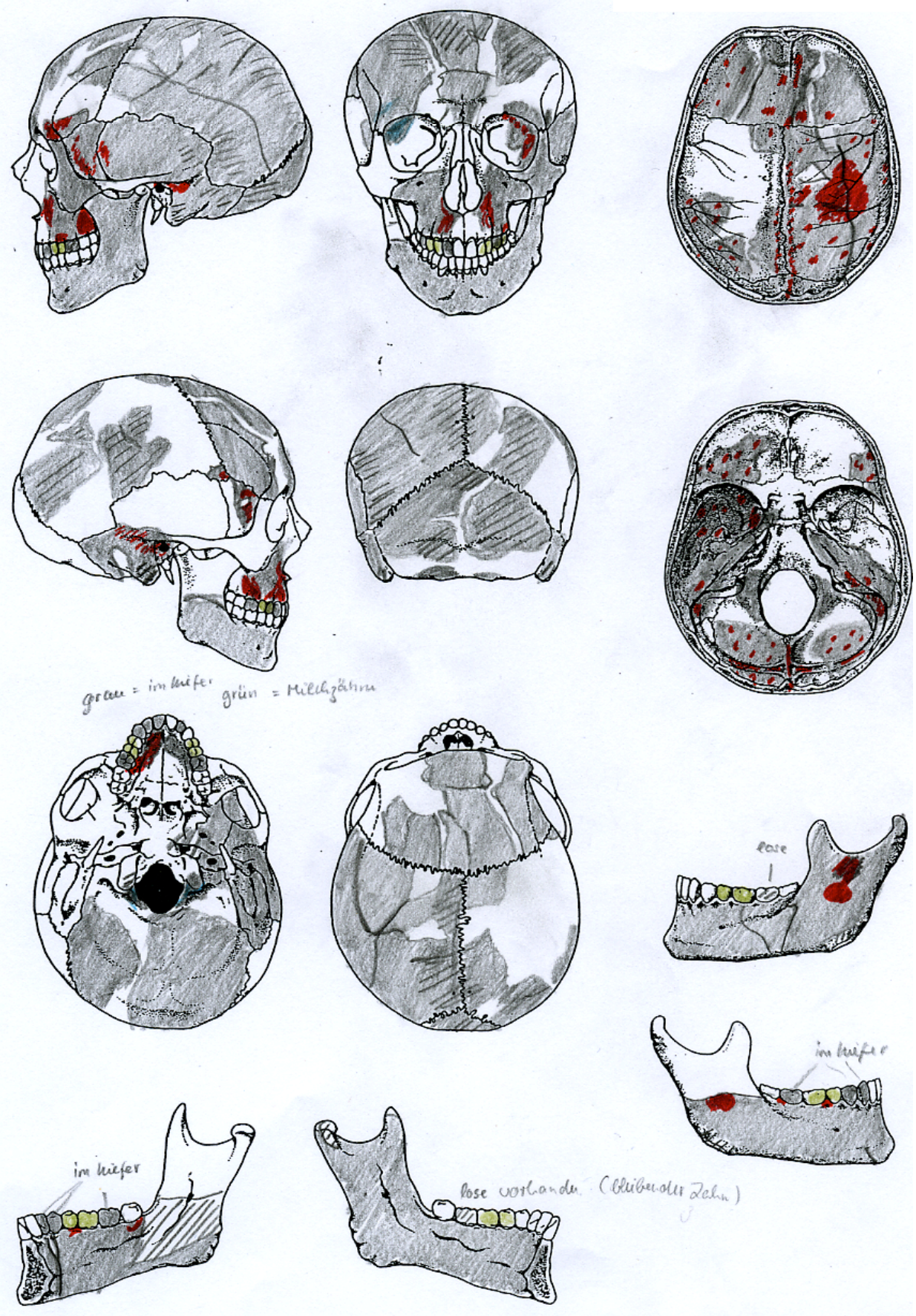


\section{Danksagung}

Mein besonderer Dank gilt Herrn Prof. Dr. Dr. M. Schultz für das mir bereitgestellte Thema dieser Arbeit und die unermüdliche und motivierende Betreuung während ihrer Fertigstellung.

Der Heinrich-Böll-Stiftung danke ich für die ideelle und materielle Förderung während meines Studiums und der Erstellung dieser Arbeit.

Der Arbeitsgruppe Paläopathologie Göttingen möchte ich für die anregende Zusammenarbeit danken, insbesondere Dr. J. Nováček, Dr. J. Gresky, Dr. K. Koel, Dr. I. Wanner und Dr. N. Roumelis.

Dr. S. Flohr danke ich außerdem für die Hilfe bei der Anfertigung der Röntgenbilder.

Herrn M. Brandt und Frau I. Hettwer-Steeger möchte ich für die Anfertigung neuer und Bereitstellung alter Knochendünnschliffpräparate danken.

Für die Bereitstellung von Literatur danke ich Dr. G. J. R. Maat (Abteilung für Anatomie, Universität Leiden, Niederlande).

Den ungarischen Anthropologen der Universität Szeged A. Marcsik, Z. Bereczki, G. Lovász und E. Molnár danke ich für die Auswahl und Bereitstellung der Skelete, die Betreuung während meines Ungarnaufenthaltes sowie für die Bereitstellung und Übersetzung von Literatur.

Für die Durchsicht der statistischen Teile danke ich Herrn Prof. Dr. Dr. R. Hilgers (Abteilung Medizinische Statistik der Universität Göttingen).

Dr. J. Klompen, Dr. L. Schorbach, K. Brosig, J. Klessen-Gödde und M. Gödde danke ich für praktische Hilfestellungen.

Für die Hilfe bei der Erstellung der Bildtafeln und Diagramme danke ich Dr. M. FischerGödde und M. Ahmedi (Institut für Planetologie der Universität Münster). 


\section{Lebenslauf}

Geboren wurde ich als Johanna Gödde am 06.01.1983 in Duisburg, wo ich bis zum achten Lebensjahr aufwuchs. Dann zog meine Familie nach Neukirchen-Vluyn am linken Niederrhein. Dort machte ich 2002 am Julius-Stursberg-Gymnasium das Abitur.

Im selben Jahr begann ich das Studium für Humanmedizin an der Georg-August-Universität in Göttingen. Die Ärztliche Vorprüfung legte ich 2004 ab.

Im Jahr 2006 heiratete ich und nahm den Namen Fischer-Gödde an, kurz darauf wurde mein Sohn geboren. Das Studium setzte ich ohne Unterbrechung fort und wechselte im Dezember 2007 an die Charité in Berlin, wo ich die letzten beiden Tertiale des Praktischen Jahrs absolvierte. 2008 wurde meine erste Tochter geboren.

Im Frühjahr 2009 bestand ich die Ärztliche Prüfung in Berlin. Zunächst arbeitete ich in der Abteilung für Innere Medizin am St. Josefs-Krankenhaus in Potsdam, bis ich 2010 nach Münster zog. Dann begann ich die Weiterbildung zur Fachärztin für Gynäkologie und Geburtshilfe am St. Franziskus-Hospital in Ahlen. Sie wurde anlässlich der Geburt meiner zweiten Tochter für ein Jahr unterbrochen, seit Anfang 2014 bin ich wieder in der Gynäkologie und Geburtshilfe tätig, ab dem 01.10.2015 im St. Franziskus-Hospital in Münster. 